\title{
IMPROVING THE DESIGN AND ANALYSIS OF SUPERCONDUCTING MAGNETS FOR PARTICLE ACCELERATORS
}
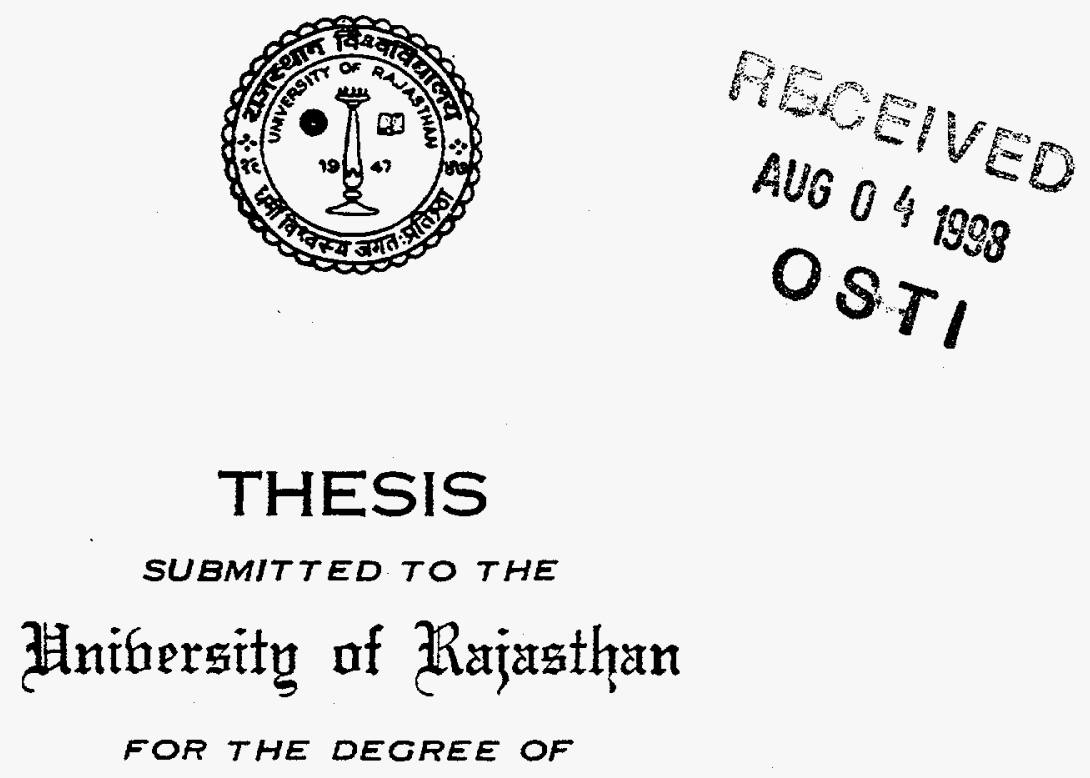

DOCTOR OF PHILOSOPHY

(PHYSICS)

By

\section{RAMESH CHANDRA GUPTA}

The University of Rajasthan and

Brookhaven National Laboratory

Under the Supervision of:

Prof. N. K. SHARMA

Emeritus Scientist (CSIR)

The University of Rajasthan, JAIPUR-302004, INDIA

and

Dr. G. H. MORGAN

Senior Scientist

Brookhaven National Laboratory, Upton, NY 11973, USA

NOVEMBER, 1996 


\section{THESIS}

DEDICATED

TO

MY FAMILY

In particular to my wife Manju who always encouraged me and took care of my obligations towards our children Ankur and Anjeli while I was busy working on evenings and weekends for several years. 


\section{DISCLAIMER}

This report was prepared as an account of work sponsored by an agency of the United States Government. Neither the United States Government nor any agency thereof, nor any of their employees, makes any warranty, express or implied, or assumes any legal liability or responsibility for the accuracy, completeness, or usefulness of any information, apparatus, product, or process disclosed, or represents that its use would not infringe privately owned rights. Reference herein to any specific commercial product, process, or service by trade name, trademark, manufacturer, or otherwise does not necessarily constitute or imply its endorsement, recommendation, or favoring by the United States Government or any agency thereof. The views and opinions of authors expressed herein do not necessarily state or reflect those of the United States Government or any agency thereof. 


\section{DISCLAIMER}

Portions of this document may be illegible in electronic image products. Images are produced from the best available original document. 


\section{SUPERVISOR'S CERTIFICATE}

This is to certify that the thesis entitled "IMPROVING THE DESIGN AND ANALYSIS OF SUPERCONDUCTING MAGNETS FOR PARTICLE ACCELERATORS" describes the original work done by Ramesh Chandra Gupta, under our supervision for the degree of Doctor of Philosophy of the University of Rajasthan, Jaipur, India. Mr. Ramesh Chandra Gupta has fulfilled the required formalities as per the university rules known to us.

(N.K. Sharma)

Emeritus Scientist (CSIR)

Department of Physics

University of Rajasthan

Jaipur 302004 INDIA

(G.H. Morgan)

Senior Scientist

Building 902

Magnet Division

Brookhaven National Laboratory

Upton, NY 11973 USA 


\section{ACKNOWLEDGMENTS}

It is my honor to use this opportunity to acknowledge Prof. N.K. Sharma for inspiring me to become a physicist and for providing a basic training in scientific methods since I was in high school. I thank my other supervisor Dr. G.H. Morgan for encouraging me to be thorough while allowing an independent style of working to be developed. I also thank Dr. Morgan for spending a large amount of time in critically examining the manuscript and for proposing several corrections to it, in particular to chapter 1.

The thesis was also reviewed by Dr. E. Willen who made several suggestions for writing it in a clearer and more professional manner. Discussions with Dr. S. Khan, Dr. G. Morgan and Dr. P. Thompson were very valuable in developing various techniques presented here. A significant amount of work on RHIC (Relativistic Heavy Ion Collider) magnets has been done in collaboration with Dr. Thompson and on SSC (Superconducting Super Collider) magnets with Dr. Morgan.

I also thank Dr. A. Jain and Dr. P. Wanderer for discussions on various topics related to magnetic measurement, testing and analysis. Most of the test data and several figures related to test results have been provided by Dr. Jain.

Advice on field quality improvements from the machine physics point of view was provided by Dr. H. Hahn, Dr. M. Harrison, Dr. S. Peggs and Dr. J. Wei. A close interaction between accelerator physicists and magnet builders was important in leading field quality improvements in the direction most critical to the performance of machine.

Discussions with the engineers in RHIC magnet production group, in particular with Mr. M. Anerella and Mr. E. Kelly, were important in incorporating various design features in magnet construction in an efficient manner. Several drawings in this manuscript are provided by the design office.

I would like to thank the University of Rajasthan, Jaipur, India for agreeing to accept the research work performed at the Brookhaven Nation Laboratory (BNL), USA, towards a Ph.D. degree after a long gap since completing my M.Sc. course work there. Finally I would like to thank the RHIC management (Drs. A. Greene, S. Ozaki, P. Wanderer and E. Willen) at BNL for their continuous support and encouragement towards the completion of this work. This work was supported by the U.S. Department of Energy under contract No. DE-AC02-98CH10886. 


\section{LIST OF PUBLICATIONS BY THE CANDIDATE}

The following is the list of external publications (i.e. the papers that are published as the conference proceedings, etc.) which are related to the research work described in this thesis. A copy of them is attached at the end of the thesis.

I. R.C. Gupta, G.H. Morgan, A Design for a High Field Combined Function Superferric Magnet, Proceedings of the 1985 Particle Accelerator Conference, Vancouver, Canada; IEEE Transaction on Nuclear Science, Volume NS-32, Oct 1985, pp. 3687-88 (1985).

II. R.C. Gupta, Modifications in the AUTOMESH and other POISSON Group Codes, Proceedings of the Workshop in Electromagnetic Field Computations, Schnectady, NY, pp. H38-H42 (1986).

III. R.C. Gupta, Improvements in the Mesh Generator of the POISSON Group Codes, Proceedings of the 1987 Particle Accelerator Conference, Washington DC, pp. 14491451 (1987).

IV. R.C. Gupta, G.H. Morgan, P.A. Thompson, A Single Layer Coil Superconducting Magnet for SSC, Proceedings of the 1987 Particle Accelerator Conference, Washington DC, pp. 1413-1415 (1987).

V. R.C. Gupta, G.H. Morgan, P.J. Wanderer, A Comparison of Calculations and Measurements of the Iron Saturation Characteristic of the SSC Design D Dipole Magnet, Proceedings of the 1987 Particle Accelerator Conference, Washington DC, pp. 14051407 (1987).

VI. R.C. Gupta, G.H. Morgan, Collarless, Close-in, Shaped Iron Aperture Designs for the SSC Dipole, Proceedings of the 1989 Particle Accelerator Conference, Chicago, pp. 500-502 (1989).

VII. R.C. Gupta, S.A. Kahn and G.H. Morgan, Coil and Iron Design for SSC $50 \mathrm{~mm}$ Magnet, Proceedings of the 1990 American Society of Mechanical Engineers (ASME) Winter Annual Meeting in Dallas (1990).

VIII. R.C. Gupta, S.A. Kahn and G.H. Morgan, A Comparison of Calculations and Measurements of the Field Harmonics as a Function of Current in the SSC Dipole Magnets, Proceedings of the 1991 IEEE Particle Accelerator Conference, San Francisco, pp. 42-44 (1991). 
IX. R.C. Gupta, et al., RHIC Insertion Magnets, Proceedings of the 1991 IEEE Particle Accelerator Conference, San Francisco, pp. 2239-2241 (1991).

X. R.C. Gupta, S.A. Kahn and G.H. Morgan, SSC $50 \mathrm{~mm}$ Dipole Cross section, Proceedings of the $3^{\text {rd }}$ International Industrial Symposium on Super Collider (IISSC), Atlanta, pp. 587-600 (1991).

XI. R.C. Gupta, Correcting Field Harmonics after Design in Superconducting Magnets, Proceedings of the $4^{\text {th }}$ International Industrial Symposium on Super Collider (IISSC), New Orleans, pp. 773-780 (1992).

XII. R. Gupta, et al., Large Aperture Quadrupoles for RHIC Interaction Regions, Proceedings of the 1993 Particle Accelerator Conference, Washington, D.C., pp. 2745-2747 (1993).

XIII. R.C. Gupta, A.K. Jain, Variation in $a_{1}$ saturation in SSC Collider Dipoles, Proceedings of the 1993 Particle Accelerator Conference, Washington, D.C., pp. 2778-2780 (1993).

XIV. R. Gupta, et al., Field Quality Improvements in Superconducting Magnets for RHIC, Proceedings of the 1994 European Particle Accelerator Conference, London, UK, pp. 2928-2930. (1994).

XV. R. Gupta, et al., Field Quality Control Through the Production Phase of RHIC Arc Dipoles, Proceedings of the 1995 International Particle Accelerator Conference, Dallas, Texas (1995).

XVI. R. Gupta, et al., Tuning Shims for High Field Quality in Superconducting Magnets, Proceedings of the Fourteenth International Conference on Magnet Technology (MT14), Tampere, Finland, June 11-16 (1995).

XVII. A. Jain, R. Gupta, et al., Skew Quadrupole in RHIC Dipole Magnets at High Fields, Proceedings of the Fourteenth International Conference on Magnet Technology (MT14), Tampere, Finland, June 11-16 (1995).

XVII. R. Gupta, Estimating and Adjusting Field Quality in Superconducting Accelerator Magnets, Proceedings of the LHC Collective Effects Workshop, Montreux, 1995; Submitted to the Particle Accelerators (1995).

XIX. R. Gupta, Field Quality in the Superconducting Magnets for Large Particle Accelerators, Proceedings of the 1996 Eruropean Particle Accelerator Conference at Sitges, Spain (1996). 


\section{CONTENTS}

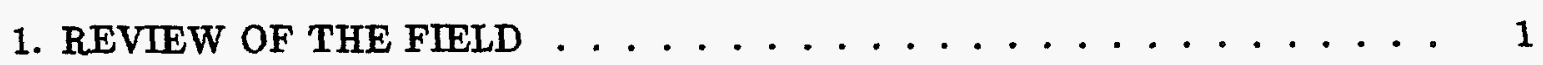

1.1. Introduction ...................... 1

1.2. Physics Potentials and Goals of RHIC . . . . . . . . . . . 2

1.3. Overview of RHIC Machine ................. 5

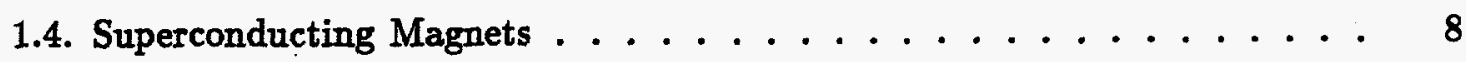

1.4.1. Introduction to the Magnet Geometry . . . . . . . . . . 8

1.4.2. Superconducting Cable ................ . 10

1.4.3. Cryogenic System . . . . . . . . . . . . . . . . . 13

1.4.4. Mechanical Design . . . . . . . . . . . . . . 14

1.4.5. Magnetic Design . . . . . . . . . . . . . 15

1.4.6. Magnet Construction . . . . . . . . . . . . . 16

1.4.7. Magnet Measurements . . . . . . . . . . . . . 17

1.5. Magnetic Field Analysis in Accelerator Magnets . . . . . . . . . . 22

1.5.1. Basic Electromagnetic Field Equations . . . . . . . . . . 22

1.5.2. Field Harmonic Definitions . . . . . . . . . . . 26

1.5.3. Analytic Expressions for Accelerator Magnets . . . . . . . . 31

1.5.3.1. Field and Vector Potential due to a Line Current . . . . . 31

1.5.3.2. Line Current in a Cylindrical Iron Cavity . . . . . . . . 36

1.5.3.3. Line Current in a Cylindrical Iron Shell . . . . . . . . . 40

1.5.3.4. Field and Harmonics due to Current Blocks in Air . . . . 4 44

1.5.3.5. Field Harmonics due to Current Blocks in a Cylindrical Iron Shell . . . . . . . . . . . . . . 47

1.5.3.6. $\operatorname{COS}(m \theta)$ Current Distribution for Ideal Fields . . . . . . . . 48

1.5.3.7. $\operatorname{Cos}(m \theta)$ Current Distribution in a Cylindrical Iron Shell . . . . . . . . . . . . . . . . 54

1.5.3.8. Intersecting Circles with a Constant Current Density for Ideal Fields . . . . . . . . . . . . . 
1.5.4. Complex Variable Method in 2-d Magnetic Field Calculations ...................... 60

1.5.4.1. Field due to an array of Line Currents . . . . . . . . . . 62

1.5.4.2. Beth's Current Sheet Theorem . . . . . . . . . . . . 63

1.5.4.3. Example - $\operatorname{Cos}(m \theta)$ current distribution . . . . . . 65

1.6. Methods Investigated for Improving Field Quality . . . . . . . . . 66

1.6.1. Improvements in the Computational and Analysis Methods ....................... 66

1.6.2. Field Quality Improvements through Yoke Design . . . . . . . . . 67

1.6.3. Field Quality Improvements through Coil Design . . . . . . . . . 67

1.6.4. Field Quality Improvements after Construction . . . . . . . . . . 68

1.6.5. Optimized Cross section Designs . . . . . . . . . . . . . . 69

2. IMPROVEMENTS IN THE COMPUTATIONAL AND ANALYSIS

METHODS ....................... 70

2.1. Introduction ..................... 70

2.2. Computer Aided Cross-section Measurement and Analysis . . . . . . 71

2.3. IMPROVEMENTS IN THE POISSON GROUP CODES . . . . . . . . 78

2.3.1. Upgraded AUTOMESH - Input Method No. 1 . . . . . . . . . . 79

2.3.2. Upgraded AUTOMESH - Input Method No. $2 \ldots \ldots$

2.3.3. Upgraded AUTOMESH - Input Method No. $3 \ldots$. . . . . . . 88

2.4. Conclusions on the Improvements in the Computational and

Analysis Methods . . . . . . . . . . . . . . . . 91

3. FIELD QUALITY IMPROVEMENTS THROUGH YOKE DESIGN $\ldots$. . . . . 92

3.1. Introduction ...................... 93

3.2. Reduction in Saturation Induced Allowed Harmonics . . . . . . . . . 102

3.2.1. Varying the yoke inner radius . . . . . . . . . . . 103

3.2.2. Varying the yoke outer radius . . . . . . . . . . . . . . . 111

3.2.3. Varying the location of the helium bypass hole. in the yoke ............................ 116 
3.2.4. Additional Saturation suppressor holes in the iron yoke . . . . . 123

3.2.5. Yoke-yoke alignment keys . . . . . . . . . . . . . . . . 129

3.2.6. Yoke collaring keys . . . . . . . . . . . . . . . . . 132

3.2.7. Tooth at the midplane of the yoke aperture . . . . . . . . . . 135

3.2.8. Cutout or Bump in the iron aperture . . . . . . . . . . . . 139

3.2.9. Elliptical iron aperture . . . . . . . . . . . . . . . 145

3.2.10. Two radius aperture yoke . . . . . . . . . . . . . . . 149

3.3. Saturation Induced Allowed Harmonics in RHIC Arc Dipoles . . . . . 153

3.4. Reduction in the Saturation-induced Non-allowed Harmonics . . . . . 169

3.4.1. $b_{1}$ saturation - Cross talk . . . . . . . . . . . . . . 170

3.4.2. $a_{1}$ saturation - Cryostat and other sources . . . . . . . . 176

3.5. $a_{1}$ Saturation in SSC Dipole Magnets . . . . . . . . . . . . 182

3.5.1. $\delta a_{1}$ variation with axial position within a magnet . . . . . . . 182

3.5.2. Magnet to magnet variations in the integral $\delta a_{1} \ldots \ldots$. . . . . 185

3.5.3. Compensation of the saturation induced $a_{1}$ in SSC magnets . . . . . . . . . . . . . . 188

3.6. $a_{1}$ Saturation in RHIC Dipole Magnets . . . . . . . . . . . . . 190

3.6.1. Magnet to magnet variation in $a_{1}$ saturation . . . . . . . . 190

3.6.2. Reduction in $a_{1}$ saturation in RHIC dipoles . . . . . . . . . . . . 194

3.7. Conclusions on the Field Quality Improvements through Yoke

Design ... . . . . . . . . . . . . . . 200

4. FIELD QUALITY IMPROVEMENTS THROUGH COIL DESIGN . . . . . . 206

4.1. Introduction ...................... 207

4.2. Sources of Harmonics Allowed by the Magnet Geometry . . . . . . . 209

4.3. Reduction in the Allowed Harmonics through Wedges . . . . . . . 210

4.4. Reduction in the Allowed Harmonics in RHIC Arc Dipoles by

Changing the Midplane Gap . . . . . . . . . . . . . . . 212

4.5. Reduction in $b_{3}$ in RHIC Quadrupoles with Midplane Gaps . . . . . . . . 214 
4.6. Coil Cross-section Iterations without Changing Wedges . . . . . . . . . 216

4.7. Conclusions on the Field Quality Improvements through Coil

Design

5. FIELD QUALITY IMPROVEMENTS AFTER CONSTRUCTION . . . . . . 220

5.1. Introduction ..................... 221

5.2. Tuning Shims in Magnet Body for Extra High Field Quality . . . . . 222

5.2.1. Tuning Shims in the RHIC Interaction Region

Quadrupoles .................. 222

5.2.2. Tuning Shim and the Magnet Design . . . . . . . . . . . . . 224

5.2.3. Procedure for Implementing the Tuning Shim Correction . . . . . 227

5.2.4. Calculations for Tuning Shim Corrections . . . . . . . . . . 229

5.2.4.1. Approximate Analytic Expressions for Low Field

Estimate ................. 230

5.2.4.2. Numerical Calculations for Low Field Correction . . . . . 233

5.2.4.3. Numerical Calculations for High Field Correction . . . . . . . 243

5.2.5. Symmetries in the Harmonics Generated by Tuning

Shims .................... . . 249

5.2.6. Independent and Coupled Changes in Harmonics Correc-

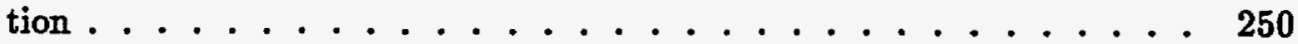

5.2.7. Comparison with the Measurements . . . . . . . . . . 250

5.2.8. Tuning Shim Correction Vs. External Correctors . . . . . . . . 255

5.3. Tuning Yoke Length at Magnet Ends for Field Correction . . . . . . . . . 258

5.3.1. Yoke Length for Integral $a_{1}$ Correction . . . . . . . . . . . . 260

5.3.2. Yoke Length for Integral Transfer Function Correction . . . . . . 262

5.4. Conclusions on the Field Quality Improvements after Construc-

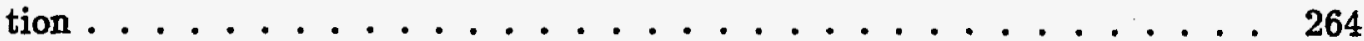

6. OPTMMIZED CROSS SECTION DESIGNS . . . . . . . . . . 265

6.1. Introduction . . . . . . . . . . . . . . . 265

6.2. SSC $50 \mathrm{~mm}$ Aperture Collider Dipole Magnet Cross-section . . . . . . . 266 
6.2.1. Coil Design . . . . . . . . . . . . . . . 266

6.2.2. Low Field Harmonics . . . . . . . . . . . . . . 268

6.2.3. Iron Yoke Design . . . . . . . . . . . . . . 270

6.2.4. Expected Quench Performance . . . . . . . . . . . . 278

6.2.5. Effect of Manufacturing Errors on the Allowed Harmonics ..................... 280

6.2.6. Stored Energy and Inductance Calculations . . . . . . . . . . 282

6.2.7. Lorentz Force Calculations . . . . . . . . . . . . . . 283

6.2.8. Summary of the Design . . . . . . . . . . . . . . . 284

6.3. RHIC $130 \mathrm{~mm}$ Aperture Interaction Region Quadrupole Crosssection .................... . . 286

6.3.1. Basic Construction ... . . . . . . . . . . 286

6.3.2. Coil Cross Section . . . . . . . . . . . . . . . 287

6.3.3. Yoke Cross Section . . . . . . . . . . . . . . 293

6.3.4. Expected Quench Performance . . . . . . . . . . . . 300

6.4. Conclusions on the Optimized Cross Section Designs . . . . . . . . . 301

7. CONCLUSIONS AND SUGGESTIONS FOR FUTURE WORK . . . . . . 302

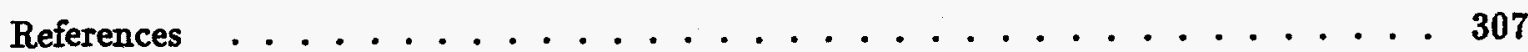

List of Figures . . . . . . . . . . . . . . . . . . 322

List of Tables . . . . . . . . . . . . . . . . . . . 328

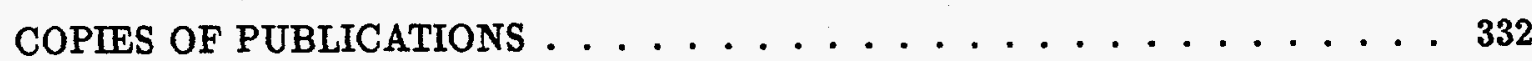




\section{Chapter 1. REVIEW OF THE FIELD}

\subsection{Introduction}

High energy particle accelerators are now the primary means of discovering the basic building blocks of matter and understanding the forces between them. In order to minimize the cost of building these machines, superconducting magnets are used in essentially all present day high energy proton and heavy ion colliders. The cost of superconducting magnets is typically in the range of $20-30 \%$ of the total cost of building such machines. The circulating particle beam goes through these magnets a large number of times (over hundreds of millions). The luminosity performance and life time of the beam in these machines depends significantly on the field quality in these magnets. Therefore, even a small error in the magnetic field shape may create a large cumulative effect in the beam trajectory to throw the particles out of the magnet aperture. The superconducting accelerator magnets must, therefore, be designed and constructed so that these errors are small. In this thesis the research and development work will be described which has resulted in a significant improvements in the field quality of the superconducting magnets for the Relativistic Heavy Ion Collider (RHIC) [140]. The design and the field quality improvements in the prototype of the main collider dipole magnet for the Superconducting Super Collider (SSC) [143] will also be presented.

RHIC will accelerate and collide two counter rotating beams of heavy ions up to 100 $\mathrm{GeV} / \mathrm{u}$ and protons up to $250 \mathrm{GeV}$. It is expected [112] that RHIC will create a hot, dense quark-gluon plasma and the conditions which, according to the Big Bang theory, existed in the early universe. 


\subsection{Physics Potentials and Goals of RHIC}

The physics potentials and goals of RHIC have been extensively discussed in a number of places $[11,112,125,136,153,173]$. The following summary is based on these and some other reports.

Two high energy heavy ion beams will collide at RHIC. This will create nuclear matter whose temperature and density will be much higher than ever produced in a controlled environment. The baryon density will be over ten times that in normal matter. Baryons are strongly interacting particles which are made of 2 quarks (in mesons) or 3 quarks (in hadrons). According to present theories, the total number of quarks is six, namely, up ( $u$ ), down $(d)$, strange $(s)$, charm $(c)$, bottom $(b)$ and top $(t)$. The interaction between the quarks is carried by gluons and the theory describing these interactions [145] is called Quantum Chromodynamics (QCD). According to some calculations based on QCD, a quark-gluon plasma (QGP) should be observed at RHIC. In a quark-gluon plasma, the usually confined interacting quarks and gluons move around freely. Such conditions are predicted when the baryon density is about ten times that in the normal conditions or when the temperature is about $2 \times 10^{12}$ degree kelvin $(\sim 150 \mathrm{MeV})$.

The protons and neutrons (of which stable nuclei are made) are dominantly made of up and down quarks; contributions from gluons and quark-anti-quark pairs from the fermi sea are also present. However, according to the theory strange quarks are much easier to create in a quark-gluon plasma and the particles/resonances based on them are expected to be made in abundance. In normal conditions the interaction between quarks is strong and attractive. Moreover, the attractive force grows rapidly in strength as the separation between the quarks increases. This leads to the confinement theories according to which it is impossible to observe a quark in a free state. However, in extreme thermodynamic conditions of temperature and density, a phase transition is predicted. In this new thermodynamic phase of the quark gluon plasma (QGP), the conditions will be such that the attractive forces which bind quark-anti-quark pairs in mesons and three quarks in hadrons will be overcome. This will lead to a deconfined state of high-energy-density matter (plasma) where the quarks will move freely. The lifetime of this phase should be long enough $\left(\sim 10^{-22} \mathrm{sec}\right)$ to be observed in RHIC experiments. The primary goals of RHIC experiments [112] will be to confirm these predictions and to study the properties of this new state of matter. A detailed investigation of this state, the formation of it and the other states to follow from it [153] will also be interesting. A phase diagram [112] of nuclear matter is 


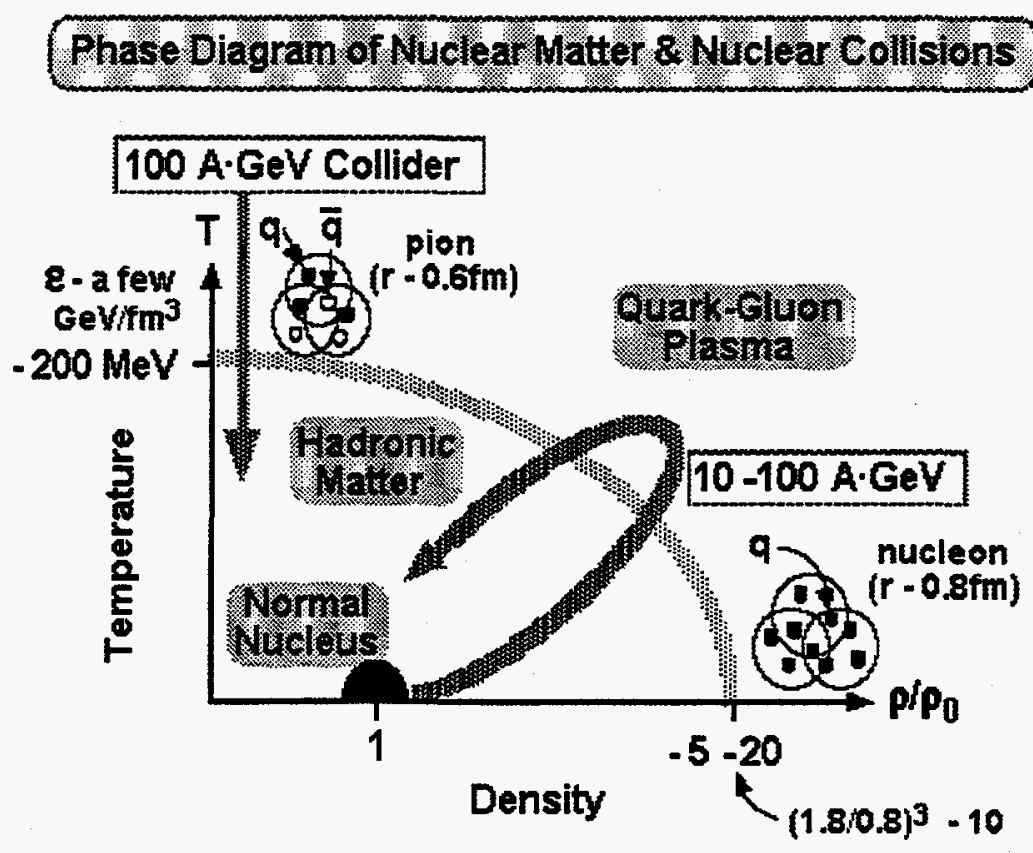

Figure 1.2.1: Phase Diagram

shown in Fig. 1.2.1, where temperature is plotted vs. net baryon density for an extended volume of nuclear matter in thermal equilibrium.

According to QCD theories, the lowest energy state is not the vacuum with no particles (and hence no interaction) in it. The lowest energy state is actually the vacuum filled with quark-anti-quark pairs (fermi sea). The relative ratio (mix) of densities between various type of quark-anti-quark pairs is determined by QCD. A preference of one type of mix over another is attributed to the spontaneous breaking of chiral symmetry in the normal phase. However, in the new thermodynamic phase of a quark gluon plasma chiral symmetry is restored with no preference given to one mix of quark-anti-quark pairs over another. Moreover, according to theories, a large number of otherwise rare strange quarks and strange anti-quarks will be generated. These quarks will eventually decay but the process is slow (on a nuclear time scale) and the secondary particles generated from them will signal the existence of such a situation to RHIC detectors. 
It is expected that a quark-gluon plasma will be created in RHIC. This state has not been created before in a laboratory environment and perhaps does not exist any where in the world today. According to theories this state existed for only 5-10 microseconds after the Big Bang and was crucial to determining the structure of the universe as we see today. This state may give a new insight to our understanding of the world and may even create phenomena which are not expected from the present theories. The use of large heavy ions in colliders allows this state to persist for a relatively long time since the lifetime is expected to be at least (or even much greater than) the radius of the nuclei divided by the velocity of light. In this respect RHIC (Relativistic Heavy Ion Collider) is in a unique situation in which the beam (heavy ions including gold with a radius of 7 fermi), the machine and the detectors are all designed with this new phase in mind.

A number of detectors and experiments are designed to study the various aspects of the quark-gluon plasma and other physics expected from RHIC. In addition to observing the strongly interacting particles, the detectors will also use the leptons and photons to obtain a signal from the quark gluon plasma. RHIC will also be studying spin physics based on $250 \mathrm{GeV}$ on $250 \mathrm{GeV}$ collisions between polarized protons. There are plans for installing four detectors in four interaction regions. These detectors are STAR (Solenoidal Tracker At RHIC) [146], PHENIX [134], PHOBOS [112] and BRAHMS (Broad Range Hadron Measuring Spectrometer) [9]. 


\subsection{Overview of RHIC Machine}

A major nuclear physics facility, the Relativistic Heavy Ion Collider (RHIC), is being built at the Brookhaven National Laboratory. A brief description and its present status is described in recent papers [84,85]. A complete description is given in the RHIC design manual [140]. The following description of RHIC is primarily based on these reports.

RHIC will accelerate heavy ions (for example gold) to a nominal maximum energy of $100 \mathrm{GeV} / \mathrm{u}$ and protons to $250 \mathrm{GeV}$. A brief list of the major parameters [140] of the RHIC machine is given in Table 1.3.1. RHIC has two rings with the orbit of the two counter rotating beams switching alternatively between the inner and outer rings at the six beam crossing points. The circumference of the machine will be $3.834 \mathrm{~km}$ and it has six interaction regions for experiments where the beam can be collided head-on or at a small angle. Each interaction region has a free space of \pm 9 meter on the either side of the crossing point for detectors before a magnet of the RHIC lattice appears. The design storage time for gold ions at the maximum energy is 10 hours. The design luminosity for gold ions at the top energy is $2 \times 10^{26} \mathrm{~cm}^{-2} \mathrm{sec}^{-1}$. The luminosity will be lower at lower energies and the storage time may well be decreased.

The injection energy of beams to $\mathrm{RHIC}$ will be in the range of $10.8 \mathrm{GeV} / \mathrm{u}$ (for gold ions) to $28.3 \mathrm{GeV}$ (for protons). In order for this beam to reach RHIC a series of beam transfer lines and existing accelerators will be used. The heavy ions will come from the existing Tandem Van de Graff which uses a pulsed sputter ion source. The gold beam leaving the Tandem Van de Graff facility will have a charge state of +14 and a kinetic energy of $1 \mathrm{MeV} / \mathrm{u}$. This beam will be transported through a long $(\sim 550 \mathrm{~m})$ transfer line and injected into the Booster. The Booster (circumference $201.781 \mathrm{~m}$ ) will accelerate this beam to $72 \mathrm{MeV} / \mathrm{u}$ energy after which the beam will pass through a stripping target for removing more electrons from the nuclei. The gold ions having a charge state of +77 will be selected for further transmission. This beam will be injected into the Alternating Gradient Synchrotron (AGS, circumference $807.125 \mathrm{~m}$ ) through a beam transport line. The AGS will accelerate this beam to an energy of $10.8 \mathrm{GeV} / \mathrm{u}$. The extracted beam from the AGS will go through another target and the nuclei will be completely stripped of all electrons to reach the final charge state of +79 . The final beam transport line from AGS to RHIC will split into two lines to inject beams into the two RHIC rings. The protons will come from the existing linear accelerator (LINAC) facility. A $200 \mathrm{Mev}$ polarized (low intensity) or unpolarized (high intensity) $\mathrm{H}^{-}$beam will be injected into the Booster. The stripper 
Table 1.3.1: Major Parameters for RHIC

\begin{tabular}{|c|c|}
\hline Kinetic Energy, Injection-Top (each beam), Au & $10.8-100 \mathrm{GeV} / \mathrm{u}$ \\
\hline protons & $28.3-250 \mathrm{GeV}$ \\
\hline Luminosity, $\mathrm{Au}-\mathrm{Au}$ at $100 \mathrm{GeV} / \mathrm{u} \& 10 \mathrm{~h}$ av. & $\sim 2 \times 10^{26} \mathrm{~cm}^{-2} \mathrm{sec}^{-1}$ \\
\hline No. of bunches/ring & 60 \\
\hline No. of Au-ions/bunch & $1 \times 10^{9}$ \\
\hline Storage time for Au at $\gamma>30$ & $\sim 10$ hours \\
\hline Diamond length & $180 \mathrm{~mm} \mathrm{rms}$ \\
\hline Circumference, $4-3 / 4 C_{A G S}$ & $3833.845 \mathrm{~m}$ \\
\hline Beam separation in arcs & $900 \mathrm{~mm}$ \\
\hline Number of crossing points & 6 \\
\hline Free space at crossing point & $\pm 9 \mathrm{~m}$ \\
\hline Beta at crossing, horizontal/vertical & $10 \mathrm{~m}$ \\
\hline low-beta insertion & $1 \mathrm{~m}$ \\
\hline Crossing angle, nominal (maximum) & $0(<1.7) \mathrm{mrad}$ \\
\hline Betatron tune, horizontal/vertical & $28.19 / 29.18$ \\
\hline Transition Energy, $\gamma_{T}$ & 22.89 \\
\hline Magnetic Rigidity, B $\rho$ at injection & $97.5 \mathrm{~T} \cdot \mathrm{m}$ \\
\hline Magnetic Rigidity, B $\rho$ at top energy & $839.5 \mathrm{~T} \cdot \mathrm{m}$ \\
\hline Bending radius, arc dipole & $242.781 \mathrm{~m}$ \\
\hline No. of dipoles (192/ring +12 common) & 396 \\
\hline No. of quadrupoles (276 arc +216 insertion) & 492 \\
\hline Dipole field at $100 \mathrm{GeV} / \mathrm{u}, \mathrm{Au}$ & $3.46 \mathrm{~T}$ \\
\hline Arc dipole length, effective & $9.45 \mathrm{~m}$ \\
\hline Arc Dipole length, physical & $9.73 \mathrm{~m}$ \\
\hline Dipole current & $5.1 \mathrm{kA}$ \\
\hline Arc quadrupole gradient & $\sim 71 \mathrm{~T} / \mathrm{m}$ \\
\hline Arc quadrupole length, effective & $1.11 \mathrm{~m}$ \\
\hline Coil i.d. arc magnets & $80 \mathrm{~mm}$ \\
\hline Beam tube i.d. & $69 \mathrm{~mm}$ \\
\hline Operating temperature, Helium refrigerant & $<4.6 \mathrm{~K}$ \\
\hline Refrigeration capacity at $4 \mathrm{~K}$ & $24.8 \mathrm{~kW}$ \\
\hline Cooldown time, entire system & $\sim 7$ days \\
\hline Vacuum, warm beam tube sections & $\sim 7 \times 10^{-10}$ mbar \\
\hline Filling time (each ring) & $<1$ min \\
\hline Injection kicker strength (95 nsec) & $\sim 0.18 \mathrm{~T} \cdot \mathrm{m}$ \\
\hline Beam stored energy & $\sim 200 \mathrm{~kJ}$ \\
\hline If voltage, $h=360$ & $600 \mathrm{kV}$ \\
\hline If voltage, $h=2520$ & $6 \mathrm{MV}$ \\
\hline Acceleration time & $\sim 1 \min$ \\
\hline
\end{tabular}


inside the Booster (to facilitate charge exchange injection) will convert $H^{-}$ions to protons which will then be accelerated to an energy of $1.5 \mathrm{GeV}$. From this point onward the beam will follow the same path as described above for gold. The AGS will accelerate protons to an energy of $28.3 \mathrm{GeV}$ which will then be injected into two RHIC rings.

As per the present design, each RHIC ring will have 60 bunches injected in 20 cycles with three bunches from the AGS in each cycle. It will take about 1 minute to fill the rings. The separation between bunches will be $64 \mathrm{~m}$ (or $213 \mathrm{nsec}$ in time). The arc magnet power supply will be ramped at a rate of $70 \mathrm{~A} / \mathrm{sec}$ from a current of $\sim 0.57 \mathrm{kA}(0.4 \mathrm{~T})$ at injection to a maximum current of $\sim 5.1 \mathrm{kA}(3.46 \mathrm{~T})$ at the top energy. Therefore, it takes about one minute to accelerate the beam from injection energy to top energy. At the time of injection the radio-frequency ( $\mathrm{rf}$ ) system, which operates at $\sim 28.2 \mathrm{MHz}$ will have a voltage of approximately $200 \mathrm{kV}$. During acceleration this voltage will be adiabaticlly raised to $\sim 300 \mathrm{kV}$. The storage system uses a different rf system, one that operates at $197 \mathrm{MHz}$ frequency.

The RHIC lattice is designed to be identical for the two counter rotating beam. It is composed of six circular arcs and six straight sections. The lattice has three super periods with each super period consisted of inner arc, insertion, outer arc and insertion. Each arc consists of 11 FODO cells (22 dipoles, 11 focusing and 11 defocusing quadrupoles) and each insertion has twelve dipoles and 18 quadrupoles. The nominal horizontal and vertical operating tune of the machine are $\nu_{H}=28.19$ and $\nu_{V}=29.18$. The transition energy $\left(\gamma_{T}\right)$ is 22.89. During injection and acceleration the size of the beam at the crossing point will be large $\left(\beta^{*}=10\right)$. The beam size will be gradually reduced $\left(\beta^{*}=1\right)$ for higher luminosity. At injection the performance of the machine will be dominated by the field errors in the 80 mm aperture arc dipole magnets and at storage the luminosity performance of RHIC will be dominated by the field errors in the $130 \mathrm{~mm}$ aperture insertion quadrupole magnets. 


\subsection{Superconducting Magnets}

In this section a brief description of the cosine theta superconducting magnets is given. A more complete description can be found elsewhere $[86,128,144,175,177,179]$. Type II superconductors, which allow penetration of magnetic field lines, are used in all superconducting magnets. They can retain their superconducting state up to a field of $\sim 20$ tesla and are being currently used in designing magnets in the range of 3 to 15 tesla. Type $I$ superconductors, which were discovered first and which completely exclude field lines, lose superconductivity at a much lower field of 0.18 tesla or below and therefore are not suitable for such applications. Despite the promising prospects of high temperature superconductors [93] they are not yet suitable for accelerator magnets.

In superconducting magnets which are mostly intended for operation above 3 tesla maximum field, the field shape is primarily determined by the superconducting coils. Superferric magnets are a hybrid version of superconducting and iron dominated room temperature magnets. In superferric magnets though, superconducting coils are used but the iron plays an important role in shaping the field. The research work described here is limited to superconducting magnets.

The two main design goals for superconducting magnets are to obtain (a) a good quench performance (a quench implies the loss of superconductivity in the cable) and (b) a good field quality. After an overall introduction to the magnet geometry, the superconducting cable, the cryogenic system, the magnetic design, the mechanical design, the magnet construction and the magnet measurements will be briefly described.

\subsubsection{Introduction to the Magnet Geometry}

Superconducting accelerator magnets are basically long cylindrical magnets whose cross section is mostly uniform along the length except at the two ends. The cross section of the $80 \mathrm{~mm}$ aperture RHIC arc dipole magnet inside the cryostat is shown in Fig. 1.4.1. The overall size of superconducting magnets is usually much larger then the aperture required for the particle beam. As compared to the $80 \mathrm{~mm}$ coil inner diameter and $69 \mathrm{~mm}$ beam tube inner diameter, the outside diameter of the RHIC cryostat is $610 \mathrm{~mm}$. Similarly, in the SSC main dipole magnet design, for a coil aperture of $50 \mathrm{~mm}$, the cryostat outside diameter was $660 \mathrm{~mm}$. 


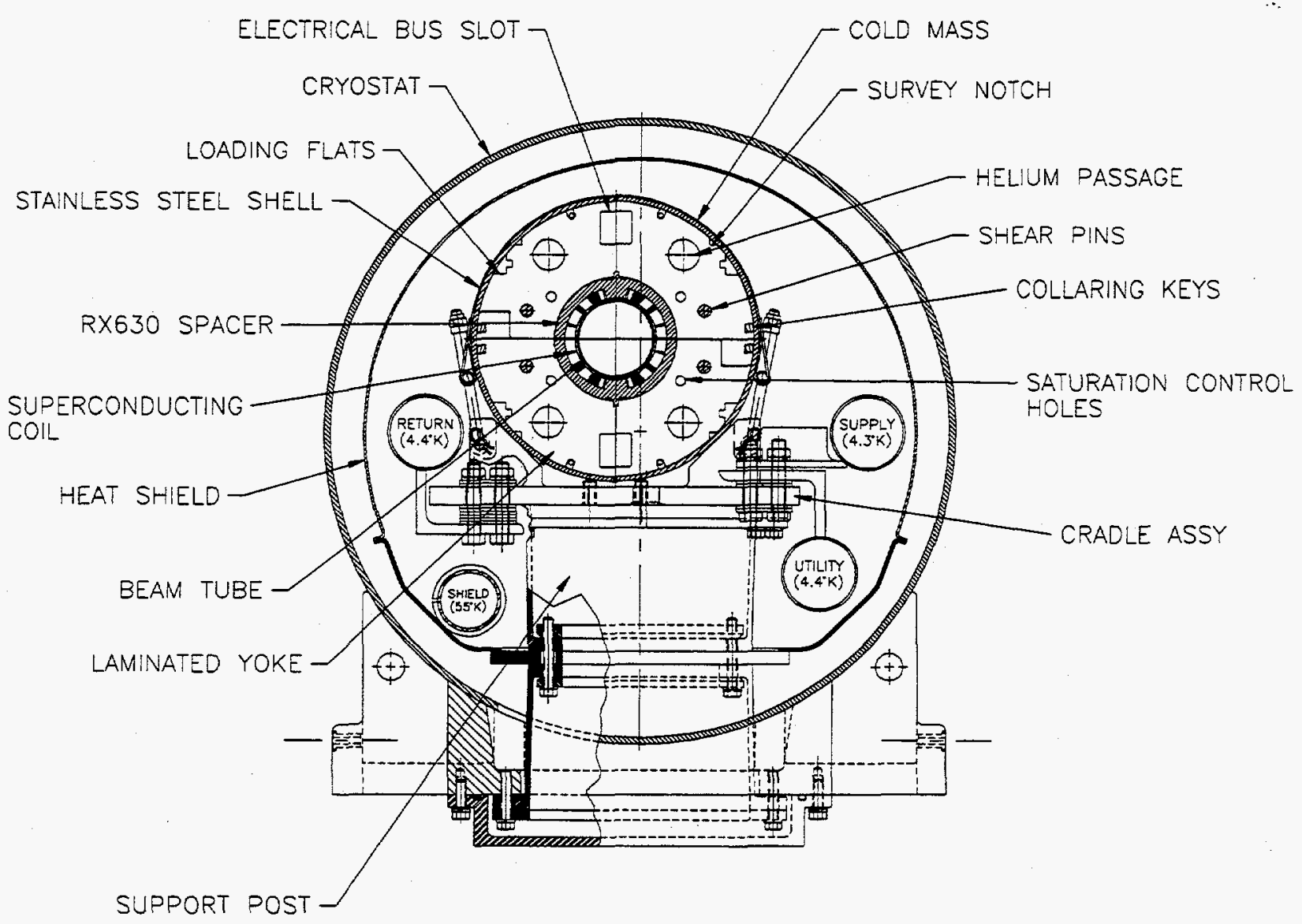

SK/S00608ML

Figure 1.4.1: A cross section of the $80 \mathrm{~mm}$ aperture RHIC arc dipole magnet with the important magnetic, mechanical and cryogenic system components marked. The cold mass is asymmetrically located inside the cryostat. The cross section shown here is at the axial center. 
The superconducting coils are made of $\mathrm{Nb}$ - $\mathrm{Ti}$ superconductor configured in a "Rutherford Type" [154,176] cable. The coils are kept below a temperature of 4.65 kelvin. The cryogenic system is designed to minimize the heat leak to room temperature outside the cryostat. The coils are kept under compression to minimize conductor motion under Lorentz forces when the magnet is energized. In RHIC magnets the space between the superconducting coils and the yoke is filled with RX630 phenolic spacers and in SSC magnets with stainless steel collars. The purpose of the yoke is to provide magnetic shielding and additional field in the magnet aperture. The yoke has several features (see Fig. 1.4.1) to serve a variety of purposes. These features include (a) loading flats to provide compression on the coil through a heavy press (b) holes for helium flow, saturation control and yoke pins (c) cutouts for electrical bus work, collaring keys, tabs which align the RX630 spacers to define the coil pole location and survey notches for aligning the magnet in the cryostat. A stainless steel shell, which also provides radial pressure, is put outside the yoke for helium containment.

The part of the magnet assembly described above (superconducting coils, iron yoke and stainless steel) is called the "coldmass" which remains below 4.65 degree kelvin in RHIC and SSC designs. The coldmass is put inside the cryostat which is a vacuum vessel. A number of components between the coldmass and the cryostat are required for structural and thermal purposes.

\subsubsection{Superconducting Cable}

In most magnet designs the superconducting wire is made of $\mathrm{NbTi}$ filaments embedded in a copper matrix. NbTi has good mechanical properties (ductility) but is generally limited to producing $\sim 7.5$ tesla at 4.5 kelvin and $\sim 10.5$ tesla at 1.8 kelvin. A higher field can be reached with the more expensive $\mathrm{Nb}_{3} \mathrm{Sn}$ superconductor. However, $\mathrm{Nb}_{3} \mathrm{Sn}$ does not have similarly good mechanical properties and therefore coil manufacturing becomes much more complicated. The titanium in $\mathrm{NbTi}$ alloy is generally about $46 \%$ by weight. The measured critical current density (the current density at which the wire looses its superconducting properties) as a function of applied field at $4.2 \mathrm{~K}$ is shown in Fig. 1.4.2 (courtesy A. Ghosh) for the NbTI wire used in RHIC corrector magnets. A similar $B-J$ performance is obtained in the superconducting cable used in the other types of RHIC magnets. In addition to the superconductor, the cable contains copper to provide stability against quench and for 


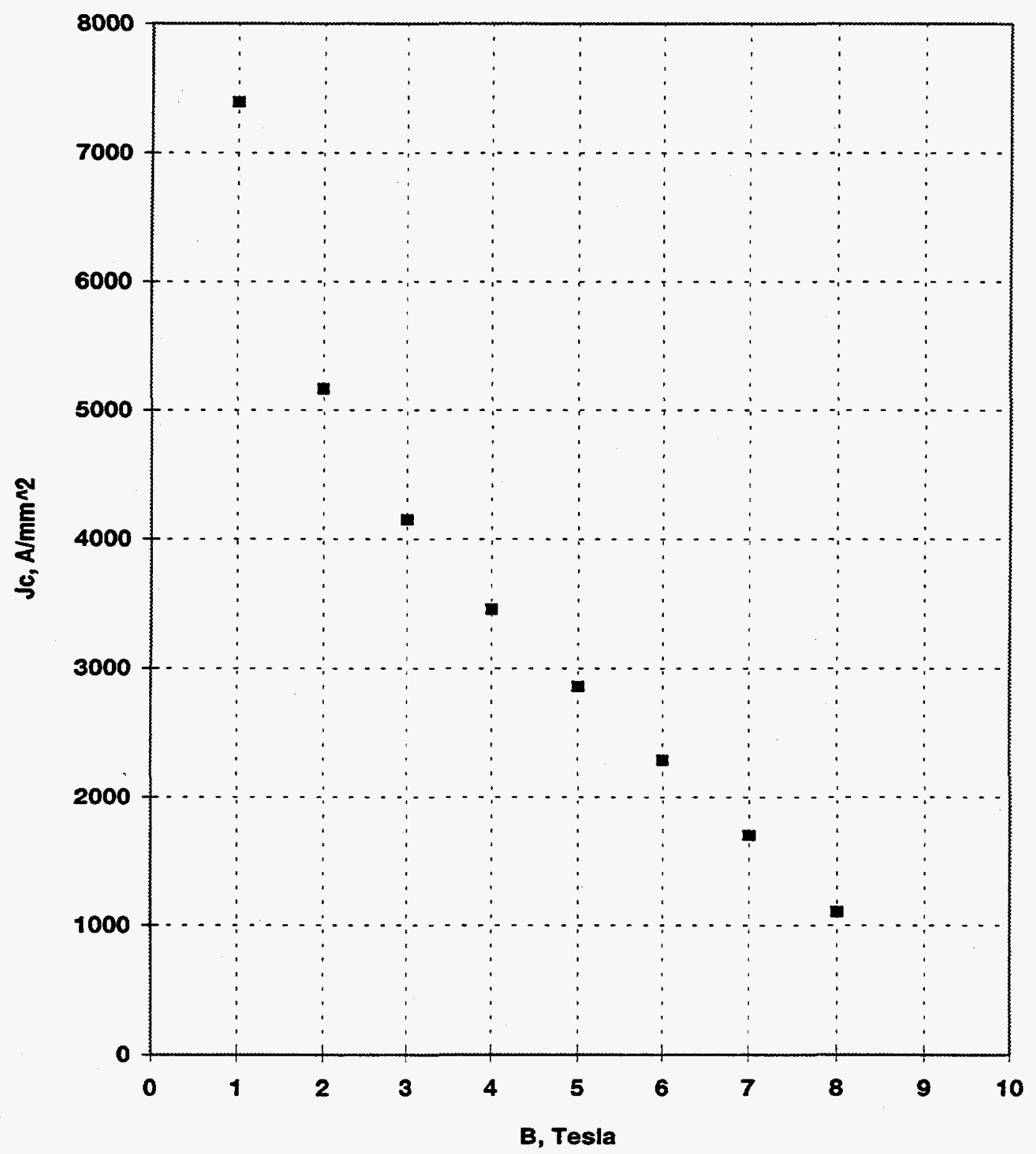

Figure 1.4.2: The measured (courtesy A. Ghosh) critical current density $\left(J_{c}\right)$ at $4.2 \mathrm{~K}$ in the superconductor of the wire used in the RHIC corrector magnets as a function of applied field $(B)$. 
heat conduction. The amount of copper in the cable is usually more than the amount of superconductor. The superconducting cable, used in RHIC magnets, has a copper to superconductor ratio (by volume) of 3.0 in corrector magnets, of 2.5 in trim quadrupole and sextupole magnets, of 2.2 in the $80 \mathrm{~mm}$ and $100 \mathrm{~mm}$ aperture magnets and 1.8 in the $130 \mathrm{~mm}$ aperture and $180 \mathrm{~mm}$ aperture insertion magnets. The cable used in the inner layer of the SSC dipole had a copper to superconductor ratio of 1.3 and the one used in the outer layer had a copper to superconductor ratio of 1.8. The filament size in the superconducting wire used in the RHIC and SSC dipole magnet design is $6 \mu \mathrm{m}$ except for the wire used in the RHIC corrector, sextupole and trim quadrupole magnets where it is $10 \mu \mathrm{m}$. There are about 3500 filaments in each wire of the RHIC dipole cable. The wire (strand) diameter is about $0.65 \mathrm{~mm}$. The parameter list of the cable used in RHIC dipole magnet is given in Table 1.4.1.

Table 1.4.1: Design specifications of the superconducting cable for the $80 \mathrm{~mm}$ aperture $\mathrm{RHIC}$ arc dipole and quadrupole magnets.

\begin{tabular}{|c|c|}
\hline Cable parameters & Value \\
\hline Filament diameter & $6.0 \mu \mathrm{m}$ \\
Filament spacing & $>1.0 \mu \mathrm{m}$ \\
Number of flaments per wire & $3510 \pm 20$ \\
Copper to Superconductor Ratio & $(2.25 \pm 0.1): 1$ \\
\hline Strand (wire) diameter & $0.648 \pm 0.003 \mathrm{~mm}$ \\
No. of strands in cable & 30 \\
Critical current in wire at 5 T, 4.2 K & $264 \mathrm{~A}$ \\
Critical current in cable at 5 T, 4.2 K & $7524 \mathrm{~A}$ \\
\hline Cable width & $9.73 \pm 0.03 \mathrm{~mm}$ \\
Cable mid-thickness & $1.166 \pm 0.006 \mathrm{~mm}$ \\
Cable keystone angle & $1.2 \pm 0.1 \mathrm{degree}$ \\
Cable lay pitch & $74 \pm 5 \mathrm{~mm}$ \\
\hline
\end{tabular}


"Rutherford" cable is used in most large scale production of accelerator magnets. This type of cable is wide and flat and is made of a number of wires (strands) twisted together in a spiral shape. The cable is asymmetrically compressed across the flat side with one edge being thinner than the other. This provides a "keystone" angle in the cable which helps the coils to conform to a circular geometry with each turn lying approximately on a radius. In a fully keystoned cable, the ratio of thickness of the two edges of the cable is the same as that between the coil inner radius and outer radius. The cable is electrically insulated to deal with the high voltage (over $1 \mathrm{kV}$ ) that is created during a quench when one turn is in the superconducting phase and the other in the normal. The RHIC design uses all-Kapton (Kapton is a registered trademark of Dupont Corporation) insulation which has good electrical break down, cryogenic and stability in ionizing radiation properties and has good dimensional tolerance. Another type of insulation used on superconducting cables is fiberglass tape impregnated with B-stage epoxy.

The superconducting cable produced for the RHIC magnet program is the result of significant R\&D and a close collaboration with industry. The standard deviation in the variation in the cable thickness and other mechanical dimensions has been generally kept to about the $5 \mu \mathrm{m}$ level. This has been crucial to providing good field quality and proper compression on the coils in the magnet. Moreover, the variation in the critical current density is also kept to about $2 \%$ to minimize the variation in the field harmonics associated with superconductor effects.

\subsubsection{Cryogenic System}

Cooling is provided by supercritical helium. A small radial gap between the beam tube and the superconducting coil provides a space for helium flow which partly cools the coil and removes the heat deposited during machine operation or instability in the magnet. The major portion of the heat removal and helium flow takes place in the four helium holes in the yoke. In order to reduce the heat load on the cryogenic system, the design of the coldmass and cryostat is optimized to minimize heat leak. To deal with a large thermal gradient between the low temperature in the superconducting coils and room temperature outside the cryostat, either one or two staged thermal shields are used where the heat is intercepted and removed. In the SSC dipole design two stage heat removal was planned (a) at 20 kelvin by gaseous helium and (b) at 80 kelvin by liquid nitrogen. In the RHIC 
dipole design, heat leakage is removed at 55 kelvin. Since the radiation heat leak loss goes as the difference between the fourth power of the two temperatures, only a small difference is expected between the two and one shield cases. A blanket of thermal insulation is placed just inside the vacuum vessel wall and between and interior to the shields.

The coldmass is put inside the vessel on a few support posts. The location of the support posts is chosen to reduce sagging of the coldmass. To minimize the heat leak through them, they are made as long as possible and to accommodate that larger length without increasing the cryostat outer diameter, the coldmass is positioned above the center of the cryostat (see Fig. 1.4.1). In both RHIC and SSC magnets the cryostat is made of low carbon magnetic steel which reduces the exterior field. However, a systematic offset between the vertical center of the cryostat and the center of the coldmass creates a skew quadrupole harmonic in the dipole at high field.

\subsubsection{Mechanical Design}

Good quench performance of the superconducting magnets is closely related to a good mechanical design which minimizes the motion of the superconducting wires. The design must be structurally sound to deal with the Lorentz forces when the magnet is energized, the thermal forces during cool-down and the mechanical forces during transportation of the magnet.

A large magnetic field in superconducting magnets generates a large Lorentz forces on the superconducting coils which may cause a small amount of conductor motion. This conductor motion generates heat which may start a quench in the magnet. The direction of the Lorentz forces in the cross section of the magnet is such that compression of the coil from the coil pole to the coil midplane is by the azimuthal component and compression outward is by the radial component. To deal with this situation, the coils are pre-compressed with a large mechanical compressive force which counters the Lorentz forces and thus minimizes conductor movement. In the SSC magnets, this compression on the coil is provided by stainless steel collars and in RHIC magnets by the yoke itself. In these designs, a significant part of the compression applied at room temperature is lost when the magnet is brought to a lower temperature. This is because of a difference in the coefficients of thermal expansion of superconducting coils, stainless steel and the yoke iron. Therefore, in order to assure an adequate compression when the magnet is cold, a much higher (a factor of two to three) 
compression is applied at room temperature. There are some alternate design concepts which have been tested in some magnets, where this loss of compression is avoided $[41,127]$.

Finally a stainless steel shell is welded on the yoke outer diameter to contain the helium. This also provides a radial pressure on the coil-yoke assembly and in the RHIC-type design the radial component of the Lorentz forces are finally transmitted to it. To deal with the outward axial component of the Lorentz forces, the ends are restrained and sometimes even compressed (loaded) axially. A detailed description of the mechanical design and analysis of the SSC magnets can be found elsewhere [44].

\subsubsection{Magnetic Design}

The main goal of the magnetic design is to optimize the geometry of the coil and iron shape to produce a highly uniform field. In addition, it is beneficial to minimize the maximum field on the conductor (peak field) and to maximize the transfer function (tesla per ampere) to obtain a high quench field (computed from cable short sample measurements). The design must also fulfill all mechanical and cryogenic requirements and a magnet based on this design should be as simple as possible to manufacture.

The coils are made of a number of turns of superconducting cable which are grouped in several current blocks. A cosine theta current distribution produces an ideal dipole field. Copper wedges are placed between the blocks of turns to approximate the cosine theta current distribution. In designs which use partially keystoned cables, the wedges also serve an important mechanical purpose in providing a proper arc shape to the current blocks in the circular coil geometry. The size of the current blocks and the copper wedges are parameters used to minimize the field harmonics and to maximize the quench field.

The iron yoke provides magnetic shielding. In addition, the magnetized yoke gives an extra contribution to the central field which in most RHIC magnets is $\sim 50 \%$ of the coil field. However, at high field, the magnetization in the iron yoke is not proportional to the current in the coils, so the yoke geometry must be carefully designed to maintain a good field quality at all fields.

The coil end design is complicated. The cable must be bent carefully to bring it from one side of the coil to the other side. Spacers are inserted between the blocks of turns in the end not only to minimize the peak field and field harmonics but also for the mechanical purpose of reducing the strain on the cable. In most RHIC magnet designs the ends are 
enclosed by the iron yoke laminations but in SSC and in most other magnet designs the iron laminations are replaced by stainless steel laminations to minimize the peak field in the ends.

The field errors in magnets are described in terms of field harmonics (see next section Eq. (1.5.17)) which are also referred to as multipoles. The are generally divided in the following three categories based on their sources :

1. Geometric multipoles

2. Persistent current multipoles

3. Saturation multipoles

The geometric multipoles are related to the magnet geometry. An error (or a departure) from the ideal geometry would create harmonics other than those desired. The persistent current multipoles are related to the persistent current in the superconductors. As the field in magnet is changed, the persistent (shielding) currents are induced in a direction to oppose the changing field [144]. Unlike in normal conductors, these currents persist for a long time in superconductors and contribute significantly to the field errors at low fields where their relative contribution is high. The saturation multipoles are related to the saturation magnetization of iron at high field. A non-uniform field and hence a non-uniform (as a function of azimuth) relative contribution of the iron distorts the field shape and field errors are thereby introduced.

\subsubsection{Magnet Construction}

The construction of the superconducting magnets is a long and complex process which requires a high level of engineering and quality control to assure consistently good quality in large scale magnet production [6]. The magnet manufacturing tooling must itself be carefully designed to realize the computed field quality and quench performance. Some of the major steps of the manufacturing process are briefly described here in the case of RHIC arc dipole magnets.

The superconducting cable and the copper wedges are insulated with Kapton layers. The magnet coils are wound on a precision convex surface with a winding machine feeding the cable continuously. The copper wedges in the coil cross section and the wedge tips in the coil ends are periodically inserted as required by the optimized design. The Kapton tape, wound around the cable, is coated on one side with a dry adhesive which is activated (cured) 
by heat at a temperature of about $130 \mathrm{C}$ while under compression. The coils are cured in a curing fixture (mold) and the temperature and curing pressure described above may contain several curing cycles with different combinations of curing temperature and curing pressure. Once cooled, the coil is firmly fixed in the shape determined by the dimensions of the curing mold.

The coils are installed in the iron yoke together with the RX630 phenolic spacers and other parts. The following is the sequence of the steps required in this operation: (a) the laminations for the lower yoke half are stacked (b) the RX630 spacers are put in place (c) the lower coil is installed (d) the beam tube is inserted (e) the upper coil, pre-assembled with the RX630 insulator, is put in place and ( $f$ ) the laminations for the upper yoke half are stacked on this assembly. The coil is compressed with a press applying pressure on the yoke collar at the loading flats. The keys are inserted to retain this compression. The stainless steel shell is welded with the required sagitta (axial curvature of the magnet) in the coldmass. The stainless steel end plates are welded and coil end force is applied before the electrical installation is completed. Then the coldmass is placed inside the cryostat on the support posts. All cryogenic piping and heat shields are also installed.

\subsubsection{Magnet Measurements}

Apart from a variety of mechanical and electrical measurements at the various stages of magnet construction, the two measurements which define the final quality of magnets for machine operation are (a) the quench performance and (b) the field harmonics. For these measurements the magnets must be tested at cryogenic temperatures. It is, however, expensive and time consuming to test each and every magnet cold (in the superconducting phase). Therefore, in the RHIC magnet program only a part of the magnets are tested cold before they are installed in the tunnel [170]. The selection of the magnets chosen for cold testing is carefully made to minimize the risk of not testing all magnets cold. The required maximum operating current for $\mathrm{RHIC}$ is about $5 \mathrm{kA}$ and the design margin over the machine requirements for most magnets for the computed quench current is over $30 \%$. The measured performance of the magnets tested cold show that most magnets require only a few (2-5) quenches to reach the computed quench current [170].

Warm magnetic measurements are performed on all magnets. At room temperature, the current in the cable is carried by the copper intermixed with the superconductor. There is an 
expected systematic difference between warm and cold harmonics because of (a) a change in the magnet geometry due to thermal contraction during cool-down (b) the persistent current effects in the superconductor and (c) the saturation effects due to the non-linear properties of the iron yoke. Based on those magnets that are tested both warm and cold, a good warm-to-cold correlation in the field harmonics has been obtained [170]. This correlation is used to estimate the field quality in those magnets that are not tested cold.

The magnetic measurements are carried out with an array of windings having a certain radius [175], mounted on a rotating cylinder which intercept the field in the magnet aperture. The geometry of these coils is chosen so that the Fourier analysis of the voltage induced in the various windings determine the field harmonics. The accuracy of the measurements depends on the accuracy of the measuring coil geometry, the electronic signal measurement and the analysis of the measured signal.

In the long curved RHIC magnets, the complete measurements are made in 10 steps with a meter long measuring coil system which is referred to as a mole [49]. In addition, the integral of the field along the axis is also measured with a long stationary coil. For a more accurate measurement of the field strength at a point an NMR (Nuclear Magnetic Resonance) probe is also used.

The measured field quality in $80 \mathrm{~mm}$ aperture $\mathrm{RHIC}$ arc dipoles is shown in Table 1.4 .2 and Table 1.4.3. Measuring the field harmonics accurately and managing it in a large number of magnets ( 1740 are required for RHIC) is a quite complex task.

The "Mean" of the distribution for the harmonic $b_{n}$ (which is also sometimes referred to as the systematic value of $b_{n}$ ) in $N$ magnets is defined as follows :

$$
<b_{n}>=\frac{1}{N} \sum_{k=1}^{N}\left(b_{n}\right)_{k},
$$

where $\left(b_{n}\right)_{k}$ is the value of harmonic $b_{n}$ in the $k^{\text {th }}$ magnet. The "SIGMA" $(\sigma)$, also the $R M S$ (Root Mean Square) deviation from the "Mean" $b_{n}$, is defined as follows :

$$
\sigma\left(b_{n}\right)=\sqrt{\left.\frac{1}{N} \sum_{k=1}^{N}\left[\left(b_{n}\right)_{k}-<b_{n}\right\rangle\right]^{2}} .
$$

The primary purpose of the harmonic measurements is to verify that the machine requirements needed for beam stability and design beam life time in RHIC are satisfied. In addition, the field harmonics are also used as a tool to detect possible manufacturing defects in the magnets or a drift in the mechanical dimensions of the components used in 
the manufacturing process. Since the harmonics are the analysis of the field created by the geometry of the coil and yoke, they are a reflection of magnet geometry. The accuracy of the magnetic measurements is sufficient to find a $100 \mu \mathrm{m}$ or less error in most of the critical components used in manufacturing the magnets. 
Table 1.4.2: The measured integral transfer function (ITF) in tesla.m/kA, body transfer function (SSTF) in tesla/kA and normal field harmonics $\left(b_{n}\right.$, defined elsewhere) at $25 \mathrm{~mm}$ reference radius in the $9.45 \mathrm{~m}$ long $80 \mathrm{~mm}$ aperture RHIC arc dipole magnets (data courtesy Jain and Wanderer). The current at which measurements are made and the number of magnets (shown in parenthesis) used in arriving at the statistics are shown here. The 30 A measurements are made when the magnet is warm (room temperature), in the non-superconducting state and the current is carried by the copper in the cable. $\left\langle b_{n}\right\rangle$ is the mean and $\sigma\left(b_{n}\right)$ is the standard deviation of harmonic $b_{n}$ in the number of magnets measured.

\begin{tabular}{|c|c|c|c|c|}
\hline & $\left\langle b_{n}> \pm \sigma\left(b_{n}\right)\right.$ & $\left\langle b_{n}> \pm \sigma\left(b_{n}\right)\right.$ & $\left\langle b_{n}\right\rangle \pm \sigma\left(b_{n}\right)$ & $<b_{n}> \pm \sigma\left(b_{n}\right)$ \\
\hline & $30 \mathrm{~A}(296)$ & $660 \mathrm{~A}(63)$ & $1450 \mathrm{~A}(61)$ & $5000 \mathrm{~A}(62)$ \\
\hline ITF & $6.6545 \pm 0.0021$ & $6.6698 \pm 0.0027$ & $6.6769 \pm 0.0021$ & $6.4180 \pm 0.0024$ \\
\hline SSTF & $0.7042 \pm 0.00021$ & $0.7078 \pm 0.0003$ & $0.7080 \pm 0.00028$ & $0.6798 \pm 0.00034$ \\
\hline$b_{1}$ & $0.25 \pm 0.37$ & $0.08 \pm 0.28$ & $0.04 \pm 0.27$ & $0.10 \pm 0.28$ \\
\hline$b_{2}$ & $3.54 \pm 1.74$ & $-0.17 \pm 2.22$ & $2.18 \pm 1.77$ & $0.83 \pm 1.76$ \\
\hline$b_{3}$ & $-0.03 \pm 0.10$ & $0.00 \pm 0.08$ & $0.00 \pm 0.08$ & $0.01 \pm 0.08$ \\
\hline$b_{4}$ & $0.22 \pm 0.44$ & $-0.33 \pm 0.57$ & $-0.15 \pm 0.58$ & $0.15 \pm 0.59$ \\
\hline$b_{5}$ & $0.01 \pm 0.03$ & $0.00 \pm 0.03$ & $0.00 \pm 0.03$ & $-0.03 \pm 0.04$ \\
\hline$b_{6}$ & $0.12 \pm 0.11$ & $-0.13 \pm 0.13$ & $-0.02 \pm 0.14$ & $1.19 \pm 0.14$ \\
\hline$b_{7}$ & $0.00 \pm 0.01$ & $-0.01 \pm 0.01$ & $-0.01 \pm 0.01$ & $-0.01 \pm 0.01$ \\
\hline$b_{8}$ & $0.09 \pm 0.11$ & $0.14 \pm 0.12$ & $0.13 \pm 0.12$ & $0.12 \pm 0.12$ \\
\hline$b_{9}$ & $0.00 \pm 0.01$ & $0.02 \pm 0.02$ & $0.02 \pm 0.02$ & $0.02 \pm 0.02$ \\
\hline$b_{10}$ & $-0.53 \pm 0.02$ & $-0.58 \pm 0.02$ & $-0.56 \pm 0.02$ & $-0.58 \pm 0.02$ \\
\hline & & & & \\
\hline
\end{tabular}


Table 1.4.3: The measured $a_{n}$ (skew harmonics) at $25 \mathrm{~mm}$ reference radius in the $9.45 \mathrm{~m}$ long $80 \mathrm{~mm}$ aperture $\mathrm{RHIC}$ arc dipole magnets (data courtesy Jain and Wanderer). The current at which the measurements are made and the number of magnets (shown in parenthesis) used in arriving at the statistics are shown here. The $30 \mathrm{~A}$ measurements are made when the magnet is warm (room temperature), non-superconducting and the current is carried by the copper in the cable. $\left\langle a_{n}\right\rangle$ is the mean and $\sigma\left(a_{n}\right)$ is the standard deviation $a_{n}$ in the number of magnets measured.

\begin{tabular}{|c|c|c|c|c|}
\hline & $\left\langle a_{n}> \pm \sigma\left(a_{n}\right)\right.$ & $\left\langle a_{n}> \pm \sigma\left(a_{n}\right)\right.$ & $\left\langle a_{n}\right\rangle \pm \sigma\left(a_{n}\right)$ & $\left.<a_{n}\right\rangle \pm \sigma\left(a_{n}\right)$ \\
\hline & $30 \mathrm{~A}(296)$ & $660 \mathrm{~A}(63)$ & $1450 \mathrm{~A}(61)$ & $5000 \mathrm{~A}(62)$ \\
\hline$a_{1}$ & $-0.20 \pm 1.62$ & $0.28 \pm 1.53$ & $0.21 \pm 1.52$ & $-1.51 \pm 1.51$ \\
\hline$a_{2}$ & $-1.11 \pm 0.20$ & $-1.03 \pm 0.17$ & $-1.03 \pm 0.17$ & $-1.07 \pm 0.18$ \\
\hline$a_{3}$ & $-0.01 \pm 0.49$ & $-0.03 \pm 0.42$ & $-0.02 \pm 0.42$ & $-0.36 \pm 0.41$ \\
\hline$a_{4}$ & $0.18 \pm 0.07$ & $0.21 \pm 0.06$ & $0.21 \pm 0.06$ & $0.20 \pm 0.06$ \\
\hline$a_{5}$ & $-0.01 \pm 0.17$ & $0.02 \pm 0.15$ & $0.01 \pm 0.15$ & $-0.06 \pm 0.16$ \\
\hline$a_{6}$ & $-0.11 \pm 0.03$ & $-0.10 \pm 0.02$ & $-0.10 \pm 0.02$ & $-0.10 \pm 0.02$ \\
\hline$a_{7}$ & $0.00 \pm 0.05$ & $-0.01 \pm 0.05$ & $-0.01 \pm 0.05$ & $-0.01 \pm 0.05$ \\
\hline$a_{8}$ & $0.02 \pm 0.01$ & $0.02 \pm 0.01$ & $0.02 \pm 0.01$ & $0.02 \pm 0.01$ \\
\hline$a_{9}$ & $0.00 \pm 0.01$ & $0.04 \pm 0.02$ & $0.04 \pm 0.02$ & $0.04 \pm 0.02$ \\
\hline$a_{10}$ & $-0.01 \pm 0.00$ & $-0.01 \pm 0.01$ & $-0.01 \pm 0.01$ & $-0.01 \pm 0.01$ \\
\hline & & & & \\
\hline
\end{tabular}




\subsection{Magnetic Field Analysis in Accelerator Magnets}

In this section an outline of the formalism and theory used in carrying out the field calculations in the superconducting magnets is given. Starting from first principles, basic expressions are developed which are used in designing and describing the magnetic fields in the accelerator magnets.

The uniformity of the magnetic field is very important since it determines the performance of the machine. A typical requirement for the field quality in the accelerator magnets is that the deviation from the ideal shape should be within a few parts in $10^{4}$. The uniformity of the field is expressed in terms of the Fourier harmonic components.

\subsubsection{Basic Electromagnetic Field Equations}

The calculation of the magnetic field in accelerator magnets is too complex to be done directly by solving Maxwell's equations. However, the most complicated formulae describing the field shape in the magnets are derived primarily from them. In this section, Maxwell's equations and other commonly used expressions of electro-magnetic theory $[95,129,150]$ are briefly described. Although the magnetic field in the accelerator magnets is not static in time, the effects of time variation are by and large negligible in the problems to be addressed during the course of this work. Therefore, most of the detailed analysis is limited to the magneto-static case only.

The four Maxwell's equations are :

$$
\begin{aligned}
\nabla \cdot \vec{D} & =\rho, \\
\nabla \cdot \vec{B} & =0, \\
\nabla \times \vec{E}+\frac{\partial \vec{B}}{\partial t} & =0, \\
\nabla \times \vec{H} & =\vec{J}+\frac{\partial \vec{D}}{\partial t} .
\end{aligned}
$$

Here $\vec{B}$ is the magnetic field, $\vec{E}$ is the electric field, $\vec{B}$ is the magnetic induction and $\vec{D}$ is the displacement vector. $\rho$ denotes the charge density and $\vec{J}$ the current density, and these two are related by the following continuity equation,

$$
\nabla \cdot \vec{J}+\frac{\partial \rho}{\partial t}=0
$$


Furthermore, $\vec{B}$ and $\vec{H}$ are related by the following equations:

$$
\begin{aligned}
\frac{\vec{B}}{\mu_{o}} & =\vec{H}+\vec{M}, \\
\frac{\vec{B}}{\mu \mu_{o}} & =\vec{H},
\end{aligned}
$$

where $\mu_{0}$ is the permeability of the vacuum $\left(\mu_{0}=4 \pi \times 10^{-7}\right.$ henry/meter) and $\mu$ is the relative permeability of the medium (relative with respect to that of vacuum). Often, $\mu$ is simply referred to as the permeability (which is in fact the case in CGS units) and the same convention is followed here unless otherwise explicitly mentioned. $\vec{M}$ denotes the magnetization (or magnetic polarization) of the medium. In free space (vacuum) $\vec{M}$ is 0 . In an isotropic medium $\vec{H}, \vec{B}$ and $\vec{M}$ are parallel to each other.

Furthermore, $\vec{D}$ and $\vec{E}$ are related by the following equations:

$$
\begin{aligned}
& \vec{D}=\epsilon_{o} \vec{E}+\vec{P}, \\
& \vec{D}=\epsilon \epsilon_{o} \vec{E},
\end{aligned}
$$

where $\vec{P}$ is the electric polarization and $\epsilon_{o}$ is the permittivity in vacuum $\left(\epsilon_{o}=8.854 \times 10^{-12}\right.$ farad/meter). - is the relative permittivity of the medium. In free space (vacuum), the electric polarization is 0 .

The constants $\epsilon_{o}$ and $\mu_{o}$ are related through the relation

$$
\epsilon_{o} \mu_{o}=\frac{1}{c^{2}}
$$

where $c$ is the velocity of light $\left(c=2.998 \times 10^{8} \mathrm{~m} / \mathrm{s}\right)$. Since $\vec{B}$ has a zero divergence, it may be expressed in term of a magnetic vector potential $\vec{A}$ as

$$
\vec{B}=\nabla \times \vec{A}
$$

The vector potential $\vec{A}$ can be obtained at any point $(\vec{r})$ due to a current density $\vec{J}\left(\overrightarrow{r^{\prime}}\right)$ with the help of the following integral equation :

$$
\vec{A}(\vec{r})=\frac{\mu \mu_{o}}{4 \pi} \int_{V} \frac{\vec{J}\left(\overrightarrow{r^{\prime}}\right)}{\left|\vec{r}-\overrightarrow{r^{\prime}}\right|} d v
$$

where $\vec{r}$ and $\overrightarrow{r^{\prime}}$ are three dimensional coordinates and $d v$ is the three dimensional volume element. 
The components of the field in Eqs. (1.5.5) in Cartesian coordinates are given by

$$
\begin{aligned}
& B_{x}=\frac{\partial A_{z}}{\partial y}-\frac{\partial A_{y}}{\partial z} \\
& B_{y}=\frac{\partial A_{x}}{\partial z}-\frac{\partial A_{z}}{\partial x} \\
& B_{z}=\frac{\partial A_{y}}{\partial x}-\frac{\partial A_{z}}{\partial y}
\end{aligned}
$$

and in cylindrical coordinates by

$$
\begin{aligned}
& B_{r}=\frac{1}{r}\left(\frac{\partial A_{z}}{\partial \theta}\right)-\frac{\partial A_{\theta}}{\partial z}, \\
& B_{\theta}=\frac{\partial A_{r}}{\partial z}-\frac{\partial A_{z}}{\partial r}, \\
& B_{z}=\frac{1}{r}\left(\frac{\partial\left(r A_{\theta}\right)}{\partial r}-\frac{\partial A_{r}}{\partial \theta}\right) .
\end{aligned}
$$

The research work to be described is restricted to static magnetic fields only and electric fields are not considered. During the accelerating cycle of the machine, the magnetic field does change with time in the superconducting magnets. However, for the problems to be discussed during the course of this work, the change in magnetic field has negligible effect on field quality. Therefore the following two Maxwell's equations for the magnetostatic case are used in developing various formulae

$$
\begin{aligned}
\nabla \cdot \vec{B} & =0 \\
\nabla \times \vec{H} & =\vec{J}
\end{aligned}
$$

Ampère's law

$$
\oint_{S} \vec{H} \cdot d s=I,
$$

can be obtained from Eqs. (1.5.8b) by integrating and using Stoke's theorem :

$$
\oint_{C} \vec{V} \cdot d \vec{l}=\int_{S}(\nabla \times \vec{V}) \cdot \vec{n} d a
$$

where $\vec{V}$ is a well behaved vector field, $S$ is an open arbitrary surface, $C$ is the closed curve bounding $\mathrm{S}, \mathrm{d} \vec{l}$ is a line element of $\mathrm{C}$, and $\vec{n}$ is a vector element normal to $\mathrm{S}$. The right hand side of the equation simply states that $I=\int \vec{J} \cdot \vec{n} d a$ is the total current flowing through the area.

Poisson's equation for the vector potential is derived here under the assumptions that $\vec{B}=\mu_{0} \mu \vec{H}$, the medium is homogeneous (i.e. $\mu$ is constant over a finite space) and isotropic. Using $\vec{B}=\mu_{0} \mu \vec{H}$ and $\vec{B}=\nabla \times \vec{A}$ in Eqs. (1.5.8), one obtains :

$$
\nabla \times \nabla \times \vec{A}=\mu_{0} \mu \vec{J}
$$


The following identity is used to simplify the above equation :

$$
\nabla^{2} \vec{A}=\nabla(\nabla \cdot \vec{A})-\nabla \times \nabla \times \vec{A}
$$

In Cartesian coordinates the above Laplacian operator $\left(\nabla^{2}\right)$ can be applied to a vector $\vec{A}$ whose $i^{\text {th }}$ component is $\nabla^{2} A_{i}$. In other coordinate systems Eq. (1.5.11) must be used to determine the expression for $\nabla^{2} \vec{A}$. In the cylindrical coordinate system :

$$
\nabla^{2} \vec{A}_{z}=\frac{1}{r} \frac{\partial}{\partial r}\left(r \frac{\partial A_{z}}{\partial r}\right)+\frac{1}{r^{2}} \frac{\partial^{2} A_{z}}{\partial \theta^{2}}
$$

when $A_{r}=A_{\theta}=0$ by symmetry (axial symmetric case).

The choice of $\nabla \cdot \vec{A}$ has thus far has been arbitrary and it is made zero in the Coulomb gauge (in the magnetostatic case). In that case Eq. (1.5.10) leads to Poisson's Equation as

$$
\nabla^{2} \vec{A}=-\mu_{0} \mu \vec{J}
$$

In the 2-dimensional case, when the direction of current flow is parallel to the z-axis, $J_{x}=J_{y}=0$. This implies that $A_{x}=A_{y}=0$ and $\frac{\partial A_{x}}{\partial z}=0$. Therefore, the above expression becomes,

$$
\nabla^{2} A_{z}=-\mu_{0} \mu J_{z}
$$

which in the Cartesian coordinate system gives :

$$
\frac{\partial^{2} A_{z}}{\partial x^{2}}+\frac{\partial^{2} A_{z}}{\partial y^{2}}=-\mu_{0} \mu J_{z}
$$

In the case of axial symmetry, the Eq. (1.5.14) in cylindrical coordinates becomes :

$$
\frac{1}{r} \frac{\partial}{\partial r}\left(r \frac{\partial A_{z}}{\partial r}\right)+\frac{1}{r^{2}} \frac{\partial^{2} A_{z}}{\partial \theta^{2}}=-\mu_{0} \mu J_{z},
$$

on using Eq. (1.5.12). 


\subsubsection{Field Harmonic Definitions}

It is useful to describe the magnetic field inside the aperture of accelerator magnets in terms of harmonic coefficients $[96,140,144,175]$. The discussion will be limited to 2dimensional analysis, which describes the field in the body (or straight section) of a long magnet. When the magnetic field is evaluated a few aperture diameters away from the two ends of the magnet, the axial component of the field is negligible. The accelerator magnets examined here are those in which the field is consists of one fundamental harmonic which is several orders of magnitude larger (usually $10^{4}$ ) than any other harmonic present.

The skew $\left(a_{n}\right)$ and the normal $\left(b_{n}\right)$ field harmonics are defined through the following relation :

$$
B_{y}+i B_{x}=10^{-4} B_{R_{0}} \sum_{n=0}^{\infty}\left[b_{n}+i a_{n}\right][\cos (n \theta)+i \sin (n \theta)]\left(\frac{r}{R_{0}}\right)^{n},
$$

where $B_{x}$ and $B_{y}$ are the horizontal and vertical components of the magnetic field at $(\mathrm{r}, \theta)$ and $i=\sqrt{-1} . R_{0}$ is the normalization radius. The magnets for the Relativistic Heavy Ion Collider (RHIC) have a coil radius ranging from $40 \mathrm{~mm}$ to $90 \mathrm{~mm}$. In most of these magnets, the normalization radius is taken to be $\frac{5}{8}$ of the coil radius. The value of the normalization radius is $25 \mathrm{~mm}$ for the $80 \mathrm{~mm}$ aperture diameter of the RHIC arc dipoles and quadrupoles, $40 \mathrm{~mm}$ for the $130 \mathrm{~mm}$ aperture of the RHIC insertion quadrupoles, $31 \mathrm{~mm}$ for the $100 \mathrm{~mm}$ aperture of the $\mathrm{RHIC}$ insertion dipoles and $60 \mathrm{~mm}$ for the $180 \mathrm{~mm}$ aperture $\mathrm{RHIC}$ insertion dipoles [140]. $B_{R_{0}}$ is the magnitude of the field due to the fundamental harmonic at the reference radius on the midplane. In the dipoles, $B_{R_{0}}=B_{0}$ (the field at the center of the magnet), in the quadrupoles, $B_{R_{0}}=G \times R_{0}$ ( $G$ being the field gradient at the center of the magnet), and in general for a $2(m+1)^{\text {th }}$ pole magnet,

$$
B_{R_{0}}=\frac{R^{m}}{m !}\left[\frac{\partial^{m} B_{y}}{\partial x^{m}}\right]_{x=0, y=0} .
$$

Eq. (1.5.17) can be re-written in several other forms using complex variables. In this section $z$ represents the complex coordinate and $B(z)$ represents the complex field as follows:

$$
\begin{aligned}
z & =x+i y \\
(x+i y)^{n} & =r^{n}(\cos [n \theta]+i \sin [n \theta]) \\
B(z) & =B_{y}+i B_{x} \\
c_{n} & =b_{n}+i a_{n}
\end{aligned}
$$


Thus :

$$
\begin{aligned}
B_{y}+i B_{x} & =10^{-4} B_{R_{0}} \sum_{n=0}^{\infty}\left[b_{n}+i a_{n}\right][x+i y]^{n}\left(\frac{1}{R_{0}}\right)^{n} \\
B(z) & =10^{-4} B_{R_{0}} \sum_{n=0}^{\infty} c_{n}\left(\frac{z}{R_{0}}\right)^{n}
\end{aligned}
$$

The harmonics used so far $\left(a_{n}, b_{n}, c_{n}\right)$ are all dimensionless coefficients. However, in another representation, the field is expressed in terms of coefficients which carry the units of magnetic field. These are usually distinguished from the harmonics $a_{n}$ and $b_{n}$ given in Eq. (1.5.17) by the use of the uppercase alphabet. The two are related as follows:

$$
\begin{aligned}
& A_{n+1}=10^{-4} B_{R_{0}} a_{n}, \\
& B_{n+1}=10^{-4} B_{R_{0}} b_{n}, \\
& C_{n+1}=10^{-4} B_{R_{0}} c_{n} .
\end{aligned}
$$

Using these, Eq. (1.5.20) can be written as :

$$
B(z)=\sum_{n=1}^{\infty} C_{n}\left(\frac{z}{R_{0}}\right)^{n-1}
$$

In this case the summation begins from $n=1$ instead of $n=0$. Sometimes $C_{n}$ is also written as $C(n)$.

The definition for the field harmonics used so far is the one which is more common in U.S. laboratories. The European laboratories (such as CERN and HERA) use a slightly different definition [179]. The two are related as follows :

$$
\begin{aligned}
& \left(a_{n+1}\right)_{E \text { urope }}=-10^{-4}\left(a_{n}\right)_{U S} \\
& \left(b_{n+1}\right)_{E u r o p e}=10^{-4}\left(b_{n}\right)_{U S}
\end{aligned}
$$

Yet another representation of field harmonic is used in beam dynamics calculations where the particle trajectory is studied in the machine [25]. For this purpose, the field is expressed in the form of a Taylor series. The vertical component of the field on the median plane is expressed as

$$
B_{y}(x, 0)=\sum_{n=0}^{\infty} \frac{1}{n !}\left[\frac{d^{n} B_{y}}{d x^{n}}\right]_{0} x^{n},
$$

where the subscript 0 implies that the derivatives are evaluated at the equilibrium orbit (which is generally at the center of the magnet). $\mathbf{n}=0$ gives the vertical component of the field at the center of the magnet, which is represented as $B_{0}$ and the above equation becomes

$$
B_{y}(x, 0)=B_{0}+\sum_{n=1}^{\infty} \frac{1}{n !}\left[\frac{d^{n} B_{y}}{d x^{n}}\right]_{0} x^{n},
$$


Similarly, the horizontal component of the field $\left(B_{x}\right)$ on the horizontal axis (X-axis) is expressed as :

$$
B_{x}(x, 0)=\sum_{n=0}^{\infty} \frac{1}{n !}\left[\frac{d^{n} B_{x}}{d x^{n}}\right]_{0} x^{n}
$$

where, the subscript 0 implies that the derivatives are evaluated at the equilibrium orbit. $\mathbf{n}=\mathbf{0}$ gives the horizontal component of the field at the center of the magnet, which is ideally zero in the magnets considered here.

The following are defined :

$$
\begin{aligned}
& k_{n}=\frac{1}{B_{0} \rho}\left[\frac{d^{n} B_{y}}{d x^{n}}\right]_{0}, \\
& h_{n}=\frac{1}{B_{0} \rho}\left[\frac{d^{n} B_{x}}{d x^{n}}\right]_{0},
\end{aligned}
$$

with $\rho$ as the bending radius of the particle in the magnet and $\left(B_{0} \rho\right)$ as the magnetic rigidity. Therefore, the Eq. (1.5.24) and Eq. (1.5.25) become

$$
\begin{aligned}
& B_{y}(x, 0)=B_{0} \rho\left(\frac{1}{\rho}+\sum_{n=1}^{\infty} \frac{1}{n !} k_{n} x^{n}\right), \\
& B_{x}(x, 0)=B_{0} \rho\left(\sum_{n=0}^{\infty} \frac{1}{n !} h_{n} x^{n}\right) .
\end{aligned}
$$

$k_{n}$ and $h_{n}$ used in the above equations can be related to $a_{n}$ and $b_{n}$ given in Eq. (1.5.19) when the horizontal and vertical components of the field are evaluated on the horizontal axis, respectively. Therefore, with $b_{0}=10^{4}$ and $B_{R_{0}}=B_{0}$, one obtains

$$
\begin{aligned}
& h_{n}=\frac{10^{-4} n !}{\rho R_{0}^{n}} a_{n}, \\
& k_{n}=\frac{10^{-4} n !}{\rho R_{0}^{n}} b_{n} .
\end{aligned}
$$

The expressions for the horizontal and vertical component of the field in Eq. (1.5.17) can be separated out as

$$
\begin{aligned}
& B_{x}=10^{-4} B_{R_{0}} \sum_{n=0}^{\infty}\left[b_{n} \sin (n \theta)+a_{n} \cos (n \theta)\right]\left(\frac{r}{R_{0}}\right)^{n}, \\
& B_{y}=10^{-4} B_{R_{0}} \sum_{n=0}^{\infty}\left[b_{n} \cos (n \theta)-a_{n} \sin (n \theta)\right]\left(\frac{r}{R_{0}}\right)^{n} .
\end{aligned}
$$

The radial and azimuthal components of the field can be computed by using the following relations : 


$$
\left(\begin{array}{l}
B_{r} \\
B_{\theta}
\end{array}\right)=\left(\begin{array}{cc}
\cos (\theta) & \sin (\theta) \\
-\sin (\theta) & \cos (\theta)
\end{array}\right) \quad\left(\begin{array}{l}
B_{x} \\
B_{y}
\end{array}\right)
$$

Therefore, the radial and azimuthal components of the field can be written as :

$$
\begin{aligned}
& B_{r}=10^{-4} B_{R_{0}} \sum_{n=0}^{\infty}\left[b_{n} \sin [(n+1) \theta]+a_{n} \cos [(n+1) \theta]\right]\left(\frac{r}{R_{0}}\right)^{n}, \\
& B_{\theta}=10^{-4} B_{R_{0}} \sum_{n=0}^{\infty}\left[b_{n} \cos [(n+1) \theta]-a_{n} \sin [(n+1) \theta]\right]\left(\frac{r}{R_{0}}\right)^{n} .
\end{aligned}
$$

In order to represent the vector potential in terms of harmonics, the following relations can be used :

$$
B_{r}=\frac{1}{r} \frac{\partial A_{z}}{\partial \theta} \quad \text { and } \quad B_{\theta}=-\frac{\partial A_{z}}{\partial r}
$$

since in the 2-dimensional case $A_{x}=A_{y}=0$. Therefore, on integrating Eqs. (1.5.31) one obtains

$$
A_{z}=-10^{-4} B_{R_{0}} \sum_{n=0}^{\infty}\left(\frac{R_{0}}{n+1}\right)\left[b_{n} \cos [(n+1) \theta]-a_{n} \sin [(n+1) \theta]\right]\left(\frac{r}{R_{0}}\right)^{n+1} .
$$

The inverse transform can be used to obtain individual field harmonics at a reference radius $R_{0}$ in terms of field or vector potential. For this, first a component of the field or vector potential is evaluated at a radius $r$ and then the integration is performed over the azimuth as follows :

$$
\begin{aligned}
a_{n} & =-\frac{10^{4}}{\pi B_{R_{0}}}\left(\frac{R_{0}}{r}\right)^{n} \int_{0}^{2 \pi} B_{y}(r, \theta) \sin (n \theta) d \theta \\
& =\frac{10^{4}}{\pi B_{R_{0}}}\left(\frac{R_{0}}{r}\right)^{n} \int_{0}^{2 \pi} B_{x}(r, \theta) \cos (n \theta) d \theta \\
& =\frac{10^{4}}{\pi B_{R_{0}}}\left(\frac{R_{0}}{r}\right)^{n} \int_{0}^{2 \pi} B_{r}(r, \theta) \cos ((n+1) \theta) d \theta \\
& =-\frac{10^{4}}{\pi B_{R_{0}}}\left(\frac{R_{0}}{r}\right)^{n} \int_{0}^{2 \pi} B_{\theta}(r, \theta) \sin ((n+1) \theta) d \theta \\
& =\frac{10^{4}(n+1)}{\pi R_{0} B_{R_{0}}}\left(\frac{R_{0}}{r}\right)^{n+1} \int_{0}^{2 \pi} A_{z}(r, \theta) \sin ((n+1) \theta) d \theta \\
b_{n} & =\frac{10^{4}}{\pi B_{R_{0}}}\left(\frac{R_{0}}{r}\right)^{n} \int_{0}^{2 \pi} B_{y}(r, \theta) \cos (n \theta) d \theta, \\
& =\frac{10^{4}}{\pi B_{R_{0}}}\left(\frac{R_{0}}{r}\right)^{n} \int_{0}^{2 \pi} B_{x}(r, \theta) \sin (n \theta) d \theta, \\
& =\frac{10^{4}}{\pi B_{R_{0}}}\left(\frac{R_{0}}{r}\right)^{n} \int_{0}^{2 \pi} B_{r}(r, \theta) \sin ((n+1) \theta) d \theta \\
& =\frac{10^{4}}{\pi B_{R_{0}}}\left(\frac{R_{0}}{r}\right)^{n} \int_{0}^{2 \pi} B_{\theta}(r, \theta) \cos ((n+1) \theta) d \theta \\
& =-\frac{10^{4}(n+1)}{\pi R_{0} B_{R_{0}}}\left(\frac{R_{0}}{r}\right)^{n+1} \int_{0}^{2 \pi} A_{z}(r, \theta) \cos ((n+1) \theta) d \theta .
\end{aligned}
$$


For the primary harmonic component $n=m$, when the field is perpendicular to the horizontal plane, one obtains

$$
b_{m}=10^{4} \text { and } a_{m}=0 .
$$




\subsubsection{Analytic Expressions for Accelerator Magnets}

Analytic expressions for the basic cosine theta superconducting magnet design have been previously obtained and described by several authors [12-18,144,175,179]. Superconducting accelerator magnets are usually long cylindrical magnets with the current flowing parallel to the magnet axis (z-axis). The geometry of these magnets is such that one can compute the field in the body of the magnet by assuming that the current is carried by a large number of wires parallel to the z-axis. The total field is obtained by simply superimposing the field created by these wires. For this purpose, it is suitable to carry out a 2-dimensional analysis in the cylindrical coordinate system. A three dimensional analysis will be necessary for computing the field at the ends of the magnet.

Accelerator magnets are designed to produce a well defined field in the aperture of the magnets. The field in the aperture is constant for dipoles, the first derivative of the field is constant for quadrupoles and, in general, the $\mathrm{n}^{\text {th }}$ derivative is constant for the $n^{\text {th }}$ order multipole. In the following sections, the current distributions needed to produce such multipole fields will be obtained.

\subsubsection{Field and Vector Potential due to a Line Current}

To compute the magnetic field and vector potential due to a single infinitely long wire, it is assumed to carry a current $I$ in the z-direction which is perpendicular to the plane of paper. The field outside this wire at a perpendicular distance $R$ from it will be computed. The cylindrical coordinate system is used to take advantage of the symmetry of the problem.

The magnetic field produced by this wire can be directly calculated by using the integral equation $\oint \vec{H} \cdot d s=I$ (Eqs. (1.5.9)) which gives:

$$
H=\frac{I}{2 \pi R}
$$

and in a medium having a relative permeability of $\mu$

$$
B=\frac{I \mu \mu_{0}}{2 \pi R}
$$

The components of vector potential in cylindrical and Cartesian geometry can be written as

$$
\begin{aligned}
& A_{z}=\frac{\mu \mu_{0} I}{2 \pi} \ln \left(\frac{1}{R}\right), \\
& A_{r}=A_{\theta}=0 \\
& A_{x}=A_{y}=0 .
\end{aligned}
$$


The validity of the above relation is verified when the curl of the vector potential is taken to obtain the magnetic field as per Eqs. (1.5.7). This gives $B_{r}=B_{z}=0$ and $B_{\theta}=\frac{\mu \mu_{0} I}{2 \pi R}$; which is the same as in Eqs. (1.5.34) with only one component of the field present.

In accelerator magnets, the magnetic field and vector potential are usually expressed in terms of harmonic components. To develop this formalism a line current is assumed to be located at a point " $Q$ " (at $\vec{a}$ ) and the magnetic field produced by it is computed at point "P" (at $\vec{r}$ ), as shown in Fig. 1.5.1. The distance between the two is $\vec{R}=\vec{r}-\vec{a}$ with the magnitude $|R|=\sqrt{r^{2}+a^{2}-2 \operatorname{racos}(\theta-\phi)}$.

In this section, the computations will be mostly done in a space free of magnetic material where the relative permeability $\mu$ is one. Moreover, to simplify the expressions to follow, Eq. (1.5.36a) is re-written after adding a constant :

$$
A_{z}(r, \theta)=-\frac{\mu_{o} I}{2 \pi} \ln \left(\frac{R}{a}\right) ;
$$

the addition of such a constant does not change the magnetic field which is a derivative of $A_{z}$.

Now $A_{z}(r, \theta)$ will be given in terms of a series expansion containing, in general, summation of terms like $\left(\frac{r}{a}\right)^{m}$ and $\left(\frac{a}{r}\right)^{m}$, together with trigonometric functions like $\cos (m \theta)$ and $\sin (m \theta)$. The exact solution will depend on a particular problem. For example, in the solution of the case when $r$ approaches the origin $(r \rightarrow 0)$, the $\left(\frac{a}{r}\right)^{m}$ terms can't be present. Similarly in the solution of the case when $r$ approaches infinity $(r \rightarrow \infty)$, the $\left(\frac{a}{r}\right)^{m}$ terms can't be present.

In order to obtain an expansion of the $\ln$ in Eq. (1.5.37), the following manipulation is carried out :

$$
\begin{aligned}
R^{2} & =r^{2}+a^{2}-2 r a \cos (\theta-\phi) \\
\frac{R}{a} & =\left(1-\left(\frac{r}{a}\right) e^{i(\phi-\theta)}\right)^{\frac{1}{2}} \cdot\left(1-\left(\frac{r}{a}\right) e^{-i(\phi-\theta)}\right)^{\frac{1}{2}} \\
\ln \left(\frac{R}{a}\right) & =\frac{1}{2} \ln \left(1-\left(\frac{r}{a}\right) e^{i(\phi-\theta)}\right)+\frac{1}{2} \ln \left(1-\left(\frac{r}{a}\right) e^{-i(\phi-\theta)}\right) .
\end{aligned}
$$

For $|z|<1$, the logarithmic expansion is given by

$$
\ln (1-z)=-\left[z+\left(\frac{z^{2}}{2}\right)+\left(\frac{z^{3}}{3}\right)+\ldots\right]=-\sum_{n=1}^{\infty} \frac{z^{n}}{n}
$$

Therefore, for $\mathrm{r}<\mathrm{a}$

$$
\ln \left(\frac{R}{a}\right)=-\left[\frac{1}{2} \sum_{n=1}^{\infty}\left(\frac{1}{n}\right)\left(\frac{r}{a}\right)^{n} e^{i n(\phi-\theta)}+\frac{1}{2} \sum_{n=1}^{\infty}\left(\frac{1}{n}\right)\left(\frac{r}{a}\right)^{n} e^{-i n(\phi-\theta)}\right],
$$




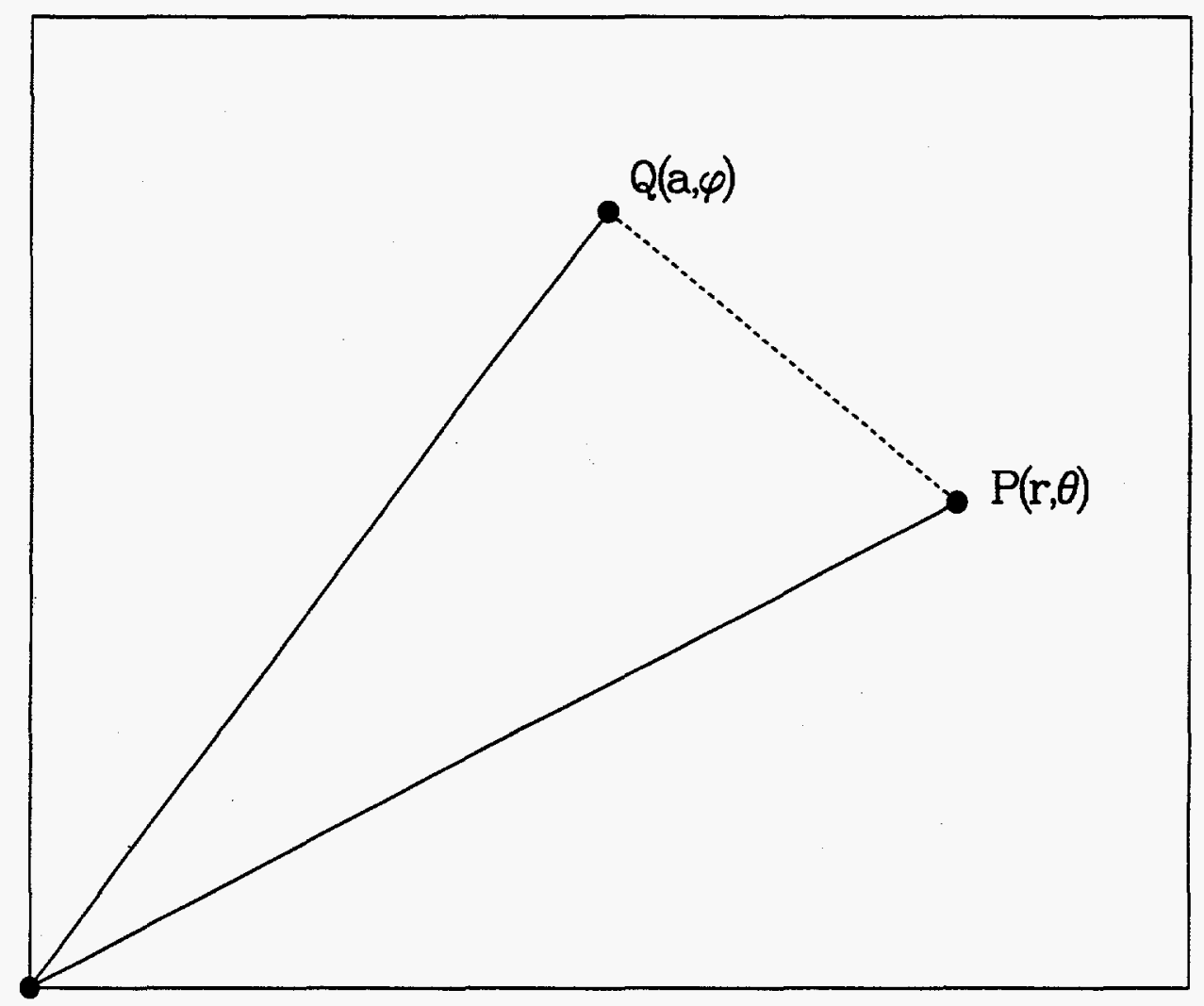

Figure 1.5.1: Computation of the field at a location "P" produced by the line current located at a position " $Q$ ".

$$
\ln \left(\frac{R}{a}\right)=-\sum_{n=1}^{\infty}\left(\frac{1}{n}\right)\left(\frac{r}{a}\right)^{n} \cos (n(\phi-\theta))
$$

Substituting Eqs. (1.5.38) in Eqs. (1.5.37) the desired expansion for the vector potential is obtained (for $r<a)$ :

$$
A_{z}(r, \theta)=\frac{\mu_{o} I}{2 \pi} \sum_{n=1}^{\infty}\left(\frac{1}{n}\right)\left(\frac{r}{a}\right)^{n} \cos (n(\phi-\theta))
$$


The magnetic field components are obtained by using Eqs. (1.5.7) and Eqs. (1.5.37) with $A_{r}=A_{\theta}=0$ :

$$
\begin{aligned}
& B_{r}=\frac{1}{r}\left(\frac{\partial A_{z}}{\partial \theta}\right), \\
& B_{\theta}=-\frac{\partial A_{z}}{\partial r} \\
& B_{z}=0 .
\end{aligned}
$$

Therefore, for $\mathrm{r}<\mathrm{a}$, one would obtain :

$$
\begin{aligned}
& B_{r}=\frac{\mu_{o} I}{2 \pi a} \sum_{n=1}^{\infty}\left(\frac{r}{a}\right)^{n-1} \sin [(n)(\phi-\theta)], \\
& B_{\theta}=-\frac{\mu_{o} I}{2 \pi a} \sum_{n=1}^{\infty}\left(\frac{r}{a}\right)^{n-1} \cos [(n)(\phi-\theta)], \\
& B_{z}=0
\end{aligned}
$$

In order to compute the harmonics components, the above equations are compared with Eqs. (1.5.31). It should be noted that there the summation starts from $n=0$ instead of $n=1$ in Eq. (1.5.39). The following expressions for the normal and skew harmonics at a reference radius $R_{0}$ are obtained for a line current located at $(a, \phi)$ :

$$
\begin{aligned}
& b_{n}=10^{4}\left(\frac{R_{0}}{a}\right)^{n} \cos [(n+1) \phi], \\
& a_{n}=-10^{4}\left(\frac{R_{0}}{a}\right)^{n} \sin [(n+1) \phi],
\end{aligned}
$$

and $B_{R_{0}}=-\frac{\mu_{0} I}{2 \pi a}$.

For $\mathbf{r}>$ a case, the following rearrangement is performed to obtain an appropriate expansion :

$$
\begin{aligned}
\frac{R}{a} & =\left(\frac{r}{a}\right)\left(1-\left(\frac{a}{r}\right) e^{i(\phi-\theta)}\right)^{\frac{1}{2}} \cdot\left(1-\left(\frac{a}{r}\right) e^{-i(\phi-\theta)}\right)^{\frac{1}{2}} \\
\ln \left(\frac{R}{a}\right) & =\ln \left(\frac{r}{a}\right)+\left[\frac{1}{2} \ln \left(1-\left(\frac{a}{r}\right) e^{i(\phi-\theta)}\right)+\frac{1}{2} \ln \left(1-\left(\frac{a}{r}\right) e^{-i(\phi-\theta)}\right)\right] \\
\ln \left(\frac{R}{a}\right) & =\ln \left(\frac{r}{a}\right)-\left[\frac{1}{2} \sum_{n=1}^{\infty}\left(\frac{1}{n}\right)\left(\frac{a}{r}\right)^{n} e^{i n(\phi-\theta)}+\frac{1}{2} \sum_{n=1}^{\infty}\left(\frac{1}{n}\right)\left(\frac{a}{r}\right)^{n} e^{-i n(\phi-\theta)}\right], \\
\ln \left(\frac{R}{a}\right) & =\ln \left(\frac{r}{a}\right)-\sum_{n=1}^{\infty}\left(\frac{1}{n}\right)\left(\frac{a}{r}\right)^{n} \cos (n(\phi-\theta)) .
\end{aligned}
$$

Therefore, for $I>$ a, one obtains the following expression for the vector potential :

$$
A_{z}(r, \theta)=-\frac{\mu_{o} I}{2 \pi} \ln \left(\frac{r}{a}\right)+\frac{\mu_{o} I}{2 \pi} \sum_{n=1}^{\infty}\left(\frac{1}{n}\right)\left(\frac{a}{r}\right)^{n} \cos (n(\phi-\theta))
$$


The magnetic field components are obtained by using Eqs. (1.5.40) :

$$
\begin{aligned}
& B_{r}=\frac{\mu_{o} I}{2 \pi a} \sum_{n=0}^{\infty}\left(\frac{a}{r}\right)^{n+1} \sin (n(\phi-\theta)), \\
& B_{\theta}=\frac{\mu_{o} I}{2 \pi a} \sum_{n=0}^{\infty}\left(\frac{a}{r}\right)^{n+1} \cos (n(\phi-\theta)) \\
& B_{x}=0
\end{aligned}
$$

It may be noted that in the expression for $B_{\theta}$, the summation in $n$ starts from $n=0$ instead of $n=1$. The $\left(B_{x}, B_{y}\right)$ components of the field can be computed using the following relation:

$$
\left(\begin{array}{l}
B_{x} \\
B_{y}
\end{array}\right)=\left(\begin{array}{cc}
\cos (\theta) & -\sin (\theta) \\
\sin (\theta) & \cos (\theta)
\end{array}\right) \quad\left(\begin{array}{l}
B_{r} \\
B_{\theta}
\end{array}\right)
$$




\subsubsection{Line Current in a Cylindrical Iron Cavity}

Expressions are obtained here for the vector potential and magnetic field due to an infinitely long paraxial filament of current $I$ at a radius $a$ in a cylindrical cavity having a radius $R_{f}>a$. The iron is infinitely long and infinitely thick and has a constant relative permeability $\mu$, which is referred to here simply as permeability following the convention explained earlier. The method of image currents can be applied to include the contribution from the iron [179]. The expressions are obtained here by matching the boundary conditions at the interface of the air and iron boundary [19]. General expressions for the vector potential and the components of the field in the region $a<r<R_{f}$, are given by :

$$
\begin{aligned}
A_{z}(r, \theta)= & -\frac{\mu_{o} I}{2 \pi} \ln \left(\frac{r}{a}\right)+\frac{\mu_{o} I}{2 \pi} \sum_{n=1}^{\infty}\left(\frac{1}{n}\right)\left(\frac{a}{r}\right)^{n} \cos (n(\phi-\theta)) \\
& +\mu_{o} \sum_{n=1}^{\infty} E_{n} r^{n} \cos (n(\phi-\theta)) \\
B_{r}= & \frac{\mu_{o} I}{2 \pi a} \sum_{n=1}^{\infty}\left(\frac{a}{r}\right)^{n+1} \sin (n(\phi-\theta)) \\
& +\mu_{o} \sum_{n=1}^{\infty} n E_{n} r^{n-1} \sin (n(\phi-\theta)) \\
H_{\theta}= & \frac{I}{2 \pi a} \sum_{n=0}^{\infty}\left(\frac{a}{r}\right)^{n+1} \cos (n(\phi-\theta)) \\
& -\sum_{n=1}^{\infty} n E_{n} r^{n-1} \cos (n(\phi-\theta))
\end{aligned}
$$

and in the region $r>R_{f}$ :

$$
\begin{aligned}
A_{z}(r, \theta)= & \mu \mu_{o} F_{o} \ln \left(\frac{r}{a}\right) \\
& +\mu \mu_{o} \sum_{n=1}^{\infty} F_{n}\left(\frac{1}{r}\right)^{n} \cos (n(\phi-\theta)), \\
B_{r}= & \mu \mu_{o} \sum_{n=1}^{\infty} n F_{n}\left(\frac{1}{r}\right)^{n+1} \sin (n(\phi-\theta)), \\
H_{\theta}=-\frac{F_{o}}{r} & +\sum_{n=1}^{\infty} n F_{n}\left(\frac{1}{r}\right)^{n+1} \cos (n(\phi-\theta)),
\end{aligned}
$$

where $E_{n}$ and $F_{n}$ are coefficients which can be determined by the boundary conditions at $r=R_{f}$ that

$$
\begin{aligned}
& \left(B_{r}\right)_{a i r}=\left(B_{r}\right)_{i r o n}, \\
& \left(H_{\theta}\right)_{a i r}=\left(H_{\theta}\right)_{i r o n},
\end{aligned}
$$


i.e. the normal component of $B$ and the azimuthal component of $H$ are continuous. Therefore, the required boundary conditions at $r=R_{f}$ for $n \neq 0$ gives :

$$
\begin{aligned}
\frac{\mu_{o} I}{2 \pi a}\left(\frac{a}{R_{f}}\right)^{n+1}+n \mu_{o} E_{n} R_{f}^{n-1} & =n \mu_{0} \mu F_{n}\left(\frac{1}{R_{f}}\right)^{n+1} \\
\frac{I}{2 \pi a}\left(\frac{a}{R_{f}}\right)^{n+1}-n E_{n} R_{f}^{n-1} & =n F_{n}\left(\frac{1}{R_{f}}\right)^{n+1}
\end{aligned}
$$

which gives

$$
\begin{aligned}
& E_{n}=\frac{1}{n} \frac{\mu-1}{\mu+1} \frac{I}{2 \pi}\left(\frac{a}{R_{f}^{2}}\right)^{n} \\
& F_{n}=\frac{1}{n} \frac{2}{\mu+1} \frac{I}{2 \pi a} a^{n+1}
\end{aligned}
$$

The $n=0$ term appears only in the expression for $H_{\theta}$ and on matching the boundary condition, one obtains :

$$
\frac{I}{2 \pi R_{f}}=-\frac{F_{o}}{R_{f}}
$$

which gives

$$
F_{\circ}=-\frac{I}{2 \pi}
$$

The expressions for vector potential and field components for $a<r<R_{f}$ case are obtained when $E_{n}$ from Eq. (1.5.51a) is substituted in Eq. (1.5.47) and Eqs. (1.5.48) :

$$
\begin{aligned}
A_{z}(r, \theta)= & -\frac{\mu_{o} I}{2 \pi} \ln \left(\frac{r}{a}\right) \\
& +\frac{\mu_{o} I}{2 \pi} \sum_{n=1}^{\infty} \frac{1}{n}\left(\frac{a}{r}\right)^{n} \cos (n(\phi-\theta))\left[1+\frac{\mu-1}{\mu+1}\left(\frac{r}{R_{f}}\right)^{2 n}\right], \\
B_{r}= & \frac{\mu_{o} I}{2 \pi a} \sum_{n=1}^{\infty}\left(\frac{a}{r}\right)^{n+1} \sin (n(\phi-\theta))\left[1+\frac{\mu-1}{\mu+1}\left(\frac{r}{R_{f}}\right)^{2 n}\right] \\
B_{\theta}= & \frac{\mu_{o} I}{2 \pi r}+\frac{\mu_{o} I}{2 \pi a} \sum_{n=1}^{\infty}\left(\frac{a}{r}\right)^{n+1} \cos (n(\phi-\theta))\left[1-\frac{\mu-1}{\mu+1}\left(\frac{r}{R_{f}}\right)^{2 n}\right] .
\end{aligned}
$$

In the above equations, the second term in the square brackets is the additional contribution of the iron to the field produced by the coil.

To obtain the expressions for the vector potential and field for $r<a$ it must be noted that a current filament is present at $r=a$. However, the radial component of the field $B_{r}$ must still be continuous, i.e. at $r=a$

$$
B_{r}(i n)=B_{r}(o u t)
$$


where $B_{r}($ in $)$ and $B_{r}$ (out) are the magnetic induction for $r<a$ and $a<r<R_{f}$ respectively. The presence of the source (current), however, gives a discontinuity in the azimuthal component of the field $H_{\theta}$ with $H_{\theta}(i n)-H_{\theta}(o u t)$ determined by the current density at $r=a$. A general expression for the vector potential for $r<a$ is given by (see Eq. (1.5.39)) :

$$
A_{z}(r, \theta)=\mu_{o} \sum_{n=1}^{\infty} I_{n} r^{n} \cos (n(\phi-\theta)),
$$

where the $I_{n}$ are unknown coefficients. Using Eqs. (1.5.40):

$$
\begin{aligned}
& B_{r}=\mu_{0} \sum_{n=1}^{\infty} I_{n} n r^{n-1} \sin (n(\phi-\theta)), \\
& B_{\theta}=-\mu_{0} \sum_{n=1}^{\infty} I_{n} n r^{n-1} \cos (n(\phi-\theta)), \\
& B_{z}=0 .
\end{aligned}
$$

In order for $B_{r}$ to be continuous at $r=a$ one obtains from Eq. (1.5.56a) and Eq. (1.5.54a) :

$$
I_{n}=\frac{I}{2 \pi}\left(\frac{1}{n}\right)\left(\frac{1}{a}\right)^{n}\left[1+\frac{\mu-1}{\mu+1}\left(\frac{a}{R_{f}}\right)^{2 n}\right] .
$$

Using this in Eqs. (1.5.56) gives the expressions for the field and vector potential for $r<a$ as :

$$
\begin{aligned}
A_{z}(r, \theta) & =\frac{\mu_{o} I}{2 \pi} \sum_{n=1}^{\infty}\left(\frac{1}{n}\right)\left(\frac{r}{a}\right)^{n} \cos (n(\phi-\theta))\left[1+\frac{\mu-1}{\mu+1}\left(\frac{a}{R_{f}}\right)^{2 n}\right] . \\
B_{r} & =\frac{\mu_{o} I}{2 \pi a} \sum_{n=1}^{\infty}\left(\frac{r}{a}\right)^{n-1} \sin (n(\phi-\theta))\left[1+\frac{\mu-1}{\mu+1}\left(\frac{a}{R_{f}}\right)^{2 n}\right], \\
B_{\theta} & =-\frac{\mu_{o} I}{2 \pi a} \sum_{n=1}^{\infty}\left(\frac{r}{a}\right)^{n-1} \cos (n(\phi-\theta))\left[1+\frac{\mu-1}{\mu+1}\left(\frac{a}{R_{f}}\right)^{2 n}\right], \\
B_{z} & =0 .
\end{aligned}
$$

To compute the field harmonics the procedure of Eqs. (1.5.42) is repeated. As before, the summation over $n$ in the above is now changed so that it starts from $n=0$ instead of $\mathrm{n}=1$.

$$
\begin{aligned}
& b_{n}=10^{4}\left(\frac{R_{0}}{a}\right)^{n} \cos ((n+1) \phi)\left[1+\frac{\mu-1}{\mu+1}\left(\frac{a}{R_{f}}\right)^{2(n+1)}\right] \\
& a_{n}=-10^{4}\left(\frac{R_{0}}{a}\right)^{n} \sin ((n+1) \phi)\left[1+\frac{\mu-1}{\mu+1}\left(\frac{a}{R_{f}}\right)^{2(n+1)}\right]
\end{aligned}
$$


All expressions derived so far reproduce the results obtained from the method of images [179] which says that the effect of iron can be replaced by an additional line current $I^{\prime}$ located at $\left(a^{\prime}, \phi\right)$ with

$$
\begin{aligned}
& I^{\prime}=\left(\frac{\mu-1}{\mu+1}\right) I \\
& a^{\prime}=\frac{R_{f}^{2}}{a}
\end{aligned}
$$

The expressions for vector potential and field components for $r>R_{f}$ case are obtained when $F_{n}$ from Eq. (1.5.516) and Eq. (1.5.52) are substituted in Eqs. (1.5.49) and Eqs. (1.5.50) :

$$
\begin{aligned}
A_{z}(r, \theta) & =-\frac{\mu \mu_{o} I}{2 \pi} \ln \left(\frac{r}{a}\right)+\frac{2 \mu}{\mu+1} \frac{\mu_{o} I}{2 \pi} \sum_{n=1}^{\infty}\left(\frac{1}{n}\right)\left(\frac{a}{r}\right)^{n} \cos (n(\phi-\theta)) \\
B_{r} & =\frac{2 \mu}{\mu+1} \frac{\mu_{o} I}{2 \pi a} \sum_{n=1}^{\infty}\left(\frac{a}{r}\right)^{n+1} \sin (n(\phi-\theta)) \\
B_{\theta} & =\frac{\mu \mu_{o} I}{2 \pi r}+\frac{2 \mu}{\mu+1} \frac{\mu_{o} I}{2 \pi a} \sum_{n=1}^{\infty}\left(\frac{a}{r}\right)^{n+1} \cos (n(\phi-\theta)) .
\end{aligned}
$$




\subsubsection{Line Current in a Cylindrical Iron Shell}

In deriving the expressions for the vector potential and field due to a line current inside an cylindrical iron it was assumed in the last section that the iron outer boundary extends to infinity. This is, however, not the case in practice. If the outer diameter of the cylindrical iron shell is $R_{a}$, then the general expressions for the vector potential in the various regions are given by :

$$
\begin{aligned}
& A_{z}(r, \theta)=\mu_{o} \sum_{n=1}^{\infty} I_{n}^{\prime} r^{n} \cos (n(\phi-\theta)), \quad[\text { for } \quad \mathrm{r}<\mathrm{a}] \\
& A_{z}(r, \theta)=-\frac{\mu_{o} I}{2 \pi} \ln \left(\frac{r}{a}\right)+\frac{\mu_{o} I}{2 \pi} \sum_{n=1}^{\infty}\left(\frac{1}{n}\right)\left(\frac{a}{r}\right)^{n} \cos (n(\phi-\theta)) \\
& +\mu_{o} \sum_{n=1}^{\infty} E_{n}^{\prime} r^{n} \cos (n(\phi-\theta)), \quad\left[\text { for } \quad a<r<R_{f}\right] \\
& A_{z}(r, \theta)=\mu \mu_{o} F_{o}^{\prime} \ln \left(\frac{r}{a}\right) \\
& +\mu \mu_{o} \sum_{n=1}^{\infty} F_{n}^{\prime}\left(\frac{1}{r}\right)^{n} \cos (n(\phi-\theta)) \\
& +\mu \mu_{o} \sum_{n=1}^{\infty} G_{n}^{\prime} r^{n} \cos (n(\phi-\theta)), \quad\left[\text { for } \quad R_{\mathfrak{f}}<r<R_{a}\right] \\
& A_{z}(r, \theta)=\mu_{o} H_{o}^{\prime} \ln \left(\frac{r}{a}\right) \\
& +\mu_{o} \sum_{n=1}^{\infty} H_{n}^{\prime}\left(\frac{1}{r}\right)^{n} \cos (n(\phi-\theta)), \quad\left[\text { for } \quad \mathrm{r}>\mathrm{R}_{\mathrm{a}}\right]
\end{aligned}
$$

Following an approach similar to one used in previous section, the five coefficients $\left(E_{n}^{\prime}, F_{n}^{\prime}, G_{n}^{\prime}, H_{n}^{\prime}, I_{n}^{\prime}\right)$ are obtained by matching the five boundary conditions $\left(B_{r}\right.$ is continuous at $r=a, r=R_{f}$ and $r=R_{a}$ and $B_{\theta}$ is continuous at $r=R_{f}$ and $r=R_{a}$ ). The results of that exercise for $n>0$ are given here :

$$
\begin{aligned}
& I_{n}^{\prime}=\frac{I}{2 \pi}\left(\frac{1}{n}\right)\left(\frac{1}{a}\right)^{n}\left[1+\frac{\mu-1}{\mu+1}\left(\frac{a}{R_{f}}\right)^{2 n} \frac{\left[1-\left(\frac{R_{f}}{R_{a}}\right)^{2 n}\right]}{\left[1-\left(\frac{\mu-1}{\mu+1}\right)^{2}\left(\frac{R_{f}}{R_{a}}\right)^{2 n}\right]}\right], \\
& E_{n}^{\prime}=\frac{1}{n} \frac{\mu-1}{\mu+1} \frac{I}{2 \pi}\left(\frac{a}{R_{f}^{2}}\right)^{n} \frac{\left[1-\left(\frac{R_{f}}{R_{a}}\right)^{2 n}\right]}{\left[1-\left(\frac{\mu-1}{\mu+1}\right)^{2}\left(\frac{R_{f}}{R_{a}}\right)^{2 n}\right]}, \\
& F_{n}^{\prime}=\frac{1}{\mu+1} \frac{I}{n \pi} \frac{a^{n}}{\left[1-\left(\frac{\mu-1}{\mu+1}\right)^{2}\left(\frac{R_{f}}{R_{a}}\right)^{2 n}\right]},
\end{aligned}
$$




$$
\begin{aligned}
& G_{n}^{\prime}=-\frac{(\mu-1)}{(\mu+1)^{2}} \frac{I}{n \pi} \frac{\left(\frac{a}{R_{a}^{2}}\right)^{n}}{\left[1-\left(\frac{\mu-1}{\mu+1}\right)^{2}\left(\frac{R_{f}}{R_{a}}\right)^{2 n}\right]}, \\
& H_{n}^{\prime}=\frac{2 \mu}{(\mu+1)^{2}} \frac{I}{n \pi} \frac{a^{n}}{\left[1-\left(\frac{\mu-1}{\mu+1}\right)^{2}\left(\frac{R_{f}}{R_{a}}\right)^{2 n}\right]},
\end{aligned}
$$

and for $n=0$, the terms are:

$$
F_{o}^{\prime}=H_{o}^{\prime}=-\frac{I}{2 \pi}
$$

Therefore, the expressions for the vector potential and field components in various regions due to a line current $I$ at $(a, \theta)$ inside a cylindrical iron shell having inner radius $R_{f}$ and outer radius $R_{a}$ are given as follows (in each case $B_{z}(r, \theta)=0$ ) :

\section{Inside Coil $(\mathbf{r}<\mathbf{a})$}

$$
\begin{gathered}
A_{z}(r, \theta)=\frac{\mu_{o} I}{2 \pi} \sum_{n=1}^{\infty}\left(\frac{1}{n}\right)\left[1+\frac{\mu-1}{\mu+1}\left(\frac{a}{R_{f}}\right)^{2 n} \frac{\left[1-\left(\frac{R_{f}}{R_{a}}\right)^{2 n}\right]}{\left[1-\left(\frac{\mu-1}{\mu+1}\right)^{2}\left(\frac{R_{f}}{R_{a}}\right)^{2 n}\right]}\right] \times \\
\left(\frac{r}{a}\right)^{n} \cos (n(\phi-\theta)) \cdot \\
B_{r}=\frac{\mu_{o} I}{2 \pi a} \sum_{n=1}^{\infty}\left[1+\frac{\mu-1}{\mu+1}\left(\frac{a}{R_{f}}\right)^{2 n} \frac{\left[1-\left(\frac{R_{f}}{R_{a}}\right)^{2 n}\right]}{\left[1-\left(\frac{\mu-1}{\mu+1}\right)^{2}\left(\frac{R_{f}}{R_{a}}\right)^{2 n}\right]}\right] \times \\
\left.\left.B_{\theta}=-\frac{\mu_{o} I}{2 \pi a} \sum_{n=1}^{\infty}\left[1+\frac{\mu-1}{\mu+1}\right)^{n-1} \sin (n(\phi-\theta)), \frac{a}{R_{f}}\right)^{2 n} \frac{\left[1-\left(\frac{R_{f}}{R_{a}}\right)^{2 n}\right]}{\left[1-\left(\frac{\mu-1}{\mu+1}\right)^{2}\left(\frac{R_{f}}{R_{a}}\right)^{2 n}\right]}\right] \times \\
\left(\frac{r}{a}\right)^{n-1} \cos (n(\phi-\theta)),
\end{gathered}
$$

$\underline{\text { Between Coil and Iron }\left(a<r<\mathbf{R}_{f}\right)}$

$$
\begin{gathered}
A_{z}(r, \theta)=-\frac{\mu_{o} I}{2 \pi} \ln \left(\frac{r}{a}\right)+\frac{\mu_{o} I}{2 \pi} \sum_{n=1}^{\infty}\left(\frac{1}{n}\right)\left(1+\frac{\frac{\mu-1}{\mu+1}\left(\frac{r}{R_{f}}\right)^{2 n}\left[1-\left(\frac{R_{f}}{R_{a}}\right)^{2 m}\right]}{1-\left(\frac{\mu-1}{\mu+1}\right)^{2}\left(\frac{R_{f}}{R_{a}}\right)^{2 n}}\right) \times \\
\quad\left(\frac{a}{r}\right)^{n} \cos (n(\phi-\theta)), \\
B_{r}=\frac{\mu_{o} I}{2 \pi a} \sum_{n=1}^{\infty}\left(1+\frac{\frac{\mu-1}{\mu+1}\left(\frac{r}{R_{f}}\right)^{2 n}\left[1-\left(\frac{R_{f}}{R_{a}}\right)^{2 m}\right]}{1-\left(\frac{\mu-1}{\mu+1}\right)^{2}\left(\frac{R_{f}}{R_{a}}\right)^{2 n}}\right) \times
\end{gathered}
$$




$$
\begin{gathered}
\left(\frac{a}{r}\right)^{n+1} \sin (n(\phi-\theta)), \\
B_{\theta}=\frac{\mu_{o} I}{2 \pi r}+\frac{\mu_{o} I}{2 \pi a} \sum_{n=1}^{\infty}\left(1-\frac{\frac{\mu-1}{\mu+1}\left(\frac{r}{R_{f}}\right)^{2 n}\left[1-\left(\frac{R_{f}}{R_{a}}\right)^{2 m}\right]}{1-\left(\frac{\mu-1}{\mu+1}\right)^{2}\left(\frac{R_{f}}{R_{a}}\right)^{2 n}}\right) \times \\
\left(\frac{a}{r}\right)^{n+1} \cos (n(\phi-\theta)) .
\end{gathered}
$$

Inside Iron $\left(\mathbf{R}_{\mathbf{f}}<\mathbf{r}<\mathbf{R}_{\mathbf{a}}\right)$

$$
\begin{gathered}
A_{z}(r, \theta)=-\frac{\mu \mu_{o} I}{2 \pi} \ln \left(\frac{r}{a}\right) \\
+\frac{\mu \mu_{o} I}{\pi(\mu+1)} \sum_{n=1}^{\infty}\left(\frac{1}{n}\right) \frac{1-\frac{\mu-1}{\mu+1}\left(\frac{r}{R_{a}}\right)^{2 n}}{1-\left(\frac{\mu-1}{\mu+1}\right)^{2}\left(\frac{R_{f}}{R_{a}}\right)^{2 n}} \times \\
\quad\left(\frac{a}{r}\right)^{n} \cos (n(\phi-\theta)), \\
B_{r}=\frac{\mu \mu_{o} I}{\pi a(\mu+1)} \sum_{n=1}^{\infty} \frac{1-\frac{\mu-1}{\mu+1}\left(\frac{r}{R_{a}}\right)^{2 n}\left(\frac{\mu-1}{\mu+1}\right)^{2}\left(\frac{R_{f}}{R_{a}}\right)^{2 n} \times}{\left(\frac{a}{r}\right)^{n+1} \sin (n(\phi-\theta)),} \\
B_{\theta}=\frac{\mu \mu_{o} I}{2 \pi r}+\frac{\mu \mu_{o} I}{\pi a(\mu+1)} \sum_{n=1}^{\infty} \frac{1+\frac{\mu-1}{\mu+1}\left(\frac{r}{R_{a}}\right)^{2 n}}{1-\left(\frac{\mu-1}{\mu+1}\right)^{2}\left(\frac{R_{f}}{R_{a}}\right)^{2 n}} \times \\
\left(\frac{a}{r}\right)^{n+1} \cos (n(\phi-\theta)) \cdot
\end{gathered}
$$

Outside Iron $\left(\mathbf{r}>\mathbf{R}_{\mathrm{a}}\right)$

$$
\begin{aligned}
A_{z}(r, \theta)= & -\frac{\mu_{o} I}{2 \pi} \ln \left(\frac{r}{a}\right)+\frac{2 \mu \mu_{o} I}{\pi(\mu+1)^{2}} \sum_{n=1}^{\infty} \frac{1}{1-\left(\frac{\mu-1}{\mu+1}\right)^{2}\left(\frac{R_{f}}{R_{a}}\right)^{2 n}} \times \\
& \left(\frac{1}{n}\right)\left(\frac{a}{r}\right)^{n} \cos (n(\phi-\theta)) \\
B_{r}= & \frac{2 \mu}{(\mu+1)^{2}} \frac{\mu_{o} I}{\pi a} \sum_{n=1}^{\infty} \frac{1}{1-\left(\frac{\mu-1}{\mu+1}\right)^{2}\left(\frac{R_{f}}{R_{a}}\right)^{2 n}} \times \\
& \left(\frac{a}{r}\right)^{n+1} \sin (n(\phi-\theta)), \\
B_{\theta}= & \frac{\mu_{o} I}{2 \pi r}+\frac{2 \mu}{(\mu+1)^{2}} \frac{\mu_{o} I}{\pi a} \sum_{n=0}^{\infty} \frac{1}{1-\left(\frac{\mu-1}{\mu+1}\right)^{2}\left(\frac{R_{f}}{R_{a}}\right)^{2 n}} \times \\
& \left(\frac{a}{r}\right)^{n+1} \cos (n(\phi-\theta)) .
\end{aligned}
$$




\section{Field Harmonics}

The field harmonics are given by :

$$
\begin{aligned}
b_{n}=10^{4}\left(\frac{R_{0}}{a}\right)^{n} \cos ((n+1) \phi) & {\left[1+\frac{\mu-1}{\mu+1}\left(\frac{a}{R_{f}}\right)^{2(n+1)} \frac{1-\left(\frac{R_{f}}{R_{a}}\right)^{2(n+1)}}{1-\left(\frac{\mu-1}{\mu+1}\right)^{2}\left(\frac{R_{f}}{R_{a}}\right)^{2(n+1)}}\right], } \\
a_{n}=-10^{4}\left(\frac{R_{0}}{a}\right)^{n} \sin ((n+1) \phi) & {\left[1+\frac{\mu-1}{\mu+1}\left(\frac{a}{R_{f}}\right)^{2(n+1)} \frac{1-\left(\frac{R_{f}}{R_{a}}\right)^{2(n+1)}}{1-\left(\frac{\mu-1}{\mu+1}\right)^{2}\left(\frac{R_{f}}{R_{a}}\right)^{2(n+1)}}\right] . }
\end{aligned}
$$




\subsubsection{Field and Harmonics due to Current Blocks in Air}

The expressions derived for the line current in the section 1.5.3.1 are extended here for one or more blocks of current. The geometry of the problem is such that a wire is replaced by a radial block between radii $\rho_{1}$ and $\rho_{2}$ and angle $\phi_{1}$ and $\phi_{2}$. The block has a constant current density $J$ such that the total current is still $I$ with $I=\frac{1}{2} J\left(\rho_{2}^{2}-\rho_{1}^{2}\right)\left(\phi_{2}-\phi_{1}\right)$. To compute the vector potential and component of field at $(r, \theta)$ Eq. (1.5.36) and Eqs. (1.5.41) should be integrated $[179]$ as $\left(\right.$ for $\left.r<\rho_{1}\right)$ :

$$
\begin{aligned}
& A_{z}(r, \theta)=\sum_{n=1}^{\infty} \int_{\rho_{1}}^{\rho_{2}} \frac{\mu_{o} J}{2 \pi}\left(\frac{1}{n}\right)\left(\frac{r}{a}\right)^{n} a d a \int_{\phi_{1}}^{\phi_{2}} \cos [n(\phi-\theta)] d \phi, \\
& B_{r}(r, \theta)=\sum_{n=1}^{\infty} \int_{\rho_{1}}^{\rho_{2}} \frac{\mu_{o} J}{2 \pi a}\left(\frac{r}{a}\right)^{n-1} a d a \int_{\phi_{1}}^{\phi_{2}} \sin [n(\phi-\theta)] d \phi, \\
& B_{\theta}(r, \theta)=-\sum_{n=1}^{\infty} \int_{\rho_{1}}^{\rho_{2}} \frac{\mu_{o} J}{2 \pi a}\left(\frac{r}{a}\right)^{n-1} a d a \int_{\phi_{1}}^{\phi_{2}} \cos [n(\phi-\theta)] d \phi .
\end{aligned}
$$

The integration of the above equations for the vector potential and the field components gives :

$$
\begin{aligned}
A_{z}(r, \theta)=\frac{\mu_{o} J r}{2 \pi}\left(\rho_{2}-\rho_{1}\right)\left[\sin \left(\phi_{2}-\theta\right)-\sin \left(\phi_{1}-\theta\right)\right] & \\
& +\frac{\mu_{o} J r^{2}}{8 \pi} \ln \left(\frac{\rho_{2}}{\rho_{1}}\right)\left[\sin \left(2\left(\phi_{2}-\theta\right)\right)-\sin \left(2\left(\phi_{1}-\theta\right)\right)\right] \\
& -\frac{\mu_{o} J}{2 \pi} \sum_{n=3}^{\infty} \frac{r^{n}}{n^{2}(n-2)}\left(\frac{1}{\rho_{2}^{n-2}}-\frac{1}{\rho_{1}^{n-2}}\right) \times \\
B_{r}(r, \theta)=- & \frac{\mu_{o} J}{2 \pi}\left(\rho_{2}-\rho_{1}\right)\left[\cos \left(\phi_{2}-\theta\right)-\cos \left(\phi_{1}-\theta\right)\right] \\
& -\frac{\mu_{o} J r}{4 \pi} \ln \left(\frac{\rho_{2}}{\rho_{1}}\right)\left[\cos \left(2\left(\phi_{2}-\theta\right)\right)-\cos \left(2\left(\phi_{1}-\theta\right)\right)\right] \\
& +\frac{\mu_{o} J}{2 \pi} \sum_{n=3}^{\infty} \frac{r^{n-1}}{n(n-2)}\left(\frac{1}{\rho_{2}^{n-2}}-\frac{1}{\rho_{1}^{n-2}}\right) \times \\
& -\frac{\mu_{o} J}{2 \pi}\left(\rho_{2}-\rho_{1}\right)\left[\sin \left(\phi_{2}-\theta\right)-\sin \left(\phi_{1}-\theta\right)\right] \\
& -\frac{\mu_{o} J r}{4 \pi} \ln \left(\frac{\rho_{2}}{\rho_{1}}\right)\left[\sin \left(2\left(\phi_{2}-\theta\right)\right)-\sin \left(2\left(\phi_{1}-\theta\right)\right)\right] \\
& \left.+\frac{\mu_{o} J}{2 \pi} \sum_{n=3}^{\infty} \frac{r^{n-1}}{n(n-2)}\left(\frac{1}{\rho_{2}^{n-2}}-\frac{1}{\rho_{1}^{n-2}}\right) \times \sin \left(n\left(\phi_{2}-\theta\right)\right)-\sin \left(n\left(\phi_{1}-\theta\right)\right)\right]
\end{aligned}
$$

Now the harmonics components $a_{n}$ and $b_{n}$ (the dimensionless coefficients as defined in Eqs. (1.5.31)) are computed due to the field from a single current block. It should be 
noted that the summation of $a_{n}$ and $b_{n}$ starts from $n=0$ instead of $n=1$ in Eq. (1.5.78). For $n>1$ and harmonics normalized to the dipole field, the following expressions for the normal and skew harmonics at a reference radius $R_{o}$ are obtained using the procedure of Eqs. (1.5.42) :

$$
\begin{aligned}
& b_{n}=\frac{-10^{4} R_{0}^{n}}{\left(n^{2}-1\right)}[\left.\left(\frac{1}{\rho_{2}^{n-1}}-\frac{1}{\rho_{1}^{n-1}}\right) /\left(\rho_{2}-\rho_{1}\right)\right] \\
& \frac{\sin \left((n+1) \phi_{2}\right)-\sin \left((n+1) \phi_{1}\right)}{\sin \left(\phi_{2}\right)-\sin \left(\phi_{1}\right)} \\
& a_{n}=\frac{-10^{4} R_{0}^{n}}{\left(n^{2}-1\right)}\left[\begin{array}{c}
\left.\left(\frac{1}{\rho_{2}^{n-1}}-\frac{1}{\rho_{1}^{n-1}}\right) /\left(\rho_{2}-\rho_{1}\right)\right] \\
\frac{\cos \left((n+1) \phi_{2}\right)-\cos \left((n+1) \phi_{1}\right)}{\sin \left(\phi_{2}\right)-\sin \left(\phi_{1}\right)}
\end{array}\right.
\end{aligned}
$$

and the harmonic expressions for $n=1$ are

$$
\begin{aligned}
& b_{n}=\frac{10^{4} R_{0} \ln \left(\frac{\rho_{2}}{\rho_{1}}\right)}{\rho_{2}-\rho_{1}} \frac{\sin \left(2 \phi_{2}\right)-\sin \left(2 \phi_{1}\right)}{\sin \left(\phi_{2}\right)-\sin \left(\phi_{1}\right)}, \\
& a_{n}=\frac{10^{4} R_{0} \ln \left(\frac{\rho_{2}}{\rho_{1}}\right)}{\rho_{2}-\rho_{1}} \frac{\cos \left(2 \phi_{2}\right)-\cos \left(2 \phi_{1}\right)}{\sin \left(\phi_{2}\right)-\sin \left(\phi_{1}\right)} .
\end{aligned}
$$

To compute $A_{n}$ and $B_{n}$ (having the dimensions of field and defined in Eqs. (1.5.21)) one derives the expressions for field components from Eqs. (1.5.78) at a reference radius $R_{0}$ in the form of :

$$
\begin{aligned}
& B_{r}=\sum_{n=1}^{\infty}\left(\frac{r}{R_{o}}\right)^{n-1}\left[B_{n} \sin (n \theta)+A_{n} \cos (n \theta)\right] \\
& B_{\theta}=\sum_{n=1}^{\infty}\left(\frac{r}{R_{o}}\right)^{n-1}\left[B_{n} \cos (n \theta)-A_{n} \sin (n \theta)\right]
\end{aligned}
$$

to obtain

$$
\begin{aligned}
& A_{1}=-\frac{\mu_{o} J}{2 \pi}\left(\rho_{2}-\rho_{1}\right)\left[\cos \left(\phi_{2}\right)-\cos \left(\phi_{1}\right)\right], \\
& A_{2}=-\frac{\mu_{o} J R_{o}}{2 \pi} \ln \left(\frac{\rho_{2}}{\rho_{1}}\right)\left[\cos \left(2 \phi_{2}\right)-\cos \left(2 \phi_{1}\right)\right],
\end{aligned}
$$

for $\mathbf{n} \geq 3$

$$
A_{n}=\frac{\mu_{o} J}{2 \pi} \frac{R_{o}^{n-1}}{n(n-2)}\left(\frac{1}{\rho_{2}^{n-2}}-\frac{1}{\rho_{1}^{n-2}}\right)\left[\cos \left(n \phi_{2}\right)-\cos \left(n \phi_{1}\right)\right]
$$

and

$$
\begin{aligned}
& B_{1}=-\frac{\mu_{o} J}{2 \pi}\left(\rho_{2}-\rho_{1}\right)\left[\sin \left(\phi_{2}\right)-\sin \left(\phi_{1}\right)\right], \\
& B_{2}=-\frac{\mu_{o} J R_{o}}{2 \pi} \ln \left(\frac{\rho_{2}}{\rho_{1}}\right)\left[\sin \left(2 \phi_{2}\right)-\sin \left(2 \phi_{1}\right)\right],
\end{aligned}
$$

for $\mathrm{n} \geq 3$

$$
B_{n}=\frac{\mu_{o} J}{2 \pi} \sum_{n=3}^{\infty} \frac{R_{o}^{n-1}}{n(n-2)}\left(\frac{1}{\rho_{2}^{n-2}}-\frac{1}{\rho_{1}^{n-2}}\right)\left[\sin \left(n \phi_{2}\right)-\sin \left(n \phi_{1}\right)\right]
$$


In a typical superconducting magnet several current blocks are used to generate the desired multipolar field. In order to compute the harmonics due to several current blocks, the field and field harmonics $A_{n}$ and $B_{n}$ (coefficients having the dimension of field) can be directly superimposed. However, $a_{n}$ and $b_{n}$ (dimensionless coefficients) can not be directly added and they must be obtained from $A_{n}$ and $B_{n}$ as follows :

$$
\begin{aligned}
& b_{n}=10^{4} \frac{\sum_{k}\left(B_{n+1}\right)_{k}}{\sum_{k}\left(B_{m+1}\right)_{k}} \\
& a_{n}=10^{4} \frac{\sum_{k}\left(A_{n+1}\right)_{k}}{\sum_{k}\left(B_{m+1}\right)_{k}}
\end{aligned}
$$

where the summation $k$ is carried over all $k$ blocks with the $k^{\text {th }}$ block carrying a current density of $J_{k}$ and located between radii $\rho_{1 k}$ and $\rho_{2 k}$ and angles $\phi_{1 k}$ and $\phi_{2 k}$. The $A_{n}$ and $B_{n}$ for each current blocks are computed using the expressions given above. The harmonics are defined such that the fundamental harmonic $b_{m}$ is normalized to $10^{4}$.

The field components outside a current block $\left(r>\rho_{2}\right)$ are obtained similarly by integrating Eqs. (1.5.78) and the results are given below

$$
\begin{aligned}
& B_{r}(r, \theta)=-\frac{\mu_{o} J}{2 \pi} \sum_{n=1}^{\infty} \frac{\rho_{2}^{n+1}-\rho_{1}^{n+1}}{n(n+2) r^{n+1}}\left[\cos \left(n\left(\phi_{2}-\theta\right)\right)-\cos \left(n\left(\phi_{1}-\theta\right)\right)\right], \\
& B_{\theta}(r, \theta)=\frac{\mu_{o} J}{2 \pi} \sum_{n=1}^{\infty} \frac{\rho_{2}^{n+1}-\rho_{1}^{n+1}}{n(n+2) r^{n+1}}\left[\sin \left(n\left(\phi_{2}-\theta\right)\right)-\sin \left(n\left(\phi_{1}-\theta\right)\right)\right]
\end{aligned}
$$

The field inside a current block $\left(\rho_{1}<r<\rho_{2}\right)$ can be obtained by dividing the current block in two parts (a) from radius $\rho_{1}$ to radius $r$ and (b) from radius $r$ to radius $\rho_{2}$. Then the superimposition principle can be used to determine the field components with the (a) part evaluated from Eqs. (1.5.78) with $\rho_{2}$ replaced by $r$ and the (b) part from Eqs. (1.5.85) with $\rho_{1}$ replaced by $r$. 


\subsubsection{Field Harmonics due to Current Blocks in a Cylindrical Iron Shell}

As shown in a previous section (Eqs. (1.5.67) for $r<a$ ), the expressions for the field component due to current blocks get modified when they are placed inside an iron shell having an iron inner radius of $R_{f}$ and outer radius of $R_{a}$. The harmonic coefficients $A_{n}$ and $B_{n}$ are enhanced by :

$$
K_{n}=\left[1+\frac{\mu-1}{\mu+1}\left(\frac{a}{R_{f}}\right)^{2 n}\right] \frac{\left[1-\left(\frac{R_{f}}{R_{a}}\right)^{2 n}\right]}{\left[1-\left(\frac{\mu-1}{\mu+1}\right)^{2}\left(\frac{R_{f}}{R_{a}}\right)^{2 n}\right]},
$$

To give

$$
A_{n}^{\prime}=K_{n} \times A_{n}
$$

and

$$
B_{n}^{\prime}=K_{n} \times B_{n}
$$

The harmonics coefficients $a_{n}$ and $b_{n}$ given in Eqs. (1.5.84) are modified to :

$$
\begin{aligned}
& b_{n}=10^{4} \frac{\sum_{k}\left(K_{n+1} B_{n+1}\right)_{k}}{\sum_{k}\left(K_{m+1} B_{m+1}\right)_{k}} \\
& a_{n}=10^{4} \frac{\sum_{k}\left(K_{n+1} A_{n+1}\right)_{k}}{\sum_{k}\left(K_{m+1} B_{m+1}\right)_{k}}
\end{aligned}
$$




\subsubsection{6. $\operatorname{COS}(m \theta)$ Current Distribution for Ideal Fields}

In this section, it is demonstrated that an ideal $2 m$ ( $m=1$ for dipole) multipolar field shape in accelerator magnets can be produced by a $\operatorname{COS}(m \theta)$ current distribution. In the last section the expressions for the field and vector potential produced by a line current were obtained. The field in the cross section of the magnet can be described by superimposing the field produced by a large number of such wires.

A cylindrical current sheet [12-18] at a radius of $a$ is shown in Fig. 1.5.2, where the angular current density $I(\phi)$ in Amperes/radian as a function of angle $\phi$ is given by the relation

$$
I(\phi)=I_{0} \cos (m \phi) \text {. }
$$

[In the case of skew harmonics the current distribution is $I(\phi)=I_{0} \sin (m \phi)$ ].

It will be demonstrated that a pure dipole field is created by $m=1$, quadrupole by $m=2$, sextupole by $m=3$, etc. The total current required (Ampere-turns) per pole for generating a $2 m$-pole field is given by

$$
I_{\text {pole }}=\int_{0}^{\pi / 2 m} I_{0} \cos (m \phi) d \phi=\frac{I_{0}}{m} .
$$

In Eqs. (1.5.39), the vector potential produced by a single wire at any position is computed. To obtain the vector potential at $(r, \theta)$ inside the sheet (i.e. $I<a$ ), the expression is integrated over $\phi$

$$
A_{z}(r, \theta)=\frac{\mu_{o} I_{o}}{2 \pi} \sum_{n=1}^{\infty}\left(\frac{1}{n}\right)\left(\frac{r}{a}\right)^{n} \int_{0}^{2 \pi} \cos (m \phi) \cos (n(\phi-\theta)) d \phi
$$

to obtain

$$
A_{z}(r, \theta)=\frac{\mu_{o} I_{o}}{2 m}\left(\frac{r}{a}\right)^{m} \cos (m \theta)
$$

where the following trigonometric relations have been used

$$
\begin{gathered}
\cos [n(\phi-\theta)]=\cos (n \phi) \cos (n \theta)+\sin (n \phi) \sin (n \theta) \\
\int_{0}^{2 \pi} \cos (m \phi) \cos (n \phi) d \phi=\pi \delta_{m, n}, \\
\int_{0}^{2 \pi} \cos (m \phi) \sin (n \phi) d \phi=0 .
\end{gathered}
$$

The field components inside the current sheet are obtained by using Eqs. (1.5.40)

$$
\begin{aligned}
& B_{\theta}(r, \theta)=-\frac{\mu_{o} I_{o}}{2 a}\left(\frac{r}{a}\right)^{m-1} \cos (m \theta) \\
& B_{r}(r, \theta)=-\frac{\mu_{o} I_{o}}{2 a}\left(\frac{r}{a}\right)^{m-1} \sin (m \theta) \\
& B_{z}(r, \theta)=0
\end{aligned}
$$




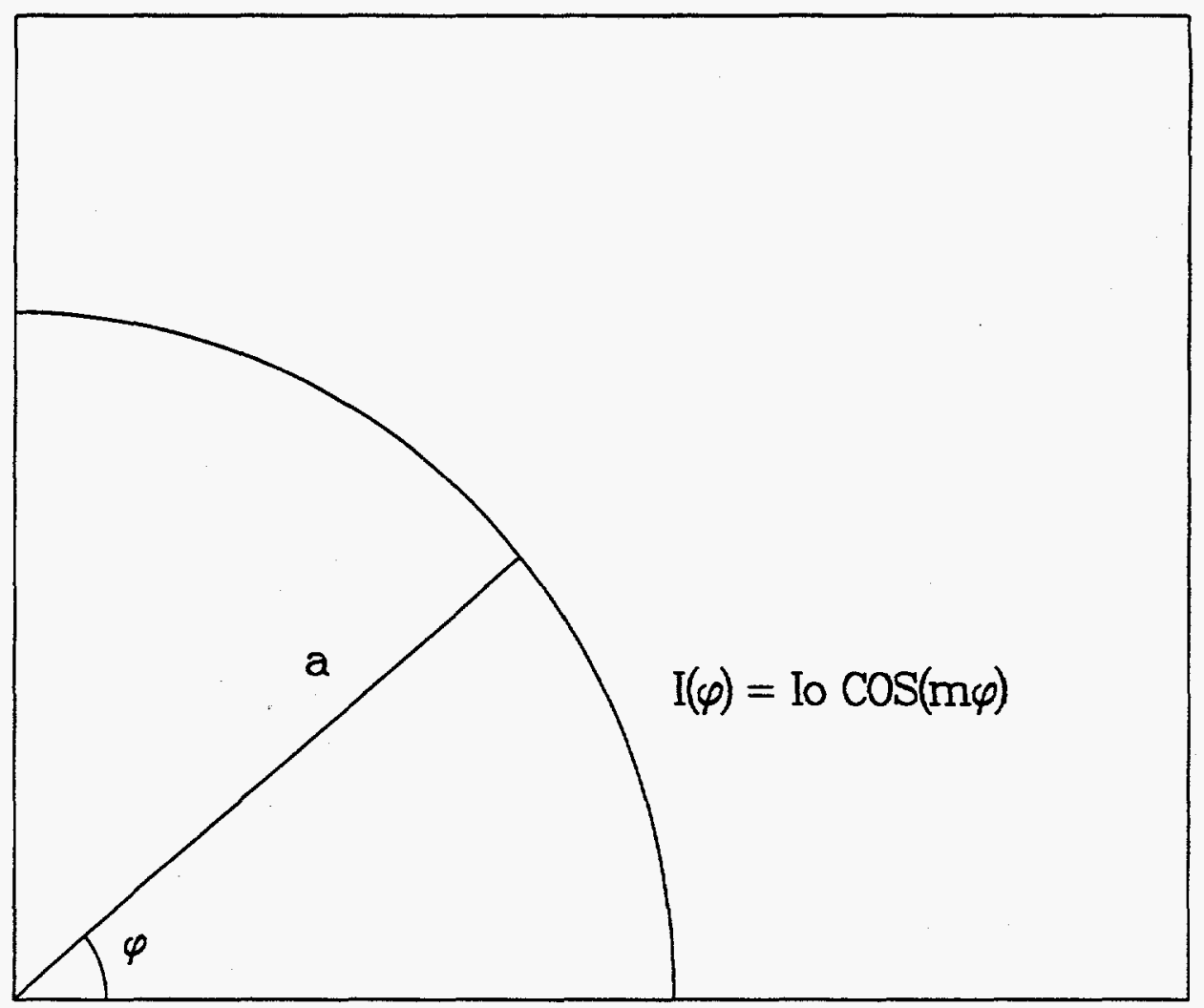

[GUPTA.THESIS. TGUREJWPHI.DAT:1

12:36:49, 21-4AY-24 GPLOT

Figure 1.5.2: Computation of the field at $(r, \theta)$ produced by a current sheet at a radius $a$ in which the current density varies as a function of angle given by $I(\phi)=I_{0} \cos (m \phi)$.

It may be noted that the magnitude of the field $|B|$ is independent of $\theta$. On using Eqs. (1.5.46)

$$
\begin{aligned}
& B_{x}(r, \theta)=-\frac{\mu_{o} I_{o}}{2 a}\left(\frac{r}{a}\right)^{m-1} \sin ((m-1) \theta) \\
& B_{y}(r, \theta)=-\frac{\mu_{o} I_{o}}{2 a}\left(\frac{r}{a}\right)^{m-1} \cos ((m-1) \theta) .
\end{aligned}
$$


For the $m=1$ case, this generates a pure dipole field, as the field components from $E$ qs. (1.5.92) reduce to

$$
\begin{aligned}
& B_{\theta}(r, \theta)=-\frac{\mu_{o} I_{o}}{2 a} \cos (\theta), \\
& B_{r}(r, \theta)=-\frac{\mu_{o} I_{o}}{2 a} \sin (\theta),
\end{aligned}
$$

and, from Eqs. (1.5.93)

$$
\begin{aligned}
& B_{x}=0 \\
& B_{y}=-\frac{\mu_{o} I_{o}}{2 a} .
\end{aligned}
$$

This implies that a cylindrical current sheet with a cosine $\theta$ current distribution would create a uniform vertical field inside it. This basic result is widely used in designing superconducting accelerator dipole magnets, although the actual current distribution is somewhat modified for practical reasons.

Likewise, for $m=2$, a pure quadrupole field is generated

$$
\begin{aligned}
& B_{\theta}(r, \theta)=-\frac{\mu_{o} I_{o} r}{2 a^{2}} \cos (2 \theta), \\
& B_{r}(r, \theta)=-\frac{\mu_{o} I_{o} r}{2 a^{2}} \sin (2 \theta),
\end{aligned}
$$

and, from Eqs. (1.5.93)

$$
\begin{aligned}
& B_{x}=g y, \\
& B_{y}=g x,
\end{aligned}
$$

with $g=-\left(\mu_{o} I_{o}\right) /\left(2 a^{2}\right)$.

Similarly, for $m=3$, a pure sextupole field is generated

$$
\begin{aligned}
& B_{\theta}(r, \theta)=-\frac{\mu_{o} I_{o} r^{2}}{2 a^{3}} \cos (3 \theta), \\
& B_{r}(r, \theta)=-\frac{\mu_{o} I_{o} r^{2}}{2 a^{3}} \sin (3 \theta),
\end{aligned}
$$

and, from Eqs. (1.5.93)

$$
\begin{aligned}
& B_{x}=2 S x y, \\
& B_{y}=S\left(x^{2}-y^{2}\right),
\end{aligned}
$$

with $S=-\left(\mu_{o} I_{o}\right) /\left(2 a^{3}\right)$. 
In general, a $\cos (m \theta)$ current distribution gives a $2 m$ order multipole with field components given by Eqs. (1.5.93).

On the $\mathrm{x}$-axis (midplane), $\theta=0$, these components become

$$
\begin{aligned}
& B_{x}(x, 0)=0 \\
& B_{y}(x, 0)=-\frac{\mu_{o} I_{o}}{2 a}\left(\frac{x}{a}\right)^{m-1},
\end{aligned}
$$

and on the $y$-axis

$$
\begin{aligned}
& B_{x}(0, y)=0, \quad \text { for } m=1,3,5, \ldots \\
& = \pm \frac{\mu_{o} I_{o}}{2 a}\left(\frac{y}{a}\right)^{m-1}, \quad \text { for } \quad m=2,4,6, \ldots \\
& B_{y}(0, y)= \pm \frac{\mu_{o} I_{o}}{2 a}\left(\frac{y}{a}\right)^{m-1}, \quad \text { for } \quad m=1,3,5, \ldots \\
& =0 . \quad \text { for } m=2,4,6, \ldots
\end{aligned}
$$

To obtain the field outside the current sheet $(r>a)$, Eqs. (1.5.44) is integrated using the trigonometric relations given in Eq. (1.5.90)and Eqs. (1.5.91)

$$
\begin{aligned}
A_{z}(r, \theta)= & -\frac{\mu_{o} I_{o}}{2 \pi} \ln \left(\frac{r}{a}\right) \int_{0}^{2 \pi} \cos (m \phi) d \phi \\
& +\frac{\mu_{o} I_{o}}{2 \pi} \sum_{n=1}^{\infty}\left(\frac{1}{n}\right)\left(\frac{a}{r}\right)^{n} \int_{0}^{2 \pi} \cos (m \phi) \cos (n(\phi-\theta)) d \phi, \\
\text { therefore, } A_{z}(r, \theta)= & \frac{\mu_{o} I_{o}}{2 m}\left(\frac{a}{r}\right)^{m} \cos (m \theta) .
\end{aligned}
$$

The field components for $\mathrm{I}>\mathrm{a}$ are obtained using Eqs. (1.5.40)

$$
\begin{aligned}
& B_{\theta}(r, \theta)=\frac{\mu_{o} I_{o}}{2 a}\left(\frac{a}{r}\right)^{m+1} \cos (m \theta) \\
& B_{r}(r, \theta)=-\frac{\mu_{o} I_{o}}{2 a}\left(\frac{a}{r}\right)^{m+1} \sin (m \theta) \\
& B_{z}(r, \theta)=0
\end{aligned}
$$

and the $\left(B_{x}, B_{y}\right)$ components of the field are obtained as :

$$
B_{x}=B_{r} \cos (\theta)-B_{\theta} \sin (\theta)
$$

and

$$
B_{y}=B_{r} \sin (\theta)+B_{\theta} \cos (\theta)
$$

therefore,

$$
\begin{aligned}
& B_{x}=-\frac{\mu_{o} I_{o}}{2 a}\left(\frac{a}{r}\right)^{m+1} \sin [(m+1) \theta] \\
& B_{y}=\frac{\mu_{o} I_{o}}{2 a}\left(\frac{a}{r}\right)^{m+1} \cos [(m+1) \theta]
\end{aligned}
$$


In the case of the dipole $(m=1)$, the field components outside the current sheet, fall as $\frac{1}{r^{2}}$, and are given by :

$$
\begin{aligned}
& B_{\theta}(r, \theta)=\frac{\mu_{o} I_{o} a}{2 r^{2}} \cos [\theta], \\
& B_{r}(r, \theta)=-\frac{\mu_{o} I_{o} a}{2 r^{2}} \sin [\theta], \\
& B_{x}(r, \theta)=-\frac{\mu_{o} I_{o} a}{2 r^{2}} \sin [2 \theta], \\
& B_{y}(r, \theta)=\frac{\mu_{o} I_{o} a}{2 r^{2}} \cos [2 \theta] .
\end{aligned}
$$

In deriving the above expressions, for simplicity it is assumed that the current is localized in a sheet. However, in accelerator magnets, the current is present between two radii $a_{1}$ and $a_{2}$. It is assumed that the current density in Amperes $/ m^{2}$ is given by

$$
J(\phi)=J_{0} \cos (m \phi)
$$

For a sheet of infitesimal thickness $d a, J_{o}$ is related to the angular current density $\left(I_{o}\right)$ as

$$
I_{0}=J_{0} a d a
$$

In this case the expression for the vector potential and field components for $\mathrm{I}<\mathrm{a}$ are by integrating Eqs. (1.5.39):

$$
A_{z}(r, \theta)=\frac{\mu_{o} J_{o}}{2 \pi} \sum_{n=1}^{\infty}\left(\frac{r^{n}}{n}\right) \int_{a_{1}}^{a_{2}} \frac{1}{a^{n}} a d a \int_{0}^{2 \pi} \cos (m \phi) \cos (n(\phi-\theta)) d \phi,
$$

Therefore,

$$
\begin{aligned}
& A_{z}(r, \theta)=\frac{\mu_{o} J_{o} r^{m}}{2 m} \cos (m \theta) \int_{a_{1}}^{a_{2}} \frac{1}{a^{m-1}} d a, \\
& B_{\theta}(r, \theta)=-\frac{\mu_{o} J_{o} r^{m-1}}{2} \cos (m \theta) \int_{a_{1}}^{a_{2}} \frac{1}{a^{m-1}} d a, \\
& B_{r}(r, \theta)=-\frac{\mu_{o} J_{o} r^{m-1}}{2} \sin (m \theta) \int_{a_{1}}^{a_{2}} \frac{1}{a^{m-1}} d a, \\
& B_{z}(r, \theta)=0 .
\end{aligned}
$$

Except for $m=2$ case (the quadrupole case, for which the expressions are given later), one obtains :

$$
\begin{aligned}
& A_{z}(r, \theta)=\frac{\mu_{o} J_{o} a_{1}^{2}}{2 m(m-2)} \cos (m \theta)\left(\frac{r}{a_{1}}\right)^{m}\left(1-\left(\frac{a_{1}}{a_{2}}\right)^{m-2}\right), \\
& B_{\theta}(r, \theta)=-\frac{\mu_{o} J_{0} a_{1}}{2(m-2)} \cos (m \theta)\left(\frac{r}{a_{1}}\right)^{m-1}\left(1-\left(\frac{a_{1}}{a_{2}}\right)^{m-2}\right),
\end{aligned}
$$




$$
\begin{aligned}
& B_{r}(r, \theta)=-\frac{\mu_{o} J_{o} a_{1}}{2(m-2)} \sin (m \theta)\left(\frac{r}{a_{1}}\right)^{m-1}\left(1-\left(\frac{a_{1}}{a_{2}}\right)^{m-2}\right), \\
& B_{y}(r, \theta)=-\frac{\mu_{o} J_{o} a_{1}}{2(m-2)} \cos ((m-1) \theta)\left(\frac{r}{a_{1}}\right)^{m-1}\left(1-\left(\frac{a_{1}}{a_{2}}\right)^{m-2}\right), \\
& B_{x}(r, \theta)=-\frac{\mu_{o} J_{o} a_{1}}{2(m-2)} \sin ((m-1) \theta)\left(\frac{r}{a_{1}}\right)^{m-1}\left(1-\left(\frac{a_{1}}{a_{2}}\right)^{m-2}\right) .
\end{aligned}
$$

In the case of the dipole $(m=1)$, this gives a vertical field

$$
B_{y}=-\mu_{o} J_{o}\left(\frac{a_{2}-a_{1}}{2}\right)=-\mu_{o} J_{o}\left(\frac{\Delta a}{2}\right) \text {. }
$$

For $m=2$ (quadrupole), the integration of Eqs. (1.5.104) gives :

$$
\begin{aligned}
& A_{x}(r, \theta)=\frac{\mu_{o} J_{o} r^{2}}{4} \cos (2 \theta) \ln \left(\frac{a_{2}}{a_{1}}\right) \\
& B_{\theta}(r, \theta)=-\frac{\mu_{o} J_{o} r}{2} \cos (2 \theta) \ln \left(\frac{a_{2}}{a_{1}}\right) \\
& B_{r}(r, \theta)=-\frac{\mu_{o} J_{o} r}{2} \sin (2 \theta) \ln \left(\frac{a_{2}}{a_{1}}\right) \\
& B_{y}(r, \theta)=-\frac{\mu_{o} J_{o} r}{2} \cos (\theta) \ln \left(\frac{a_{2}}{a_{1}}\right) \\
& B_{x}(r, \theta)=-\frac{\mu_{o} J_{o} r}{2} \sin (\theta) \ln \left(\frac{a_{2}}{a_{1}}\right)
\end{aligned}
$$

If the sheet thickness $\Delta a=a_{2}-a_{1}$ is very small compared to the the average radius $\bar{a}=$ $\frac{\left(a_{2}+a_{1}\right)}{2}$, then the expressions in Eqs. (1.5.106) for $r<a$ may be simplified to the following equations since the integral in Eq. (1.5.103) and Eqs. (1.5.104) can be approximated as $\left(\Delta a / \bar{a}^{m-1}\right)$ :

$$
\begin{aligned}
& A_{z}(r, \theta)=\frac{\mu_{0} J_{o} r \Delta a}{2 m}\left(\frac{r}{\bar{a}}\right)^{m-1} \cos (m \theta), \\
& B_{\theta}(r, \theta)=-\frac{\mu_{o} J_{o} \Delta a}{2}\left(\frac{r}{\bar{a}}\right)^{m-1} \cos (m \theta), \\
& B_{r}(r, \theta)=-\frac{\mu_{o} J_{o} \Delta a}{2}\left(\frac{r}{\bar{a}}\right)^{m-1} \sin (m \theta) .
\end{aligned}
$$




\subsubsection{7. $\operatorname{Cos}(m \theta)$ Current Distribution in a Cylindrical Iron Shell}

In superconducting accelerator magnets, the coils are frequently placed inside a cylindrical iron yoke to (a) reduce the stray magnetic field outside the magnet and (b) as an added benefit to enhance the field in the aperture of the magnet. Due to the non-linear properties of the iron, the fraction of field generated by the iron at any current depends on how much the yoke is magnetized. This is too complex a problem to solve analytically. However, one can obtain simple expressions if one assumes that the permeability $(\mu)$ of the iron is constant everywhere in the yoke. Expressions for the vector potential and the field are given for the case in which a $\operatorname{COS}(m \theta)$ current sheet at radius $a$ is inside in an iron shell with inner radius of $R_{f}$ and outer radius of $R_{a}$.

In this case, the method of matching the boundary conditions at the air and iron interfaces, as described in the last section, can be used to include the contribution from the iron. This is equivalent to the method of images when the effect of the iron is replaced by the equivalent image currents.

In the presence of a cylindrical iron yoke, the vector potential and the field components given in Eqs. (1.5.89) and Eqs. (1.5.92), for $r<a$, are modified to

$$
\begin{aligned}
A_{z}(r, \theta)= & \frac{\mu_{o} I_{o}}{2 m} \cos (m \theta)\left(\frac{r}{a}\right)^{m} \times \\
& {\left[1+\frac{\mu-1}{\mu+1}\left(\frac{a}{R_{f}}\right)^{2 m} \frac{\left[1-\left(\frac{R_{f}}{R_{a}}\right)^{2 m}\right]}{\left[1-\left(\frac{\mu-1}{\mu+1}\right)^{2}\left(\frac{R_{f}}{R_{a}}\right)^{2 m}\right]}\right] } \\
B_{\theta}(r, \theta)=- & \frac{\mu_{o} I_{o}}{2 a} \cos (m \theta)\left(\frac{r}{a}\right)^{m-1} \times \\
B_{r}(r, \theta)=- & \left.\frac{\mu_{o} I_{o}}{2 a} \sin (m \theta)\left(\frac{r}{a}\right)^{m-1} \times \frac{\mu-1}{\mu+1}\left(\frac{a}{R_{f}}\right)^{2 m} \frac{\left[1-\left(\frac{R_{f}}{R_{a}}\right)^{2 m}\right]}{\left[1-\left(\frac{\mu-1}{\mu+1}\right)^{2}\left(\frac{R_{f}}{R_{a}}\right)^{2 m}\right]}\right] \\
& {\left[1+\frac{\mu-1}{\mu+1}\left(\frac{a}{R_{f}}\right)^{2 m} \frac{\left[1-\left(\frac{R_{f}}{R_{a}}\right)^{2 m}\right]}{\left[1-\left(\frac{\mu-1}{\mu+1}\right)^{2}\left(\frac{R_{f}}{R_{a}}\right)^{2 m}\right]}\right] }
\end{aligned}
$$

The other components are obtained using Eqs. (1.5.46)

$$
B_{x}(r, \theta)=-\frac{\mu_{o} I_{o}}{2 a} \sin ((m-1) \theta)\left(\frac{r}{a}\right)^{m-1} \times
$$




$$
\begin{aligned}
& {\left[1+\frac{\mu-1}{\mu+1}\left(\frac{a}{R_{f}}\right)^{2 m} \frac{\left[1-\left(\frac{R_{f}}{R_{a}}\right)^{2 m}\right]}{\left[1-\left(\frac{\mu-1}{\mu+1}\right)^{2}\left(\frac{R_{f}}{R_{a}}\right)^{2 m}\right]}\right],} \\
& B_{y}(r, \theta)=-\frac{\mu_{o} I_{0}}{2 a} \cos ((m-1) \theta)\left(\frac{r}{a}\right)^{m-1} \times \\
& \\
& {\left[1+\frac{\mu-1}{\mu+1}\left(\frac{a}{R_{f}}\right)^{2 m} \frac{\left[1-\left(\frac{R_{f}}{R_{a}}\right)^{2 m}\right]}{\left[1-\left(\frac{\mu-1}{\mu+1}\right)^{2}\left(\frac{R_{f}}{R_{a}}\right)^{2 m}\right]}\right],} \\
& B_{z}(r, \theta)=0 .
\end{aligned}
$$

Similarly, the vector potential and field outside the current sheet but inside the iron, i.e. $a<r<R_{f}$, is given by :

Between Coil and Iron $\left(\mathbf{a}<\mathbf{r}<\mathbf{R}_{\mathbf{f}}\right)$

$$
\begin{aligned}
& A_{z}(r, \theta)=\frac{\mu_{o} I_{o}}{2 m}\left(1+\frac{\mu-1}{\mu+1}\left(\frac{r}{R_{f}}\right)^{2 m} \frac{\left[1-\left(\frac{R_{f}}{R_{a}}\right)^{2 m}\right]}{\left[1-\left(\frac{\mu-1}{\mu+1}\right)^{2}\left(\frac{R_{f}}{R_{a}}\right)^{2 m}\right]}\right) \times \\
& \left(\frac{a}{r}\right)^{m} \cos (m \theta), \\
& B_{r}=-\frac{\mu_{o} I_{o}}{2 a}\left(1+\frac{\mu-1}{\mu+1}\left(\frac{r}{R_{f}}\right)^{2 m} \frac{\left[1-\left(\frac{R_{f}}{R_{\mathrm{a}}}\right)^{2 m}\right]}{\left[1-\left(\frac{\mu-1}{\mu+1}\right)^{2}\left(\frac{R_{f}}{R_{a}}\right)^{2 m}\right]}\right) \times \\
& \left(\frac{a}{r}\right)^{m+1} \sin (m \theta), \\
& B_{\theta}=\frac{\mu_{o} I_{o}}{2 a}\left(1-\frac{\mu-1}{\mu+1}\left(\frac{r}{R_{f}}\right)^{2 m} \frac{\left[1-\left(\frac{R_{f}}{R_{a}}\right)^{2 m}\right]}{\left[1-\left(\frac{\mu-1}{\mu+1}\right)^{2}\left(\frac{R_{f}}{R_{a}}\right)^{2 m}\right]}\right) \times \\
& \left(\frac{a}{r}\right)^{m+1} \cos (m \theta) .
\end{aligned}
$$

Inside Iron $\left(\mathbf{R}_{\mathrm{f}}<\mathbf{r}<\mathbf{R}_{\mathrm{a}}\right)$

$$
\begin{gathered}
A_{z}(r, \theta)=\frac{\mu \mu_{o} I_{o}}{m(\mu+1)}\left(\frac{1-\frac{\mu-1}{\mu+1}\left(\frac{r}{R_{a}}\right)^{2 m}}{1-\left(\frac{\mu-1}{\mu+1}\right)^{2}\left(\frac{R_{f}}{R_{a}}\right)^{2 m}}\right) \times \\
\left(\frac{a}{r}\right)^{m} \cos (m \theta), \\
B_{r}=-\frac{\mu \mu_{o} I_{o}}{a(\mu+1)}\left(\frac{1-\frac{\mu-1}{\mu+1}\left(\frac{r}{R_{a}}\right)^{2 m}}{1-\left(\frac{\mu-1}{\mu+1}\right)^{2}\left(\frac{R_{f}}{R_{a}}\right)^{2 m}}\right) \times
\end{gathered}
$$




$$
\begin{gathered}
\left(\frac{a}{r}\right)^{m+1} \sin (m \theta), \\
B_{\theta}=\frac{\mu \mu_{o} I_{o}}{a(\mu+1)}\left(\frac{1+\frac{\mu-1}{\mu+1}\left(\frac{r}{R_{a}}\right)^{2 m}}{1-\left(\frac{\mu-1}{\mu+1}\right)^{2}\left(\frac{R_{f}}{R_{a}}\right)^{2 m}}\right) \times \\
\left(\frac{a}{r}\right)^{m+1} \cos (m \theta) .
\end{gathered}
$$

Outside Iron $\left(\mathbf{r}>\mathbf{R}_{\mathbf{a}}\right)$

$$
\begin{aligned}
A_{z}(r, \theta) & =\frac{2 \mu \mu_{o} I_{o}}{m(\mu+1)^{2}} \frac{1}{1-\left(\frac{\mu-1}{\mu+1}\right)^{2}\left(\frac{R_{f}}{R_{a}}\right)^{2 m}}\left(\frac{a}{r}\right)^{m} \cos (m \theta), \\
B_{r} & =-\frac{2 \mu \mu_{o} I_{o}}{a(\mu+1)^{2}} \frac{1}{1-\left(\frac{\mu-1}{\mu+1}\right)^{2}\left(\frac{R_{f}}{R_{a}}\right)^{2 m}}\left(\frac{a}{r}\right)^{m+1} \sin (m \theta), \\
B_{\theta} & =\frac{2 \mu \mu_{o} I_{o}}{a(\mu+1)^{2}} \frac{1}{1-\left(\frac{\mu-1}{\mu+1}\right)^{2}\left(\frac{R_{f}}{R_{a}}\right)^{2 m}}\left(\frac{a}{r}\right)^{m+1} \cos (m \theta)
\end{aligned}
$$




\subsubsection{Intersecting Circles with a Constant Current Density for Ideal Fields}

It has been shown [137] that a pure dipole field can be created simply by two intersecting circles carrying constant current densities in opposite directions. To demonstrate this, the field is evaluated inside and outside a circular conductor with a radius $a$ and carrying a constant current density $J$ in the direction of the axis (perpendicular to the plane of paper). For a radius $R>a$ (outside the conductor), Ampere's law gives

$$
2 \pi R \cdot H=\pi a^{2} J
$$

Therefore,

$$
H=\frac{J a^{2}}{2 R}
$$

The direction of the magnetic field is azimuthal, with $(x, y)$ components of the field at any point outside the conductor given by

$$
\begin{aligned}
& H_{x}=-\frac{J a^{2}}{2 R} \sin (\theta)=-\frac{J}{2}\left(\frac{a}{R}\right)^{2} y, \\
& H_{y}=\frac{J a^{2}}{2 R} \cos (\theta)=\frac{J}{2}\left(\frac{a}{R}\right)^{2} x .
\end{aligned}
$$

The field inside the conductor $(R<a)$ can be obtained as

$$
\begin{aligned}
2 \pi R \cdot H & =\pi R^{2} J, \\
\text { i.e., } \quad H & =\frac{J R}{2} .
\end{aligned}
$$

with the components of the field being given by

$$
\begin{aligned}
& H_{x}=-\frac{J}{2} R \sin (\theta)=-\frac{J}{2} y, \\
& H_{y}=\frac{J}{2} R \cos (\theta)=\frac{J}{2} x .
\end{aligned}
$$

Now expressions will be derived for the field produced by the conductors in two intersecting circles. The coordinate system is defined such that the $\mathrm{x}$-axis passes through the centers of the two circles with the origin of the new coordinate system $\left(x^{\prime}, y^{\prime}\right)$ being in the middle of the two. The distance between the centers of the two circles is $s$ with circle 2 to the right such that $x^{\prime}=x_{1}-\frac{s}{2}=x_{2}+\frac{s}{2}$ and $y_{1}=y_{2}=y^{\prime}$. The direction of the current is opposite in the two circles, with constant current densities $J_{1}$ and $-J_{2}$ respectively. The components of the field inside the region created by the two intersecting circles can be computed by superimposing the field produced by the conductors in the two circles

$$
\begin{aligned}
& H_{x}=\frac{y^{\prime}}{2}\left(J_{2}-J_{1}\right), \\
& H_{y}=\frac{x^{\prime}}{2}\left(J_{1}-J_{2}\right)+\frac{s}{4}\left(J_{1}+J_{2}\right) .
\end{aligned}
$$




\section{Two intersecting circles}

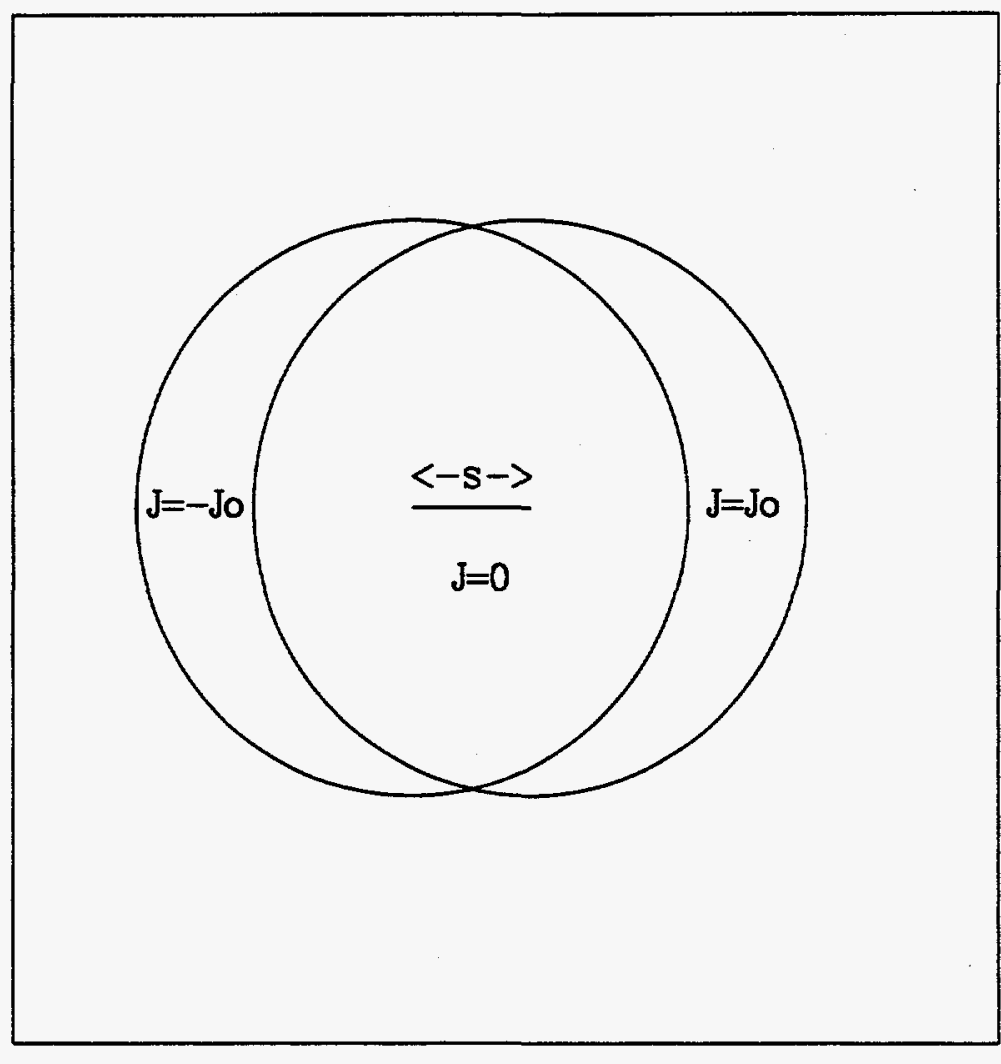

1 [GUPTA.THESIS. MTGUREXYROROO1.DAT:O

16:12:67, 29-MAY-94 GPLOT

Figure 1.5.3: This figure shows the two intersecting circles of equal size with one carrying a current with a constant density $J=-J_{0}$ and the other $J=J_{o}$. The two circles are separated by a distance $s$. In the intersection region of the two circles, the net current density is zero and therefore it can be replaced by a current free region. It is demonstrated that this configuration produces a vertical dipole field given by $\frac{J_{0}}{2} s$.

A special case comes when the magnitude of the current densities in the two circles is $J_{0}$ but the direction is opposite as shown in Fig. 1.5.3. This means that the intersection region is a current free region which can be used as an aperture for the particle beam and and the aperture has a constant vertical magnetic field given by $H_{y}=\frac{J_{0}}{2} s$. 
It can be shown [14] that four intersecting circles create a quadrupole field and in general $2 m$ intersecting circles create a $2 m$-order multipole. The treatment has been also been extended to ellipses by a number of authors (see for example Beth [14]). 


\subsubsection{Complex Variable Method in 2-d Magnetic Field Calculations}

The method of complex variable is found very useful in deriving many expressions in superconducting magnets. [12-18,81] These methods can be applied to 2-dimensional field computations, which is the case for the most part of long superconducting magnets. Mills and Morgan [115] have shown that the complex method can also be extended throughout the ends, however, to the field integral $\left(\int B . d z\right)$. The complex variables have two parts (real and imaginary) and the following variables will be used :

$$
\begin{aligned}
z & =x+i y \\
H(z) & =H_{y}+i H_{x} \\
B(z) & =B_{y}+i B_{x} \\
W(z) & =-(A+i \phi)+\text { constant. }
\end{aligned}
$$

where $W$ is the complex potential having $\phi$ and $A$ (scalar and vector potentials) as the two components, and $i=\sqrt{-1} . z^{*}$ is the complex conjugate of $z$ with

$$
z^{*}=x-i y
$$

In the 2-d case the following relations are valid :

$$
\begin{aligned}
B_{x} & =\frac{\partial A}{\partial y} \\
B_{y} & =-\frac{\partial A}{\partial x}, \\
\text { with } \quad B_{z} & =\mu_{0} \mu H_{x}, \\
\text { and } \quad B_{y} & =\mu_{0} \mu H_{y} .
\end{aligned}
$$

Moreover, in air $(\mu=1)$,

$$
\begin{aligned}
& H_{x}=-\frac{1}{\mu_{0}} \frac{\partial \phi}{\partial x}=\frac{1}{\mu_{0}} \frac{\partial A}{\partial y}, \\
& H_{y}=-\frac{1}{\mu_{0}} \frac{\partial \phi}{\partial y}=-\frac{1}{\mu_{0}} \frac{\partial A}{\partial x} .
\end{aligned}
$$

The Cauchy-Riemann equations are the necessary and sufficient conditions for a function to be analytic in Z-plane. For a function $F_{v}=u+i v$, these conditions are:

$$
\begin{aligned}
& \frac{\partial u}{\partial x}=\frac{\partial v}{\partial y} \\
& \frac{\partial u}{\partial y}=-\frac{\partial v}{\partial x}
\end{aligned}
$$


In a medium free of magnetic material with $\mu=1$, Eqs. (1.5.124) gives

$$
\begin{aligned}
& \frac{\partial A}{\partial x}=\frac{\partial \phi}{\partial y} \\
& \frac{\partial A}{\partial y}=-\frac{\partial \phi}{\partial x}
\end{aligned}
$$

which are the Cauchy-Riemann conditions for $W(z)=-(A+i \phi)+$ constant to be analytic. In the same way, $B(z)$ (and similarly $H(z)$ ) is analytic if :

$$
\begin{aligned}
& \frac{\partial B_{y}}{\partial x}=\frac{\partial B_{x}}{\partial y} \\
& \frac{\partial B_{y}}{\partial y}=-\frac{\partial B_{x}}{\partial x}
\end{aligned}
$$

which are just Maxwell's equations in a current free region. It may be noted that the choice of variable $B(z)$ as $B(z)=B_{y}+i B_{x}$ is important since $B_{x}$ and $B_{y}$ do not the satisfy the Cauchy-Rieman conditions if the variable is $B_{x}+i B_{y}$.

Since $W(z)$ is analytic, the derivative of $W(z)$ gives the the complex field function :

$$
\frac{d W}{d z}=-\frac{\partial A}{\partial x}-i \frac{\partial \phi}{\partial x}=i \frac{\partial A}{\partial y}-\frac{\partial \phi}{\partial y}=H_{y}+i H_{x}=H(z)
$$

To deal with a region with current, a new analytic function is defined as follows :

$$
F(z)=B(z)-\frac{1}{2} \mu_{o} J z^{*}=\left(B_{y}-\frac{1}{2} \mu_{o} J x\right)+i\left(B_{x}+\frac{1}{2} \mu_{o} J y\right)
$$

where the current density $J$ is constant throughout the region. The Cauchy-Riemann conditions become :

$$
\begin{aligned}
\frac{\partial B_{y}}{\partial x}-\frac{1}{2} \mu_{o} J & =\frac{\partial B_{x}}{\partial y}+\frac{1}{2} \mu_{o} J \\
\Longrightarrow \quad \frac{\partial B_{y}}{\partial x}-\frac{\partial B_{x}}{\partial y} & =\mu_{o} J \\
& \text { and }, \quad \frac{\partial B_{y}}{\partial y}=-\frac{\partial B_{x}}{\partial x} \\
\Longrightarrow \quad \frac{\partial B_{x}}{\partial x}+\frac{\partial B_{y}}{\partial y} & =0
\end{aligned}
$$

which are Maxwell's equations in the presence of current. 


\subsubsection{Field due to an array of Line Currents}

The complex potential at a point $z$, due a current flowing in a direction perpendicular to the Z-plane at $z=z_{o}$, is given by :

$$
W(z)=\frac{I}{2 \pi} \log \left(z-z_{o}\right)+\text { constant }
$$

and the magnetic field is given by :

$$
H(z)=\frac{d W}{d z}=\frac{I}{2 \pi\left(z-z_{0}\right)} .
$$

The direction of the field is that of $\left(z-z_{0}\right)^{*}$, which is perpendicular to the vector $\left(z-z_{0}\right)$. The superposition principle can be used to obtain the field due to $n$ filaments with the $k^{\text {th }}$ filament carrying $I_{k}$ amperes and located at $z=z_{k}$ :

$$
H(z)=\sum_{k=1}^{n} \frac{I_{k}}{2 \pi\left(z-z_{k}\right)}
$$

Cauchy's Residue Theorem gives [29]

$$
\oint_{C} f(z) d z=2 \pi i \sum_{k} \operatorname{Res}\left(a_{k}\right)
$$

where $\operatorname{Res}\left(a_{k}\right)$ are the residues which are defined as the coefficients of $\frac{1}{z-z_{k}}$ inside the contour $C$ over which the contour integral of the function $f(z)$ is taken. Applying this to Eq. (1.5.129) while taking the contour integral of the field around the wires in the Z-plane, one obtains

$$
\oint H(z) d z=i \sum_{k=1}^{n} I_{k} .
$$

which is basically Ampere's law.

The Cauchy integral formula [29] gives :

$$
f\left(z_{o}\right)=\frac{1}{2 \pi i} \oint_{C} \frac{f(z)}{\left(z-z_{o}\right)} d z
$$

where the function $f(z)$ is analytic everywhere within and on a closed contour $C$ and $f\left(z_{0}\right)$ is the value of $f(z)$ at $z=z_{0}$. 


\subsubsection{Beth's Current Sheet Theorem}

Beth's "Current Sheet Theorem" [12-18] can be derived from Eq. (1.5.131). As shown in Fig. 1.5.4 the current sheet is made up of a number of filaments carrying a total current $\Delta I$ perpendicular to the Z-plane along the curve from $z$ to $z+\Delta z$. A contour integral on a closed path enclosing the current sheet will give

$$
\oint H(z) d z=i \Delta I
$$

Now if the path is squeezed from the right and left sides (indicated by the subscripts $R$ and $L$ ) on to the current sheet, then in the limiting case one obtains

$$
H_{R}\left(z_{\circ}\right)-H_{L}\left(z_{o}\right)=i \frac{d I}{d z}
$$

where $H_{R}\left(z_{o}\right)$ and $H_{L}\left(z_{o}\right)$ are the limits of the analytic functions $H_{R}(z)$ and $H_{L}(z)$ when $z$ approaches $z_{o}$ from the right and left and $\frac{d I}{d z}$ is the limit of $\frac{\Delta I}{\Delta z}$ when $\Delta z$ approaches 0 at any $z$.

The above equation Eqs. (1.5.133) is called Beth's current sheet theorem. To obtain another equation in potential form this equation is integrated to give

$$
W_{R}\left(z_{o}\right)-W_{L}\left(z_{o}\right)=i I+\text { Constant }
$$

where $W_{R}\left(z_{0}\right)$ and $W_{L}\left(z_{0}\right)$ are the limits of the analytic functions $W_{R}(z)$ and $W_{L}(z)$ when $z$ approaches $z_{o}$ from the right and left. 


\section{Beth's Current Sheet}

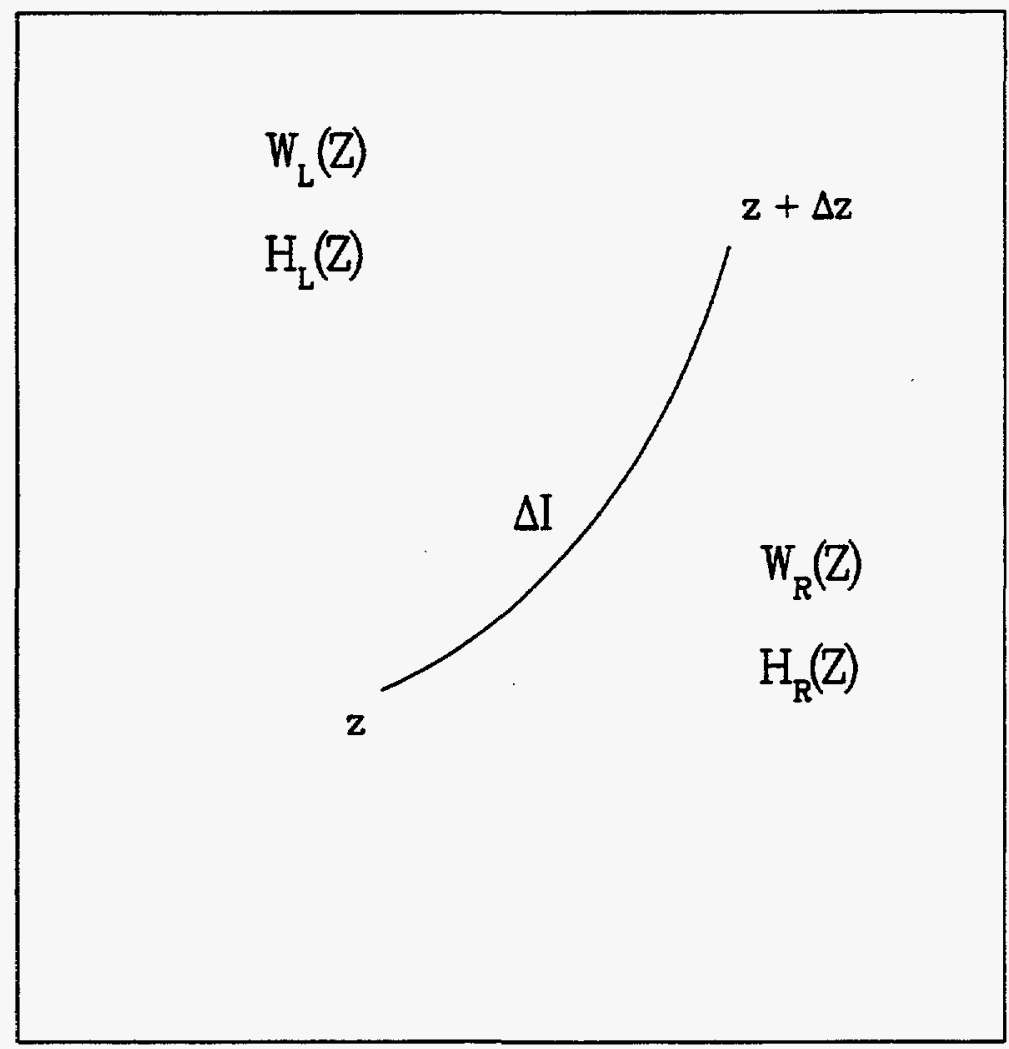

[GUPTA.THESIS.FIGURE] POROOL.DAT:7

14:38:14 , 29-MAY-94 GPLOT

Figure 1.5.4: Beth's current sheet is shown here, which is made up of a number of filaments, carrying a total current $\Delta I$ perpendicular to the Z-plane along the curve from $z$ to $z+\Delta z$. The sub-script " $\mathrm{R}$ " denotes the right side and " $L$ " denotes the left side to the sheet. 


\subsubsection{Example - $\operatorname{Cos}(m)$ current distribution}

As an example of use of the complex variable methods, expressions are derived here for the field due to a cylindrical current sheet at a radius $r=a$, as shown in Fig. 1.5.2. An angular current density distribution, mentioned earlier, is :

$$
\frac{d I}{d \phi}=I_{0} \cos (m \phi)
$$

In complex coordinates, the above current sheet is located at $z=a e^{i \phi}$. Then,

$$
\frac{d I}{d z}=\left(\frac{d I}{d \phi}\right) /\left(\frac{d z}{d \phi}\right)=\frac{I_{0} \cos (m \phi)}{i a e^{i \phi}}
$$

Since $H(z)$ is analytic both inside and outside the current sheet, a general expression for the field to remain finite inside the current sheet $(r<a)$ is $H_{i n}=\sum_{n} a_{n} z^{n}$ and for outside the current sheet $(r>a)$ is $H_{o u t}=\sum_{n} b_{n} z^{-n}$. To obtain the coefficients $a_{n}$ and $b_{n}$, the fields $\left(H_{i n}\right)$ and $\left(H_{\text {out }}\right)$ are linked using Beth's current sheet theorem (Eqs. (1.5.133)) as follows :

$$
\begin{aligned}
H_{\text {out }}-H_{\text {in }} & =I_{o} \frac{\cos (m \phi)}{a e^{i \phi}} \\
& =\frac{I_{0}}{2 a}\left[e^{-i(m+1) \phi}+e^{i(m-1) \phi}\right] \\
& =\frac{I_{o}}{2 a}\left[\left(\frac{a}{z}\right)^{m+1}+\left(\frac{z}{a}\right)^{m-1}\right]
\end{aligned}
$$

The right hand side of the above equation gives the field on the current sheet and it acts as a boundary condition which must match interior and exterior solutions. Hence $a_{n}=0$ for $\mathrm{n} \neq \mathrm{m}-1$ and $b_{n}=0$ for $\mathrm{n} \neq \mathrm{m}+1$, giving

$$
\begin{aligned}
H_{\text {in }}=\frac{-I_{0}}{2 a}\left(\frac{z}{a}\right)^{m-1} & |z|<a, \\
H_{\text {out }}=\frac{I_{o}}{2 a}\left(\frac{a}{z}\right)^{m+1} & |z|>a .
\end{aligned}
$$




\subsection{Methods Investigated for Improving Field Quality}

This section gives a brief summary of the research and development work performed during the course of this work for improving the field quality in superconducting magnets. The work is presented in more detail in chapters 2 through 6 . The two major components of the magnet which influence the field quality are the coil and yoke designs. A major effort has been made to develop and apply various methods of obtaining the field quality required in large particle accelerators. The field quality is further improved by using tuning shims for correcting errors after construction. Improvements in the techniques used to analyze the magnets have also been made. In the end, optimized designs for the cross section of the SSC collider dipole and RHIC insertion quadrupole magnet are described. The research work is divided into five chapters. The following is a brief description of each chapter.

\subsubsection{Improvements in the Computational and Analysis Methods}

A technique has been developed for a computer-aided mechanical measurement of the cross section. This can be used to study the geometry of the coil and iron cross section in an actual magnet as built. It can be used to understand the magnetic performance of a design and also to examine the mechanical deformation in a magnet resulting from the large mechanical forces applied during the magnet assembly. This technique is described in chapter 2.

The computer code POISSON has been extensively used to carry out the calculations presented during the course of this work. In order to make a precise computer model of a magnet and in order to obtain the desired accuracy, this program has been extensively modified. A significant effort has been made developing the techniques used in setting up the mesh which describes the problem, to a desired accuracy. These improvements are also described in chapter 2. 


\subsubsection{Field Quality Improvements through Yoke Design}

An iron yoke is used in superconducting magnets to reduce the fringe field outside the physical boundary of the magnet. In addition, the magnetized yoke iron also contributes to the field at the center of the magnet. However, the magnetization is not proportional (linear) to the current in the coil. This contribution becomes non-linear when the field in the iron is above 1.2 Tesla. This non-linearity in iron properties (referred to commonly as saturation effects) complicates the design and limits the field quality of the magnet at high field. The iron saturation changes the azimuthal distribution of the yoke magnetization. This in turn changes the uniformity of the field in the magnet aperture.

In the magnet designs described in this research work, the saturation effects are minimized by controlling the iron saturation across the azimuth. The path of the magnetic field lines is controlled with the help of a number of techniques so that the iron saturates uniformly throughout the yoke.

The field in the accelerator magnets is represented by the harmonic components and they change with the azimuthal distribution of the field. In an ideal magnet all except one harmonic is zero. In RHIC dipole magnets these yoke design techniques have resulted in reducing the saturation induced harmonics by about an order of magnitude. These involve (a) optimizing the locations of certain holes in the yoke which must be there, or (b) putting in some additional holes which are dedicated to controlling the saturation or (c) putting in cutouts or bumps to shape the aperture or (d) using a non-circular aperture having multiple circular arcs with different radii. The field quality improvements obtained through the yoke designs are described in chapter 3.

\subsubsection{Field Quality Improvements through Coil Design}

For a variety of reasons a significant difference is observed between the design and measured values of harmonics in the magnets. Moreover, sometimes, there are also differences between the component dimensions as used in the original design computations and the component dimensions as delivered for use in the magnets. A systematic study of this is made in chapter 4 . To handle such errors the coil cross section is generally iterated. This is also studied in chapter 4. This approach, however, requires a long time to incorporate in the next design of the magnet and is relatively inflexible. In the RHIC interaction region quadrupole magnets, the cross section iterations are accomplished by changing the size of 
the midplane shims (thin material at the coil midplane) and the size of the pole shims (thin material at the coil pole). The major advantage of this approach is the fast turn-around time and an ability to iterate the cross section after the coils are made.

An adjustment in the midplane gap has been used in the industry-built $80 \mathrm{~mm}$ aperture $\mathrm{RHIC}$ arc dipole magnet to minimize the critical $b_{4}$ harmonic. This is also described in chapter 4.

An ideal dipole cross section has 2-fold symmetry and an ideal quadrupole has 4-fold symmetry. The RHIC arc and insertion quadrupoles are collared like dipoles to save cost and for design simplicity reasons. This, however, breaks the ideal 4-fold symmetry in the quadrupoles. Earlier RHIC quadrupoles thus built had large undesired harmonics. The harmonics have been compensated in the present design using a method described in chapter 4. This basically involves creating another deliberate asymmetry between the horizontal and vertical planes by making the gap between the two coils asymmetric at the midplane.

\subsubsection{Field Quality Improvements after Construction}

The ultimate luminosity performance of $\mathrm{RHIC}$ is determined by the field quality in the $130 \mathrm{~mm}$ aperture interaction region quadrupoles. To overcome the limitations in obtaining a good field quality due to practical manufacturing and assembling tolerances, the method of Tuning Shims has been developed. This is described in detail in chapter 5. The method is referred to as the Tuning Shim Method because it corrects the measured harmonics in an individual magnet by adjusting the strength of harmonics generated by each of several tuning shims.

The basic principle is as follows : when a magnetic material (shim) is placed inside the yoke aperture, it becomes magnetized and changes the shape of the field at the center of the magnet. This change can be expressed in terms of harmonic components. By properly choosing the location and size of these magnetic shims one can cancel out as many measured harmonics as the number of shims. In the $130 \mathrm{~mm}$ aperture $\mathrm{RHIC}$ interaction region quadrupoles, the number of tuning shims is eight. They are used to compensate eight critical harmonics. Calculations will be compared with measurements.

In addition to the above method, which corrects the harmonics in the body of the magnet, an integral correction can also be made in the two ends of the magnet. This 
scheme was examined for correcting the top-bottom asymmetry in the SSC collider dipole magnets. This is also described in chapter 5.

\subsubsection{Optimized Cross section Designs}

The ultimate purpose of the above research and development in the design and analysis methods is to obtain optimized cross section designs which produce the required good field quality in superconducting accelerator magnets. A detailed description of the magnetic design of the cross section of the prototype of the $50 \mathrm{~mm}$ aperture SSC collider dipole magnets and the $130 \mathrm{~mm}$ aperture RHIC insertion quadrupole magnets is given in chapter 6. This chapter also describes the design philosophy and other considerations used in developing the final optimized sets of parameters. Moreover, detailed computations of the field harmonics at various currents and computations of other quantities like the maximum expected field at which the magnet would remain superconducting (quench field), etc. are also given. 


\section{Chapter 2.}

\section{IMPROVEMENTS IN THE COMPUTATIONAL AND ANALYSIS METHODS}

The research work described in this chapter is partly based on the following papers :

- R.C. Gupta, Modifications in the AUTOMESH and other POISSON Group Codes, Proceedings of the Workshop in Electromagnetic Field Computations, Schnectady, NY (1986).

- R.C. Gupta, Improvements in the Mesh Generator of the POISSON Group Codes, Proceedings of the 1987 Particle Accelerator Conference, Washington DC (1987).

- R.C. Gupta, POISSON-BNL, Computer Codes for Particle Accelerator Design and Analysis: A Compendium, Los Alamos Accelerator Code Group, Second Edition, LA-UR-90-1766 (1990).

\subsection{Introduction}

In order to produce the desired high field quality in superconducting magnets, the computational and analysis techniques should be developed to a level which are reliable to an accuracy of $\sim 10^{-4}$. Moreover, the source of differences between the expected and measured harmonics should be understood to assure that it (the source) will not change and that the desired change in harmonics is obtained after an iteration in the design. A method that permits analysis of the actual shape and size of the geometry of various components in the magnet is a useful tool to understand the source of those differences.

The first section of this chapter describes the development of a computer aided cross section measurement and analysis technique which can be used to find the deviation in the coil and iron shape from the ideal design. This method makes use of personal computers (PC) and commercially available hardware and software for them. The second section describes improvements in the computational techniques, in particular the modelling of the cross section with the code POISSON [135]. Since the geometry of superconducting magnets is too complex for the field to be obtained by analytic formulas, numerical methods such as those used in POISSON are essential to obtain the desired results. With the help of the modelling techniques described here, it is possible to define the magnet geometry with improved accuracy. 


\subsection{Computer Aided Cross-section Measurement and Analysis}

The shape of the coil and yoke undergoes significant deformation when a large compressive force is applied on the superconducting coils in the magnet. Typically, the coil and yoke cross section deforms from a circular to an elliptical shape and bends inward on the horizontal plane. Moreover, during the coil curing process, there may be a small change from design in the turn-to-turn spacing (or effective thickness) of the insulated cable. These deformations affect the field quality in the magnet. In order to incorporate these deformations in a magnetic model, the actual mechanical cross section of the coil and iron geometry in the magnet after it is built must be known. Commercially available software such as ANSYS [7] can compute the deformation under the applied force based on a certain model. The results of such calculations [101] depend on the details of the model and are usually not reliable to the desired $25 \mu m$ accuracy. In any case, a confirmation of the mechanical model would be desirable before relying on the accuracy of magnetic field calculations based on the model.

The following method is presently followed [159] to obtain an actual mechanical description of the coil and yoke cross section in the magnet. The magnet is physically cut perpendicular to the axis into a thin slice showing the cross section of the magnet on the two sides of the slice. The slice is referred to as a "cookie" and it is etched to enhance the contrast between the insulation, copper and superconductor of the wires. This procedure obviously destroys the magnet. An alternate method [10] was proposed at the SSC laboratory where $\gamma$-ray tomography and spectroscopy is used to construct a 3-dimensional image without cutting the magnet. The method, though very appealing and promising, did not in practice reach the required resolution. The schemes which are presently used at BNL still rely on disecting the magnet. These require a direct mechanical measurement [159] of several thousand wire centers as seen on the cookie or a photograph of it. This task is very demanding in terms of time and human concentration and is subjected to various practical errors. Moreover, it is almost impossible to revisit those measurements since the data processing and other analysis must be done off-line (after the measurements).

The method proposed here is also based on using a cookie to study the magnet cross section. In this method an image of the magnet cross section is digitally stored (rasterized) in a computer where study of the image and data analysis can be efficiently done. The method is based on commercial hardware and software generally used for other purposes. In any case, these are readily available and are relatively inexpensive. The first step in 
this method is to scan a photograph of the cookie, or the cookie itself, directly using a commercial scanner such as the HP ScanJet [92]. Such a scanner converts the object into a rasterized image. This rasterized image can be stored to a desired resolution in a variety of popular formats such as TIFF. The above digitization could also be obtained directly using a video camera and special software. The desired accuracy in this step is at the level of 25-50 $\mu \mathrm{m}$. The issue of overall positional accuracy in the method will be discussed later.

In Fig. 2.2.1, a portion of the cross section of the coil in the $40 \mathrm{~mm}$ aperture SSC dipole DSS005 is shown. This was obtained when a photograph of a cookie was scanned. In order to enhance the contrast on the photographic image the cookie was etched and the photographic techniques were optimized for this application. Though in reality one would work on the whole image at a time, here only a part of the 2 layer coil cross section is shown to elaborate the details. One can clearly see the two layers separated by darker insulation. The insulation also separates the various turns (cables) in each layer. The copper appears light gray and it can be seen in the wedge and also at the center of each wire in the inner layer cable. A number of superconducting wires (strands) can be seen in each cable.

This image can now be examined with a number of software packages. A simple software package like HP Paintbrush [91] can be used to manually obtain the location of wire centers in Fig. 2.2.1. Alternately, this image can be transported to more advanced programs like AUTOCAD [8] for further analysis. A commercially available program, "CAD Overi.; GS [26] converts the TIFF format file to an IGES format file and overlays this image onto AUTOCAD. This conversion maintains the grayscale which is crucial to determining the center of each wire. This software works as an add-on to AUTOCAD, and thus all of the important analytical tools of AUTOCAD software can be utilized. For example, to perform the simple task of obtaining the coordinates of the center of all wires, one would manually mark them with a mouse and at the end of the run, the output file containing the information where the mouse was clicked could be saved. This would create an ASCII file containing the center of the wires. This file can then be post-processed by other programs which, for example, can compute the field harmonics, etc.

The information provided by this method, even at this stage, has already exceeded that which is obtained by other BNL methods. In addition, one can now re-examine the object (image), if desired, and be assured of repeatability since the image can be placed exactly as before. This re-examination could be used, for example, to verify the center position of a particular wire. This repeatability also permits the measurements to be made in several 


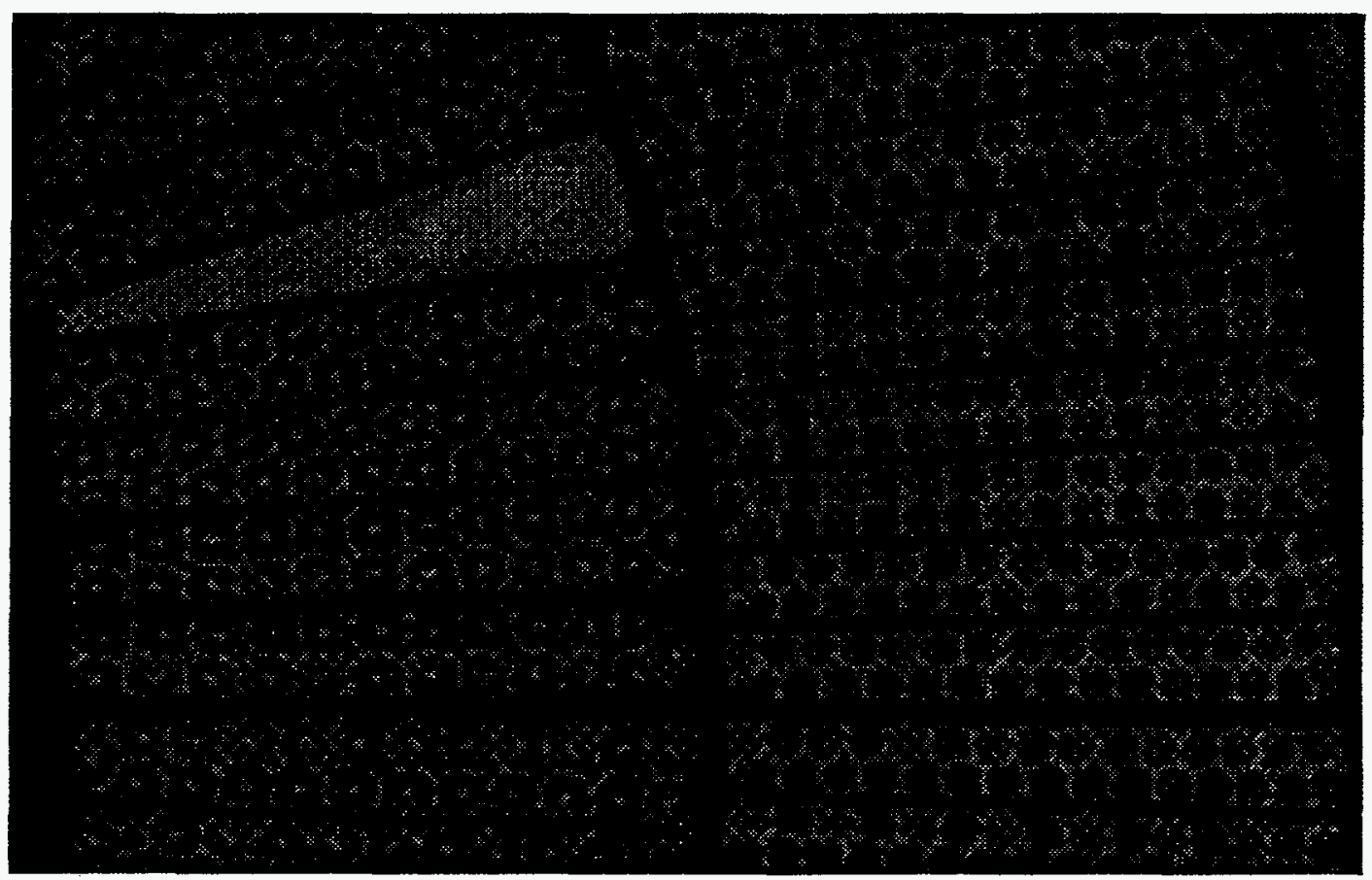

Figure 2.2.1: A raster image of a part of the actual cross-section of the two-layer SSC $40 \mathrm{~mm}$ aperture dipole DSS005. After the normal testing, the magnet was physically cut perpendicular to the magnet axis and across the cross section into a small axial length to make what is referred to as a "cookie". A photograph of the cookie was made after etching. The photograph was scanned and this raster image was electronically saved. The purpose of this exercise is to further study this image with software like "AUTOCAD" [8] to understand the mechanical deformation in the geometry of the coil cross section in the magnet and to compare the measured harmonics with those computed from this geometry (the actual geometry of the magnet as built). 
shifts. One can also easily check, for example, whether all the wires were included in the analysis or not, because those wires which were not included would not have a mark which is left by the click of the mouse. These debugging and advanced features were not possible in the earlier methods. One can also zoom the image to any desired magnification to get better accuracy in finding the wire center or to see visually any type of distortion in the magnet geometry.

An important issue in determining the reliability or accuracy of the method is a loss in linearity when the object is translated into a digital image through the photography and scanning process. These non-linear effects, in principle, can be removed by overlaying a high precision grid image which would go through a similar distortion. In the present method the non-linearity is experimentally determined by examining the distortion in the scanned grid scale and then this information can be used to remove the non-linearity in the image. Kahn [101] examined the extent of loss of linearity and observed that the linearity of a high precision scale is maintained within measurement accuracy and other errors.

The next issue which determines the accuracy of the process is that of resolution in the final digitized image. The photographic image itself has a high resolution and therefore the use of a photograph instead of the real object does not incur any loss in resolution. The practical limit in the past was in the image size following the scanning and post-processing. The HP ScanJet scanner used here had a resolution of 1500 DPI (dots per inch). The desired accuracy in the process is $25 \mu m\left(1 \mathrm{mil}\right.$ or $\left.0.001^{\prime \prime}\right)$ which would require $1000 \mathrm{DPI}$ or more resolution. The memory required for the large photograph when digitized with a high resolution tends to become large. For example, a 10 inch $\times 8$ inch image would use 1.2 megabytes of storage space if $1500 \mathrm{DPI}$ resolution is used. Kahn [101] used 600 DPI resolution scan to test the accuracy of the method - with each pixel representing 1.67 mil. The typical deviation in the position of any point was 1 or 2 pixel with the maximum being 3 pixel. These errors were found to be of a random nature and therefore should not produce systematic errors (particularly since the statistical sample is large for a few thousand wires) in the results obtained based on these measurements. Moreover this error is consistent with that expected from the error in the scale used and also with the quality of the image used in this calibration. In some applications, however, the errors with 600 DPI resolution may be a factor of two higher then desired. In order to maintain the required accuracy in further analysis, the post processing programs (such as AUTOCAD) should be able to read and accept the images with a resolution matching the images produced in the 
scanner. The available resolution (image size in Megabites) was one of the limitations in the study made by Kahn. This should not be a problem now with the advancement in personal computer (PC) technology. Significant advances have been made in recent years not only in the hardware but also in the software, which should result in a significant improvement in the accuracy of the method.

As mentioned earlier, once the image is processed in the AUTOCAD software, one can do a variety of useful online analyses. For example, one can get coil pole angle, size and location of the wedges and individual turns, etc. Moreover, the distortion in the coil or yoke shape can be easily obtained with the help of AUTOCAD. The image can be overlayed on top of the design cross section to compare the measured and mechanical deformations/deviations in the shape, etc. The use of the built-in zooming facilities in the software is helpful in such investigations. These mechanical measurements can be used to verify the mechanical and magnetic modelling and hence to improve the quality of analysis and construction of the magnet.

A cable consists of a large number of wires (23, 30 or 36$)$. In another method the position of a turn (cable) is determined from measurements of the position of all of these individual wires. The proposed AUTOCAD based method provides an option where one can obtain the position of a turn from a single measurement by using a straight line or box fit on the cable surfaces. A turn (insulated cable) can be seen clearly on the screen because of good contrast between the insulation and superconducting wires (see Fig. 2.2.1). In cases where this accuracy is acceptable there is no need to obtain the center position of a large number of wires in the whole magnet. The field harmonic calculations give practically the same results whether one computes the field by integrating over the individual wires in the magnet or over all the cables (each of which contains many wires) in the magnet.

One can also measure the collar, spacer or yoke, etc. by directly putting those objects (or a photograph of them) on the ScanJet. By comparing an image of them before and after the magnet is assembled, one can obtain the deformation at various places which would be useful in checking the results of a mechanical analysis. A partial image of a pair of yoke laminations used in the prototype $80 \mathrm{~mm}$ aperture RHIC arc dipoles is shown in Fig. 2.2.2. This raster image was obtained by directly placing the yoke lamination on the scanner. A comparison of the shape of this lamination outside the assembled magnet (as shown here) and inside the assembled magnet (as obtained from the cookie) would give the change in 


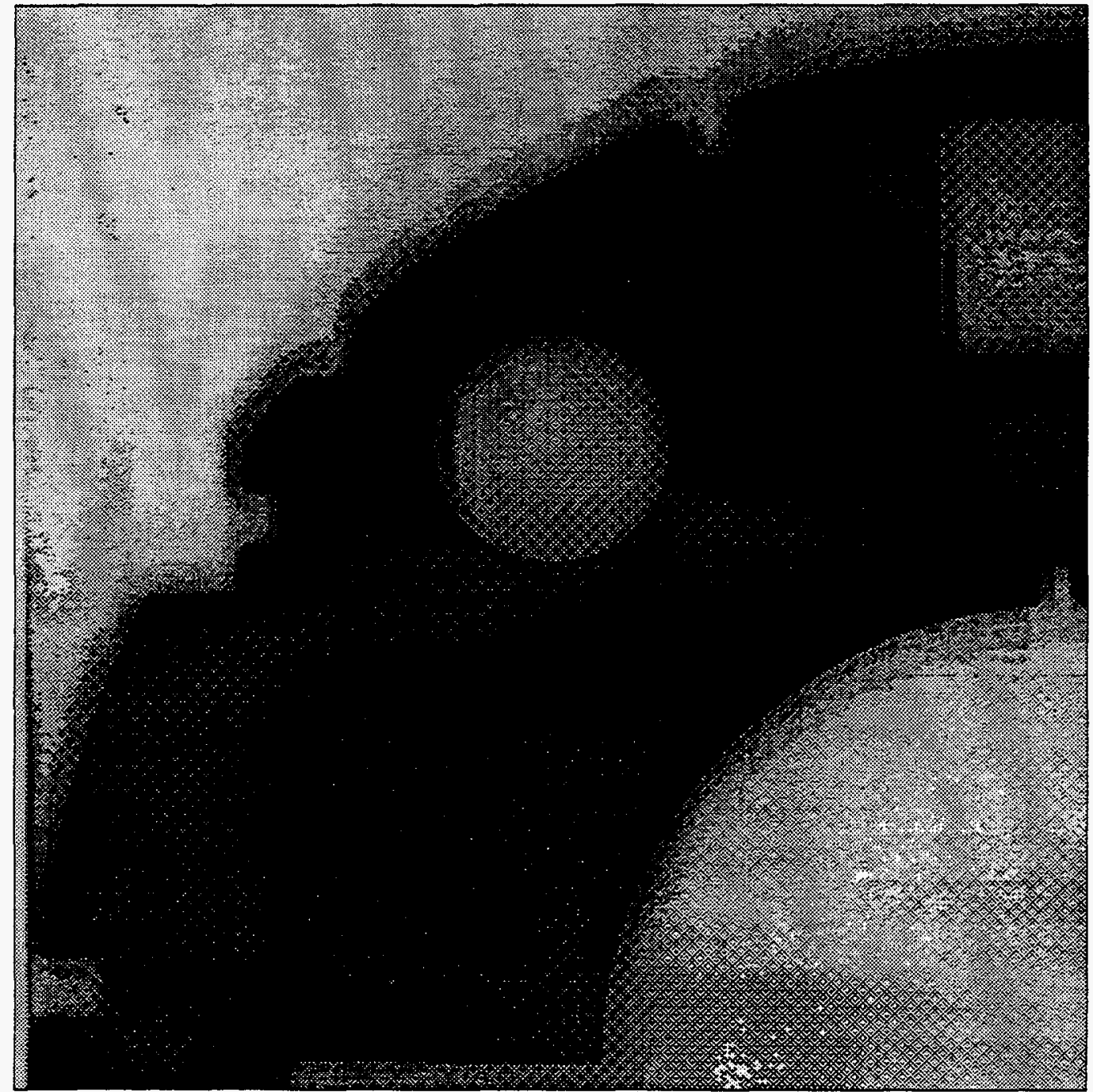

Figure 2.2.2: A raster image of about one-half of a yoke lamination used in a prototype of the RHIC $80 \mathrm{~mm}$ aperture arc dipole. A comparison of the shape of this lamination outside the assembled magnet and inside the assembled magnet gives information about the change in yoke shape due to the large compressive forces used during the magnet assembly. 
the shape and the dimensions of the yoke due to the large compressive forces used in the magnet assembly.

This method (the use of digitized images) has been brought to reality with discussion and help from R. Gottschalk, S. Kahn, R. Hogue, K. Power and M. Rehak. 


\subsection{IMPROVEMENTS IN THE POISSON GROUP CODES}

The 2-dimensional POISSON Group computer codes are used to perform electromagnetic field computations for magnets and radio-frequency cavities. They include four separate major programs : AUTOMESH, LATTICE, POISSON and TEKPLOT written in the computer language FORTRAN. The POISSON Group codes were written and developed by Holsinger and Halbach [79,90] and further modified by other authors $[27,56,57,63,135]$. These programs require a discretization of the geometry with a large number of mesh points (generally over 10,000). The model geometry is defined with the help of nonuniform triangles. The sides of these triangles approximate the curvilinear boundaries of the physical problem. The regions of various material properties and current carrying coils are defined by these boundaries. Program AUTOMESH produces the minimum necessary points on the boundary to complete the description of the bounding surfaces of the regions and assigns them the appropriate properties. The interior mesh points for each region are generated by program LATTICE. Program POISSON finds the solution in terms of the vector potential by the successive point over-relaxation method. Program TEKPLOT is used to graphically display the model, the mesh and the field lines. A more detailed description of these codes can be found in Ref. 135 .

The accuracy of the calculations depends on how well the computer model approximates the actual geometry of the problem, especially in the area where the desired accuracy of the solution is high. This is particularly critical when the geometry includes curved boundaries and when the separation between the boundaries of the various regions is relatively small. To deal with such cases one must (a) have a high mesh density in these critical regions and (b) have control on the way the mesh is generated so that the details of the geometry can be incorporated by refining the mesh. An increase in the total number of mesh points (and hence the mesh density everywhere) may be limited not only by the computer resources but would also excessively increase the computational time. To overcome these problems, the POISSON Group codes (in particular AUTOMESH and LATTICE) have been extensively enhanced with over 10,000 lines of additional code. Improved techniques for generating a more optimized mesh have been developed and three new methods of giving AUTOMESH input are described which exploit the use of those techniques. An example will be given for each of these methods that explains these techniques. These improvements enable one to have full control over the way the mesh is generated and in particular the way the mesh density is distributed throughout the model. A higher mesh density in certain 
regions coupled with successively lower mesh density in others keeps the accuracy of the field computation high and the requirements on the computer time and computer memory low.

Following is a brief description of the improved AUTOMESH. A detailed description and the improvements in LATTICE and POISSON can be found in Ref. 56 and Ref. 57.

\subsubsection{Upgraded AUTOMESH - Input Method No. 1}

An input to the new AUTOMESH based on this method is given in Fig. 2.3.1. The model is for a proposed design of a $40 \mathrm{~mm}$ aperture 2-in-1 SSC dipole magnet [139]. Though the cross section of the coil is the same in the two apertures, the magnitude of the current may be different. Therefore, a model must be made for one half of the magnet. The model geometry is shown in Fig. 2.3.2. The input conventions and various NAMELIST parameters are compatible with those described in the User's Guide to the POISSON Group codes [135]. Only the use of new NAMELIST parameters will be discussed here. Throughout this section variables starting with $X$ and $Y$ refer to the physical coordinates and $K$ and $L$ to the logical coordinates.

One of the major motivations for improving AUTOMESH was to be able to use a variable mesh size efficiently. This method offers complete control of the mesh size with the help of new NAMELIST parameters (XSTR,XEND), (KSTR,KEND) and/or (YSTR,YEND), (LSTR,LEND). The points at XSTR are assigned the logical coordinate KSTR and at XEND the logical coordinate KEND. The physical increment (DX) between two consecutive logical coordinates is (XEND-XSTR)/(KEND-KSTR). A similar concept is adopted for (YSTR,YEND) and (LSTR,LEND). The scale, once so determined, is used in subsequent regions until changed. Any such scale can be stored by the NAMELIST parameter ICARY =1 and may be recalled by specifying ICARY=-1. ICARY=0 (Default) uses the current scale. In addition to providing complete control of the mesh size in each region, the method also permits a change in mesh size within the same region. This is accomplished by breaking a region into a number of contiguous sub-regions. The NAMELIST parameter $\mathrm{ICONT}=1$ indicates that the next region is a continuation of the present region. Once a region is broken into several sub-regions, one can use the techniques described above to use 
different scales. ICONT $=0$ signals the end of the continuation. The NAMELIST parameter NEXT is used (in the first region) to store information about the number of such extra regions.

In the example of Fig. 2.3.1 and Fig. 2.3.2, a very dense mesh is used in the coil regions. The maximum accuracy in the field calculations is desired at the center of the coil. The mesh size is reduced gradually in the regions away from the coils where the magnetic field is also lower and therefore an error in the geometry has only a small effect. This strategy of change in mesh size keeps the number of mesh points low and, therefore, the computation time low without significantly compromising the accuracy of the results. The coils in the example have two layers - inner and outer. The mesh size in the coil region is sufficient to separate different regions (blocks) of coils within the inner and outer layers. It is, however, not enough to produce a separation between the two layers and to devise a small midplane gap. To incorporate these details the coils are moved away by one unit in the logical coordinates - in physical space the coils do not move [56].

A more detail description of this model and method can be found in [56]. 


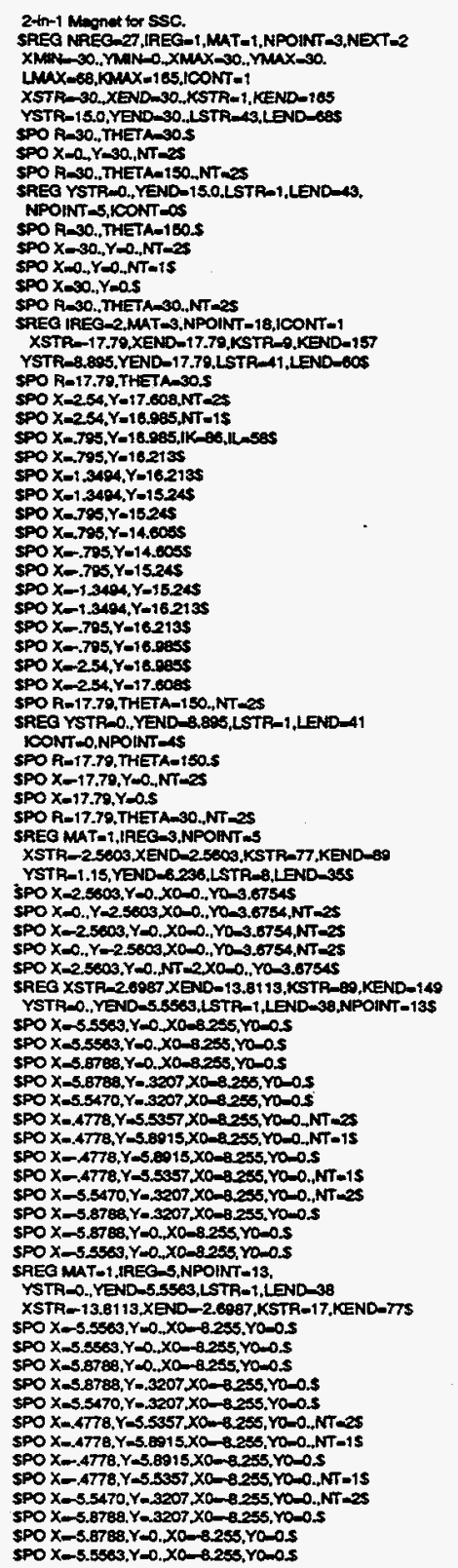

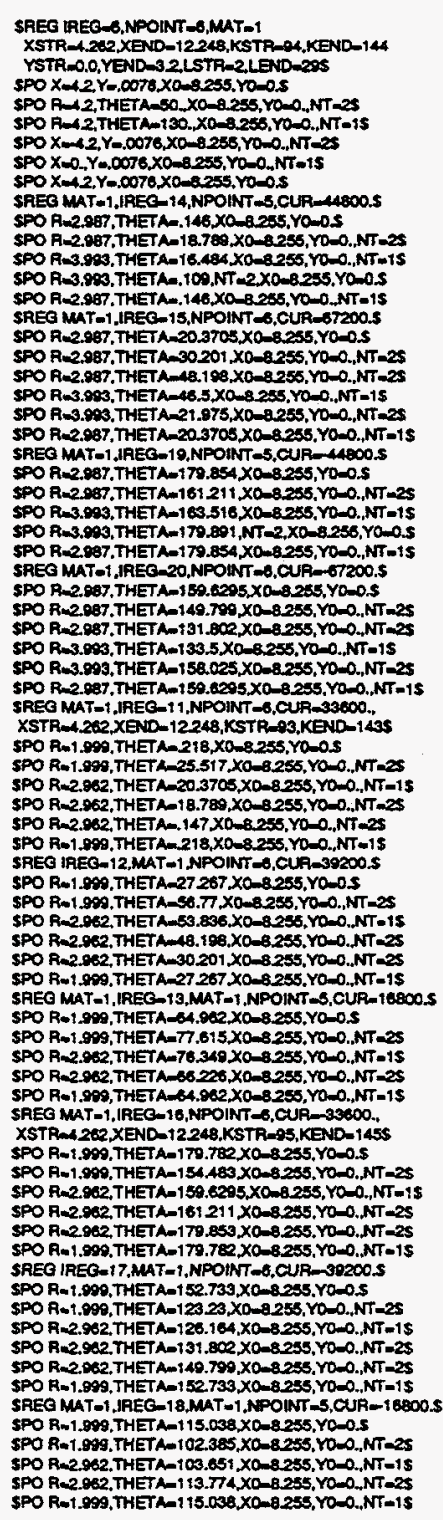

SAEG IREQ - S.NPOINT -0, MAT-1 YSTA-0.0, YEND-32.LTTR-2.LEND-20S $5 P 0 \times 0+2, Y-, 0078 \times 0-0.255, Y 0-0.5$ SPO R-12,THETA-130.,X0-8.256.Y0-0.NT-1S SPO Xins, $2, Y=.0078, X 0-8235, Y 0-0 . N T-25$

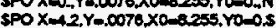
SAEQ MAT -1,IREQ - 14,NPOINT - $\$$, CUR 4800 . SPO R-2.987,THETA-18.780.X0-8255,Y0-0.NT-2

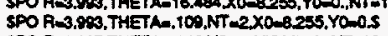

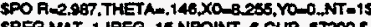
SPO P. 2.087 THETh-203705 $\times 0-0255$ Y YoSPO A-2.987.THETAM-30201, X0-82.255, YO-0.,NT-2S SPO RSPO A-3.903.THETA-21.975, X0-0255, YO-0.,NT 22 SPO RM 2.987. THETA-20.3708,XO-8.255,YO-O.NTT SPO R-2.087.THETA-179.854 X0-8.255,YO-0.5 SPO R-2.287.THETA-161.211, X0-8.255.YO-O.NT-2S SPO A-3.003.THETA-170

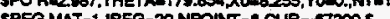

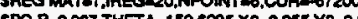

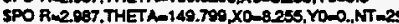

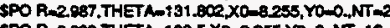
SPO Ri3..903.THETA-158.025, X0-8.255.Y0-0.,NT-25

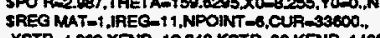
XSTRM, 262, XEND-12248, KSTR-03,KEND

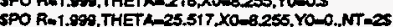
SPO R-2.262.THETA=20.3705 X0-8.255, Y0-0.NT-15

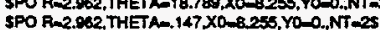
SPO R-1.999, THETA-218 X0-8.255, YO-O.NT-19 SPEO Ro1 SPO Ri 1.900 ,THETA-SO.77 X0-82.255, Y0-0.NT-25

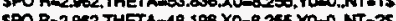
SPO R- 0.002 THETA SPO R-1.099.THETA-27267 X0-8255.YO-O.NT-1S

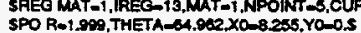
SPO R-1.999, THETA=77.615. X0-8255,Y0-0.NT-2S SPO RSPO R-1.908, THETA-A. 062 , X0-8255, Y0-0.,NT-1S $X S T R$ and 2ER, XEND

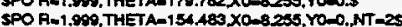
SPO P-2.962, THETA-159.6295, X0-0255, YO-0.NT-1 SPO R-2.962. THETA-161 211 X0-8.255.Y0-0.NT -2S

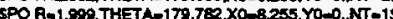
SREQ IREG =17.MAT-1.NPONT-6.CUR-30200

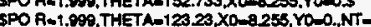
SPO R-2.962.THET A-125.164, X0-8255, Y0-0.NT-1S SPO RN2.926.THETA=131.800, X0-8255, YO- YN.NT-25 SPO R-1099 THETA-158739X0.825

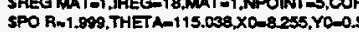
SPO RM2.962.THETA-103.651, X0-8255, Y0-0.NT-15

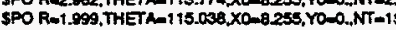

SREQ MAT-1,IREQG-7,NPOINT-6,CUR-O., XSTR=12.248,XEND-4262,KSTR-22,KEND-72S SPO X $-12,2.0 .0075 \times 0-8.255, Y 0-0.5$ SPO RAd.2. THETA-50. X0-0.265.YO-O,NT-2S SPO R-1.2,THETA 130.X0-8.235.YO-O.NT-1 SPO X $=0.4$ Y $=0.0076 \times 00-0.265$ Y Y SPOX X-4.2,Y $-0.0078, X 0-8.255, Y 0-0.5$ SREQ MAT-1, IREO-24,NPOINT -5, CUR-44800s SPO R-2.087, THETA 14.146 , X0-8.253, YO-0.5 SPO R-2.087, THETA-18.780,X0-8.255,Y0-0,NT-2S SPO R-3.903, THETA-16.464, X0-8.255,YO-O.NT-1

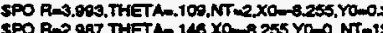

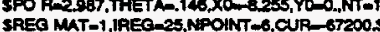
SREO MAT-1, IREO-25,NPOINT-6,CUR-67200.5 SPO R-2.087. THETA-30.201,X0-8255, YO-O.NT-2

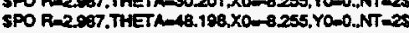
SPO R-3.003.THETTA-46.5, X0-8.255,YO-0,NT-15

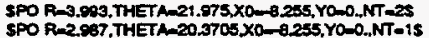
SPO R-2.987, THET A-20.3705 X0-0.255,Y0-0.NT-1
SREO MAT-1,IAEQ-20,NPOINT 5 , CUR -48200.S

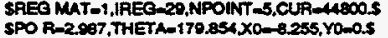

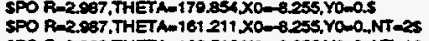

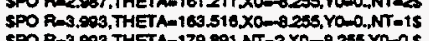
SPO R-3.003,THETA-170.891, NT-2, $\times 0-9.235$, Yo- 0.5 SPO R-2.267, THEFA-170.854, XD-8.255, YO- .,NTSREG MAT-1, IAEG-30,NPOINT -6, CUR-67200.5 SPO R-2.087, THETA=159.6205. X0-8.255, Y0-0.5 SPO R-2.097,THETA-149.799X0-8255, Y0-0.NT-2S

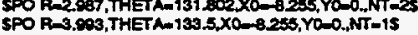
SPO R-3.903, THETA-158,Ce5, X0-8.255, Y0.0.,NT-25 SPO R-2087, THETA-150.6205, X0-8.255, Y000.NT-15 SPES MAT -1 .IREQ 21. NFOINT -6 . CUP 33600 .

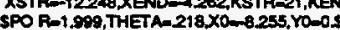
SPO R=1.009, THETM-25.517, X0-8.255, YO-0.,NT-2S SPO R-2.062.THETA $20.3705 . X 0-8255$, Y0-0..NT- IS SPO R-2.062,THETA-18.789.X0-8.255,YO-O.NT-25

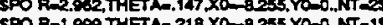
SPO R-1 909,THETA $218, \times 0-8255$, YO-0.NT-1S SRE RE SPO R-1.009.THET A $27.267 \times 00-8.255$, YO-OS SPO R-2062 THETA-50.836 X0-8.255.YO-O NT-15

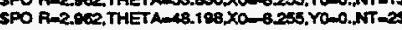
SPO R-2 D02.THETA-30.201, X0-8255, YO-O.NT-2 SPO R-1.900.THETA-27.267. X0-8.255.YO-0.NT-15 SREQ MAT-1. IREQ-23.MAT-1.NPOINT-S.CUFT-16800. SPO R-1.008.THETA-64.962, X0-8.255, Y0=0.5

SPO R-1.999.7HET A -77.615.X0-8255, YO-O.NT-25

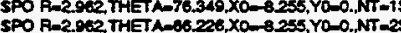
SPO R-1.009.THETA-64.962. X0-8.255, YO-N.NT-1S SREQ MAT-1, ,REO-26, NPONNT -S, CUR-33800 XSTR-12.248,XEND 6 262,KSTR-23,KEND-73, SPO A-1.099,THETA-179.782, X0-8.255,Y0-0.5 SPO R-1.999.THETA-156.483.X0-8255. YO-0.NT m 2S SPO A-2.002,THETA $150.6203, \times 0-6.255, Y 0-0 . N T=15$

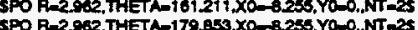
SPO R-1 SREQ IREO-27.MATA. NPOINT - B.CUR-30200.5 SPO R-1.099.THETA-152.733. X0-8.255, Y0-0.5 SPO R-1.000.THETA-12323, X0-8255, Y0-0,NT-2s SPO R-2.962, THETA-126.164, X0-8.255, Y0-0.,NT-15 SPO R-2.962.THETA-131.802 X0-8.255,YV-0.NT-25 SPO RM2.962.THETA-149.798.X0-8.255, Y0-0.NTT-2S

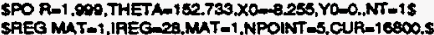

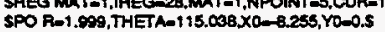
SPO R-1.999.THETA-100.385.X0-8255, YO-0.NT-25 SPO A-2.962.THETA=103.651 X0-8.255. Y0-0.NTT-1S

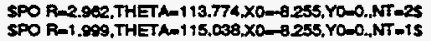

Figure 2.3.1: An input to AUTOMESH of the POISSON Group codes for creating a mesh based on the upgraded AUTOMESH - Input Method No. 1. The model is for a proposed design of a $40 \mathrm{~mm}$ aperture 2-in-1 SSC dipole magnet. 


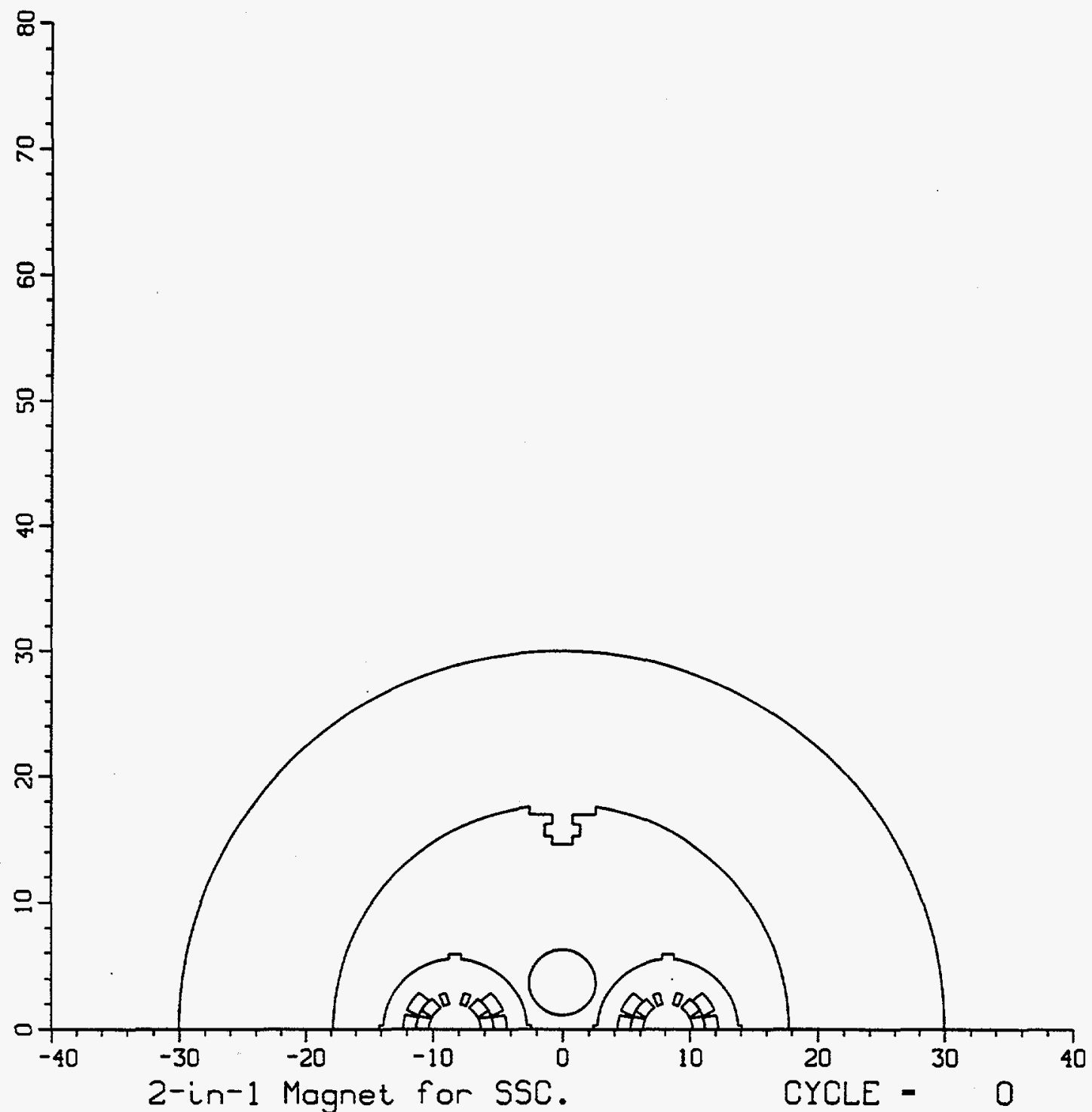

Figure 2.3.2: A POISSON model for the example based on the $A U$ TOMESH Input Method No. 1. The model is for a proposed design of a $40 \mathrm{~mm}$ aperture 2-in-1 SSC dipole magnet. 


\subsubsection{Upgraded AUTOMESH - Input Method No. 2}

In this method, the distribution of the mesh density is managed with the help of several special regions, referred to here as SREG (normal regions are referred to as REG). The purpose of these special regions is to generate a preliminary but complete mesh with appropriate calls to LATTICE before the actual run of AUTOMESH starts. The input to AUTOMESH contains NSREG special regions in addition to the NREG normal regions, as shown in the example given in Fig. 2.3.3. The special regions must be given before the normal regions and most of the rules and conventions which apply to normal regions apply to these special regions as well. However, there are the following three exceptions :

- Instead of using the NAMELIST variables MAT, CUR etc., one would use new NAMELIST variables for defining the mesh size and other related quantities. The use of these variables SX0,SX,NX0,NX, SY0,SY,NY0,NY (having zero default values) is explained below by an example :

$\$$ SREG $\mathrm{SX}=10 ., \mathrm{NX}=10, \mathrm{SY} 0=2 ., \mathrm{SY}=5 ., \mathrm{NY} 0=8, \mathrm{NY}=6 \$$

This will make $10(\mathrm{NX})$ divisions between 0.0 (SX0) and 10.0 (SX) in the $\mathrm{X}$ dimension and 6 (NY) divisions between 2.0 (SY0) and 5.0 (SY) in the $Y$ dimension; moreover 8 (NY0) mesh points will be left before 2.0 (SY).

- The first special region will be automatically generated as a rectangular region with the following points in the specification of the first region :

$$
\text { (SX0,SY0), (SX,SY0), (SX,SY), (SX0,SY), (SX0,SY0). }
$$

In the example given in Fig. 2.3.3, these points are : (0.,0.), (0.,25.), (25.,25.), $(25 ., 0$.$) and (0 ., 0$.$) . The next NSREG regions must be explicitly given but$ they need not, however, have a rectangular shape.

- These special regions should either be closed regions or their end points should lie on a region boundary of a previously defined special region.

The implementation of the mesh density management system into the program is described here. In each special region the program freshly computes parameters such as XMIN, XMAX, DX,... with the help of NAMELIST variables SX0, SX, NX0, NX,... The necessary points on the boundary of each special region are found and the region data are generated. An intermediate file TAPE74.DAT;1 (similar to TAPE73.DAT) is created with 
the NSREG+1 number of special regions. At this stage the program calls several subroutines of LATTICE to generate a complete mesh using the file TAPE74.DAT;1 as input. This mesh is later used to find the logical coordinates in the normal regions.

In addition to the above modifications, the four new variables XADD, YADD, RADD, TADD in the \$REG NAMELIST statement are also included. These variable are used to bias (shift) the path of finding the logical coordinates in a certain preferred direction. They are essential in resolving two curves when the separation between them is less than a mesh spacing.

An example which demonstrates the complete use of this method is given in Fig. 2.3.3 which is the input to AUTOMESH. The model geometry of this problem is shown in Fig. 2.3.4. The mesh generated by the above input of AUTOMESH is shown in Fig. 2.3.5. The model described here is for a coldmass of the $40 \mathrm{~mm}$ aperture SSC dipole magnet [139]. Because of the dipole symmetry, only a quadrant of the dipole magnet need be included in the model.

To resolve a small separation between the inner and the outer layers, a negative value $(-0.06)$ of RADD for the coil regions in the inner layer (IREG=11, IREG=12, IREG=13) and a positive value $(+0.06)$ for the regions in the outer layer (IREG=14 and IREG=15) is used. It logically increases the separation between the two layers by $0.12 \mathrm{~cm}$. Similarly, to resolve an angular separation between the first and second blocks in both inner and outer layers, an angular bias is used with the variable TADD in the second blocks (region number 12 and 15). A very small midplane gap is incorporated with the help of parameter YADD in the first blocks of these two layers (see IREG = 11 and IREG =14).

The accuracy of the field computations at the center of the aperture depends on a good coil description. Therefore, as shown in Fig. 2.3.5, a very dense mesh is used in the coil region. To keep the total number of mesh points low the mesh density is successively reduced in the outer regions.

A more detail description of this model and method can be found in Ref. 57. 


\author{
A Superconducting Dipole Magnet \\ SREG NSREG $=3, N R E G=11, \mathrm{~S} X=25 ., \mathrm{NX}=70, \mathrm{SY}=25 ., \mathrm{NY}=70$ S \\ \$SREG SX $=14.0, N X=62, S Y=14.0, N Y=62, N P O I N T=2 S$ \\ \$SPO $X=14.0, Y=0 . \$$ \\ $\$ S P O X=0 ., Y=14.0, N T=2 S$ \\ SSREG $S X=6.0, N X=40, S Y=6.0, N Y=40, N P O I N T=2 S$ \\ \$SPO $X=6.0, Y=0 . \$$ \\ $\$ S P O X=0 ., Y=6.0, N T=2 \$$ \\ $\$ S R E G$ SX $=4.20, N X=30, S Y=3.25, N Y=30, N P O I N T=3 \$$ \\ \$SPO $X=4.2, Y=0 . \$$ \\ $\$ S P O R=4.2, T H E T A=51 ., N T=2 \$$ \\ SSPO $X=0 ., Y=3.25 \$$ \\ SREG IREG $=1, M A T=1, C U R=0, N P O I N T=4 S$ \\ \$PO $X=25 ., Y=0 . \$$ \\ SPO $X=0 ., Y=25 ., N T=2 S$ \\ \$PO $X=0 ., Y=0 . \$$ \\ $\$ P O X=25 ., Y=0 . \$$ \\ $\$ R E G$ IREG $=2, M A T=3, C U R=0, N P O I N T=8 S$ \\ $\$ P O X=12.9791, Y=0 . \$$ \\ SPO $X=12.9791, Y=1.905 \$$ \\ SPO $X=13.1995, Y=1.905 S$ \\ SPO $X=2.159, Y=13.1604, N T=2 S$ \\ SPO $X=2.159, Y=10.6172 S$ \\ SPO $X=0 ., Y=10.6172 \$$ \\ SPO $X=0 ., Y=0 . \$$ \\ SPOX $=12.9791, Y=0 . \$$ \\ SREG MAT $=1$, IREG $=3, \mathrm{NPOINT}=4 \$$ \\ SPO $R=1.4605, T H E T A=0 ., X 0=5.08, Y O=9.525 \$$ \\ SPO $R=1.4605$, THETA=120.,NT $=2, X 0=5.08, Y O=9.525 \$$ \\ SPO $R=1.4605, T H E T A=240 ., N T=2, X O=5.08, Y O=9.525 \$$ \\ SPO $R=1.4605$, THETA $=0 ., N T=2, X 0=5.08, Y 0=9.525 \mathrm{~S}$ \\ SREG MAT $=1$,IREG $=4, \mathrm{NPO} \mid \mathrm{NT}=4 \mathrm{~S}$ \\ SPO R $=0.4832$, THETA $=0 ., X 0=10.16, Y 0=6.915$ \\ SPO $R=0.4832$, THETA $=120 ., N T=2, X O=10.16, Y 0=6.915 S$ \\ SPO $R=0.4832$, THETA $=240 ., N T=2, X O=10.16, Y O=6.915 \mathrm{~S}$ \\ SPO $R=0.4832, T H E T A=0 ., N T=2, X O=10.16, Y O=6.915 \$$ \\ SREG IREG $=5, M A T=1, N P O I N T=8 \$$ \\ SPO $X=5.8865, Y=0 . S$ \\ \$PO $X=5.8865, Y=.3207 \$$ \\ $\$ P O X=5.5470, Y=.3207 \$$ \\ SPO $X=0.254, Y=5.5505, N T=2 \$$ \\ SPO $X=0.254, Y=5.823, N T=1 \mathrm{~S}$ \\ SPO X $=0 ., Y=5.823 \mathrm{~s}$ \\ SPO $X=0 ., Y=0 . \$$ \\ $\$ P O X=5.8865, Y=0 . \$$
}

\author{
\$REG NPOINT $=3, \mid R E G=6 \$$ \\ SPO $X=4.2, Y=0 . \$$ \\ $\$ P O R=4.2, \mathrm{THETA}=51 ., \mathrm{NT}=2 \mathrm{~S}$ \\ \$PO $X=0 ., Y=3.25 \mathrm{~S}$ \\ SREG MAT $=1$, IREG $=11$, NPOINT $=5$, \\ CUR $=35400 ., R A D D=0.06, Y A D D=0.08 S$ \\ \$PO $R=1.999$, THETA $=0.218 \$$ \\ SPO R=1.999, THETA $=25.517, \mathrm{NT}=2 \$$ \\ \$PO $R=2.962, \mathrm{THETA}=20.463, \mathrm{NT}=1 \$$ \\ SPO $R=2.962, T H E T A=0.15, N T=2 \$$ \\ SPO $R=1.999$, THETA $=0.218 \$$ \\ SREG IREG $=12$, MAT $=1$, NPOINT $=5$ \\ CUR $=41300 .$, RADD $=0.06, T A D D=2.5 \mathrm{~s}$ \\ SPO $R=1.999, T H E T A=27.267 \mathrm{~S}$ \\ SPO $R=1.999$, THETA=56.77, $\mathrm{NT}=2 \$$ \\ SPO $R=2.962$, THETA $=53.836, N T=15$ \\ SPO $R=2.962$, THETA $=30.201, N T=25$ \\ \$PO R=1.999,THETA=27.267\$ \\ \$REG MAT $=1$, REEG $=13, M A T=1$, NPOINT $=5$, \\ CUR $=17700 ., \mathrm{RADD}=0.06 \mathrm{~S}$ \\ SPO R=1.999, THETA $=64.962 \mathrm{~S}$ \\ SPO R=1.999,THETA $=77.615, \mathrm{NT}=2 \mathrm{~S}$ \\ SPO R=2.962, THETA $=76.349, \mathrm{NT}=1 \mathrm{~S}$ \\ SPO R=2.962, THETA $=66.226, \mathrm{NT}=2 \$$ \\ \$PO R=1.999, THETA=64.962\$ \\ SREG MAT $=1$, IREG $=14$, NPOINT $=5$, \\ CUR $=47200 ., \mathrm{RADD}=0.06, \mathrm{YADD}=0.08 \mathrm{~s}$ \\ SPO R $=2.987$, THETA $=0.15 \$$ \\ SPO R=2.987, $T H E T A=18.789, \mathrm{NT}=2 \mathrm{~S}$ \\ SPO R=3.993, $T H E T A=16.484, \mathrm{NT}=1 \mathrm{~S}$ \\ SPO $R=3.993$, THETA $=0.11, N T=2 S$ \\ SPO R=2.987, THETA=0.15, NT $=1 \$$ \\ SREG MAT $=1, \mathrm{IREG}=15, \mathrm{NPOINT}=5$, \\ CUR $=70800 ., \mathrm{RADD}=0.06, \mathrm{TADD}=2.0 \mathrm{~s}$ \\ \$PO $R=2.987$. THETA $=20.278$ \$ \\ SPO $R=2.987, T H E T A=48.198, N T=2 S$ \\ SPO $R=3.993, \mathrm{THETA}=46.5, \mathrm{NT}=1 \$$ \\ $\$ P O R=3.993, T H E T A=21.975, \mathrm{NT}=2 \$$ \\ \$PO R=2.987, $\mathrm{THETA}=20.278, \mathrm{NT}=1 \$$
}

Figure 2.3.3: An input to AUTOMESH of the POISSON Group codes for creating a mesh based on the upgraded AUTOMESH - Input Method No. 2. The model described here is for the coldmass of the $40 \mathrm{~mm}$ aperture SSC dipole magnet. 


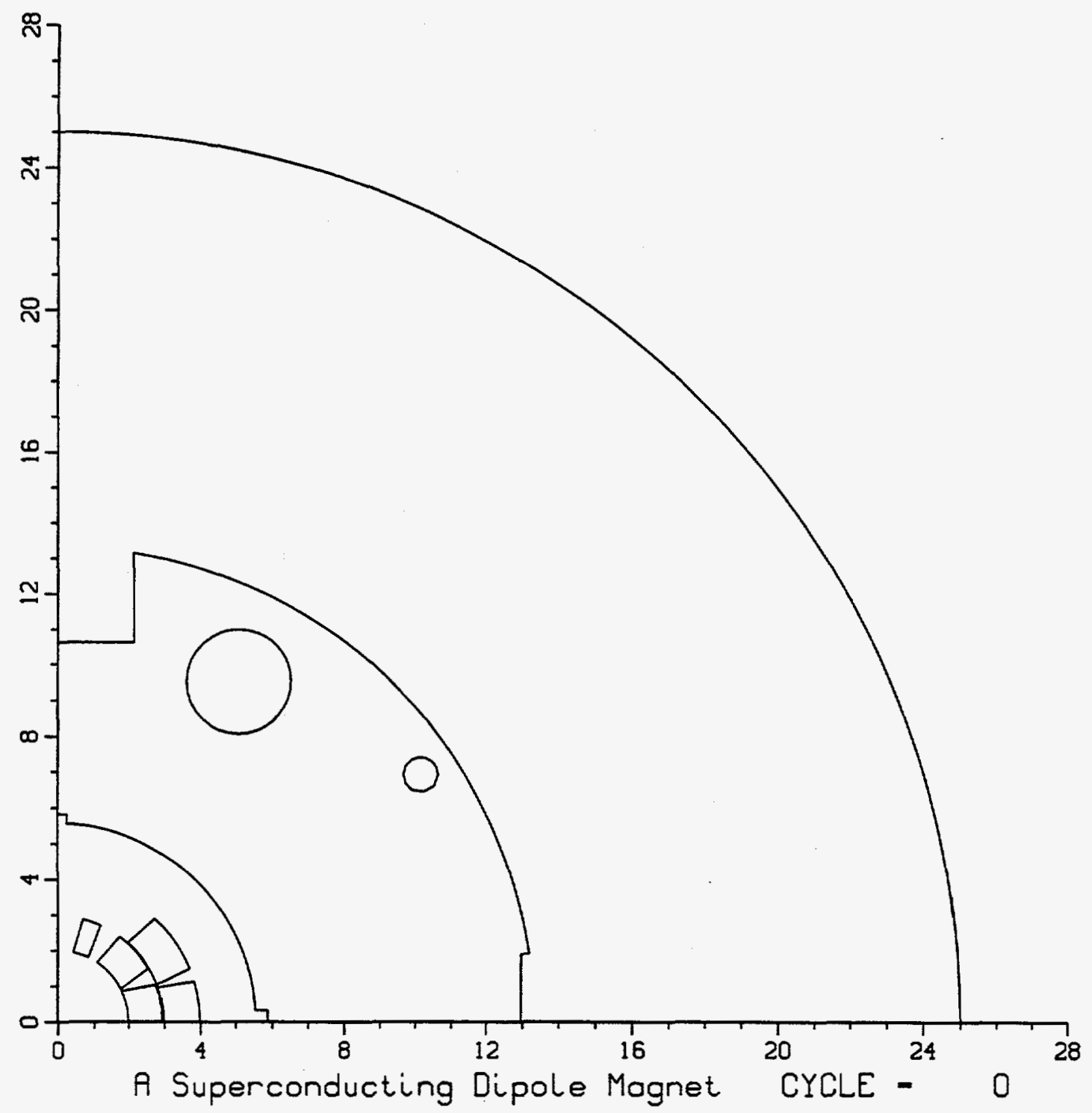

Figure 2.3.4: The POISSON model for the example given, using the AUTOMESH Input Method No. 2. The model is for a $40 \mathrm{~mm}$ aperture SSC dipole magnet. 


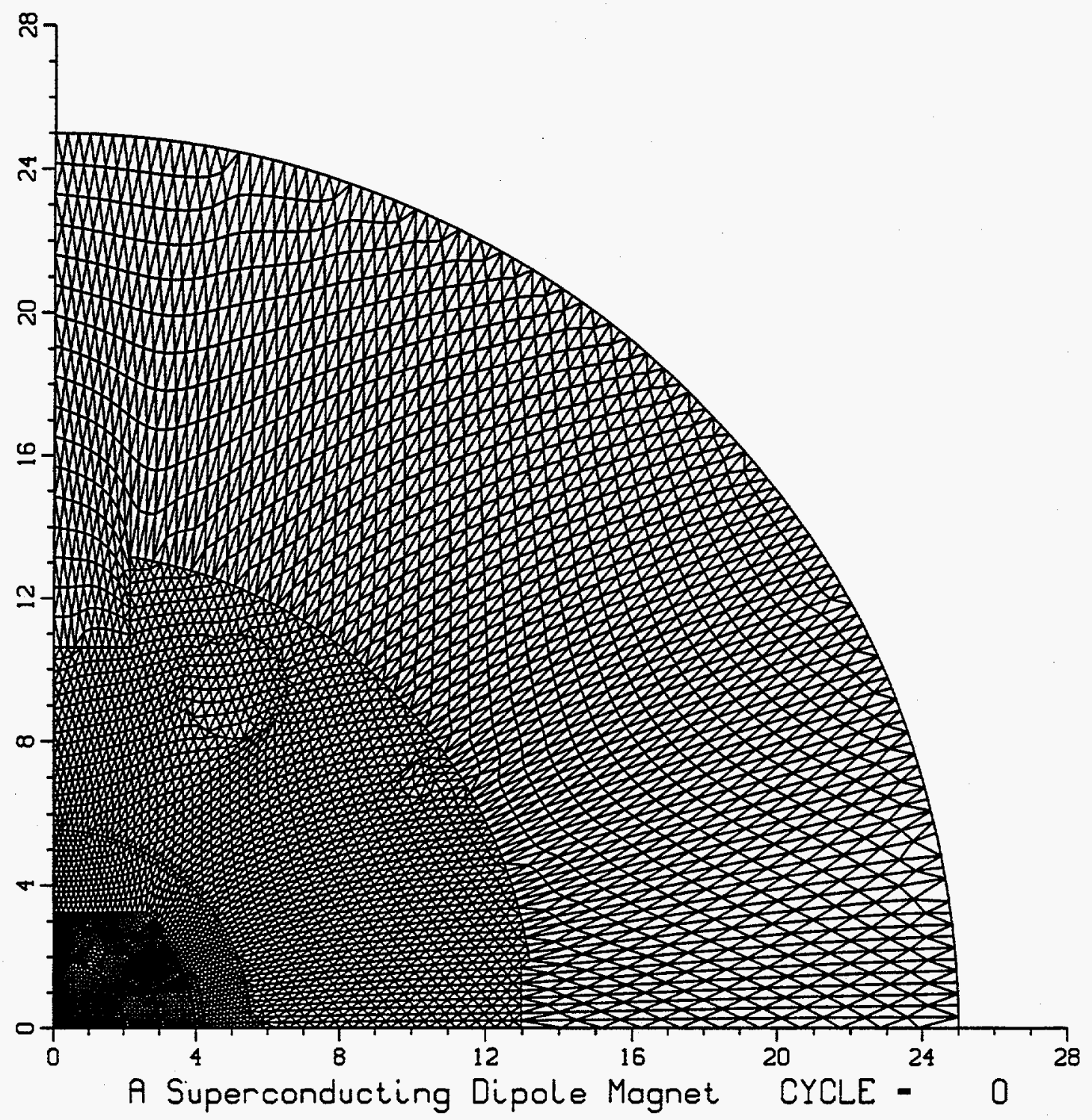

Figure 2.3.5: The mesh for a POISSON model generated by the AUTOMESH Input Method No. 2. The model is for a $40 \mathrm{~mm}$ aperture SSC dipole magnet. A higher mesh density is used in the aperture region and a gradually decreasing mesh density in the regions away away from it. This combination increases the accuracy of the field calculations in the magnet aperture without proportionally increasing the number of mesh points. 


\subsubsection{Upgraded AUTOMESH - Input Method No. 3}

From a user point of view, conceptually this method is an extension and generalization of the original XREG method for varying the mesh size in the problem. However, in the original method, one was allowed to have only three different mesh sizes with the restriction that the ratio of the different mesh sizes should exactly be 2:1. In the method described here, one can have up to 18 different mesh sizes with no restrictions. This is true in both the horizontal and vertical planes and as described below one can use both Cartesian and polar coordinates to define the variable mesh size.

The mesh size is defined by new namelist variables (XCARn, KCARn) and (YCARn, LCARn) in Cartesian coordinates and by (RPOLn, KPOLn) and (TPOLn, LPOLn) in polar coordinates (here " $n$ " is a number whose value can be between 1 and 9 ). There can be an offset in the scale in the case of polar coordinates. This offset is defined by the variables X0Pn and YOPn. These polar coordinates are converted into Cartesian ones by simple trigonometric relations.

This method is explained here with the example of input shown in Fig. 2.3.6. The input is for a CBA 2-in-1 magnet [20] and the model based on this input is shown in Fig. 2.3.7 First the working of XCARn and KCARn is discussed. This example illustrates the use of XCARn and KCARn with $X C A R 1=-23.139, X C A R 2=-21.412, X C A R 3=-2.7686, \ldots$ and $K C A R 1=15, K C A R 2=20, K C A R 3=83 \ldots$ This means that $\mathrm{K}$ is 15 for $\mathrm{X}=-23.139$, and $\mathrm{K}$ is 20 for $\mathrm{X}=-21.142$. If this portion ( $\mathrm{X}=-23.139$ to $\mathrm{X}=-21.412)$ is not further divided, then the computed DX (the average spacing between mesh points on the horizontal plane) between XCAR1 and XCAR2 will be $(-21.412+23.139) /(20-15)=0.3454$. Similarly between XCAR2 and XCAR3 the DX will be $(-2.7686+21.412) /(83-20)=0.2959$. A similar concept is used for producing the desired mesh size DY with the variables YCARn and LCARn.

The polar coordinate specification is given with the help of RPOLn and TPOLn. For RPOL5 $=8.678$, TPOL5 $=180$. $, \mathrm{XOP} 5=12.065, \mathrm{KPOL} 5=108$ and LPOL5 $=1$, the computed $\mathrm{X}$ will be RPOL5*COS(TPLO5) $+\mathrm{XO}=3.387$, and $\mathrm{K}$ will be KPOL5 $=108$. Similarly, the computed Y will be RPOL5*SIN(TPLO5) + Y0=0., and L will be LPOL5 $=1$. 


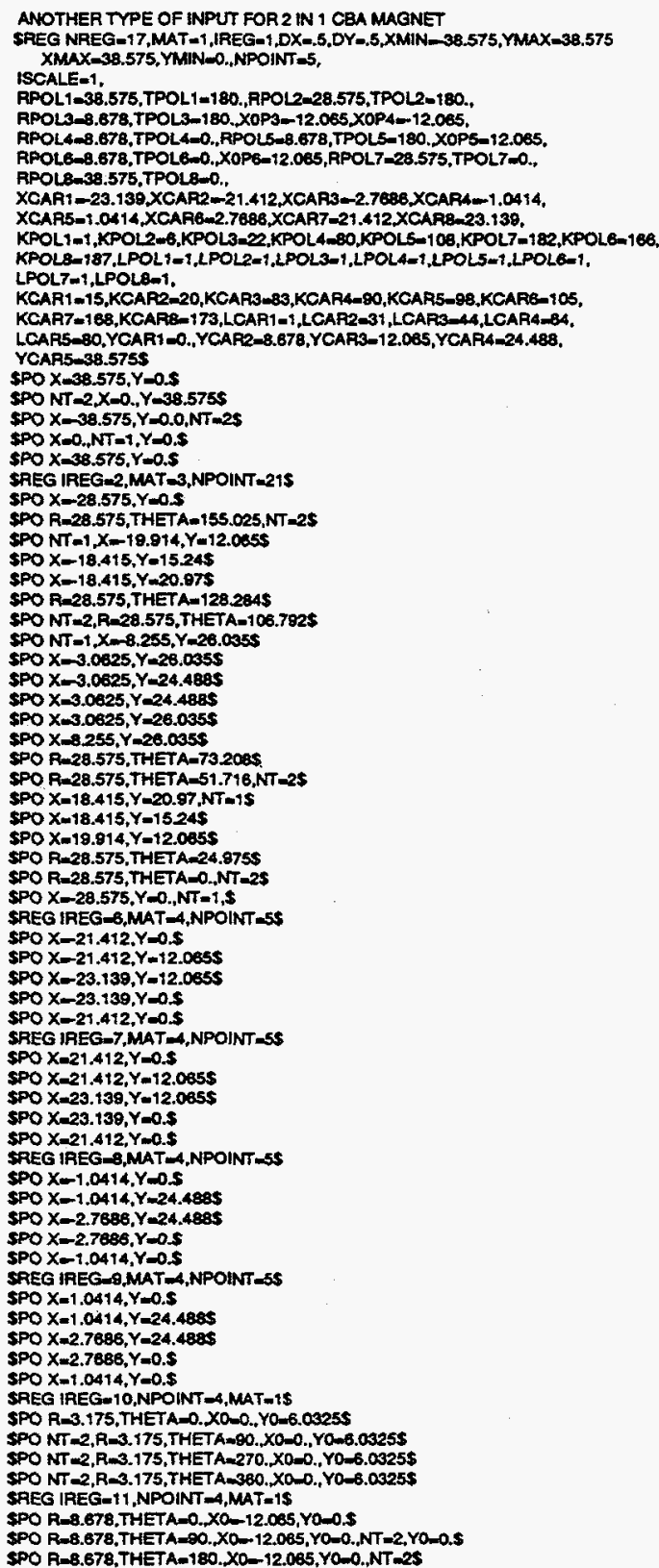




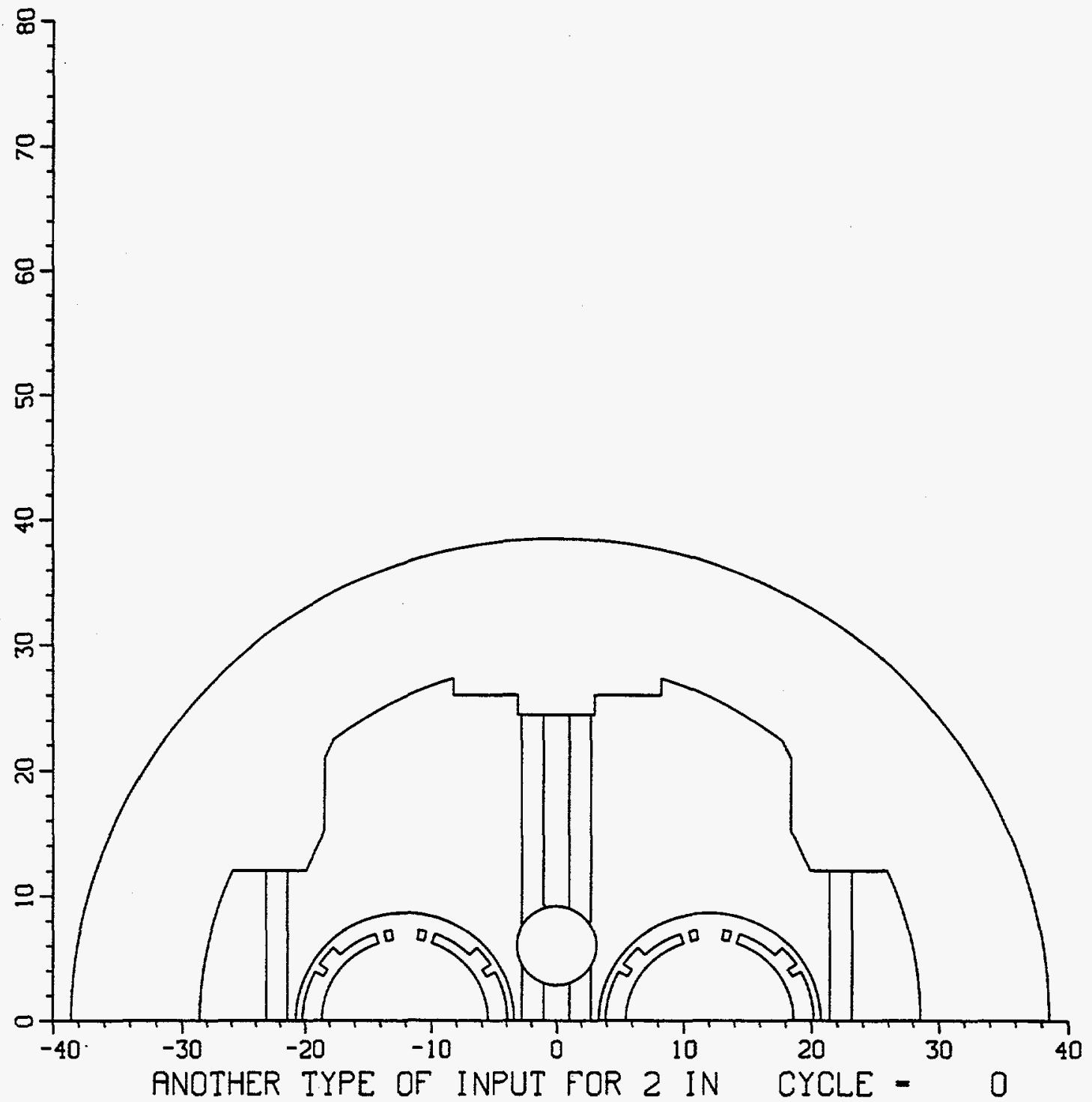

Figure 2.3.7: A POISSON model for the example based on the AUTOMESH Input Method No. 3. The model is for a $130 \mathrm{~mm}$ aperture 2-in-1 CBA dipole magnet. 


\subsection{Conclusions on the Improvements in the Computational and Analy- sis Methods}

A computer aided cross section measurement and analysis method has been developed to evaluate the mechanical deformation on the coil and yoke cross section under large compressive forces. The method creates a digital image of the cross section which can be compared with the original design or with expected mechanical deformations. This method was not used during the R\&D phase of the RHIC magnet program in a significant way because at that time the resolution of the image in the support software was marginal and other methods were in use. However, the advances in computer technology over the last several years makes this method quite attractive now. The major advantage of this method is the availability of a digitized image on the computer. Powerful commercial software like AutoCAD can work with that image to do a variety of analyses.

The POISSON Group codes have been extensively improved to enhance their capability for making computer models. The use of these improved techniques allows an efficient use of the mesh points to define the problem. A high mesh density in the critical regions coupled with successively lower mesh density further away keeps the accuracy of the field calculations high and the computation time low. The POISSON Group codes have been used throughout this research work to perform most of the field calculations, especially in analyzing the contribution to the magnetic field from the iron yoke. 


\section{Chapter 3.}

\section{FIELD QUALITY IMPROVEMENTS THROUGH YOKE DESIGN}

The research work described in this chapter is partly based on the following papers :

- R.C. Gupta, G.H. Morgan, A Design for a High Field Combined Function Superferric Magnet, Proceedings of the 1985 Particle Accelerator Conference, Vancouver; IEEE Transaction on Nuclear Science, Volume NS-32, Oct 1985, pp. 3687-3688 (1985).

- R.C. Gupta, G.H. Morgan, P.A. Thompson, A Single Layer Coil Superconducting Magnet for SSC, Proceedings of the 1987 Particle Accelerator Conference, Washington DC, pp. 14131415(1987).

- R.C. Gupta, G.H. Morgan, P.J. Wanderer, A Comparison of Calculations and Measurements of the Iron Saturation Characteristic of the SSC Design D Dipole Magnet, Proceedings of the 1987 Particle Accelerator Conference, Washington DC, pp. 1405-1407 (1987).

- R.C. Gupta, G.H. Morgan, Collarleas, Close-in, Shaped Iron Aperture Designs for the SSC Dipole, Proceedings of the 1989 Particle Accelerator Conference, Chicago, pp. 500-502 (1989).

- R.C. Gupta, S.A. Kahn and G.H. Morgan, Coil and Iron Design for SSC $50 \mathrm{~mm}$ Magnet, Proceedings of the 1990 American Society of Mechanical Engineers (ASME) Winter Annual Meeting in Dallas (1990).

- R.C. Gupta, S.A. Kahn and G.H. Morgan, A Comparison of Calculations and Measurements of the Field Harmonics as a Function of Current in the SSC Dipole Magnets, Proceedings of the 1991 IEEE Particle Accelerator Conference, San Francisco, pp. 42-44 (1991).

- R.C. Gupta, et al., RHIC Insertion Magnets, Proceedings of the 1991 IEEE Particle Accelerator Conference, San Francisco, pp. 2239-2241 (1991).

- R.C. Gupta, S.A. Kahn and G.H. Morgan, SSC $50 \mathrm{~mm}$ Dipole Cross section, Proceedings of the International Industrial Symposium on Super Collider (IISSC), Atlanta, pp. 587-600 (1991).

- R.C. Gupta, Correcting Field Harmonics after Design in Superconducting Magnets, Proceedings of the $4^{\text {th }}$ International Industrial Symposium on Super Collider (IISSC), New Orleans, pp. 773-780 (1992).

- R. Gupta, et al., Large Aperture Quadrupoles for RHIC Interaction Regions, Proceedings of the 1993 Particle Accelerator Conference, Washington, D.C., pp. 2745-2747 (1993).

- R.C. Gupta, A.K. Jain, Variation in a saturation in SSC Collider Dipoles, Proceedings of the 1993 Particle Accelerator Conference, Washington, D.C., pp. 2778-2780 (1993).

- R. Gupta, et al., Field Quality Improvements in Superconducting Magnets for RHIC, Proceedings of the 1994 European Particle Accelerator Conference, London, UK, pp. 2928-2930 (1994).

- R. Guptz, et al., Field Quality Control Through the Production Phase of RHIC Arc Dipoles, Proceedings of the 1995 International Particle Accelerator Conference, Dallas, Texas (1995).

- A. Jain, R. Gupta, et al., Skew Quadrupole in RHIC Dipole Magnets at High Fields, Proceedings of the Fourteenth International Conference on Magnet Technology (MT-14), Tampere, Finland, June 11-16 (1995).

- R. Gupta, Field Quality in the Superconducting Magnets for Large Particle Accelerators, Proceedings of the 1996 European Particle Accelerator Conference at Sitges, Spain (1996). 


\subsection{Introduction}

An iron yoke plays an important role in superconducting magnets. The superconducting coils are placed in a cylindrical yoke having one or more cavities for the coils. The yoke reduces the fringe field outside the magnet structure by several orders of magnitude and more importantly it shields the field in the aperture of the magnet from the surroundings, which may contain some magnetic material. Moreover, the yoke iron is magnetized by the magnetic field produced by the coil and this magnetized iron, in turn, contributes to the field at the center of the magnet. This enhancement of the field will be computed later for a constant $\mu$ in the iron.

In accelerator magnets, the central field is changed during the accelerating cycle by changing the current in the coil. Whereas, the contribution to the central field from the coil is proportional (linear) to the current in the coil, the contribution from the iron becomes non-linear when the iron magnetization $(M)$ approaches the saturation magnetization $\left(M_{t}\right)$, which is around 2.1 Tesla for the most common type of iron used in accelerator magnets. This phenomena is shown in Fig. 3.1.1 and Table 3.1.1, where $B$ is the magnetic induction or flux density and $H$ is the magnetic field intensity. It may be recalled that $\vec{B} / \mu_{o}=\vec{B}+\vec{M}$ and $H$ is proportional to the current in the coil. It may be noted that there is a non-linearity in the curve at low field also but here the discussion will be limited to the high field case where the non-linearity is commonly referred to as iron saturation, since the magnetization in the iron reaches a maximum saturated value that can not be exceeded.

The saturation magnetization $\left(M_{t}\right)$ is primarily determined [100] by the impurities (such as nitrogen, sulphur, aluminium, etc.) in the iron. The relative contribution of the field from the iron is further reduced at high field by the presence of small gaps between the laminations. The packing factor (or filling factor by which the iron laminations are packed in the given space) in RHIC and SSC magnets was about $97.5 \%$.

This non-linearity in the iron properties complicates the design and performance of the magnet since the azimuthal distribution of the magnetization in the iron yoke changes with the field. This in turn changes the azimuthal distribution of the field in the aperture of the magnet as represented by circular harmonic components. The magnet must be designed in such a way that this change in field harmonics as a function of current is minimized.

The contribution of the iron shell with yoke inner radius $R_{f}$ and yoke outer radius $R_{a}$ to the field produced by $a \cos (m \theta)$ current sheet at radius $a$ is computed for constant $\mu$ iron. For easy reference the expressions for the field in the coil aperture $(r<a)$ in the presence of 


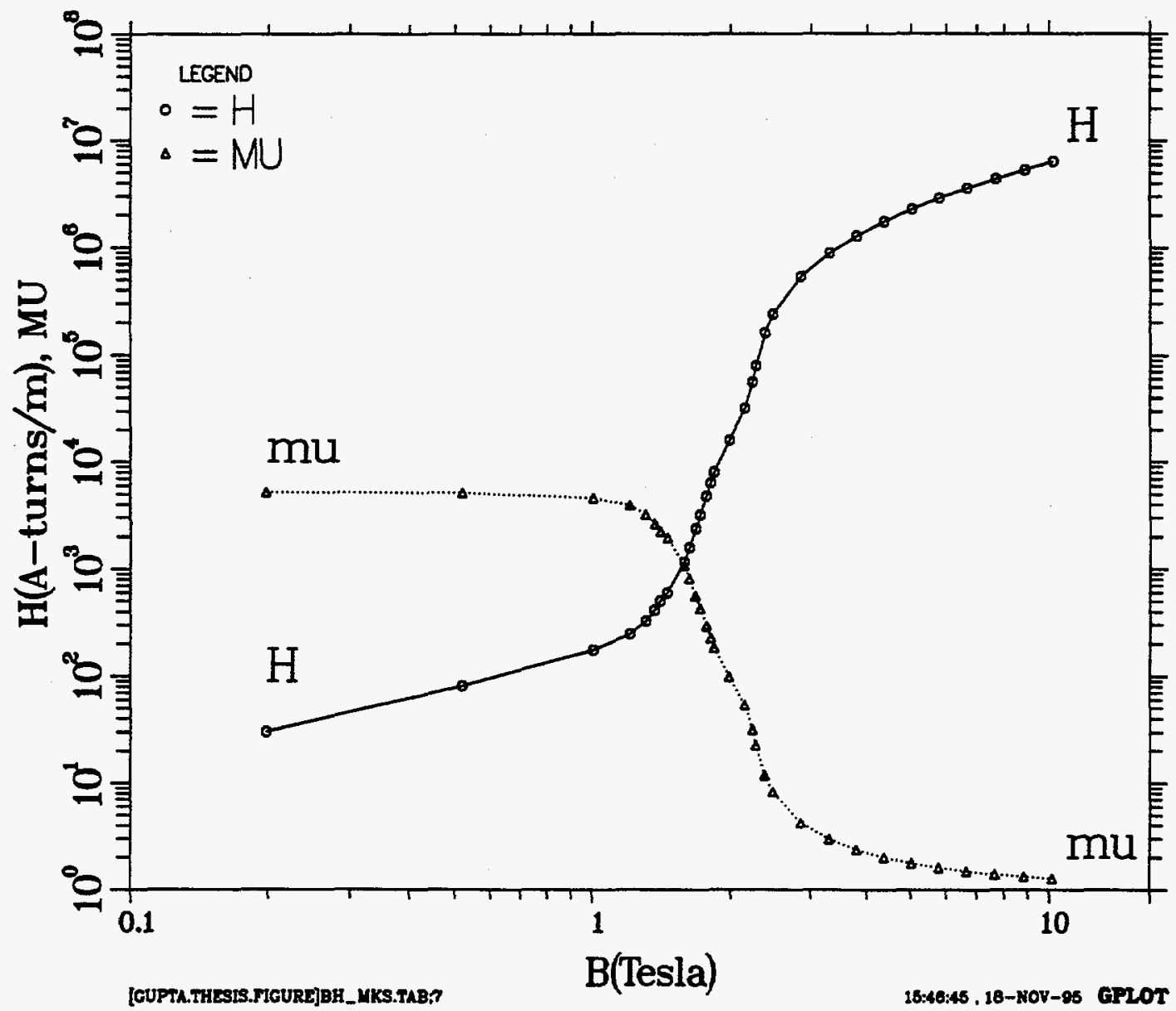

Figure 3.1.1: B-H table used in the calculations carried out with the computer code POISSON. One can note the non-linear relationship between the magnetic induction (B) and magnetic field (H). The relative permeability $\mu(\mathrm{mu})$ is also plotted.

the yoke (which were obtained before in Eqs. (1.5.112)) are given here again. However, first a saturation parameter is defined as follows :

$$
S_{\mu}=\frac{\mu-1}{\mu+1} \frac{\left[1-\left(\frac{R_{f}}{R_{a}}\right)^{2 m}\right]}{\left[1-\left(\frac{\mu-1}{\mu+1}\right)^{2}\left(\frac{R_{f}}{R_{a}}\right)^{2 m}\right]} .
$$

The value of this saturation parameter is 1.0 when $\mu$ is large. A value of one indicates no saturation. A reduction in the value of saturation parameter from 1.0 indicates the onset of 
Table 3.1.1: The following B-H table is used in the calculations carried out with the computer code POISSON. Here, $B$ is the magnetic induction, $H$ is the magnetic field, $\mu$ is the relative permeability and $\gamma=\frac{1}{\mu}=\frac{\mu_{0} H}{B}$.

\begin{tabular}{|c|c|c|c|c|}
\hline $\mathbf{N}$ & B(Tesla) & $\mathrm{H}(\mathrm{A}$-turns $/ \mathrm{m})$ & $\mu(\mathrm{MU})$ & $\gamma($ GAMMA) \\
\hline 1 & 0.000521 & 0.00 & 5210.00 & 1.920E-04 \\
\hline 2 & 0.198 & $3.024 \mathrm{E}+01$ & 5194.12 & $1.930 \mathrm{E}-04$ \\
\hline 3 & 0.52 & $8.117 \mathrm{E}+01$ & 5098.04 & $1.960 \mathrm{E}-04$ \\
\hline 4 & 1.008 & $1.751 \mathrm{E}+02$ & 4581.82 & 2.180E-04 \\
\hline 5 & 1.21 & $2.467 \mathrm{E}+02$ & 3903.23 & $2.560 \mathrm{E}-04$ \\
\hline 6 & 1.31 & $3.263 \mathrm{E}+02$ & 3195.12 & $3.130 \mathrm{E}-04$ \\
\hline 7 & 1.37 & $4.138 \mathrm{E}+02$ & 2634.62 & $3.800 \mathrm{E}-04$ \\
\hline 8 & 1.41 & $5.014 \mathrm{E}+02$ & 2238.1 & $4.470 \mathrm{E}-04$ \\
\hline 9 & 1.46 & $5.968 \mathrm{E}+02$ & 1946.67 & 5.140E-04 \\
\hline 10 & 1.59 & $1.170 \mathrm{E}+03$ & 1081.63 & $9.250 \mathrm{E}-04$ \\
\hline 11 & 1.63 & $1.592 \mathrm{E}+03$ & 815 & $1.227 \mathrm{E}-03$ \\
\hline 12 & 1.682 & $2.387 E+03$ & 560.67 & $1.784 \mathrm{E}-03$ \\
\hline 13 & 1.722 & $3.183 \mathrm{E}+03$ & 430.5 & 2.323E-03 \\
\hline 14 & 1.774 & $4.775 \mathrm{E}+03$ & 295.67 & $3.382 \mathrm{E}-03$ \\
\hline 15 & 1.812 & $6.366 \mathrm{E}+03$ & 226.5 & $4.415 \mathrm{E}-03$ \\
\hline 16 & 1.842 & $7.958 \mathrm{E}+03$ & 184.2 & $5.429 \mathrm{E}-03$ \\
\hline 17 & 1.988 & $1.592 \mathrm{E}+04$ & 99.4 & $1.006 \mathrm{E}-02$ \\
\hline 20 & 2.272 & $7.958 \mathrm{E}+04$ & 22.72 & $4.401 \mathrm{E}-02$ \\
\hline 21 & 2.38 & $1.592 \mathrm{E}+05$ & 11.9 & 8.403E-02 \\
\hline 22 & 2.481 & $2.387 E+05$ & 8.27 & $1.209 \mathrm{E}-01$ \\
\hline 23 & 2.852 & $5.332 \mathrm{E}+05$ & 4.26 & 2.349E-01 \\
\hline 24 & 3.292 & $8.833 E+05$ & 2.97 & 3.372E-01 \\
\hline 25 & 3.792 & $1.281 \mathrm{E}+06$ & 2.36 & $4.246 \mathrm{E}-01$ \\
\hline 26 & 4.362 & $1.735 \mathrm{E}+06$ & 2 & $4.998 \mathrm{E}-01$ \\
\hline 27 & 5.022 & $2.260 \mathrm{E}+06$ & 1.77 & $5.655 \mathrm{E}-01$ \\
\hline 28 & 5.782 & $2.865 \mathrm{E}+06$ & 1.61 & 6.226E-01 \\
\hline 29 & 6.662 & $3.565 \mathrm{E}+06$ & 1.49 & $6.725 \mathrm{E}-01$ \\
\hline 30 & 7.672 & $4.371 E+06$ & 1.4 & $7.159 \mathrm{E}-01$ \\
\hline 31 & 8.842 & $5.302 E+06$ & 1.33 & $7.534 \mathrm{E}-01$ \\
\hline 32 & 10.182 & $6.366 \mathrm{E}+06$ & 1.27 & 7.857E-01 \\
\hline
\end{tabular}


iron saturation and a value of 0.0 indicates complete saturation. Complete saturation takes place when the field in the yoke iron is so high that $\mu$ approaches 1 (which is the value of $\mu$ in air). A reduction in $R_{a}$ also causes a reduction in the saturation parameter and in the limiting case when $R_{a}$ approaches $R_{f}$, the saturation parameter approaches zero.

The use of the saturation parameter in Eqs. (1.5.112)) gives :

$$
\begin{aligned}
& A_{z}(r, \theta)=\frac{\mu_{o} I_{o}}{2 m} \cos (m \theta)\left(\frac{r}{a}\right)^{m}\left[1+S_{\mu}\left(\frac{a}{R_{f}}\right)^{2 m}\right], \\
& B_{\theta}(r, \theta)=-\frac{\mu_{o} I_{o}}{2 a} \cos (m \theta)\left(\frac{r}{a}\right)^{m-1}\left[1+S_{\mu}\left(\frac{a}{R_{f}}\right)^{2 m}\right], \\
& B_{r}(r, \theta)=-\frac{\mu_{o} I_{o}}{2 a} \sin (m \theta)\left(\frac{r}{a}\right)^{m-1}\left[1+S_{\mu}\left(\frac{a}{R_{f}}\right)^{2 m}\right] .
\end{aligned}
$$

For the $m^{\text {th }}$ order multipole ( $m=1$ for dipole), the ratio of the field provided by the iron yoke to the field provided by the current sheet is given by :

$$
\frac{B_{f e}}{B_{c}}=S_{\mu}\left(\frac{a}{R_{f}}\right)^{2 m} .
$$

The theoretical maximum value of the above is 1.0 at low field (large $\mu$ ) when $R_{f}=a$. In RHIC arc dipole magnets this ratio is $\sim 0.55(55 \%)$ at low field and $\sim 0.50(50 \%)$ at high field.

The field enhancement factor $B_{\text {enhc }}$ due to the iron is defined as the field provided by the iron to the total field and is given by :

$$
B_{e n h c}=\frac{S_{\mu}\left(\frac{a}{R_{f}}\right)^{2 m}}{\left[1+S_{\mu}\left(\frac{a}{R_{f}}\right)^{2 m}\right]} .
$$

The enhancement in the field due to the iron yoke is in the range of $20 \%$ to $40 \%$ in the magnets examined in this thesis.

The vector potential and the field components at the inside surface of the iron in air at $r=R_{f}$ are obtained from Eqs. (1.5.115) as :

$$
\begin{aligned}
& A_{z}\left(R_{f}, \theta\right)=\frac{\mu_{o} I_{o}}{2 m} \cos (m \theta)\left(\frac{a}{R_{f}}\right)^{m}\left[1+S_{\mu}\right], \\
& B_{\theta}\left(R_{f}, \theta\right)=\frac{\mu_{o} I_{o}}{2 a} \cos (m \theta)\left(\frac{a}{R_{f}}\right)^{m+1}\left[1-S_{\mu}\right] \\
& B_{r}\left(R_{f}, \theta\right)=-\frac{\mu_{o} I_{o}}{2 a} \sin (m \theta)\left(\frac{a}{R_{f}}\right)^{m+1}\left[1+S_{\mu}\right] .
\end{aligned}
$$


For large $\mu$ (low field), $S_{\mu}$ is $\simeq 1$; therefore the magnetic field at $r=R_{f}$ would have only a radial component (field perpendicular condition) with its magnitude doubled over its value in the absence of the yoke. The magnitude of field for any $\mu$ is obtained as $|B|=\sqrt{\left(B_{r}^{2}+B_{\theta}^{2}\right)}$. Therefore,

$$
\left|B\left(R_{f}, \theta\right)\right|=\frac{\mu_{o} I_{o}}{2 a}\left(\frac{a}{R_{f}}\right)^{m+1} \sqrt{1+S_{\mu}^{2}-2 S_{\mu} \cos (2 m \theta)}
$$

In a magnet, the normal poles are at $\theta=(2 n-1) \frac{\pi}{2 m}$, with $n=1,2, \ldots, 2 \mathrm{~m}$ (m is 1 for dipoles and 2 for quadrupoles, etc.) and the coil midplanes are at $\theta=(n-1) \frac{x}{m}$. At the poles Eq. (3.1.6) reduces to

$$
\mid B\left(R_{f}, \text { poles }\right) \mid=\frac{\mu_{o} I_{o}}{2 a}\left(\frac{a}{R_{f}}\right)^{m+1}\left(1+S_{\mu}\right)
$$

and at the midplanes to

$$
\mid B\left(R_{f}, \text { midplanes }\right) \mid=\frac{\mu_{o} I_{o}}{2 a}\left(\frac{a}{R_{f}}\right)^{m+1}\left(1-S_{\mu}\right) .
$$

Since $S_{\mu}$ is nearly one at low fields and nearly 0 at high fields, the ratio $B / I_{o}$ at the yoke inner surface decreases at the poles and increases at the midplanes as the field increases from low to high.

To study a relative change in the azimuthal field distribution, two limiting cases are examined. First for the case when the iron saturation is negligible (low field and hence large $\mu$ in iron) and the saturation parameter $\left(S_{\mu}\right)$ is one. In this case the expression given in Eq. (3.1.6) reduces to :

$$
\left|B\left(R_{f}, \theta\right)\right|=\frac{\mu_{o} I_{o}}{a}|\sin (m \theta)|\left(\frac{a}{R_{f}}\right)^{m+1},
$$

and then the case when the saturation parameter $\left(S_{\mu}\right)$ is zero (the maximum saturation case with $\mu \simeq 1$ ), the expression given in Eq. (3.1.6) reduces to

$$
\left|B\left(R_{f}, \theta\right)\right|=\frac{\mu_{o} I_{o}}{a}\left(\frac{a}{R_{f}}\right)^{m+1} .
$$

A comparison of Eq. (3.1.9) and Eq. (3.1.10) shows that there is a change in the azimuthal distribution of the field at the yoke inner surface in the two cases. Whereas, at low fields, the magnitude of the field at the yoke inner radius changes as $\sin (m \theta)$, it is completely independent of the angle at high fields. Therefore, yoke saturation causes a change in the azimuthal field distribution which in turn would cause a change in field harmonics. 
The actual situation is much more complex than the expressions derived here under the assumption of constant $\mu$ in the iron everywhere. In a realistic case $\mu$ is not constant in the iron and varies as the field varies as a function of radius and angle. A local change in field, therefore, also causes a local change in $\mu$ which is not accounted in the above expressions. That situation is too complex to be solved analytically. However, most qualitative conclusions reached under the constant $\mu$ assumptions remain valid and are useful to develop strategies to minimize the dependence of field harmonics on iron saturation.

At low and medium field, the magnetization in the iron is such that it is maximum at the poles $\left(90^{\circ}\right.$ and $270^{\circ}$ in dipoles and $45^{\circ}$, etc. in quadrupoles) and minimum at the midplane $\left(0^{\circ}\right.$ and $180^{\circ}$ in dipoles and $0^{\circ}, 90^{\circ}$, etc. in quadrupoles). However, most of the flux from the aperture returns through the iron yoke at the midplane and at high field this changes the distribution of flux lines such that the iron may be more magnetized at the midplane depending on the yoke thickness. This is the basis of the statement given earlier that the azimuthal distribution of the magnetization in the iron is a function of the central field. The harmonics in the aperture of the magnet change with central field if the iron magnetization approaches the saturation magnetization and if there is a significant variation in the azimuthal distribution of this iron magnetization, particularly near the yoke inner surface. During the course of this work, the yoke inner surface (opening) will be referred as the yoke aperture also.

In a simple magnet design where no steps are taken to force an early saturation elsewhere, the poles will saturate first with increasing excitation. This saturation effectively reduces the field at the poles of the magnet giving a positive contribution to $b_{2}$ in dipoles, $b_{5}$ in quadrupoles and $b_{8}$ in sextupoles. The pole saturation also gives a negative contribution to the next allowed harmonic which is $b_{4}$ in dipoles, $b_{9}$ in quadrupoles and $b_{14}$ in sextupoles. At sufficiently high field, because of the limited yoke thickness, the iron at the midplane will saturate and this effectively reduces the field at the midplane. The midplane saturation gives a negative contribution to all allowed harmonics.

In an improved design the harmonics induced due to iron saturation can be minimized by controlling the iron saturation across the azimuth from midplane to pole. In the simplest of such schemes the return flux can be manipulated such that the midplane saturates sooner than it otherwise would and the change in the first allowed harmonic is minimized balancing the negative midplane saturation against the positive pole saturation. This, however increases the saturation in the next allowed harmonic ( $b_{4}$ in dipoles and $b_{9}$ in quadrupoles) for 
which the contribution from the midplane and pole saturation have the same sign. The drop in transfer function (field/current) is determined by the change in the relative permeability $\mu$ in the iron, or more precisely by $\frac{\mu-1}{\mu+1}$ (see Eqs. (3.1.3)).

In a still more sophisticated design the field lines are controlled such that the iron saturates uniformly along the yoke aperture. The goal is to minimize the variation in $\frac{\mu-1}{\mu+1}$ as a function of angle at different levels of excitation. This azimuthal minimization in variation is more important in the iron near the yoke inner radius since that contributes to the central field much more than the iron near the yoke outer radius. A minimization in the variation of $\frac{\mu-1}{\mu+1}$ minimizes the relative change in the azimuthal distribution of field, which by definition minimizes the change in field harmonics. (This argument will be visited again at the conclusion of this chapter, using the RHIC arc dipole magnet as an example). A further benefit of controlling the saturation induced harmonics is that the iron can be brought closer to the coil. This increases the field in the aperture of the magnet for a given amount of superconductor, which is much more expensive than the iron. Moreover, a reduction in saturation induced harmonics reduces the need for multipole corrector magnets, which increase the cost and complexity of the accelerator.

For reference, the field lines in the present design of the $80 \mathrm{~mm}$ aperture $\mathrm{RHIC}$ arc dipoles are shown in Fig. 3.1.2 at $1000 \mathrm{~A}$ (0.7 Tesla central field) and in Fig. 3.1.3 at 7000 A (4.5 Tesla central field). The influence of holes, notch and cutout can be clearly seen. 


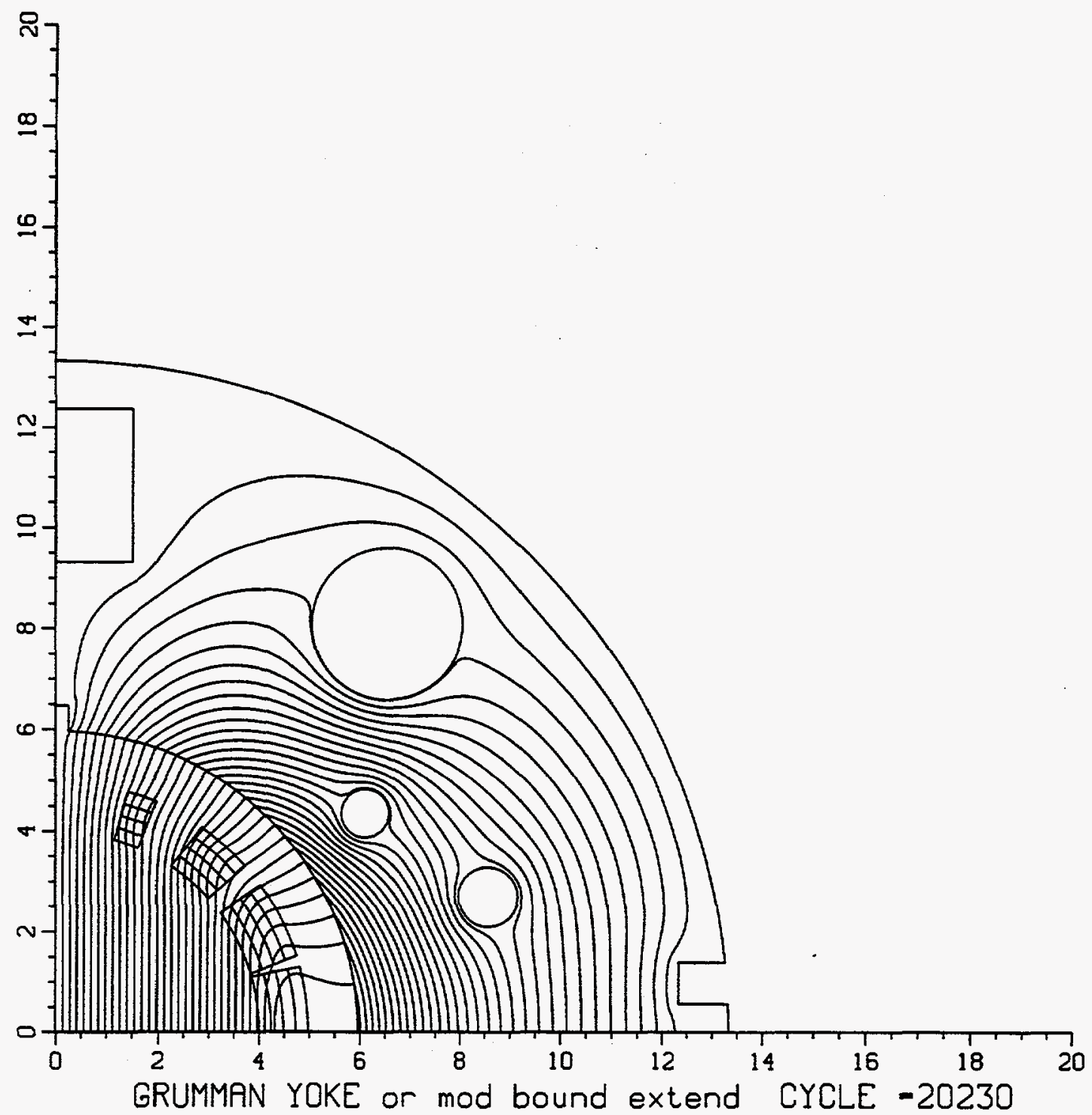

Figure 3.1.2: The field lines at $1000 \mathrm{~A}(\sim 0.7$ Tesla central field $)$ in the present design of the $80 \mathrm{~mm}$ aperture RHIC arc dipoles. 


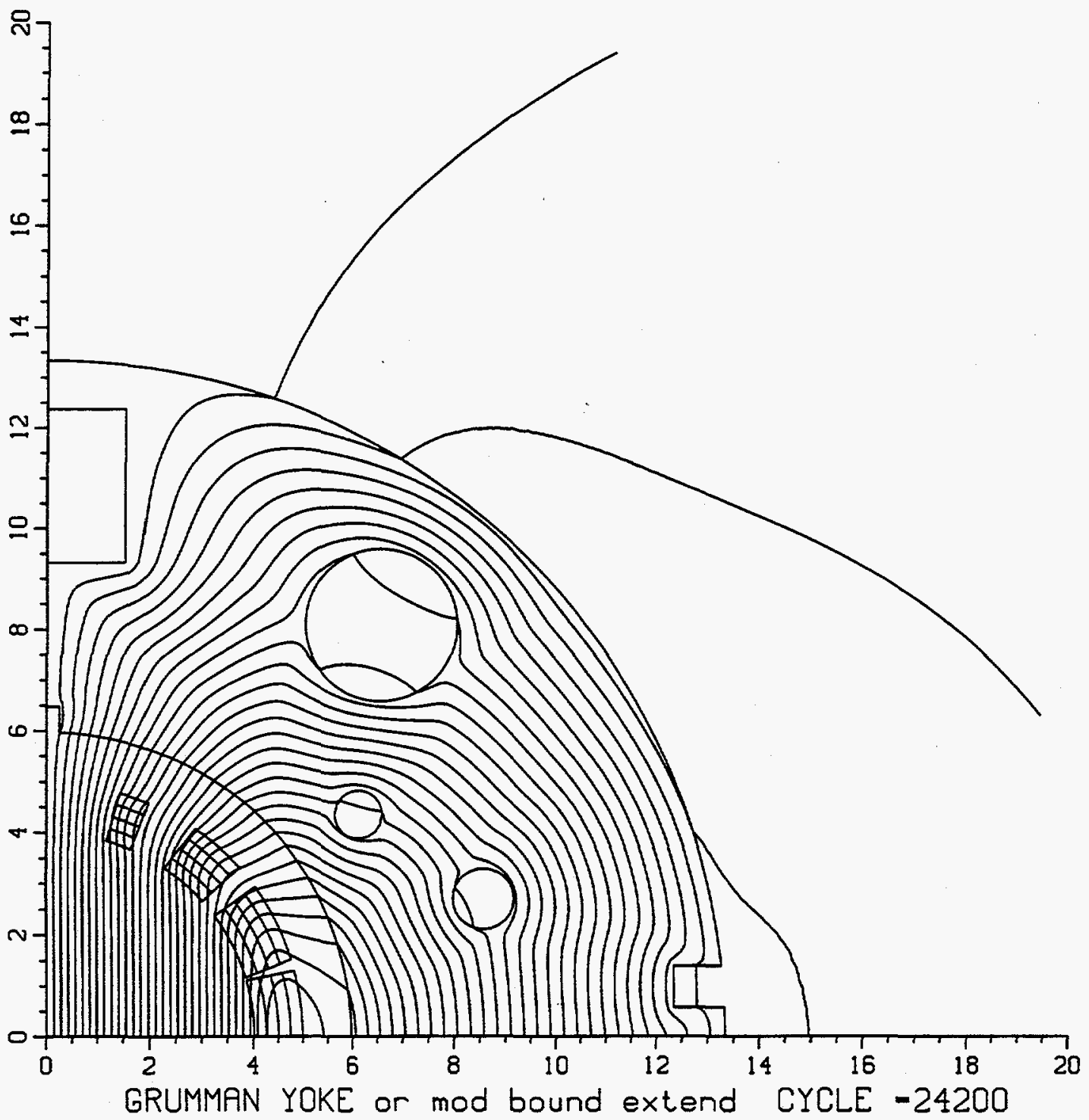

Figure 3.1.3: The field lines at $7000 \mathrm{~A}$ ( $\sim 4.5$ Tesla central field) in the present design of the $80 \mathrm{~mm}$ aperture RHIC arc dipoles. 


\subsection{Reduction in Saturation Induced Allowed Harmonics}

A number of schemes are examined here for reducing the saturation induced harmonics in superconducting magnets. In many cases, similar work has been done by other authors $[21,30,118,119,157]$. The methods described here are built upon theirs and developed further to achieve optimum results in RHIC and SSC magnets. The saturation induced harmonics are defined as the difference between the low field and high field values of harmonics due to the non-linear properties of the iron yoke. The effectiveness of an individual method depends on the type of magnet (dipole, quadrupole, etc.), on the size of the magnet and on the field in the aperture of the magnet. The methods to be described have been examined $[67,71,72]$ in the magnets for RHIC and SSC. A computer model for the cross section of a quadrant of the cold mass (i.e. coil and iron) of the $80 \mathrm{~mm}$ aperture RHIC arc dipole is shown in Fig. 3.2.1 and of the $50 \mathrm{~mm}$ aperture SSC main collider dipole magnet in Fig. 3.2.2. For dipoles, one needs to make a computer model only for a quadrant of the magnet since the rest is symmetric. In these two cases, the cold mass itself is off-center inside a circular cryostat (vacuum tank), which breaks the four fold symmetry of the model. This has a small influence on the non-allowed harmonics which will be discussed in the next section. For the purpose of studying the allowed harmonics only, this small effect is neglected here. 


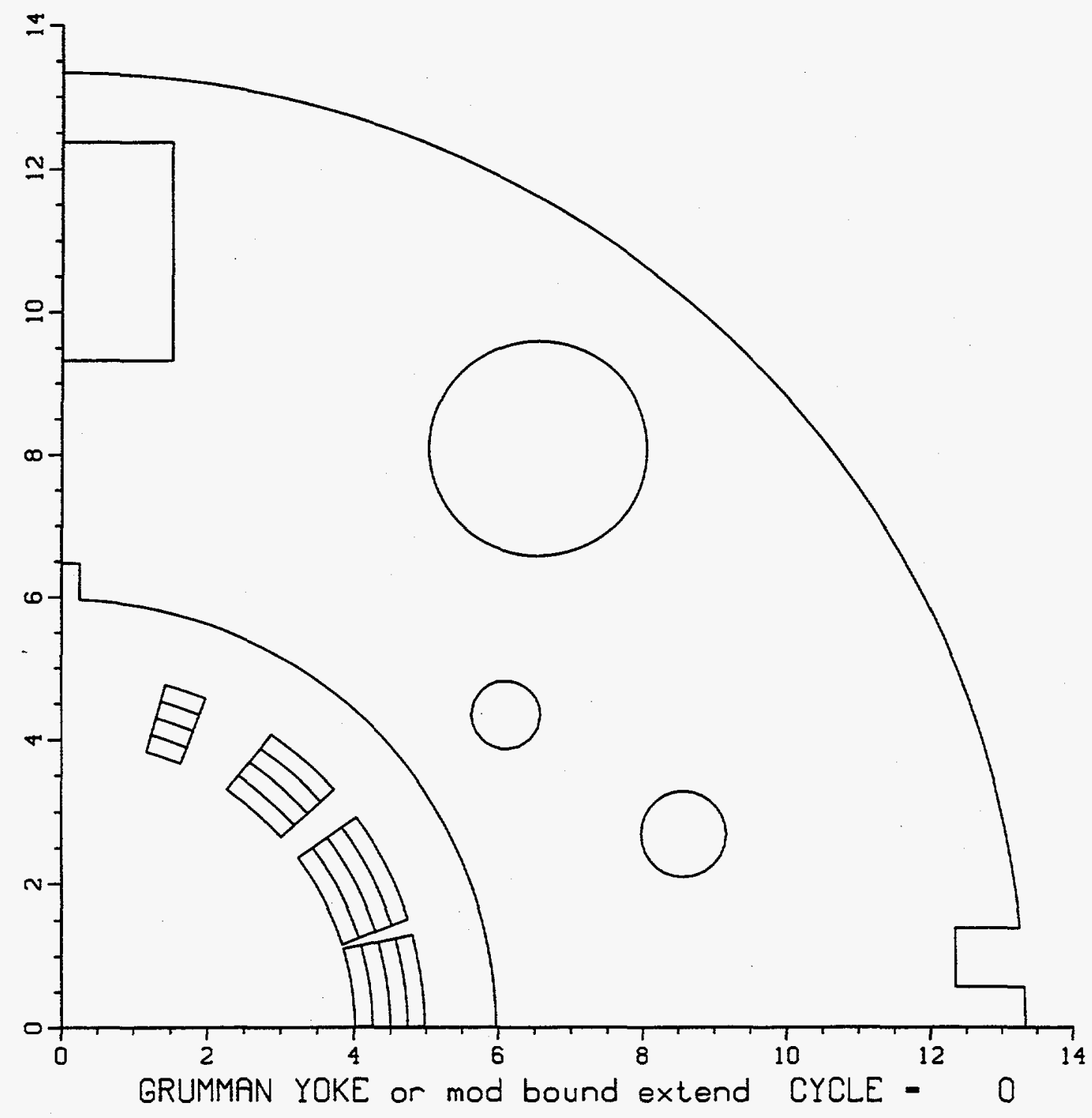

Figure 3.2.1: A computer model of the cross section of the cold mass of the $80 \mathrm{~mm}$ aperture $\mathrm{RHIC}$ arc dipole. 


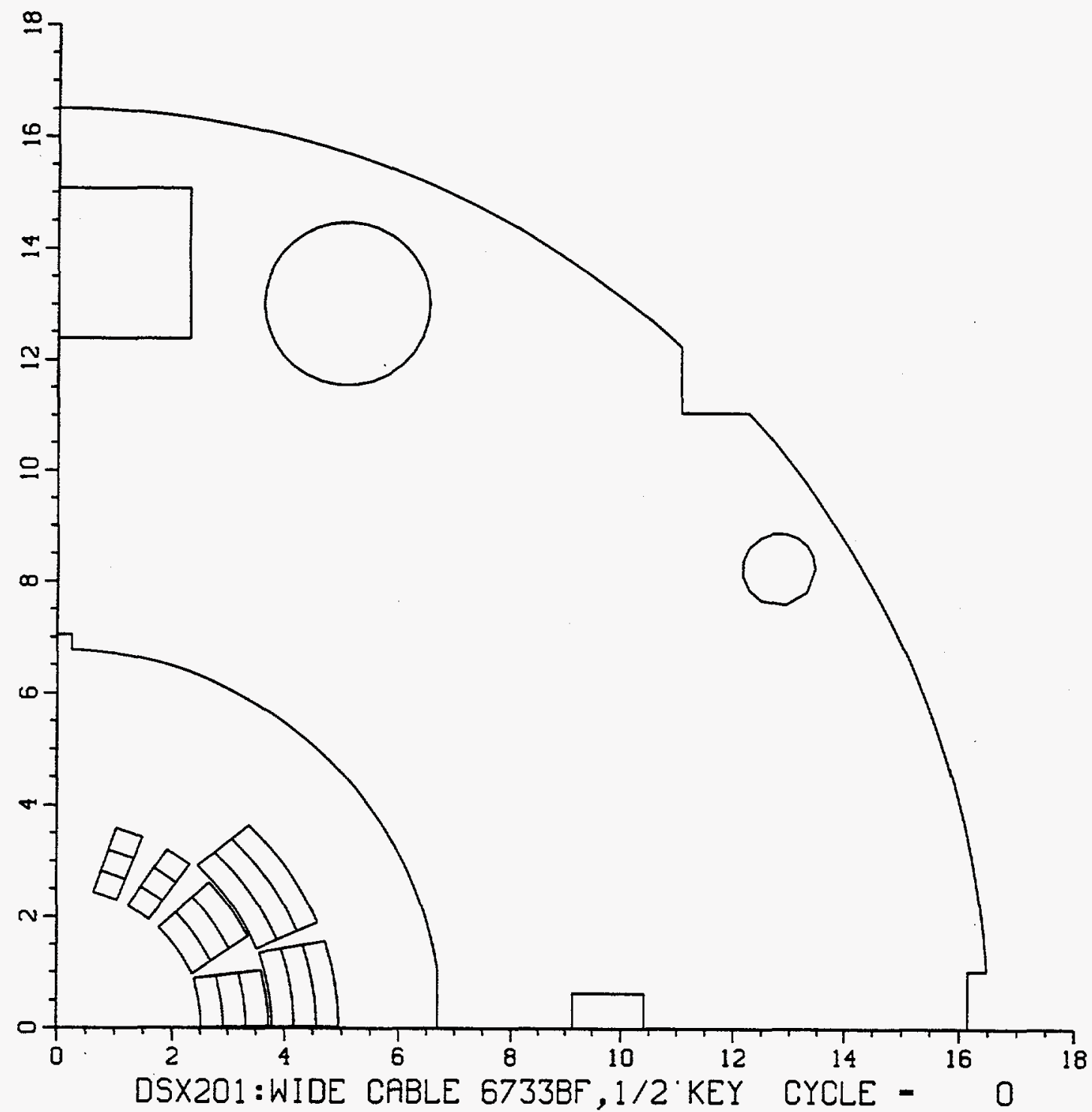

Figure 3.2.2: A computer model of the cross section of a quadrant of the $50 \mathrm{~mm}$ aperture SSC main collider dipole. 


\subsubsection{Varying the yoke inner radius}

The yoke inner radius has a large influence [21] on the iron saturation, particularly when the gap between the coil and iron is small. The magnetic field for the $m^{\text {th }}$-pole outside the coil is proportional to $\left(\frac{1}{r}\right)^{m+1}$ ), where $m$ is 1 for dipole, 2 for quadrupole, etc. Thus the magnetic field falls rapidly as a function of radius and a larger yoke inner radius would therefore mean a lower field in the yoke. This in turn means a lower effect of the saturating iron on the field at the center of the magnet. However, a larger yoke inner radius implies that the yoke is further away from the coil, which reduces the iron contribution to the magnetic field at the center of the magnet (see Eqs. (3.1.4)). For these reasons, one would like to make the yoke inner radius as small as possible as long as the saturation induced harmonics are acceptable.

In Fig. 3.2.3 the transfer function (field divided by current) computed using code POISSON, at $1 \mathrm{kA}$ and at $5 \mathrm{kA}$ is plotted as a function of the yoke inner radius in the RHIC arc dipole magnet. The design yoke inner radius is $59.69 \mathrm{~mm}$ and the above variations are made in the present yoke design with all holes and cutouts preserved. The maximum operating current for the $\mathrm{RHIC}$ arc dipole is $5 \mathrm{kA}$. The yoke outer radius is kept constant at its nominal value of $133.35 \mathrm{~mm}$. The values of the transfer function at $1 \mathrm{kA}$ and $5 \mathrm{kA}$ as a function of radius are given in Table 3.2.1. In Fig. 3.2.4 the computed saturation induced sextupole, decapole and 14-pole harmonics $\left(b_{2}, b_{4}, b_{6}\right)$ are plotted as a function of yoke inner radius at $5 \mathrm{kA}$, and the values are given in Table 3.2.2. Recall that in dipoles, the $b_{i}$ are $10^{4} \frac{B_{i}}{B_{0}}$, where $B_{i}$ is the magnitude of field due to the $i^{\text {th }}$ harmonic at the reference radius and $B_{o}$ is the central field. In the RHIC arc dipoles, the reference radius is $25 \mathrm{~mm}$.

In Fig. 3.2.5 the transfer function computed using code POISSON, at $1 \mathrm{kA}$ and at 6.6 kA is plotted as a function of the yoke inner radius in the SSC $50 \mathrm{~mm}$ aperture collider dipole magnet. The design yoke inner radius is $67.8 \mathrm{~mm}$ and the above variations are made in the present yoke design with all holes and cutouts preserved. The maximum operating current for this magnet is $6.6 \mathrm{kA}$. The yoke outer radius is kept constant at its nominal value of $165.1 \mathrm{~mm}$. In Fig. 3.2.6 the computed saturation induced sextupole and decapole harmonics are plotted as a function of yoke inner radius at $6.6 \mathrm{kA}$. The reference radius is $10 \mathrm{~mm}$.

Fig. 3.2.3 and Fig. 3.2.5 show that the transfer function is increased as the yoke inner radius is reduced, or in other words as the yoke is brought closer to the coil by reducing the coil to iron gap. However, Fig. 3.2.4 and Fig. 3.2.6 show that this increases the saturation 

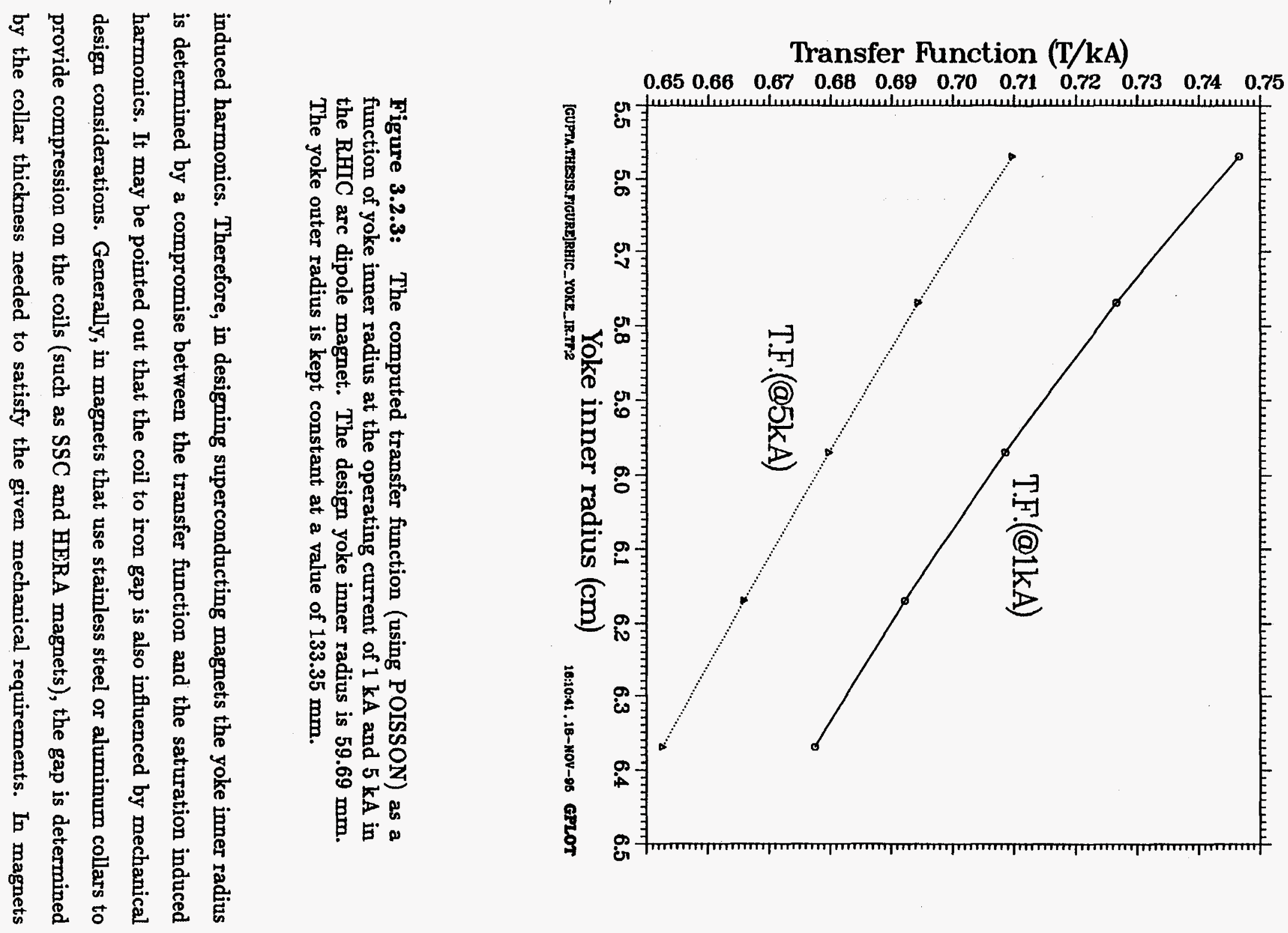


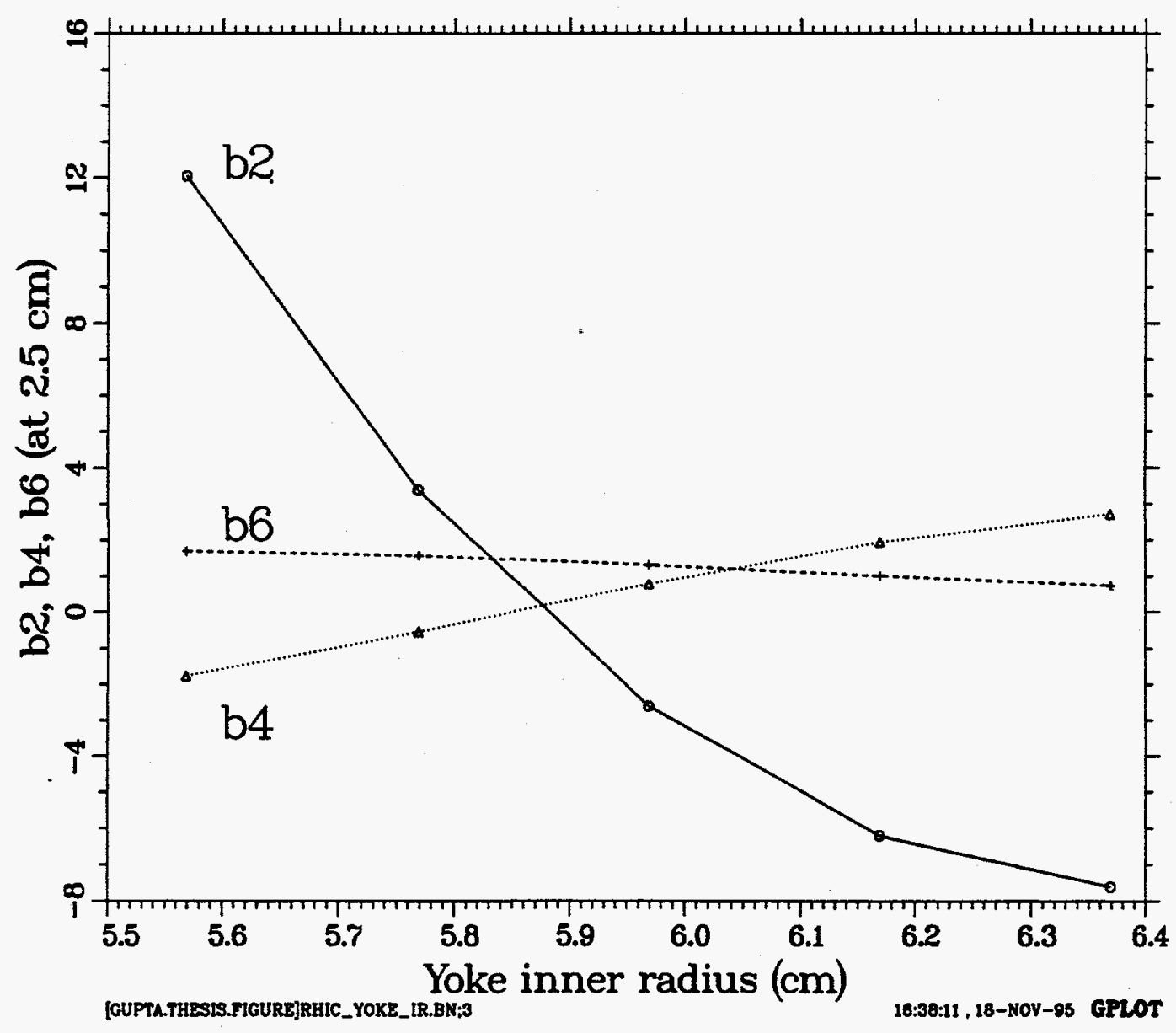

Figure 3.2.4: The computed saturation induced harmonics, $b_{2}, b_{4}$ and $b_{6}$ (using POISSON) as a function of yoke inner radius at a maximum design operating current of $5 \mathrm{kA}$ in the RHIC arc dipole magnet. The design yoke inner radius is $59.69 \mathrm{~mm}$ and the reference radius is $25 \mathrm{~mm}$. The saturation induced harmonics are defined as the difference between the low field and high field values of harmonics due to the non-linear properties of the iron yoke. The yoke outer radius is kept constant at a value of $133.35 \mathrm{~mm}$. 
where the yoke also acts as the collar (such as RHIC), the coil to iron gap can be significantly reduced. This gives a higher transfer function and also higher saturation induced harmonics. These saturation induced harmonics can be reduced by using methods described in the subsequent sections.

Table 3.2.1: The computed transfer function (TF) as a function of yoke inner radius at the operating currents of $1 \mathrm{kA}$ and $5 \mathrm{kA}$ in the $\mathrm{RHIC}$ arc dipole magnet. $\delta \mathrm{TF}(\%)$ is the computed percentage change (loss) in transfer function at $5 \mathrm{kA}$ from its low field value at $1 \mathrm{kA}$. The yoke outer radius is kept constant at a value of $133.35 \mathrm{~mm}$ in this analysis.

\begin{tabular}{|c|c|c|c|}
\hline Inner Radius(mm) & $\mathrm{TF}(@ 1 \mathrm{kA})$ & $\mathrm{TF}(@ 5 \mathrm{kA})$ & $\delta \mathrm{TF}(\%)$ \\
\hline 55.69 & 0.746528 & 0.709736 & 4.928 \\
\hline 57.69 & 0.72657 & 0.69443 & 4.423 \\
\hline 59.69 & 0.70857 & 0.67983 & 4.056 \\
\hline 61.69 & 0.69229 & 0.66592 & 3.809 \\
\hline 63.69 & 0.67751 & 0.65272 & 3.660 \\
\hline
\end{tabular}

Table 3.2.2: The computed saturation induced harmonics, $b_{2}, b_{4}$ and $b_{6}$ as a function of yoke inner radius at a maximum design operating current of $5 \mathrm{kA}$ in the RHIC arc dipole magnet. The yoke outer radius is kept constant at a value of $133.35 \mathrm{~mm}$.

\begin{tabular}{|c|c|c|c|}
\hline Inner Radius $(\mathrm{mm})$ & $b_{2}$ & $b_{4}$ & $b_{6}$ \\
\hline 55.69 & 12.056 & -1.77 & 1.68 \\
\hline 57.69 & 3.382 & -0.55 & 1.55 \\
\hline 59.69 & -2.602 & 0.78 & 1.30 \\
\hline 61.69 & -6.195 & 1.94 & 1.01 \\
\hline 63.69 & -7.614 & 2.72 & 0.74 \\
\hline
\end{tabular}




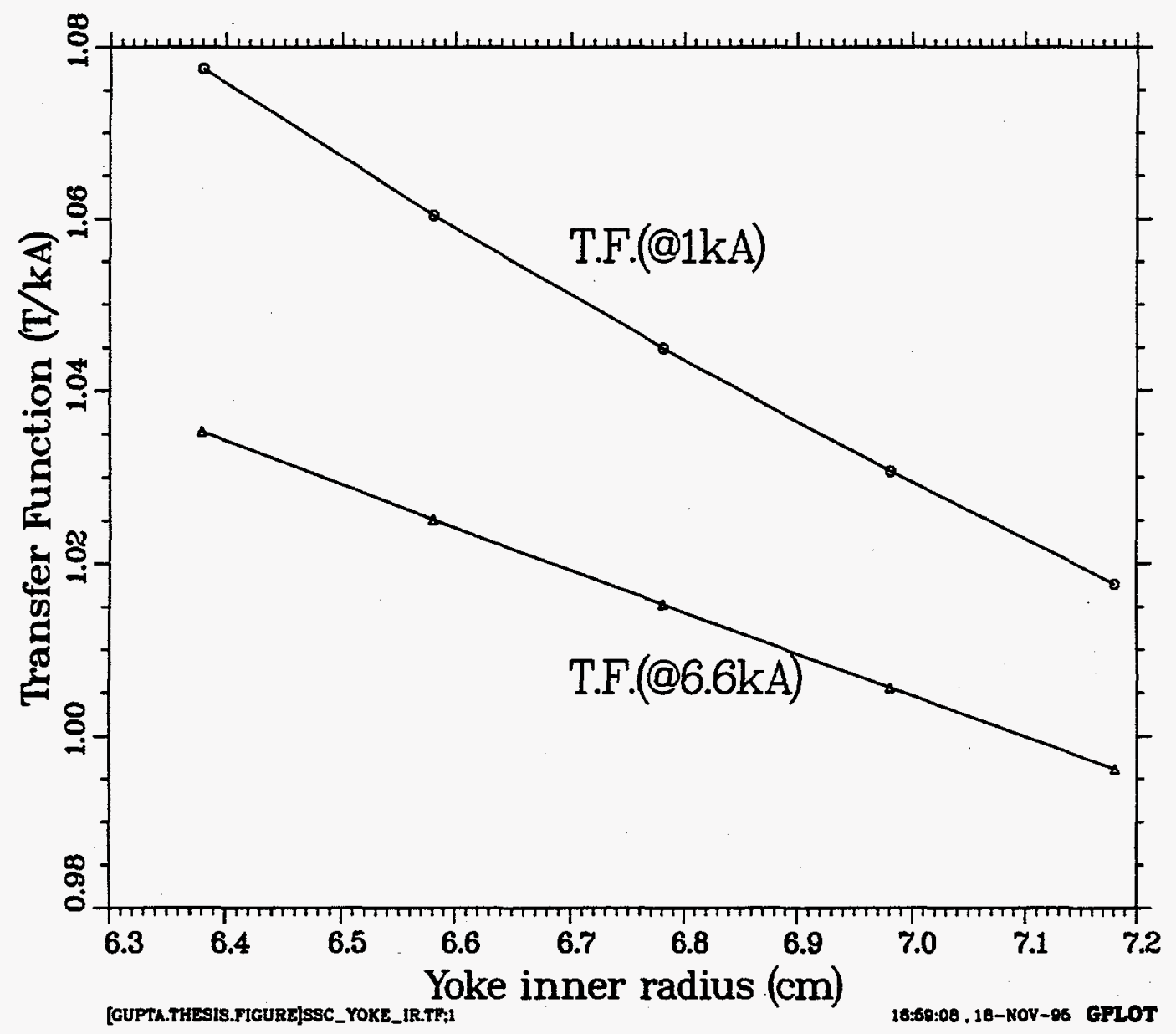

Figure 3.2.5: The computed transfer function as a function of yoke inner radius at the operating currents of $1 \mathrm{kA}$ and $6.6 \mathrm{kA}$ in the $S S C 50 \mathrm{~mm}$ aperture collider dipole magnet. The design yoke inner radius is $67.8 \mathrm{~mm}$. The yoke outer radius is kept constant at a value of $165.1 \mathrm{~mm}$. 


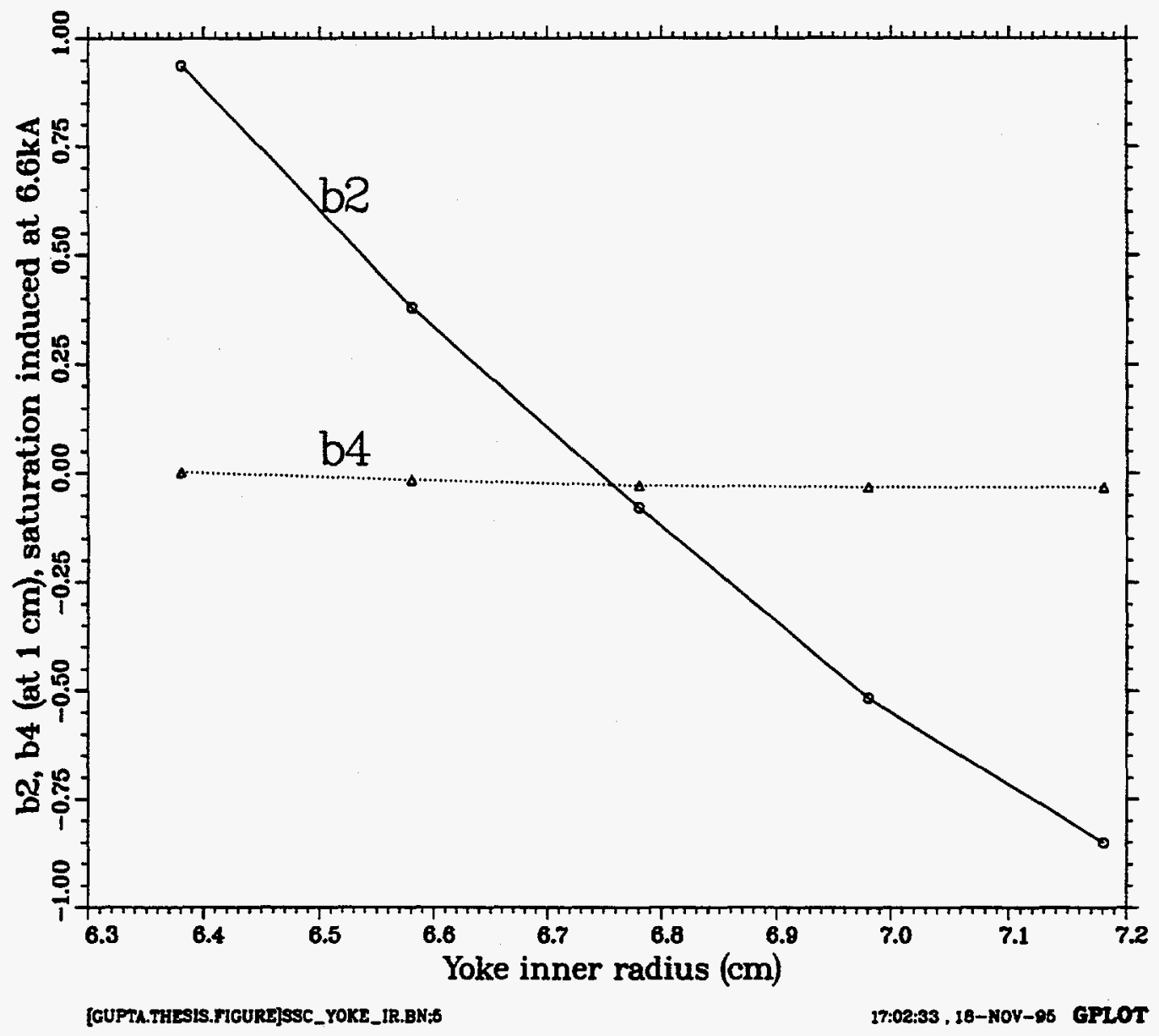

Figure 3.2.6: The computed saturation induced harmonics, $b_{2}$ and $b_{4}$ as a function of yoke inner radius at a maximum design operating current of $6.6 \mathrm{kA}$ in the SSC $50 \mathrm{~mm}$ aperture collider dipole magnet. The saturation induced harmonics are defined as the difference between the low field and high field values of harmonics due to the non-linear properties of the iron yoke. The design yoke inner radius is $67.8 \mathrm{~mm}$ and the reference radius is $10 \mathrm{~mm}$. The yoke outer radius is kept constant at a value of $165.1 \mathrm{~mm}$. 


\subsubsection{Varying the yoke outer radius}

The outer yoke radius affects the field only at high current and has little effect at low current. At high field, in addition to changing the transfer function and the saturation induced harmonics, a change in outer yoke radius may also have a significant effect on the fringe field, i.e., the field outside the magnet. Changing the outer yoke radius reduces the yoke thickness which provides a return path for the magnetic field lines or magnetic flux. If the yoke thickness is not enough, the iron at the midplane will saturate. As mentioned earlier, the midplane saturation gives a negative contribution to all allowed harmonics. However, in dipole magnets, the pole saturation gives a positive contribution to the sextupole, a negative decapole, etc. Therefore, the iron saturation, particularly in the first allowed harmonic after fundamental, which is the sextupole in dipole magnets, can be significantly controlled by optimizing the midplane thickness of the yoke. This could be done either by making the yoke outer surface elliptical or by changing the iron outer radius. For simplicity and construction reasons, the yoke outer surface is kept circular while the optimization of the yoke outer radius is examined.

In Fig. 3.2.7, the variation in the saturation induced sextupole and decapole harmonics and the percentage drop in transfer function (from the low field value of transfer function) of the SSC $50 \mathrm{~mm}$ dipole magnet are plotted as a function of yoke outer radius at the design current of $6.6 \mathrm{kA}$. The inner radius of the yoke is kept constant at $67.81 \mathrm{~mm}$ and the computer model contains all the holes and cutouts of the optimized design [66]. The values are given in Table 3.2.3.

Table 3.2.3: Saturation induced harmonics and the percentage drop in Transfer Function $(\delta T F)$ at $6.6 \mathrm{kA}$ as a function of yoke outer radius in the SSC $50 \mathrm{~m}$ aperture collider dipole magnets.

\begin{tabular}{|c|c|c|c|}
\hline Outer Radius (mm) & $b_{2}$ & $b_{4}$ & $\delta T F(\%)$ \\
\hline 145.1 & -3.303 & -0.032 & 5.579 \\
\hline 155.1 & -1.768 & -0.042 & 3.886 \\
\hline 165.1 & -0.073 & -0.028 & 2.819 \\
\hline 175.1 & 1.291 & -0.008 & 2.114 \\
\hline 185.1 & 2.1854 & 0.008 & 1.699 \\
\hline
\end{tabular}




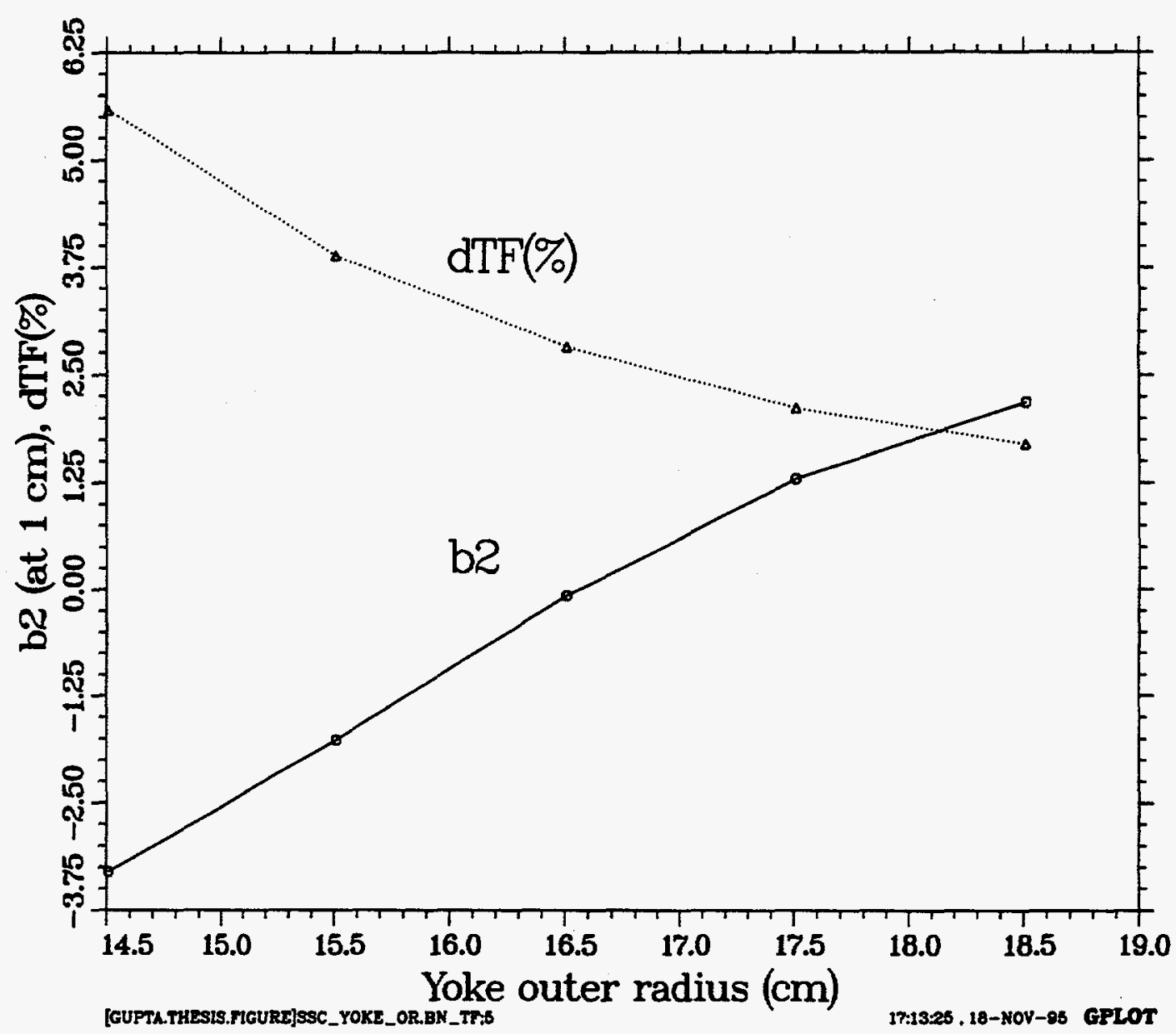

Figure 3.2.7: The effects of varying the outer yoke radius in the SSC $50 \mathrm{~mm}$ collider dipole magnet. The nominal value in the design is 165.1 $\mathrm{mm}$. These are the saturation induced harmonics at $6.6 \mathrm{kA}$, the maximum operating current. $\mathrm{dTF}(\%)$ is the percentage drop in transfer function at $6.6 \mathrm{kA}$ as compared to its low field value.

The values of the fringe field (the field outside the yoke) on the horizontal plane are given in Table 3.2 .4 for an outer radius of $165.1 \mathrm{~mm}$ at the maximum operating current of 6.6 kA. In Fig. 3.2.8, the fringe field of the SSC $50 \mathrm{~mm}$ dipole outside the yoke on the midplane and in Fig. 3.2 .9 on the vertical plane is plotted as a function of distance from the magnet center at a current of $6.6 \mathrm{kA}$ when the yoke outer radius is respectively 14.51 , 
Table 3.2.4: Fringe field at $6.5 \mathrm{ka}$ on the $\mathrm{X}$-axis in the SSC $50 \mathrm{~mm}$ aperture collider dipole magnet at the maximum design current of $6.6 \mathrm{kA}$. The distance is measured from the magnet center. The yoke outer radius is $165.1 \mathrm{~mm}$.

\begin{tabular}{|c|c|c|}
\hline $\mathrm{X}(\mathrm{mm})$ & $B_{y}$ (Gauss) & $\frac{\partial B_{y}}{\partial x}\left(\frac{\text { Gaube }}{c m}\right)$ \\
\hline 170 & 1526.160 & $-4.7933 E+02$ \\
\hline 180 & 1030.736 & $-4.2402 E+02$ \\
\hline 190 & 723.291 & $-2.1871 \mathrm{E}+02$ \\
\hline 200 & 557.745 & $-1.2484 \mathrm{E}+02$ \\
\hline 210 & 456.130 & $-8.2494 \mathrm{E}+01$ \\
\hline 220 & 386.219 & $-5.9519 \mathrm{E}+01$ \\
\hline 230 & 334.205 & $-4.5438 \mathrm{E}+01$ \\
\hline 240 & 293.840 & $-3.5949 \mathrm{E}+01$ \\
\hline 250 & 261.480 & $-2.9184 \mathrm{E}+01$ \\
\hline 260 & 234.872 & $-2.4258 \mathrm{E}+01$ \\
\hline 270 & 212.594 & $-2.0477 \mathrm{E}+01$ \\
\hline 280 & 193.659 & $-1.7528 \mathrm{E}+01$ \\
\hline 290 & 177.367 & $-1.5135 \mathrm{E}+01$ \\
\hline 300 & 163.236 & $-1.3251 \mathrm{E}+01$ \\
\hline 310 & 150.831 & $-1.1625 \mathrm{E}+01$ \\
\hline 320 & 139.879 & $-1.0328 \mathrm{E}+01$ \\
\hline 330 & 130.137 & $-9.1954 \mathrm{E}+00$ \\
\hline 340 & 121.439 & $-8.2198 E+00$ \\
\hline 350 & 113.653 & $-7.4616 \mathrm{E}+00$ \\
\hline 360 & 106.702 & $-6.7260 \mathrm{E}+00$ \\
\hline 370 & 100.562 & $-5.7218 \mathrm{E}+00$ \\
\hline 380 & 94.340 & $-6.6750 \mathrm{E}+00$ \\
\hline 390 & 89.080 & $-3.5703 \mathrm{E}+00$ \\
\hline 400 & 85.332 & $-4.0159 \mathrm{E}+00$ \\
\hline
\end{tabular}

16.51 (nominal) and $185.1 \mathrm{~mm}$. In both cases, the fringe field outside the iron falls as $\frac{1}{r^{2}}$ as expected for a dipole magnet. By comparing the last two figures, one can note that the leakage (fringe) field is much more on the horizontal plane (X-axis) than on the vertical plane (Y-axis). It is clear from these plots, that though the outer radius is a useful parameter to minimize the saturation induced sextupole harmonic, care must be taken so that the yoke outer radius is not made so small as to increase the fringe field above a tolerable limit. In the above calculations, the influence of the cryostat, which is made of magnetic steel, is not included. It can contain a part of the flux outside the yoke. 


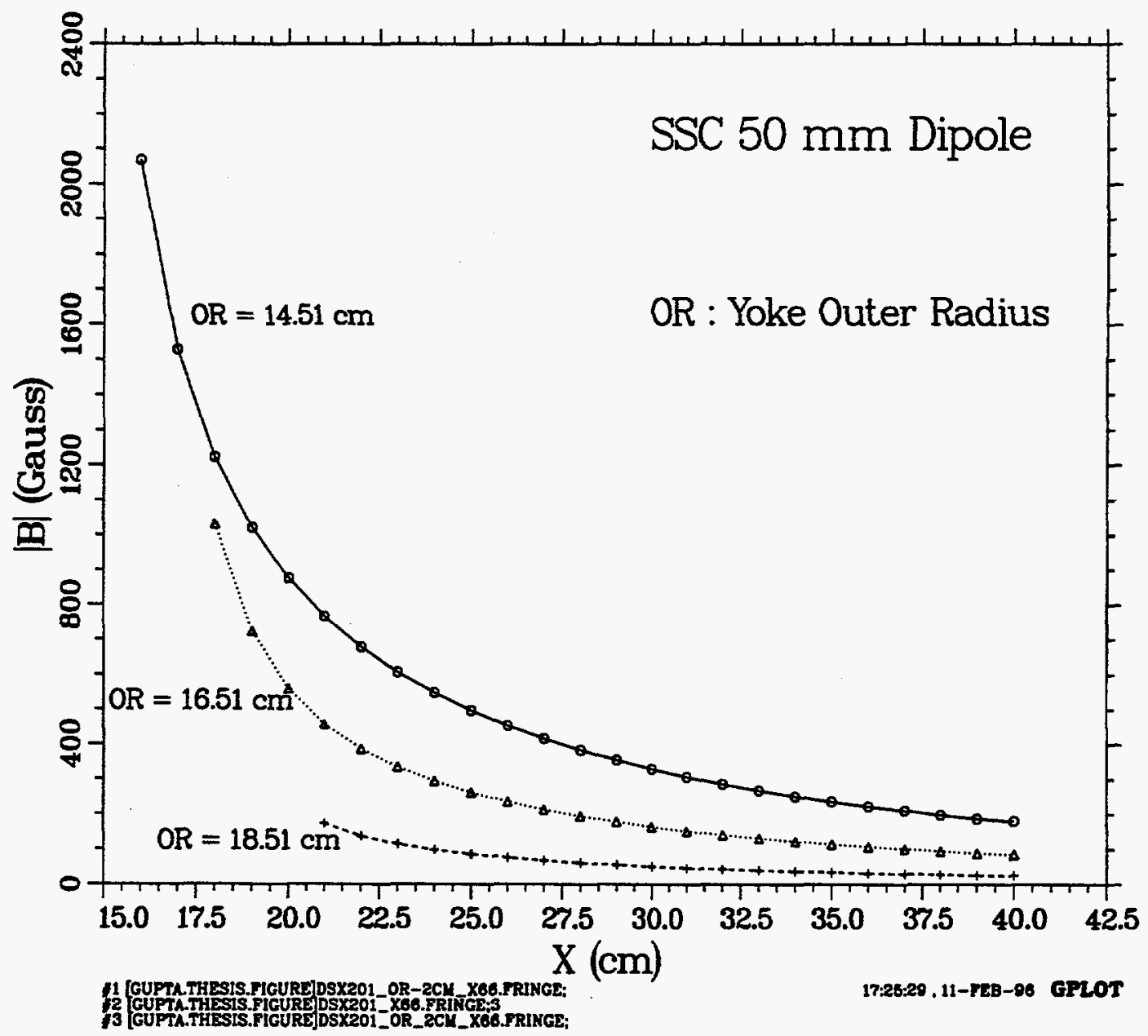

Figure 3.2.8: The effects of varying the outer yoke radius in the SSC $50 \mathrm{~mm}$ collider dipole magnet on the fringe field on the horizontal plane (X-axis). The nominal value of the yoke outer radius in the design is 165.1 $\mathrm{mm}$. The cryostat is not present in these model calculations. 


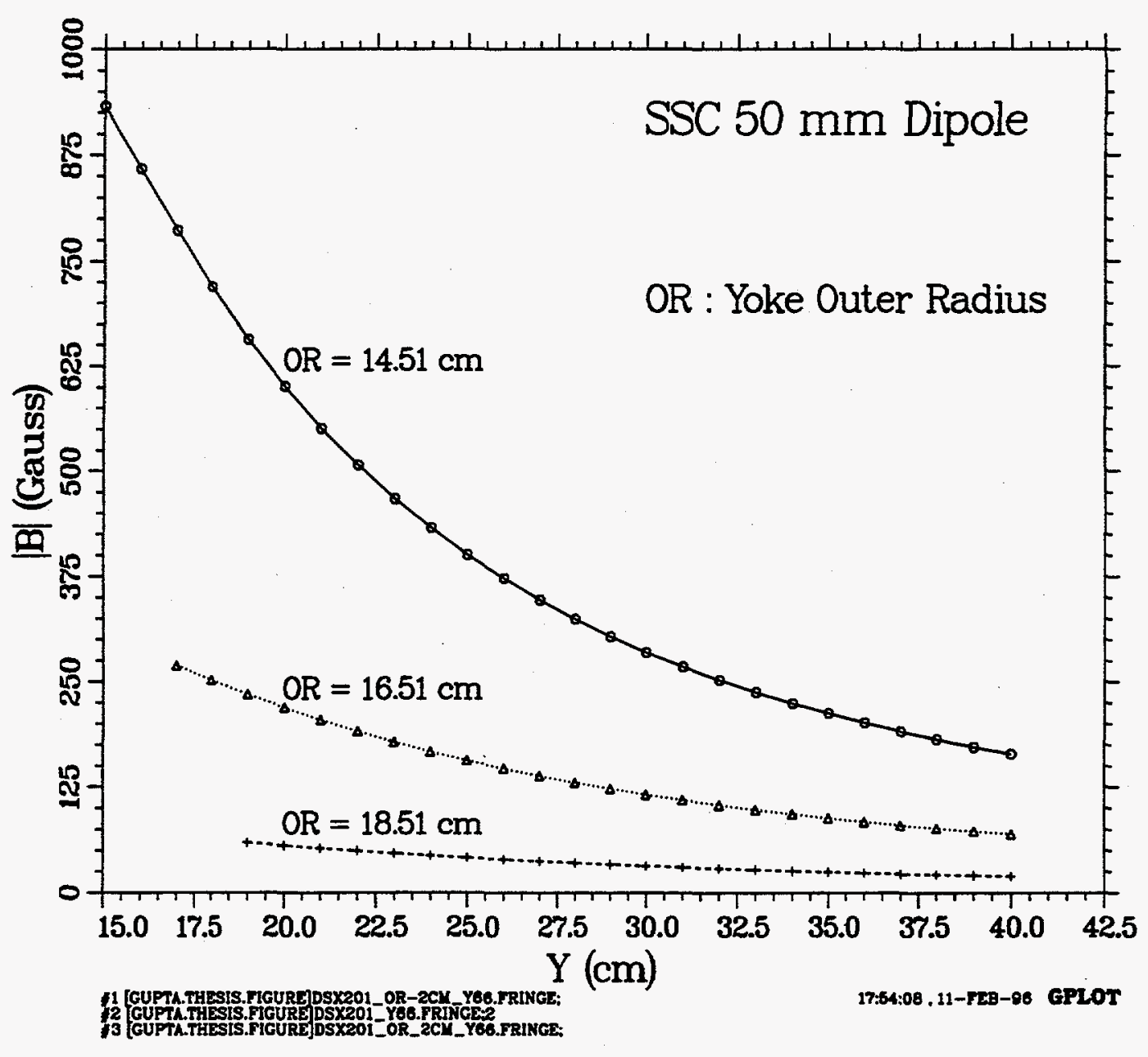

Figure 3.2.9: The influence of varying the outer yoke radius in the SSC $50 \mathrm{~mm}$ collider dipole magnet on the fringe field on the vertical plane ( $Y$ axis). The nominal value of the yoke outer radius in the design is 165.1 $\mathrm{mm}$. The cryostat is not present in these model calculations. 


\subsubsection{Varying the location of the helium bypass hole in the yoke}

In superconducting accelerator magnets, the helium coolant must be transported through spaces in the coil and its vicinity and also through several holes in the yoke. The cross section and size of these holes are computed from the cooling requirements. In the RHIC and SSC magnets these holes, which are known as helium bypass holes, have a significantly large cross section. Because of their large size, their effect on the iron saturation can be sizable. In earlier designs they were placed at passive locations (at the poles where the flux density is low) such that their presence would not cause any additional saturation in the yoke. However, it is shown here that by placing them at a proper active location (so that their presence changes the saturation characteristic of the yoke), one can effectively reduce or cancel a few saturation induced harmonics. This method does not have the drawback of significantly increasing the fringe field or of reducing the transfer function.

In the RHIC arc dipole magnets, the location of these holes has been optimized to reduce saturation induced $b_{2}$ and $b_{4}$ harmonics. There are four holes of equal size and they are placed in the four quadrant of the yoke so that they maintain the basic dipole symmetry. If the hole in the first quadrant is located at a radius $R$ and at an angle $\theta$, the other three must be located at the same radius but at the angles of $(\pi-\theta),(\pi+\theta)$ and $(-\theta)$, respectively. However, in a computer model of the dipoles, only one quarter of the problem needs to be defined. The rest of the geometry is implicitly included by the boundary conditions. Therefore, in the subsequent discussion, the location of only one hole in the first quadrant will be given - the other three holes in other quadrants are implicit as explained above.

In Fig. 3.2.10, the saturation induced $b_{2}$ and $b_{4}$ harmonics are plotted as a function of the angular position of the helium bypass hole in the RHIC arc dipole at $5 \mathrm{kA}$ current. The values are given in Table 3.2.5. The variation in hole location is performed on a computer model which retained the other holes and cutouts of the present design. The radius of the helium bypass hole is $15.075 \mathrm{~mm}$. Though the radial location of the hole has a significant impact on the saturation induced harmonics, in this figure it is always kept at a radius of $104.1 \mathrm{~mm}$ and only the angular position is varied. The final optimized location of the helium bypass hole in the RHIC arc dipole magnet is $R=104.1 \mathrm{~mm}$ and $\theta=51^{\circ}$. The three other holes required by dipole symmetry are placed at the angles of $129^{\circ}, 231^{\circ}$ and $309^{\circ}$, respectively. 


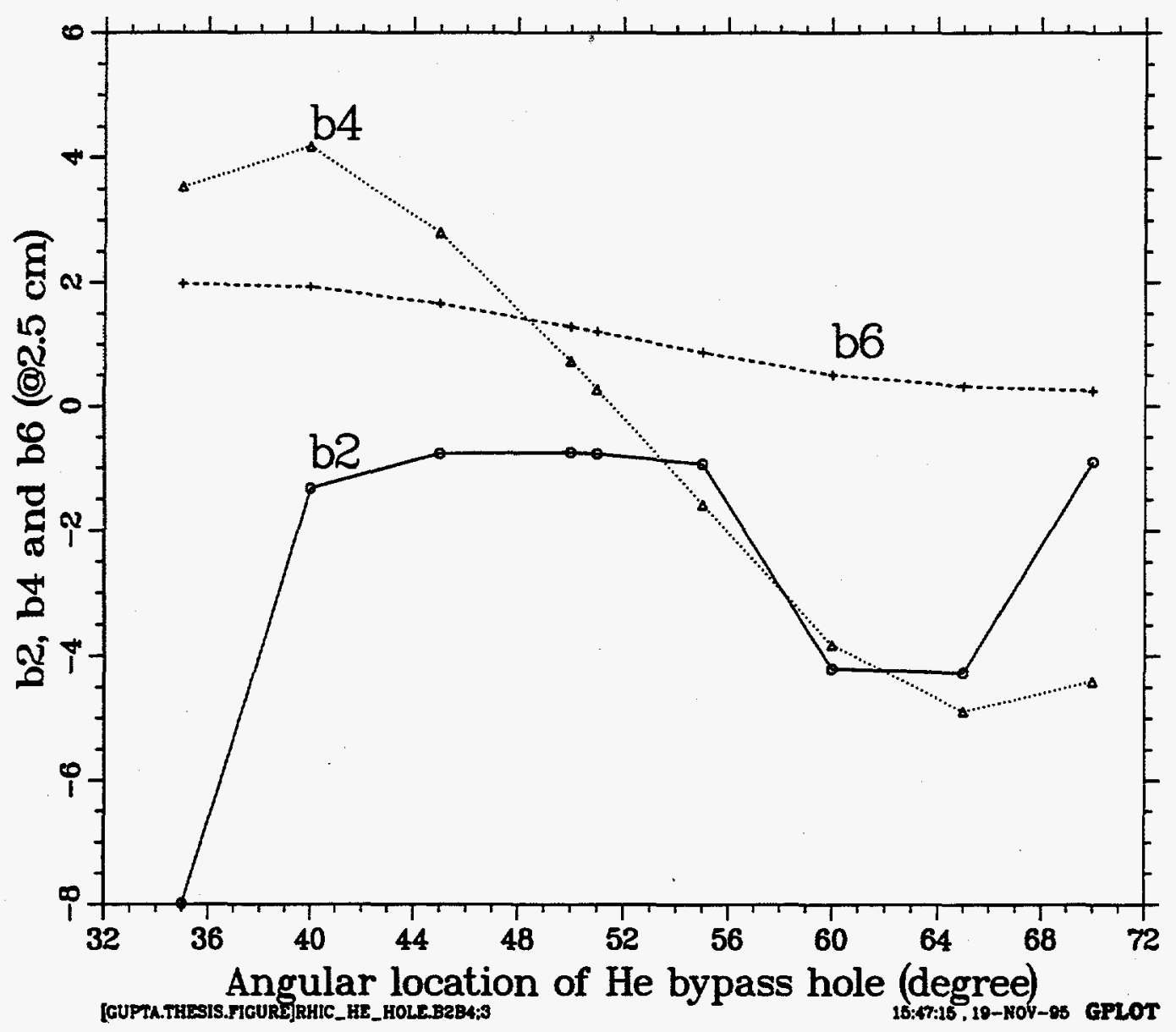

Figure 3.2.10: The influence of the angular location of the helium bypass hole on the saturation induced sextupole $\left(b_{2}\right)$, decapole $\left(b_{4}\right)$ and 14-pole $\left(b_{6}\right)$ harmonics at $5 \mathrm{kA}$ in the $\mathrm{RHIC}$ arc dipole magnet. The radial position of this hole is kept at $104.13 \mathrm{~mm}$. There are four such holes in the magnet; their location is determined by the dipole symmetry.

The radial location of the helium hole was optimized in the $100 \mathrm{~mm}$ aperture $\mathrm{RHIC}$ insertion dipole magnet $D O$. A model for the cross section of a quadrant of this magnet is shown in Fig. 3.2.11. In Fig. 3.2.12, the saturation induced $b_{2}$ harmonic is plotted and in Fig. 3.2.13 the saturation induced $b_{4}$ and $b_{6}$ harmonics are plotted at $4.5 \mathrm{kA}$ (near the nominal maximum operating current) as a function of the angular location of the helium 
Table 3.2.5: The influence of the variation in the angular location of the helium bypass hole on the saturation induced harmonics at $5.0 \mathrm{kA}$ in the RHIC arc dipole magnet. The radial position of this hole is kept at 104.13 mm.

\begin{tabular}{|c|c|c|c|c|c|c|c|c|}
\hline Location & $\mathrm{Bo}(\mathrm{T})$ & $\delta \mathrm{TF}(\%)$ & $\delta b_{2}$ & $\delta b_{4}$ & $\delta b_{6}$ & $\delta b_{8}$ & $\delta b_{10}$ & $\delta b_{12}$ \\
\hline $35^{\circ}$ & 3.36241 & -5.09321 & -7.989 & 3.540 & 1.973 & 0.040 & -0.047 & -0.016 \\
\hline $40^{\circ}$ & 3.37448 & -4.75252 & -1.333 & 4.178 & 1.924 & 0.004 & -0.052 & -0.016 \\
\hline $45^{\circ}$ & 3.38795 & -4.37232 & -0.769 & 2.803 & 1.657 & -0.008 & -0.046 & -0.015 \\
\hline $50^{\circ}$ & 3.40612 & -3.85946 & -0.752 & 0.725 & 1.281 & -0.019 & -0.035 & -0.012 \\
\hline $51^{\circ}$ & 3.41005 & -3.74853 & -0.774 & 0.268 & 1.199 & -0.020 & -0.033 & -0.012 \\
\hline $55^{\circ}$ & 3.42589 & -3.30144 & -0.936 & -1.581 & 0.870 & -0.027 & -0.024 & -0.010 \\
\hline $60^{\circ}$ & 3.43560 & -3.02736 & -4.201 & -3.819 & 0.502 & -0.032 & -0.014 & -0.007 \\
\hline $65^{\circ}$ & 3.44521 & -2.75612 & -4.264 & -4.879 & 0.322 & -0.035 & -0.008 & -0.006 \\
\hline $70^{\circ}$ & 3.44024 & -2.89639 & -0.901 & -4.405 & 0.247 & -0.044 & -0.007 & -0.006 \\
\hline
\end{tabular}

bypass hole for three radial locations, namely $100 \mathrm{~mm}, 110 \mathrm{~mm}$ and $120 \mathrm{~mm}$. The helium bypass hole itself has a radius of $15.075 \mathrm{~mm}$. The reference radius for the $D O$ dipole is 31 mm. During these variations, the two smaller holes shown in Fig. 3.2.11 were not present. The results of the calculations are also shown in Table 3.2.6. In the final design the bypass hole is located at $\mathrm{R}=106 \mathrm{~mm}$ and $\theta=46^{\circ}$.

The differences in the variation of the harmonics as a function of the angular position of helium bypass hole between $80 \mathrm{~mm}$ aperture RHIC arc dipoles (see Fig. 3.2.10) and 100 $\mathrm{mm}$ aperture RHIC insertion dipoles DO (see Fig. 3.2.12 and Fig. 3.2.13) is attributed to the following factors : (a) in the case of the arc dipole magnet model used in this study, all other holes and cutouts beside the helium bypass hole were retained whereas in the $D O$ magnet model they were not and (b) the relative yoke thickness (i.e. yoke outer radius minus yoke inner radius divided by the coil aperture) is larger in the arc dipoles than in the $D O$ dipoles. The maximum operating field is about the same ( $\sim 3.5$ tesla) in the two magnets. 


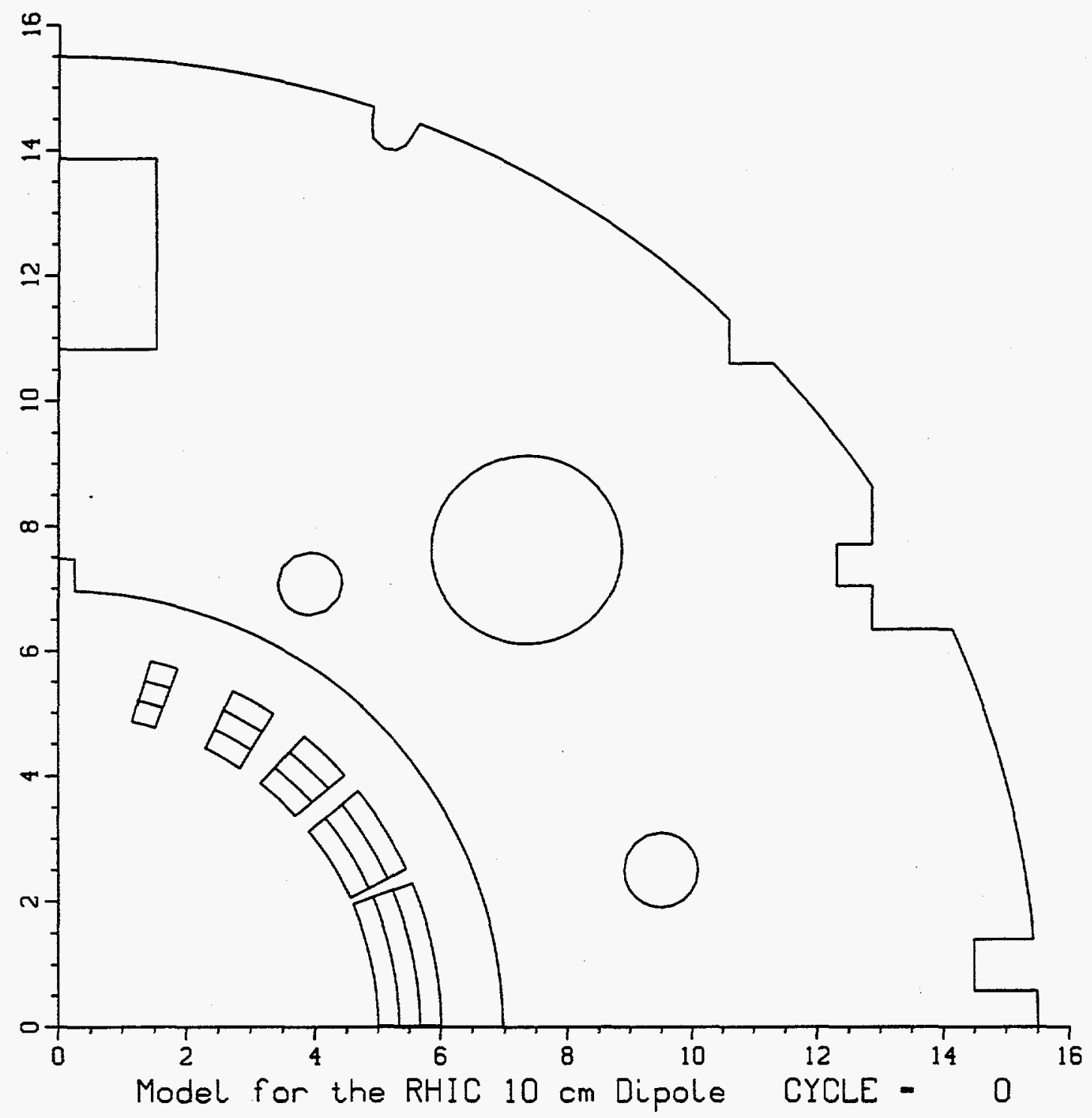

Figure 3.2.11: A computer model of the cold mass of a quadrant of the $100 \mathrm{~mm}$ aperture RHIC insertion dipole $D O$. Two cold masses will be put inside a common cryostat (not shown here). 


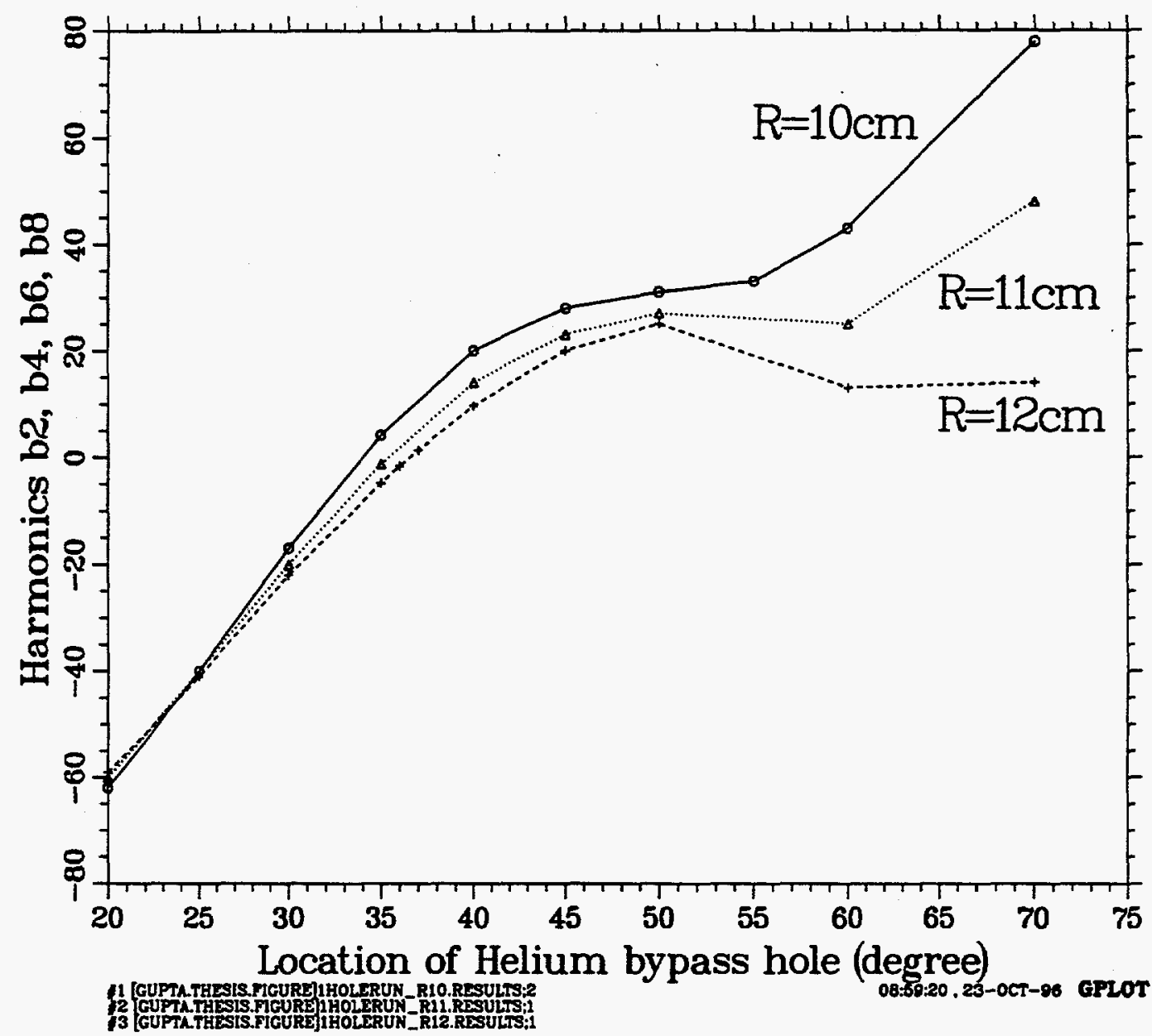

Figure 3.2.12: The influence of the angular location of the helium bypass hole on the saturation induced sextupole harmonic at $4.5 \mathrm{kA}$ in the $100 \mathrm{~mm}$ aperture $\mathrm{RHIC}$ insertion dipole $D O$. The influence is examined at three radial positions of this hole, namely $100 \mathrm{~mm}, 110 \mathrm{~mm}$ and $120 \mathrm{~mm}$. There are four such holes in the magnet; the location of the three others is determined by dipole symmetry. 


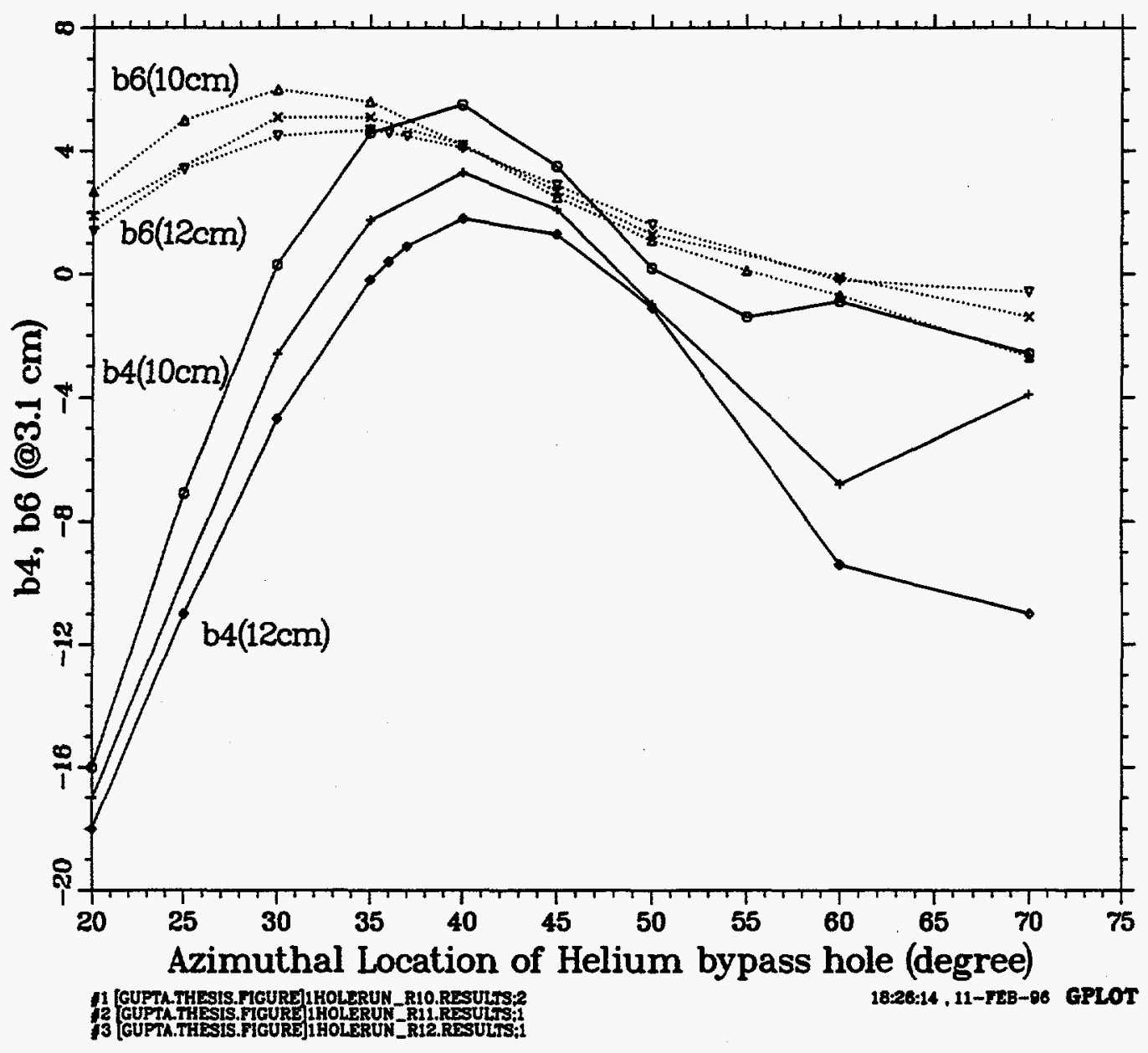

Figure 3.2.13: The influence of the angular location of the helium bypass hole on the saturation induced $b_{4}$ and $b_{6}$ harmonics at $4.5 \mathrm{kA}$ in the $100 \mathrm{~mm}$ aperture $\mathrm{RHIC}$ insertion dipole $D O$. The influence is examined at three radial positions of this hole, namely $100 \mathrm{~mm}, 110 \mathrm{~mm}$ and $120 \mathrm{~mm}$. There are four such holes in the magnet; the location of the three others is determined by the dipole symmetry. 
Table 3.2.6: Saturation induced harmonics at $4.5 \mathrm{kA}$ in the $100 \mathrm{~mm}$ aperture $\mathrm{RHIC}$ insertion dipole D0 for various locations of the Helium Bypass Hole. The reference radius for computing field harmonics in is 31 $\mathrm{mm} . \mathbf{R}$ and $\theta$ in the table below indicates the location of the hole.

\begin{tabular}{|c|c|c|c|c|c|c|}
\hline $\begin{array}{c}R \\
\mathrm{~mm}\end{array}$ & $\begin{array}{c}\theta \\
\text { degrees }\end{array}$ & $\begin{array}{c}\delta \mathrm{TF} \\
\%\end{array}$ & $\begin{array}{c}b_{2} \\
\text { Units }\end{array}$ & $\begin{array}{c}b_{4} \\
\text { Units }\end{array}$ & $\begin{array}{c}b_{6} \\
\text { Units }\end{array}$ & $\begin{array}{c}b_{8} \\
\text { Units }\end{array}$ \\
\hline 100 & 20 & 6.68 & -62 & -16 & 2.7 & 1.1 \\
\hline 100 & 25 & 6.37 & -40 & -7.1 & 5.0 & 1.4 \\
\hline 100 & 30 & 5.97 & -17 & 0.3 & 6.0 & 1.1 \\
\hline 100 & 35 & 5.50 & 4.1 & 4.6 & 5.6 & 0.6 \\
\hline 100 & 40 & 5.00 & 20 & 5.5 & 4.2 & 0.02 \\
\hline 100 & 45 & 4.52 & 28 & 3.5 & 2.5 & -0.4 \\
\hline 100 & 50 & 4.13 & 31 & 0.2 & 1.1 & -0.5 \\
\hline 100 & 55 & 4.04 & 33 & -1.4 & 0.12 & -0.6 \\
\hline 100 & 60 & 4.23 & 43 & -0.9 & -0.7 & -0.7 \\
\hline 100 & 70 & 4.81 & 78 & -2.6 & -2.7 & -0.4 \\
\hline 110 & 20 & 6.50 & -60 & -17 & 1.9 & 0.8 \\
\hline 110 & 30 & 5.87 & -20 & -2.6 & 5.1 & 1.0 \\
\hline 110 & 35 & 5.46 & -1.3 & 1.75 & 5.1 & 0.7 \\
\hline 110 & 40 & 5.00 & 14 & 3.3 & 4.2 & 0.2 \\
\hline 110 & 45 & 4.53 & 23 & 2.1 & 2.7 & -0.2 \\
\hline 110 & 50 & 4.07 & 27 & -1.0 & 1.3 & -0.4 \\
\hline 110 & 60 & 3.53 & 25 & -6.8 & -0.1 & -0.4 \\
\hline 110 & 70 & 4.16 & 48 & -3.9 & -1.4 & -0.5 \\
\hline 120 & 20 & 6.38 & -59 & -18 & 1.4 & 0.7 \\
\hline 120 & 25 & 6.14 & -41 & -11 & 3.4 & 1.0 \\
\hline 120 & 30 & 5.81 & -22 & -4.7 & 4.5 & 1.0 \\
\hline 120 & 35 & 5.42 & -4.9 & -0.2 & 4.7 & 0.8 \\
\hline 120 & 36 & 5.34 & -1.7 & 0.4 & 4.6 & 0.6 \\
\hline 120 & 37 & 5.26 & 1.3 & 0.9 & 4.5 & 0.5 \\
\hline 120 & 40 & 5.00 & 9.6 & 1.8 & 4.1 & 0.3 \\
\hline 120 & 45 & 4.56 & 20 & 1.3 & 2.9 & -0.1 \\
\hline 120 & 50 & 4.12 & 25 & -1.1 & 1.6 & -0.3 \\
\hline 120 & 60 & 3.78 & 13 & -9.4 & -0.2 & -0.3 \\
\hline 120 & 70 & 3.66 & 14 & -11. & -0.6 & -0.3 \\
\hline
\end{tabular}




\subsubsection{Additional Saturation suppressor holes in the iron yoke}

In the last section, the location of the helium bypass holes was adjusted to alter the saturation characteristics of the yoke. In this section, the use of additional holes is investigated for controlling several saturation induced harmonics. In principle, a sufficient number of holes could reduce all required saturation induced harmonics. However, in practice, they can not be placed in any arbitrary position and the location and size of them may be restricted by structural (mechanical) considerations. In the magnet designs examined during the course of this work, an optimized solution was always found such that the mechanical integrity of the magnet was not compromised. These holes do not change the transfer function at low field and their effect is minimal at high field unless they are very large in size.

The calculated predictions were compared with the results of measurements in a RHIC short model of the arc dipole magnet. This magnet, DRS006, was re-built adding one saturation suppressor hole per quadrant. The angular location of this saturation suppressor hole in the first quadrant was $\theta=33^{\circ}$ at a radial location of $R=75 \mathrm{~mm}$ with a size of $4.76 \mathrm{~mm}$. For reference, the location of the helium bypass hole discussed here and in the other cases throughout the rest of this section, is fixed at $R=104.1 \mathrm{~mm}$ and $\theta=51^{\circ}$. The diameter of the helium bypass hole is $30.15 \mathrm{~mm}$. The measurements on DRS006 with saturation suppressor holes were compared with those of another short model magnet DRS008 which did not have these holes but was otherwise built with the same design. These results are shown in Fig. 3.2.14 where the saturation induced harmonics are plotted as a function of current. A significant reduction in the variation in sextupole $\left(b_{2}\right)$ and decapole $\left(b_{4}\right)$ harmonics can be seen. In this particular case, there is an increase in $\left(b_{6}\right)$, however, this is of no significant consequence to machine performance.

In Table 3.2.7, the change in harmonics in the DRS008 design due to the additional saturation suppressor hole is given as a function of its size and location at the maximum design operating current of $5 \mathrm{kA}$ in the RHIC arc dipoles. In Fig. 3.2.15, the computed change in the saturation induced $b_{2}, b_{4}$ and $b_{6}$ harmonics due to a circular hole are plotted as a function of its angular location at $5 \mathrm{kA}$ in the $\mathrm{RHIC}$ arc dipoles. The radius of this hole is $5 \mathrm{~mm}$ and it is always located at a radius of $75 \mathrm{~mm}$. The change in the field harmonics is computed by taking the difference in the saturation induced harmonics between a magnet with and without saturation suppressor holes. The inner radius of the yoke is $59.69 \mathrm{~mm}$. In Fig. 3.2.16, the angular location of the hole is kept at $\theta=35^{\circ}$ but the radial location 


\section{Saturation with and without "Saturation suppressor holes"}

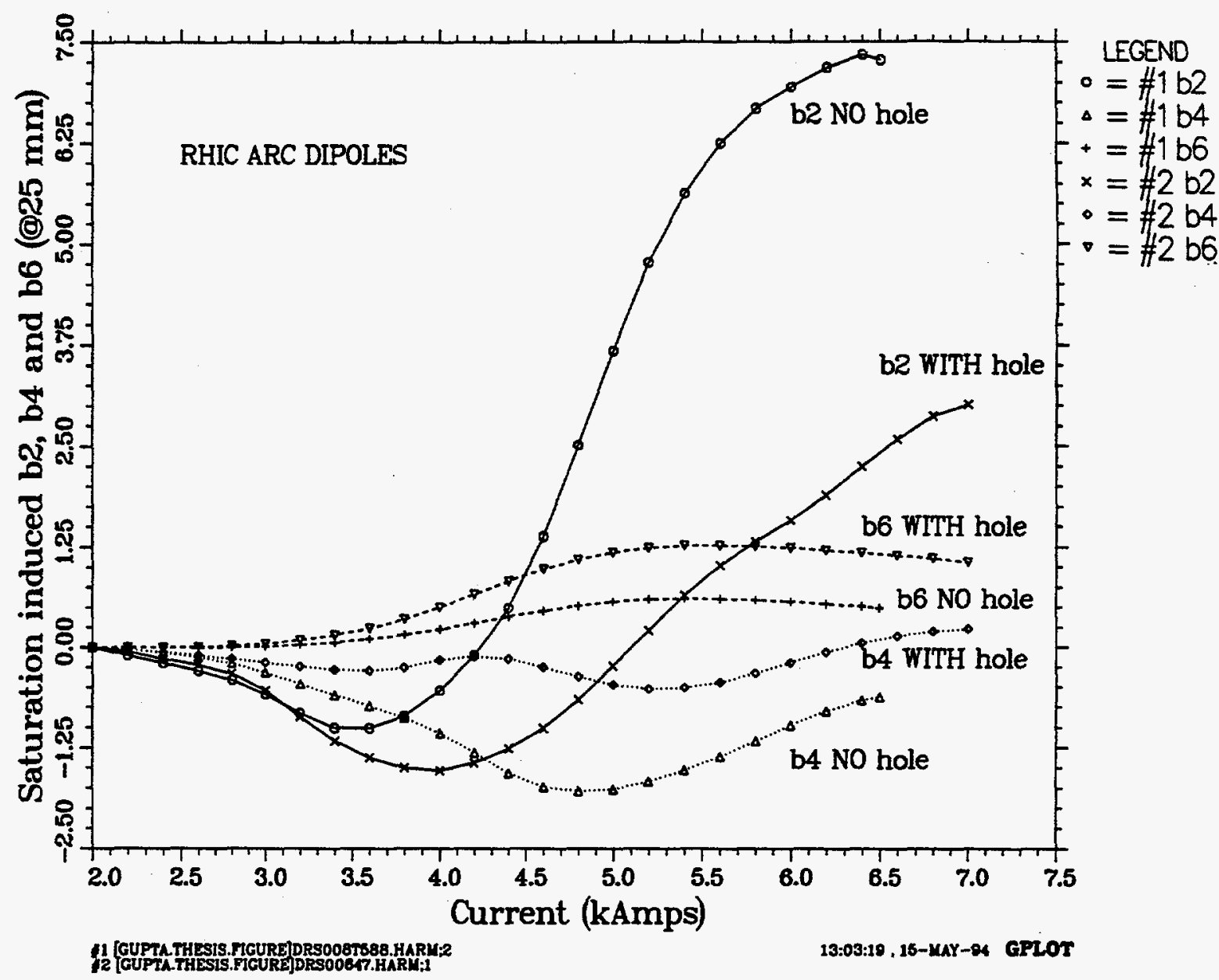

Figure 3.2.14: Saturation induced $b_{2}, b_{4}$ and $b_{6}$ harmonics as a function of current in model magnets for RHIC arc dipoles with and without the saturation suppressor holes. Magnet DRS006 was built with these holes and had much smaller saturation as compared to magnet DRS008, which did not have these holes. The harmonics are the average of the measured up and down ramp and they are shifted to start from zero at $2 \mathrm{kA}$ for easy comparison.

is varied. The radius of the hole is still $5 \mathrm{~mm}$. In Fig. 3.2.17, the size of the hole is changed by changing its radius but the location is fixed at $R=75 \mathrm{~mm}$ and $\theta=35^{\circ}$. These plots show that the angular location of the hole determines how much each harmonic will change and the radial location and size of the hole determines the magnitude of this change. 
Table 3.2.7: Change in the saturation induced harmonics due to variations in the location of the saturation suppressor holes in an $80 \mathrm{~mm}$ aperture RHIC arc dipole at the maximum design operating current of $5 \mathrm{kA}$. In this table the effect of changing the radial size $(R)$, the radial location $\left(R_{0}\right)$ and the angular location $\left(\theta_{0}\right)$ of the saturation suppressor holes is respectively examined.

\begin{tabular}{|c|c|c|c|c|c|c|c|c|}
\hline$R(m m)$ & $R_{0}(m m)$ & $\theta_{0}$ & $\delta B_{o}$ & $\% \delta(\mathrm{TF})$ & $\delta b_{2}$ & $\delta b_{4}$ & $\delta b_{6}$ & $\delta b_{8}$ \\
\hline 5 & 75 & $10^{\circ}$ & -0.02540 & -0.71693 & -17.34 & -3.238 & -0.491 & -0.056 \\
\hline 5 & 75 & $15^{\circ}$ & -0.03044 & -0.85919 & -17.10 & -2.083 & -0.057 & 0.053 \\
\hline 5 & 75 & $20^{\circ}$ & -0.02441 & -0.68899 & -9.695 & 0.128 & 0.418 & 0.125 \\
\hline 5 & 75 & $25^{\circ}$ & -0.01431 & -0.40390 & -4.419 & 0.867 & 0.483 & 0.108 \\
\hline 5 & 75 & $30^{\circ}$ & -0.01758 & -0.49620 & -7.500 & 0.667 & 0.577 & 0.119 \\
\hline 5 & 75 & $35^{\circ}$ & -0.02311 & -0.65229 & -4.622 & 2.161 & 0.719 & 0.076 \\
\hline 5 & 75 & $40^{\circ}$ & -0.02756 & -0.77790 & 2.914 & 3.868 & 0.544 & -0.030 \\
\hline 5 & 75 & $45^{\circ}$ & -0.02590 & -0.73104 & 11.132 & 4.247 & 0.054 & -0.115 \\
\hline 5 & 75 & $50^{\circ}$ & -0.01717 & -0.48463 & 14.543 & 2.794 & -0.407 & -0.110 \\
\hline 5 & 75 & $55^{\circ}$ & -0.00708 & -0.19983 & 9.621 & 0.889 & -0.394 & -0.040 \\
\hline 5 & 75 & $60^{\circ}$ & -0.00286 & -0.08072 & 2.191 & 0.293 & -0.085 & -0.006 \\
\hline 5 & 75 & $65^{\circ}$ & -0.00383 & -0.10811 & 2.775 & 0.301 & -0.102 & -0.001 \\
\hline 5 & 75 & $70^{\circ}$ & -0.00448 & -0.12645 & 3.828 & 0.172 & -0.127 & 0.007 \\
\hline 5 & 70 & $35^{\circ}$ & -0.02705 & -0.76350 & -2.180 & 3.255 & 0.796 & 0.042 \\
\hline 5 & 75 & $35^{\circ}$ & -0.02311 & -0.65229 & -4.622 & 2.161 & 0.719 & 0.076 \\
\hline 5 & 80 & $35^{\circ}$ & -0.01877 & -0.52979 & -5.461 & 1.336 & 0.592 & 0.085 \\
\hline 5 & 85 & $35^{\circ}$ & -0.01419 & -0.40052 & -4.864 & 0.794 & 0.438 & 0.073 \\
\hline 5 & 90 & $35^{\circ}$ & -0.00983 & -0.27746 & -3.141 & 0.543 & 0.284 & 0.050 \\
\hline 5 & 95 & $35^{\circ}$ & -0.00745 & -0.21028 & -1.148 & 0.609 & 0.185 & 0.025 \\
\hline 5 & 100 & $35^{\circ}$ & -0.00766 & -0.21621 & -1.111 & 0.601 & 0.173 & 0.024 \\
\hline 3 & 75 & $35^{\circ}$ & -0.00886 & -0.25008 & -2.737 & 0.699 & 0.318 & 0.043 \\
\hline 4 & 75 & $35^{\circ}$ & -0.01508 & -0.42564 & -3.896 & 1.298 & 0.509 & 0.063 \\
\hline 5 & 75 & $35^{\circ}$ & -0.02311 & -0.65229 & -4.622 & 2.161 & 0.719 & 0.076 \\
\hline 6 & 75 & $35^{\circ}$ & -0.03272 & -0.92354 & -4.898 & 3.264 & 0.940 & 0.085 \\
\hline 7 & 75 & $35^{\circ}$ & -0.04354 & -1.22895 & -4.899 & 4.529 & 1.171 & 0.090 \\
\hline 8 & 75 & $35^{\circ}$ & -0.05549 & -1.56624 & -4.771 & 5.924 & 1.413 & 0.095 \\
\hline & & & & & & & & \\
\hline 5 & 75 & 75 & & & & & &
\end{tabular}

In the $80 \mathrm{~mm}$ aperture $\mathrm{RHIC}$ arc dipoles, this method has been used in the final design for optimizing the yoke. By putting a small additional saturation suppressor hole having a radius of $4.76 \mathrm{~mm}$ at $R=75 \mathrm{~mm}$ and $\theta=35.5^{\circ}$ in addition to the other holes and cutout 


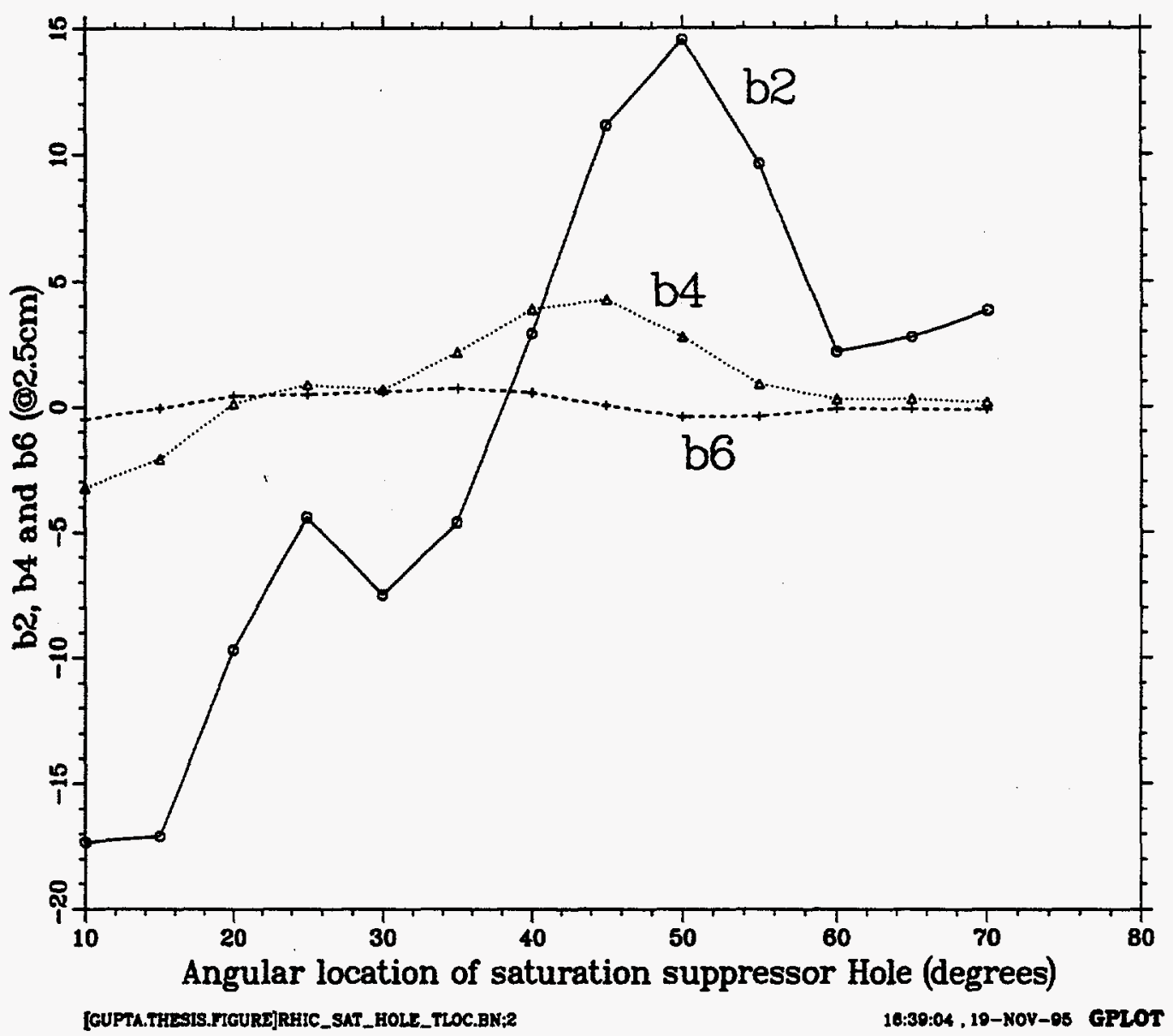

Figure 3.2.15: Saturation induced $b_{2}, b_{4}$ and $b_{6}$ harmonics generated at $5 \mathrm{kA}$ by a $10 \mathrm{~mm}$ diameter saturation suppressor hole as a function of its angular location in the first quadrant. The radial location of this hole was kept at $75 \mathrm{~mm}$. The location of three other holes is determined by the basic dipole symmetry. These are the changes in the harmonics which are computed by taking the difference in the saturation induced harmonics when (a) these holes were present and (b) were not present in the computer model.

required for various purpose (see Fig. 3.2.1), the $b_{2}$ saturation is kept below 3 unit and $b_{4}$ saturation below 0.6 unit in the entire range of operation. 


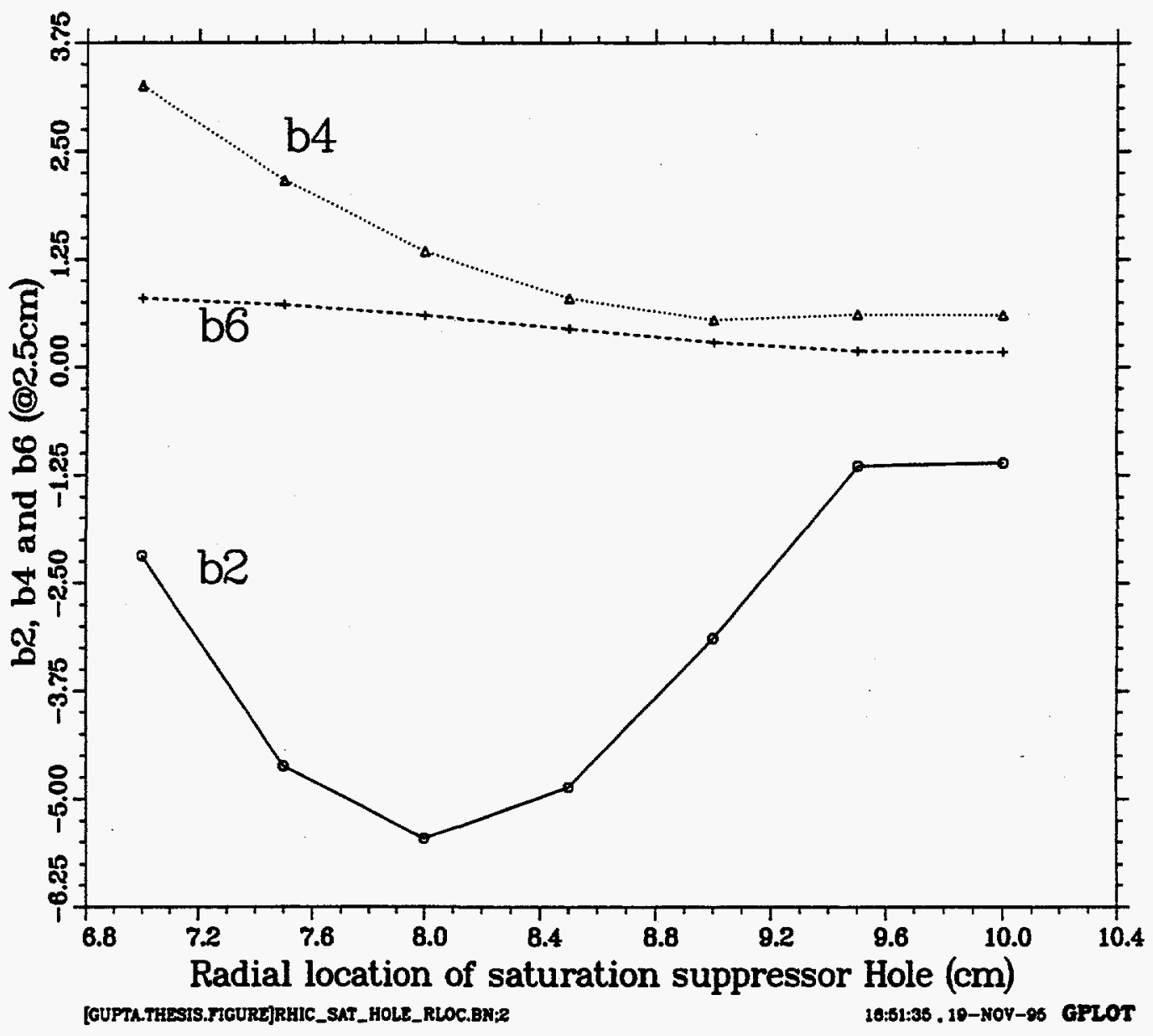

Figure 3.2.16: Saturation induced $b_{2}, b_{4}$ and $b_{6}$ harmonics generated at $5 \mathrm{kA}$ by a $10 \mathrm{~mm}$ diameter saturation suppressor hole as a function of its radial location. The angular location of this hole was kept at $35^{\circ}$. These are the changes in the harmonics which are computed by taking the difference in the saturation induced harmonics when (a) these holes were present and (b) were not present in the computer model.

In the $100 \mathrm{~mm}$ aperture RHIC insertion $D O$ dipoles, a similar hole (having a radius of $10 \mathrm{~mm}$ ) has been placed at $R=81 \mathrm{~mm}$ and $\theta=61^{\circ}$. This, in addition to properly positioning the helium bypass hole, has reduced $b_{2}, b_{4}$ and $b_{6}$ saturation to less then 3,1 , 0.5 unit respectively. The saturation in higher order harmonics is less than $\mathbf{0 . 2}$ unit. 


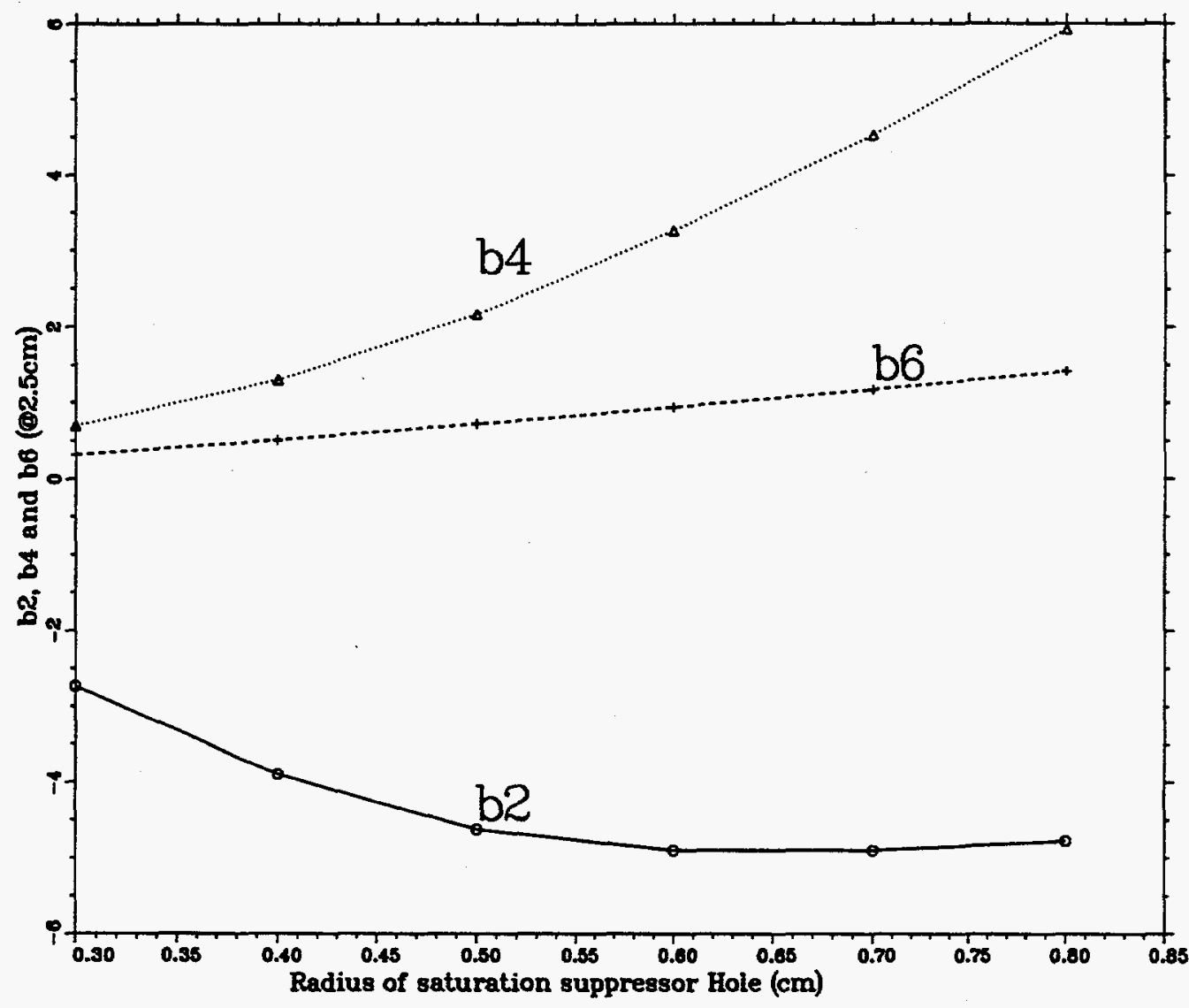

[GUPTA.THESIS.FIGURE]RHIC_SAT_HOLE_RSIZE.BN:2

16:56:51 , 10-NOV-98 GPLOT

Figure 3.2.17: Saturation induced $b_{2}, b_{4}$ and $b_{6}$ harmonics generated at $5 \mathrm{kA}$ by a saturation suppressor hole as a function of its size (radius). The center of this hole was kept at a radius $75 \mathrm{~mm}$ and at an angle of $35^{\circ}$. These are the changes in the harmonics which are computed by taking the difference in the saturation induced harmonics when (a) these holes were present and (b) were not present in the computer model. 


\subsubsection{Yoke-yoke alignment keys}

The SSC magnets built at BNL were based on a horizontally split yoke design. In such designs the top and bottom yoke halves of the magnet are aligned with the help of two keys at the midplane, the slot for one of which is shown in Fig. 3.2.2 . At high field, the saturation induced harmonics depend on the type of material used in the keys. These keys are usually made of low carbon magnetic steel to provide the maximum magnetic material in the return path for the flux at the yoke midplane. However, the structural requirement is satisfied by keys made of either low carbon magnetic steel or non-magnetic stainless steel. When the keys are made of non-magnetic stainless steel, the return flux density on the midplane is greater. This makes the midplane saturate more and at a relatively lower field than otherwise it would have. Since the midplane saturation gives a negative $b_{2}$, which is opposite to the sign of $b_{2}$ given by the pole saturation, one can optimize the size and/or location of the stainless steel keys to minimize this harmonic. A similar saturation control can be achieved by adjusting the yoke outer diameter, with an undesirable increase in the fringe field if the diameter is decreased.

In SSC magnets, a stainless steel key of $12.7 \mathrm{~mm} \times 12.7 \mathrm{~mm}$ cross section was placed at $x=91.44 \mathrm{~mm}$ to keep $b_{2}$ saturation under 0.4 unit over the entire range of operation. As shown in Fig. 3.2.18, the $b_{2}$ saturation is first positive and then negative but the magnitude is always under 0.4 unit. In the Fermilab-built SSC magnets, the yoke is vertically split and therefore the yoke-yoke alignment key is located on the vertical axis. To obtain the BNL-type saturation control in the Fermilab design, a square cutout was placed at the yoke midplane.

In Fig. 3.2.18, the saturation induced sextupole and decapole harmonics are plotted as a function of current in the SSC $50 \mathrm{~mm}$ aperture dipole magnets when these keys were made of either magnetic or non-magnetic material. The values are given in Table 3.2.8 in the case of non-magnetic keys and in Table 3.2.9 in the case of magnetic keys. Though in the nominal design, the material of the keys was non-magnetic stainless steel, a magnet was built at KEK in which the keys were made of low carbon magnetic steel. Good agreement was found between the calculations and measurements in these two cases. 


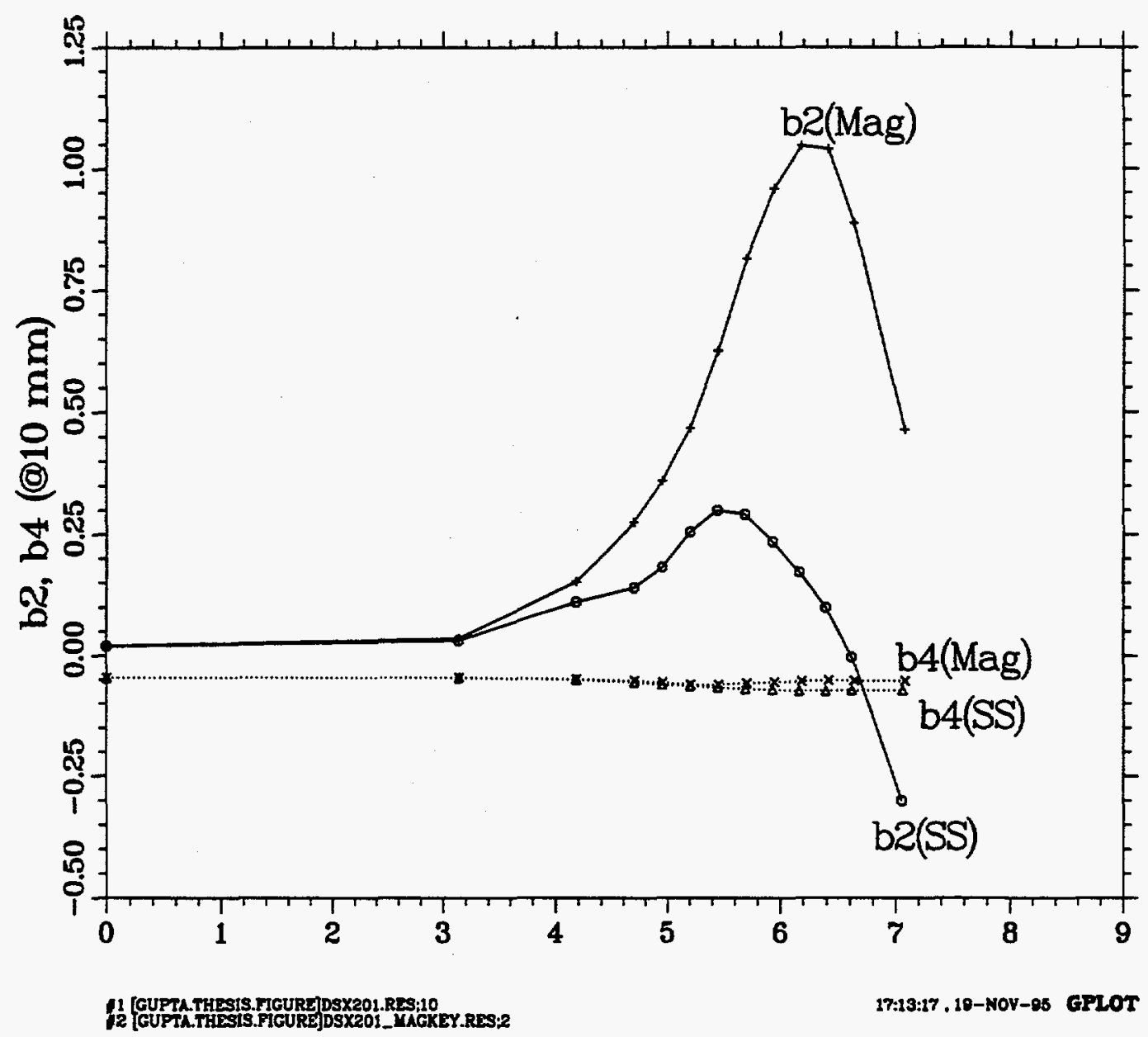

Figure 3.2.18: The computed saturation induced harmonics as a function of current in the Brookhaven design of the SSC collider arc dipole magnets when the material of the yoke-yoke alignment keys is (a) stainless steel (non-magnetic) or (b) low carbon steel (magnetic). The material of the key has been used as the primary method of reducing the saturation induced harmonics in the SSC $40 \mathrm{~mm}$ aperture and SSC $50 \mathrm{~mm}$ dipole magnets. In the Fermilab vertically split yoke design, a cutout was introduced in the midplane of the yoke since the yoke-yoke alignment key is on the vertical plane. 
Table 3.2.8: The computed harmonics as a function of current in the BNL-built SSC $50 \mathrm{~mm}$ aperture dipoles in the case when the yoke-yoke alignment keys are made of non-magnetic stainless steel (ss).

\begin{tabular}{|c|c|c|c|c|c|c|}
\hline $\mathrm{I}(\mathrm{kA})$ & $B_{o}(\mathrm{~T})$ & $\mathrm{TF}(\mathrm{T} / \mathrm{kA})$ & $\left(b_{2}\right)_{\text {s. }}$ & $\left(b_{4}\right)_{s s}$ & $\left(b_{6}\right)_{\text {.s }}$ & $\left(b_{8}\right)_{\text {.s. }}$ \\
\hline 0.00 & 0.00 & 1.04493 & 0.020 & -0.046 & 0.000 & 0.047 \\
\hline 3.00 & 3.13413 & 1.04471 & 0.031 & -0.046 & 0.001 & 0.047 \\
\hline 4.00 & 4.17625 & 1.04406 & 0.111 & -0.050 & 0.001 & 0.047 \\
\hline 4.50 & 4.69208 & 1.04268 & 0.140 & -0.055 & 0.001 & 0.047 \\
\hline 4.75 & 4.94641 & 1.04135 & 0.182 & -0.060 & 0.001 & 0.047 \\
\hline 5.00 & 5.19846 & 1.03969 & 0.255 & -0.063 & 0.001 & 0.047 \\
\hline 5.25 & 5.44536 & 1.03721 & 0.299 & -0.066 & 0.001 & 0.047 \\
\hline 5.50 & 5.68711 & 1.03402 & 0.291 & -0.069 & 0.001 & 0.048 \\
\hline 5.75 & 5.92405 & 1.03027 & 0.235 & -0.071 & 0.001 & 0.048 \\
\hline 6.00 & 6.15729 & 1.02621 & 0.172 & -0.073 & 0.000 & 0.048 \\
\hline 6.25 & 6.38679 & 1.02189 & 0.100 & -0.073 & 0.000 & 0.048 \\
\hline 6.50 & 6.61214 & 1.01725 & -0.003 & -0.072 & 0.000 & 0.048 \\
\hline 7.00 & 7.05133 & 1.00733 & -0.300 & -0.072 & 0.000 & 0.049 \\
\hline
\end{tabular}

Table 3.2.9: The computed harmonics as a function of current in the BNL-built SSC $50 \mathrm{~mm}$ aperture dipoles in the case when the yoke-yoke alignment keys are made of magnetic low carbon steel (mag).

\begin{tabular}{|c|c|c|c|c|c|c|}
\hline $\mathrm{I}(\mathrm{kA})$ & $B_{o}(\mathrm{~T})$ & $\mathrm{TF}(\mathrm{T} / \mathrm{kA})$ & $\left(b_{2}\right)_{m a g}$ & $\left(b_{4}\right)_{m a g}$ & $\left(b_{6}\right)_{\text {mag }}$ & $\left(b_{8}\right)_{\text {mag }}$ \\
\hline 0.00 & 0.00 & 1.04493 & 0.020 & -0.046 & 0.000 & 0.047 \\
\hline 3.00 & 3.13418 & 1.04473 & 0.035 & -0.046 & 0.001 & 0.047 \\
\hline 4.00 & 4.17703 & 1.04426 & 0.152 & -0.049 & 0.001 & 0.047 \\
\hline 4.50 & 4.69495 & 1.04332 & 0.275 & -0.052 & 0.001 & 0.047 \\
\hline 4.75 & 4.95052 & 1.04221 & 0.360 & -0.056 & 0.001 & 0.047 \\
\hline 5.00 & 5.20167 & 1.04033 & 0.468 & -0.060 & 0.001 & 0.047 \\
\hline 5.25 & 5.45140 & 1.03836 & 0.626 & -0.060 & 0.001 & 0.047 \\
\hline 5.50 & 5.69941 & 1.03626 & 0.815 & -0.057 & 0.001 & 0.048 \\
\hline 5.75 & 5.94138 & 1.03328 & 0.959 & -0.055 & 0.001 & 0.048 \\
\hline 6.00 & 6.17865 & 1.02978 & 1.048 & -0.053 & 0.001 & 0.048 \\
\hline 6.25 & 6.41005 & 1.02561 & 1.041 & -0.051 & 0.001 & 0.048 \\
\hline 6.50 & 6.63365 & 1.02056 & 0.889 & -0.052 & 0.000 & 0.048 \\
\hline 7.00 & 7.07118 & 1.01017 & 0.465 & -0.052 & 0.001 & 0.049 \\
\hline
\end{tabular}




\subsubsection{Yoke collaring keys}

All RHIC magnets are based on a horizontally split yoke design. Moreover, in most of these magnets, the yoke also acts as the collar providing pre-compression on the coils. Yoke collaring keys are inserted on the outer periphery near the midplane to hold the applied compression on the coil. The present RHIC design uses a total of four keys in the complete $360^{\circ}$ cross section; the slot for one of which is shown in Fig. 3.2.1. These collaring keys can be made of either low carbon magnetic steel or non-magnetic stainless steel. As in the case of yoke-yoke alignment keys, the saturation induced harmonics depends on the type of material used in the keys. However, in this case there is less flexibility in choosing their size and location. Nonetheless, it is a useful tool.

In Fig. 3.2.19, the saturation induced sextupole and decapole harmonics are plotted as a function of current in the RHIC magnets when these keys were made of magnetic or non-magnetic material. The values are given in Table 3.2.10 in the case of non-magnetic keys and in Table 3.2.11 in the case of magnetic keys. The final design of the RHIC arc dipole magnets uses the stainless steel keys. 
Table 3.2.10: The computed harmonics as a function of current in the $80 \mathrm{~mm}$ aperture $\mathrm{RHIC}$ arc dipoles in the case when the yoke collaring keys are made of non-magnetic stainless steel.

\begin{tabular}{|c|c|c|c|c|c|c|}
\hline $\mathrm{I}(\mathrm{kA})$ & $B_{o}(\mathrm{~T})$ & $\delta \mathrm{TF}(\%)$ & $b_{2}(\mathrm{ss})$ & $b_{4}(\mathrm{ss})$ & $b_{6}(\mathrm{ss})$ & $b_{8}(\mathrm{ss})$ \\
\hline 0.0 & 0.00000 & 0.00000 & 0.000 & 0.000 & 0.000 & 0.000 \\
\hline 1.0 & 0.70837 & -0.02780 & 0.049 & 0.013 & 0.003 & 0.001 \\
\hline 2.0 & 1.41669 & -0.03190 & 0.009 & 0.020 & 0.006 & 0.001 \\
\hline 3.0 & 2.12224 & -0.16338 & 0.241 & 0.148 & 0.083 & -0.005 \\
\hline 4.0 & 2.79470 & -1.39662 & -0.369 & 1.037 & 0.603 & -0.030 \\
\hline 4.5 & 3.10599 & -2.58986 & -0.819 & 1.242 & 1.037 & -0.037 \\
\hline 5.0 & 3.39914 & -4.05648 & -2.600 & 0.778 & 1.303 & -0.014 \\
\hline 5.5 & 3.67461 & -5.71010 & -6.822 & 0.106 & 1.271 & 0.005 \\
\hline 6.0 & 3.93935 & -7.34050 & -10.690 & -0.235 & 1.125 & 0.013 \\
\hline 7.0 & 4.44724 & -10.33785 & -13.925 & -0.367 & 0.862 & 0.024 \\
\hline
\end{tabular}

Table 3.2.11: The computed harmonics as a function of current in the $80 \mathrm{~mm}$ aperture $\mathrm{RHIC}$ arc dipoles in the case when the yoke collaring keys are made of magnetic low carbon steel.

\begin{tabular}{|c|c|c|c|c|c|c|}
\hline $\mathrm{I}(\mathrm{kA})$ & $B_{o}(\mathrm{~T})$ & $\delta \mathrm{TF}(\%)$ & $b_{2}(\mathrm{mag})$ & $b_{4}(\mathrm{mag})$ & $b_{6}(\mathrm{mag})$ & $b_{8}(\mathrm{mag})$ \\
\hline 0.0 & 0.000 & 0.000 & 0.000 & 0.000 & 0.000 & 0.000 \\
\hline 1.0 & 0.70838 & -0.02752 & 0.050 & 0.014 & 0.004 & 0.001 \\
\hline 2.0 & 1.41670 & -0.03119 & 0.019 & 0.021 & 0.006 & 0.001 \\
\hline 3.0 & 2.12239 & -0.15633 & 0.346 & 0.161 & 0.085 & -0.005 \\
\hline 4.0 & 2.79757 & -1.29536 & 1.471 & 1.322 & 0.643 & -0.026 \\
\hline 4.5 & 3.11114 & -2.42835 & 2.303 & 1.720 & 1.103 & -0.031 \\
\hline 5.0 & 3.40729 & -3.82644 & 2.184 & 1.539 & 1.410 & -0.002 \\
\hline 5.5 & 3.68807 & -5.36471 & 1.080 & 1.475 & 1.474 & 0.034 \\
\hline 6.0 & 3.95648 & -6.93758 & -0.848 & 1.534 & 1.393 & 0.053 \\
\hline 7.0 & 4.46426 & -9.99470 & -4.910 & 1.235 & 1.098 & 0.057 \\
\hline
\end{tabular}




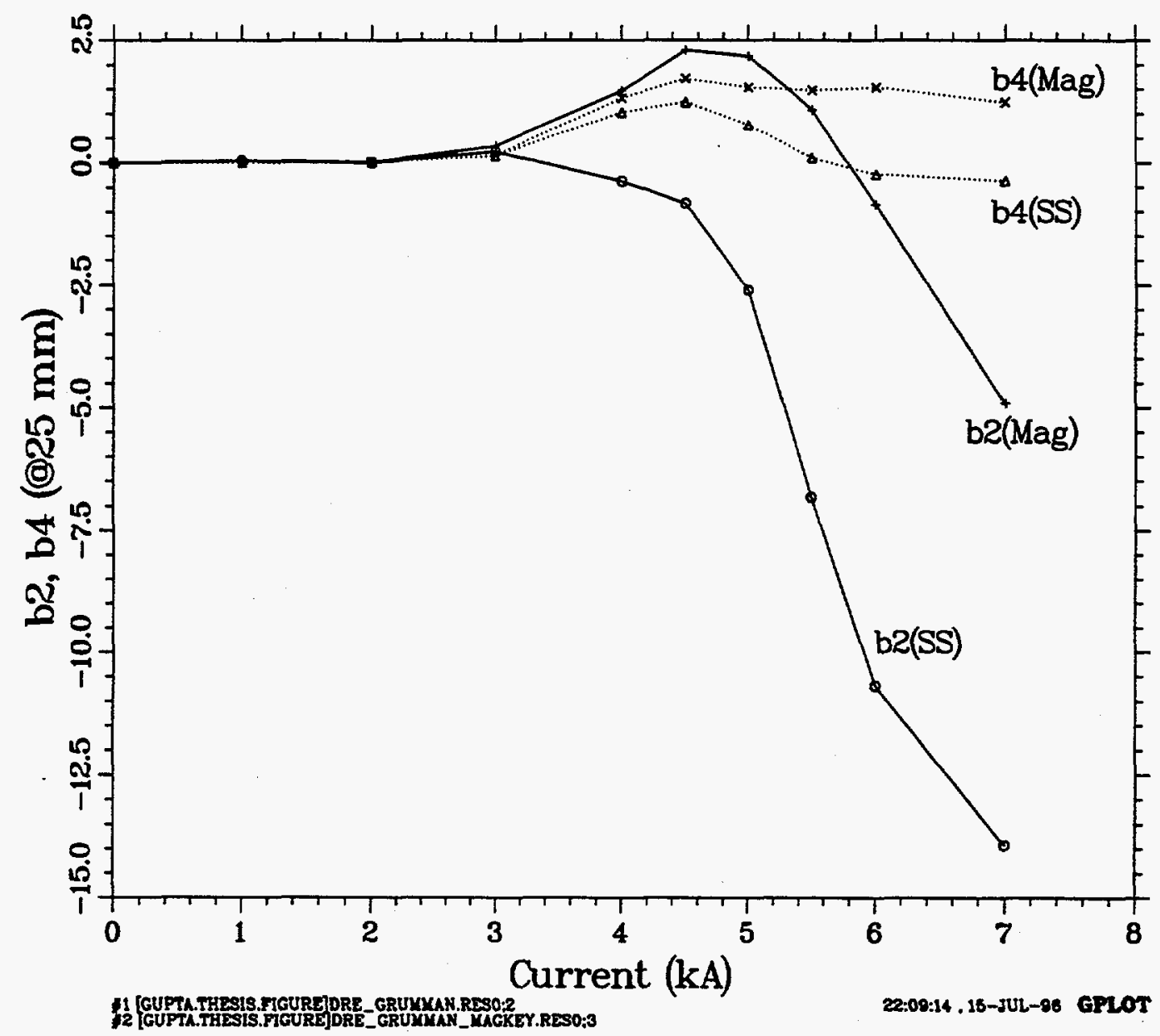

Figure 3.2.19: The computed saturation induced harmonics as a function of current in the RHIC arc dipoles when the material of the yoke collaring keys is either stainless steel (non-magnetic) or low carbon steel (magnetic). The material of the key has been used to reduce the saturation induced harmonics. 


\subsubsection{Tooth at the midplane of the yoke aperture}

The center and orientation of the coil must be aligned to that of the yoke. The geometry of the coil in the magnet is controlled and defined by the structure between the yoke and the coil. In SSC magnets, it is a stainless steel collar whose function has been explained earlier. In RHIC magnets, it is a molded phenolic (brand name RX630) spacer, whose purpose, in addition to providing electrical isolation, is to fill the space such that the geometry of the coil is well defined with respect to the yoke. Both of these materials are non-magnetic.

The earlier designs of both SSC and RHIC magnets had a notch in the yoke inner diameter at the midplane for the purpose of alignment. This notch, however, can be put either at the midplane or at the pole. In both cases a part of the magnetic material (yoke) that is close to the magnetic center is removed. A midplane location for the notch is preferred over the pole location for magnetic reasons, since the magnetic field at the pole is a maximum and a notch there increases the pole saturation significantly. However, a pole location is preferred for mechanical reasons since that location gives better control in defining the coil location in the magnet. Since the pole saturation is compensated with other methods, in the final production design of RHIC magnets the pole location was used.

One can obtain the required mechanical alignment between the coil and yoke if the notch is put in the stainless steel collar or phenolic spacer instead of the yoke. In this case the iron has a tooth which protrudes inwards towards the coil. Although the notch and tooth provide a similar mechanical function, there is a significant difference in the magnetic properties of the two. In one case the iron is taken away from the midplane in the yoke aperture whereas in the other case, iron is added to the midplane. Because of this difference, the notch gives a negative $b_{2}$ and $b_{4}$ due to saturation, whereas the tooth gives a positive $b_{2}$ and $b_{4}$ due to saturation, as compared to the saturation in these harmonics when neither of the two is present.

A comparison of the effects of a tooth or a notch was seen in the measurements of RHIC long model magnets DRB005 and DRB006 having a notch at the midplane with that of short model magnet DRS003 having a tooth at the midplane. In the dipoles DRB005 and DRB006, the value of $b_{4}$ saturation was larger than desired. Calculations showed that if the midplane notch in the yoke were replaced by a tooth at the midplane, the $b_{4}$ saturation would be reduced from -5.8 unit to -3.1 unit. In Fig. 3.2 .20 , the saturation induced $b_{2}$ and $b_{4}$ harmonics are plotted in the presence of tooth or notch in magnets DRB005 and DRB006. The values are shown in Table 3.2.12 and in Table 3.2.13. A short magnet DRS003 
was built to test these calculations and the measurements showed that the difference in saturation between this and magnets DRB005 and DRB006 was the same as predicted by the calculations. This feature was, however, not incorporated in the final RHIC arc dipole design because for mechanical reasons the preferred place for the notch or tooth is the pole instead of midplane. A tooth instead of notch would be undesirable at the pole location as that position would result in very large saturation harmonics. Hence, in the final design for the RHIC production magnets, a pole notch was used and the saturation induced harmonics created by it were compensated by the modifications in the yoke which are discussed in the other sections of this chapter. 


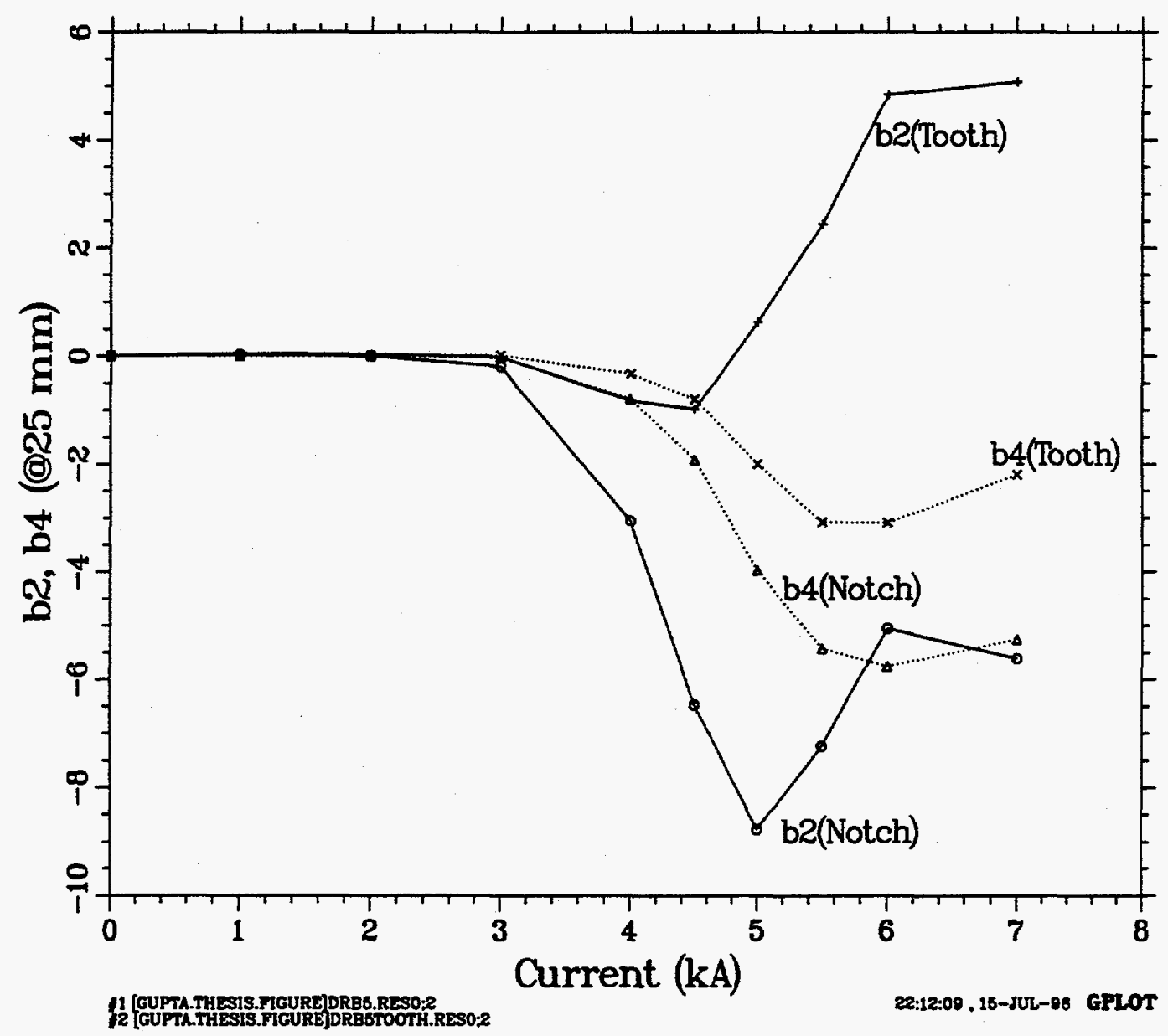

Figure 3.2.20: The computed variation in the $b_{2}$ and $b_{4}$ harmonics as a function of current when a notch is present at the midplane or when instead a tooth is present at the midplane. There is a significant improvement in the $b_{4}$ saturation when the notch is replaced by a tooth. There is also a significant change in the behavior of the $b_{2}$ saturation. 
Table 3.2.12: The computed saturation induced harmonics in the RHIC arc dipoles DRB005 and DRB006 (as built per Drawing No. 22-398.05-5) which has notches in the yoke inner surface at the midplane.

\begin{tabular}{|c|c|c|c|c|c|c|}
\hline $\mathrm{I}(\mathrm{kA})$ & $B_{0}(\mathrm{~T})$ & $\delta \mathrm{TF}(\%)$ & $b_{2}$ & $b_{4}$ & $b_{6}$ & $b_{8}$ \\
\hline 0.0 & 0.00 & 0.00 & 0.000 & 0.000 & 0.000 & 0.000 \\
\hline 1.0 & 0.70745 & -0.02261 & 0.020 & 0.001 & 0.003 & 0.001 \\
\hline 2.0 & 1.41485 & -0.02600 & -0.003 & -0.002 & 0.001 & 0.001 \\
\hline 3.0 & 2.12181 & -0.04791 & -0.196 & -0.031 & -0.003 & -0.001 \\
\hline 4.0 & 2.82004 & -0.36729 & -3.051 & -0.787 & -0.103 & -0.019 \\
\hline 4.5 & 3.15414 & -0.94530 & -6.476 & -1.918 & -0.208 & -0.048 \\
\hline 5.0 & 3.46817 & -1.97496 & -8.774 & -3.974 & -0.383 & -0.082 \\
\hline 5.5 & 3.76650 & -3.22082 & -7.239 & -5.422 & -0.599 & -0.101 \\
\hline 6.0 & 4.04390 & -4.75201 & -5.051 & -5.753 & -0.748 & -0.121 \\
\hline 7.0 & 4.54494 & -8.24351 & -5.612 & -5.254 & -0.791 & -0.127 \\
\hline
\end{tabular}

Table 3.2.13: The computed saturation induced harmonics when the midplane notches in the RHIC arc dipoles DRB005 and DRB006 are replaced by the tooths in magnet DRS003.

\begin{tabular}{|c|c|c|c|c|c|c|}
\hline $\mathrm{I}(\mathrm{kA})$ & $B_{0}(\mathrm{~T})$ & $\delta \mathrm{TF}(\%)$ & $b_{2}$ & $b_{4}$ & $b_{6}$ & $b_{8}$ \\
\hline 0.0 & 0.00 & 0.00 & 0.000 & 0.000 & 0.000 & 0.000 \\
\hline 1.0 & 0.70752 & -0.02219 & 0.040 & 0.006 & 0.004 & 0.001 \\
\hline 2.0 & 1.41500 & -0.02473 & 0.027 & 0.007 & 0.004 & 0.001 \\
\hline 3.0 & 2.12216 & -0.04074 & -0.023 & 0.007 & 0.004 & 0.001 \\
\hline 4.0 & 2.82350 & -0.25435 & -0.819 & -0.326 & -0.005 & 0.001 \\
\hline 4.5 & 3.16289 & -0.67977 & -0.981 & -0.795 & 0.031 & 0.000 \\
\hline 5.0 & 3.48314 & -1.56103 & 0.629 & -1.999 & 0.046 & 0.010 \\
\hline 5.5 & 3.78086 & -2.86090 & 2.446 & -3.072 & -0.044 & 0.025 \\
\hline 6.0 & 4.05747 & -4.44131 & 4.834 & -3.083 & -0.087 & 0.033 \\
\hline 7.0 & 4.55936 & -7.96098 & 5.076 & -2.197 & -0.027 & 0.049 \\
\hline
\end{tabular}




\subsubsection{Cutout or Bump in the iron aperture}

The saturation induced harmonics are significantly modified when a cutout or bump is introduced in the iron aperture. Coupland [30] has studied cutouts in the yoke aperture in superconducting magnets. The use of a cutout in the RHIC arc dipole magnets and a bump in the SSC collider dipole magnets is examined here. The bumps can also be visualized as shimming the yoke inner surface with some extra iron and the notch as trimming the yoke inner surface. A cutout and a bump are shown schematically in Fig. 3.2.21. The change in saturation in the RHIC arc dipoles due to a midplane notch or tooth was discussed in the last section. Those (located either at the midplane or pole) were special cases of a more general method of controlling saturation with the help of a cutout or bump at the yoke inner surface as discussed in this section.

The cutout and bump not only change the saturation induced harmonics, but also change the harmonics at all fields including those at low fields. A low field is defined as the field when the magnetization in the iron is far below its saturation magnetization. The coil cross section must be designed to remove the low field harmonics generated by a cutout or bump in the yoke aperture. Bumps and notches also change the transfer function, as shown in Fig. 3.2.22. At low field the change in transfer function is large due to a bump, particularly when it is near the pole. However, a bump like this saturates very rapidly and most of the increase is lost at high field.

The change in the value of an individual harmonic at saturation and the change in the value of that harmonic at low field depend on the size and the angular location of the cutout or bump. In Fig. 3.2.23, the low field $b_{2}$ harmonic and the saturation induced $b_{2}$ at 5000 amperes are plotted as a function of angle for a $5 \mathrm{~mm} \times 5 \mathrm{~mm}$ bump or notch in the aperture of the RHIC arc dipoles. The saturation suppressor holes, which were mentioned in an earlier section, are not present in these computer models. In Fig. 3.2.24, the $b_{4}$ harmonic has been plotted for the same parameters and in Fig. 3.2.25 the $b_{6}$ harmonic.

It may be noted that the magnitude of the change in the saturation induced harmonic is quite large in this method. However, once the coil is designed, it can't be tuned to further optimize the saturation without iterating the coil cross section as well since it creates a rather large value of the low field harmonic as well. 


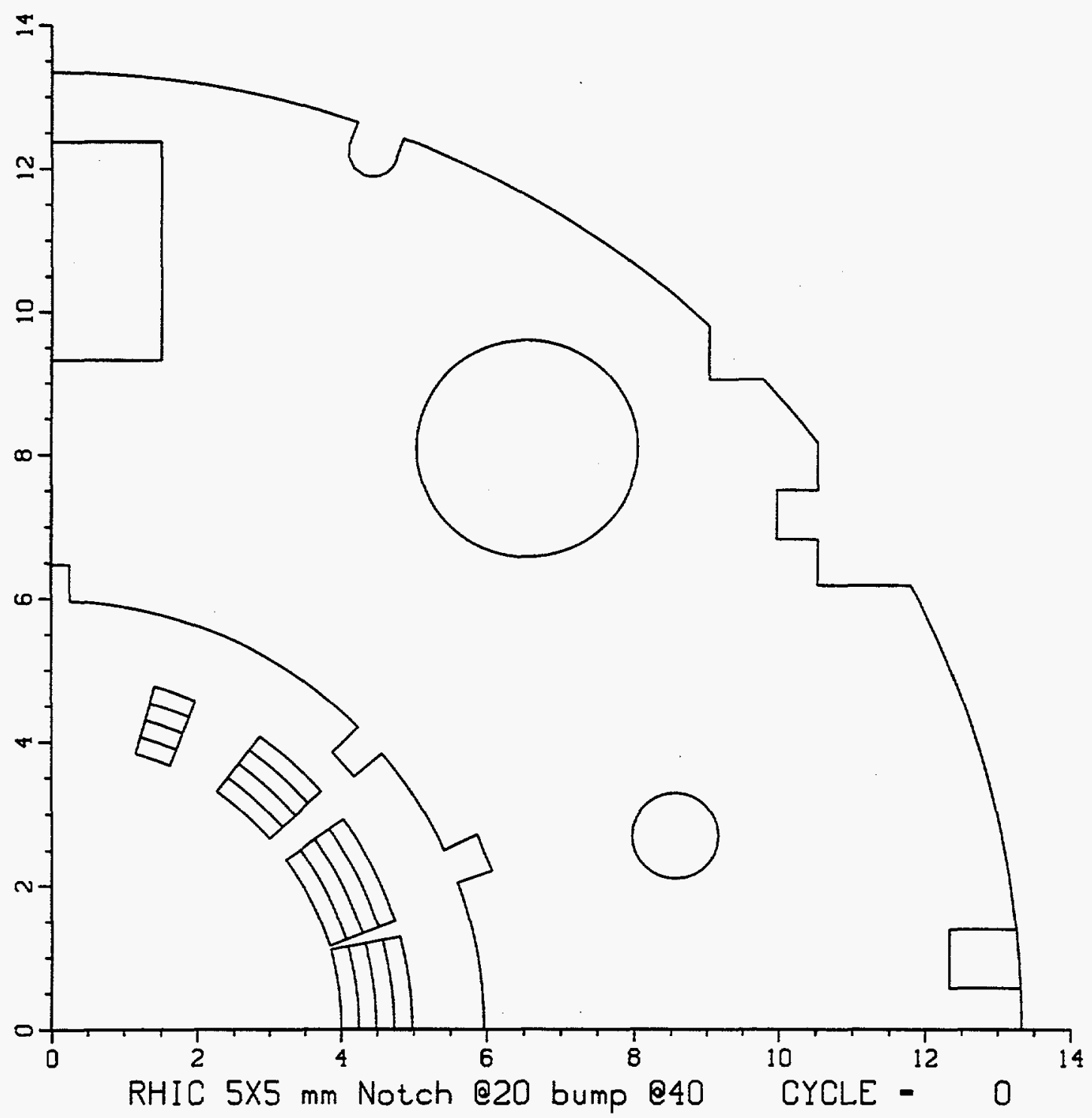

Figure 3.2.21: A computer model of a notch and a cutout in the iron aperture. The notch begins at $\theta=20^{\circ}$ and the bump at $\theta=40^{\circ}$. The notch cuts into the iron and the bump into the collar or spacer between the superconducting coil and the iron. Since the notch reduces the amount of iron it lowers the transfer function whereas the bump, because it adds iron, increases the transfer function. A $5 \mathrm{~mm} \times 5 \mathrm{~mm}$ notch at the pole $\left(90^{\circ}\right)$ is part of the standard design. 


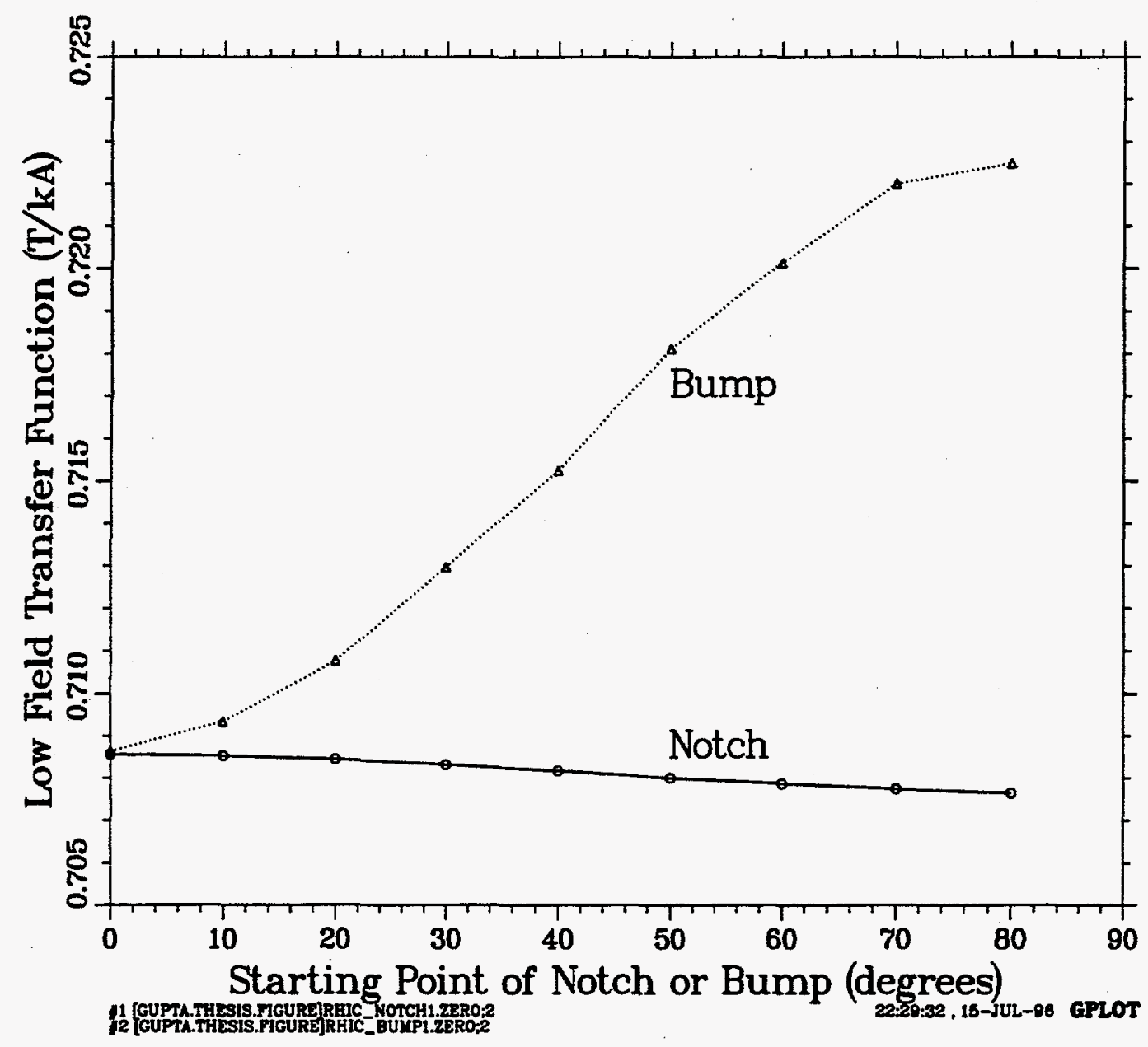

Figure 3.2.22: The transfer function at low field as a function of angle for a $5 \mathrm{~mm} \times 5 \mathrm{~mm}$ bump or notch in the aperture of the RHIC arc dipoles. The notch (or bump) is in addition to a $5 \mathrm{~mm} \times 5 \mathrm{~mm}$ notch at the pole $\left(90^{\circ}\right)$ which is part of the standard design. 
$5 \mathrm{~mm} X 5 \mathrm{~mm}$ Notch or Bump in the Aperture of RHIC arc dipole

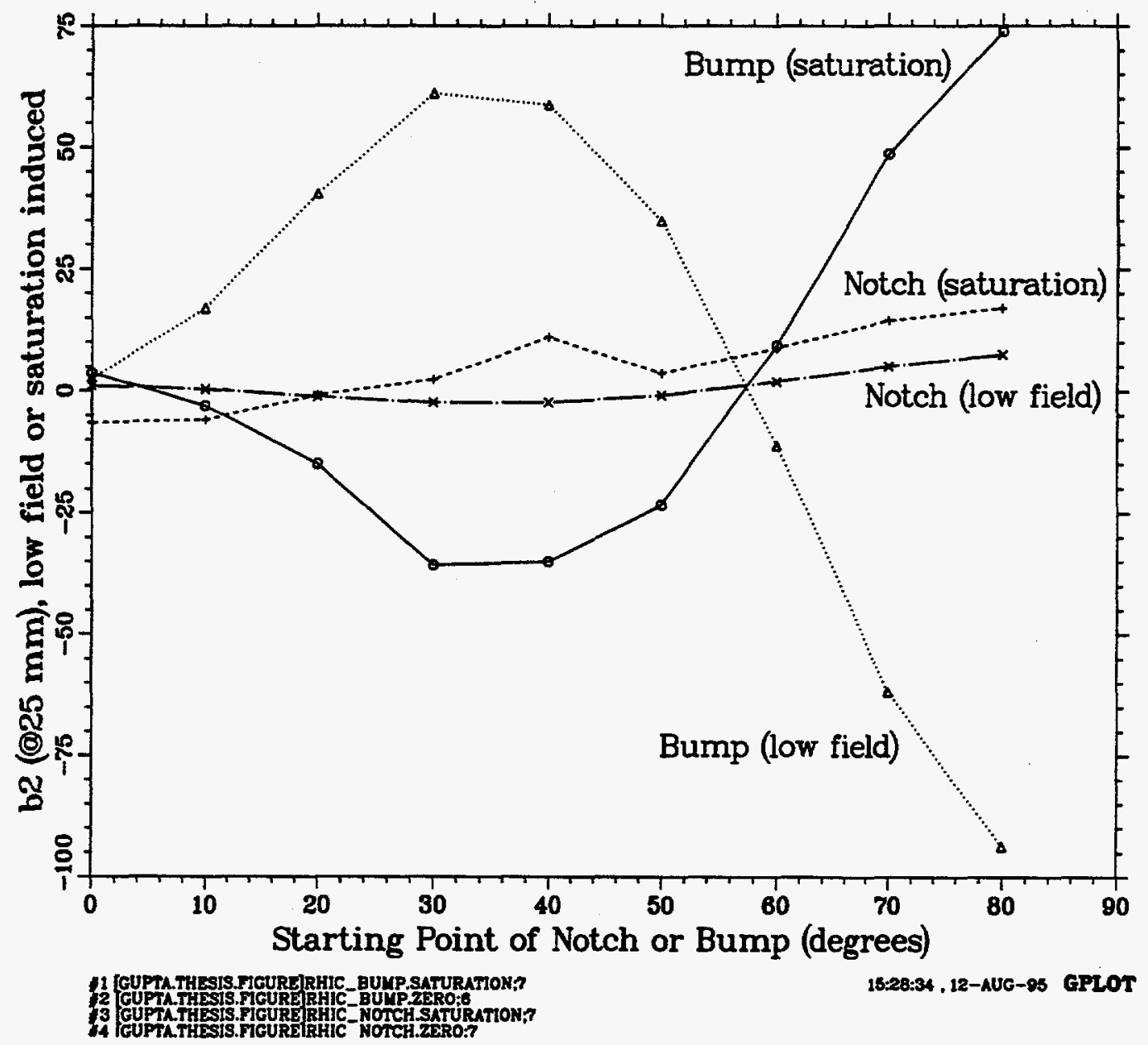

Figure 3.2.23: The low field and saturation induced $b_{2}$ harmonic at 5000 amperes as a function of angle for a $5 \mathrm{~mm} \times 5 \mathrm{~mm}$ bump or notch in the aperture of the RHIC arc dipoles. The above notch (or bump) is in addition to a $5 \mathrm{~mm} \times 5 \mathrm{~mm}$ notch at the pole $\left(90^{\circ}\right)$ which is part of the standard design. 

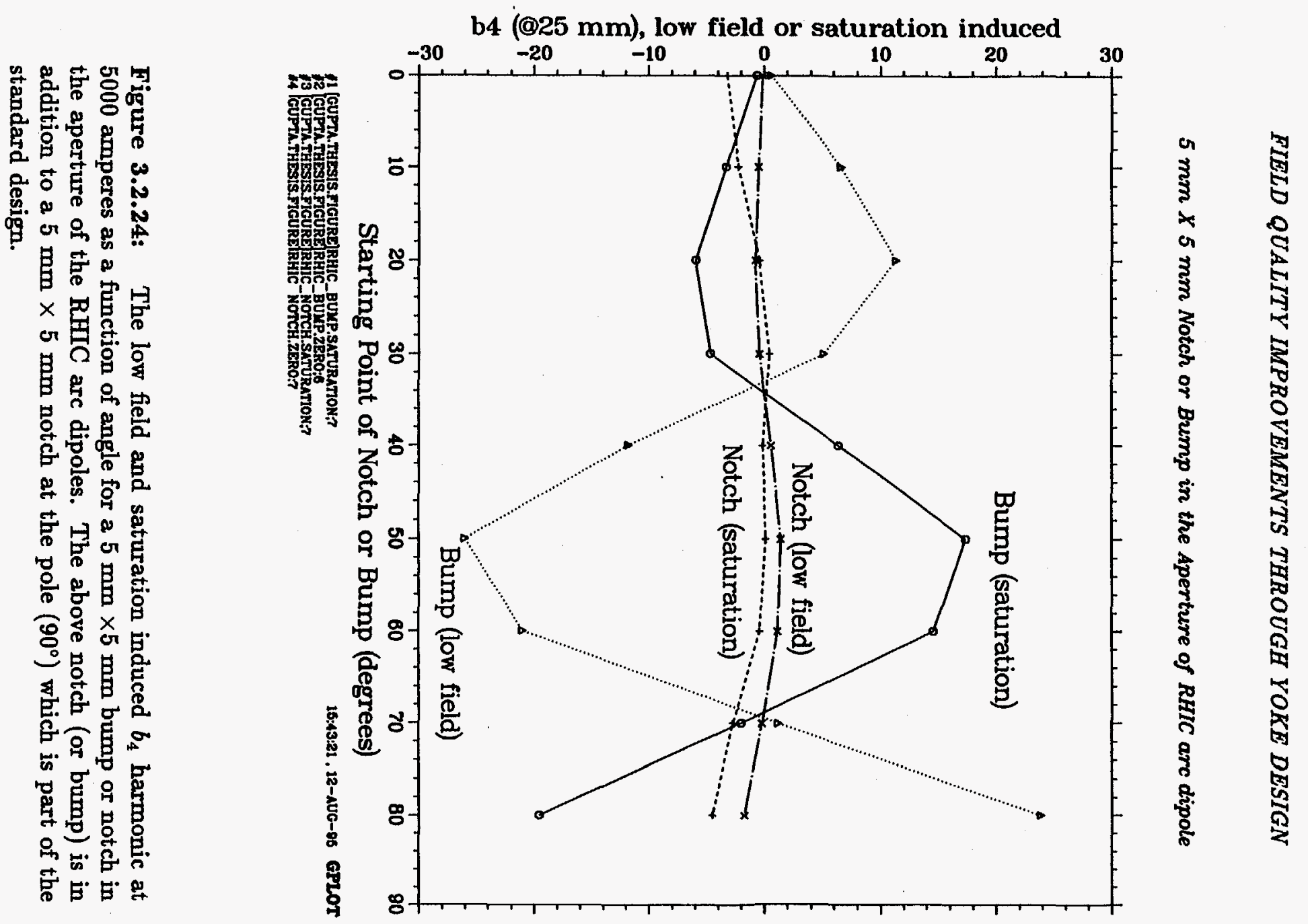
$5 \mathrm{~mm} X 5 \mathrm{~mm}$ Notch or Bump in the Aperture of RHIC arc dipole

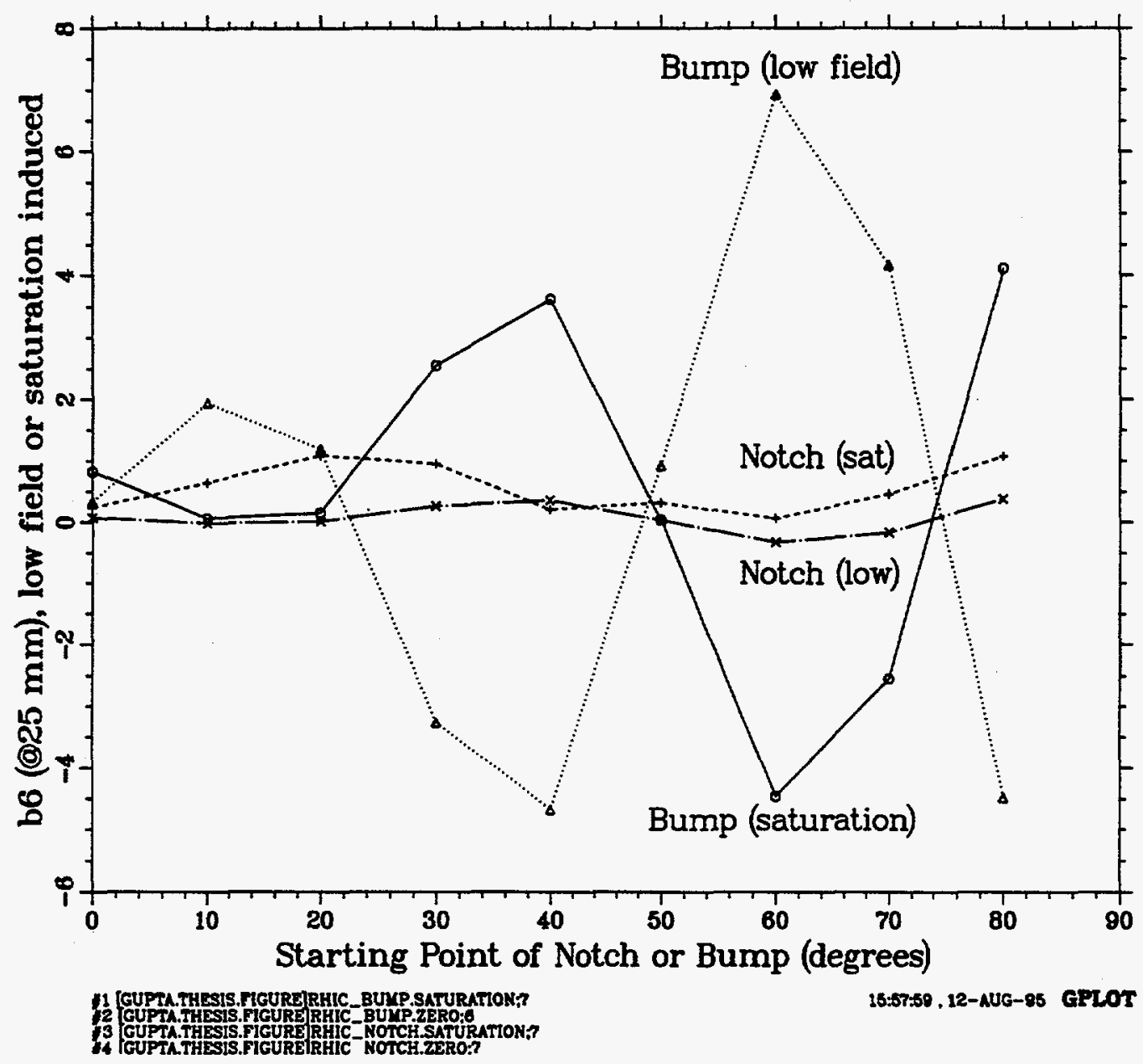

Figure 3.2.25: The low field and saturation induced $b_{6}$ harmonic at 5000 amperes as a function of angle for a $5 \mathrm{~mm} \times 5 \mathrm{~mm}$ bump or notch in the aperture of the RHIC arc dipoles. The above notch (or bump) is in addition to a $5 \mathrm{~mm} \times 5 \mathrm{~mm}$ notch at the pole $\left(90^{\circ}\right)$ which is part of the standard design. 


\subsubsection{Elliptical iron aperture}

Morgan [118] has noted that the use of an elliptical iron aperture in the RHIC arc dipole can reduce the saturation induced harmonics. The way in which an elliptical aperture can reduce these saturation induced harmonics may be understood as follows: The iron is further away from the magnet center at the pole as compared to that at the midplane. Since the pole region is the first and by far the most to saturate, this effectively removes the saturating iron and therefore reduces the saturation induced harmonics. Though removing iron from the pole reduces the transfer function as compared to that in the circular aperture case, the reduction in saturation allows the iron to be brought closer to the coil elsewhere. This, in turn reduces the average yoke radius and hence increases the transfer function. The overall result is an increased transfer function with reduced saturation. The simple elliptical aperture mainly reduces the $b_{2}$ saturation. Morgan [118] has further investigated higher order deformations in the elliptical aperture to reduce the $b_{4}, b_{6}$ and other harmonics due to saturation.

These non-circular shapes change the values of low field harmonics by a significant amount. Therefore, the coil must be designed to cancel these harmonics. This method is examined here for an alternate design of the SSC $40 \mathrm{~mm}$ aperture dipole magnet. The shape of the aperture is shown in Fig. 3.2.26. The saturation induced harmonics are plotted in Fig. 3.2.27. The values are given in Table 3.2.14. As compared to the standard design, the increase in transfer function in this design was $6.6 \%$ at low field and $4.9 \%$ at the operating field together with a reduction in the values of saturation induced harmonics. This method was not adopted (or tested) in any SSC or RHIC magnet built at BNL due to mechanical complications. However, Dell'Orco, Caspi, et al. [41] at Lawrence Berkeley National Laboratory (LBNL) have designed, built and tested a magnet based on the basic elliptical aperture concept and thus the validity of the method has been verified. 


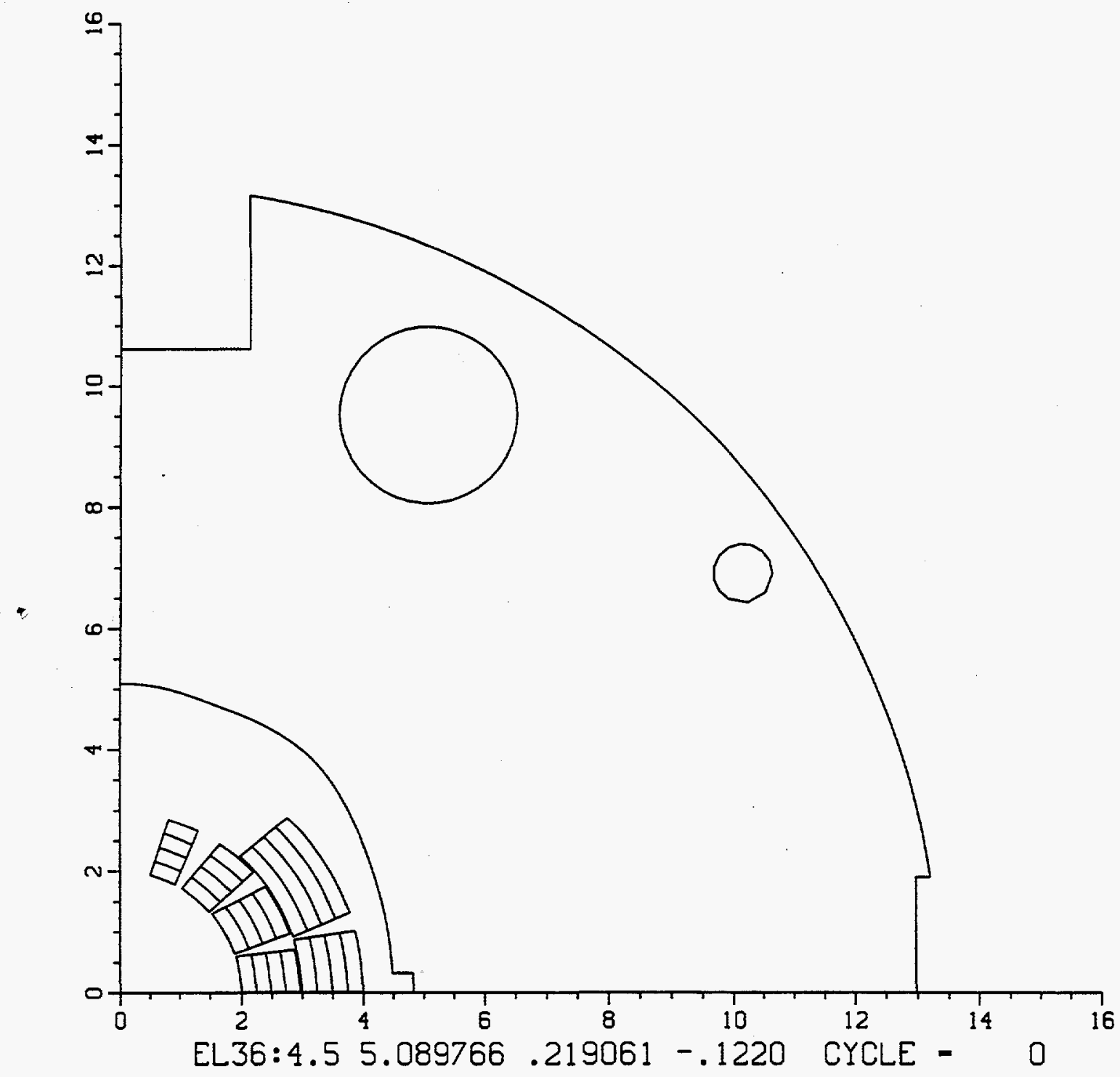

Figure 3.2.26: A computer model of an elliptical aperture dipole magnet. The shape of the aperture is optimized to increase the transfer function and reduce the field harmonics. 


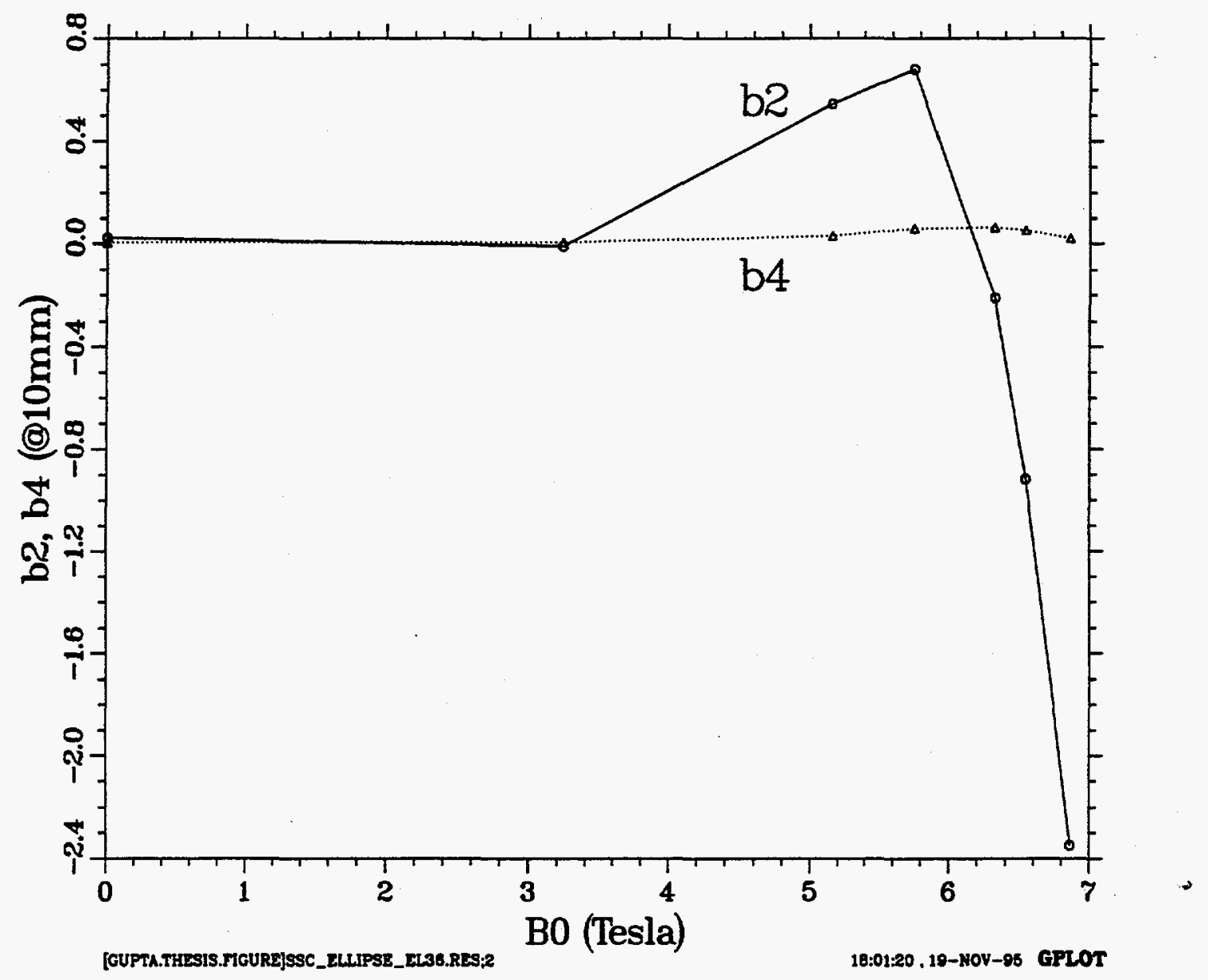

Figure 3.2.27: The variation in the sextupole $\left(b_{2}\right)$ and decapole $\left(b_{4}\right)$ harmonics as a function of current in the elliptical aperture design of the $40 \mathrm{~mm}$ coil inner diameter SSC dipole magnet. 
Table 3.2.14: Saturation induced harmonics in the proposed SSC 40 mm Elliptical aperture dipole (EL36).

\begin{tabular}{|c|c|c|c|c|c|c|}
\hline$I(\mathrm{kA})$ & $B_{o}(\mathrm{~T})$ & $\delta \mathrm{TF}(\%)$ & $b_{2}$ & $b_{4}$ & $b_{6}$ & $b_{8}$ \\
\hline 0.000 & 0.00000 & 1.105 & 0.02231 & 0.00423 & 0.02857 & 0.00963 \\
\hline 2.950 & 3.24194 & 1.099 & -0.01008 & 0.00474 & 0.02905 & 0.00985 \\
\hline 4.720 & 5.15805 & 1.093 & 0.54434 & 0.02992 & 0.02635 & 0.01000 \\
\hline 5.310 & 5.75570 & 1.084 & 0.68019 & 0.05964 & 0.02678 & 0.01008 \\
\hline 5.900 & 6.32511 & 1.072 & -0.21213 & 0.06409 & 0.02766 & 0.01042 \\
\hline 6.136 & 6.54452 & 1.067 & -0.91737 & 0.05291 & 0.02768 & 0.01057 \\
\hline 6.490 & 6.86319 & 1.058 & -2.34910 & 0.02028 & 0.02748 & 0.01077 \\
\hline
\end{tabular}




\subsubsection{Two radius aperture yoke}

In superconducting magnets, the yoke aperture is usually circular. The saturation characteristics of the yoke can be drastically altered if the yoke aperture consists of circular arcs with two radii instead of one. It has been mentioned earlier that the field near the surface of the iron is maximum at the poles and minimum at the midplanes. The radius of the circular arc is made larger at the poles as compared to that at the midplanes.

The basic principle behind this method is explained here. Yoke saturation at the pole induces a change in the allowed harmonics at high fields with the sign of them changing alternatively with increasing harmonic number (i.e. $+\delta b_{2},-\delta b_{4},+\delta b_{6},-\delta b_{8}, \ldots$ in dipoles and $+\delta b_{5},-\delta b_{9},+\delta b_{13},-\delta b_{17}, \ldots$ in quadrupoles) whereas yoke saturation at the midplane induces a negative change in all allowed harmonics. In a typical magnet design, the onset of iron saturation is observed by an increase (a positive change) in the first allowed harmonic as the level of excitation is raised. The harmonic referred to here is $b_{2}$ in dipoles and $b_{5}$ in quadrupoles. A larger yoke inner radius at the pole regions reduces a positive saturation in this harmonic and a smaller inner radius at the midplane further compensates it. This, however, increases the net (negative) saturation in the next allowed harmonic. The harmonic referred to here is $b_{4}$ in dipoles and $b_{9}$ in quadrupoles as the contribution is negative from both the midplane and pole saturation. Therefore, making the yoke inner radius smaller at the midplane and larger at the pole reduces the natural compensation and gives a larger negative saturation at high field in this harmonic. The third region which generates a significant change in the saturation induced allowed harmonics is the place where an abrupt transition from one radius to another radius takes place. This saturation can be used to further control the saturation induced allowed harmonics.

The two-radius method method has been successfully used in designing the $130 \mathrm{~mm}$ aperture quadrupole for the RHIC interaction region. The first allowed harmonic in the quadrupole is $b_{5}$ and by using this technique it was drastically reduced from 15 units to less than 1 unit. This two-radius method has been chosen over the elliptical aperture method in these magnets.

There are three parameters, in this method, which can be used to minimize the iron saturation. These are : (a) and (b) the values of the two radii and (c) the angle at which the radius of the aperture changes from one value to the other. The variation in the saturation induced $b_{5}$ and $b_{9}$ harmonics at a $40 \mathrm{~mm}$ reference radius at the maximum operating current of $5 \mathrm{kA}$ and the percentage drop in transfer function relative to its low field value in the 
Table 3.2.15: Values of the transfer function at $5 \mathrm{kA}$ and percentage drop in transfer function relative to its low field value in the case of $130 \mathrm{~mm}$ aperture RHIC insertion quadrupoles as a function of the angle at which the yoke inner radius changes from $87 \mathrm{~mm}$ to $92 \mathrm{~mm}$. The saturation induced allowed harmonics at $\mathbf{4 0} \mathrm{mm}$ reference radius are also given.

\begin{tabular}{|c|c|c|c|c|c|}
\hline $\begin{array}{c}\text { Angle } \\
\text { (degrees) }\end{array}$ & $\begin{array}{c}\text { T.F. } \\
(\mathrm{T} / \mathrm{m} / \mathrm{kA})\end{array}$ & $\begin{array}{c}\delta \text { T.F. } \\
(\%)\end{array}$ & $\begin{array}{c}\delta b_{5} \\
\text { (units) }\end{array}$ & $\begin{array}{c}\delta b_{9} \\
\text { (units) }\end{array}$ & $\begin{array}{c}\delta b_{13} \\
\text { (units) }\end{array}$ \\
\hline 5 & 46.1625 & -0.13002 & 0.638 & -0.009 & 0.001 \\
\hline 10 & 46.2516 & -0.12849 & 0.501 & -0.017 & 0.001 \\
\hline 15 & 46.4231 & -0.15174 & -0.236 & -0.026 & 0.003 \\
\hline 20 & 46.6801 & -0.23275 & -1.394 & 0.062 & 0.010 \\
\hline 25 & 47.0162 & -0.38814 & -1.768 & 0.273 & 0.006 \\
\hline 30 & 47.4166 & -0.61767 & -0.132 & 0.425 & -0.014 \\
\hline 35 & 47.8620 & -0.90488 & 3.808 & 0.289 & -0.024 \\
\hline 40 & 48.3406 & -1.12132 & 8.224 & -0.116 & -0.004 \\
\hline
\end{tabular}

case of the RHIC $130 \mathrm{~mm}$ aperture quadrupoles is given in Fig. 3.2 .28 as a function of the angle at which the yoke inner radius changes. The yoke inner radius is always larger $(92 \mathrm{~mm})$ at the pole and smaller $(87 \mathrm{~mm})$ at the midplane and the two circular arcs are connected by a radial line. A larger value of this angle gives a larger transfer function which is desirable. The values of the transfer function at the maximum operating current $(5 \mathrm{kA})$, the percentage drop in transfer function relative to its low value and the saturation induced allowed harmonics at a $40 \mathrm{~mm}$ reference radius as a function of angle at which the yoke inner radius changes are given in Table 3.2.15. The model used in this study is similar to the one shown in Fig. 3.2.29 except for the absence of the notch at the midplane and except that in this case the two radii are connected by a radial line.

In Fig. 3.2.29, a computer model of an octant of the $130 \mathrm{~mm}$ aperture RHIC insertion quadrupole is shown. The yoke inner radius is $87 \mathrm{~mm}$ at the midplane and $92 \mathrm{~mm}$ at the pole. The two radii are connected by a straight line (face) which is either parallel to the horizontal axis or to the vertical axis depending on the location. As shown in the figure, the two radii are connected by a vertical line in this octant. The change-over from one radius 


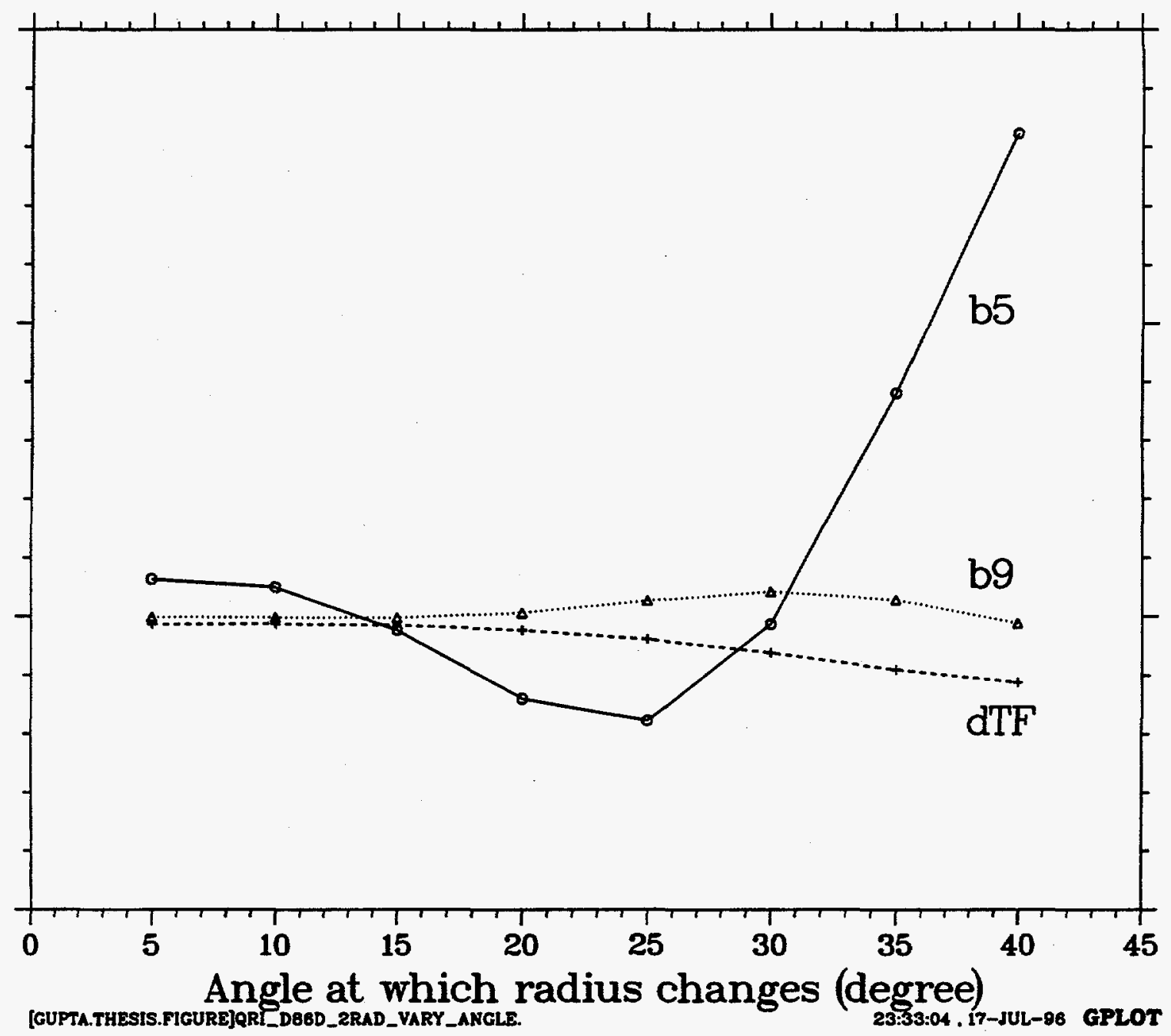

Figure 3.2.28: The variation in the saturation induced $b_{5}$ and $b_{9}$ harmonics at $5 \mathrm{kA}$ at a reference radius of $40 \mathrm{~mm}$ as a function of angle at which the yoke inner radius changes from $87 \mathrm{~mm}$ to $92 \mathrm{~mm}$ in the case of the $130 \mathrm{~mm}$ aperture $\mathrm{RHIC}$ insertion quadrupole. The yoke inner radius is larger ( $92 \mathrm{~mm}$ ) at the pole. The two circular are connected by a radial line. 
to another radius takes place at an angle of about 30 degree in the first octant. The angles in the other octants are determined by quadrupole symmetry.

Fig. 3.2.30, Fig. 3.2.31 and Fig. 3.2.32 show the variation in the transfer function, $b_{5}$ and $b_{9}$ harmonics, respectively, as a function of current in the $130 \mathrm{~mm}$ aperture $\mathrm{RHIC}$ insertion quadrupoles. The three cases in each figure represent the aperture described (a) by one 87 mm radius for which the computed saturation induced harmonics are given in Table 3.2.16, (b) by one $92 \mathrm{~mm}$ radius (data in Table 3.2.17) and (c) by $87 \mathrm{~mm}$ at the midplane and by $92 \mathrm{~mm}$ radius at the pole (data in Table 3.2.18), with the change-over in the yoke inner radius taking place at $\theta=30^{\circ}$ in the first octant.

One can see from Fig. 3.2.31 that the saturation induced $b_{5}$ is smaller in the two radii case even as compared to the case when the radius was larger all over (inner radius = 92 $\mathrm{mm}$ ). However, as shown in Fig. 3.2.32, the net $b_{9}$ saturation has increased now. This was expected from the explanation given earlier in this section. This increase is however too small to be of concern to RHIC operation. The transfer function (Fig. 3.2.30) is maximum when the radius is $87 \mathrm{~mm}$. However, at high current in the two radius case, the transfer function approaches the transfer function of the $87 \mathrm{~mm}$ case. This is because in the lower yoke inner radius case, the pole is highly saturated and the permeability in the yoke is close to that of air, anyway. 


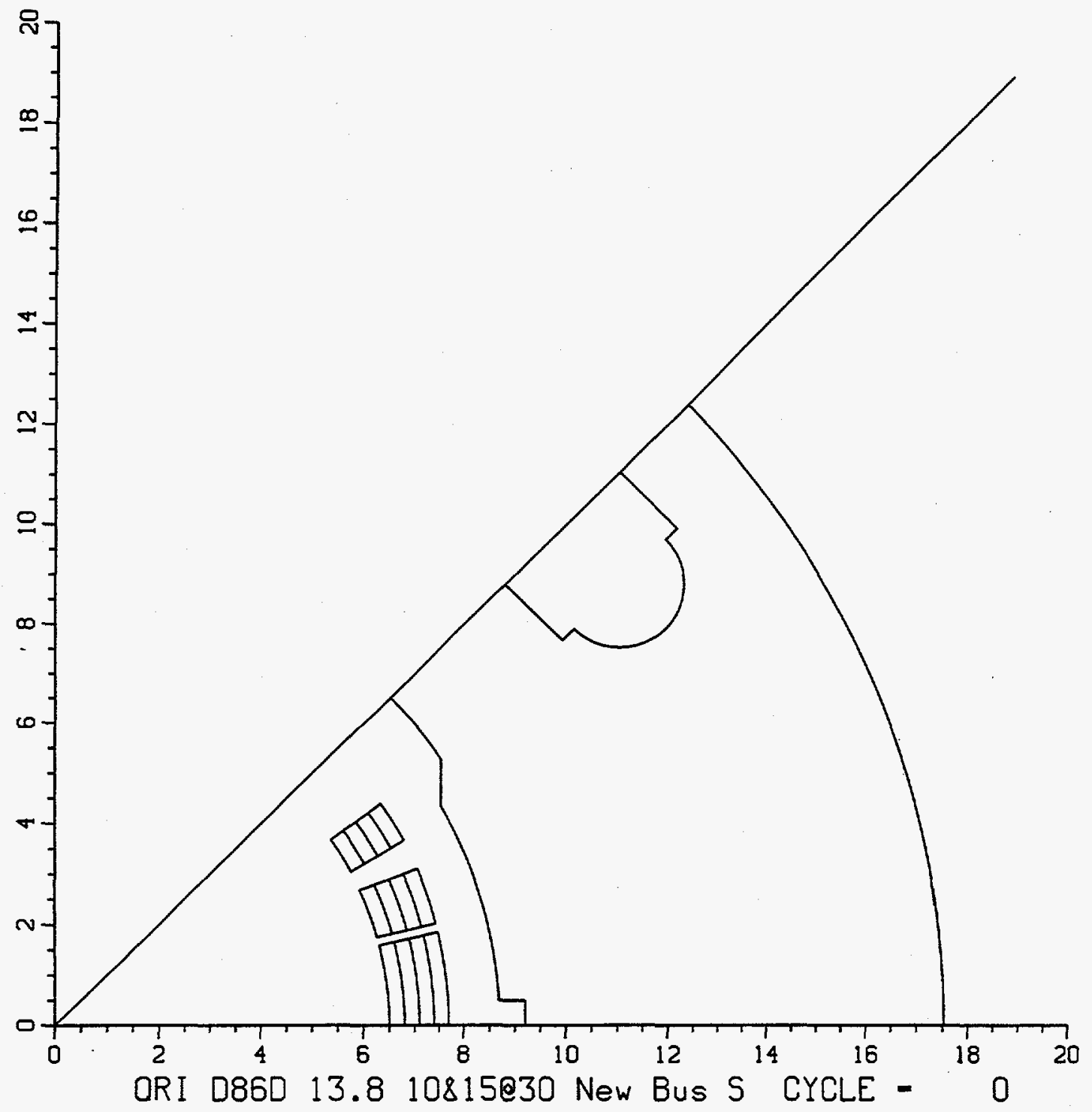

Figure 3.2.29: The computer model of the $130 \mathrm{~mm}$ aperture RHIC insertion dipole. The yoke inner surface is described by circular arcs having radii of $87 \mathrm{~mm}$ and $92 \mathrm{~mm}$. The change over from $87 \mathrm{~mm}$ to $92 \mathrm{~mm}$ takes place around $\theta=30^{\circ}$. The method has been found to be very efficient in reducing the saturation induced harmonic in quadrupoles. 


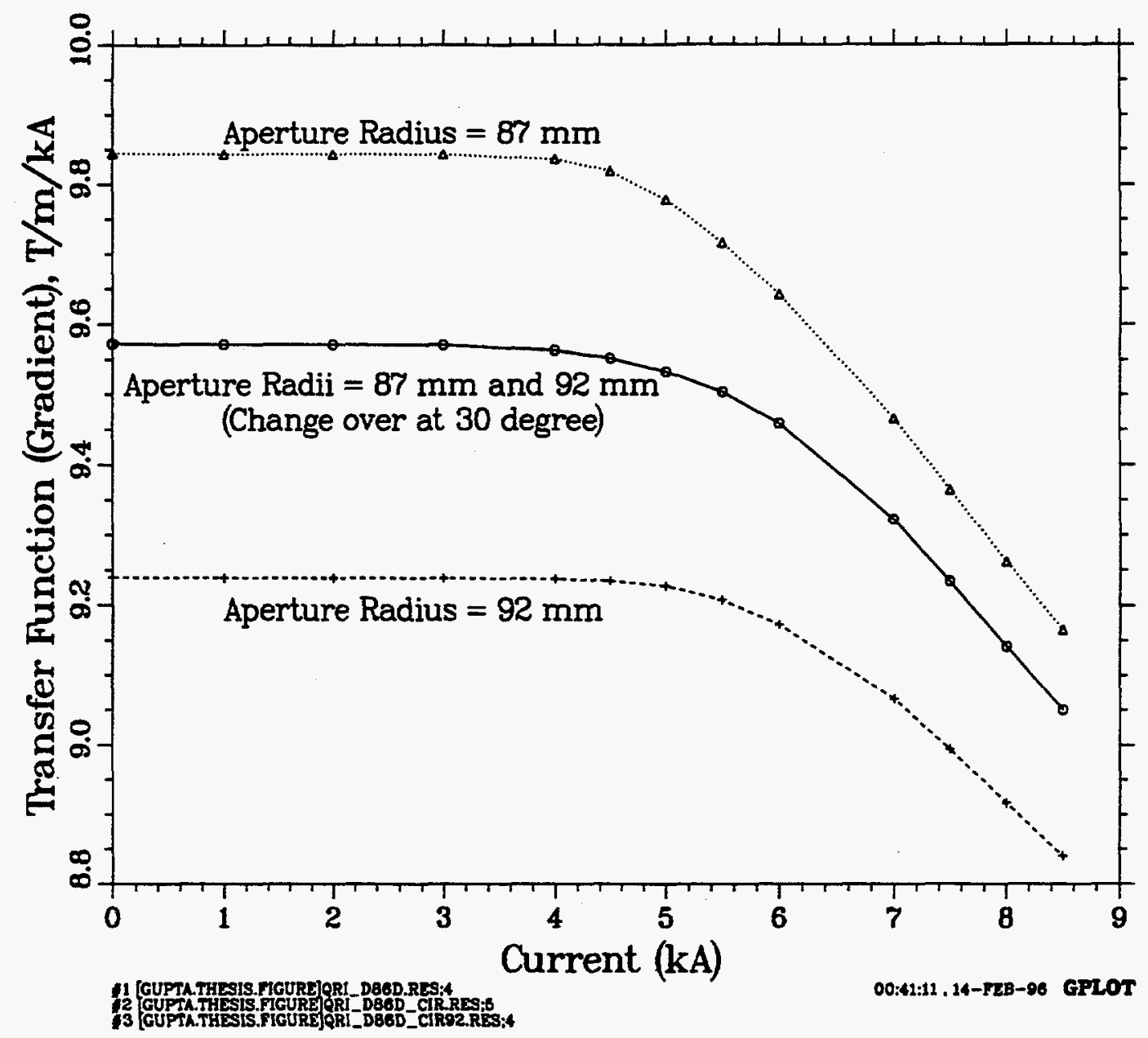

Figure 3.2.30: The variation in the transfer function as a function of current in the $130 \mathrm{~mm}$ aperture RHIC insertion quadrupole in the three cases of the yoke inner surface. The yoke inner surface is defined either by one single circle or by two circular arcs. The radii of these circles are indicated on the curves for the three cases. 
Saturation induced b5 in various cases

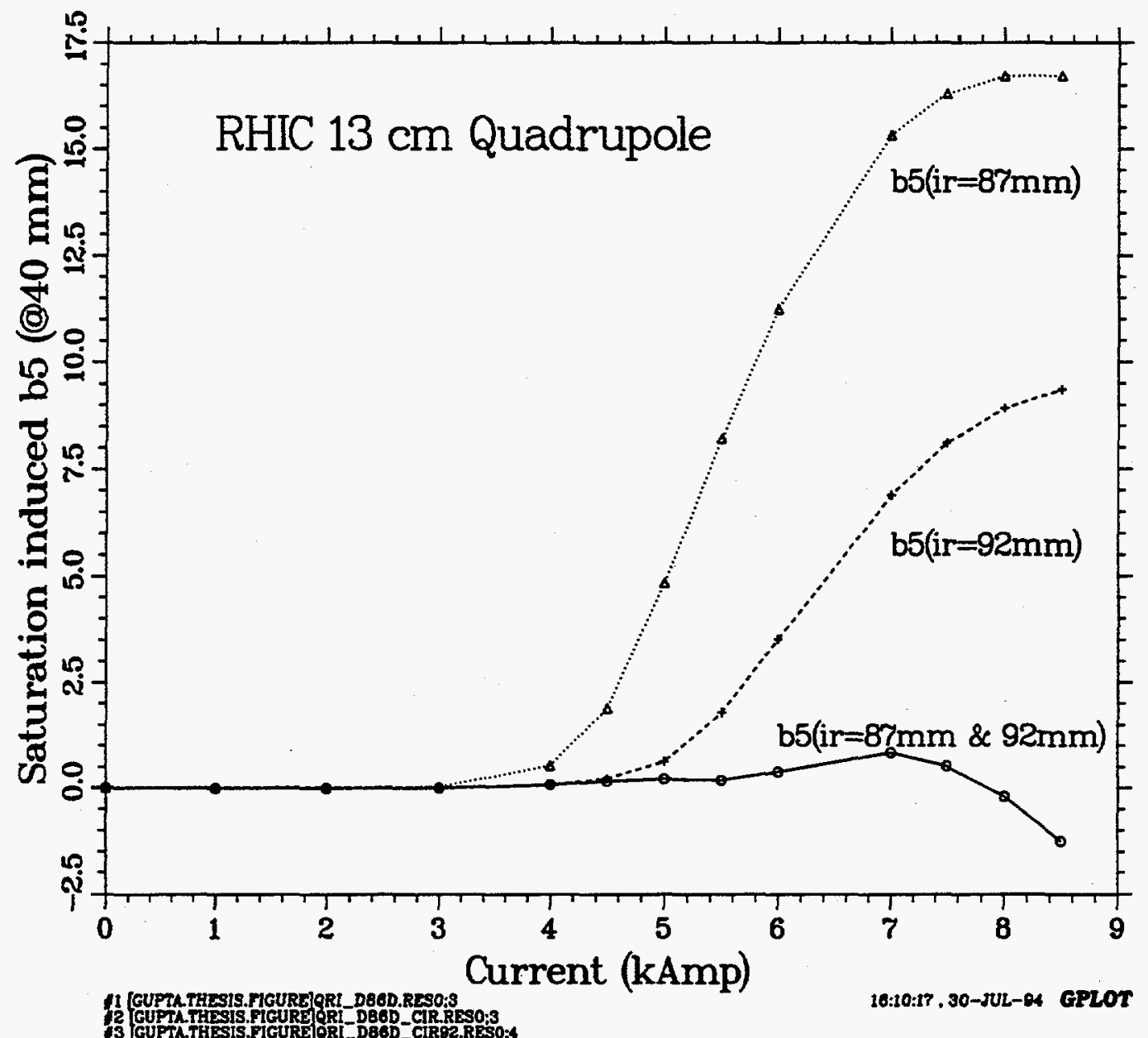

Figure 3.2.31: The variation in the dodecapole $\left(b_{5}\right)$ harmonic as a function of current in the $130 \mathrm{~mm}$ aperture RHIC insertion quadrupole in the three cases of the yoke inner surface. The yoke inner surface is defined either by one single circle or by two circular arcs. The radii of these circles are indicated on the curves in various cases. 


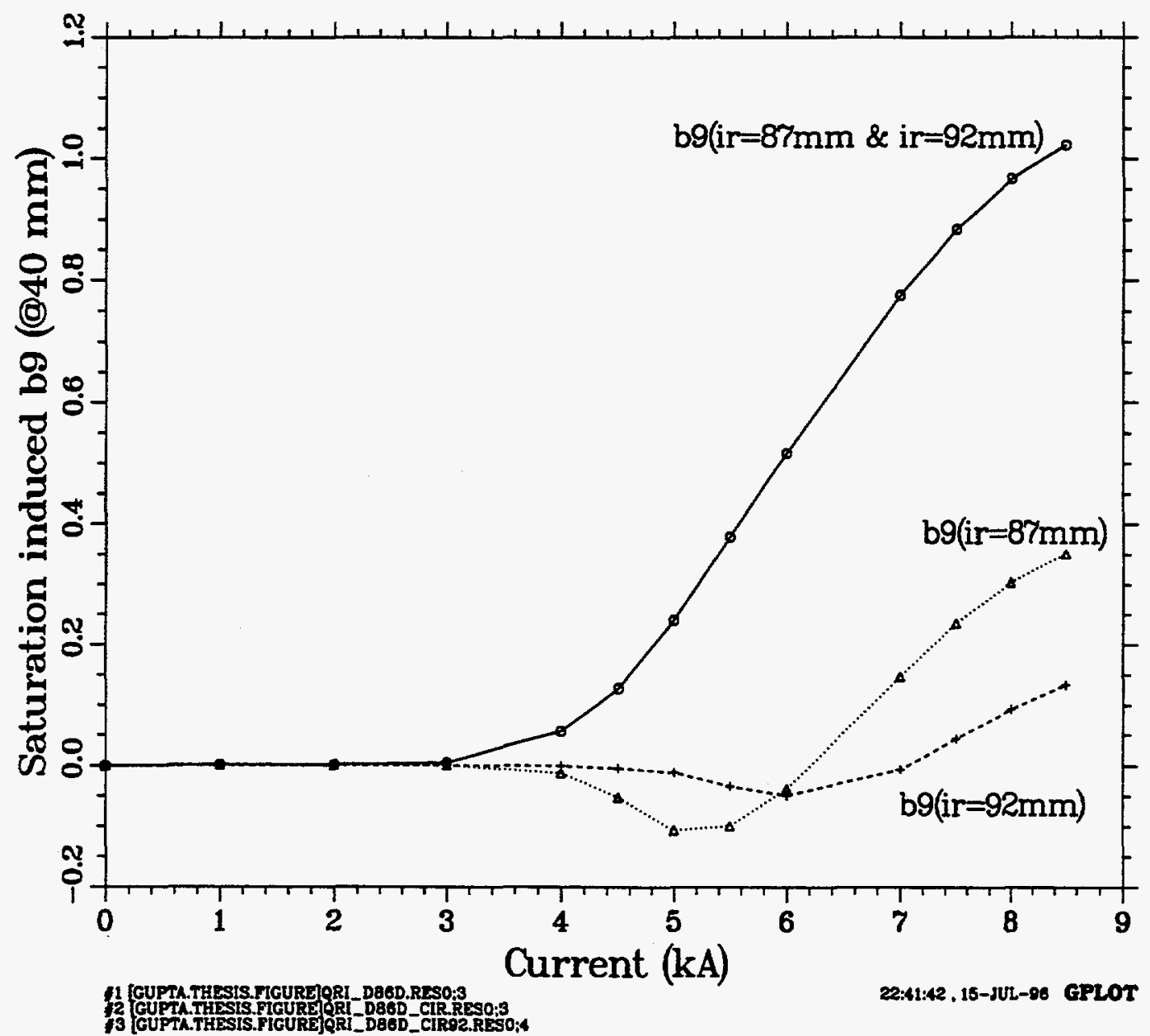

Figure 3.2.32: The variation in the $b_{9}$ harmonic as a function of current in the $130 \mathrm{~mm}$ aperture RHIC insertion quadrupole in the three cases of the yoke inner surface. The yoke inner surface is defined either by one single circle or by two circular arcs. The radii of these circles are indicated on the curves in various cases. 
Table 3.2.16: Current dependence in field harmonics when the yoke aperture has a radius $R=87 \mathrm{~mm}$.

\begin{tabular}{|c|c|c|c|c|c|}
\hline $\mathrm{I}(\mathrm{kA})$ & Gradient $(\mathrm{T} / \mathrm{m})$ & $\delta \mathrm{TF}(\%)$ & $\delta b_{5}$ & $\delta b_{9}$ & $\delta b_{13}$ \\
\hline 0.0 & 0.00 & 0.00 & 0.0 & 0.0 & 0.0 \\
\hline 1.0 & 9.84301 & -0.01341 & 0.008 & 0.001 & 0.0 \\
\hline 2.0 & 19.6859 & -0.01402 & 0.007 & 0.001 & 0.0 \\
\hline 3.0 & 29.5274 & -0.01893 & 0.023 & 0.001 & 0.0 \\
\hline 4.0 & 39.3427 & -0.08791 & 0.530 & -0.012 & 0.001 \\
\hline 4.5 & 44.1844 & -0.25979 & 1.882 & -0.052 & 0.002 \\
\hline 5.0 & 48.8889 & -0.67602 & 4.840 & -0.106 & 0.003 \\
\hline 5.5 & 53.4417 & -1.29676 & 8.205 & -0.099 & 0.002 \\
\hline 6.0 & 57.8534 & -2.05292 & 11.238 & -0.038 & 0.004 \\
\hline 7.0 & 66.2596 & -3.84661 & 15.325 & 0.147 & 0.012 \\
\hline 7.5 & 70.2309 & -4.87803 & 16.298 & 0.236 & 0.018 \\
\hline 8.0 & 74.1027 & -5.90688 & 16.715 & 0.304 & 0.024 \\
\hline 8.5 & 77.9053 & -6.89738 & 16.724 & 0.351 & 0.029 \\
\hline
\end{tabular}

Table 3.2.17: Current dependence in field harmonics when the yoke aperture has a radius $R=92 \mathrm{~mm}$.

\begin{tabular}{|c|c|c|c|c|c|}
\hline $\mathrm{I}(\mathrm{kA})$ & Gradient(T/m) & $\delta \mathrm{TF}(\%)$ & $\delta b_{5}$ & $\delta b_{9}$ & $\delta b_{13}$ \\
\hline 0.0 & 0.00 & 0.00 & 0.0 & 0.0 & 0.0 \\
\hline 1.0 & 9.23875 & -0.01212 & 0.005 & 0.001 & 0.000 \\
\hline 2.0 & 18.47740 & -0.01266 & 0.004 & 0.001 & 0.000 \\
\hline 3.0 & 27.71560 & -0.01447 & 0.003 & 0.001 & 0.000 \\
\hline 4.0 & 36.94950 & -0.02700 & 0.060 & 0.000 & 0.000 \\
\hline 4.5 & 41.55540 & -0.05776 & 0.225 & -0.004 & 0.000 \\
\hline 5.0 & 46.13710 & -0.13474 & 0.623 & -0.011 & 0.001 \\
\hline 5.5 & 50.64210 & -0.34865 & 1.779 & -0.034 & 0.002 \\
\hline 6.0 & 55.03640 & -0.72660 & 3.518 & -0.049 & 0.003 \\
\hline 7.0 & 63.46360 & -1.87926 & 6.879 & -0.006 & 0.006 \\
\hline 7.5 & 67.45400 & -2.66241 & 8.099 & 0.044 & 0.009 \\
\hline 8.0 & 71.33440 & -3.49648 & 8.919 & 0.093 & 0.012 \\
\hline 8.5 & 75.14050 & -4.32702 & 9.363 & 0.133 & 0.016 \\
\hline
\end{tabular}


Table 3.2.18: Current dependence in field harmonics when the yoke aperture consists of arcs having two radii : $R=87 \mathrm{~mm}$ at the midplane and $R=92 \mathrm{~mm}$ at the pole. The radius changes at an angle of $30^{\circ}$ in the first quadrant.

\begin{tabular}{|c|c|c|c|c|c|}
\hline $\mathrm{I}(\mathrm{kA})$ & Gradient $(\mathrm{T} / \mathrm{m})$ & $\delta \mathrm{TF}(\%)$ & $\delta b_{5}$ & $\delta b_{9}$ & $\delta b_{13}$ \\
\hline 0.0000 & 0.00000 & 0.00000 & 0.000 & 0.000 & 0.000 \\
\hline 1.0000 & 9.57088 & -0.01264 & -0.010 & 0.002 & 0.000 \\
\hline 2.0000 & 19.14160 & -0.01348 & -0.013 & 0.002 & 0.000 \\
\hline 3.0000 & 28.71020 & -0.02114 & -0.010 & 0.005 & 0.000 \\
\hline 4.0000 & 38.25020 & -0.09967 & 0.075 & 0.056 & -0.003 \\
\hline 4.5000 & 42.98180 & -0.21499 & 0.160 & 0.127 & -0.006 \\
\hline 5.0000 & 47.66230 & -0.41402 & 0.214 & 0.240 & -0.009 \\
\hline 5.5000 & 52.26810 & -0.71875 & 0.183 & 0.379 & -0.011 \\
\hline 6.0000 & 56.75130 & -1.18615 & 0.388 & 0.517 & -0.010 \\
\hline 7.0000 & 65.25230 & -2.61525 & 0.834 & 0.775 & -0.004 \\
\hline 7.5000 & 69.25570 & -3.53106 & 0.521 & 0.884 & 0.001 \\
\hline 8.0000 & 73.13450 & -4.49513 & -0.203 & 0.967 & 0.006 \\
\hline 8.5000 & 76.92910 & -5.44925 & -1.255 & 1.022 & 0.010 \\
\hline
\end{tabular}




\subsection{Saturation Induced Allowed Harmonics in RHIC Arc Dipoles}

In this section the saturation induced allowed harmonics are reviewed in various full length RHIC arc dipoles. This includes the earlier designs for the R\&D phase of the program through the final production design [72] used by industry (the Northrop Grumman Corporation) in building the machine magnets. The cross section of the production design is shown in Fig. 3.3.1. The original yoke and magnetic design of this magnet was carried out by Thompson [156] and most of the research work described in this section has been performed in collaboration with him. With several iterations using the techniques described in the earlier sections, the saturation induced sextupole $\left(b_{2}\right)$ and decapole $\left(b_{4}\right)$ harmonics in the present design are reduced by over an order of magnitude as compared to that in the initial design. A comparison of the calculations and measurements in the various yoke designs will be made.

The measured current dependence in the harmonics comes not only from the non-linear properties of iron (saturation induced) but also from (a) the persistent currents in the superconductor (whose fractional contribution is more at low current) and (b) deformation of the superconducting coils due to Lorentz forces on them. In a typical measurement of the current dependence, the harmonics are measured during the up ramp (when the current is increased) and also during the down ramp (when it is decreased). The sign of the harmonics created by the persistent currents is opposite in the up and down ramps. Moreover, it has been found that in the RHIC operating range, the magnitude of the persistent current harmonics is about the same during up and down ramp. Therefore, the persistent current effects can be mostly removed from the data by taking the average of the measurements during the up and down ramp. However, there is no easy method of removing the harmonics produced by the coil deformation due to Lorentz forces and therefore when comparing calculations and measurements, this effect contributes to the differences between the two. However, in a RHIC dipole magnet built to specifications, this effect is rather small.

During the R\&D phase of the program, usually two magnets were built with the same yoke design. It has been found (both in RHIC and SSC magnets) that the variation in the magnet-to-magnet current dependence of the saturation induced harmonics, in magnets built with the same yoke design, is rather small. Therefore, for clarity, the measurements from only one magnet per design will be included in the tables and plots which show the current dependence of the field harmonics. In Fig. 3.3.2, Fig. 3.3.3 and Fig. 3.3.4 the measured current dependence in the sextupole $\left(b_{2}\right)$, decapole $\left(b_{4}\right)$ and 14-pole $\left(b_{6}\right)$ 


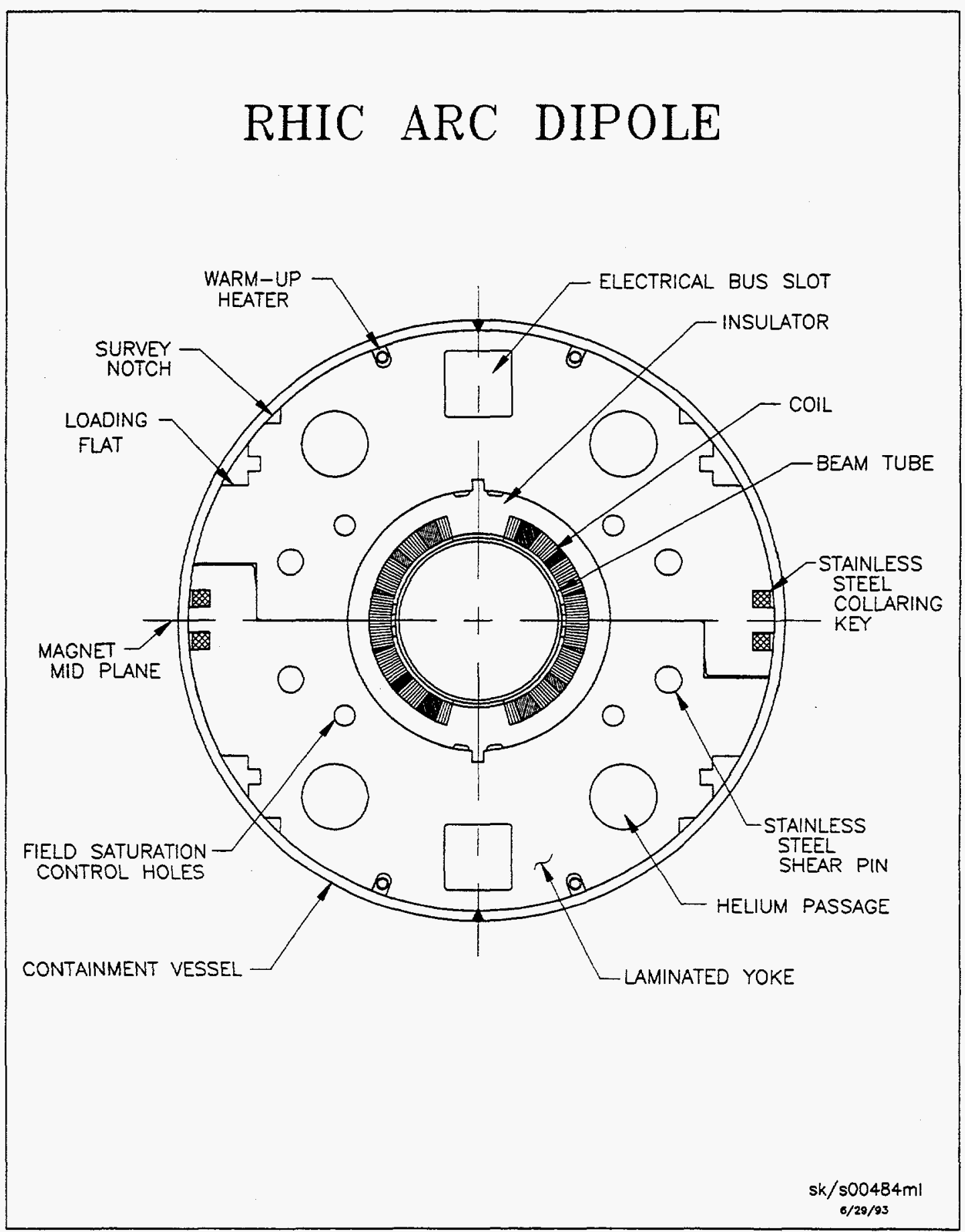

Figure 3.3.1: The cross section of the production series of the cold mass of the $80 \mathrm{~mm}$ aperture RHIC arc dipoles. This cold mass is put inside a cryostat (not shown here). 
harmonics, respectively, is shown. The magnet nomenclature used here (for example in DRE011) is such that " $D$ " is for dipole, " $R$ " for $R H I C$, " $E$ " for design series and "011" for the magnet number. The first digit was 0 in $R \& D$ magnets and is non-zero in production magnets. As mentioned earlier, the measured current dependence is the average of the up and down ramps. Moreover, in order to make an easy comparison in the relative current dependence, a proper offset is added so that each curve starts from zero at $2 \mathrm{kA}$.

One can see from Fig. 3.3.2 and Fig. 3.3.3, that the current dependence over the design range (up to $\sim 5 \mathrm{KA}$ ) in the sextupole harmonic $\left(b_{2}\right)$ and decapole $\left(b_{4}\right)$ harmonic is reduced by over an order of magnitude between the first $D R A$ design and the present $D R G$ design. Two production series magnets (DRG) have been tested up to $7 \mathrm{kA}$ and they show that the current dependence remains small up to that current. In the first long ( 9.5 meter) magnet, DRA001, the maximum variation in the $b_{2}$ harmonic between $2 \mathrm{kA}$ and $5 \mathrm{kA}$ was over 42 units and in DRG101 magnet this variation is reduced to about 2 units. Similarly, one can see from Fig. 3.3.3 that the $b_{4}$ variation has also been reduced by over an order of magnitude. In DRA001, the maximum variation in the $b_{4}$ harmonic between the $2 \mathrm{kA}$ and $5 \mathrm{kA}$ current was over 8 units and in DRG101 this variation is reduced to 0.6 unit. However, as shown in Fig. 3.3.4, the variation in the $b_{6}$ harmonic in DRG101 has become more than in some intermediate prototype magnets though its magnitude is still about the same as it was in DRA101. This harmonic was not minimized as it does not adversely affect RHIC performance. In the recent yoke design of the $10 \mathrm{~cm}$ aperture insertion dipole D0, this harmonic was included in the optimization and it was also made small using similar techniques. Very little current dependence ( $<0.3$ unit) has been observed in $b_{8}$ and higher order harmonics in all magnets.

The series of yoke iterations done to achieve the above mentioned improvements will now be described. The discussion will be restricted to only those features in yoke design which influence the saturation characteristics of the cross section.

DRA001, DRA002 and DRA003 dipoles were constructed with a small (5mm) radial gap between coil outer diameter and yoke together with pole notches in the yoke aperture. Both of these features give a large iron saturation and over 42 units of $b_{2}$ saturation and -8 units of $b_{4}$ saturation were observed. These are the maximum values of saturation induced harmonics between $2 \mathrm{kA}$ and $5 \mathrm{kA}$. Magnet DRA004 was built with the same basic features except for some changes near the outer surface of the iron which reduced $b_{2}$ saturation from 42 units to 35 units and $b_{4}$ saturation from -8.5 units to -5.7 units. 
Saturation induced $b 2=(b 2 u p+b 2 d n) / 2$

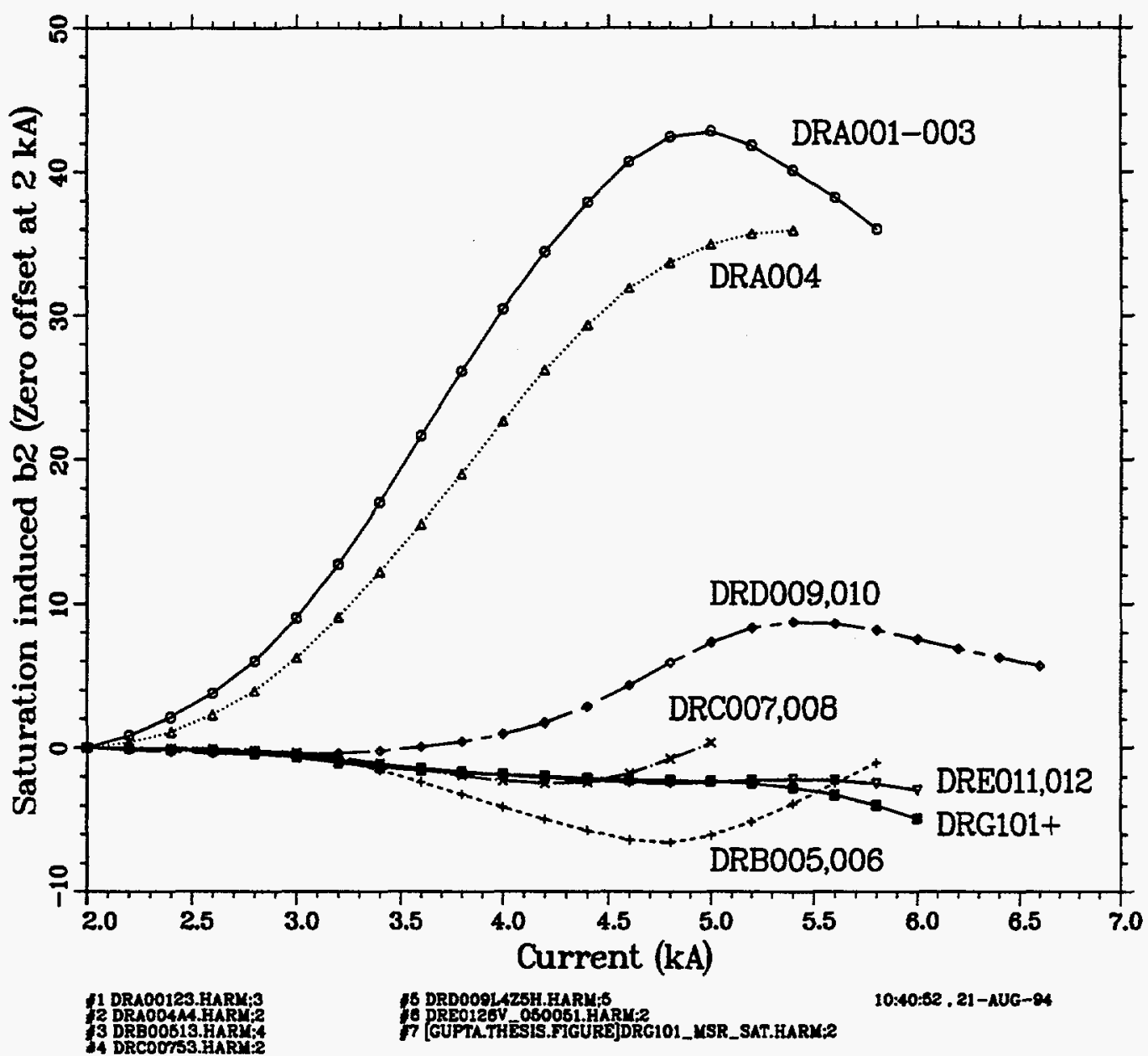

Figure 3.3.2: Measured saturation induced sextupole harmonic $\left(b_{2}\right)$ as a function of current. The $b_{2}$ harmonic shown here is the average of $b_{2}$ measured during an up and down ramp. This removes the persistent current $b_{2}$ to first order in the operating range. Moreover, in order to make an easy relative comparison between various designs, the value of $b_{2}$ in each magnet is biased so that each curve starts from a zero value at $2 \mathrm{kA}$. 


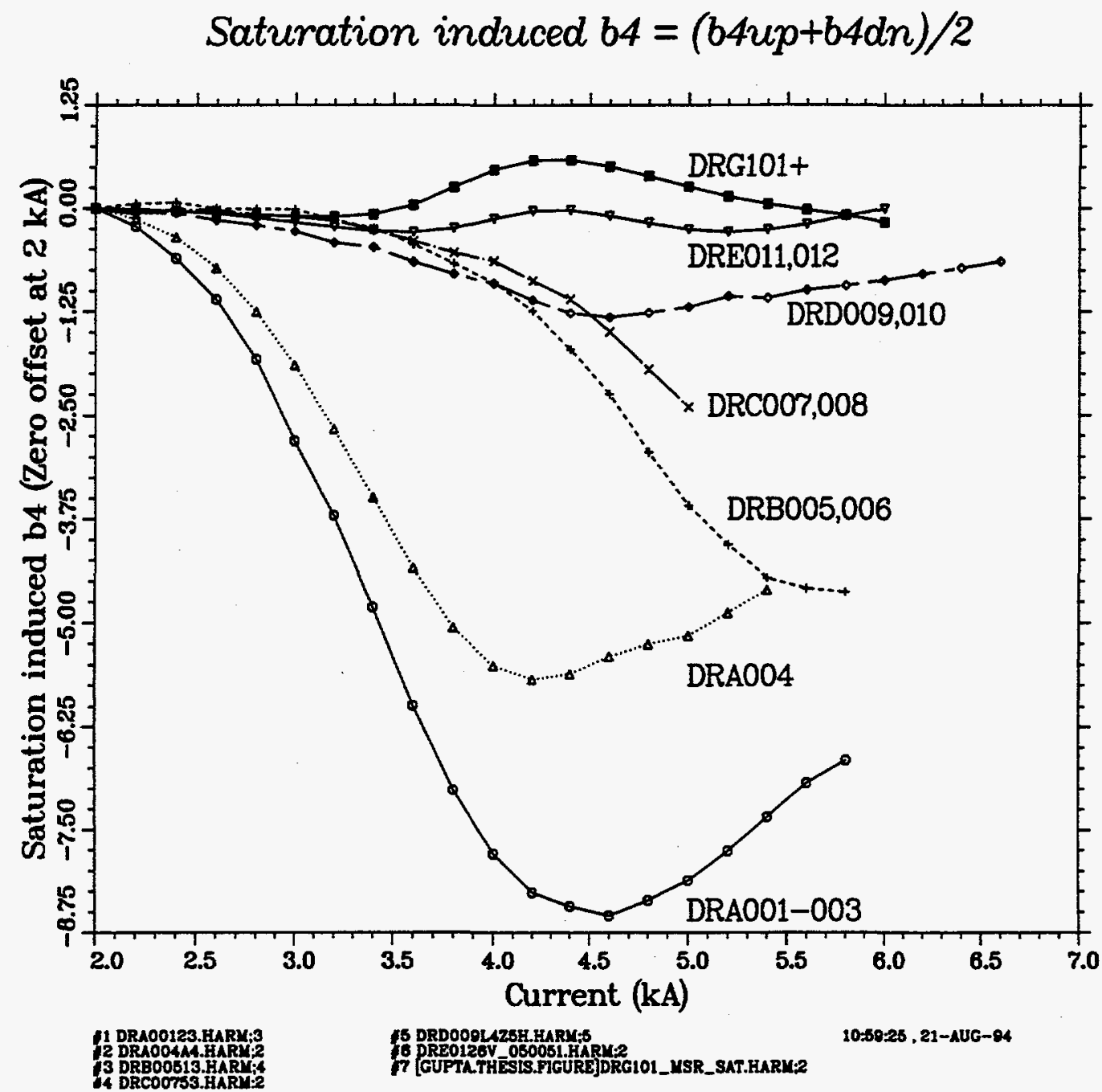

Figure 3.3.3: Measured saturation induced decapole harmonic $\left(b_{4}\right)$ as a function of current. The $b_{4}$ harmonic shown here is the average of $b_{4}$ measured during an up and down ramp. This removes the persistent current $b_{4}$ to first order in the operating range. Moreover, in order to make an easy relative comparison between various designs, the value of $b_{4}$ in each magnet is biased so that each curve starts from a zero value at $2 \mathrm{kA}$. 


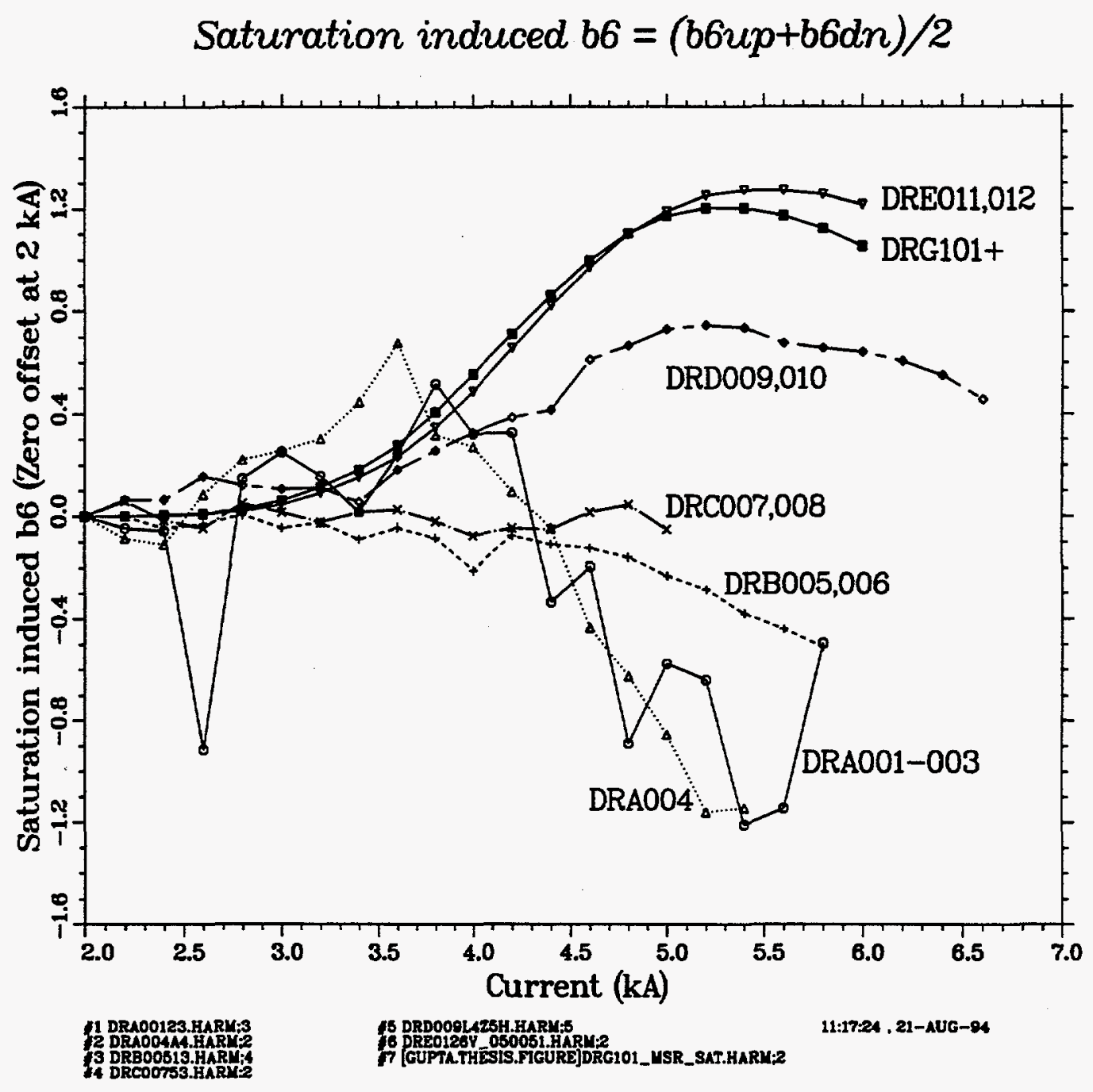

Figure 3.3.4: Measured saturation induced $b_{6}$ harmonic as a function of current. The $b_{6}$ harmonic shown here is the average of $b_{6}$ measured during an up and down ramp. This removes the persistent current $b_{6}$ to first order in the operating range. Moreover, in order to make an easy relative comparison between various designs, the value of $b_{6}$ in each magnet is biased so that each curve starts from a zero value at $2 \mathrm{kA}$. 
To make a significant reduction in the saturation, the radial gap between the coil and yoke was increased from $5 \mathrm{~mm}$ to $10 \mathrm{~mm}$ and the coil-yoke locating notch was moved from the pole to the midplane in magnets DRB005 and DRB006. This brought $b_{2}$ saturation down from +35 units to -6 units and $b_{4}$ saturation from -5.7 units to -3.6 units. These $D R B$ magnets were built with non-magnetic stainless steel yoke midplane keys. In magnets DRC007 and DRC008, the material of this key was changed from non-magnetic stainless steel to low carbon magnetic steel. This increased the effective magnetic thickness of the yoke at the midplane and therefore modified the saturation induced harmonics. This change reduced $b_{2}$ saturation from -8.5 units to -2.5 units and $b_{4}$ saturation from -3.6 units to -2.4 units.

Though the midplane notch (along with magnetic keys) gave the best saturation performance thus far, these features were not desired in the production magnets. It has been believed that a pole locating notch would be better able to define the pole location of the coil and hence would give less variation in the harmonics produced by coil geometry. These harmonics are commonly referred to as the geometric multipoles. Moreover, the goal at that time was to reduce $b_{4}$ saturation to less than 2 units. As mentioned earlier, a pole notch is bad magnetically. That made the above observed $b_{4}$ saturation of -2.4 units even worse. Therefore, a detailed study was undertaken to modify the cross section to significantly change the magnetic properties of the yoke, while retaining the desired mechanical properties. After examining a large number of options in the yoke design, it was found that simply a relocation of the helium bypass hole was very effective in controlling saturation induced harmonics. This, despite the presence of the pole notch, significantly reduced the saturation in $b_{4}$ harmonic. Magnets DRD009 and DRD010 were built on this cross section. They were built with stainless steel keys and as in all previous magnets, the shear pins (two $9.52 \mathrm{~mm}$ diameter rivets which are used to hold each pair of yoke laminations together) were made with low carbon magnetic steel. The crucial $b_{4}$ saturation in this "DRD" series magnets now became -1.3 units (an improvement from the previous value of -2.4 units in "DRC" series magnets). This improvement came however at the cost of $b_{2}$ saturation which now increased to +7.3 units (the value of $b_{2}$ saturation in the "DRC" series was 0.4 unit. with a maximum change of $\mathbf{- 2 . 4}$ units at an intermediate current).

Finally, saturation suppressor holes were added in magnets DRE011 and DRE012. The size and location of the holes were optimized to reduce $b_{2}$ and $b_{4}$ saturation to values that can be tolerated in the machine (i.e. $b_{2}$ saturation about 2 units and $b_{4}$ saturation about $\frac{1}{2}$ 
unit). The optimized azimuthal location of a $9.52 \mathrm{~mm}$ diameter hole in the first quadrant was $33^{\circ}$ when located at a radial distance of $75 \mathrm{~mm}$ from the magnet center. In addition to this change the material of the shear pins was changed from magnetic low carbon steel to non-magnetic stainless steel for mechanical reasons. To deal with this change, the material of the yoke keys was changed to magnetic low carbon steel. This inter-change of material produced little change in the saturation induced harmonics. The goal of minimizing $b_{2}$ and $b_{4}$ due to saturation at this level requires a careful analysis and inclusion of those small effects that were previously ignored (because of their relatively minor contributions) was necessary. The calculations and measurements and their differences, are given in Table 3.3.1.

Table 3.3.1: The saturation induced values of $b_{2}$ and $b_{4}$ harmonics at $5 \mathrm{kA}$ in $\mathrm{RHIC}$ long dipoles. The model used in these calculations did not include the cryostat, which induces a change in $b_{2}$ of $\sim+1.5$ units and $b_{4}$ of - 0.1 unit at $5 \mathrm{kA}$. Since very little variation has been observed in the saturation induced harmonics in multiple magnets built with the same yoke cross section, only one from each series is listed here.

\begin{tabular}{|c|c|c|c|c|c|c|}
\hline Magnet & $b_{2}$ & $b_{2}$ & Difference & $b_{4}$ & $b_{4}$ & Difference \\
Name & Measured & Computed & Meas-Comp & Measured & Computed & Meas-Comp \\
\hline DRA001 & 42.8 & 36.0 & +6.8 & -8.1 & -7.0 & -1.1 \\
\hline DRA004 & 34.5 & 35.5 & -1.1 & -5.2 & -5.8 & +0.6 \\
\hline DRB005 & -6.1 & -8.8 & +2.7 & -3.6 & -4.0 & +0.4 \\
\hline DRC007 & 0.4 & 0.8 & -0.4 & -2.4 & -2.4 & 0.0 \\
\hline DRD009 & 7.3 & 9.4 & -2.1 & -1.2 & -0.4 & -0.8 \\
\hline DRE012 & -2.4 & 0.1 & -2.5 & -0.3 & 0.7 & -1.0 \\
\hline DRG101 & -2.3 & -2.6 & +0.3 & 0.3 & 0.8 & -0.5 \\
\hline
\end{tabular}

Some possible sources of the differences between the calculations and the measurements are :

(a) The calculations did not include the effect of the cryostat around the yoke. The inclusion of the cryostat in a computer model means that a larger geometry is included in the model which requires lowering the mesh density in the yoke region. This is in conflict with the requirement that, to 
obtain a high accuracy in the change in harmonics due to yoke saturation, the model in the yoke region should have sufficient mesh density. However, based on a typical calculation, the change in the saturation induced $b_{2}$ and $b_{4}$ due to the cryostat at $5 \mathrm{kA}$ is respectively $\sim+1.5$ units and $\sim-0.1$ unit.

(b) The calculations ignore a small wedge-shaped gap at the mating plane of the top and bottom yoke halves. The average width of this gap is about $0.0254 \mathrm{~mm}$ with a maximum value at the yoke outer radius. This small gap is difficult to include in a reliable finite element computer model. This gap, when included in the model, results in a mesh in which two angles of the triangles defining the mesh in the gap are very small and the third is very large. Such triangles give large errors in this region and therefore the results of the computations are not very reliable. However, some model calculations suggest that this wedge-shaped gap may give as much as 2 units of $b_{2}$ and 0.2 unit of $b_{4}$ at $5 \mathrm{kA}$.

(c) As mentioned earlier, calculations are done here only for the saturation induced harmonics. Harmonics due to coil deformation as a result of the Lorentz force on the coil are not included. The deformation is radially outward at the horizontal midplane (which could be significant above a medium current of $\sim 3 \mathrm{kA}$ ) and azimuthally from pole to midplane (which could be significant above a high current of $\sim 4.5 \mathrm{kA}$, after the compression from the Lorentz forces has overcome the initial pole pre-compression). In order to compute these effects, the coil deformation must be known to $0.0254 \mathrm{~mm}$ level. Mechanical modelling based on finite element codes is perhaps not reliable to this accuracy. However, the estimated current dependence caused by these Lorentz forces is about 2 units in $b_{2}$ and 0.2 unit in $b_{4}$ in the design operating current range of RHIC arc dipoles. This deformation is not separable in the measured harmonics from the change in harmonics due to iron saturation, unless the coil deformation is very large. This was found to be the cause of a much larger than expected current dependence in $b_{2}$ harmonic in some SSC magnets [64]. The source was traced to a radial gap of about $100 \mu m$ between the stainless steel collar and yoke which allowed an extra deflection of the collared coil at the yoke midplane. 
(d) The differences shown in Table 3.3.1 are the differences between the measured and computed values at $5 \mathrm{kA}$. The ideal goal would be to minimize the peak rather than the value at $5 \mathrm{kA}$. In general the calculations and measurements do not peak at the same current and sometimes the two peaks are separated by a few hundred amperes of current. The difference between the two in the operating range may be as much as 2 units for $b_{2}$ and about 0.3 unit for $b_{4}$. This may give a small error when the differences between the calculations and measurements are being empirically removed.

Therefore, when the goal is to optimize $b_{2}$ saturation to within a few units and $b_{4}$ saturation to within a few tenths of a unit, the uncertainties in the current dependence in the harmonics due to computer modelling and magnet manufacturing are appreciable. In order to overcome these limitations, first the differences between the calculations and measurements in each harmonic, $\delta b_{n}=b_{n}$ (computed) $-b_{n}$ (measured), are obtained. These differences are then empirically removed while optimizing the location of the saturation suppressor holes by making the target to be $-\delta b_{n}$ instead of zero. It is important to note that in order for this scheme to work as desired, no other significant change should be simultaneously made in the magnet. This approach is validated by the small measured values of $b_{2}$ and $b_{4}$ due to saturation as shown in Fig. 3.3.2 and Fig. 3.3.3. in magnets DRE011 and DRE012.

In the final design of the production series magnet (the $D R G$ series), it was decided to change back the material of the collaring keys to non-magnetic stainless steel (see Fig. 3.3.1). This change was made to match the thermal contraction (during cool down) of the stainless steel shell with the thermal contraction of the collaring keys in the axial direction. Therefore, in this design both the keys and the shear pins are made of non-magnetic material. This made a significant impact on the saturation characteristic of the magnet. In order to maintain small values of $b_{2}$ and $b_{4}$ due to saturation, the saturation suppressor hole was moved from $33^{\circ}$ to $35.5^{\circ}$. Moreover the value of the small yoke gap (described in item (b) above) was also changed. This change was not included in the computer modelling. The final $D R G$ design for the production magnets gave a measured $b_{2}$ saturation of $\sim 2$ units and $b_{4}$ saturation of $\sim 0.6$ unit in the entire range of operation. 


\subsection{Reduction in the Saturation-induced Non-allowed Harmonics}

In superconducting accelerator magnets, the radial thickness of the yoke is seldom adequate to contain all the magnetic return flux exterior to the coil at the maximum design field. Therefore the presence of magnetic material outside the yoke may influence the field uniformity in the aperture of the magnet. These effects are seen only after a certain threshold field which is usually close to the maximum operating field. The harmonics created are the reflection of the relative geometry of the yoke and the outside magnetic material.

In the RHIC and SSC dipole magnets, these effects can be separated in to two major classes. The first is the presence of another magnet in a colliding beam accelerator. This is more important in the interaction region magnets, where the separation between the inner and outer ring is a minimum. The left-right symmetry in RHIC dipoles is broken, introducing a current dependence in the odd normal harmonics $\left(b_{1}, b_{3}, b_{5}\right.$, etc.) at high field.

The second class is the influence of the cylindrical vacuum tank containing the yoke which also serves as the cryostat wall. In the RHIC and SSC dipole designs the center of the cryostat does not coincide with the center of the yoke or cold mass. The cold mass refers to the part of the magnet mass that is at low temperature ( $4.5 \mathrm{kelvin}$ in RHIC) and it includes the assembly of superconducting coils, yoke, etc. The center of the cold mass is located above the horizontal midplane of the cryostat to allow a maximum space for the support post. This reduces the heat load on the cryo-system at the expense of breaking the top-bottom symmetry. An absence of top-bottom symmetry (but the presence of left-right symmetry) introduces the odd skew harmonics $\left(a_{1}, a_{3}, a_{5}\right.$, etc.). This effect is seen only at high field levels where the yoke is not able to contain the field lines. Moreover, in RHIC dipoles the left-right symmetry is also broken to some extent. This is because the cryostat is straight but the cold mass is curved to follow the beam, which is bent significantly by the dipole field. This generates a small but noticeable current dependence in the $b_{1}$ harmonic as a function of axial position since the yoke center does not always coincide with the cryostat.

In addition, a current dependence in the non-allowed harmonics may also be generated by a difference in the weight of the two yoke halves and by the influence of the currents in the bus (see Fig. 3.3.1) when the yoke at high field does not provide sufficient magnetic shielding. These and other sources will be discussed in this section. 


\subsection{1. $b_{1}$ saturation - Cross talk}

In colliding beam accelerators, the magnets guiding the counter-rotating beams in the two rings are near each other. The magnets are usually closest to each other near the interaction regions where the two beam are brought together to collide. At bigh field, when the permeability of the iron yoke is too low to provide adequate magnetic shielding, the field in the aperture of one gets influenced by the field of the other. This condition is commonly referred to as "cross talk". If the two magnets lie on the same horizontal plane it generates odd normal harmonics like $b_{1}$ and if they lie on the same vertical plane it generates odd skew harmonics like $a_{1}$. In RHIC, the magnets lie on the same horizontal plane and thus the cross talk generates $b_{1}, b_{3}, b_{5}, \ldots$ The $b_{1}$ generated would be much larger in "2-in-1" superconducting magnets where the coils of the two magnets are contained in a common yoke. In these "2-in-1" designs the separation between the two apertures and hence the thickness of the yoke providing the magnetic shielding between the two is generally small. In this case the field of one dipole could affect the field in the aperture of the other dipole at high field. This is also referred to as cross talk and it may generate a significant amount of $b_{1}$ and other odd normal harmonics at high fields.

The amount of cross talk induced $b_{1}$ depends on $(a)$ the center-to-center distance between the two magnets, (b) the field in the aperture of the magnet, (c) the yoke thickness and (d) the coil aperture. The cross talk problem is examined here in the RHIC $100 \mathrm{~mm}$ aperture insertion dipole DO. A cross section of the cold mass of $D O$ is shown in Fig. 3.4.1. Two such magnets are placed in a common cryostat. The center-to-center distance between the two cold masses is determined by the machine layout. The distance between the $t$ wo varies axially and it is smallest (and hence the magnetic coupling between the two is largest) on the end that is towards the beam crossing. The direction of the field in the two DO dipoles is the same since the two counter-rotating beams have the same sign of charge.

In Fig. 3.4.2, $b_{1}$ is plotted as a function of field for $100 \mathrm{~mm}, 105 \mathrm{~mm}$ and $110 \mathrm{~mm}$ coil apertures when the magnitude of the field in the two dipoles is the same. In Fig. 3.4.3, $b_{1}$ is plotted as a function of field when the ratio of the field in the two dipoles is $2.5: 1$, which is the maximum difference in this ratio. For a constant separation between the centers of two magnets, a smaller aperture allows a thicker yoke shielding between the two and hence reduces the cross talk induced harmonics at high field. The final choice of $100 \mathrm{~mm}$ aperture was made to keep cross-talk induced harmonics to a reasonable value at the design field of 3.5 Tesla. 


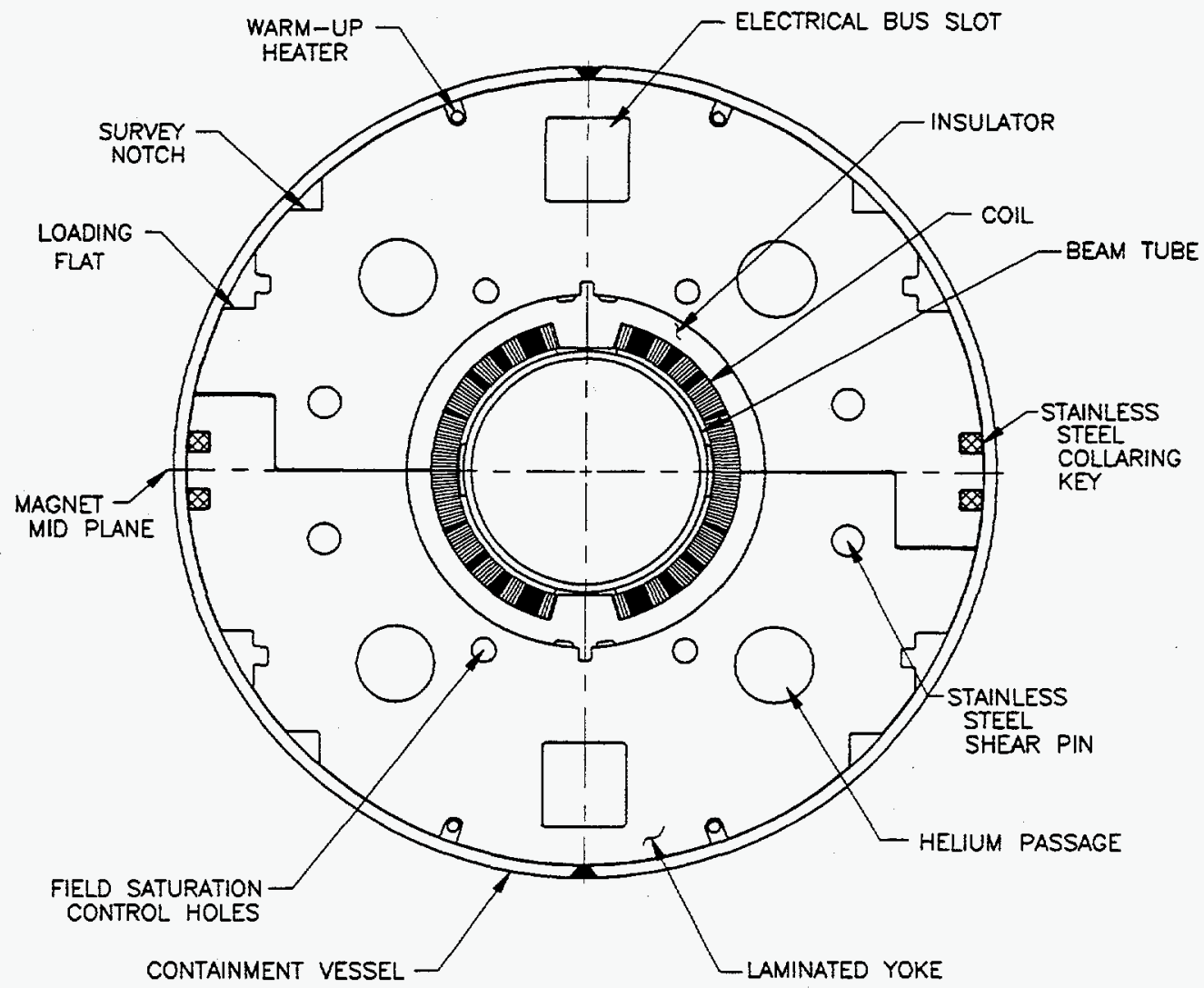

Figure 3.4.1: A cross section of the $100 \mathrm{~mm}$ aperture $\mathrm{RHIC}$ insertion dipole $D O$ cold mass. Two such cold masses will be inside a common cryostat (not shown here). 
EQUAL CURRENT in $100 \mathrm{~mm}, 105 \mathrm{~mm}$ and $110 \mathrm{~mm}$ aperture $D 0$ magnets

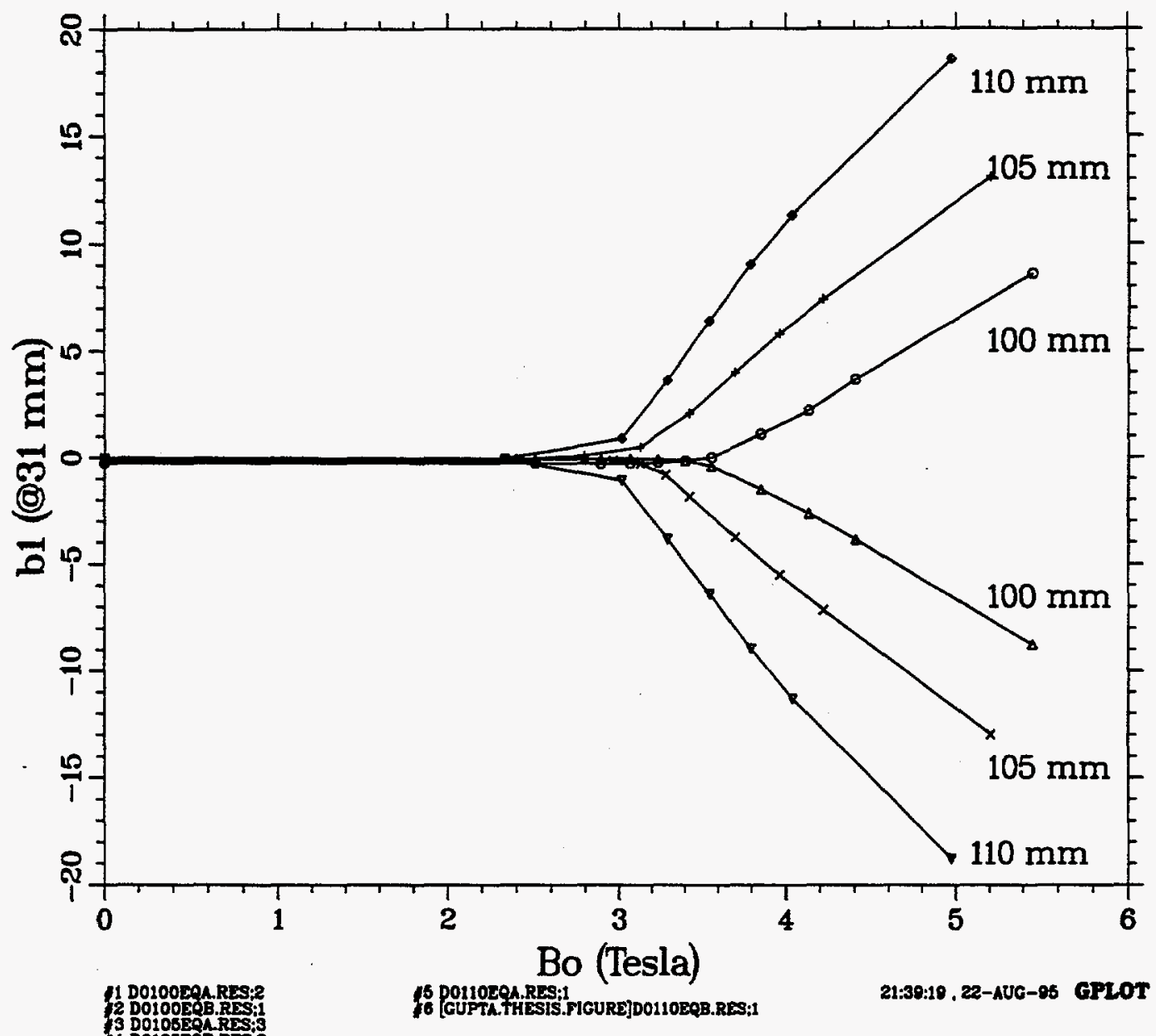

Figure 3.4.2: $b_{1}$ as a function of field in the $100 \mathrm{~mm}, 105 \mathrm{~mm}$ and 110 $\mathrm{mm}$ aperture designs of the $D O$ magnets. This is the cross talk induced harmonic in the two apertures when both the magnitude and direction of the field in the two dipoles is the same. The sign of $b_{1}$ is positive in the magnet on the right hand side and negative on the left hand side. 
$2.5: 1$ current ratio in $100 \mathrm{~mm}, 105 \mathrm{~mm} \& 110 \mathrm{~mm}$ Do magnets

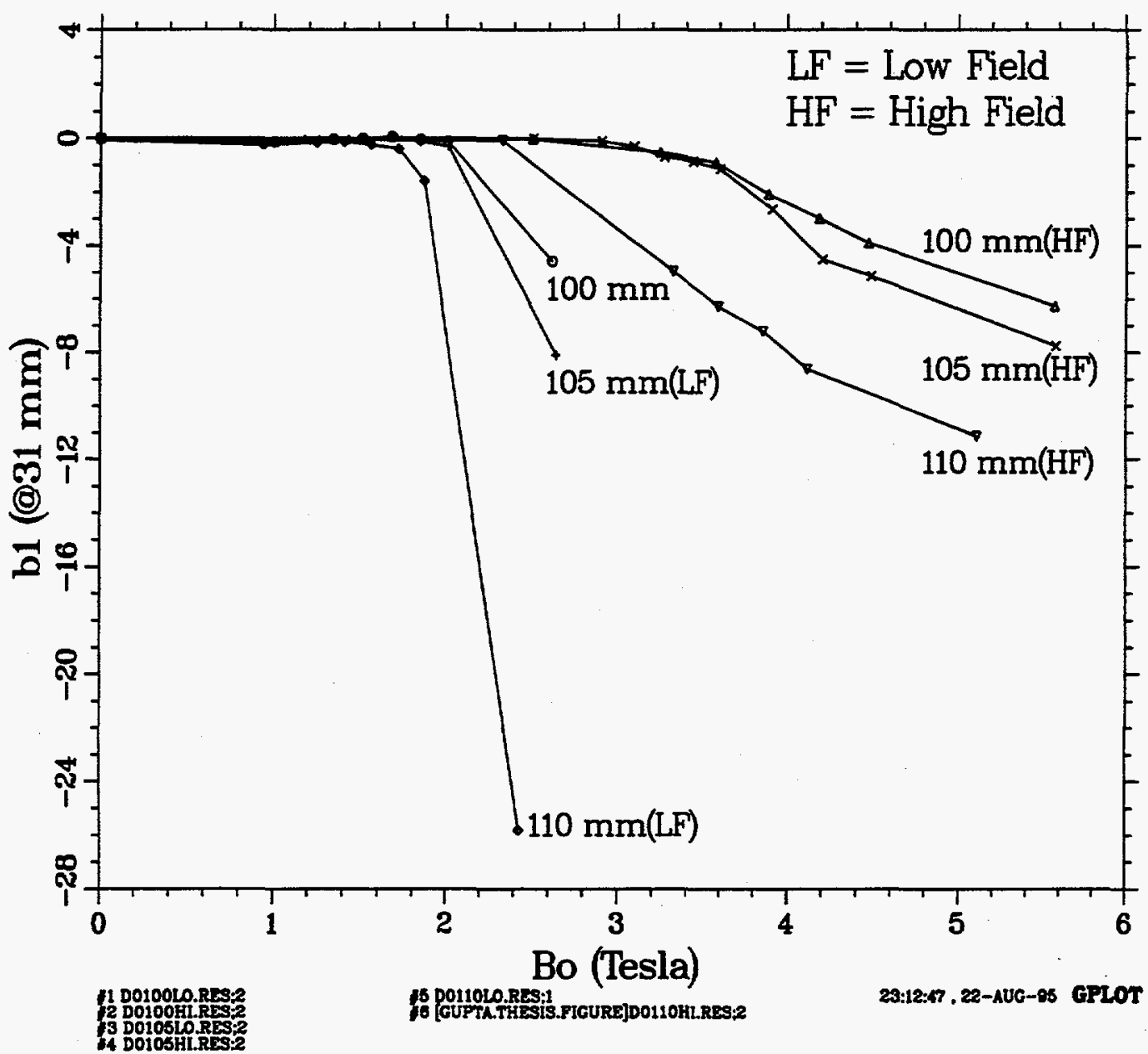

Figure 3.4.3: $b_{1}$ as a function of field in the $100 \mathrm{~mm}, 105 \mathrm{~mm}$ and 110 $\mathrm{mm}$ aperture designs of the $D O$ magnets. This is the cross talk induced harmonic in the two magnets when the direction of the field in the two is the same and the current is in the ratio of 2.5:1. The maximum high field in operation is 3.5 tesla. 
In Fig. 3.4.4 a POISSON model and field lines are shown for a $40 \mathrm{~mm}$ aperture SSC 2-in-1 magnet. The field lines are shown when the field in both aperture is equal to the design field of 6.6 tesla. A quadrupole harmonic $\left(b_{1}\right)$ is created at this field since the field in the iron and hence iron saturation is not left-right symmetric about the vertical axis of either aperture. A left-right asymmetry in the field lines across the vertical axis of the two apertures ( $y$-axis) can be seen in Fig. 3.4.4. 


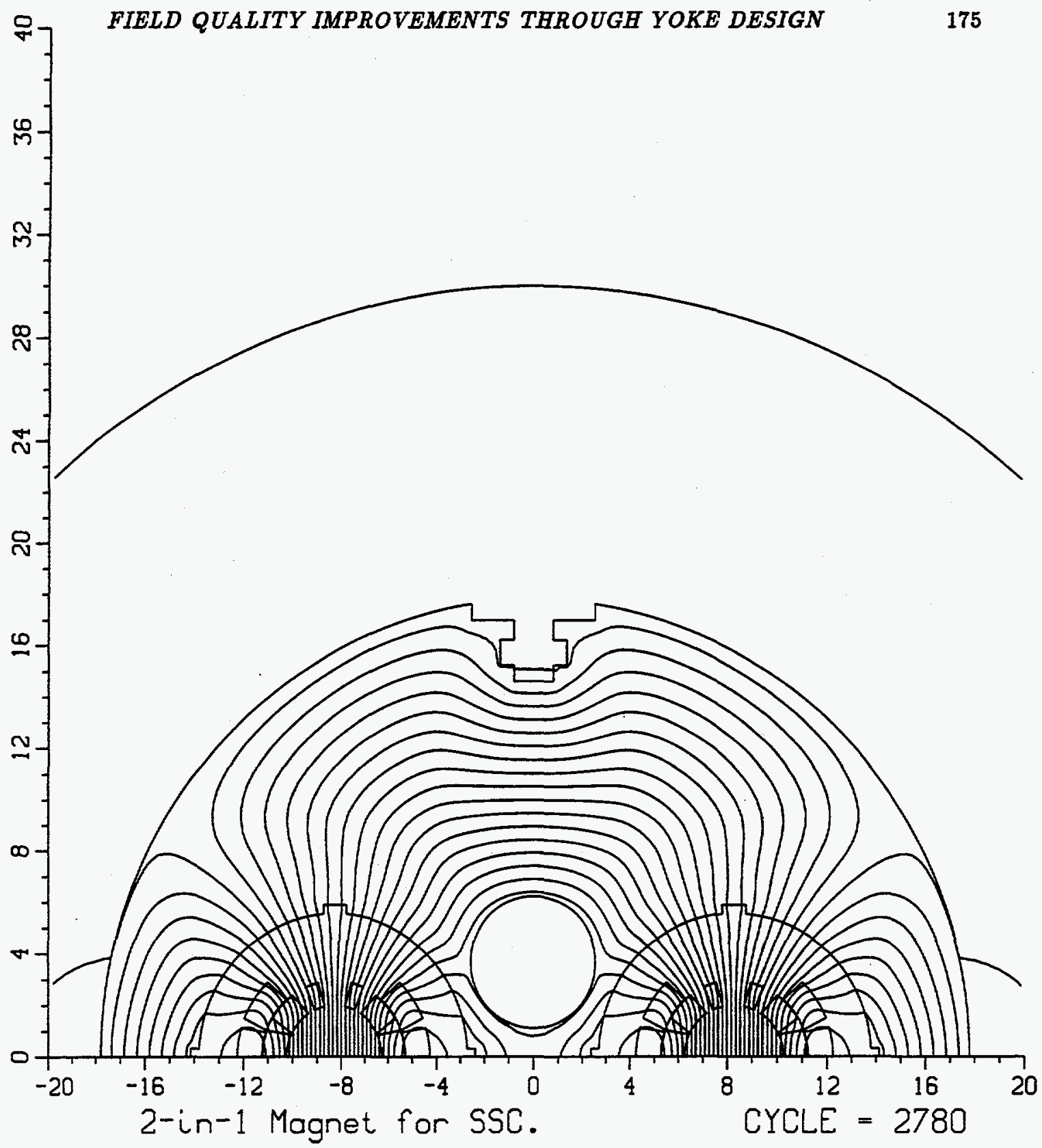

Figure 3.4.4: POISSON model and field lines in the upper half of the $40 \mathrm{~mm}$ aperture SSC 2-in-1 magnet. The field lines are shown when the field in both apertures is equal to the design field of 6.6 tesla. 


\subsection{2. $a_{1}$ saturation - Cryostat and other sources}

A large current dependence in the skew quadrupole harmonic $\left(a_{1}\right)$ has been observed in both SSC and RHIC dipole magnets. The variation of $a_{1}$ with current at high field is commonly referred to as " $a_{1}$ saturation" even though a number of sources other than the offcentered cryostat (discussed earlier) are responsible for it. The effects and their magnitude in SSC magnets will be discussed in detail. The change in $a_{1}$ at the design current (6600 A for SSC magnets and 5000 A for RHIC magnets) with respect to its geometric value at low current (2000 A for SSC magnets and 1450 A for RHIC magnets) will be evaluated by the following quantity :

$$
\begin{gathered}
S S C \rightarrow \quad \delta a_{1}=\left[a_{1}(@ 6600 A)-a_{1}(@ 2000 A)\right], \\
R H I C \rightarrow \quad \delta a_{1}=\left[a_{1}(@ 5000 A)-a_{1}(@ 1450 A)\right] .
\end{gathered}
$$

The low current value for the geometric harmonic is chosen so that it is below where iron saturation sets in and yet high enough that $a_{1}$ induced by the persistent currents has been significantly reduced.

The current dependence measured [70] with a 1 meter-long measuring coil in several 15 meter long BNL-built SSC magnets is shown in Fig. 3.4.5. To facilitate comparison, the curves are offset along the $\mathrm{y}$-axis such that the value of $a_{1}$ is zero at $2 \mathrm{kA}$ for all the magnets.

In the rest of this section the sources responsible for the variation in the current dependence of $a_{1}$ are discussed. For each of these sources, an estimate of $\delta a_{1}$ in SSC magnets is also given.

(a) Off-centered cryostat : At high current when the flux lines are not contained in the iron cross section, they leak outside the yoke. The magnetic iron in the cryostat (vacuum vessel) provides an additional magnetic path to return the flux lines. However, since the center of the cryostat is below the center of the yoke, an up-down asymmetry is introduced. Calculations for the SSC magnets show a noticeable $a_{1}$ current dependence beyond 6.0 tesla and the computed $\delta a_{1}$ is $\sim-0.2$ unit. A computer model for the code POISSON with the field lines at the maximum design current for this problem is shown in Fig. 3.4.6. Calculations and measurements 


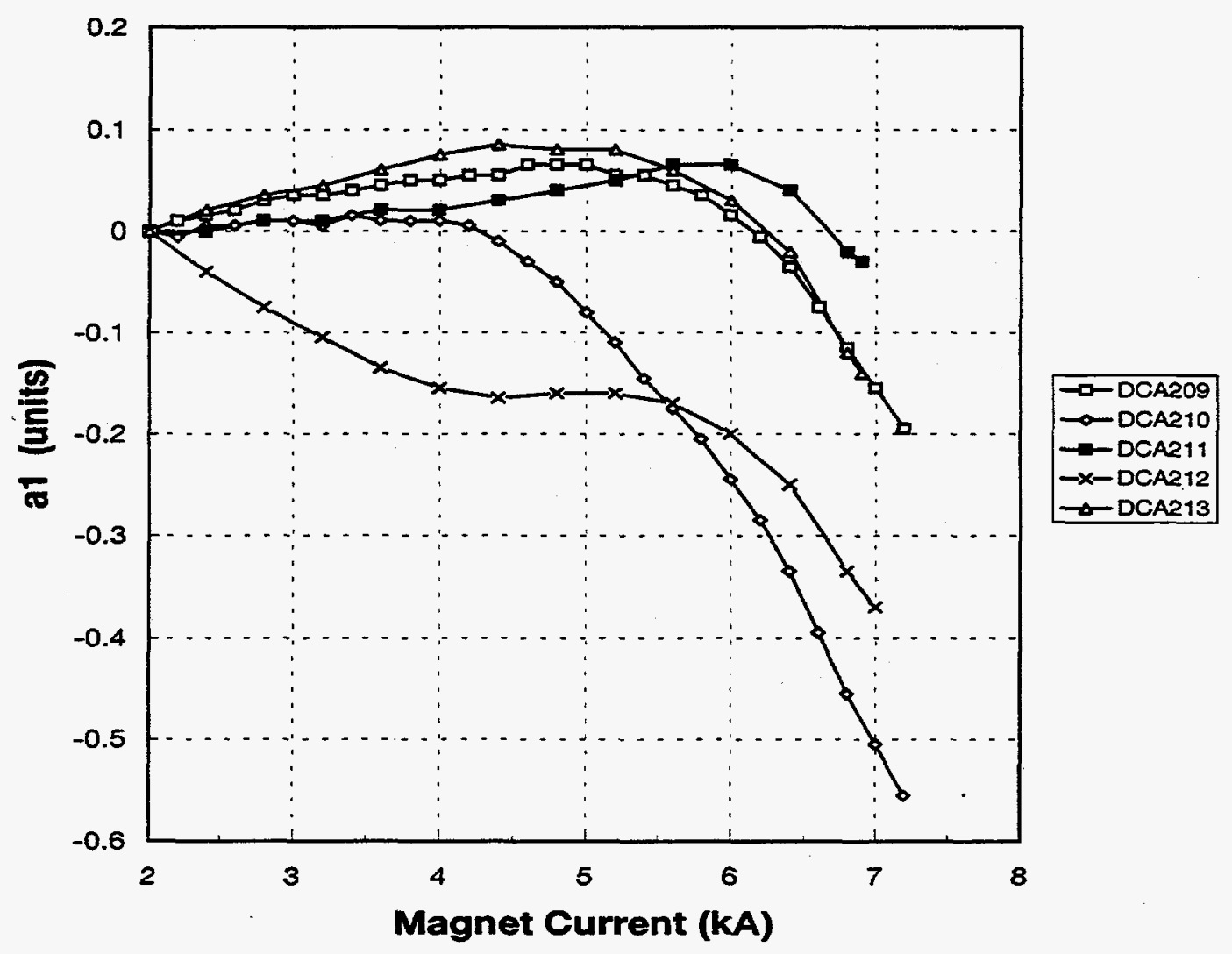

Figure 3.4.5: Current dependence of the skew quadrupole $\left(a_{1}\right)$ harmonic in the SSC $50 \mathrm{~mm}$ aperture prototype dipoles DCA209 to DCA213. The curves are offset such that $a_{1}$ at $2 \mathrm{kA}$ is zero for all magnets. 


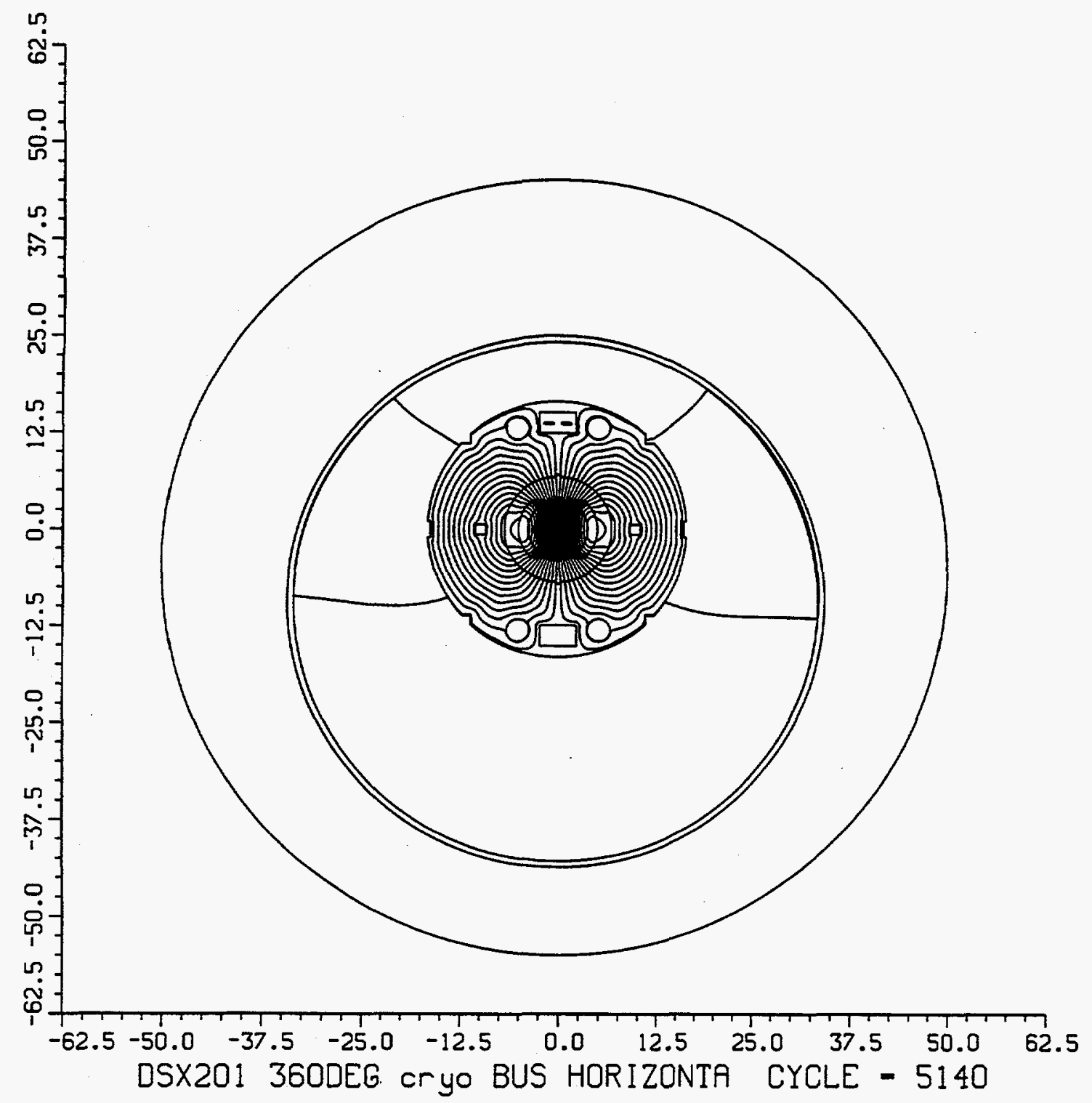

Figure 3.4.6: A computer model of the SSC $50 \mathrm{~mm}$ aperture dipole coldmass in the cryostat. The field lines are shown at the maximum design current of $6600 \mathrm{~A}$. The center of the coldmass is located above the center of the cryostat and at high field when the yoke can not contain the field lines (one line per quadrant in the above diagram), this asymmetry creates a current dependence in the value of the skew quadrupole harmonic. The upper bus slot (between two helium holes near the perimeter of yoke) contains lead and return current cables. A proper placement of them can partly compensate the above asymmetry. 
for the RHIC arc dipole magnets also show an $a_{1}$ saturation of $\sim-2.0$ units at the design field of 3.45 tesla. This will be discussed in more detail later.

(b) Difference in the packing factor between the upper and lower yoke halves : The packing factor is defined here as the ratio between the volume occupied by the iron to the theoretical maximum in the given cross section (i.e. the holes, etc. are not included in the volume calculations). The relative variation in it can be evaluated by comparing the yoke weight per unit length in various lamination packs. Though the overall difference in the packing factor between the top and bottom yoke halves is well controlled, there may be some local variations along the length of the magnet as these have not been controlled. In the BNL-built SSC magnets, the iron weight of each $76.2 \mathrm{~mm}$ long block (a riveted pack containing 12 laminations) used in the top and bottom yoke halves has been measured. Since the length of the measuring coil is one meter, a top bottom weight difference in the yoke in a 1 meter region (the region of field measurements) may be seen in the field harmonics. The 2-d calculations for SSC magnets show that a $0.1 \%$ higher packing factor in the upper yoke half would give $\sim-0.1$ unit of $\delta a_{1}$. This is the net change at $6600 \mathrm{~A}$ but the effect begins to appear at $3000 \mathrm{~A}$. In RHIC magnets the calculations show that a $\mathbf{0 . 1 \%}$ higher packing factor in the upper yoke half would give $\sim \mathbf{- 0 . 3}$ unit of $a_{1}$ at the design field. The 2-d calculations, however, may be overestimating this effect because in reality the field lines would not only move from bottom to top (as reflected in the calculations) but they may also move in the axial direction (if the iron density in the neighbouring pack is different) giving a lesser $\delta a_{1}$. This will be discussed in more detail later in this section.

(c) Off-centered coil in the yoke : If the coil center is above or below the yoke center, an additional $\delta a_{1}$ will be seen. This will also give a geometric $a_{1}$. Calculations for the SSC magnets show that for a $25 \mu \mathrm{m}$ off-centered coil above the midplane there would be an additional $\delta a_{1} \sim+0.1$ unit and the geometric $a_{1}$ would be $\sim-0.12$ unit.

(d) Difference in the top-bottom coil sizes : A difference between the top and bottom coil sizes gives a geometric $a_{1}$. Calculations for the SSC 
magnets show that if the upper coil half is $25 \mu \mathrm{m}$ larger in size (which means that the midplane is shifted down by half of this amount), the geometric $a_{1}$ would be $\sim+0.7$ unit. It also gives a small additional contribution to the saturation induced $\delta a_{1}$, which is about $1 \%$ of the geometric $a_{1}$.

There is a second effect of coil size differences. An asymmetric coil package (midplane shifted downward or upward) produces an asymmetric Lorentz force (which is basically $I^{2}$ dependent). As a result, the asymmetry would be enhanced at high field by Lorentz forces. Mechanical calculations to compute the amount of displacement have not been done but it may be pointed out that merely a $2.5 \mu \mathrm{m}$ additional displacemen$t$ in the coil center would give a contribution of about 0.14 unit to the observed $\delta a_{1}$.

(e) Special purpose holes in some yoke packs : At about 5 meter from the lead end, strain gauges were installed in the SSC prototype magnets built at BNL. In order to bring the wiring out, two $\sim 10 \mathrm{~mm}$ diameter holes were drilled in one yoke pack from the iron inner radius to the two $H e$ bypass holes. This was done only in the bottom half of the magnet. This gave a large local $a_{1}$ saturation, which has been measured. As mentioned earlier the measuring coil is one 1 meter long and the estimated value of this effect is an additional $\delta a_{1}$ of $\sim-0.15$ in SSC magnets.

(f) Persistent Currents : Persistent currents in the superconductor of a magnet change when the current in the coils is changed. An up-down asymmetry either in the geometry of the coil (which also gives geometric $a_{1}$ ) or in the properties of the superconducting cables used in the top and bottom coils may generate an $a_{1}$ due to persistent currents. Since the magnitude of persistent current induced $a_{1}$ decreases at higher field, the difference between $5000 \mathrm{~A}$ and $2000 \mathrm{~A}$ values would contribute to the observed $\delta a_{1}$. The calculation of this effect is beyond the scope of this work. However, measurements show that $\delta a_{1}$ from this source is relatively small (under 0.05 unit) in SSC magnets.

(g) Electrical Bus near the perimeter of the yoke: A large number of magnets in the accelerator are connected in series so that a common power 
supply and control can be used for all. The current from one magnet to the next is carried by a bus that is placed in a rectangular cutout near the perimeter of the yoke (see Fig. 3.4.6). The cutout also contains a bus for the return current, which depending on its configuration with the lead (supply) bus, may nearly cancel the lead bus contribution. A saturated iron yoke at high field does not provide adequate magnetic shielding at the center of the magnet against the field lines produced by the two currents in the bus. In the SSC and RHIC dipole magnets the bus is placed at the top of the yoke. This breaks an up-down symmetry and produces a current dependent $a_{1}$ at high field. The amount of $\delta a_{1}$ depends on the exact configuration of the two conductors in the bus; a significant $\delta a_{1}$ may be produced if the conductors in the bus are separated. The position of this bus and the configuration of the two conductors is chosen such that it has a minimum influence on the field at the center of the magnet and produces a small $\delta a_{1}$. 


\section{5. $a_{1}$ Saturation in SSC Dipole Magnets}

In this section the variations in $\delta a_{1}$ are examined in SSC magnets (both within a magnet and magnet to magnet). Several methods for compensating a large systematic $\delta a_{1}$ due to an off-centered cryostat are proposed.

\subsection{1. $\delta a_{1}$ variation with axial position within a magnet}

Axial scans of the field harmonics have been made, using a one meter long measuring coil [70], in the prototype SSC magnets DCA209 through DCA213 at 2000A and 6600A current. The difference between these two values at each position gives $\delta a_{1}$ ( $a_{1}$ saturation). It has been found that $\delta a_{1}$ varies significantly along the length of a magnet. This is shown in Fig. 3.5.1. Amongst the various mechanisms discussed in the previous section, the offcentered yoke in the cryostat $(a)$ and electrical bus $(g)$ give a constant $\delta a_{1}$ and thus can't account for the variation with position. The magnitude of the persistent current effects $(f)$ is rather small. Of the remaining mechanisms, a difference in the packing factor $(b)$ between the upper and lower yoke halves can be examined from the data on individual yoke block weights. As pointed out earlier, a difference in upper and lower yokes could affect $\delta a_{1}$.

The local asymmetry in the top and bottom yoke block weights is defined as :

$$
\text { asymmetry }=\frac{\text { Weight of bottom block }- \text { Weight of top block }}{\text { Average of top and bottom weights. }} \times 100 \%
$$

The yoke weight in each block and the location of each block was carefully recorded [122] by BNL engineers. Fig. 3.5.2 shows the local asymmetry in the top and bottom yoke block weights averaged over the length of the measuring coil (one meter), as a function of block number (position along the magnet) in magnet DCA213. As can be seen from the figure, although the average asymmetry for the magnet is nearly zero, there can be a local asymmetry of up to $\pm 0.2 \%$, when average values over one meter length are considered. The asymmetry is most prominent when the measuring coil center is located around block numbers 3,60 and 90. Fig. 3.5.2 also shows the variation of $\delta a_{1}$ with position. A good correlation between the yoke asymmetry and $\delta a_{1}$ is seen. A similar correlation has been seen in other SSC prototype magnets. This leads to the conclusion that a major cause of $\delta a_{1}$ variation along the length of the magnet is a local top-bottom asymmetry in the iron weight (packing factor). A similar variation in the vertically split prototype magnets [133] 


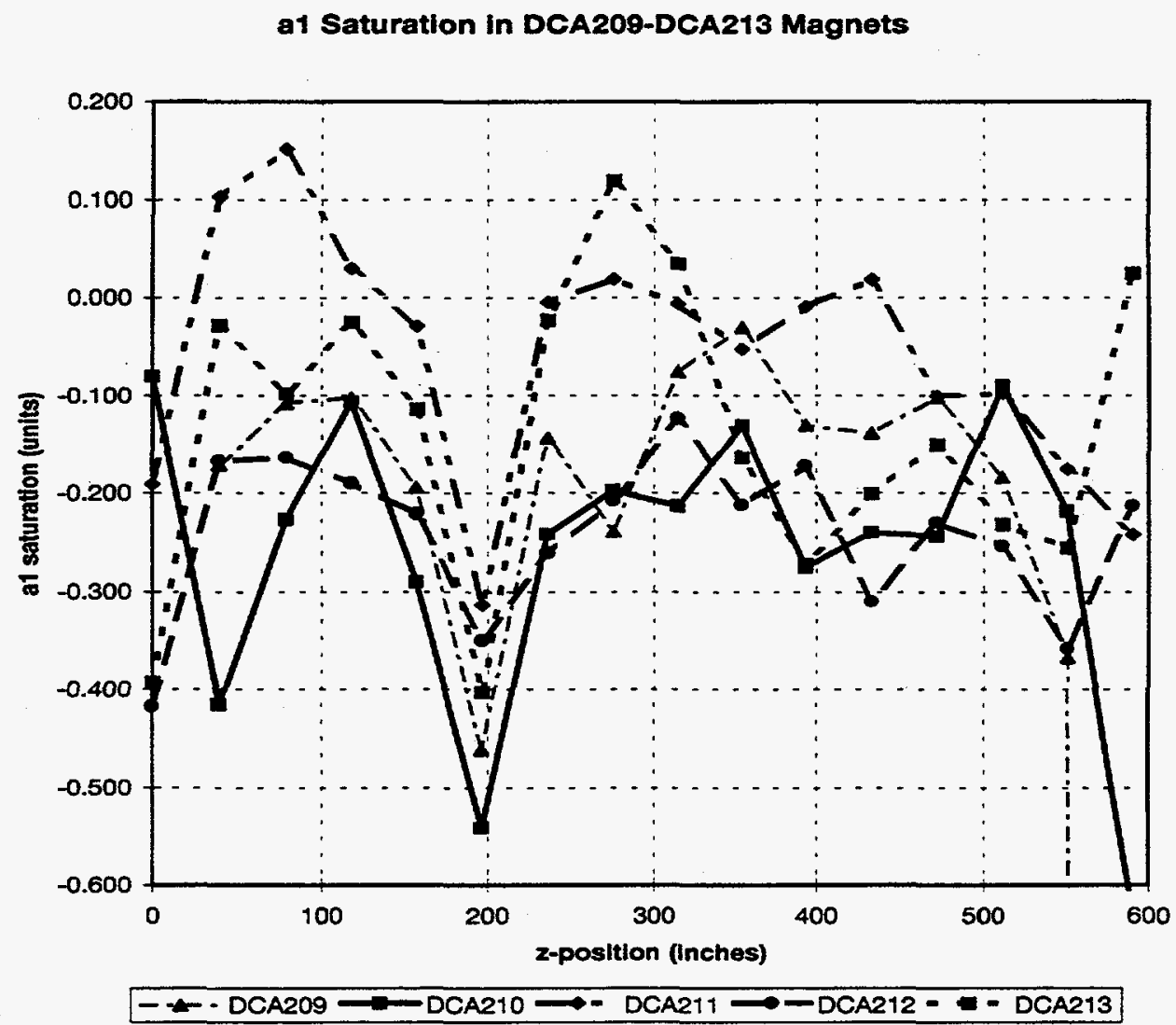

Figure 3.5.1: Variation in $a_{1}$ saturation along the length of the five, 15 meter long, SSC $50 \mathrm{~mm}$ aperture prototype dipole magnets DCA209DCA213. 


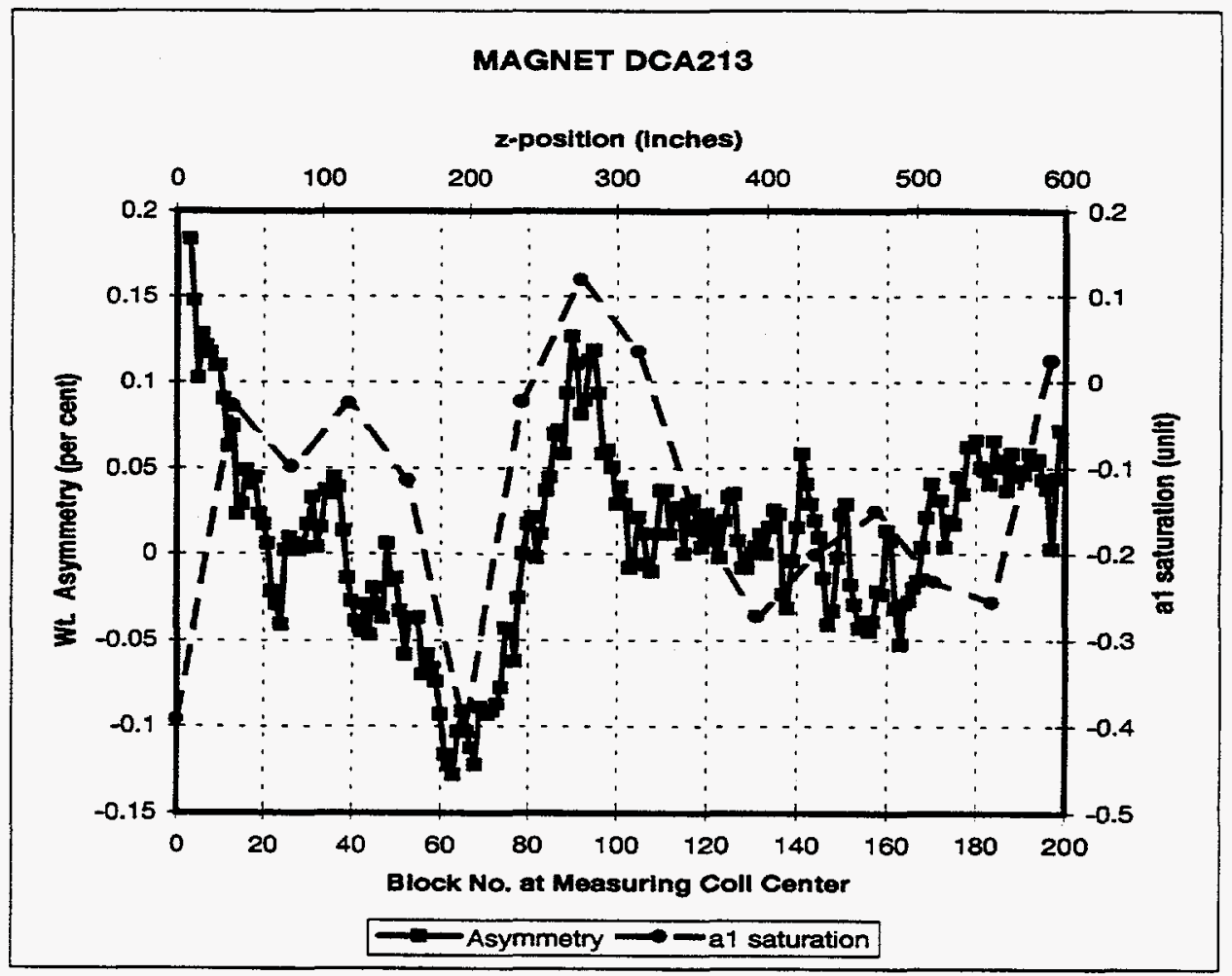

Figure 3.5.2: Variation of top-bottom weight asymmetry and $a_{1}$ saturation $\left(\delta a_{1}\right)$ along the length of the SSC prototype magnet DCA213. 
built at Fermilab, has been observed in $b_{1}$ saturation, which could be associated with a left-right asymmetry in the packing factor.

An examination of the $a_{1}$ saturation profiles for all the magnets (Fig. 3.5.1) shows a large $\delta a_{1}$ at about 5 meter (200 inches) in all the magnets. This can be linked to item (d) described in the previous section, which refers to the presence of a $\sim 10 \mathrm{~mm}$ diameter radial hole in the lower yoke block at this location. All SSC $50 \mathrm{~mm}$ aperture prototype magnets built at BNL had this hole to route the wiring for the strain gauges. Though the effect of this hole on the weight when averaged over 1 meter is negligible, the local perturbation on the field harmonics is much larger due to its geometry. Therefore, based on the computed estimates and measured systematic value of $\delta a_{1}$, it is correlated with the radial hole, rather than the weight asymmetry alone. It may be pointed out that the hole would not have been a part of the design of production magnets.

\subsubsection{Magnet to magnet variations in the integral $\delta a_{1}$}

Since the total yoke weight in the top and bottom halves is well controlled, one would not expect a magnet to magnet variation in $\delta a_{1}$ integrated over the entire length of the magnet. Table 3.5.1 lists the average values and RMS variations in $a_{1}(@ 2000 \mathrm{~A}), a_{1}(@ 6600 \mathrm{~A})$ and $\delta a_{1}$ in the straight sections of the SSC magnets DCA209-213 measured with the one meter long measuring coil.

Table 3.5.1 shows that the integral $\delta a_{1}$ does have a significant magnet to magnet variations. Thus, the observed $\delta a_{1}$ averaged over a magnet depends on factors other than the total yoke weight alone. A strong correlation is seen between the geometric $a_{1}\left[a_{1}(2000 \mathrm{~A})\right]$ and the integral $\delta a_{1}$. Please see Table 3.5.1 and Fig. 3.5.3 which shows the integral $\delta a_{1}$ as a function of geometric $a_{1}$ in magnets DCA209-213. A linear dependence of $\delta a_{1}$ on $a_{1}$ is observed and it is parameterized as [70]

$$
\delta a_{1}=-0.209+0.104 \times a_{1}(2000 \mathrm{~A})
$$

The constant term agrees with the value of an -0.2 unit calculated for the effect of the off-centered yoke in the cryostat in the absence of any other asymmetry. The second term gives the dependence on the geometric $a_{1}$. A coefficient of 0.104 implies that there is a $\sim 10 \%$ enhancement in coil asymmetry (and hence in $a_{1}$ ) at $6600 \mathrm{~A}$. The enhancement may have been caused by (a) asymmetric Lorentz forces due to asymmetry in the coil geometry, 
Table 3.5.1: Integral Skew Quadrupole in DCA209-213

\begin{tabular}{|c|c|c|c|c|}
\hline $\begin{array}{c}\text { Magnet } \\
\text { Magnet }\end{array}$ & $\begin{array}{c}<a_{1}> \pm \sigma\left(a_{1}\right) \\
(2000 \mathrm{~A})\end{array}$ & $\begin{array}{c}<a_{1}> \pm \sigma\left(a_{1}\right) \\
(6600 \mathrm{~A})\end{array}$ & $\begin{array}{c}<\delta a_{1}> \pm \sigma\left(\delta a_{1}\right) \\
(6600 \mathrm{~A}-2000 \mathrm{~A})\end{array}$ & $\begin{array}{c}\delta a_{1} \\
(\max -\min )\end{array}$ \\
\hline DCA209 & $0.261 \pm 0.408$ & $0.086 \pm 0.420$ & $-0.175 \pm 0.115$ & 0.43 \\
\hline DCA210 & $-0.229 \pm 0.227$ & $-0.474 \pm 0.211$ & $-0.245 \pm 0.118$ & 0.45 \\
\hline DCA211 & $1.758 \pm 0.623$ & $1.724 \pm 0.706$ & $-0.034 \pm 0.115$ & 0.46 \\
\hline DCA212 & $-0.187 \pm 0.200$ & $-0.417 \pm 0.220$ & $-0.230 \pm 0.070$ & 0.24 \\
\hline DCA213 & $0.607 \pm 0.335$ & $0.477 \pm 0.351$ & $-0.130 \pm 0.139$ & 0.52 \\
\hline Average & $0.442 \pm 0.812^{* *}$ & $0.279 \pm 0.897^{* *}$ & $-0.163 \pm 0.085^{* *}$ & $0.42 \pm 0.11$ \\
\hline
\end{tabular}

** Sigma refers to magnet to magnet variation.

(b) saturation due to asymmetric placement of the coil in the yoke and (c) contribution of the persistent current induced $a_{1}$ in the coil. Using the above equation, one can predict the saturation $\delta a_{1}$ for any BNL built, horizontally split, $50 \mathrm{~mm}$ SSC dipole magnet. 


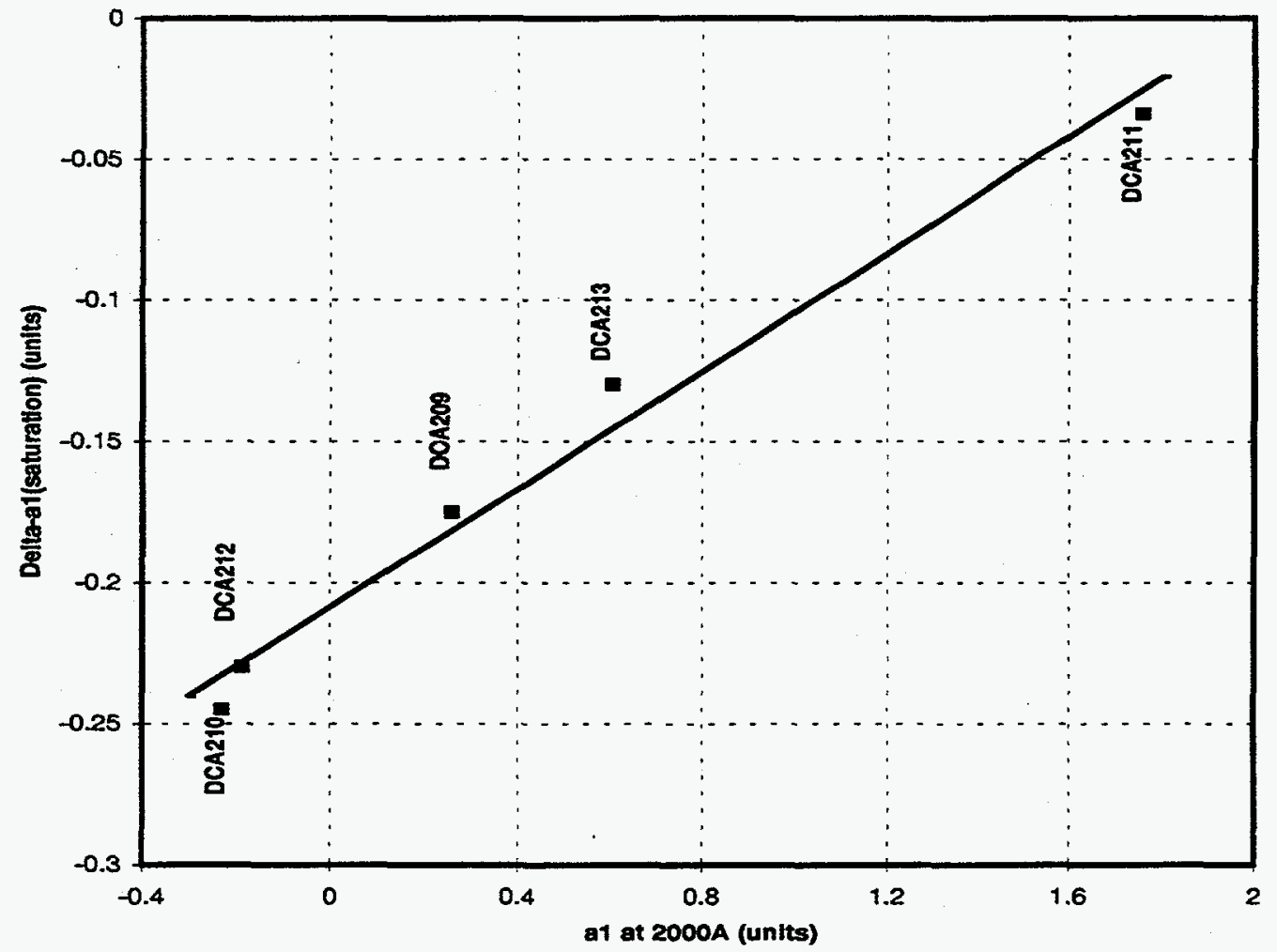

Figure 3.5.3: Correlation between geometric $a_{1}$ ( $a_{1}$ at $2000 \mathrm{Amp}$ ) and $a_{1}$ saturation $\left(\delta a_{1}\right)$. 


\subsubsection{Compensation of the saturation induced $a_{1}$ in SSC magnets}

As discussed in the previous sections, both the calculations and measurements show a significant variation in $a_{1}$ (skew quadrupole) as a function of current beyond 6 tesla central field in SSC dipoles. In this section the discussion is restricted to the $a_{1}$ caused by the saturating iron in the yoke, the center of which is located asymmetrically $93.7 \mathrm{~mm}$ above the horizontal axis of the magnetic cryostat vessel. The effect on $a_{1}$ is several times the specification, a maximum of 0.04 unit for the systematic $a_{1}$. In the SSC $50 \mathrm{~mm}$ dipole the computed $a_{1}$ saturation is 0.2 unit at $6600 \mathrm{~A}$. A few methods are discussed here which reduce this systematic change in $a_{1}$ and bring it within the specification.

(a) Placing the conductors in a computed location in the bus slot: It has been mentioned earlier that a saturated iron yoke does not provide adequate magnetic shielding against the field lines produced by currents in the bus conduit (item $(g)$ in the previous section). Since the two conductors in the bus slot (see Fig. 3.4.6) carry currents in opposite directions, a dipole field is created by them. However, the direction and the magnitude of the field produced at the magnet center depends on their orientation, the separation between them and on the saturation of the yoke between the bus slot and magnet aperture. At high field, a $\delta a_{1}$ may be created, which then may be used to partly compensate the $\delta a_{1}$ produced by the cryostat. A maximum $\delta a_{1}$ is generated in the configuration when the conductors in the bus slot are oriented in such a way that they produce a vertical field (side-by-side configuration) and a minimum when they produce a horizontal field (up-down configuration). POISSON calculations show that in the side-by-side configuration if the spacing between the midpoint of the two conductors is $10 \mathrm{~mm}$, the net $\delta a_{1}$ in the magnet stays within the specified tolerance of 0.04 unit. In the given configuration, the relative direction of the current in the bus conductors should be opposite to that of the direction of the current in the main magnet coil in the aperture of the magnet.

(b) Iron weight difference between the upper and lower yoke halves: In the BNL-built SSC dipole magnets the yoke is split in two halves at the horizontal plane. If the number of magnetic laminations or their weight 
is different between the top and bottom halves of the magnet, $\delta a_{1}$ is created (see item $(b)$ in previous section). This could be used to significantly compensate the $\delta a_{1}$ produced by the cryostat. One practical way to implement this scheme in a magnet could be that some of the low carbon steel (magnetic) laminations are replaced by stainless steel (non-magnetic) laminations in the upper yoke-half. If the number of non-magnetic laminations is a small fraction of the magnetic laminations, the situation can be simulated in a computer program by using a difference in packing factor between the top and bottom halves of the yoke. POISSON calculations show that $\sim 0.1 \%$ difference in packing factor is adequate to bring the net $a_{1}$ within the specified tolerance of 0.04 unit.

(c) Placing extra magnetic steel at the bottom of yoke: Since the saturation $a_{1}$ is caused by the proximity of the iron cryostat wall at the top half of the magnet, a natural solution to this problem would be to put some extra iron on the opposite side of it. Several configurations have been examined to put (or attach) this extra iron at the bottom half of the magnet. POISSON calculations show that a $1 \mathrm{~mm}$ thick iron strip from 180 degree to 360 degree on the yoke outer radius (just outside the stainless steel shell) would be adequate to produce the required compensation. If the strip is put from 225 degree to 315 degree (width $=90$ degree) the thickness required would be $10 \mathrm{~mm}$.

General Dynamics has examined [76] placing this extra iron inside the magnet coldmass and have found that would also generate an adequate compensation. 


\section{6. $a_{1}$ Saturation in RHIC Dipole Magnets}

In the RHIC arc dipoles, as in the case of the SSC dipoles discussed above, the center of the cold mass lies above the center of the cryostat (see Fig. 3.6.1). This introduces a systematic top-bottom asymmetry leading to a skew quadrupole $\left(a_{1}\right)$ harmonic at high fields [97]. The magnitude of this $a_{1}$ depends on the current (field). Moreover, like the SSC dipoles built at BNL, the RHIC dipoles also have a horizontally split yoke design and the weight of the top and bottom yoke is not always the same. This may create an additional current dependence in the skew quadrupole harmonics. These two effects are studied here. An attempt is made to compensate the $a_{1}$ created by the cryostat with an $a_{1}$ created by a deliberate asymmetry between the top and bottom yoke weights. The results of this attempt will be discussed.

\subsubsection{Magnet to magnet variation in $a_{1}$ saturation}

The field quality in all the RHIC dipoles is measured at room temperature using a one meter long rotating coil system. [155] The integral harmonics are determined from an axial scan of the magnet in one meter steps. The first 30 dipoles were also measured cold with the rotating coil system. Subsequently, only about $8 \%$ of the dipoles are tested cold. The field measurements in cold magnets consist of axial scans at $660 \mathrm{~A}$ (near injection), 1450A (near transition) and 5000A (near storage). In addition, the complete current dependence from $50 \mathrm{~A}$ to $6000 \mathrm{~A}$ is studied at one location in the center of the magnet. The dependence of the skew quadrupole term on current, measured in the axial center of two of the RHIC dipoles, is shown in Fig. 3.6.2. In order to facilitate comparison between the two magnets, the geometric skew quadrupole term is removed by subtracting the value at $1450 \mathrm{~A}$. The possible sources leading to variation of $a_{1}$ with current in the SSC have been discussed earlier and those sources play a similar role in RHIC magnets.

The major systematic source of the current dependence is the same as in SSC magnets, which is the cryostat. The major source of magnet to magnet variation could be the differences in persistent currents in the cable used in the upper and lower coils and the differences in yoke weights between the upper and lower halves. The skew quadrupole contribution at $1450 \mathrm{~A}$ due to superconducting cable magnetization is less than 0.1 unit in the magnets measured so far. 


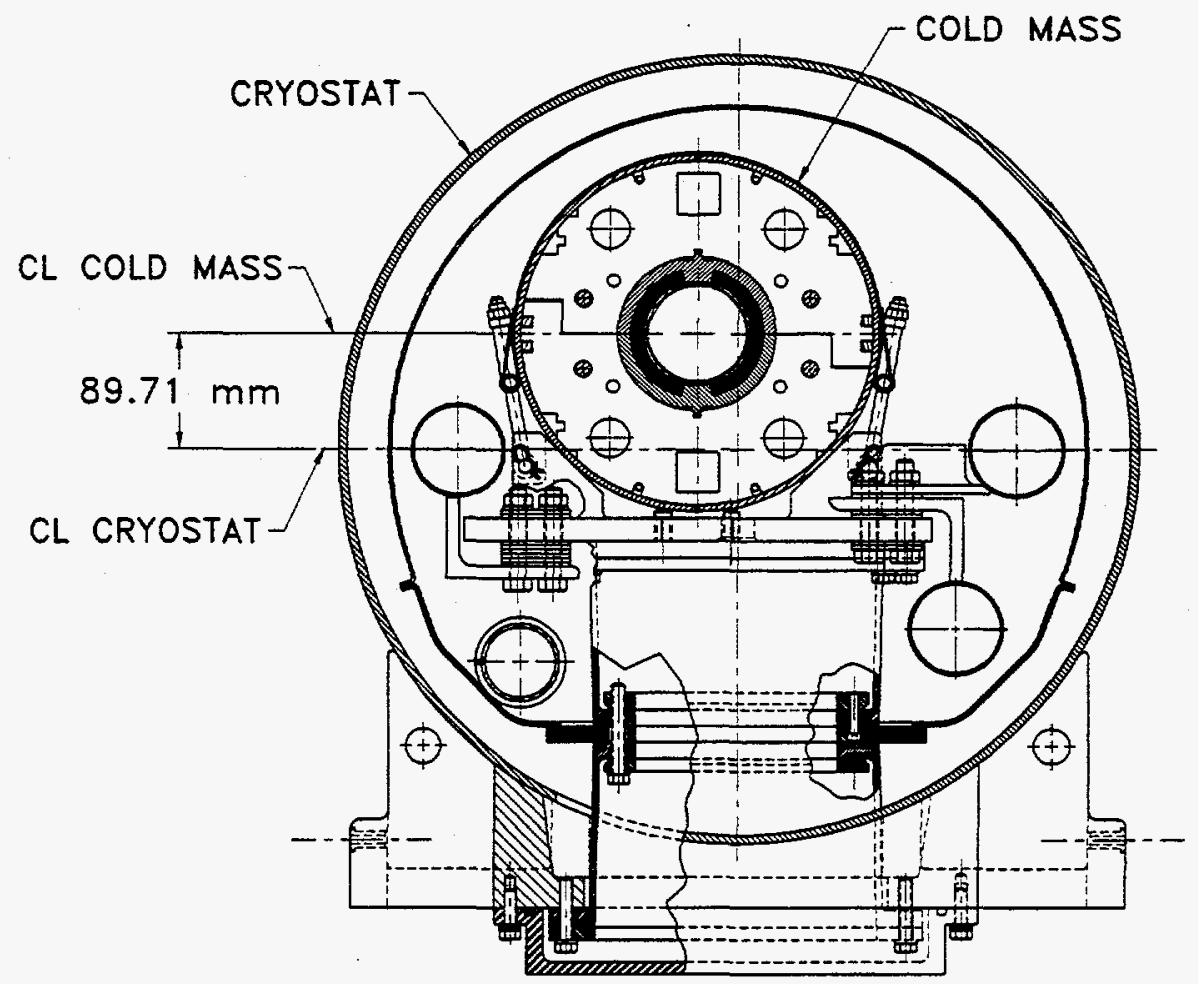

Figure 3.6.1: A cross section of the RHIC arc dipole showing the asymmetrically located cold mass in the cryostat. The top-bottom asymmetry generates a skew quadrupole harmonic $\left(a_{1}\right)$ at high fields. The left-right asymmetry generates a normal quadrupole harmonic $\left(b_{1}\right)$ at high fields. The latter asymmetry, which varies along the axis, is generated because the cold mass is curved to match the beam curvature and the cryostat is not. The cross section shown above is at the axial center. 


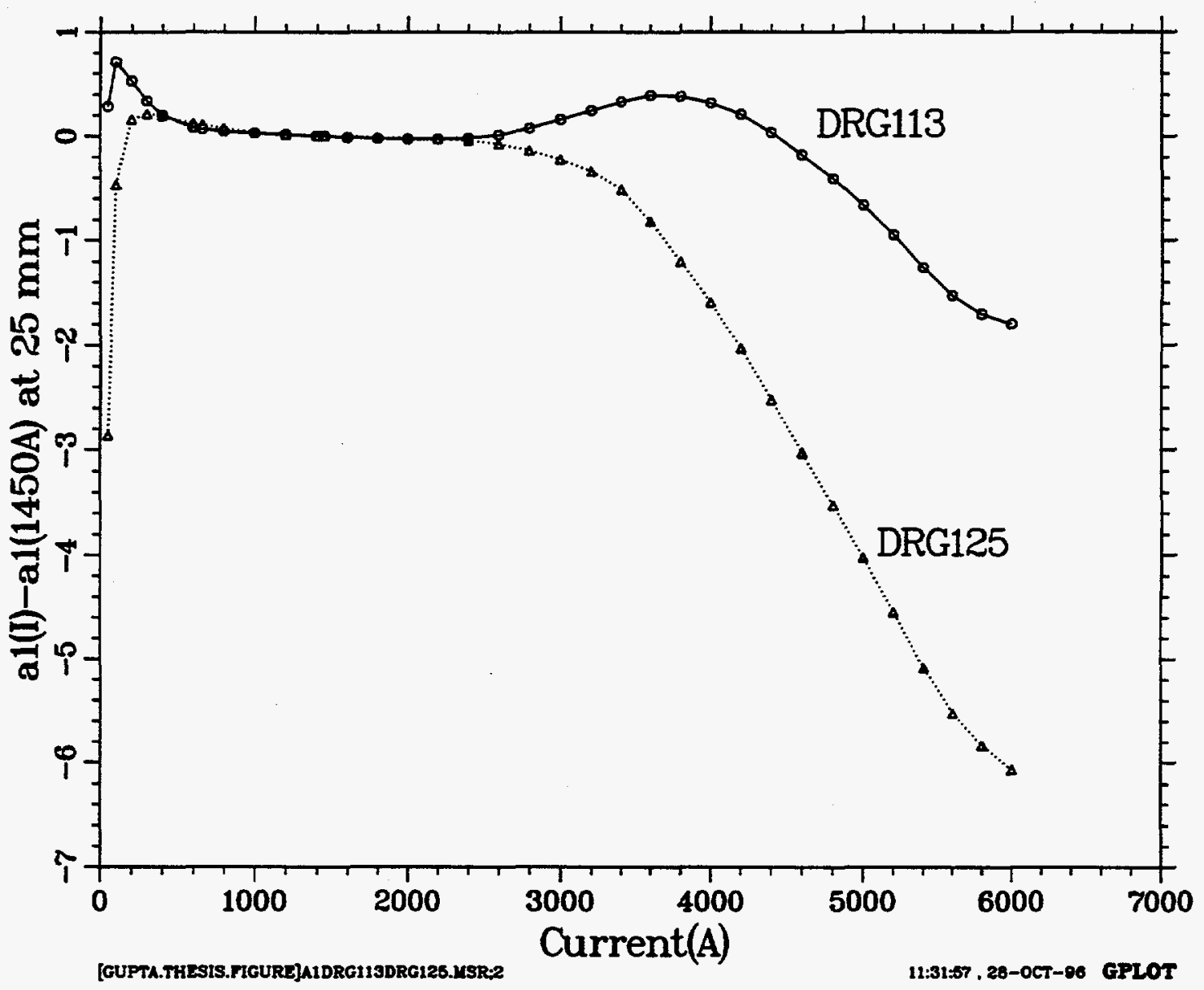

Figure 3.6.2: Dependence of the skew quadrupole term on current in the dipoles DRG113 and DRG125. The magnitude of change between low currents and 5000A is larger in DRG125 and is smaller in DRG113. 
The two magnets for which data are shown in Fig. 3.6.2 exhibit the largest (DRG125, filled boxes) and among the smallest (DRG113, solid line) skew quadrupole due to saturation. In SSC dipole prototypes, a good correlation was found between the top-bottom yoke weight asymmetry and the $a_{1}$ saturation. In RHIC dipoles, the upper and the lower iron yoke weights are available in three different axial sections. The values of the skew harmonic terms are integrated over each of these three sections in a magnet by appropriately summing the values measured in axial scans. Using axial scans at $1450 \mathrm{~A}$ and $5000 \mathrm{~A}$, the $a_{1}$ saturation in each of the three sections is computed.

Fig. 3.6.3 shows the correlation between the yoke weight asymmetry and $a_{1}$ saturation in RHIC dipoles. The yoke weight asymmetry is defined here as [97]

$$
\text { asymmetry }=\frac{\text { Weight of Top block }- \text { Weight of Bottom block }}{\text { Average of top and bottom weights }}
$$

There are a total of 38 magnets in the plot shown in Fig. 3.6.3, for a total of $114(=3 \times 38)$ points in the plot. A good correlation is seen between the yoke weight asymmetry and the saturation in skew quadrupole, as expected. The solid line shows a linear fit to the data points. A few of the data points are seen to lie away from the line. This lack of correlation in certain sections of a few magnets could be due to incorrect recording of the yoke weight. The linear fit shown excludes such data points (seven points in all, belonging to six different magnets). The linear fit gives an $a_{1}$ saturation of -1.9 units for zero asymmetry in yoke weight, in good agreement with the computed value of -2.0 units. Furthermore, the slope of the line gives a change of -2.8 units in $a_{1}$ saturation for a $1 \%$ asymmetry in the iron yoke weight. This is also close to the computed value. The calculated current dependence of the skew quadrupole term is shown in Fig. 3.6.4 for various values of top-bottom weight asymmetry. The calculated curves are similar to the measured current dependences shown in Fig. 3.6.2. 


$$
y=-0.028 x-1.915 \text { (107 points) }
$$

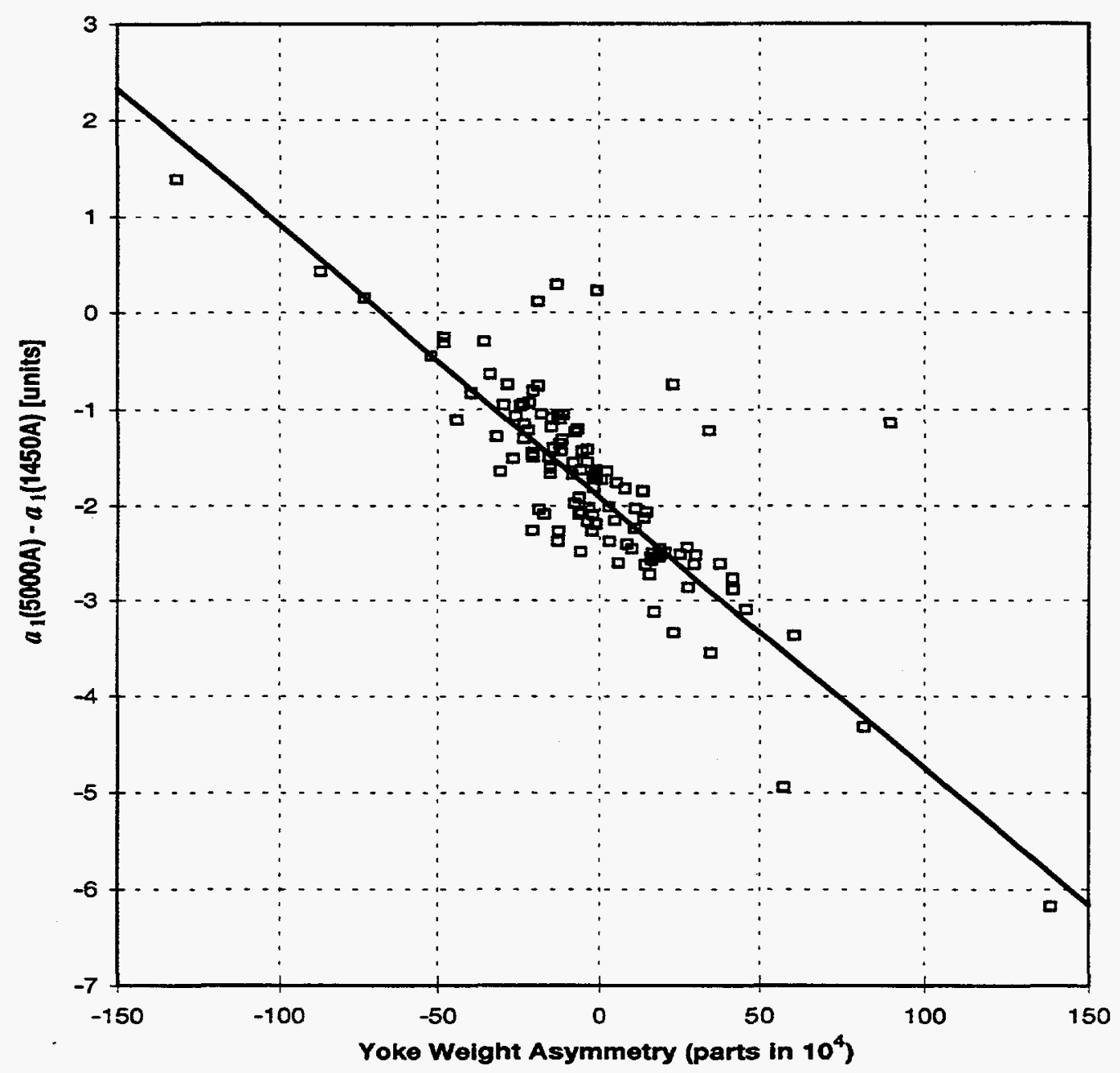

Figure 3.6.3: Correlation between the RHIC dipole yoke weight asymmetry and the saturation in the skew quadrupole term. There are three data points for each magnet corresponding to the three sections of the yoke blocks for which the weights are known. The equation on the top of the figure is obtained by a least square fit with $x$ representing the yoke weight asymmetry and $y$ the change in $a_{1}(@ 2.5 \mathrm{~cm})$ between $5000 \mathrm{~A}$ and $1450 \mathrm{~A}$. (Courtesy A. Jain). 


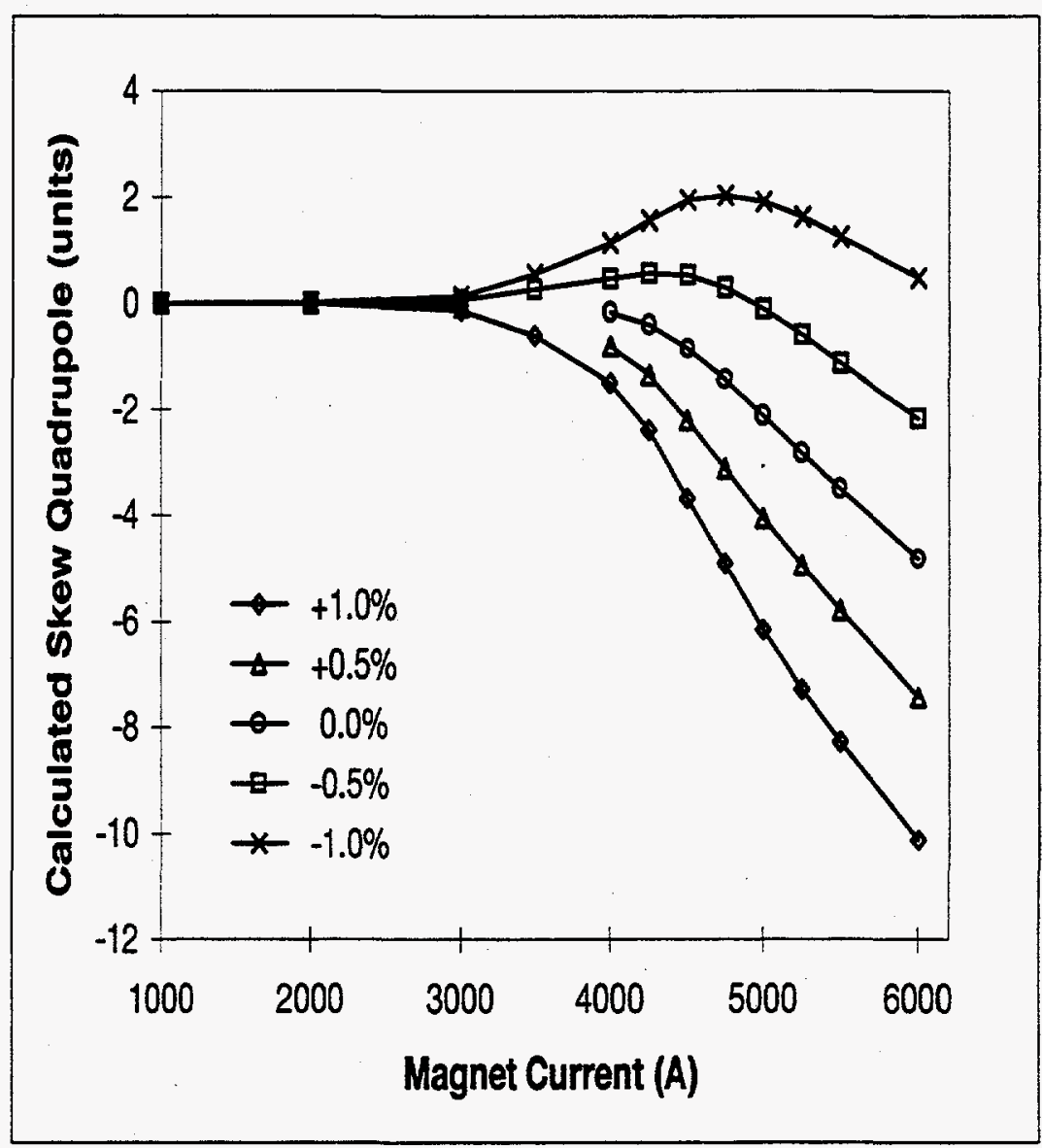

Figure 3.6.4: The calculated current dependence of the skew quadrupole term for various values of the asymmetry between the top and the bottom halves of the yoke. 


\subsubsection{Reduction in $a_{1}$ saturation in RHIC dipoles}

As seen from Fig. 3.6.3, there is considerable magnet to magnet variation in $a_{1}$ saturation. This variation is a result of asymmetry in the top and bottom yoke weights. The production specifications for the RHIC dipoles specify that the total weight of the yoke be held constant within $\pm 2 \mathrm{~kg}$. The total yoke weight is approximately $2764 \mathrm{~kg}$. Ideally, the weights of the yoke halves should be controlled with little magnet to magnet variation. However, such a requirement is difficult to fulfill in practice. Since the yoke laminations are $6.35 \mathrm{~mm}$ thick with a tolerance of $0.25 \mathrm{~mm}$, the weights of the yoke packs are expected to differ somewhat. The systematic value of the skew quadrupole measured at low fields in the RHIC production dipoles is close to zero. If the upper and the lower yoke blocks are exactly matched in weight, this would imply a systematic $a_{1}$ of -1.9 units at $5000 \mathrm{~A}$ due to the cryostat. It is possible to reduce this systematic $a_{1}$ at high fields by counteracting the asymmetry of the cryostat by some other means. Various possible means to achieve this have been discussed above.

The control of $a_{1}$ saturation essentially requires that additional iron be available in the bottom half of the yoke compared to the top half. A study of yoke weight data in the first $\sim 50$ RHIC dipole magnets showed that there was a significant variation in the weights of yoke blocks. This natural variation in the yoke weights has been utilized to create a deliberate top-bottom asymmetry to compensate the asymmetry created by the off-centered cryostat. In practical terms, this reduces the net $a_{1}$ saturation without incurring any additional cost in the production. In the dipoles now under production at the Northrop Grumman Corporation, the yoke blocks are assigned in such a way that the heavier blocks are used for the bottom half and the lighter ones for the top half. A top-bottom weight difference of $0.5 \%$ of the total (top + bottom) yoke weight (or an asymmetry of 0.01 as defined in Eqs. (3.6.1)) is targeted to counteract the -1.9 units of systematic $a_{1}$ saturation.

Fig. 3.6.5 shows the asymmetry in the total upper and the lower yoke weights in all the dipoles delivered so far. The current scheme of yoke block assignment was implemented starting with dipole sequence number 63 . For dipoles 1 through 62 , no attempt was made to control the upper and the lower yoke weights separately. As can be seen in Fig. 3.6.5, most magnets in this group had a positive asymmetry, which only added to the effect from the cryostat. For dipoles numbered 63 onwards, the lower half of the yoke was made heavier than the top half as much as allowed by the actual weights of the yoke block in each magnet up to a limit of 0.01 in asymmetry. This is reflected in the negative values of asymmetry 


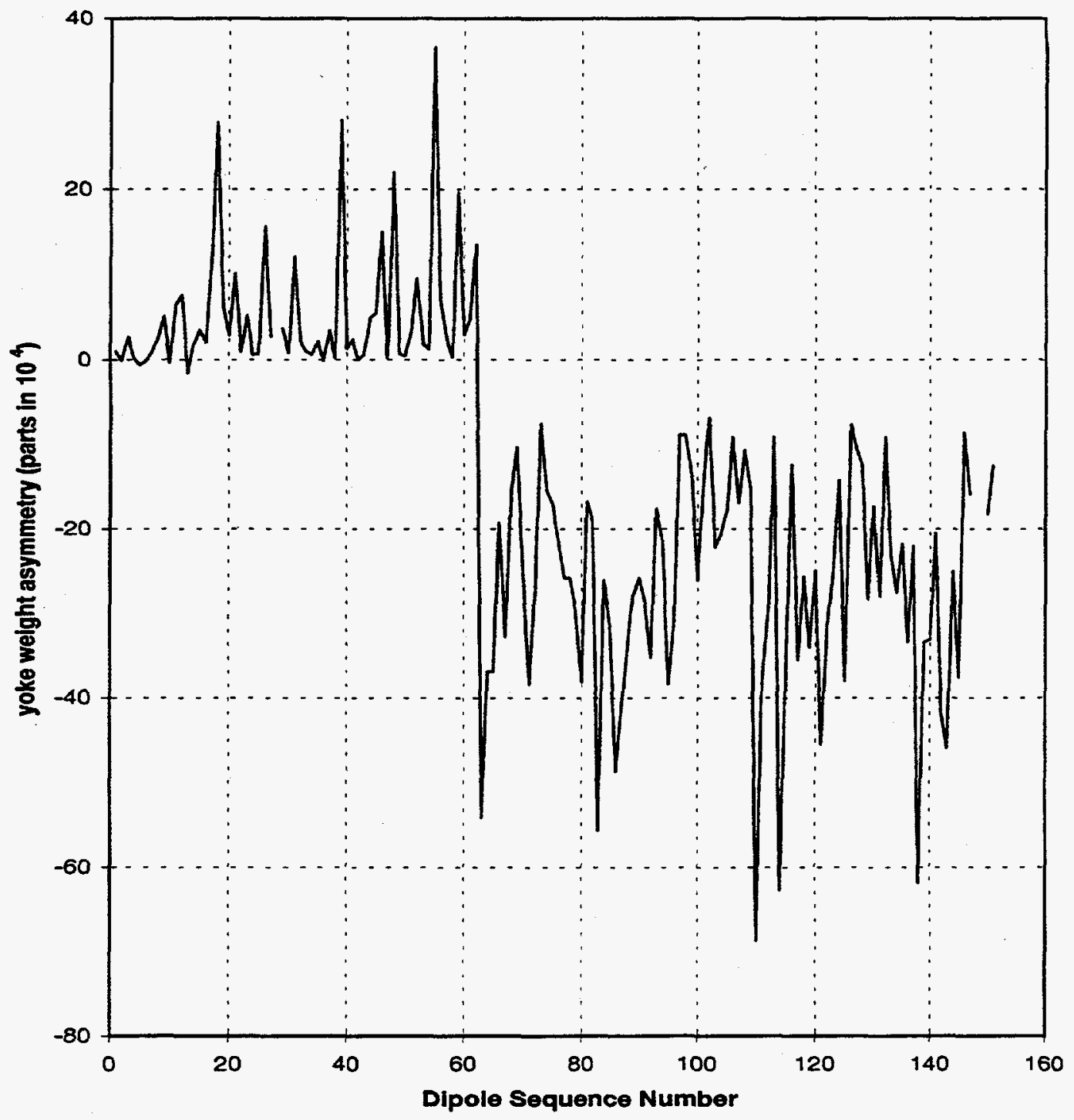

Figure 3.6.5: The asymmetry in the weights of the upper and the lower yoke halves in the RHIC arc dipoles. Starting with magnet sequence number 63, the lower yoke half was made heavier than the top half. (Courtesy A. Jain). 
in Fig. 3.6.5 for sequence number 63 and higher. The additional iron on the bottom is expected to counteract the effect of the cryostat proximity on the top.

The integral values of $a_{1}$ in the magnets were obtained by summing the fields measured in the axial scans. The integral values of $a_{1}$ saturation were calculated using the integral values from the axial scans at $1450 \mathrm{~A}$ and $5000 \mathrm{~A}$. The correlation between the integral $a_{1}$ saturation and the asymmetry in the total upper and the lower weights is shown in Fig. 3.6.6 for both the intial magnets (open boxes), and the current production (filled boxes). Once again, the correlation with iron weights can be seen to hold for most of the magnets. The solid line shows a linear fit to the data. Some of the points do not fall close to the line. It should be noted that most of these points belong to the same magnets that did not show a good correlation in Fig. 3.6.3.

It is clear from Fig. 3.6.5 that the magnets in the new scheme (production sequence 63 and higher) have a negative top-bottom asymmetry in yoke weight on an average. This has resulted in a correspondingly lower $a_{1}$ saturation (see Fig. 3.6.6). The average $a_{1}$ saturation in the magnets 1 through 62 (cold data in 33 magnets) is -1.95 units, whereas the corresponding average for the magnets 63 onwards (cold data in 7 magnets) is only -0.83 units. Thus, on the average a reduction of about 1.1 unit in $a_{1}$ saturation has been achieved. In principle, the average $a_{1}$ saturation could have been made zero by applying a proper yoke weight asymmetry. In RHIC magnets, since it was not critical to the machine performance, the extra complication and effort did not justify reaching this goal. It may be mentioned again that the above reduction in $a_{1}$ saturation is obtained without incurring an extra cost or excessive complications during the magnet production; only the scheme of assigning the location of the eight yoke blocks in each magnet has been modified. 


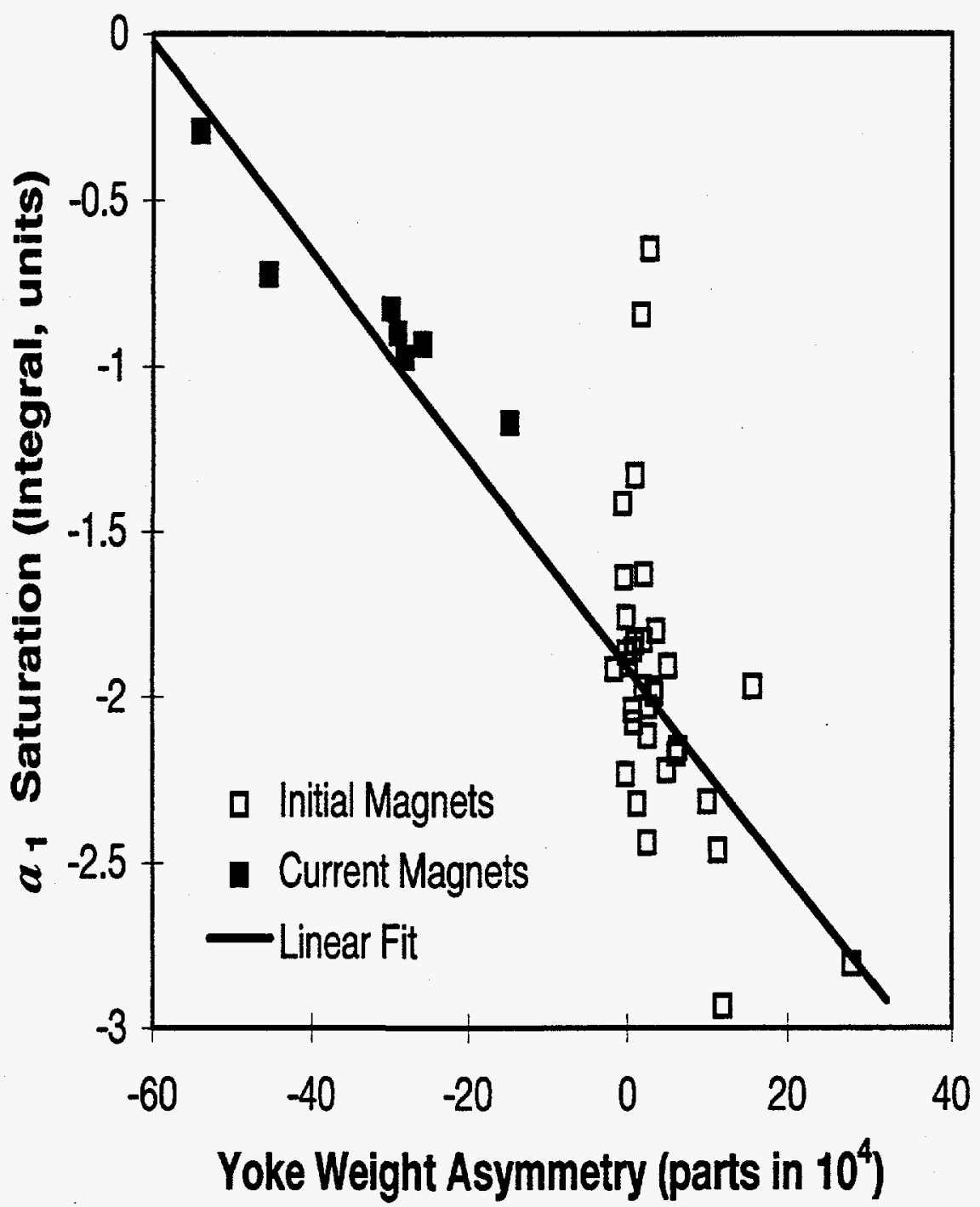

Figure 3.6.6: Correlation between the integral $a_{1}$ saturation and the asymmetry in the total weights of the upper and the lower yoke halves. (Courtesy A. Jain). 


\subsection{Conclusions on the Field Quality Improvements through Yoke Design}

The magnetic field (or current) dependence in harmonics due to iron saturation could be a major problem particularly in the magnets such as those for RHIC where the iron contributes a significant fraction of the total field. Several methods have been examined and developed here to minimize this current dependence to a level where it is of little concern to the beam dynamics in the entire operating range of the machine. It has been demonstrated here that by a proper yoke design one can eliminate the need for external corrector magnets which may otherwise be needed to compensate for the harmonics generated by iron saturation.

The methods examined here can be put in three major categories : (a) optimization of the yoke radial thickness at the midplane by choosing the yoke inner and outer radius, (b) optimization of the yoke inner surface geometry such as by the use of a 2-radius aperture design or an elliptical aperture design and selecting an average gap between the coil and iron and (c) the optimization (and addition) of the size and location of a number of holes and cutouts in the yoke geometry to control the yoke saturation. The efficiency of a particular approach depends on the design details.

These approaches have significantly (about an order of magnitude) reduced the current dependence of the $b_{2}$ and $b_{4}$ harmonics in the $80 \mathrm{~mm}$ aperture RHIC arc dipole magnets as shown in Fig. 3.3.2 and Fig. 3.3.3. The field dependence of the average (mean) values of allowed harmonics in the production dipole magnets built by Northrop Grumman Corporation for the RHIC machine is given in Fig. 3.7.1. Since not all magnets are tested cold, the current dependence is obtained from a one meter long section in the body (straight section) of the magnet DRG107 and it is superimposed over the measured mean of the warm measurements in all magnets based on a warm-cold correlation. The harmonics are given at $25 \mathrm{~mm}$ reference radius. The current dependence in the two ends of the magnet is somewhat different from that observed in the body and it gives a small positive contribution to the integral $b_{2}$ harmonic at fields higher than 2.5 tesla. The current dependence in $b_{2}$ (see Fig. 3.7.1) at lower fields (below 1.6 tesla) is due to persistent currents. The current dependence above 1.6 tesla is primarily due to the saturation of the yoke iron and also due to the coil deformation as a result of Lorentz forces. Only a high field saturation induced current dependence is minimized here. As a result, one can see that the field harmonics remain small even beyond the operating field of 3.46 tesla. The geometric $b_{2}$ in the magnet is optimized so that $b_{2}$ is small at $3.46 \mathrm{~T}(5 \mathrm{kA})$. The actual variation in the mean value of 


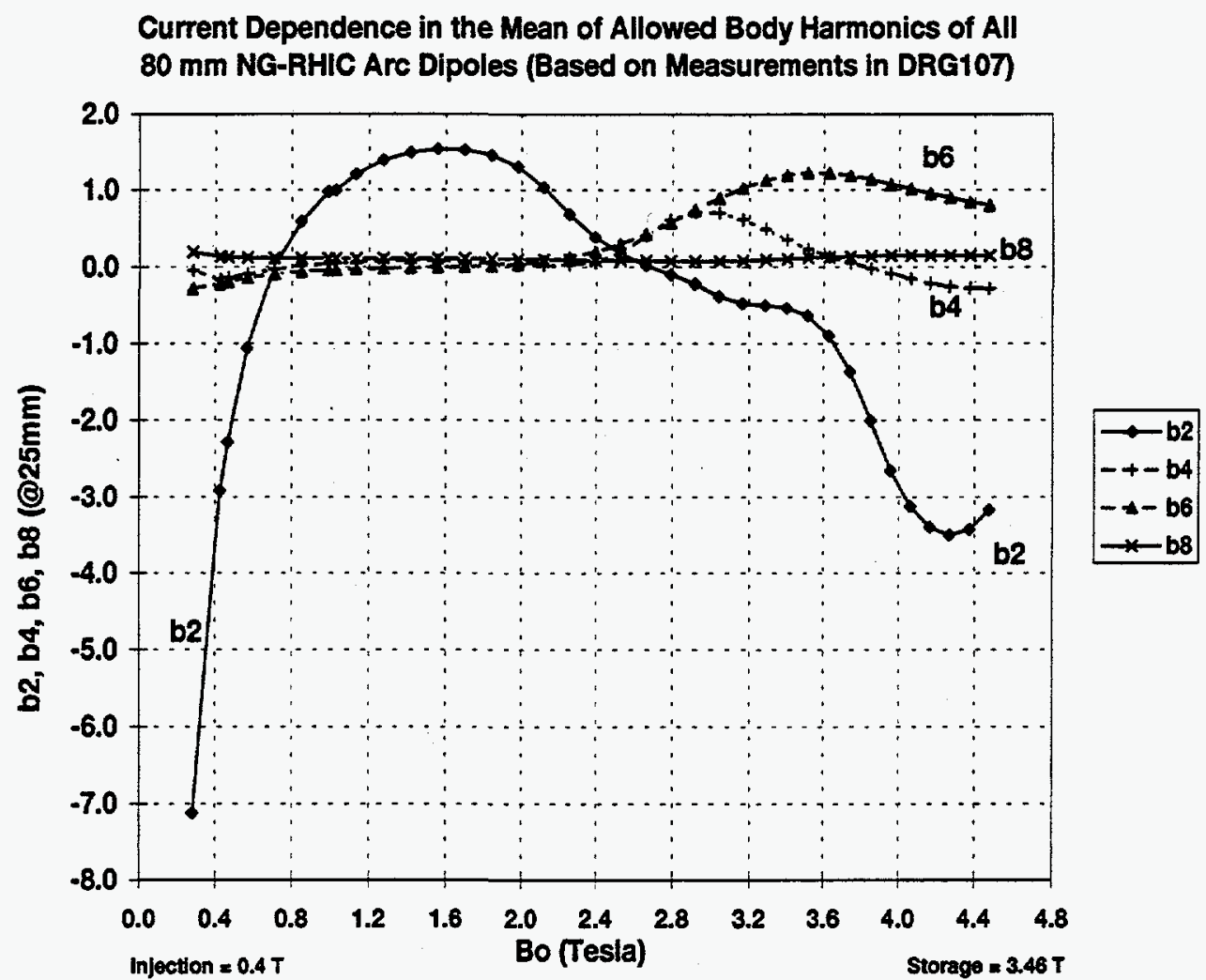

Figure 3.7.1: The current dependence of the average values of the allowed field harmonics in the Northrop Grumman built RHIC $80 \mathrm{~mm}$ aperture arc dipole magnets based on the measured current dependence in DRG107. A Warm-cold correlation is used here to shift the measurements in DRG107 to the average values of the allowed harmonics in all magnets. 
the integral $b_{2}$ (Fig. 3.7.1 shows only that for the body of the magnet) in the entire series of $\mathrm{RHIC}$ dipole production magnets remains within \pm 3 unit from injection $(0.4 \mathrm{~T}$ or $570 \mathrm{~A})$ to quench ( $\sim 4.5 \mathrm{~T}$ or $\sim 7.2 \mathrm{kA}$ which is $\sim 40 \%$ over the maximum design operating current of $5.1 \mathrm{kA}$ ). Most accelerator magnets have a large saturation in sextupole harmonics beyond their operating current. This unusually small current dependence beyond operating current is attributed to the methods used in optimizing the yoke design of RHIC magnets.

To minimize the saturation induced harmonics at high fields one should minimize the variation of iron magnetization or $|B|$ at the yoke inner surface. Although in RHIC magnets the variation in the current dependence of field harmonics was minimized, a more direct approach could have been to minimize the azimuthal variation of $\frac{\mu-1}{\mu+1}$. The function $\left(\frac{\mu-1}{\mu+1}\right)$ lies in the range of 0 to 1 and it reflects the iron contribution to the total field. The function is 1 when $\mu$ is large ( $\mu$ is a few thousand at low fields when the iron is not saturated) and is 0 when $\mu$ is one (at high fields when the iron is completely saturated). The importance of this function is clear from Eqs. (1.5.60) which gives the harmonics $b_{n}$ and $a_{n}$ due to a conductor in a cylindrical iron aperture. In Fig. 3.7.2, the variation in the magnitude of the field $(|B|)$ is shown in the RHIC $80 \mathrm{~mm}$ aperture dipole in the case of (a) the circular aperture yoke without any holes or cutouts and (b) the yoke having holes and cutouts used in the present optimized design. These figures are given at $7 \mathrm{kA}$ current (or $4.5 \mathrm{~T}$ field), which is significantly above the $5.1 \mathrm{kA}(\sim 3.46 \mathrm{~T})$ operating current. As mentioned earlier, the saturation remains small even at these high fields. A high field is chosen here to enhance the contrast between the two cases. In Fig. 3.7.3, the variation in the function $\frac{\mu-1}{\mu+1}$ is shown for the same two cases. The computer model shown in Fig. 3.7.2 and in Fig. 3.7.3 was made by $P$. Thompson and the design work for the RHIC arc dipole magnets has been carried out in collaboration with him. A significant reduction in the azimuthal variation of $|B|$ and in $\frac{\mu-1}{\mu+1}$ can be seen, particularly in the iron near the yoke inner surface. This reduction is made possible by optimizing the location and size of various holes and cutouts and by putting additional holes dedicated to controlling the iron saturation.

The design of the $130 \mathrm{~mm} \mathrm{RHIC}$ insertion quadrupole is based on the two radius method for controlling iron saturation in the yoke. This method has resulted in reducing $b_{5}$ by an order of magnitude (see Fig. 3.2.31). In quadrupoles, the 2-radius saturation control method has been found to be more efficient than the hole method used effectively in dipoles. The primarily reason for this is that the amount of flux per pole in quadrupoles is half of that in dipoles and therefore manipulating that smaller amount of flux to force saturation at the 

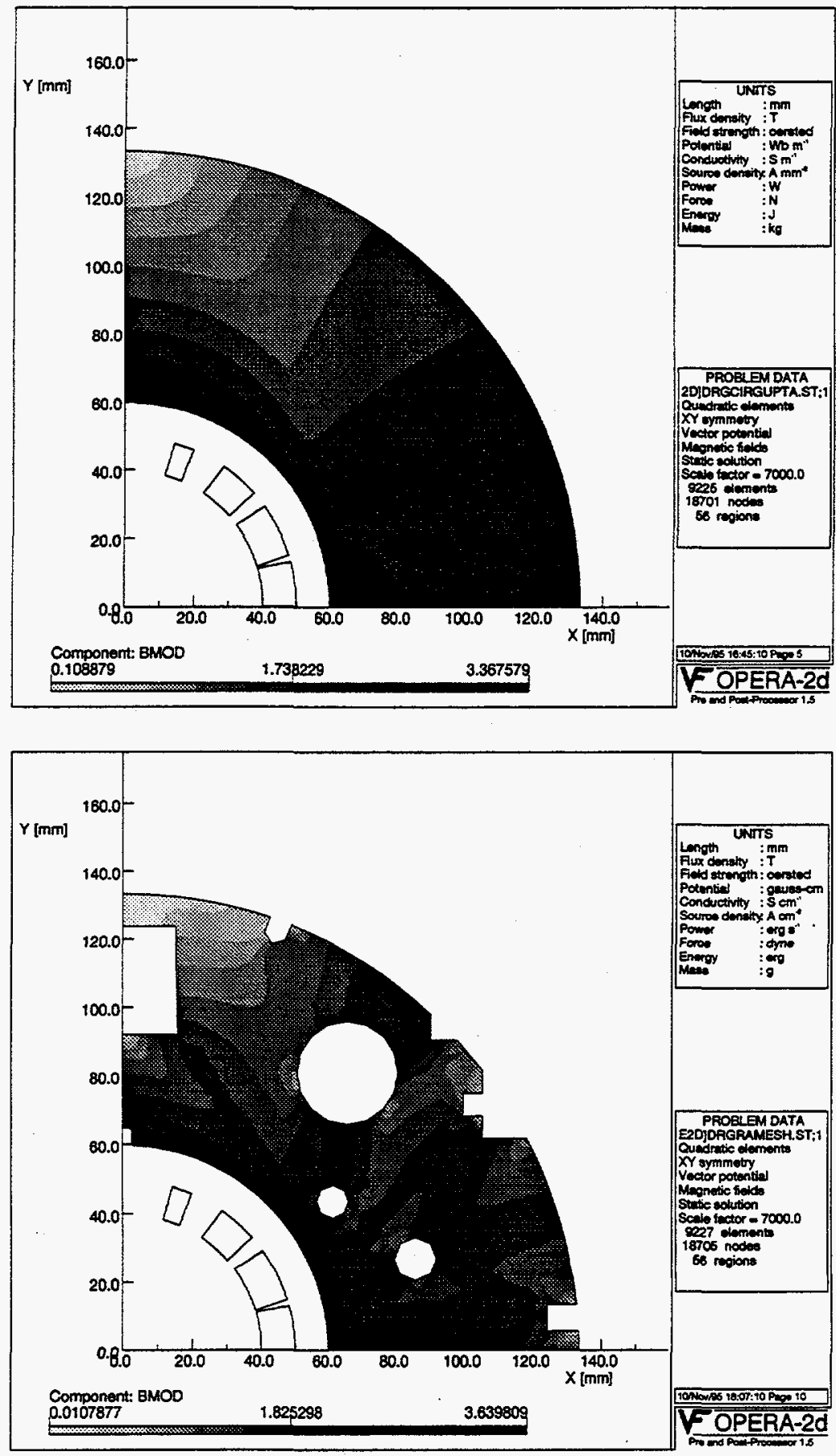

Figure 3.7.2: The magnitude of the field in the iron yoke of the RHIC $80 \mathrm{~mm}$ aperture arc dipole magnet at $7.0 \mathrm{kA}$ in (a) circular aperture case (upper figure) with no holes, etc. and in (b) the optimized design (lower figure) with all holes and other structures. A reduction in the variation, particularly at the yoke inner surfaces may be seen. The design operating current in these magnets is $\sim 5.1 \mathrm{kA}$. 

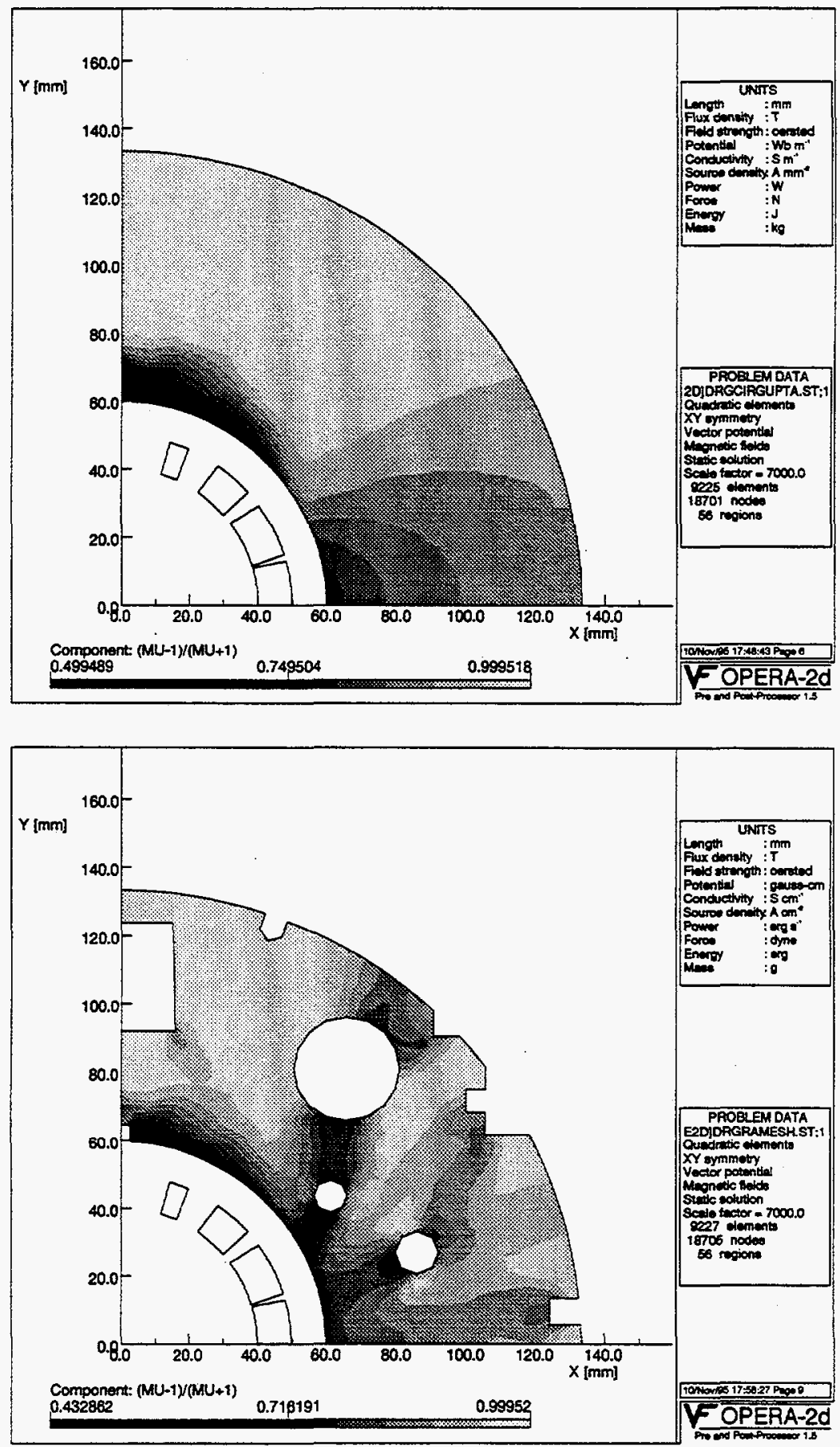

Figure 3.7.3: $\frac{\mu-1}{\mu+1}$ in the iron yoke of the RHIC $80 \mathrm{~mm}$ aperture arc dipole magnet at $7.0 \mathrm{kA}$ in (a) circular aperture case (upper figure) with no holes, etc. and in (b) the optimized design (lower figure) with all holes and other structures. $\frac{\mu-1}{\mu+1}$ is related to the "yoke-air" boundary condition. A reduction in the variation of this function, particularly at the yoke inner surface, reduces the saturation induced harmonics. 
yoke inner surface is not as efficient unless the yoke outer radius is reduced by an excessive amount.

An asymmetric placement of the cold mass in the cryostat results in the introduction of a large $a_{1}$ at high field when the field lines are not contained in the iron yoke. This has been found in both the SSC and the RHIC dipoles. Moreover, if there is a difference in the yoke weights between the top and bottom halves, an additional current dependence in saturation $a_{1}$ is also generated. In the RHIC arc dipoles, the effect of an off-centered cryostat on the saturation $a_{1}$ has been compensated by deliberately making the lower yoke half heavier than the upper. This has resulted in reducing the average saturation induced $a_{1}$ at the design current by about a factor of 2 (from about -2 units to within -1 unit). 


\section{Chapter 4.}

\section{FIELD QUALITY IMPROVEMENTS THROUGH COIL DESIGN}

The research work described in this chapter is partly based on the following papers :

- R.C. Gupta, S.A. Kahn and G.H. Morgan, Coil and Iron Design for SSC $50 \mathrm{~mm}$ Magnet, Proceedings of the 1990 American Society of Mechanical Engineers (ASME) Winter Anmual Meeting in Dallas (1990).

- R.C. Gupta, S.A. Kahn and G.H. Morgan, A Comparison of Calculations and Measurements of the Field Harmonics as a Function of Current in the SSC Dipole Magnets, Proceedings of the 1991 IEEE Particle Accelerator Conference, San Francisco, Pp. 42-44 (1991).

- R.C. Gupta, S.A. Kahn and G.H. Morgan, SSC $50 \mathrm{~mm}$ Dipole Cross section, Proceedings of the International Industrial Symposium on Super Collider (IISSC), Atlanta, pp. 587-600 (1991).

- R.C. Gupta, Correcting Field Harmonics after Design in Superconducting Magnets, Proceedings of the $4^{\text {th }}$ International Industrial Symposium on Super Collider (IISSC), New Orleans, pp. 773-800 (1992).

- R. Gupta, et al., Large Aperture Quadrupoles for RHIC Interaction Regions, Proceedings of the 1993 Particle Accelerator Conference, Washington, D.C., pp. 2745-2747 (1993).

- R. Gupta, et al., Field Quality Improvements in Superconducting Magnets for RHIC, Proceedings of the 1994 European Particle Accelerator Conference, London, UK, pp. 2928-2930 (1994).

- R. Gupta, et al., Field Quality Control Through the Production Phase of RHIC Arc Dipoles, Proceedings of the 1995 International Particle Accelerator Conference, Dallas, Texas (1995).

- R. Gupta, Field Quality in the Superconducting Magnets for Large Particle Accelerators, Proceedings of the 1996 European Particle Accelerator Conference at Sitges, Spain (1996). 


\subsection{Introduction}

An iteration in coil cross section is usually carried out to change the allowed harmonics by a constant amount at all currents. To help understand the following discussion, a coil cross section is shown in Fig. 4.1.1. This coil cross section is used in the optimized design for the $100 \mathrm{~mm}$ aperture RHIC insertion dipole $D O$.

The details of the coil cross section design and optimization process are described in Chapter 6, "OPTIMIZED CROSS SECTION DESIGNS". In the present chapter only iterations of a previously optimized design will be discussed. These iterations are required to remove the differences between the design and measured harmonics in the initial magnets. These differences are present because of (a) a difference between the parts used in the design and in the actual magnet (b) a deformation in the mechanical shape of the coil and iron cross section as a result of the large compressive forces applied on the coils during the magnet assembly (c) deviations in the tooling and manufacturing (assembly) process from the initial ones used, etc. The cross section of the coil is iterated in an attempt to remove systematic values of field harmonics due to such sources. This is usually accomplished by changing the copper wedges in the coil, an approach which is discussed later in this chapter. This method, however, requires a long time to implement since a new magnet must be built. Moreover, in addition to a wedge itself, several other associated components (particularly in the coil ends) may also require a change. In RHIC magnets, an alternate approach to coil cross section iteration is used which does not require changes in the wedges (and associated components). A symmetric gap at the coil midplane has been used to effectively reduce the crucial decapole harmonic in RHIC arc dipoles. Moreover, the coil pole shims can be used as additional parameters to adjust a number of field harmonics. A large octupole harmonic in RHIC quadrupoles (which like dipoles are collared in a 2-fold symmetric way) is removed by using unequal coil to midplane gaps between horizontal and vertical planes. These methods are discussed in separate sections. The calculations presented in this chapter are mostly performed with the computer code PAR2DOPT [130] which computes field harmonics analytically based on coils made of constant current density, trapezoidal conductors in an infinite permeability circular iron cylinder. 


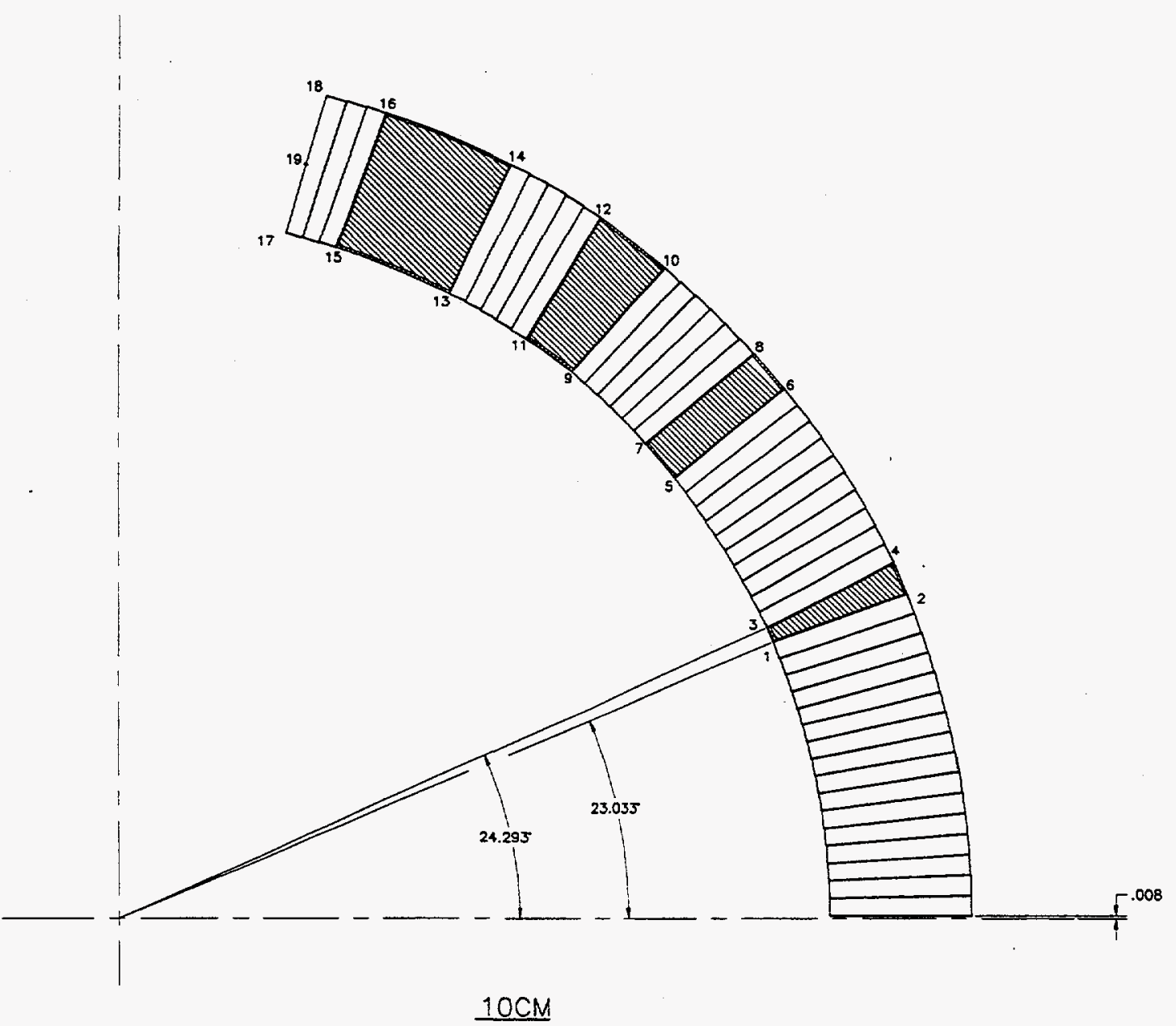

Figure 4.1.1: The coil cross section used in the design of the $100 \mathrm{~mm}$ aperture RHIC insertion dipole $D 0$. This shows only one half of the upper coil, the other half is symmetric with respect to the vertical plane. The shaded parts represent copper wedges and the non-shaded part, the insulated superconducting cable. The first wedge is between the points marked 1, 2, 3 and 4 and angles 23.033 and 24.293 degrees. ".008" represents the coil midplane gap of $0.008^{\prime \prime}(\sim 0.2 \mathrm{~mm})$ as measured from the horizontal axis of the magnet. Point 19 represents the location where the coil pole angle is defined. 


\subsection{Sources of Harmonics Allowed by the Magnet Geometry}

A cross section iteration is generally carried out to remove only allowed harmonics. The allowed harmonics are those which are allowed by the symmetry of the magnet cross section, for example, $b_{2}, b_{4}, b_{6}, \ldots$ in dipoles. Dipole coils have a reflection symmetry with respect to both the horizontal and vertical plane which effectively generates a four fold symmetry. Because of this basic symmetry all harmonics except $b_{2}, b_{4}, b_{6}, \ldots$ will be zero. The presence of harmonics other then these means that the ideal dipole symmetry is broken. Similarly quadrupoles have eight fold symmetry and $b_{5}, b_{9}, b_{13}$, etc. are the only allowed harmonics.

The allowed harmonics are primarily caused by (a) systematic errors in components used in the magnet (b) an imperfect design which is limited by the number of parameters used in cross section optimization and (c) the difference between the design and the realized cross section due to mechanical and thermal deformations discussed below. The value of the allowed harmonics is primarily controlled with copper wedges. These wedges are placed between the blocks of superconducting cables and they primarily determine where an individual turn will go. To obtain a high field quality, as desired in high energy accelerator magnets, the conductors must be placed at an appropriate location to an accuracy of 50 $\mu m$. However, after the coils are wound, they go through a significant deformation during the curing, collaring and cool down processes and there is no direct control on where an individual turn will actually end up (although, the overall coil dimensions are constrained by the cavity in which the coil must fit). In principle, one could compute these mechanical changes and incorporate them in the original design. In practice this is not done since the reliability of mechanical model calculations is generally less than the required accuracy of 25-50 $\mu \mathrm{m}$ in geometric deformation. The mechanical errors due to these effects do not alter the basic magnet symmetry and therefore only the allowed harmonics are generated in the process. These harmonics are removed by coil cross section iterations.

In order to simplify the magnet construction and to save the cost of tooling used in the collaring process, all RHIC quadrupoles are collared like dipoles. This collaring process creates an elliptical deformation in the coil and iron shape (which creates the allowed harmonics $b_{2}, b_{4}, b_{6}, \ldots$ in dipoles). However, the elliptical shape does not have a reflection symmetry at the quadrupole poles, which are at $45^{\circ}, 135^{\circ}, 225^{\circ}$ and $315^{\circ}$. As a result, in addition to the quadrupole allowed harmonics $b_{5}, b_{9}, b_{13}, \ldots$, the normally non-allowed harmonics $b_{3}, b_{7}, b_{9}, \ldots$ are also generated. 


\subsection{Reduction in the Allowed Harmonics through Wedges}

The most common method of removing the systematic values of allowed harmonics measured in the magnet is to change the size of the wedges used in the initial design. The possible sources of them and limitations in predicting these harmonics accurately in the initial design have been discussed in the last two sections. To overcome those limitations, the measured systematic values of allowed harmonics are empirically removed by making a new cross section such that the design harmonics have the same magnitude but opposite sign to the measured harmonics. Care is taken so that the change in wedge thickness and other mechanical component is small. A small change assures that the mechanics of the cross section will not change significantly and the iterated cross section will go through a mechanical deformation similar to the original one. This approach has been found quite successful in RHIC magnets [71,72].

In this paragraph the SSC $50 \mathrm{~mm}$ aperture dipole magnet cross section (Fig. 3.2.2) is examined. This cross section is described in detail in chapter "OPTIMIZED CROSS SECTION DESIGNS". The basic coil cross section consists of two layers of coils with three wedges in the inner layer and one in the outer. In the first four rows of Table 4.3.1, the computed change in the transfer function and field harmonics are given at a reference radius of $10 \mathrm{~mm}$ for a change in the wedge thickness of $+25 \mu \mathrm{m}$. The changes in the values of harmonics higher than $b_{6}$ is small and they are not given here. In these calculations the pole width is held constant while the wedge thickness is changed. This means that the effective cable thickness is reduced by an appropriate amount. The wedge number counting starts in the inner layer and in each layer in the wedge which is closest to the midplane. The pole width, which was kept constant in these calculations, however, need not be constant in general; the effect of increasing the pole width by $+25 \mu \mathrm{m}$ in the inner or outer layer is given in the final two rows.

In Table 4.3.2, the change in transfer function and in field harmonics is given at the 25 $\mathrm{mm}$ reference radius for $\mathrm{a}+25 \mu \mathrm{m}$ change in wedge thickness, pole width and coil midplane gap in the $80 \mathrm{~mm}$ aperture RHIC arc dipoles [72] whose model is shown in Fig. 3.2.1.

When an iteration in the coil design is performed following the guidelines given earlier (that the mechanical changes are small during iteration), the differences in field harmonics between calculation and measurement are within the errors expected from the mechanical tolerances [71]. 
Table 4.3.1: The effect of $a+25 \mu m$ change in a wedge thickness or pole width on the transfer function and the field harmonics in the SSC $50 \mathrm{~mm}$ aperture dipole magnet. The field harmonics are calculated with a $10 \mathrm{~mm}$ reference radius. The numbering of the wedges is counter-clockwise from the midplane. The pole width is measured from the vertical axis.

\begin{tabular}{|c|c|c|c|c|}
\hline $\begin{array}{c}\text { Parameter } \\
\text { changed }\end{array}$ & $\begin{array}{c}\delta T F \\
10^{-4} \frac{T}{k .}\end{array}$ & $\begin{array}{c}\delta b_{2} \\
10^{-4}\end{array}$ & $\begin{array}{c}\delta b_{4} \\
10^{-4}\end{array}$ & $\begin{array}{c}\delta b_{6} \\
10^{-4}\end{array}$ \\
\hline Wedge No. 1 & -0.78 & -0.24 & 0.01 & 0.005 \\
Wedge No. 2 & 0.42 & 0.30 & 0.03 & -0.005 \\
Wedge No. 3 & 1.16 & 0.36 & -0.02 & 0.00 \\
Wedge No. 4 & -0.29 & -0.06 & 0.00 & 0.00 \\
\hline Pole Width (inner) & 2.0 & 0.23 & -0.03 & 0.005 \\
Pole Width (outer) & 1.13 & 0.21 & 0.00 & 0.000 \\
\hline
\end{tabular}

Table 4.3.2: The computed change in the transfer function and field harmonics produced by a $+25 \mu \mathrm{m}\left(0.001^{\prime \prime}\right)$ change in the wedge thickness, pole width or midplane gap in the RHIC $80 \mathrm{~mm}$ aperture arc dipoles. The field harmonics are calculated with a $25 \mathrm{~mm}$ reference radius. The numbering of the wedges starts at the midplane. The pole width and midplane gap are measured from the vertical and horizontal axis, respectively.

\begin{tabular}{|c|c|c|c|c|c|}
\hline $\begin{array}{c}\text { Parameter } \\
\text { changed }\end{array}$ & $\begin{array}{c}\delta T F \\
10^{-4} \frac{T}{k A}\end{array}$ & $\begin{array}{c}\delta b_{2} \\
10^{-4}\end{array}$ & $\begin{array}{c}\delta b_{4} \\
10^{-4}\end{array}$ & $\begin{array}{c}\delta b_{6} \\
10^{-4}\end{array}$ & $\begin{array}{c}\delta b_{8} \\
10^{-4}\end{array}$ \\
\hline Wedge 1 & -0.6 & -0.98 & -0.122 & 0.061 & 0.043 \\
\hline Wedge 2 & 0.1 & 0.69 & 0.423 & 0.022 & -0.050 \\
\hline Wedge 3 & 1.1 & 1.42 & -0.090 & -0.068 & 0.041 \\
\hline Pole Width & 1.7 & 1.11 & -0.154 & 0.039 & -0.014 \\
\hline Midplane Gap & -0.9 & -1.68 & -0.557 & -0.156 & -0.050 \\
\hline
\end{tabular}




\subsection{Reduction in the Allowed Harmonics in RHIC Arc Dipoles by Chang- ing the Midplane Gap}

The Northrop Grumman Corporation (NGC) started building the $80 \mathrm{~mm}$ aperture RHIC arc dipoles based on a design previously tested in prototype magnets built at BNL. Due to differences in tooling and in the details of the manufacturing process between the magnets built at BNL and NGC, a small change in the harmonics (particularly the first few allowed ones) was expected. Such differences are normally removed by changing the wedges in the coil cross section in an iterated design as discussed in the last section. The most critical allowed harmonic in $80 \mathrm{~mm}$ RHIC arc dipoles is $b_{4}$, since the sextupole correctors incorporated in the machine have a more than adequate capacity for changes in $b_{2}$. Moreover, $b_{6}$ and higher harmonics were not expected to deviate much from the value measured in prototype magnets.

To adjust only the $b_{4}$ harmonic by a small amount, a more efficient scheme than the wedge-based iteration was incorporated in the production RHIC arc dipole design. In these magnets, the measured $b_{4}$ is removed through an adjustment in the gap between the coil and the midplane. In the original BNL-built prototype magnets, $\sim 0.10 \mathrm{~mm}$ Kapton "caps" were placed on the top and bottom coils at the midplane. These Kapton caps are used to provide electric insulation between the two coils. In the production dipole design, this gap was increased to $\sim 0.15 \mathrm{~mm}$ [71]. The purpose of this increase was to permit an adjustment, if needed, of $\pm 0.05 \mathrm{~mm}(\sim 0.10 \mathrm{~mm}$ to $\sim 0.20 \mathrm{~mm})$ which would change $b_{4}$ by $\sim \mp 1$ unit at 25 $\mathrm{mm}$ reference radius (see Table 4.4.1). In principle, a similar adjustment can be obtained by a change in the coil pole shims, an adjustment which has been routinely used in the past for other purposes. However, as shown in Table 4.3.2, the change required in the pole shim to produce a similar adjustment in $b_{4}$ is over three times the change required in the midplane gap and it also creates a larger change in the $b_{2}$ harmonic. Such a large change in pole width would also cause a bigger change in the compression on the coils, which could affect the quench performance of the magnets.

The midplane gap adjustment method was experimentally tested earlier in the RHIC short dipole DRS009. The magnet was originally built with $0.0045^{\prime \prime}(0.114 \mathrm{~mm})$ thick midplane caps. This magnet was later rebuilt with $0.0063^{\prime \prime}(0.160 \mathrm{~mm})$ thick midplane caps. The total change was $0.0018^{\prime \prime}(0.046 \mathrm{~mm})$. The computed and measured change in harmonics is given in Table 4.4.1. Good agreement between the calculations and measurements can be seen. 
Table 4.4.1: The computed and measured change in field harmonics at $25 \mathrm{~mm}$ reference radius due to a change in the coil midplane gap. The midplane gap was increased from $0.114 \mathrm{~mm}$ to $0.16 \mathrm{~mm}$ in the rebuilt $80 \mathrm{~mm}$ aperture RHIC model dipole magnet DRS009. In the production magnets, the midplane gap was changed back to $0.114 \mathrm{~mm}$ from $0.16 \mathrm{~mm}$ to adjust the $b_{4}$ harmonic.

\begin{tabular}{|l|c|c|c|c|}
\hline & $\Delta b_{2}$ & $\Delta b_{4}$ & $\Delta b_{6}$ & $\Delta b_{8}$ \\
\hline Computed & -3.0 & -1.0 & -0.28 & -0.09 \\
\hline Measured & -3.0 & -1.0 & -0.29 & -0.12 \\
\hline
\end{tabular}

Magnetic measurements in the first few industry-built magnets showed that this adjustment in $b_{4}$ harmonic was indeed needed. After building 19 dipoles with a $0.16 \mathrm{~mm}$ midplane gap, a change to a $0.114 \mathrm{~mm}$ midplane gap [72] was decided on for the next 86 magnets. This adjustment could be quickly implemented in the production as it did not require any change in the wedges or in the coil-ends and already-built coils could be used in the new series. 


\subsection{Reduction in $b_{3}$ in RHIC Quadrupoles with Midplane Gaps}

As mentioned earlier, in order to simplify construction and to save the cost of tooling, the RHIC arc quadrupoles [160] and RHIC insertions quadrupoles [69] are collared like dipoles. This creates an elliptical deformation of the yoke aperture which breaks the quadrupole symmetry and therefore generates the non-allowed octupole harmonic $b_{3}$ (also $b_{7}, b_{11}$, etc.). In early prototype production of RHIC quadrupoles (both $80 \mathrm{~mm}$ aperture and $130 \mathrm{~mm}$ aperture) $\sim 7$ units of $b_{3}$ was observed.

The ideal way to correct this problem would be to start width a pre-ovalized noncircular yoke aperture which would become circular after the deformation during collaring. This would require a new yoke die, an option that is both expensive and time consuming. The amount of pre-ovalization would be based on mechanical calculations. Given that the reliability of those calculations is generally 25-50 $\mu \mathrm{m}$ an additional design iteration may be required to remove the residual $b_{3}$ harmonic.

An alternate method to the above is outlined here. Recall that $b_{3}$ is caused when the quadrupole is not symmetric between the horizontal and vertical planes. The proposed method consists of introducing another asymmetry which is used to compensate for the above asymmetry caused by an elliptical yoke (and hence coil) deformation. This new asymmetry is a difference in the gaps between the horizontal $\left(0^{\circ}\right.$ and $\left.180^{\circ}\right)$ and vertical $\left(90^{\circ}\right.$ and $\left.270^{\circ}\right)$ planes and the coils when the four coils are assembled in the magnet. There are a total of eight such gaps in the quadrupole magnets as, for example, there are two gaps at $0^{\circ}$ - one from horizontal plane to upper coil and another from horizontal plane to lower coil. In the earlier designs these eight coil-to-midplane gaps had an identical value of $0.1 \mathrm{~mm}$. In the present designs to remove $b_{3}$, the four horizontal midplane gaps at $0^{\circ}$ and $180^{\circ}$ are increased from $0.1 \mathrm{~mm}$ to $0.2 \mathrm{~mm}$ but the four vertical gaps $90^{\circ}$ and $270^{\circ}$ are left unchanged at $0.1 \mathrm{~mm}$. This asymmetry of $0.1 \mathrm{~mm}$ between the horizontal and vertical planes generates the right amount of change in $b_{3}$, but with an undesired change in $b_{7}$. This change in midplane gap also increases the average value of the midplane gap by $0.05 \mathrm{~mm}$ which generates the allowed harmonics $b_{5}$ and $b_{9}$. The change in the allowed harmonics $b_{5}$ and $b_{9}$ is corrected in the regular coil cross section iteration. However, a $b_{7}$ would remain remain if nothing additional were done to remove it. In the $80 \mathrm{~mm}$ aperture arc quadrupoles, this $b_{7}$ can be tolerated but in the $130 \mathrm{~mm}$ aperture insertion quadrupoles, it is unacceptable and is corrected with the help of "tuning shims" (to be described in the next chapter). 
An experiment was done to test the validity of this method before incorporating it in to the large scale production. In Table 4.5.1 calculations and measurements are compared in a rebuild of the $130 \mathrm{~mm}$ aperture $\mathrm{RHIC}$ insertion quadrupole QRI002. Good agreement between the calculations and measurements can be seen.

The same method has been used in the $80 \mathrm{~mm}$ aperture arc quadrupole design. A similar experiment was done to verify the validity of this scheme in those magnets.

Table 4.5.1: The measured and computed change in field harmonics caused by an asymmetric increase in the coil-to-midplane gap in the prototype $130 \mathrm{~mm}$ aperture RHIC interaction quadrupole QRI002. The gap was increased by $0.1 \mathrm{~mm}$ in the horizontal plane only. The harmonics are given at a reference radius of $40 \mathrm{~mm}$.

\begin{tabular}{|l|c|c|c|c|}
\hline & $\Delta b_{3}$ & $\Delta b_{5}$ & $\Delta b_{7}$ & $\Delta b_{9}$ \\
\hline Computed & -6.8 & -1.3 & -0.45 & -0.16 \\
\hline Measured & -6.5 & -1.2 & -0.30 & -0.17 \\
\hline
\end{tabular}




\subsection{Coil Cross-section Iterations without Changing Wedges}

In the previous two sections, an adjustment in one critical harmonic with the help of an adjustment in midplane gaps has been discussed. However, the initial design iteration generally requires (a) an adjustment in two harmonics (sometime even more) and (b) an adjustment for a deviation in coil size from the design value either due to a change in the size of the insulated cable, or conversely, a coil size change to obtain a desired compression of the coil. In the past such adjustments have been incorporated by an iteration in the coil cross section through a change in the wedges and associated components in the ends. As mentioned earlier, this is a major design iteration and therefore it can have a significant impact on the cost and schedule.

A simpler alternative to the above approach to cross section iteration has been used in several RHIC magnet designs. In this approach, instead of changing wedges (and hence the coil cross section) both the coil midplane gap and the coil pole shims are adjusted. In a multi-layer coil magnet (all RHIC magnet designs use only a single layer of coil), the number of parameters would be twice the number of layers. In RHIC magnets these two variables (which determine the starting and the end point of the coil) are used to minimize the first two allowed harmonics ( $b_{2}$ and $b_{4}$ in dipoles and $b_{5}$ and $b_{9}$ in quadrupoles). In dipoles, an increase in the midplane gap produces a negative change in all of the allowed harmonics $\left(b_{2}, b_{4}\right.$, etc.) and an increase in the pole shim produces a positive change in $b_{2}$ and a negative change in $b_{4}$. Similarly in quadrupoles, an increase in the midplane gap gives a negative change in all of the allowed harmonics $\left(b_{5}, b_{9}\right.$, etc.) and an increase in the pole shim gives a positive change in $b_{5}$ and a negative change in $b_{9}$. In order for these two parameters to optimize the two harmonics independently, the azimuthal coil size must be allowed to change. A method was developed during the SSC and RHIC magnet $R \& D$ program [5] which permits a modest change in the coil azimuthal size during the coil curing cycle by adjusting the curing pressures and hence effective cable insulation thickness.

Since the third allowed harmonic is $b_{6}$ in dipoles and $b_{13}$ in quadrupoles, the success of this approach means that the allowed harmonics below it can be removed with this method. In the cases where this adjustment in the first two harmonics is sufficient, this is a very efficient approach. Moreover, an asymmetric adjustment in the coil-to-midplane gap and/or coil pole shim can be used to remove additional non-allowed harmonics also, as discussed in the previous section. 
The application of this approach is demonstrated in Table 4.6.1 where the desired adjustment in harmonics in the RHIC $130 \mathrm{~mm}$ insertion quadrupoles is obtained with the help of coil pole shims and midplane shims. As mentioned in the previous section, the midplane shims are made to have different values at the horizontal midplane $\left(0^{\circ}\right.$ and $\left.180^{\circ}\right)$ and at the vertical midplane $\left(90^{\circ}\right.$ and $\left.270^{\circ}\right)$ to eliminate the measured $b_{3}$ in the prototype magnets. In the design used in magnets QRI103-QRI110, the values of coil-to-midplane gap was $0.25 \mathrm{~mm}$ and $0.15 \mathrm{~mm}$ respectively at the horizontal and vertical midplanes which removed practically all $b_{3}$. However, this intial $0.1 \mathrm{~mm}$ asymmetry between the two planes in design 1 generated a relatively large $b_{7}\left(-0.32\right.$ unit). To reduce this large $b_{7}$, the asymmetry was reduced from $0.1 \mathrm{~mm}$ to $0.075 \mathrm{~mm}$ for the rest of the Q1 program (magnets QRI111QRI128), which reduced $b_{7}$ from -0.32 to -0.26 but left a $b_{3}$ of $\sim 2$ unit. This $b_{3}$ is removed by the tuning shims (which are iron shims attached to the yoke inner radius, see the next chapter). The sign of the change in $b_{3}$ and $b_{7}$ due to the asymmetric coil midplane shims and the asymmetric tuning shims is opposite and therefore when the two are used in the proper combination they can make both $b_{3}$ and $b_{7}$ small. Design 2, which is used in magnets QRI111-QRI119 and QRI122-QRI125, has on the average a larger midplane gap (0.27 mm instead of $0.2 \mathrm{~mm}$ ) and a decreased pole shim of $0.03 \mathrm{~mm}$. This was done to obtain a desired change in $b_{5}$. In design 3, which is used in the last three magnets QRI126-QRI128, the pole shims were adjusted by $0.05 \mathrm{~mm}$ to correct for an undesired change in $b_{5}$ caused by errors in other components in the magnet. Design 4 compensated for a reduction in the cable thickness of $0.009 \mathrm{~mm}(9 \mu \mathrm{m})$. Since there are 27 turns in the cross section, this changed the coil size by $0.24 \mathrm{~mm}$. This would have created unacceptable changes in the harmonics and pre-compression of the coils in the magnet. However, in this method, the decrease in cable thickness was accommodated using an increase in the coil midplane gap of $0.09 \mathrm{~mm}$ and an increase in the coil pole shim of $0.15 \mathrm{~mm}$. This proportion of coil midplane gap and coil pole shim adjustment also reduced $b_{9}$ without changing $b_{5}$. This cross section is used in two magnets QRI120 and QRI121. These two magnets were actually used to test the cross section for the longer $130 \mathrm{~mm}$ aperture quadrupoles, where a reduction in $b_{9}$ is much more beneficial because of a larger beam size. 
Table 4.6.1: A summary of designs used in various $130 \mathrm{~mm}$ aperture 1.44 meter long Q1 (QRI) quadrupoles for RHIC insertion regions. All designs used the same wedges and only the coil midplane gap and coil pole shims were changed. The cable used in all but design 4 had the same thickness. The thickness in the cable of design 4 was smaller by $0.009 \mathrm{~mm}$. Since there are 27 turns in the cross section, this would have meant a $0.24 \mathrm{~mm}$ change in the coil size. The calculated field harmonics are given at $40 \mathrm{~mm}$ reference radius. Generally good agreement has been found between the calculated and measured changes in field harmonics during these iterations. $b_{3}$ and $b_{7}$ are further reduced by tuning shims which were not included in these calculations.

\begin{tabular}{|c|c|c|c|c|}
\hline Parameter & Design 1 & Design 2 & Design 3 & Design 4 \\
\hline Magnets QRI... & $103-110$ & $\begin{array}{c}111-119 \\
122-125\end{array}$ & $126-128$ & $120-121$ \\
\hline Coil pole shim & $0.46^{\mathrm{a}} \mathrm{mm}$ & $0.43 \mathrm{~mm}$ & $0.48 \mathrm{~mm}$ & $0.63 \mathrm{~mm}$ \\
\hline Midplane gap $\left(0^{\circ}, 180^{\circ}\right)$ & $0.25 \mathrm{~mm}$ & $0.30 \mathrm{~mm}$ & $0.30 \mathrm{~mm}$ & $0.39 \mathrm{~mm}$ \\
\hline Midplane gap $\left(90^{\circ}, 270^{\circ}\right)$ & $0.15 \mathrm{~mm}$ & $0.225 \mathrm{mil}$ & $0.225 \mathrm{~mm}$ & $0.315 \mathrm{~mm}$ \\
\hline Midplane gap (asymmetry) & $0.1 \mathrm{~mm}$ & $0.075 \mathrm{~mm}$ & $0.075 \mathrm{~mm}$ & $0.075 \mathrm{~mm}$ \\
\hline Midplane gap (average) & $0.2 \mathrm{~mm}$ & $0.27 \mathrm{~mm}$ & $0.27 \mathrm{~mm}$ & $0.36 \mathrm{~mm}$ \\
\hline Body $b_{3}$ & 0.5 & 2.2 & 2.2 & 2.2 \\
\hline Body $b_{5}$ & 3.2 & 1.0 & $1.0^{b}$ & 1.0 \\
\hline Body $b_{7}$ & -0.32 & -0.2 & -0.2 & -0.2 \\
\hline Body $b_{9}$ & 0.7 & 0.5 & 0.5 & -0.05 \\
\hline
\end{tabular}

a The actual thickness of the pole shim used in these magnets was $0.53 \mathrm{~mm}$ but the thickness of the pole piece was smaller by $0.07 \mathrm{~mm}$. The value $0.46 \mathrm{~mm}$ used in the table accounts for this difference in the pole thickness between these and the subsequent magnets.

b The calculated value of $b_{5}$ was 2.5 unit; the value given above includes corrections for errors in the size of other components. 


\subsection{Conclusions on the Field Quality Improvements through Coil Design}

It has been demonstrated that the coil cross section can be reliably iterated to obtain field harmonics which are quite close to the desired values. Mechanical changes in the coil cross section should be minimized during such iterations to minimize the change in mechanical deformations between the two designs. This disciplined approach has resulted in producing desired changes in field harmonics during cross section iteration whether a change in a wedge is required or not. This is an improvement over past experience where a good field quality was not always obtained in a single coil cross section iteration.

A change in coil midplane gap has been found to be an efficient method to reduce the crucial decapole $\left(b_{4}\right)$ harmonic during the production run of the RHIC arc dipoles. An asymmetric coil midplane gap has been used to reduce the octupole harmonic which is generated in RHIC quadrupoles due to dipole-like collaring. Instead of iterating the coil cross section by changing wedges, a limited adjustment in harmonics and mechanical parameters has been obtained by adjusting the coil midplane gap and the coil pole shims. This has been used in a number of design iterations in the $130 \mathrm{~mm}$ aperture $\mathrm{RHIC}$ interaction region quadrupoles where changes in the $b_{3}, b_{5}, b_{7}$ and $b_{9}$ harmonics and/or a change in cable size is corrected. Since the wedges and the associated components in the ends are not changed, this is an efficient alternative method. In addition this method allows for using coils which have already been built. 


\section{Chapter 5.}

\section{FIELD QUALITY IMPROVEMENTS AFTER CON- STRUCTION}

The research work described in this chapter is partly based on the following papers :

- R.C. Gupta, Correcting Field Harmonics after Design in Superconducting Magnets, Proceedings of the $4^{\text {th }}$ International Industrial Symposium on Super Collider (IISSC), New Orleans, pp. 773-780 (1992).

- R. Gupta, Iron Shims to Correct the Measured Harmonics in $130 \mathrm{~mm}$ Aperture RHIC Insertion Quadrupoles, Magnet Division Internal Note 480-16 (RHIC-MD-185), Dec. 10, 1992 , Unpublished (1992).

- R. Gupta, et al., Large Aperture Quadrupoles for RHIC Interaction Regions, Proceedings of the 1993 Particle Accelerator Conference, Washington, D.C., pp. 2745-2747 (1993).

- R. Gupta, et al., Field Quality Improvements in Superconducting Magnets for RHIC, Proceedings of the 1994 European Particle Accelerator Conference, London, UK, pp. 2928-2930 (1994).

- R. Gupta, et al., Turing Shims for High Field Quality in Superconducting Magnets, Proceedings of the Fourteenth International Conference on Magnet Technology (MT-14), Tampere, Finland, June 11-16 (1995).

- R. Gupta, Field Quality in the Superconducting Magnets for Large Particle Accelerators, Proceedings of the 1996 European Particle Accelerator Conference at Sitges, Spain (1996). 


\subsection{Introduction}

The field quality in superconducting magnets is mainly determined by the following three factors :

(a) The inherent harmonic components in the magnetic design

(b) The errors in the dimensions of the parts used in making the magnets

(c) The errors (non-reproducible distortions) in the magnets

(a) was discussed in chapters 2 and 3 where the field quality improvements through yoke and coil designs were discussed. In this chapter methods to overcome the limitations posed by (b) and (c), which usually determine the ultimate field quality in superconducting magnets, are developed. The tolerances in components, in manufacturing and in the assembly are typically of the order of $25-50 \mu m$. An attempt to improve on those mechanical tolerances would be too expensive and too demanding, so for all practical purposes the field errors from them set the limit on the expected field quality in superconducting magnets. However, sometimes the performance of a machine may be limited by these harmonics.

To overcome these limitations, methods are developed here which correct or compensate for the measured harmonics after the construction of a magnet is partly complete. These corrections are applied either at the body of the magnet or lumped at the ends of the magnet. First, the "tuning shim" method is discussed, which is applied in the body of the magnet. Then in the next section, the " $a_{1}$ correction" method is discussed which is applied at the ends of the magnet. Neither of the two methods requires disassembling the already built magnet. The relative field errors $(\Delta B / B)$ in superconducting magnets are generally of the order of $10^{-4}$ at $2 / 3$ of the coil radius. These methods can reduce these relative field errors by about an order of magnitude provided that the harmonic measurements are reliable and the harmonic contents in the magnets are reproducible after quench and thermal cycles.

A correction in the field non-uniformity by attaching iron shims on the yoke surface is now routinely made in MRI (Magnetic Resonance Imaging) magnets and has also been used in window frame [39] magnets. The use of iron shim methods in superconducting accelerator magnets based on methods similar to those discussed here has been proposed elsewhere $[50,81]$. 


\subsection{Tuning Shims in Magnet Body for Extra High Field Quality}

A tuning shim correction method has been incorporated in the basic design of the 130 $\mathrm{mm}$ aperture $\mathrm{RHIC}$ insertion quadrupoles to compensate the measured field harmonics in the body of the magnet. The length of these tuning shims is the same as the mechanical length of the magnet. They are made partly of magnetic and partly of non-magnetic material with the actual composition of each shim determined by the harmonics in an individual magnet. The tuning shim method described here can reduce the targeted harmonics to a level where the value of them is determined only by the measurement and reproducibility errors in the magnets.

The subsequent discussion here is limited to the RHIC insertion quadrupoles, but the basic principle and method could apply to any superconducting magnet design. In the following sub-sections the principle of the tuning shim correction, the basic magnet and tuning shim design, the procedure for implementing it in the magnet, the calculations, and a comparison between the calculations and the measurements will be discussed.

\subsubsection{Tuning Shims in the RHIC Interaction Region Quadrupoles}

To obtain a high luminosity ( $a$ term used in specifying the probability of interaction of particle beams in collision) for a given number of particles in the beam, the beam is squeezed to a very small size at the crossing (collision) point. This is called the low $\beta^{*}$ squeeze as the $\sqrt{\beta}$ is proportional to beam size. An unavoidable consequence is an increase in beam size in the quadrupoles located on either side of the crossing point. The maximum tolerable beam size is determined by the field quality in the magnets since the physical aperture is more than the good field aperture. In RHIC the ultimate luminosity performance is determined by the field quality in the interaction region quadrupoles used in the "low $\beta^{*}$ " focussing triplet. To overcome the usual mechanical limitations on field quality a method of Tuning Shims has been developed for the $130 \mathrm{~mm}$ aperture RHIC interaction region quadrupoles. The method is referred to as the Tuning Shim Method because it corrects the measured harmonics in an individual magnet by adjusting (tuning) the amount of iron in each of several tuning shims. Such methods have been previously used to obtain a high field uniformity in room temperature, window-frame, iron-dominated magnets.

The basic principle behind this passive correction method is to place magnetic material (shims) at the inside surface of the yoke aperture. There, it is magnetized and creates 
a large local distortion in the magnetic field as well as a significant change in the shape of the field at the center of the magnet. This change in field shape can be expressed in terms of harmonic components. By properly choosing the locations and dimensions of these magnetic shims one can cancel out the field errors. The number of measured harmonics which can be independently optimized using this method is equal to or less then the number of shims used. An undesired effect of this method is that in the process of correcting lower order harmonics, higher order harmonics are also generated. However, the location of the shims can be chosen to minimize these higher order effects. The location of these shims is also chosen to minimize iron saturation effects. This requires that the tuning shims be put as close to the yoke aperture as possible.

In the $130 \mathrm{~mm}$ aperture RHIC interaction region quadrupoles, eight tuning shims are used. They are used to correct eight harmonics $-a_{2}$ through $a_{5}$ and $b_{2}$ through $b_{5}$. Physically a single tuning shim is a package of epoxied laminations. The thickness of the package is constant, but some of the laminations are made of magnetic low carbon steel and some are made of non-magnetic brass. The adjustment (tuning) of the harmonics is achieved by varying the ratio of magnetic to non-magnetic laminations in the eight shims. 


\subsubsection{Tuning Shim and the Magnet Design}

The magnetic lengths of the three interaction region quadrupoles in the focusing triplet are $1.44 \mathrm{~m}, 3.4 \mathrm{~m}$ and $2.1 \mathrm{~m}$. They all have the same cross section. The cross section of these magnets is shown in Fig. 5.2.1 which also shows the locations of the eight tuning shims $1 \mathrm{~A}$ through 4B. The complete design of this magnet will be described in detail in chapter 6. The yoke inner surface consists of two circular radii instead of the usual one radius to reduce the saturation induced $b_{5}$. The yoke inner radius is $87 \mathrm{~mm}$ at the midplane and $92 \mathrm{~mm}$ at the pole. The space between the coil and yoke is filled with a thin, glass-filled, phenolic spacer. The outer surface of the spacer also has two radii, $87 \mathrm{~mm}$ at the midplane and $92 \mathrm{~mm}$ at the pole. However, eight gaps are left between the yoke and phenolic spacer. The location of gap 2B in the first octant is about $30^{\circ}$. The location of the other seven gaps is determined by the quadrupole symmetry, for example gap $2 \mathrm{~A}$ is at $60^{\circ}, 1 \mathrm{~B}$ is at $120^{\circ}$, etc. (see Fig. 5.2.1).

A schematic diagram of the tuning shim in the magnet at location $1 B$ is also shown in Fig. 5.2.1. The actual tuning shim is a package of a number of low carbon steel (magnetic) and brass (non-magnetic) laminations. The use of laminations assures small eddy currents. These laminations have a width of $6.6 \mathrm{~mm}$ and a thickness of either $0.13 \mathrm{~mm}$ or $0.51 \mathrm{~mm}$. The total thickness of all laminations in each tuning shim package is always $6.1 \mathrm{~mm}$, but the proportion of iron laminations varies. The lamination pack is cured into a parallelogram shape in a fixture with epoxy between the laminations. At the end of the curing cycle the tuning shim package is quite sturdy and can be handled easily. The width of the lamination pack is $7.5 \mathrm{~mm}$ and the thickness is $6.35 \mathrm{~mm}$ (6.1 mm laminations and $0.25 \mathrm{~mm}$ epoxy). These dimensions provide a small but sufficient clearance for easy insertion of the tuning shims into the magnet. The two thicknesses of laminations (0.13 $\mathrm{mm}$ and $0.51 \mathrm{~mm})$ provide the necessary resolution in the harmonic correction while keeping the number of laminations to a reasonable value. The nominal magnetic thickness of the tuning shim is $3.175 \mathrm{~mm}$, of which $3.05 \mathrm{~mm}$ is occupied by the iron laminations; the presence of the epoxy reduces the iron packing factor by $\sim 4 \%$. A composition of the tuning shim package is shown in Fig. 5.2.2 where one side of the tuning shim is shown in more detail. The steel shims are actually the iron laminations which define the magnetic thickness of the tuning shim.

The tuning shims are placed such that the iron laminations are next to the yoke to provide a better magnetic circuit. This has been done to minimize the difference in harmonic 

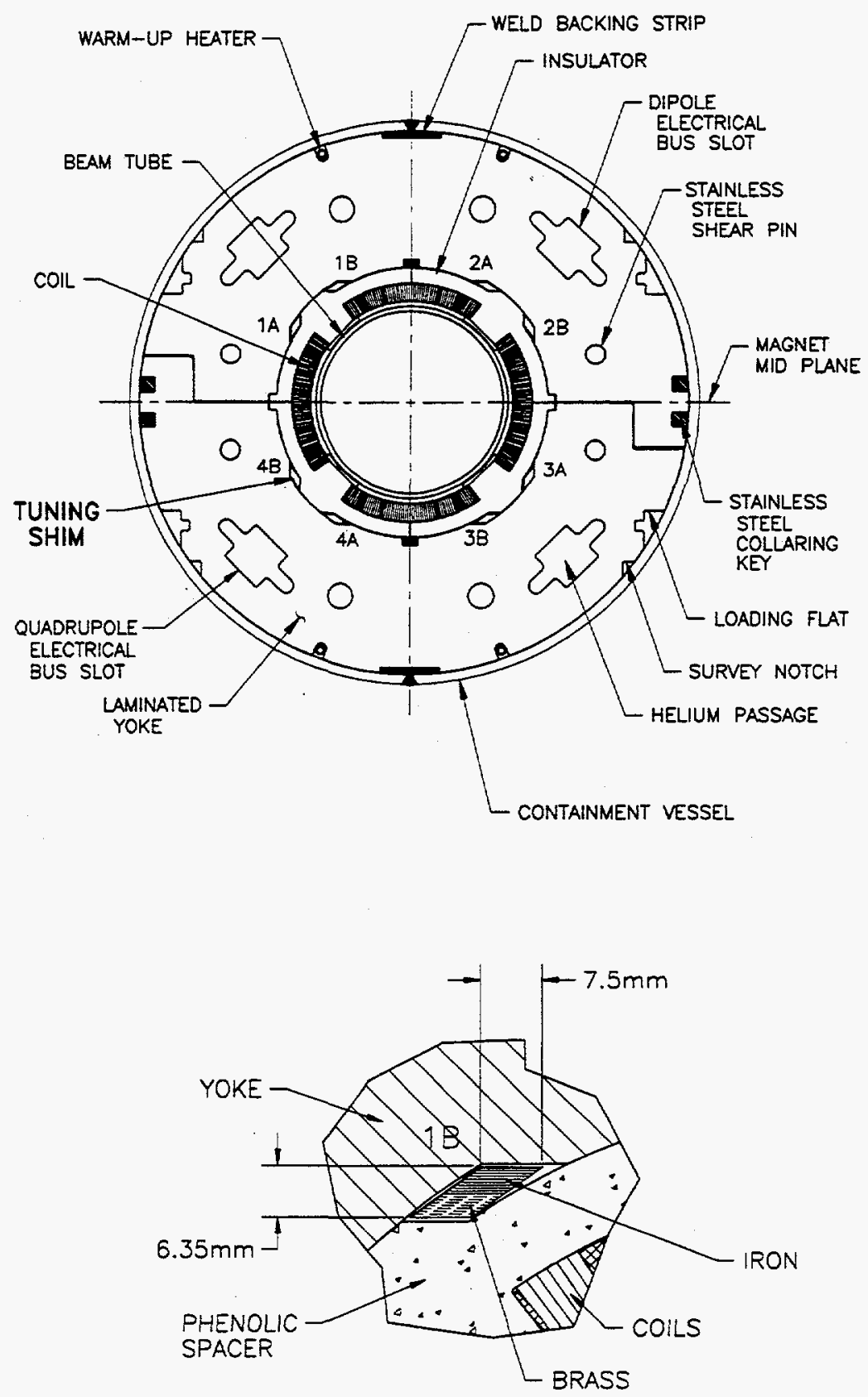

Figure 5.2.1: The upper drawing shows the cross section of the $130 \mathrm{~mm}$ aperture $\mathrm{RHIC}$ interaction quadrupoles. $1 \mathrm{~A}, 1 \mathrm{~B}$, etc. are the locations of eight gaps for tuning shims. The lower drawing shows the tuning shim placed at location $1 \mathrm{~B}$ in the magnet. There are eight such shims in every magnet, each having the same total size but a variable composition of brass and low carbon steel. 


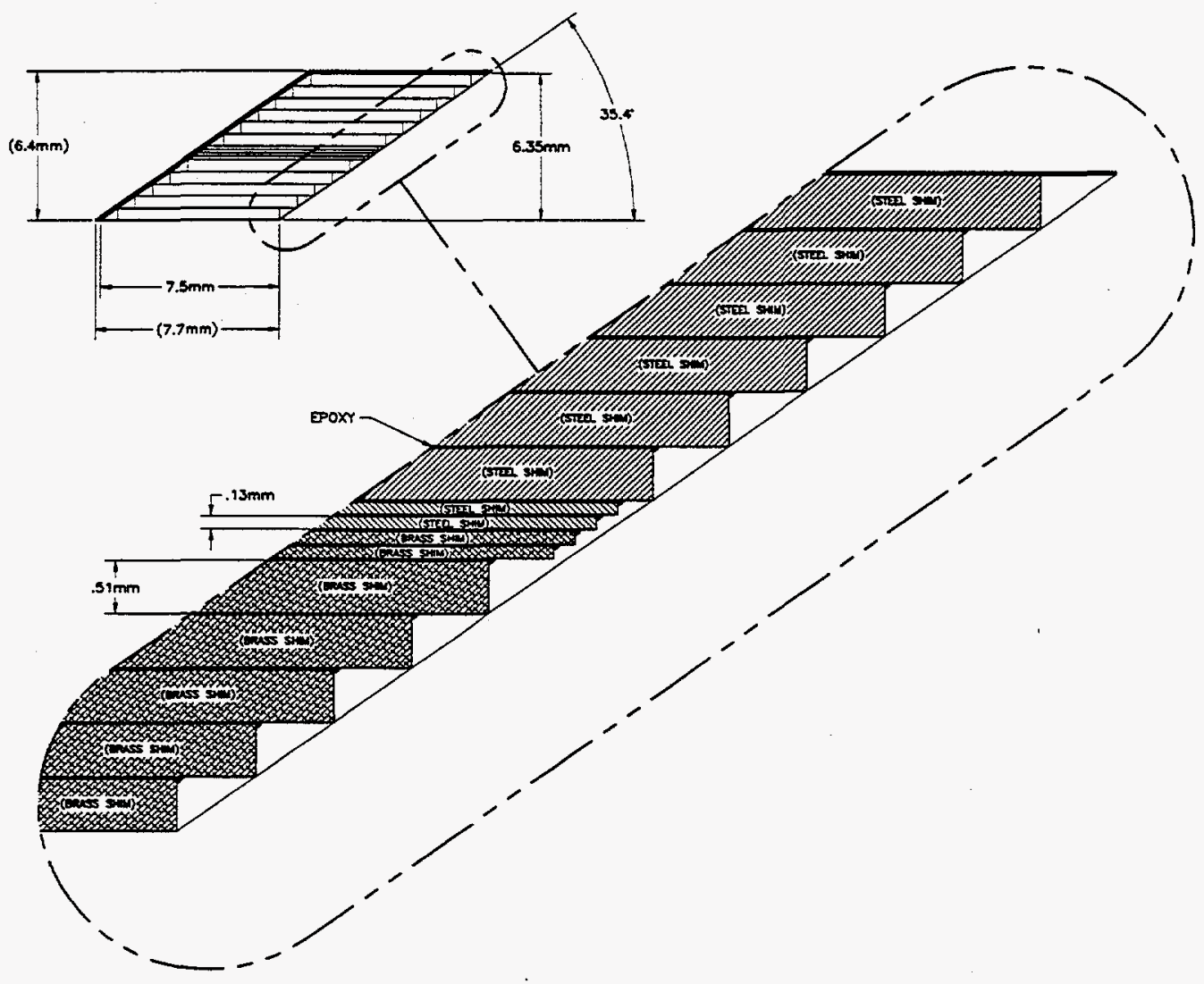

Figure 5.2.2: A composition of the tuning shim package where one side of the tuning shim is shown in more detail. The steel shims are actually the iron laminations which defines the magnetic thickness of the tuning shim. There are 11 laminations (brass or steel) having a thickness of 0.51 $\mathrm{mm}$ and four of $.13 \mathrm{~mm}$. The epoxy provides a bond between them after curing. 
compensation between low current and high current due to iron saturation. However, a small gap is left for the following reasons :

(a) a mismatch in yoke and tuning shim geometries (the yoke inner surface is circular and the defining surface of the shim package is flat)

(b) the actual shape of the tuning shim package is a staircase in which each rectangular lamination is displaced by a small amount to create a parallelogram shape.

(c) a $0.1 \mathrm{~mm}$ thick Kapton sheet is used between the tuning shim and the yoke to provide insulation against eddy currents between the two.

(d) a small clearance is needed for easy insertion of the tuning shims in the magnet. Once the quadrupole is energized, the magnetic attraction between the yoke-iron and lamination-iron pulls the tuning shims close to the yoke inner surface.

\subsubsection{Procedure for Implementing the Tuning Shim Correction}

The complete procedure for installing tuning shims to compensate the field harmonics involves the following steps:

1. Measure the harmonics in the absence of tuning shims,

2. Compute the thickness of magnetic material in each of the eight tuning shims needed to obtain the desired change in eight harmonics,

3. Make eight tuning shims. Each tuning shim is a package that consists of a number of magnetic steel and non-magnetic brass laminations cured to a fixed dimension,

4. Install the eight tuning shims in the magnet at the proper locations, and

5. Measure the field harmonics with the tuning shims installed, for verification.

Since the highest luminosity is desired at the top energy, ideally the magnetic measurements in step 1 should be performed at the maximum operating current which is about 5 kA. However, this would require a costly cryogenic test. To save on the time and cost of the initial magnetic measurements, the field harmonics are measured first at room temperature. It is known by the measurements that, except for a systematic shift (change) in the harmonics allowed by the magnetic geometry, the values of the warm and cold harmonics 
are nearly the same. Therefore, to a reasonable certainty, one is able to predict the values of field harmonics at $5 \mathrm{kA}$ from the warm measurements of the field harmonics.

The thickness of the magnetic material in the tuning shims in step 2 above is therefore, computed based on the warm measurements. The thickness of iron laminations in the eight tuning shim is computed such that they compensate for the harmonics in the straight section (body) of the magnet. As mentioned earlier, the eight tuning shims would have different proportions of iron in them to correct the eight harmonics $a_{2}$ through $a_{5}$ and $b_{2}$ through $b_{5}$. A computer code SHIMCAL [68] has been developed which computes the number of magnetic laminations needed in each of the eight tuning shim locations to produce the required change in the eight harmonics. Ideally these harmonics (except for $b_{5}$ ) should be zero at $5 \mathrm{kA}$ after the tuning shims are installed. The design value of $b_{5}$ in the straight section is -1.2 unit [171] to compensate for a large $b_{5}$ in the magnet ends. In reality, however, a small component may be left because of the limited resolution in the thickness of laminations and because of various other errors involved in the process and in harmonic measurements. However, these residual harmonics are expected to be about an order of magnitude smaller than has been achieved in previous superconducting magnets.

The procedure for manufacturing the tuning shims (step 3) has been described in the previous section.

The eight tuning shims are inserted (step 4) from the return end (non-lead end) of the magnet in the eight pre-defined spaces in the magnet as shown in Fig. 5.2.1. The tuning shims are inserted such that the magnetic laminations are closest to the yoke inner surface. The magnets are not disassembled to install the tuning shims. It may be pointed out that if the magnet is disassembled and reassembled, the field harmonics may change in the process. This is due to small differences in the mechanics involved in the collaring process, which can not be reproduced. Because of these differences, the conductors in the re-collared coil do not go exactly to the same original location. Although these differences may only be of the order of $25 \mu \mathrm{m}$, they produce harmonic changes that are larger than desired in these highly accurate magnets. Therefore, the design feature that allows the shim to be installed without opening the magnet not only makes the process more efficient but also removes the harmonics that would have been created in the reassembly process.

The harmonics are re-measured after the shims are installed (step 5). First they are measured warm for a quick check that (a) the magnetic laminations in the eight tuning shims have the correct thickness, and (b), all of the eight shims have been installed at the 
correct locations and in the correct orientation. If the warm measurements show any error, the shims can be easily taken out and installed again in a relatively short period of time as compared to the time required for a cold test. The warm measurements should show an over-compensation in field harmonics. This is necessary because a part of the compensation is lost at $5 \mathrm{kA}$ due to the saturation of the iron in the tuning shims. Finally, the actual values of field harmonics after shim correction are measured at $5 \mathrm{kA}$ after the magnet is cooled to $\sim 4.5 \mathrm{~K}$.

\subsubsection{Calculations for Tuning Shim Corrections}

In this section calculations for the field harmonics produced by magnetic tuning shims of various thickness are presented. In the nominal design, with the nominal size tuning shims, the change over from iron to brass occurs at about $30^{\circ}$, in the first octant, as shown in Fig. 5.2.1. The eight diamond-shaped tuning shims are placed in these eight locations in such a way that the two iron surfaces of the tuning shim are as close to the two yoke inner surface as possible. However, as mentioned earlier, a small gap between the two surfaces is left for various assembly reasons. Inclusion of such gaps in computer models produces a mesh that may create large errors in the numerical calculations. Moreover, no method is known to include these gaps in analytic calculations that would account for saturable iron in such a geometry. However, to help understand the magnitude of harmonics by tuning shims and to understand geometric and symmetry effects, approximate analytic expressions are obtained even though they do not quite represent the actual problem.

Analytic expressions for the field harmonics produced by the magnetic tuning shims are difficult to obtain due to the followings reasons : (a) the shims have a large size and complex shape, (b) the permeability of the iron (both in the tuning shim region and in the yoke iron) is not constant and varies by a large amount and (c) the shims are located where the yoke aperture is not defined by a simple circular geometry (for which the analytic expressions are easier to derive) but instead is described by two radii in a complex geometry. Therefore analytic methods are used only to help understand symmetry etc., and numerical methods are used for quantitative calculations. The computer code used for the numerical work is POISSON; however the calculations have been verified with results obtained with the codes PE2D and MDP. 


\subsubsection{Approximate Analytic Expressions for Low Field Estimate}

Approximate analytic expressions for the harmonics generated by an iron shim at the yoke inner surface can be derived using the first order perturbation theory such as that used by Halbach [81]. In order to obtain expressions the following assumptions and modifications are made for simplification : (a) The yoke inner surface is described by a circular geometry (rather than two radii) having a constant radius $R_{f}$ throughout the aperture, (b) the field at the yoke inner surface is low and the permeability $(\mu)$ of the iron is constant (c) the field at (inside) the yoke inner surface is perpendicular to it (d) the perturbation is small, and the shim has a radial thickness $\Delta t$ small compared to the radius $R_{f}$ and an angular width $\Delta \theta$ (between angles $\theta_{0}$ and $\theta_{0}+\Delta \theta$ ) small compared $\frac{\pi}{m}$. Though none of these assumptions are valid in the geometry (2-radius aperture) and field (high field where iron is saturated) under consideration, nevertheless, the analytic expressions are obtained to develop a conceptual understanding. For example these expressions would correctly predict the symmetry between the harmonics generated by tuning shims in various locations. Since the agreement is not good, no attempt will be made to compare the measurements with the analytic expressions; the measurements will be compared with the numerical calculations.

Following a procedure similar to that discussed by Halbach [81] the effect of a tuning - shim to first order is described by changing the radial component of the field at the iron surface. This perturbation and the inverse Fourier transformations given in Eq. (1.5.33c) and Eq. (1.5.33h) are used to compute the change in the field harmonics (convention : $b_{2}$ for normal sextupole) due to this additional iron in the tuning shim as follows :

$$
\begin{aligned}
\Delta a_{n} & =\frac{10^{4}}{\pi B_{R_{0}}} \int_{R_{f}-\Delta t}^{R_{f}}\left(\frac{R_{0}}{r}\right)^{n} \frac{d r}{r} \int_{\theta_{0}}^{\theta_{0}+\Delta \theta} \Delta B_{r}(r, \theta) \cos ((n+1) \theta) d \theta \\
\Delta b_{n} & =\frac{10^{4}}{\pi B_{R_{0}}} \int_{R_{f}-\Delta t}^{R_{f}}\left(\frac{R_{0}}{r}\right)^{n} \frac{d r}{r} \int_{\theta_{0}}^{\theta_{0}+\Delta \theta} \Delta B_{r}(r, \theta) \sin ((n+1) \theta) d \theta
\end{aligned}
$$

where $B_{R_{0}}$ is the unperturbed field on the median plane at $x=R_{0}$ and $\Delta B_{r}(r, \theta)$ is the incremental radial component of the field due to the shim iron. The incremental field is computed at the yoke inner surface where the tuning shims are placed. The radial component of the field inside the yoke inner surface (air region) in the case of an ideal $2 m$ pole magnet with a current sheet at a radius $a$ having a distribution $I(a, \theta)=I_{0} \cos (m \theta)$ is given in chapter 1 (see Eq. (1.5.115a)). The shim iron enhances this field by $\mu$ and therefore, 
the incremental field due to the shim iron is given by :

$$
\begin{gathered}
\Delta B_{r}(r, \theta)=-\frac{\mu_{o} I_{o}}{2 a}\left(1+\frac{\mu-1}{\mu+1}\left(\frac{r}{R_{f}}\right)^{2 m} \frac{\left[1-\left(\frac{R_{f}}{R_{a}}\right)^{2 m}\right]}{\left[1-\left(\frac{\mu-1}{\mu+1}\right)^{2}\left(\frac{R_{f}}{R_{a}}\right)^{2 m}\right]}\right) \times \\
(\mu-1)\left(\frac{a}{r}\right)^{m+1} \sin (m \theta) .
\end{gathered}
$$

Let

$$
F_{\mu}=\frac{\mu-1}{\mu+1} \frac{\left[1-\left(\frac{R_{f}}{R_{a}}\right)^{2 m}\right]}{\left[1-\left(\frac{\mu-1}{\mu+1}\right)^{2}\left(\frac{R_{f}}{R_{a}}\right)^{2 m}\right]},
$$

which is just $\frac{\mu-1}{\mu+1}$ for $R_{a}>>R_{f}$. Using $F \mu$ in Eq. (5.2.2) one gets :

$$
\Delta B_{r}(r, \theta)=-\frac{\mu_{o} I_{0}}{2 a}(\mu-1)\left(1+F_{\mu}\left(\frac{r}{R_{f}}\right)^{2 m}\right) \sin (m \theta)\left(\frac{a}{r}\right)^{m+1} .
$$

$B_{R_{0}}$, the field at $x=R_{0}$ is obtained from Eq. (1.5.112) as

$$
B_{R_{0}}=-\frac{\mu_{o} I_{o}}{2 a}\left(1+F_{\mu}\left(\frac{a}{R_{f}}\right)^{2 m}\right)\left(\frac{R_{0}}{a}\right)^{m-1}
$$

where $F_{\mu}$ is defined in Eq. (5.2.3).

Substituting Eq. (5.2.2) and Eq. (5.2.5) in Eqs. (5.2.1) and simplifying, one obtains :

$$
\begin{aligned}
& \Delta a_{n}=K_{n} \int_{\theta_{0}}^{\theta_{0}+\Delta \theta} \sin (m \theta) \cos ((n+1) \theta) d \theta \\
& \Delta b_{n}=K_{n} \int_{\theta_{0}}^{\theta_{0}+\Delta \theta} \sin (m \theta) \sin ((n+1) \theta) d \theta
\end{aligned}
$$

with

$$
K_{n}=\frac{(\mu-1) 10^{4} R_{0}^{n-m+1} a^{2 m}}{\pi\left(1+F_{\mu}\left(\frac{a}{R_{f}}\right)^{2 m}\right)} \int_{R_{f}-\Delta t}^{R_{f}}\left(\frac{1}{r^{n+m+1}}+\frac{F_{\mu}}{R_{f}^{2 m}} r^{m-n-1}\right) d r
$$

On integration one obtains :

$$
\begin{aligned}
K_{n}=-\frac{(\mu-1) 10^{4} R_{0}^{n-m+1} a^{2 m}}{\pi\left(1+F_{\mu}\left(\frac{a}{R_{f}}\right)^{2 m} R_{f}^{n+m}\right)} \times \\
{\left[\frac{1-\left(1-\frac{\Delta t}{R_{f}}\right)^{-(n+m)}}{n+m}-F_{\mu} \frac{1-\left(1-\frac{\Delta t}{R_{f}}\right)^{(n-m)}}{n-m}\right] . }
\end{aligned}
$$


For $\Delta t<<R_{f}$, this becomes :

$$
K_{n}=\frac{(\mu-1) 10^{4} R_{0}^{n-m+1} a^{2 m}}{\pi\left(1+F_{\mu}\left(\frac{a}{R_{f}}\right)^{2 m} R_{f}^{n+m}\right)} \times \frac{\left(1+F_{\mu}\right) \Delta t}{R_{f}}
$$

To solve Eqs. (5.2.6) completely, the integration is performed over the angle to obtain :

$$
\begin{aligned}
\Delta a_{n}=K_{n} & \left(\frac{\cos \left[(n+1+m)\left(\theta_{0}+\Delta \theta\right)\right]-\cos \left[(n+1+m) \theta_{0}\right]}{n+1+m}\right) \\
& -K_{n}\left(\frac{\cos \left[(n+1-m)\left(\theta_{0}+\Delta \theta\right)\right]-\cos \left[(n+1-m) \theta_{0}\right]}{n+1-m}\right) \\
\Delta b_{n}= & K_{n}\left(\frac{\sin \left[(n+1+m)\left(\theta_{0}+\Delta \theta\right)\right]-\sin \left[(n+1+m) \theta_{0}\right]}{n+1+m}\right) \\
& -K_{n}\left(\frac{\sin \left[(n+1-m)\left(\theta_{0}+\Delta \theta\right)\right]-\sin \left[(n+1-m) \theta_{0}\right]}{n+1-m}\right)
\end{aligned}
$$

For small $\Delta \theta$ the Eqs. (5.2.6) reduces to :

$$
\begin{array}{lll}
\Delta a_{n}=K_{n} \cos \left((n+1) \theta_{0}\right) & \sin \left(m \theta_{0}\right) & \Delta \theta \\
\Delta b_{n}=K_{n} \sin \left((n+1) \theta_{0}\right) & \sin \left(m \theta_{0}\right) & \Delta \theta
\end{array}
$$

As a reminder $n=0$ is dipole and the current distribution for creating a dipole field is $m=1$. 


\subsubsection{Numerical Calculations for Low Field Correction}

In this section, the results of calculations are given for the field harmonics produced by magnetic shims in the low current (field) case. At low field, the permeability of the iron is large and to a sufficient accuracy, the iron can be considered to have a field-perpendicular boundary condition. In this case, the harmonics do not show any current dependence. The small gap between the tuning shim and the yoke aperture is ignored. The shim thickness is changed in steps of $0.020^{\prime \prime}(0.51 \mathrm{~mm})$. The change for a shim of given thickness is computed going from the no-shim case (rather than the nominal shim case).

The computed harmonics are listed in selected cases. In Table 5.2.1 they are given for a shim thickness of $0.060^{\prime \prime}$ (1.52 mm), in Table 5.2.2 for $0.120^{\prime \prime}(3.05 \mathrm{~mm}$ ), in Table 5.2.3 for $0.180^{\prime \prime}(4.57 \mathrm{~mm})$ and and in Table 5.2.4 for a shim thickness of $0.240^{\prime \prime}(6.10 \mathrm{~mm})$ respectively. The above changes are computed going from the no-shim case. The capability of tuning shims to correct the measured harmonics is computed from the nominal case for which the average thickness of the tuning shims is $0.120^{\prime \prime}(3.05 \mathrm{~mm})$, half of the maximum value. In this shifted system, the thickness of the tuning shims is negative if the magnetic part of the shim is smaller than nominal. 
Table 5.2.1: Computed low field change in harmonics caused by 1.52 $\mathrm{mm}\left(0.060^{\prime \prime}\right)$ thick magnetic tuning shims at the given location, LOC, (see Fig. 5.2.1). The transfer function is given in $T / m / k A$.

\begin{tabular}{|c|c|c|c|c|c|c|c|c|c|c|c|}
\hline LOC & $\delta T F$ & $\delta a_{0}$ & $\delta a_{1}$ & $\delta a_{2}$ & $\delta a_{3}$ & $\delta a_{4}$ & $\delta a_{5}$ & $\delta a_{6}$ & $\delta a_{7}$ & $\delta a_{8}$ & $\delta a_{9}$ \\
\hline None & 0.000 & 0.00 & 0.00 & 0.00 & 0.00 & 0.00 & 0.00 & 0.00 & 0.00 & 0.00 & 0.00 \\
\hline $1 \mathrm{~A}$ & 0.0033 & 3.61 & -1.60 & -0.38 & 1.03 & -0.83 & 0.45 & -0.17 & 0.03 & 0.02 & -0.022 \\
\hline $1 B$ & 0.0033 & 2.32 & 1.60 & -2.58 & 1.03 & 0.26 & -0.45 & 0.17 & 0.03 & -0.06 & 0.023 \\
\hline $2 A$ & 0.0033 & 2.32 & -1.60 & -2.59 & -1.03 & 0.26 & 0.45 & 0.17 & -0.03 & -0.06 & -0.022 \\
\hline $2 B$ & 0.0033 & 3.60 & 1.59 & -0.38 & -1.03 & -0.83 & -0.45 & -0.17 & -0.03 & 0.02 & 0.024 \\
\hline $3 A$ & 0.0033 & -3.60 & -1.60 & 0.38 & 1.03 & 0.83 & 0.45 & 0.17 & 0.025 & -0.03 & -0.02 \\
\hline $3 B$ & 0.0033 & -2.32 & 1.60 & 2.58 & 1.033 & -0.26 & -0.45 & -0.17 & 0.03 & 0.058 & 0.02 \\
\hline $4 \mathrm{~A}$ & 0.0033 & -2.32 & -1.60 & 2.58 & -1.03 & -0.26 & 0.45 & -0.17 & -0.03 & 0.057 & -0.02 \\
\hline $4 B$ & 0.0033 & -3.61 & 1.60 & 0.38 & -1.03 & 0.83 & -0.45 & 0.17 & -0.03 & -0.018 & 0.02 \\
\hline ALL & 0.0267 & 0.00 & 0.00 & 0.00 & 0.00 & 0.00 & 0.00 & 0.00 & 0.00 & 0.00 & 0.00 \\
\hline LOC & TF & $\delta b_{0}$ & $b_{1}$ & $\delta b_{2}$ & $\delta b_{3}$ & $\delta b_{4}$ & $\delta b_{5}$ & $\delta b_{6}$ & $\delta b_{7}$ & $\delta b_{8}$ & $\delta b_{9}$ \\
\hline None & 9.4889 & 0.00 & $10^{4}$ & 0.00 & 0.00 & 0.00 & 0.00 & 0.00 & 0.00 & 0.00 & 0.00 \\
\hline $1 \mathrm{~A}$ & 9.4923 & -2.32 & $10^{4}$ & -2.59 & 1.18 & -0.26 & -0.12 & 0.17 & -0.12 & 0.06 & -0.02 \\
\hline 1B & 9.4923 & -3.59 & $10^{4}$ & -0.38 & -1.18 & 0.83 & -0.12 & -0.17 & 0.12 & -0.02 & -0.02 \\
\hline $2 \mathrm{~A}$ & 9.4923 & 3.60 & $10^{4}$ & 0.38 & -1.18 & -0.83 & -0.12 & 0.17 & 0.12 & 0.02 & -0.02 \\
\hline $2 B$ & 9.4923 & 2.32 & $10^{4}$ & 2.58 & 1.18 & 0.26 & -0.12 & -0.17 & -0.12 & -0.06 & -0.02 \\
\hline $3 A$ & 9.4923 & 2.32 & $10^{4}$ & 2.58 & 1.18 & 0.26 & -0.12 & -0.17 & -0.12 & -0.06 & -0.02 \\
\hline $3 B$ & 9.4923 & 3.60 & $10^{4}$ & 0.38 & -1.18 & -0.83 & -0.12 & 0.17 & 0.12 & 0.02 & -0.02 \\
\hline $4 \mathrm{~A}$ & 9.4923 & -3.60 & $10^{4}$ & -0.38 & -1.18 & 0.83 & -0.12 & -0.17 & 0.12 & -0.02 & -0.02 \\
\hline $4 B$ & 9.4923 & -2.32 & $10^{4}$ & -2.58 & 1.18 & -0.26 & -0.12 & 0.17 & -0.12 & 0.06 & -0.02 \\
\hline ALL & 9.5156 & 0.00 & $10^{4}$ & 0.00 & 0.00 & 0.00 & -0.93 & 0.00 & 0.00 & 0.00 & -0.16 \\
\hline
\end{tabular}


Table 5.2.2: Computed low field change in harmonics caused by 3.05 $\left(0.120^{\prime \prime}\right)$ thick magnetic tuning shims at the given location, LOC, (see Fig. 5.2.1). The transfer function is given in $T / m / k A$.

\begin{tabular}{|c|c|c|c|c|c|c|c|c|c|c|c|}
\hline LOC & $\delta T F$ & $\delta a_{0}$ & $\delta a_{1}$ & $\delta a_{2}$ & $\delta a_{3}$ & $\delta a_{4}$ & $\delta a_{5}$ & $\delta a_{6}$ & $\delta a_{7}$ & $\delta a_{8}$ & $\delta a_{9}$ \\
\hline None & 0.000 & 0.00 & 0.00 & 0.00 & 0.00 & 0.00 & 0.00 & 0.00 & 0.00 & 0.00 & 0.00 \\
\hline $1 \mathrm{~A}$ & 0.0075 & 7.87 & -3.31 & -1.08 & 2.43 & -1.87 & 0.96 & -0.33 & 0.03 & 0.06 & -0.055 \\
\hline $1 B$ & 0.0075 & 5.23 & 3.31 & -5.67 & 2.43 & 0.44 & -0.96 & 0.41 & 0.03 & -0.12 & 0.055 \\
\hline $2 A$ & 0.0075 & 5.23 & -3.31 & -5.67 & -2.43 & 0.44 & 0.96 & 0.41 & -0.03 & -0.12 & -0.0055 \\
\hline $2 B$ & 0.0074 & 7.85 & 3.30 & -1.08 & -2.43 & -1.87 & -0.96 & -0.33 & -0.03 & 0.06 & 0.055 \\
\hline $3 \mathbf{A}$ & 0.0075 & -7.87 & -3.31 & 1.08 & 2.43 & 1.87 & 0.96 & 0.33 & 0.03 & -0.06 & -0.055 \\
\hline 3B & 0.0075 & -5.23 & 3.31 & 5.67 & 2.43 & -0.44 & -0.96 & -0.41 & 0.03 & 0.12 & 0.055 \\
\hline $4 \mathrm{~A}$ & 0.0074 & -5.22 & -3.30 & 5.67 & -2.43 & -0.44 & 0.96 & -0.41 & -0.03 & 0.12 & -0.055 \\
\hline $4 B$ & 0.0075 & -7.87 & 3.30 & 1.08 & -2.43 & 1.87 & -0.96 & 0.33 & -0.03 & -0.06 & 0.055 \\
\hline ALL & 0.0595 & 0.00 & 0.00 & 0.00 & 0.00 & 0.00 & 0.00 & 0.00 & 0.00 & 0.00 & 0.00 \\
\hline LOC & TF & $\delta b_{0}$ & $b_{1}$ & $\delta b_{2}$ & $\delta b_{3}$ & $\delta b_{4}$ & $\delta b_{5}$ & $\delta b_{6}$ & $\delta b_{7}$ & $\delta b_{8}$ & $\delta b_{9}$ \\
\hline None & 9.4889 & 0.00 & $10^{4}$ & 0.00 & 0.00 & 0.00 & 0.00 & 0.00 & 0.00 & 0.00 & 0.00 \\
\hline $1 \mathrm{~A}$ & 9.4964 & -5.23 & $10^{4}$ & -5.67 & 2.48 & -0.44 & -0.34 & 0.42 & -0.265 & 0.12 & -0.035 \\
\hline $1 \mathrm{~B}$ & 9.4964 & -7.87 & $10^{4}$ & -1.08 & -2.48 & 1.87 & -0.34 & -0.33 & 0.265 & -0.06 & -0.035 \\
\hline $2 A$ & 9.4964 & 7.87 & $10^{4}$ & 1.08 & -2.48 & -1.87 & -0.34 & 0.33 & 0.265 & 0.06 & -0.035 \\
\hline $2 B$ & 9.4964 & 5.21 & $10^{4}$ & 5.67 & 2.48 & 0.44 & -0.34 & -0.42 & -0.265 & -0.12 & -0.035 \\
\hline $3 A$ & 9.4964 & 5.23 & $10^{4}$ & 5.67 & 2.48 & 0.44 & -0.34 & -0.42 & -0.265 & -0.12 & -0.035 \\
\hline $3 B$ & 9.4964 & 7.87 & $10^{4}$ & 1.08 & -2.48 & -1.87 & -0.34 & 0.33 & 0.265 & 0.06 & -0.035 \\
\hline $4 \mathrm{~A}$ & 9.4964 & -7.86 & $10^{4}$ & -1.08 & -2.48 & 1.87 & -0.34 & -0.33 & 0.265 & -0.06 & -0.035 \\
\hline $4 \mathrm{~B}$ & 9.4964 & -5.23 & $10^{4}$ & -5.67 & 2.48 & -0.44 & -0.34 & 0.42 & -0.265 & 0.12 & -0.035 \\
\hline ALL & 9.5484 & 0.00 & $10^{4}$ & 0.00 & 0.00 & 0.00 & -2.70 & 0.00 & 0.00 & 0.00 & -0.30 \\
\hline
\end{tabular}


Table 5.2.3: Computed low field change in harmonics caused by 4.57 $\mathrm{mm}\left(0.180^{\prime \prime}\right)$ thick magnetic tuning shims at the given location, LOC, (see Fig. 5.2.1). The transfer function is given in $T / m / k A$.

\begin{tabular}{|c|c|c|c|c|c|c|c|c|c|c|c|}
\hline LOC & $\delta T F$ & $\delta a_{0}$ & $\delta a_{1}$ & $\delta a_{2}$ & $\delta a_{3}$ & $\delta a_{4}$ & $\delta a_{5}$ & $\delta a_{6}$ & $\delta a_{7}$ & $\delta a_{8}$ & $\delta a_{9}$ \\
\hline None & 0.000 & 0.00 & 0.00 & 0.00 & 0.00 & 0.00 & 0.00 & 0.00 & 0.00 & 0.00 & 0.00 \\
\hline $1 \mathbf{A}$ & 0.0119 & 12.30 & -4.86 & -2.09 & 4.05 & -2.99 & 1.46 & -0.44 & -0.01 & 0.11 & -0.09 \\
\hline $1 B$ & 0.0119 & 8.43 & 4.85 & -8.86 & 4.05 & 0.47 & -1.46 & 0.70 & -0.01 & -0.17 & 0.09 \\
\hline $2 \mathrm{~A}$ & 0.0119 & 8.43 & -4.85 & -8.86 & -4.05 & 0.47 & 1.46 & 0.70 & 0.01 & -0.17 & -0.09 \\
\hline 2B & 0.0119 & 12.30 & 4.85 & -2.09 & -4.05 & -2.99 & -1.46 & -0.44 & 0.01 & 0.11 & 0.09 \\
\hline $3 \mathrm{~A}$ & 0.0119 & -12.30 & -4.85 & 2.09 & 4.05 & 2.99 & 1.46 & 0.44 & -0.01 & -0.11 & -0.09 \\
\hline $3 B$ & 0.0119 & -8.43 & 4.85 & 8.86 & 4.05 & -0.47 & -1.46 & -0.70 & -0.01 & 0.17 & 0.09 \\
\hline $4 \mathrm{~A}$ & 0.0119 & -8.44 & -4.85 & 8.86 & -4.05 & -0.47 & 1.46 & -0.70 & 0.01 & 0.17 & -0.09 \\
\hline $4 B$ & 0.0119 & -12.31 & 4.85 & 2.09 & -4.05 & 2.99 & -1.46 & 0.44 & 0.01 & -0.11 & 0.09 \\
\hline ALI & 0.0947 & 0.00 & 0.00 & 0.00 & 0.00 & 0.00 & 0.00 & 0.00 & 0.00 & 0.00 & 0.00 \\
\hline LOC & $\mathrm{TF}$ & $\delta b_{0}$ & $b_{1}$ & $\delta b_{2}$ & $\delta b_{3}$ & $\delta b_{4}$ & $\delta b_{5}$ & $\delta b_{6}$ & $\delta b_{7}$ & $\delta b_{8}$ & $\delta b_{9}$ \\
\hline None & 9.4889 & 0.00 & $10^{4}$ & 0.00 & 0.00 & 0.00 & 0.00 & 0.00 & 0.00 & 0.00 & 0.00 \\
\hline $1 \mathrm{~A}$ & 9.5008 & -8.44 & $10^{4}$ & -8.86 & 3.68 & -0.47 & -0.670 & 0.70 & -0.42 & 0.173 & -0.04 \\
\hline $1 \mathrm{~B}$ & 9.5008 & -12.30 & $10^{4}$ & -2.09 & -3.68 & 2.99 & -0.669 & -0.44 & 0.42 & -0.115 & -0.04 \\
\hline $2 A$ & 9.5008 & 12.29 & $10^{4}$ & 2.09 & -3.68 & -2.99 & -0.668 & 0.44 & 0.42 & 0.115 & -0.04 \\
\hline $2 B$ & 9.5008 & 8.43 & $10^{4}$ & 8.86 & 3.68 & 0.47 & -0.671 & -0.70 & -0.42 & -0.173 & -0.04 \\
\hline $3 \mathrm{~A}$ & 9.5008 & 8.43 & $10^{4}$ & 8.86 & 3.68 & 0.47 & -0.672 & -0.70 & -0.42 & -0.173 & -0.04 \\
\hline $3 B$ & 9.5008 & 12.30 & $10^{4}$ & 2.09 & -3.68 & -2.99 & -0.670 & 0.44 & 0.42 & 0.113 & -0.04 \\
\hline $4 \mathrm{~A}$ & 9.5008 & -12.31 & $10^{4}$ & -2.09 & -3.68 & 2.99 & -0.673 & -0.44 & 0.42 & -0.114 & -0.04 \\
\hline $4 B$ & 9.5008 & -8.43 & $10^{4}$ & -8.86 & 3.68 & -0.47 & -0.671 & 0.70 & -0.42 & 0.172 & -0.04 \\
\hline ALL & 9.5837 & 0.00 & $10^{4}$ & 0.00 & 0.00 & 0.00 & -5.28 & 0.00 & 0.001 & 0.00 & -0.34 \\
\hline
\end{tabular}


Table 5.2.4: Computed low field change in harmonics caused by 6.10 $\mathrm{mm}\left(0.240^{\prime \prime}\right)$ thick magnetic tuning shims at the given location, LOC, (see Fig. 5.2.1). The transfer function is given in $T / m / k A$.

\begin{tabular}{|c|c|c|c|c|c|c|c|c|c|c|c|}
\hline LOC & $\delta T F$ & $\delta a_{0}$ & $\delta a_{1}$ & $\delta a_{2}$ & $\delta a_{3}$ & $\delta a_{4}$ & $\delta a_{5}$ & $\delta a_{6}$ & $\delta a_{7}$ & $\delta a_{8}$ & $\delta a_{9}$ \\
\hline NONE & 0.000 & 0.00 & 0.00 & 0.00 & 0.00 & 0.00 & 0.00 & 0.00 & 0.00 & 0.00 & 0.00 \\
\hline $1 \mathrm{~A}$ & 0.0165 & 16.65 & -6.13 & -3.39 & 5.82 & -4.11 & 1.89 & -0.49 & -0.08 & 0.18 & -0.13 \\
\hline $1 B$ & 0.0165 & 11.78 & 6.13 & -11.97 & 5.82 & 0.33 & -1.89 & 1.01 & -0.08 & -0.21 & 0.13 \\
\hline $2 A$ & 0.0165 & 11.77 & -6.13 & -11.97 & -5.82 & 0.33 & 1.89 & 1.01 & 0.08 & -0.21 & -0.13 \\
\hline $2 B$ & 0.0165 & 16.64 & 6.13 & -3.39 & -5.82 & -4.11 & -1.89 & -0.49 & 0.08 & 0.18 & 0.13 \\
\hline $3 A$ & 0.0165 & -16.65 & -6.13 & 3.39 & 5.82 & 4.11 & 1.89 & 0.49 & -0.08 & -0.18 & -0.13 \\
\hline $3 B$ & 0.0165 & -11.78 & 6.14 & 11.97 & 5.82 & -0.33 & -1.89 & -1.01 & -0.08 & 0.21 & 0.13 \\
\hline $4 \mathrm{~A}$ & 0.0165 & -11.79 & -6.14 & 11.97 & -5.82 & -0.33 & 1.89 & -1.01 & 0.08 & 0.21 & -0.13 \\
\hline $4 B$ & 0.0165 & -16.67 & 6.13 & 3.39 & -5.82 & 4.11 & -1.89 & 0.49 & 0.08 & -0.18 & 0.13 \\
\hline ALL & 0.1304 & -0.03 & 0.01 & 0.00 & 0.00 & 0.00 & 0.00 & 0.00 & 0.00 & 0.00 & 0.00 \\
\hline LOC & $\mathrm{TF}$ & $\delta b_{0}$ & $b_{1}$ & $\delta b_{2}$ & $\delta b_{3}$ & $\delta b_{4}$ & $\delta b_{5}$ & $\delta b_{6}$ & $\delta b_{7}$ & $\delta b_{8}$ & $\delta b_{9}$ \\
\hline None & 9.4889 & 0.00 & $10^{4}$ & 0.00 & 0.00 & 0.00 & 0.00 & 0.00 & 0.00 & 0.00 & 0.00 \\
\hline $1 \mathrm{~A}$ & 9.5054 & -11.78 & $10^{4}$ & -11.97 & 4.69 & -0.33 & -1.08 & 1.01 & -0.56 & 0.21 & -0.04 \\
\hline $1 B$ & 9.5054 & -16.66 & $10^{4}$ & -3.39 & -4.69 & 4.11 & -1.08 & -0.49 & 0.56 & -0.18 & -0.04 \\
\hline $2 \mathrm{~A}$ & 9.5054 & 16.65 & $10^{4}$ & 3.39 & -4.69 & -4.11 & -1.08 & 0.49 & 0.56 & 0.18 & -0.04 \\
\hline $2 B$ & 9.5054 & 11.77 & $10^{4}$ & 11.97 & 4.69 & 0.33 & -1.08 & -1.01 & -0.56 & -0.21 & -0.04 \\
\hline $3 \mathrm{~A}$ & 9.5054 & 11.78 & $10^{4}$ & 11.97 & 4.69 & 0.33 & -1.08 & -1.01 & -0.56 & -0.21 & -0.04 \\
\hline $3 B$ & 9.5054 & 16.66 & $10^{4}$ & 3.39 & -4.69 & -4.11 & -1.08 & 0.49 & 0.56 & 0.18 & -0.04 \\
\hline $4 \mathrm{~A}$ & 9.5054 & -16.66 & $10^{4}$ & -3.39 & -4.69 & 4.11 & -1.08 & -0.49 & 0.56 & -0.18 & -0.04 \\
\hline $4 B$ & 9.5054 & -11.79 & $10^{4}$ & -11.97 & 4.69 & -0.33 & -1.08 & 1.01 & -0.56 & 0.21 & -0.04 \\
\hline ALL & 9.6193 & -0.03 & $10^{4}$ & 0.00 & 0.00 & 0.00 & -8.48 & 0.00 & 0.00 & 0.00 & -0.30 \\
\hline
\end{tabular}


In Table 5.2.5, the maximum possible correction is listed for the given thickness of tuning shims. In this case the thickness of a tuning shim is computed from the nominal value thickness of tuning shim; so that it can have either a negative or a positive value. In these calculations the magnitude of the shim thickness is the same at all eight locations (for example, it could be four tuning shims with $2 \mathrm{~mm}$ and the other four with $-2 \mathrm{~mm}$ ). For most (but not all) the change in harmonic is symmetric with respect to the sign of the tuning shim thickness. This means that in most cases if " $+x$ " mm thick gives $+b_{n}$ change in harmonics then "- $x$ " mm gives $-b_{n}$ change. However, the most notable exceptions are $b_{5}$ and $b_{9}$. The non-linearity in these harmonics is associated with the non-linearity of the trigonometric expressions $(\Delta \theta$ in Eqs. (5.2.10)) at these angles. It may be emphasized that this is the maximum possible correction. The actual value of the harmonics produced by these shims would have an opposite sign to the measured harmonics. The computed maximum corrections, thus obtained, are given in Fig. 5.2.3 for the skew and normal sextupole $\left(a_{2}\right.$ and $\left.b_{2}\right)$, skew and normal octupole $\left(a_{3}\right.$ and $\left.b_{3}\right)$, skew and normal decapole $\left(a_{4}\right.$ and $\left.b_{4}\right)$ harmonics. In Fig. 5.2.4 they are given for the skew and normal do-decapole $\left(a_{5}\right.$ and $b_{5}$ harmonics and in Fig. 5.2.5 for $a_{9}$ and $b_{9}$ harmonics. 
Table 5.2.5: Maximum possible low field correction in the harmonics as a function of shim thickness $(\mathrm{T})$ in $\mathrm{mm}$ as measured from the nominal value of $3.05 \mathrm{~mm}\left(0.012^{\prime \prime}\right)$ iron. The shim thickness can be either negative or positive. The change in transfer function $(\delta T F)$ is given in $T / m / k A$.

\begin{tabular}{|c|c|c|c|c|c|c|c|c|c|c|c|}
\hline a) & $\delta T F$ & $a_{0}$ & $\delta a_{1}$ & $\delta a_{2}$ & $\delta a_{3}$ & $\delta a_{4}$ & $\delta a_{5}$ & $\delta a_{6}$ & $\delta a_{7}$ & $\delta a_{8}$ & $\delta a_{9}$ \\
\hline 305 & 0722 & 6.85 & 24.54 & 30.74 & 23.24 & 8.87 & 7.576 & 3.004 & 0.324 & 0.792 & 521 \\
\hline-2.54 & 1 & .24 & 21.02 & 7 & 5 & 0 & 7 & 2 & & 2 & 0.452 \\
\hline-2.03 & 0474 & 39.18 & 6 & 2 & 22 & 6.22 & 5.318 & 06 & 0 & 8 & 368 \\
\hline-1.52 & 0.0354 & 29.62 & 13.01 & 15.99 & 12.04 & 4.73 & 4.048 & 1.600 & 0.033 & 0.426 & .281 \\
\hline-1.02 & 0234 & 19.93 & 8.79 & 10.74 & 8.10 & 3.19 & 2.736 & 1.085 & 0.094 & 0.288 & 0.193 \\
\hline-0.51 & 0114 & 10.0 & 4.42 & 5.38 & 4.05 & 1.59 & 1.374 & 0.547 & 0.047 & 0.146 & 0.096 \\
\hline 0.0 & 0.0 & 0.0 & 0.0 & 0.0 & 0.0 & 0.0 & 0.0 & 0.0 & 0 & 0.0 & 0 \\
\hline 0.51 & .0118 & 0 & 438 & -5.39 & 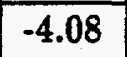 & .62 & .372 & 38 & 5 & 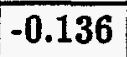 & - \\
\hline 2.08 & & 0.50 & 0.0 & . & 0.12 & 3.19 & .730 & 1.077 & & 0.284 & 0.50 \\
\hline 1.02 & 0334 & 9.62 & 3.01 & 5.97 & 12.09 & -4.74 & 4.039 & 1.599 & -0.137 & -0.419 & -0.201 \\
\hline 2.03 & .0430 & 39.16 & -17.14 & 1.12 & 15.97 & -6.23 & 5.308 & 2.102 & -0.190 & -0.549 & -0.366 \\
\hline 2.54 & .0519 & -48.25 & -21.00 & 26.04 & 9.71 & -6.38 & 6.490 & -2.564 & -0.246 & -0.670 & 0.448 \\
\hline 3.05 & .0598 & -56.88 & -24.52 & -30.72 & -23.28 & -7.57 & -7.575 & -2.987 & -0.324 & -0.784 & -0.524 \\
\hline$\Gamma(m m)$ & $\delta T F$ & $\delta b_{0}$ & $b_{1}$ & $\delta b_{2}$ & $\delta b_{3}$ & $\delta b_{4}$ & $\delta b_{5}$ & $\delta b_{6}$ & $\delta b_{7}$ & $\delta b_{8}$ & $\delta b_{9}$ \\
\hline-3.05 & 0.0720 & 56.87 & $10^{4}$ & 30.73 & 18.78 & 8.87 & 2.726 & 2.988 & 2.236 & 0.786 & .286 \\
\hline-2.54 & 0.0600 & 48.28 & $10^{4}$ & 26.03 & 16.11 & 7.60 & 2.497 & 2.562 & 1.911 & 0.669 & 0.238 \\
\hline-2.03 & 0479 & 39.19 & $10^{4}$ & 2 & 5 & 6.22 & 3 & 3 & 3 & 2 & 0.184 \\
\hline-1.52 & 0.0352 & 29.63 & $10^{4}$ & 15.98 & 10.0 & 4.72 & 1.796 & 1.602 & 1.193 & 0.417 & .127 \\
\hline-1.02 & 232 & 19.92 & $10^{4}$ & 10.75 & 6.76 & 3.18 & 1.295 & 1.076 & 0.808 & 0.284 & 0.081 \\
\hline-0.51 & 0112 & 10.01 & $10^{4}$ & 5.38 & 3.38 & 1.60 & 0.695 & 0.538 & 0.407 & 0.142 & 0.036 \\
\hline 0.0 & 0.0 & 0.0 & $10^{4}$ & 0.0 & 0.0 & 0.0 & 0.0 & 0.0 & 0.0 & 0.0 & 0.0 \\
\hline 0.51 & .0114 & -9.96 & $10^{4}$ & -5.37 & -3.38 & 1.61 & -0.795 & -0.551 & -0.400 & -0.146 & -0.029 \\
\hline 1.02 & 0225 & 19.91 & $10^{4}$ & 0.72 & -6.73 & 3.20 & 1.665 & -1.093 & -0.805 & -0.284 & -0.047 \\
\hline 1.02 & -0.0328 & -20.00 & $10^{4}$ & -10.01 & -9.98 & 4.72 & .638 & .610 & 1.188 & 3 & 0.053 \\
\hline 2.03 & -0.0432 & -39.14 & $10^{4}$ & -21.12 & -13.14 & -6.22 & -3.679 & -2.107 & -1.564 & -0.553 & -0.051 \\
\hline 2.54 & -0.0520 & -48.27 & $10^{4}$ & -26.00 & -16.06 & -6.40 & -4.789 & -2.582 & -1.917 & -0.671 & -0.041 \\
\hline 3.05 & -0.0598 & -56.88 & $10^{4}$ & -30.71 & \begin{tabular}{|l|}
-18.78 \\
\end{tabular} & -7.56 & -5.962 & -3.008 & -2.225 & -0.785 & -0.012 \\
\hline
\end{tabular}




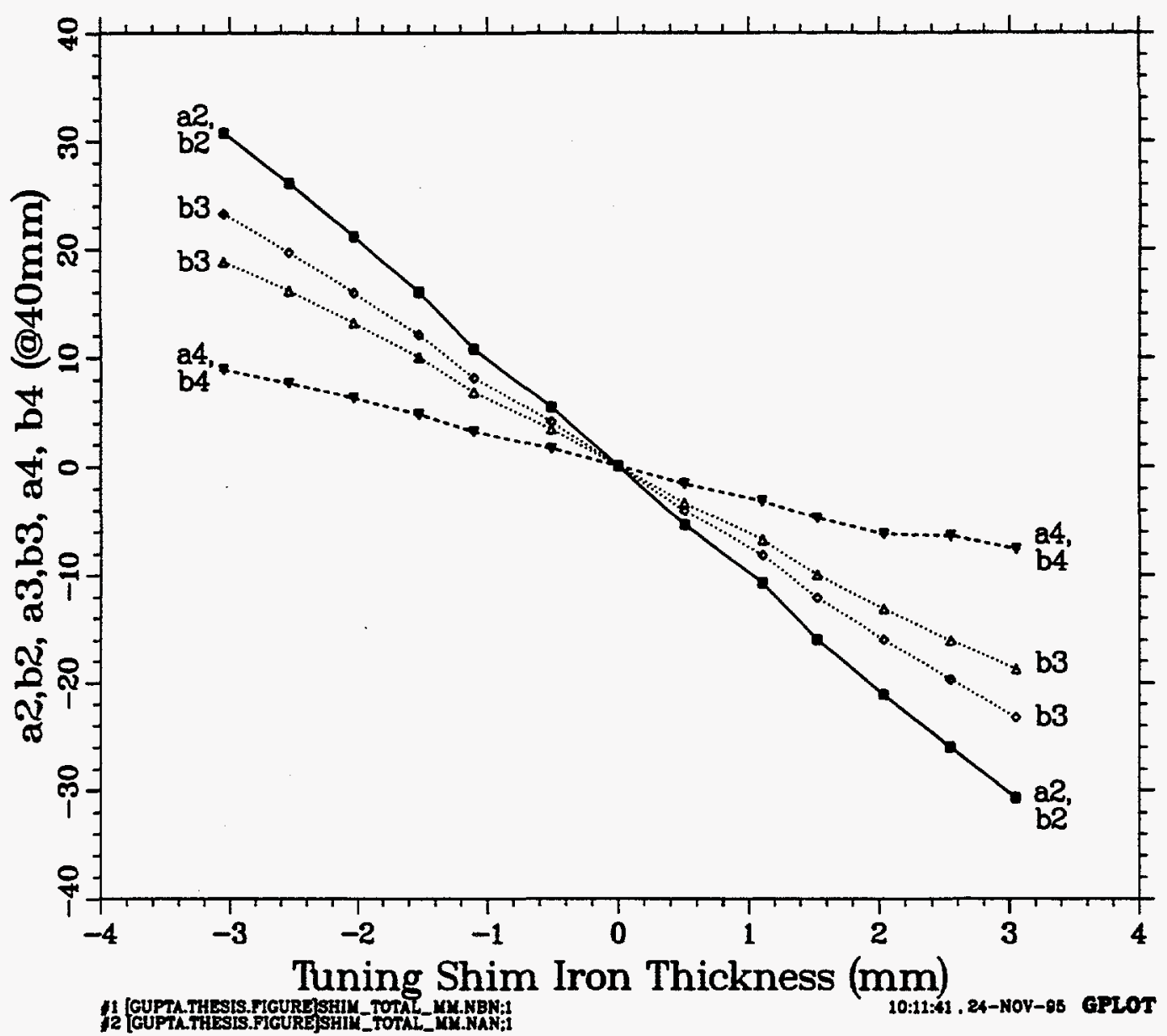

Figure 5.2.3: The computed maximum possible low field correction in the skew and normal sextupole $\left(a_{2}\right.$ and $\left.b_{2}\right)$, the skew and normal octupole $\left(a_{3}\right.$ and $\left.b_{3}\right)$, and the skew and normal decapole $\left(a_{4}\right.$ and $\left.b_{4}\right)$ harmonics as a function of tuning shim thickness $(1 \mathrm{mil}=0.0254 \mathrm{~mm})$. The tuning shim thickness is measured from its nominal value and therefore has both negative and positive values. 


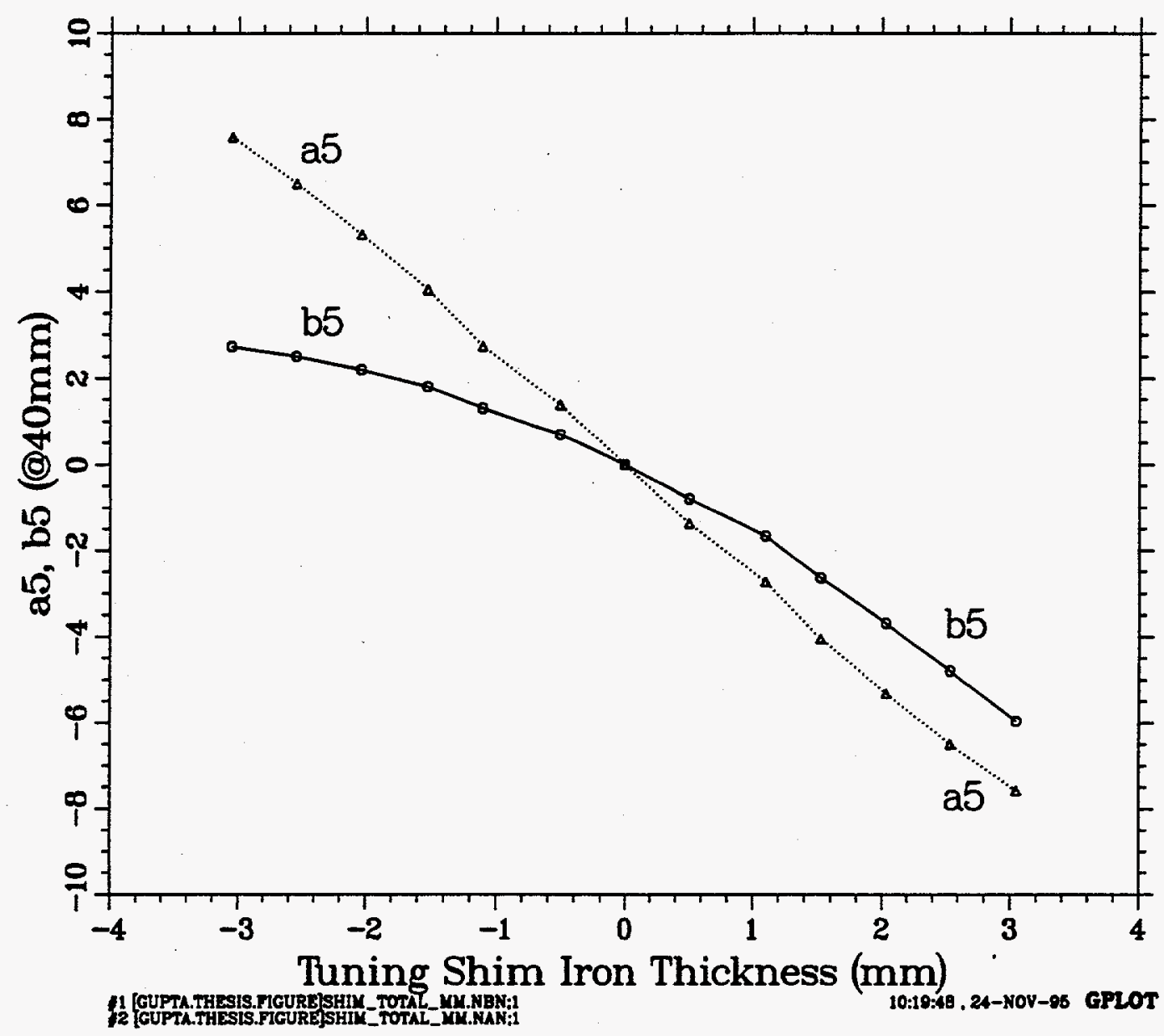

Figure 5.2.4: The computed maximum possible low field correction in the skew and normal do-decapole $\left(a_{5}\right.$ and $b_{5}$ ) harmonics as a function of tuning shim thickness $(1 \mathrm{mil}=0.0254 \mathrm{~mm})$. The tuning shim thickness is measured from its nominal value and therefore has both negative and positive values. 


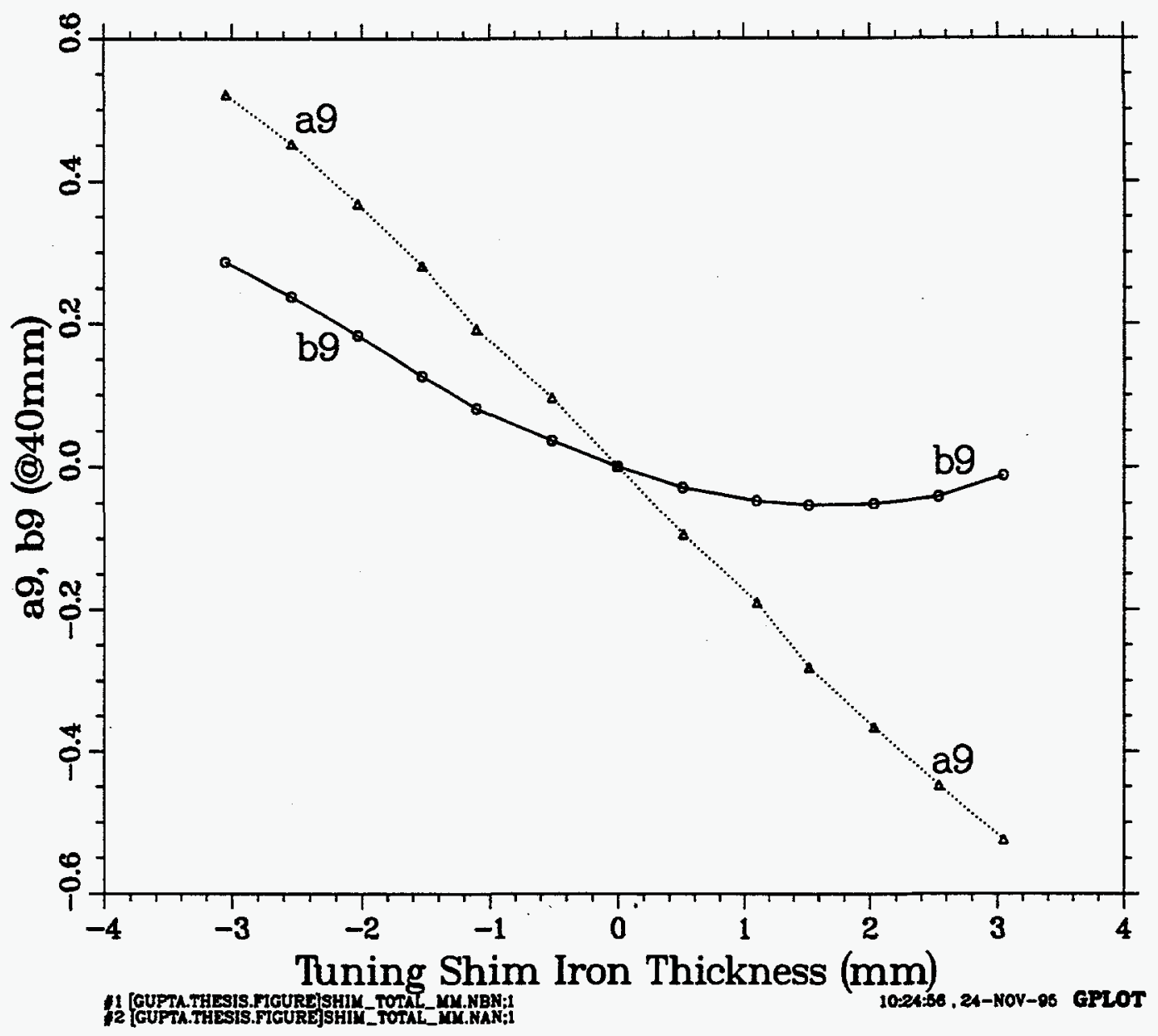

Figure 5.2.5: The computed maximum possible low field correction in the $a_{9}$ and $b_{9}$ harmonics as a function of tuning shim thickness (1mil = $0.0254 \mathrm{~mm}$ ). The tuning shim thickness is measured from its nominal value and therefore has both negative and positive values. 


\subsubsection{Numerical Calculations for High Field Correction}

At high field, the magnetization of the shims become almost constant, so their contribution to the harmonics decreases with increasing field. In this case the correction in the field harmonics produced by the tuning shim is smaller by an amount that depends on the current. The absolute loss in correction depends on the harmonic concerned and may also depend on the details of the tuning shim configuration. Since the maximum beam luminosity in RHIC is desired at the top energy, the tuning shim correction is optimized for the design current of $5 \mathrm{kA}$. This means that generally there will be an over-correction at low field.

The current dependence of the harmonic correction for particular locations of tuning shim is examined in Table 5.2.6 for the skew harmonics and Table 5.2.7 for the normal harmonics. These calculations are for the change in harmonics created by the nominal size $(3.175 \mathrm{~mm})$ tuning shim compared to the no-shim case. In these tables, the second row shows the location of the tuning shim for which the current dependence in the harmonic in the first row is given. This is one of the several locations where that particular harmonic has the maximum positive value. The last row shows the ratio of correction between $1 \mathrm{kA}$ and 5 $\mathrm{kA}$ for each harmonic. This is the over-correction factor at low current when the measured harmonics are compensated at $5 \mathbf{k A}$. The error in harmonic calculations is typically of the order of 0.01 unit for higher order harmonics $\left(b_{5} / a_{5}\right.$ and higher) and 0.1 unit in lower order harmonics. Therefore $a_{7}$ and $b_{9}$ harmonics, which have small values, will have a relatively large error, and the significantly different value of " $1 \mathrm{kA} / 5 \mathrm{kA}$ " (last row in Table 5.2.6) is not real.

The current dependence of the change in the harmonics produced by shims can also be seen in Fig. 5.2.6 and Fig. 5.2.7. Here the ratio of the shim correction at any current to that at $1 \mathrm{kA}$ is plotted for the same location as those given in Table 5.2.6 and Table 5.2.7. At $5 \mathrm{kA}$ this number is the inverse of the number given in the last row of the above tables.

The influence of this saturation on the maximum tuning shim correction range is examined here for each harmonic. Maximum range is obtained when, for each individual harmonic, all shims are put in a configuration which gives the maximum change. This is shown in Table 5.2.8. To obtain the range (i.e. maximum positive or maximum negative correction) each individual shim is either made of all iron (magnetic) laminations or all brass laminations (non-magnetic). The correction is computed at low current (1kA) and at the maximum design current ( $5 \mathrm{kA})$. The ratio, which represent the amount of over-correction 
at low field, is also computed here. The significantly different values of ratio for $a_{7}$ and $b_{9}$ harmonics are not real, as explained earlier.

Table 5.2.6: The current dependence of the change in skew harmonics generated by a nominal tuning shim at the location (listed below) which gives the maximum positive correction compared to the no-shim case. The relative computational error in $a_{7}$ is large and the increase at $5 \mathrm{kA}$ is artificial because of its smaller value.

\begin{tabular}{|c|c|c|c|c|c|c|c|c|c|c|}
\hline $\begin{array}{c}\text { Current } \\
(\mathrm{kA})\end{array}$ & $\begin{array}{c}a_{0} \\
(1 \mathrm{~A})\end{array}$ & $\begin{array}{c}a_{1} \\
(1 \mathrm{~B})\end{array}$ & $\begin{array}{c}a_{2} \\
(3 \mathrm{~B})\end{array}$ & $\begin{array}{c}a_{3} \\
(1 \mathrm{~B})\end{array}$ & $\begin{array}{c}a_{4} \\
(3 \mathrm{~A})\end{array}$ & $\begin{array}{c}a_{5} \\
(1 \mathrm{~A})\end{array}$ & $\begin{array}{c}a_{6} \\
(1 \mathrm{~B})\end{array}$ & $\begin{array}{c}a_{7} \\
(1 \mathrm{~B})\end{array}$ & $\begin{array}{c}a_{8} \\
(3 \mathrm{~B})\end{array}$ & $\begin{array}{c}a_{9} \\
(1 \mathrm{~B})\end{array}$ \\
\hline 0.0 & 7.88 & 3.32 & 5.673 & 2.428 & 1.874 & 0.963 & 0.414 & 0.026 & 0.12 & 0.055 \\
\hline 1.0 & 7.87 & 3.31 & 5.67 & 2.429 & 1.873 & 0.963 & 0.415 & 0.025 & 0.121 & 0.054 \\
\hline 3.0 & 7.83 & 3.29 & 5.651 & 2.414 & 1.867 & 0.958 & 0.411 & 0.025 & 0.119 & 0.054 \\
\hline 4.0 & 7.51 & 3.16 & 5.442 & 2.281 & 1.79 & 0.913 & 0.384 & 0.028 & 0.115 & 0.051 \\
\hline 4.5 & 7.24 & 3.07 & 5.217 & 2.169 & 1.706 & 0.871 & 0.358 & 0.034 & 0.109 & 0.046 \\
\hline 5.0 & 6.94 & 2.97 & 4.948 & 2.035 & 1.584 & 0.826 & 0.327 & 0.039 & 0.103 & 0.041 \\
\hline 5.5 & 6.49 & 2.75 & 4.578 & 1.898 & 1.457 & 0.749 & 0.293 & 0.038 & 0.093 & 0.036 \\
\hline 6.0 & 5.83 & 2.38 & 4.039 & 1.735 & 1.279 & 0.649 & 0.259 & 0.029 & 0.079 & 0.031 \\
\hline 7.0 & 4.43 & 1.76 & 3.055 & 1.386 & 0.992 & 0.493 & 0.204 & 0.019 & 0.061 & 0.024 \\
\hline 8.0 & 3.59 & 1.46 & 2.506 & 1.171 & 0.834 & 0.411 & 0.176 & 0.013 & 0.051 & 0.021 \\
\hline $1 \mathrm{kA} / 5 \mathrm{kA}$ & 1.13 & 1.11 & 1.15 & 1.19 & 1.18 & 1.17 & 1.27 & $0.64 *$ & 1.17 & 1.32 \\
\hline
\end{tabular}

*This ratio contains large errors due to smaller values of $a_{7}$ 
Table 5.2.7: The current dependence of the change in normal harmonics generated by a nominal tuning shim at the location (listed below) which gives the maximum positive correction compared to the no-shim case.

\begin{tabular}{|c|c|c|c|c|c|c|c|c|c|c|}
\hline $\begin{array}{c}\text { Current } \\
(\mathrm{kA})\end{array}$ & $\begin{array}{c}b_{0} \\
(2 \mathrm{~A})\end{array}$ & $\begin{array}{c}\delta(T F) \\
(\mathrm{ALL})\end{array}$ & $\begin{array}{c}b_{2} \\
(2 \mathrm{~B})\end{array}$ & $\begin{array}{c}b_{3} \\
(1 \mathrm{~A})\end{array}$ & $\begin{array}{c}b_{4} \\
(1 \mathrm{~B})\end{array}$ & $\begin{array}{c}b_{5} \\
(\mathrm{ALL})\end{array}$ & $\begin{array}{c}b_{6} \\
(1 \mathrm{~A})\end{array}$ & $\begin{array}{c}b_{7} \\
(1 \mathrm{~B})\end{array}$ & $\begin{array}{c}b_{8} \\
(1 \mathrm{~A})\end{array}$ & $\begin{array}{c}b_{9} \\
(\mathrm{ALL})\end{array}$ \\
\hline 0.0 & 7.87 & 0.0075 & 5.656 & 2.482 & 1.87 & -0.342 & 0.416 & 0.265 & 0.119 & -0.037 \\
\hline 1.0 & 7.87 & 0.0074 & 5.655 & 2.482 & 1.868 & -0.341 & 0.416 & 0.266 & 0.12 & -0.037 \\
\hline 3.0 & 7.84 & 0.0074 & 5.639 & 2.468 & 1.859 & -0.338 & 0.413 & 0.264 & 0.119 & -0.038 \\
\hline 4.0 & 7.58 & 0.0071 & 5.451 & 2.366 & 1.759 & -0.31 & 0.386 & 0.248 & 0.114 & -0.037 \\
\hline 4.5 & 7.31 & 0.0068 & 5.162 & 2.276 & 1.676 & -0.283 & 0.361 & 0.235 & 0.108 & -0.035 \\
\hline 5.0 & 6.99 & 0.0064 & 4.917 & 2.182 & 1.575 & -0.248 & 0.33 & 0.218 & 0.102 & -0.034 \\
\hline 5.5 & 6.52 & 0.006 & 4.552 & 1.999 & 1.445 & -0.225 & 0.298 & 0.195 & 0.092 & -0.031 \\
\hline 6.0 & 5.81 & 0.0054 & 4.051 & 1.73 & 1.277 & -0.212 & 0.263 & 0.168 & 0.078 & -0.026 \\
\hline 7.0 & 4.44 & 0.004 & 3.053 & 1.285 & 0.984 & -0.175 & 0.207 & 0.131 & 0.061 & -0.02 \\
\hline 8.0 & 3.63 & 0.0032 & 2.492 & 1.058 & 0.827 & -0.155 & 0.178 & 0.111 & 0.05 & -0.018 \\
\hline $1 \mathrm{kA} / 5 \mathrm{kA}$ & 1.13 & 1.16 & 1.15 & 1.14 & 1.19 & 1.37 & 1.26 & 1.22 & 1.18 & 1.09 \\
\hline
\end{tabular}




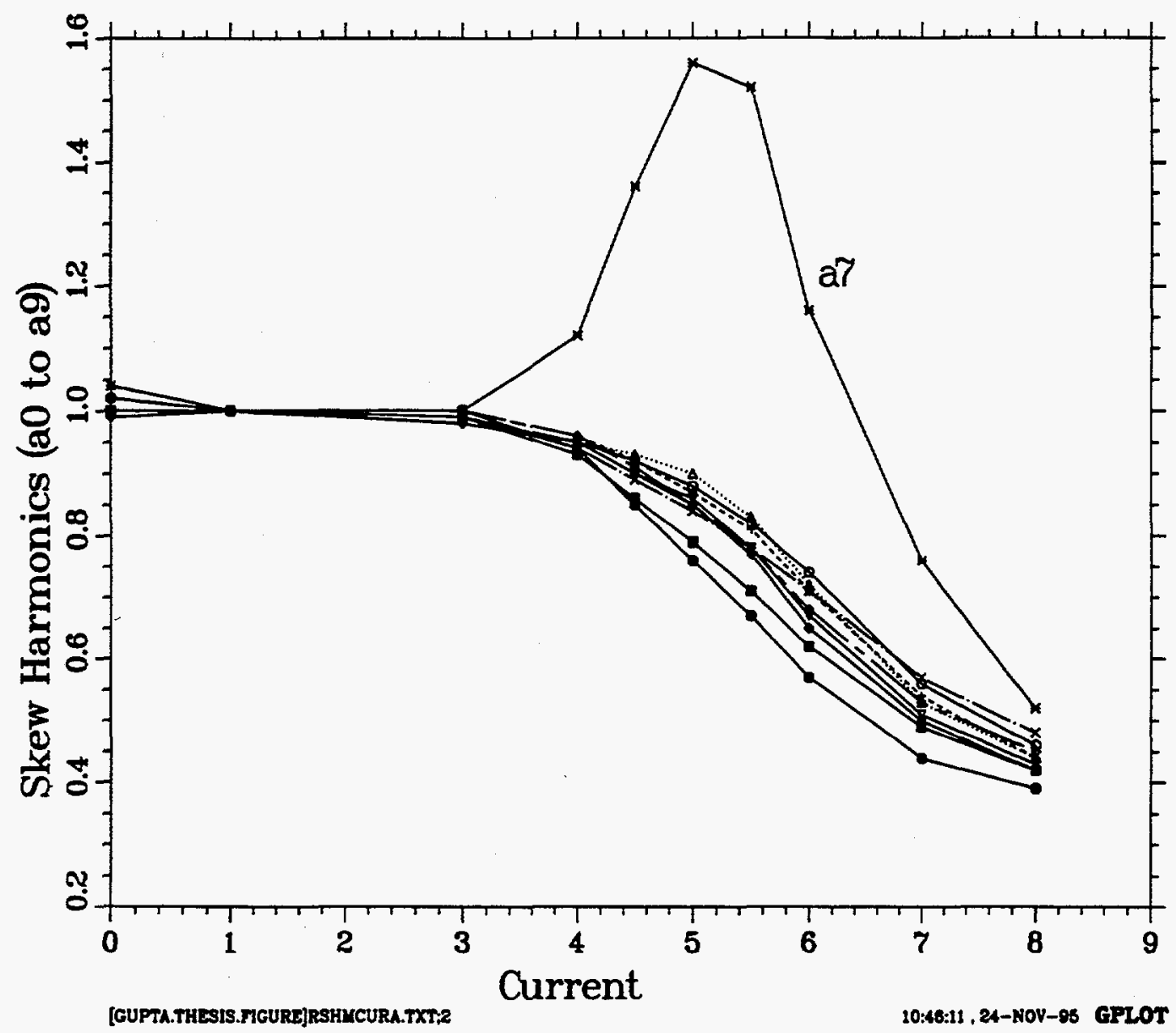

Figure 5.2.6: The ratio of the harmonics generated by a tuning shim at the given current to those at $1 \mathrm{kA}$. The design current is $5 \mathrm{kA}$. The shim location is that which gives the maximum correction in the harmonics produced by tuning shims having the nominal thickness. 


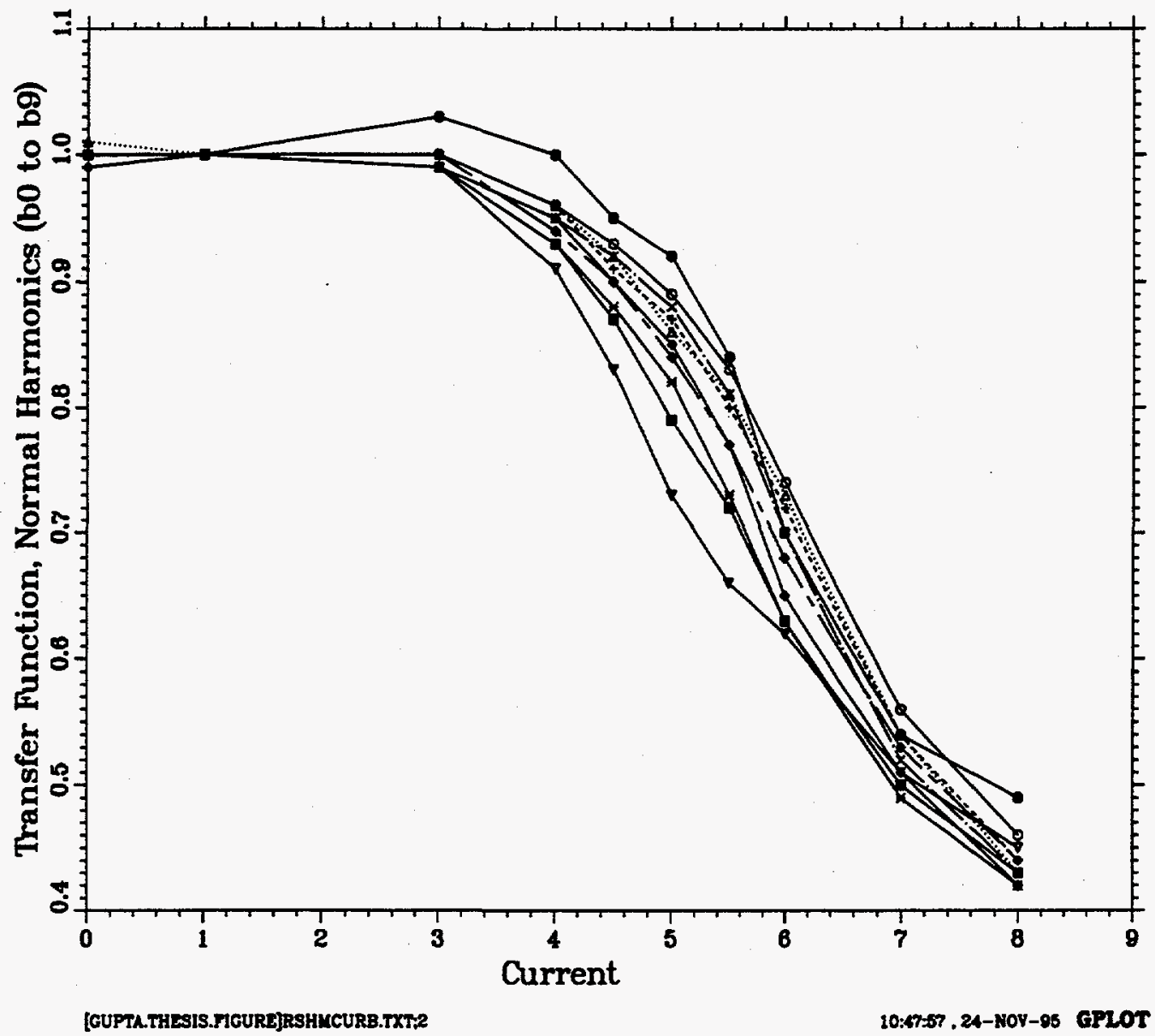

Figure 5.2.7: The ratio of the harmonics generated by a tuning shim at the given current to those at $1 \mathrm{kA}$. The design current is $5 \mathrm{kA}$. The shim location is that which gives the maximum correction in the harmonics produced by tuning shims having the nominal thickness. 
Table 5.2.8: The maximum possible change in harmonics (range) which can be made by tuning shims in the RHIC $130 \mathrm{~mm}$ aperture insertion quadrupoles. To obtain this number for each harmonic, each of eight tuning shims has either a maximum (all) or minimum (none) iron content so that the contributions add. The values are computed at low current $(1 \mathrm{kA})$ and at the nominal design current $(5 \mathrm{kA})$. Since the correction is made at $5 \mathrm{kA}$, the ratios $\mathrm{A}_{1 k A / 5 k A}$ and $\mathrm{B}_{1 k A / 5 k A}$ indicate the over-correction factor at low current. The transfer function is given in $T / m / k A$ and harmonics are given at a reference radius of $40 \mathrm{~mm}$.

\begin{tabular}{|c|c|c|c|c|c|c|c|}
\hline $\begin{array}{c}\text { Harm } \\
a_{n}\end{array}$ & $\begin{array}{l}\text { Correction } \\
\text { (Range) }_{1 k A}\end{array}$ & $\begin{array}{l}\text { Correction } \\
\text { (Range) })_{5 k A}\end{array}$ & $\begin{array}{l}\text { Ratio }_{a n} \\
\mathrm{~A}_{1 k A / 5 k A}\end{array}$ & $\begin{array}{c}\text { Harm } \\
b_{n}\end{array}$ & $\begin{array}{l}\text { Correction } \\
{\text { (Range })_{1 k A}}^{\text {Ran }}\end{array}$ & $\begin{array}{l}\text { Correction } \\
{\text { (Range })_{5 k A}}\end{array}$ & $\begin{array}{l}\text { Ratio }_{b n} \\
\mathrm{~B}_{1 k A / 5 k A}\end{array}$ \\
\hline$a_{0}$ & 113.73 & 91.82 & 1.239 & $b_{0}$ & 113.75 & 91.89 & 1.238 \\
\hline$a_{1}$ & 49.06 & 40.96 & 1.198 & TF & 0.132 & 0.1047 & 1.261 \\
\hline$a_{2}$ & 61.464 & 48.505 & 1.267 & $b_{2}$ & 61.439 & 48.372 & 1.270 \\
\hline$a_{3}$ & 46.525 & 36.104 & 1.289 & $b_{3}$ & 37.557 & 30.832 & 1.218 \\
\hline$a_{4}$ & 17.754 & 14.41 & 1.232 & $b_{4}$ & 17.745 & 14.433 & 1.229 \\
\hline$a_{5}$ & 15.151 & 12.148 & 1.247 & $b_{5}$ & 8.688 & 6.099 & 1.424 \\
\hline$a_{6}$ & 5.991 & 4.724 & 1.268 & $b_{6}$ & 5.996 & 4.734 & 1.267 \\
\hline$a_{7}$ & 0.645 & 0.196 & 3.291 & $b_{7}$ & 4.461 & 3.444 & 1.295 \\
\hline$a_{8}$ & 1.576 & 1.188 & 1.327 & $b_{8}$ & 1.571 & 1.18 & 1.331 \\
\hline$a_{9}$ & 1.045 & 0.766 & 1.364 & $b_{9}$ & 0.298 & 0.304 & 0.980 \\
\hline$a_{10}$ & 0.329 & 0.232 & 1.418 & $b_{10}$ & 0.328 & 0.235 & 1.396 \\
\hline$a_{11}$ & 0.159 & 0.135 & 1.178 & $b_{11}$ & 0.192 & 0.129 & 1.488 \\
\hline$a_{12}$ & 0.066 & 0.052 & 1.269 & $b_{12}$ & 0.068 & 0.054 & 1.259 \\
\hline$a_{13}$ & 0.0243 & 0.0137 & 1.774 & $b_{13}$ & 0.0418 & 0.0316 & 1.323 \\
\hline
\end{tabular}




\subsubsection{Symmetries in the Harmonics Generated by Tuning Shims}

The harmonics generated by the same tuning shim placed at two different locations follow certain symmetries or anti-symmetries. This means that the harmonics created by a same size (iron content) tuning shim placed in a certain pair of locations have the same magnitude. These two locations are called symmetric with respect to a particular harmonic if the sign of the harmonic change is the same and anti-symmetric if the sign is opposite.

The following rules are obtained for quadrupoles from Eqs. (5.2.10) or Eqs. (5.2.11).

If these two locations are left and right with respect to the vertical axis (for example see locations $1 \mathrm{~A}$ and 2B in Fig. 5.2.1), then the odd $b_{n}$ and even $a_{n}$ are symmetric and even $b_{n}$ and odd $a_{n}$ are anti-symmetric. This means that $b_{3}(@ 1 A)=b_{3}(@ 2 B)$ and $a_{3}(@ 1 A)=$ $-a_{3}(@ 2 B)$.

Similarly if these two locations are top and bottom (for example locations $1 \mathrm{~A}$ and $4 \mathrm{~B}$ ), then all $b_{n}$ are symmetric and all $a_{n}$ are anti-symmetric.

The remaining combination is of two locations that are on the two sides of the coil pole (for example locations $(1 \mathrm{~A}, 1 \mathrm{~B}),(2 \mathrm{~A}, 2 \mathrm{~B})$, etc.) If a relationship in the harmonics generated between these two locations is obtained, then the harmonics created by a tuning shim at one location completely determines the value of harmonics at all other locations. In this case, as explained below, the symmetries in the harmonics are somewhat complicated.

In the case of odd harmonics, two locations are symmetric for harmonics $b_{4 k+1}$ and $a_{4 k+3}$ for $\mathrm{k}=0,1,2, \ldots$ (for example $b_{5}(@ 1 \mathrm{~A})=b_{5}(@ 1 \mathrm{~B})$ or $a_{3}(@ 3 \mathrm{~A})=a_{3}(@ 3 \mathrm{~B})$ ) and two locations are anti-symmetric for harmonics $b_{4 k+3}$ and $a_{4 k+1}$ (for example $\left.b_{3}(@ 2 A)=-b_{3}(@ 2 B)\right)$. Even harmonics have a cross relationship between the normal and skew components $b_{n}$ and $a_{n}$ as follows : (a) the harmonics $b_{4 k+2}$ and $a_{4 k+2}$ are symmetric at location 1 and 3 (for example $\left.b_{2}(@ 1 A)=a_{2}(@ 1 B)\right)$ and are anti-symmetric at location 2 and 4 and (b) the harmonics $b_{4 k+4}$ and $a_{4 k+4}$ are anti-symmetric at location 1 and 3 and are symmetric at location 2 and $4\left(\right.$ for example $\left.a_{4}(@ 4 \mathrm{~A})=b_{4}(@ 4 \mathrm{~B})\right)$. 


\subsubsection{Independent and Coupled Changes in Harmonics Correction}

A consequence of correcting field harmonics with tuning shims is the creation of other harmonics in the process. These other harmonics are those that are allowed by the symmetry of the shims. Therefore, " $n$ " tuning shims can not fully compensate any arbitrary " $n$ " harmonics.

In this design, eight tuning shims correct eight harmonics. The following three rules will apply in a quadrupole :

1. of eight harmonics, four must be skew $\left(a_{n}\right)$ and four must be normal $\left(b_{n}\right)$,

2. of the four skew and four normal harmonics, two must be odd (e.g. $a_{3}$ ) and two must be even (e.g. $b_{4}$ )

3. for any change in an odd harmonic $n=2 k+1$, with $k=0,1,2, .$. the harmonic $n=2 \mathrm{k}+5$ will also change, e.g. a change in $b_{3}$ will cause a coupled change in $b_{7}$, as well.

Despite the above restrictions, the desired eight harmonics $\left(a_{2}, a_{3}, a_{4}, a_{5}\right)$ and $\left(b_{2}, b_{3}, b_{4}, b_{5}\right)$ can be independently minimized. Though the change in the other harmonics is determined by the coupling, certain couplings can be minimized in the initial yoke design by properly choosing the azimuthal locations of the tuning shims. For example, the coupling of $b_{5}$ to $b_{9}$ is small when the tuning shims are positioned anywhere within the range $\theta=30^{\circ}$ to $\theta=35^{\circ}$ (the range is fixed in the original design and can not be changed during the tuning process). In an optimized scheme the coupling in most harmonics should generally be an order of magnitude smaller than the primary harmonics. (Please refer to Table 5.2.5).

\subsubsection{Comparison with the Measurements}

Calculations are now compared with measurements of the field harmonics produced by magnetic shims. The calculations, as described in the previous section, are performed with the code POISSON. The comparison is made first in the low current case. In this case the permeability of the iron is assumed to be infinite. The low current measurements are made at room temperature (warm) when the magnet is not in a superconducting state; the current is carried by the copper fraction of the superconducting cable. In the following discussion, the shim thickness means the thickness of the low carbon steel (magnetic iron) in the tuning shim package. As mentioned earlier, the actual thickness of the package is 
constant since the rest of the space is filled by non-magnetic brass. In the calculations, the shim thickness is varied in steps of $0.51 \mathrm{~mm}\left(0.020^{\prime \prime}\right)$ and in the measurements in steps of $1.52 \mathrm{~mm}\left(0.060^{\prime \prime}\right)$. The change in harmonic (or harmonic due to the tuning shim) is obtained by subtracting out the harmonic of the no-shim case from the harmonic of the given shim thickness case. In Fig. 5.2.8, calculations and measurements of the skew and normal sextupole, the skew and normal octupole, and the skew and normal decapole harmonics are compared. In Fig. 5.2.9, calculations and measurements of the $a_{6}, b_{6}, a_{7}, b_{7}$, $a_{8}$ and $b_{8}$ harmonics are compared. In Fig. 5.2.10, calculations and measurements of the transfer function (gradient), skew and normal dodecapole and the $a_{9}$ and $b_{9}$ harmonics are compared. In the case of the skew quadrupole harmonic, only calculations are given as this harmonic is not available with present measurement techniques. It may be mentioned that in addition to the field gradient (or transfer function), $b_{5}$ and $b_{9}$ are harmonics allowed by the quadrupole geometry.

Though the symbols (measurements) and type of lines (calculations) are difficult to resolve for individual shim locations in these figures, it has been observed that the measurements always lie on the top of the computed values for each tining shim location. The difference between the calculations and measurements is quite small $(<0.05)$. It is quite clear from these figures that there is good agreement between the calculations and measurements of the field harmonics produced by the tuning shims at low currents. 

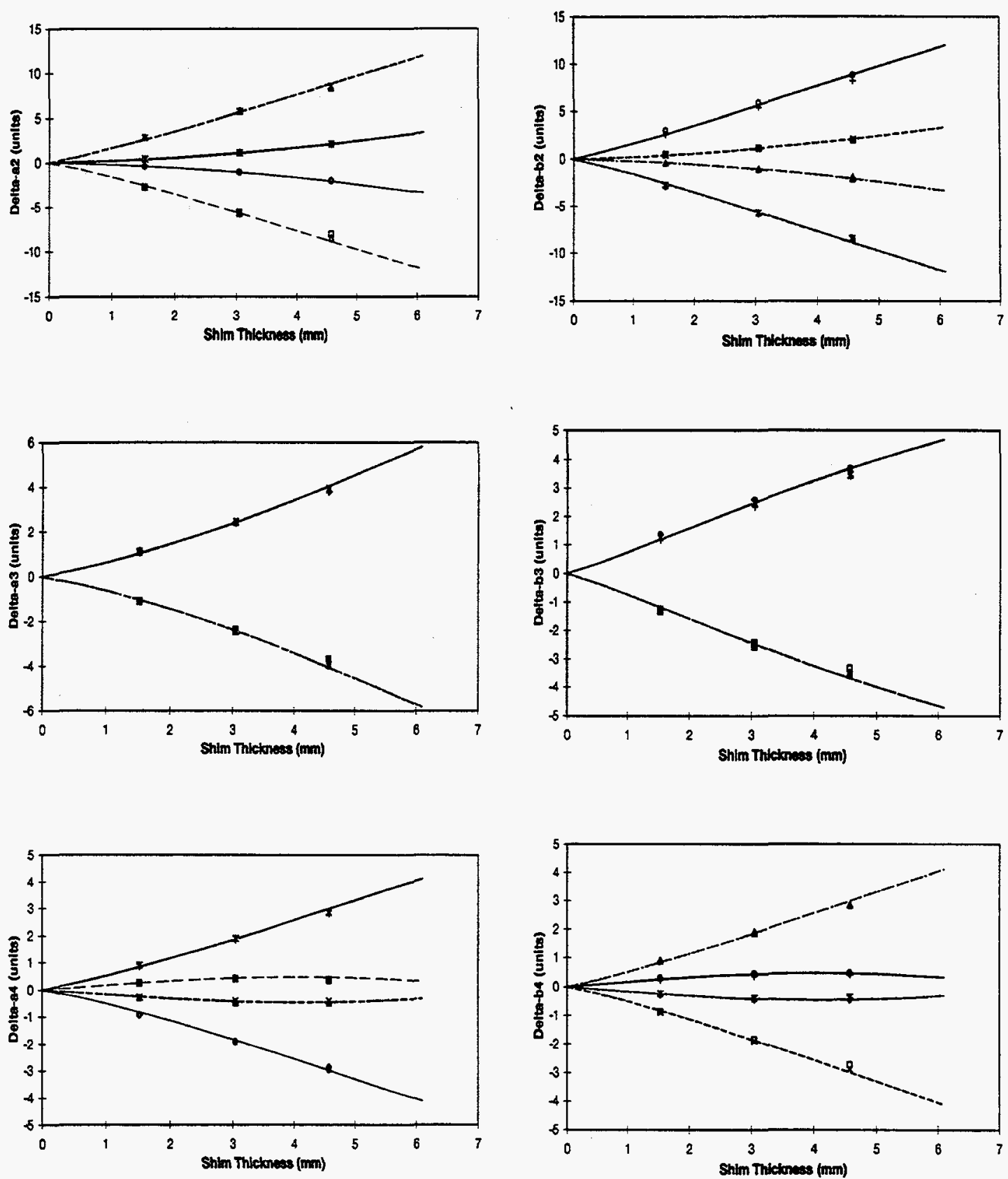

Figure 5.2.8: A comparison between calculations and measurements of the field harmonics created by the tuning shims at low fields. These are the changes in harmonics relative to the no shim or zero shim case for each shim. The eight symbols (see text) represent the measurements for the eight tuning shim locations and the eight lines (see text) are the calculations for these locations. The symbols and lines for the harmonics at two or four locations overlap as per the symmetries described in the previous section. 

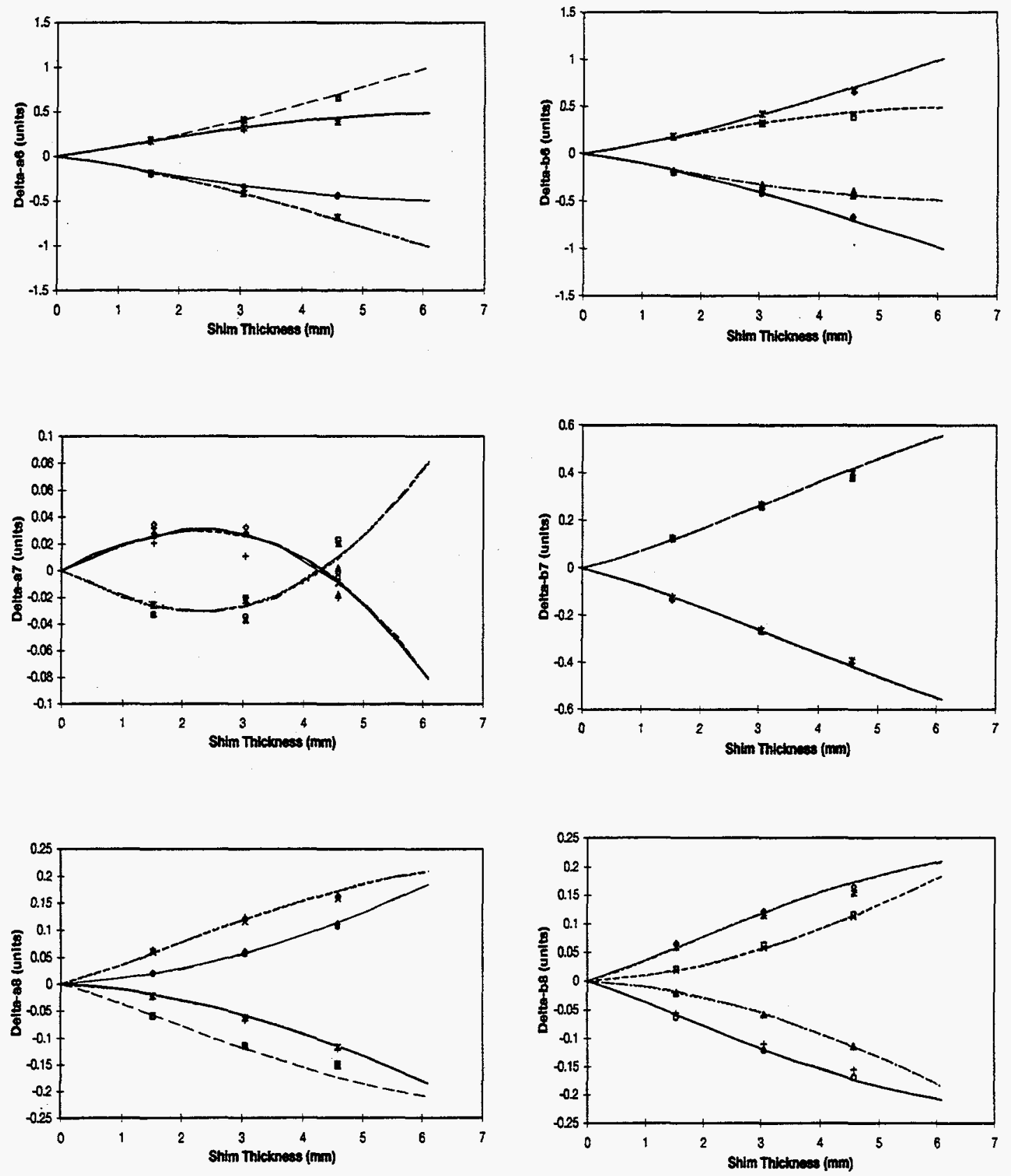

Figure 5.2.9: A comparison between calculations and measurements of the field harmonics created by the tuning shims at low fields. These are the changes in harmonics relative to the no shim or zero shim case for each shim. The eight symbols (see text) represent the measurements for the eight tuning shim locations and the eight lines (see text) are the calculations for these locations. The symbols and lines for the harmonics at two or four locations overlap as per the symmetries described in the previous section. 

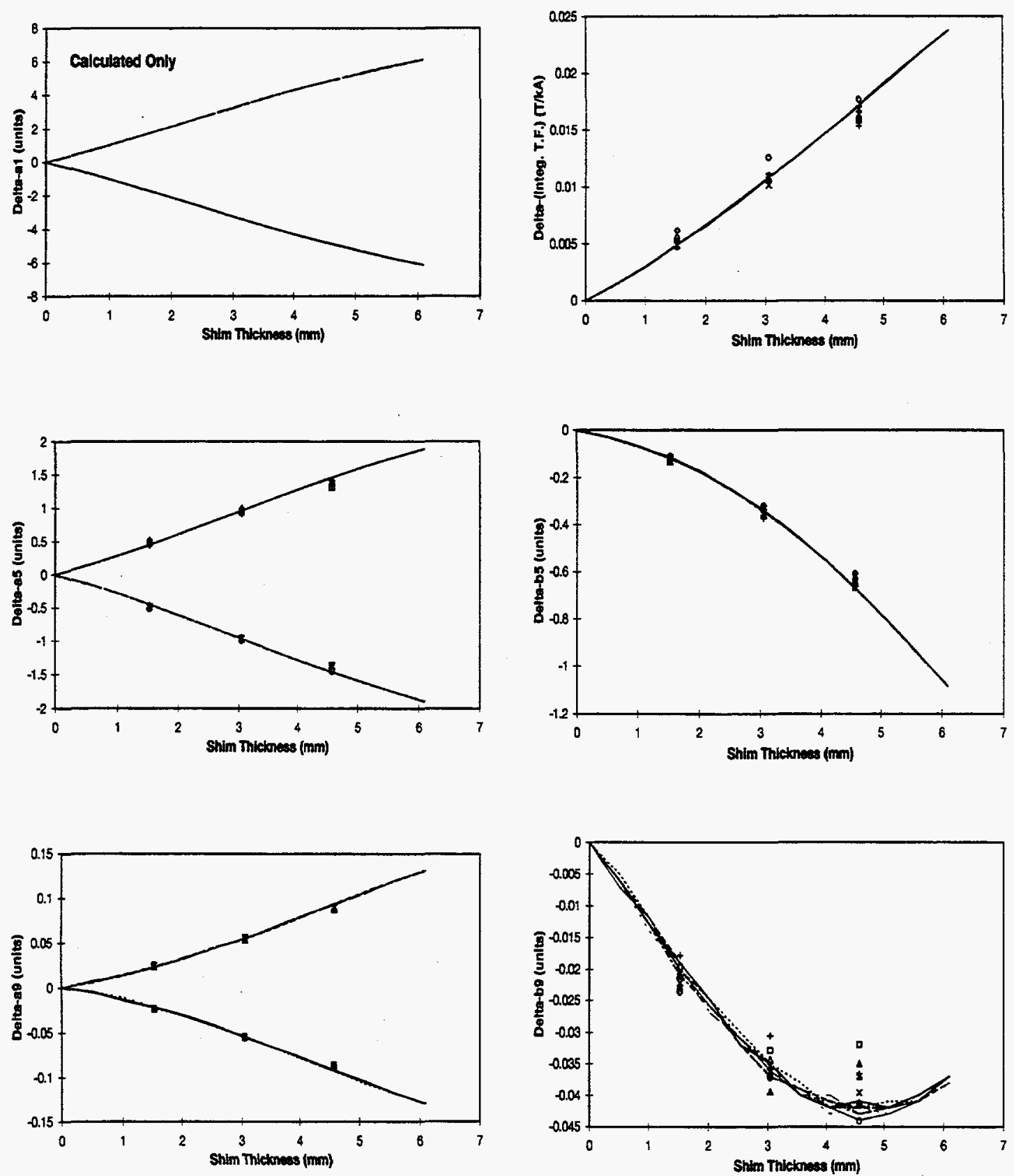

Figure 5.2.10: A comparison between calculations and measurements of the field harmonics created by the tuning shims at low fields. These are the changes in harmonics relative to the no shim or zero shim case for each shim. The eight symbols (see text) represent the measurements for the eight tuning shim locations and the eight lines (see text) are the calculations for these locations. The symbols and lines for the harmonics at two or four locations overlap as per the symmetries described in the previous section. 


\subsubsection{Tuning Shim Correction Vs. External Correctors}

Harmonic errors in magnets are usually corrected by external corrector magnets. These give a lumped compensation to the beam dynamics of the distortion caused by the harmonic errors. These harmonic correctors are either located at the end of the magnet to correct for the errors in the individual magnets or they are lumped together and placed elsewhere in the machine. Valuable space for such correctors must be provided in the machine. External correctors provide the flexibility of adjustable correction by simply changing the current in the power supplies. However, they add to the component and power supply cost and complicate the machine operation. In order to independently correct eight harmonics one must have at least eight correction coils, either in one magnet or in a number of magnets.

In contrast, the tuning shim correction scheme corrects harmonic errors locally. The tuning shims require no extra space in the machine since they are installed inside each magnet. They are relatively inexpensive and require no external power supply to operate, which simplifies machine operation. Of course, there is no flexibility in the harmonic correction after the magnets are installed in the machine. However, the harmonics are measured after the tuning shims are inserted in each magnet and if the correction is not satisfactory they can immediately be adjusted.

The crucial advantage of the tuning shim scheme is its ability to correct errors locally. To explain this point, same basic considerations of beam optics in the RHC interaction region (as set by accelerator physicists) are reviewed here together with the implications for field quality in the interaction region quadrupoles [171]. The variation in $\sqrt{\beta_{x}}$ and $\sqrt{\beta_{y}}$ ( $\sqrt{\beta_{x}}$ and $\sqrt{\beta_{y}}$ are proportional to the horizontal and vertical beam size) in the interaction region optics is shown in Fig. 5.2.11. One can note a rapid variation in the beta function, particularly in the insertion triplets, which consist of three $130 \mathrm{~mm}$ aperture quadrupoles. As a result, lumped external correctors would not be able to fully compensate the effects of distributed errors in the magnets on the orbit [171]. The tuning shim scheme, which removes the errors in eight harmonics locally, is much more effective. A larger good field region in the magnet means that the beam can be squeezed to a smaller size at the crossing point resulting in a higher luminosity. In the RHIC interaction region quadrupoles $5 \sigma$ of the beam is expected to use $71 \%$ of the coil aperture. With tuning shims, a large fraction of the physical aperture can be made a good field aperture.

The tuning shim scheme, though crucial in the interaction region quadrupoles, could also be used in a large scale production of magnets with limited harmonics. In that case, 


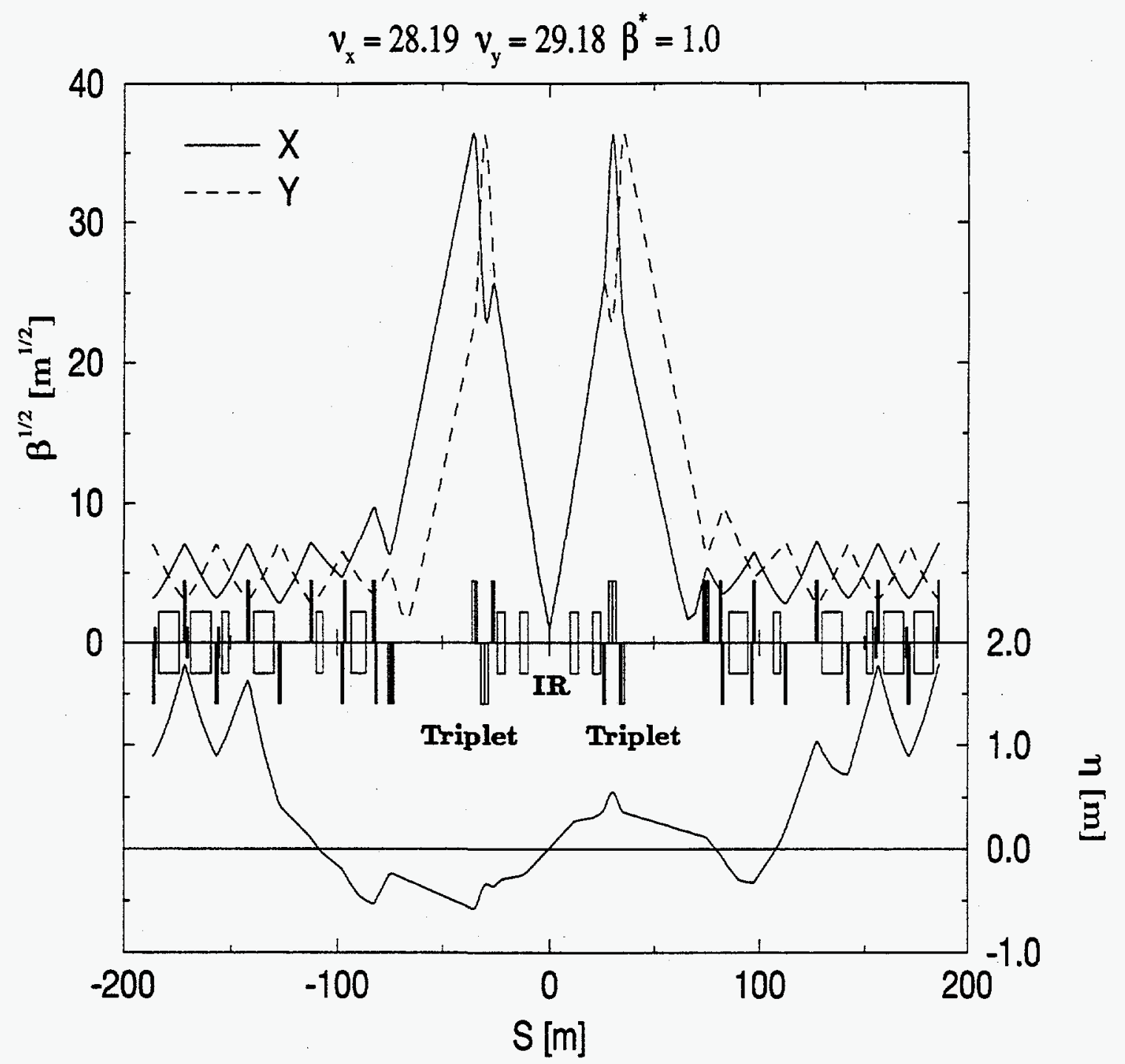

Figure 5.2.11: The variation in the square-root of the betatron functions $\left(\beta_{x}, \beta_{y}\right)$ and dispersion function $(\eta)$ in a $\mathrm{RHIC}$ insertion region for $\beta^{*}=$ 1.0 (courtesy Jie Wei). The horizontal beam size is proportional to $\sqrt{\beta_{x}}$ and $\eta$ and the vertical beam size is proportional to $\sqrt{\beta_{y}}$. A large beam size can be seen on the two sides of the insertion region (IR) where the focusing triplets are located. 
it would be essential to further simplify the logistics of implementing such a scheme in an industrial environment. The use of this scheme was examined for SSC collider dipole magnets. The SSC magnets use stainless steel collars. In this case, it would perhaps be best to measure the harmonics before the collared coil is put in the yoke. The thickness of the magnetic material in the tuning shims would be determined by the measured harmonics in the collared coil in the absence of the yoke. The shims would be installed before (or during) the collared coil assembly into the yoke. 


\subsection{Tuning Yoke Length at Magnet Ends for Field Correction}

Schemes are proposed here to correct deviations in the integral transfer function and the skew quadrupole harmonic in dipole magnets. As against the tuning shim scheme discussed earlier, the schemes proposed here are implemented only near the ends of the magnet. These schemes are based on adjusting the proportion of magnetic, low carbon steel to non-magnetic, stainless steel laminations to correct measured errors.

In the body (straight section) of the superconducting magnets, the yoke laminations (made of low carbon, magnetic steel) generally contribute $15 \%$ to $40 \%$ of the total field depending on the details of a particular design. The low carbon, magnetic steel laminations are replaced by non-magnetic, stainless steel laminations in the ends of the SSC and most other magnets, to reduce the maximum field on the conductor in an attempt to improve quench performance. However, this also reduces the integral field (or integral transfer function) of the magnet. In RHIC magnets (all except the design of the $180 \mathrm{~mm}$ aperture, insertion dipoles), the laminations in the ends are made of low carbon magnetic steel in order to maximize the integral transfer function. The proposed schemes of field adjustments are more powerful and natural to adopt in the former (SSC) type of magnet designs.

It is proposed here that while keeping the total physical length constant, the yoke magnetic length is adjusted to compensate for the following errors : (a) The total magnetic length (or the length of yoke laminations) can be adjusted to correct an error in the integral transfer function. (b) A deliberate up-down asymmetry in the magnetic yoke length between the top and bottom halves can be used to compensate for the measured skew quadrupole $\left(a_{1}\right)$ harmonic. The proposed correction would take place towards the magnet ends as shown in Fig. 5.3.1. The starting of the actual location of the correction would be in the body (straight section) of the magnet where the local magnetic field is still high. 

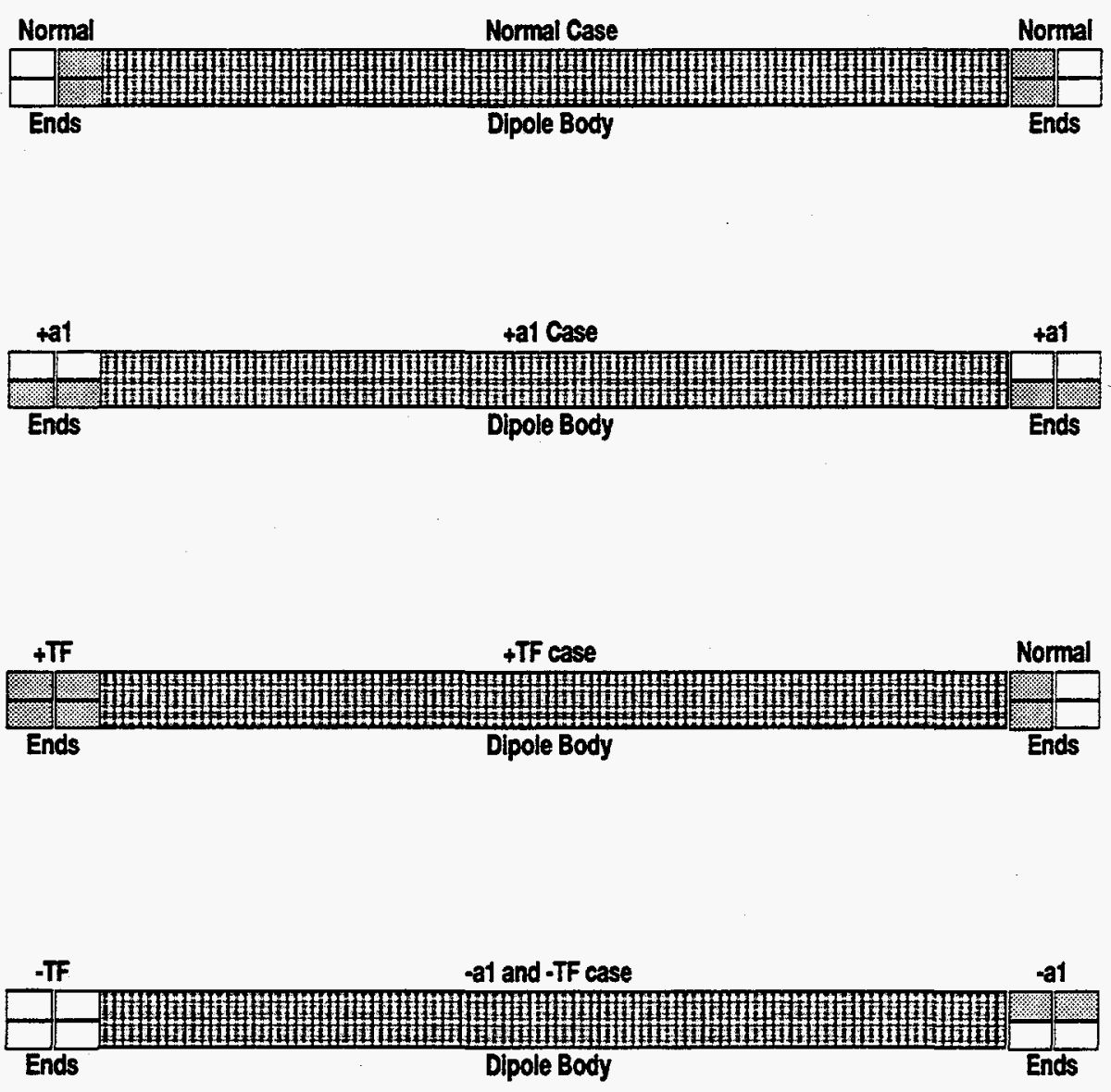

Figure 5.3.1: A conceptual diagram for correcting the integral $a_{1}$ harmonic and integral transfer function in a superconducting dipole magnet. The proposed adjustment is applied in the end region of the magnet. The actual starting point would be somewhere in the dipole body where the field is still high. In the normal case (top figure) the change between the magnetic, low carbon steel laminations [dark or filled] and non-magnetic stainless steel laminations [light or empty] occurs at a nominal location. Interchanging the stainless steel and low carbon steel laminations between top and bottom halves (second figure) creates an $a_{1}$ which can be used to compensate the measured $a_{1}$ in a magnet. Increasing the number of low carbon steel magnetic laminations increases the integral transfer function (third figure). An adjustment (decrease) in both $a_{1}$ and integral transfer function can be obtained together by mixing the two schemes in the same magnet (bottom figure). 


\subsubsection{Yoke Length for Integral $a_{1}$ Correction}

Among the non-allowed measured harmonics, $a_{1}$ (skew quadrupole) had a magnitude large enough to be of concern in SSC dipoles. The major source of geometric $a_{1}$ in the dipole magnets is an up-down asymmetry in the coil. This $a_{1}$ is primarily related to variations in the components and the manufacturing process of the coils resulting in a difference between the top and bottom. This top-bottom difference is generally of a random nature and is the major source of the random variations in the geometric $a_{1}$ in the superconducting dipoles. A good correlation is expected for the measured $a_{1}$ in the collared coils before and after they are placed in the yoke.

In this section a method is presented that compensates for the measured $a_{1}$ in the collared coil by deliberately introducing another asymmetry in the yoke magnetic length between the upper and lower halves of the magnet. The method is schematically shown in Fig. 5.3.1. This correction would be adopted near the ends of the two coils in the magnet. The location where this correction would be implemented is the place where a change from the low carbon steel laminations to the stainless steel laminations takes place in the magnet end. A difference in the magnetic length between the top and bottom yoke halves is generated by switching the two types of laminations between the upper and lower halves. In order to adopt this scheme, one would first measure the $a_{1}$ in the collared coil at room temperature. This would determine the total number of laminations needed to be switched between the top and bottom halves of the magnet.

It is estimated that to correct 1 unit of $a_{1}$ in the SSC dipole one would need to switch $\sim 25 \mathrm{~mm}$ of magnetic laminations with the same length of stainless steel laminations. The total amount of either type of the laminations used in the magnet would not change in the process. To first order, it would not change the allowed harmonics. In the 2-d cross-section of the magnet, it locally creates $\sim 200$ units of $a_{1}$ when normalized to the local field. The actual contribution is somewhat smaller since the field in this transition region is $\sim 85 \%$ of the field in the straight section of the magnet. The actual 3-d end calculations give slightly different numbers and show a change in the $a_{1}$ correction [126] at high fields due to iron saturation. Other harmonics introduced in the process are $a_{3}, a_{5}$, etc. However, they all are $\sim .01$ unit or less, which is well within their specifications. 
This $a_{1}$ correction scheme should be relatively simple to implement, particularly in the BNL-type horizontally split yoke designs where the geometry of the non-magnetic stainless steel and magnetic low carbon steel lamination is identical. It may, for example, be implemented in the following manner:

- The usual thickness of a pack of laminations in the body of BNL-built SSC prototype magnets is $76.2 \mathrm{~mm}$. This pack could be made of $6.35 \mathrm{~mm}$ thick laminations. The same thickness and cross section should be used in both the magnetic and the stainless steel laminations.

- The amount of the two types of laminations used in any magnet is always the same and it is independent of the amount of the $a_{1}$ correction to be applied. Only the location of their placement in the magnet changes.

- A mis-match of a single $6.35 \mathrm{~mm}$ thick lamination in one end corrects $\sim \frac{1}{8}$ unit of $a_{1}$. The actual switch between top and bottom creates twice the length of $a$ mis-match. Therefore each switch of a lamination corrects $\sim \frac{1}{4}$ unit of $a_{1}$.

- Make the magnetic measurement in the collared coils before they are put in a iron yoke. Decide how the two types of laminations should be distributed between the top and bottom halves of the magnet based on the measured $a_{1}$ in the collared coil. Note that if the measured $a_{1}$ is zero then the change over from magnetic to non-magnetic laminations would occur at the same axial location in the top and bottom halves of the magnet.

In the BNL-built SSC $50 \mathrm{~mm}$ dipole design, the length of the coil straight section is 14.859 meter and the length of the space occupied by low carbon steel laminations is 14.783 meter. The length of the space occupied by the stainless steel laminations is $150 \mathrm{~mm}$ in each end. With this geometry one can easily correct a few units of $a_{1}$ before getting into a region where the correction becomes highly non-linear or may significantly change the peak field distribution on the conductor. This change should give an adequate correction based on the expected maximum $a_{1}$ in most magnets or based on the measured $a_{1}$ in the prototype magnets. 


\subsubsection{Yoke Length for Integral Transfer Function Correction}

The measured integral transfer function in the magnets may be different from the value . expected from calculations. The difference in measured integral field could also be observed between two series of magnets built on the same design but by two manufacturers with two different sets of toolings. This was observed in HERA magnets [113,179]. This difference is of concern when these magnets are connected in series over the same power supply.

As mentioned earlier, the laminations used in the ends of the SSC magnets are made of non-magnetic stainless steel and the laminations used in the body (straight section) are made of magnetic low carbon steel. The place of transition from the magnetic steel to the non-magnetic steel laminations is somewhat flexible. An adjustment in the length (amount) of magnetic laminations to the stainless steel laminations for a fixed total mechanical length (amount), can be used as a parameter to adjust integral transfer function (see Fig. 5.3.1). This adjustment could either be applied to obtain a systematic change in the value to accommodate a systematic difference in transfer function between two manufacturers or to correct for a continuous upward or downward trend in transfer function during production. It could even be applied on a magnet by magnet basis after measurements (perhaps on the collared coil) to reduce the random variation in transfer function. Moreover, the proposed adjustment can be applied after the magnet tooling is made, a few magnets are constructed and magnetic measurements are performed. To reduce the random variation in transfer function, it may be adequate to measure the integral transfer function in the collared coil. The above adjustment then can be applied during the yoke assembly.

The feasibility of this scheme is examined here. The measured systematic difference in the transfer function between the two series of HERA dipole magnets $[113,179]$ manufactured by two vendors was $\sim 2 \times 10^{-3}$. The required adjustment to correct the above difference in SSC magnets is evaluated here. In SSC dipole magnets, the yoke contributes $\sim 25 \%$ to the transfer function at injection and $\sim 22 \%$ at the maximum design field. The design magnetic length of the dipole was 15 meter. Therefore to adjust $2 \times 10^{-3}$ at injection one would change the material of yoke laminations in a length by $\sim 12 \mathrm{~cm}$. To implement such a scheme in practice, one would start with a magnet design in which the total magnetic lamination length is reduced (or conversely the total stainless steel lamination length is increased) from a typical value. To contain the leakage (fringe) field in that region, if required, one could have low carbon steel laminations with an increased inner diameter. The stainless steel laminations then could be put in-between the collar and the increased 
yoke inner diameter. The later would decrease the efficiency of the proposed scheme only by a small amount as compared to the case when all end laminations were made of stainless steel only.

Methods to adjust a current dependence in the skew quadrupole harmonic $\left(a_{1}\right)$ in dipoles have been discussed earlier. To adjust a current dependence in transfer function, one could adjust the packing factor of magnetic, low carbon steel laminations in the body (straight section) of a magnet. This adjustment in the current dependence has been applied in the $80 \mathrm{~mm}$ aperture $\mathrm{RHIC}$ arc dipoles. 


\subsection{Conclusions on the Field Quality Improvements after Construction}

The methods described in this chapter show that it is possible to build superconducting magnets having relative field errors $\left(\frac{\Delta B}{B}\right)$ only a few parts in $10^{-5}$ instead of the usual errors of a few parts in $10^{-4}$ at $2 / 3$ of the coil radius. This improvement in field quality is crucial to the high luminosity performance of RHIC and could be important to all high energy colliders.

The tuning shim method described overcomes the limitations in field quality posed by manufacturing tolerances. It compensates for the field errors in the body of the magnet. The second proposed method makes a lumped correction at the magnet ends. Though this has been proposed here for compensating integral $a_{1}$, it can be used for compensating other harmonics also.

Eight tuning shims are used to independently correct eight measured harmonics in the $130 \mathrm{~mm}$ aperture RHIC insertion quadrupoles. The tuning shims are installed after the cold-mass has been assembled. This does not require opening the magnet, which not only makes the process more efficient but also more reliable. If a magnet is opened and assembled again, there may be a slight change in field harmonics which can not be neglected in these high field-quality magnets.

Good agreement has been found between the calculations and measurements based on the data available so far. A series of warm measurements have been performed to make comparison over a wide range of tuning shims. These results show that the effects can be reliably computed at low fields. Magnetic measurements at the design current $(5 \mathrm{kA})$ were also performed to complete a comparison between the calculations and measurements. The algorithm to compute the tuning shim thickness is based on these measured changes in harmonics at $5 \mathrm{kA}$.

The method of tuning shims (or a simplified version of it) can also be adopted in a large scale magnet production program. In that case the number of tuning shims can be reduced to correct only a few important harmonics. This could relieve the tolerances on parts, assembly and general manufacturing process while assuring a good field quality in a plan suitably adopted for the industrial magnet production environment. 


\section{Chapter 6. OPTIMIZED CROSS SECTION DESIGNS}

The research work described in this chapter is partly based on the following papers :

1. R.C. Gupta, S.A. Kahn and G.H. Morgan, Coil and Iron Design for SSC $50 \mathrm{~mm}$ Magnet, Proceedings of the 1990 American Society of Mechanical Engineers (ASME) Winter Annual Meeting in Dallas (1990).

2. R.C. Gupta, S.A. Kahn and G.H. Morgan, A Comparison of Calculations and Measurements of the Field Harmonics as a Function of Current in the SSC Dipole Magnets, Proceedings of the 1991 IEEE Particle Accelerator Conference, San Francisco, pp. 42-44 (1991).

3. R.C. Gupta, et al., RHIC Insertion Magnets, Proceedings of the 1991 IEEE Particle Accelerator Conference, San Francisco, pp. 2239-2241 (1991).

4. R.C. Gupta, S.A. Kahn and G.H. Morgan, SSC $50 \mathrm{~mm}$ Dipole Cross section, Proceedings of the International Industrial Symposium on Super Collider (IISSC), Atlanta, pp. 587-600 (1991).

5. R. Gupta, et al., Large Aperture Quadrupoles for RHIC Interaction Regions, Proceedings of the 1993 Particle Accelerator Conference, Washington, D.C., pp. 2745-2747 (1993).

6. R.C. Gupta, A.K. Jain, Variation in a saturation in SSC Collider Dipoles, Proceedings of the 1993 Particle Accelerator Conference, Washington, D.C., Pp. 2778-2780 (1993).

7. R. Gupta, et al., Field Quality Improvements in Superconducting Magnets for RHIC, Proceedings of the 1994 European Particle Accelerator Conference, London, UK, pp. 2928-2930 (1994).

\subsection{Introduction}

In this chapter the magnetic design and optimization process and the final design of the cross section of the $130 \mathrm{~mm}$ aperture $\mathrm{RHIC}$ insertion quadrupole and the prototype of the $50 \mathrm{~mm}$ aperture SSC dipole magnet will be described. The field harmonics of the SSC dipole magnet are specified for a reference radius of $10 \mathrm{~mm}$ and is given in units of $10^{-4}$ of the central field. The harmonics of the insertion quadrupole are specified for a reference radius of $40 \mathrm{~mm}$, in units of $10^{-4}$ of the field magnitude at this radius. 


\subsection{SSC $50 \mathrm{~mm}$ Aperture Collider Dipole Magnet Cross-section}

In this section the magnetic design of the two dimensional coil and iron cross section for the prototype $50 \mathrm{~mm}$ aperture main ring dipole magnet for the Superconducting Super Collider (SSC) is presented. Several prototype dipole magnets based on this design have been built at Brookhaven National Laboratory (BNL) and at Fermi National Accelerator Laboratory (FNAL). Except for a few minor differences (which will be discussed in more detail later), the magnetic design of the BNL and FNAL magnets is nearly the same. The computed values of the allowed field harmonics as a function of current, the quench performance predictions, the stored energy calculations, the effect of construction errors on the field harmonics and the Lorentz forces on the coil will be discussed. The yoke has been optimized to reduce the effects of iron saturation on the field harmonics. A summary of this design will also be presented.

\subsubsection{Coil Design}

The coil is made of two layers of superconducting cables. Some parameters of the cables used in the inner and outer layers are given in Table 6.2.1.

The coil is designed by placing the cables in such a way that they produce a field with a high degree of uniformity. This is done using the computer program PAR2DOPT [130] which uses analytic expressions for computing the field harmonics at the center of the magnet of coils in a circular $\infty \mu$ iron aperture. It also computes the peak field on the surface of the conductor.

A large number of configurations for the coil design were examined. The one selected has a total of 45 turns in each quadrant in two layers. The inner layer has 19 turns in four blocks (three wedges) and the outer has 26 turns in two blocks (one wedge). In the final selection of the optimized coil cross section, the peak field (the maximum magnitude of the magnetic field in the conductor) was also used as an important parameter in addition to the other magnetic and mechanical parameters. For the same transfer function, a coil design with a lower peak field will produce a magnet which will quench at a higher current. In a search for the optimum coil configuration, the number of wedges in the outer layer was kept at one whereas for the inner layer, solutions with a variable number of wedges were examined. The designs with two wedges in the inner layer were, in general, found to have a higher peak field or excessive harmonic content. For this reason, the design chosen 
Table 6.2.1: Properties of the cables used in the SSC $50 \mathrm{~mm}$ dipoles. $J_{c}$ gives the value of the critical current density which was used in the design calculations for the superconducting part of the wires (strands) and cables.

\begin{tabular}{|c|c|c|}
\hline Cable parameters & Inner layer & Outer Layer \\
\hline Filament diameter, micron & 6.0 & 6.0 \\
\hline Strand diameter, $\mathrm{mm}$ & 0.808 & 0.648 \\
\hline Strand $J_{c}(5 T, 4.2 K), A / \mathrm{mm}^{2}$ & 2750 & 2750 \\
\hline No. of strands & 30 & 36 \\
\hline $\begin{array}{c}\text { No. of strands } \times \text { Strand Area, } \mathrm{mm}^{2} \\
\text { (area of metal) }\end{array}$ & 15.382 & 11.872 \\
\hline Cable $J_{c}(5 T, 4.2 K), A / \mathrm{mm}^{2}$ & 2612.5 & 2612.5 \\
\hline Cable width, bare, mm & 12.34 & 11.68 \\
\hline Cable width, insulated, $\mathrm{mm}$ & 12.51 & 11.85 \\
\hline Cable mid-thickness, bare, $\mathrm{mm}$ & 1.458 & 1.156 \\
\hline Cable mid-thickness, insulated, mm & 1.626 & 1.331 \\
\hline Cable area, bare, $\mathrm{mm}^{2}$ & 17.99 & 13.50 \\
\hline Cable area, insulated, $\mathrm{mm}^{2}$ & 20.34 & 15.77 \\
\hline Keystone, (max-min) thickness, mm & 0.262 & 0.206 \\
\hline
\end{tabular}

has three wedges in the inner layer. However, the present coil is optimized in such a way that the two wedges nearest to the pole in the inner layer are identical and symmetric. A symmetric wedge design has a lower chance of incorrect installation as compared to a non-symmetric wedge design. The final design with symmetric wedges has performance comparable to those that did not require the wedges to be symmetric. The wedge in the outer layer is close to symmetric and in fact, in magnets built at FNAL this wedge was also made mechanically symmetric, without changing its effective size in the coil.

The cross section of the optimized coil placed in the stainless steel collar is shown in Fig. 6.2.1. 


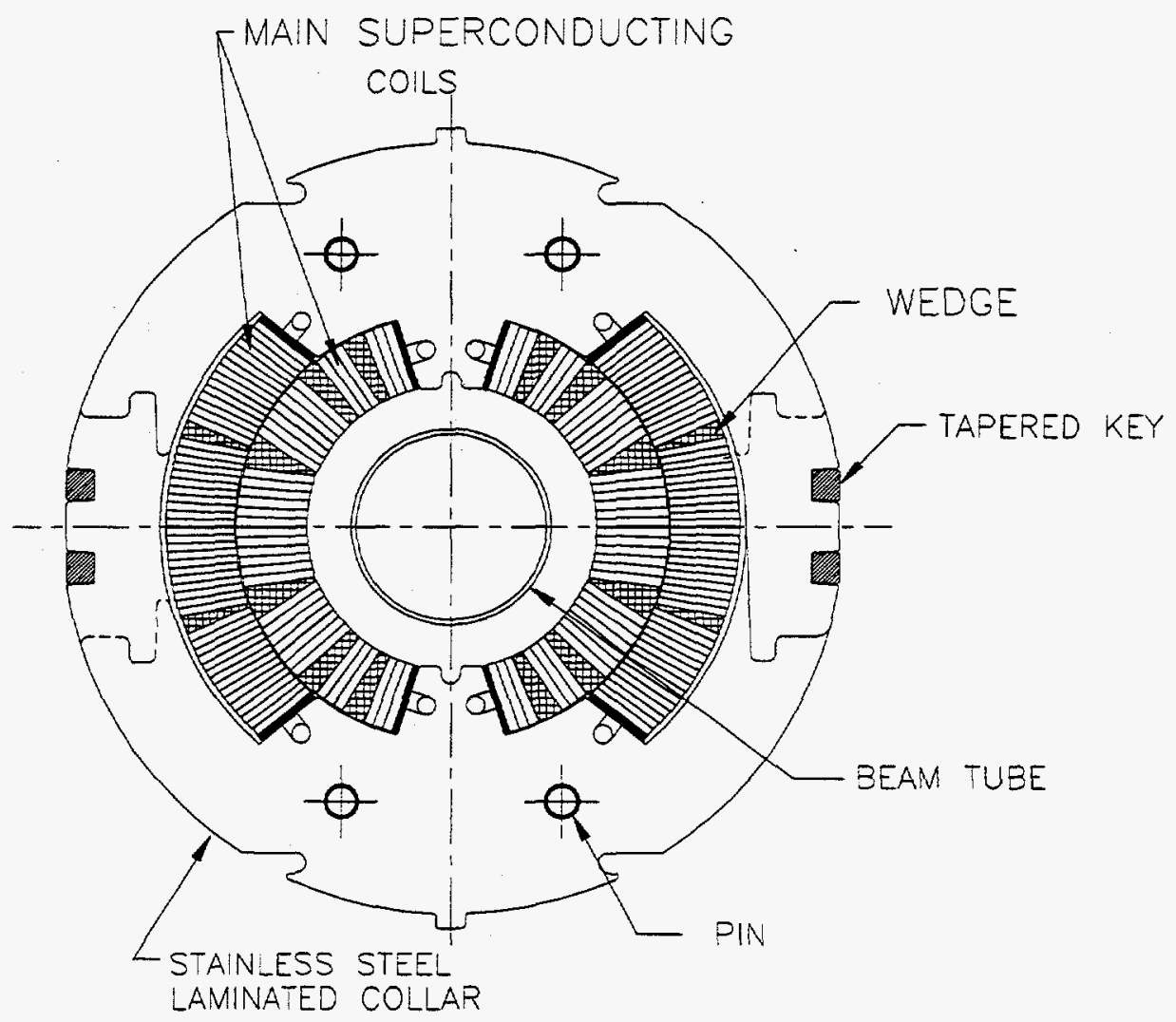

Figure 6.2.1: The cross section of the optimized coil for the prototype SSC $50 \mathrm{~mm}$ main collider dipole magnet. The coil is shown inside the stainless steel collar, which provides the compression on the coil. 


\subsubsection{Low Field Harmonics}

The iron aperture is not completely circular in this magnet. It has a pole notch and a small vertical straight face at the midplane. These features introduce small but noticeable values of the $b_{2}$ and $b_{4}$ harmonics. These harmonics should be cancelled in the coil design if the magnet is to produce zero low field harmonics. Therefore, to cancel the effects of the non-circular iron inner radius, -0.28 unit of $b_{2}$ and +0.01 of $b_{4}$ were desired in the optimized coil. In addition, a non-zero value of $b_{8}$ harmonic was desired for centering the coil during the field measurements. Since the given tolerance in $b_{8}$ was 0.05 unit at the time of design, a solution was sought which had a magnitude for this harmonic between 0.04 and 0.05 . This requirement on $b_{8}$ eliminated many coil configurations from contention. However, the final design that satisfied all of the above requirements was equal in performance to those that did not. An alternate cross section with a zero $b_{8}$ harmonic was also designed which was mechanically very close to this cross section and, moreover, had all wedges perfectly symmetric. However, no magnet was ever built with this alternate cross section.

In Table 6.2.2 the desired and optimized values of field harmonics are presented. Harmonics higher than $b_{12}$ had an optimized value of $<0.001$, as desired. In the row labelled "Desired" the allowed systematic errors are also listed. In the row "BNL magnets", the harmonics inciude the effects of the pole notch and the flat face in the iron at the midplane. These would be the expected values of low field harmonics in this magnet, not including the contributions from persistent currents in the superconductor. The size of the cable used in the actual magnets was different (inner layer cable wider and outer layer cable thinner) by a small amount from that assumed in the original design. This produced noteworthy deviations in the three lowest allowed field harmonics. The last two rows of the table, "Revised BNL" and "Revised FNAL", refer to the values of field harmonics in the magnet after this change in the cable size.

A small difference in the "Revised BNL" and "Revised FNAL" harmonics is due to the fact that (a) the pole angle in the outer layer of the FNAL cross section is $10 \mathrm{mil}(0.254 \mathrm{~mm})$ smaller than in the BNL version (the wedge size was the same therefore the effective cable thickness in the coil was reduced) and (b) the notch in the aperture of the vertically split iron is at the midplane and in the horizontally split iron is at the pole. The normalization or reference radius $\left(R_{0}\right)$ for the field harmonics is $10 \mathrm{~mm}$ and as usual the harmonics are given in units $10^{-4}$ of the central field. 
Table 6.2.2: Desired and Optimized values of low field harmonics with a circular aperture. The harmonics in "BNL magnets" include the effects of the pole notch and a flat face in the iron at the midplane. These harmonics are in units of $10^{-4}$ at $10 \mathrm{~mm}$ reference radius. The last two rows include the effects of a change in cable size.

\begin{tabular}{|c|c|c|c|c|c|c|}
\hline Values & $b_{2}$ & $b_{4}$ & $b_{6}$ & $b_{8}$ & $b_{10}$ & $b_{12}$ \\
\hline Desired & $-.28 \pm .4$ & $.01 \pm .1$ & $0 \pm .05$ & $\pm .045 \pm .05$ & $0 \pm .05$ & $0 \pm .05$ \\
\hline Optimized & -0.280 & 0.009 & -0.004 & 0.044 & 0.014 & -0.001 \\
\hline BNL magnets & 0.000 & -0.001 & -0.004 & 0.044 & 0.014 & -0.001 \\
\hline Revised BNL & 1.566 & 0.070 & -0.024 & 0.043 & 0.015 & -0.001 \\
\hline Revised FNAL & 0.165 & 0.073 & -0.021 & 0.043 & 0.015 & -0.001 \\
\hline
\end{tabular}

\subsubsection{Iron Yoke Design}

In this section, the process used in designing the iron yoke is discussed. The iron contributes about $22 \%$ to the magnetic field at 6.7 tesla (somewhat higher at lower field). Since the magnetization of the iron is not a linear function of the current in the coil and varies throughout the cross section, the uniformity of the field becomes a function of the current in the coil. The yoke is optimized to produce a minimum change in the field harmonics due to iron saturation for the maximum achievable value of transfer function at 6.7 tesla. The results of field computations with the computer codes POISSON and MDP will be presented here. The computer model of the final design and the results of field calculations with POISSON will be discussed in more detail. An iron packing factor of $97.5 \%$ has been used in these calculations.

If no special technique for controlling iron saturation were used, the change in the $b_{2}$ harmonic due to iron saturation would be over 1 unit. The following three options were considered for reducing the $b_{2}$ saturation swing. They all try to control the iron saturation at the iron aperture so that it saturates evenly.

- Reduced (shaved) iron o.d.

- Stainless Steel (non-magnetic) key at the midplane

- Shim at the iron inner surface 
The first scheme, though most straight forward, produces a larger loss in transfer function at 6.7 tesla than the other two schemes. The third scheme, though actually increasing the transfer function at 6.7 tesla due to extra iron, requires more engineering development due to its non-circular aperture. The second scheme produces very little loss in transfer function ( $0.3 \%$ at 6.7 tesla compared to a keyless or magnetic key version) for a comparatively large reduction in $b_{2}$ due to saturation ( $\frac{3}{4}$ unit). Moreover, it has the advantage of giving a way to control the $b_{2}$ due to saturation by changing the location and/or size of the key without affecting the other parts of the magnet design. It may be pointed out that besides the change due to iron saturation, $b_{2}$ and the other harmonics are also a function of current because of the coil deformation due to Lorentz forces. This has been observed in several SSC $40 \mathrm{~mm}$ aperture dipole magnets [64]. If the measured change in the $b_{2}$ harmonic is more than desired (either due to saturation or due to coil motion due to Lorentz forces), then this could be a useful and convenient method of correction.

The cross section of the cold mass (coil, collar and yoke) for the BNL-built SSC 50 $\mathrm{mm}$ prototype dipole is shown in Fig. 6.2.2. The POISSON model of this optimized cross section is given in a previous chapter as Fig. 3.2.2. The cross section for the vertically split iron used by FNAL is shown in Fig. 6.2.3. The field lines at 6500 ampere are also shown in this figure. The iron i.d. is $135.6 \mathrm{~mm}$; leaving a space of $17 \mathrm{~mm}$ for the collar, and the iron o.d. is $330.2 \mathrm{~mm}$. The stainless steel key in the horizontally split yoke design is located at $91.4 \mathrm{~mm}$ and has a size of $12.7 \mathrm{~mm} \times 12.7 \mathrm{~mm}$. In the vertically split design for the FNAL-built magnet, a cutout at the horizontal midplane is incorporated to reduce iron saturation. The size and location of this cutout is the same as in the BNL yoke. As mentioned earlier, the iron aperture is not completely circular. The BNL yoke has a pole notch of size $5.11 \mathrm{~mm} \times 2.67 \mathrm{~mm}$ and a vertical straight face at the midplane which starts at $x=67.13 \mathrm{~mm}$. The FNAL yoke has both the notch at the midplane and a vertical face at the midplane. The FNAL yoke has an additional pin located below the bus slot. This pin is made of non-magnetic steel and produces a noticeable effect on iron saturation. Other features in the two yokes are shown in the above mentioned figures.

The computed transfer function (T.F.) and $b_{2}$ as a function of current in the BNL and FNAL magnets are listed in Table 6.2.3. The $b_{2}$ harmonic has been adjusted so that it starts from zero; a non-zero value is artificial and is related to the way the computer model of a given coil and the iron geometry is set up in the two codes. The maximum computed $b_{2}$ due to saturation is about 0.3 unit. The calculations presented here, however, do not include 


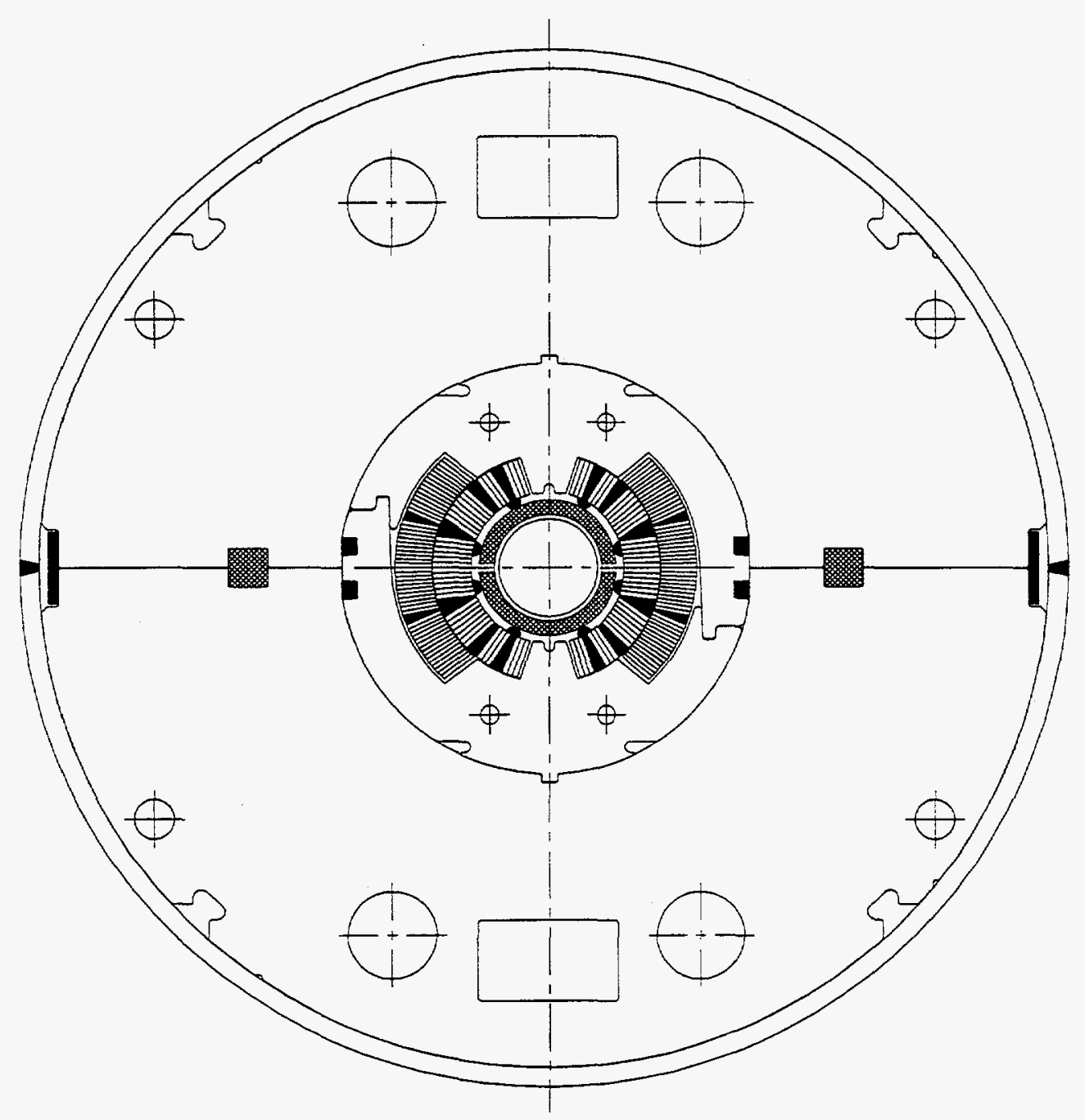

Figure 6.2.2: The cross section of the cold mass of $50 \mathrm{~mm}$ aperture horizontally split iron SSC arc dipoles. This cross section has been used in BNL built prototype magnets for SSC. The above cold mass is put inside a cryostat (not shown here). 


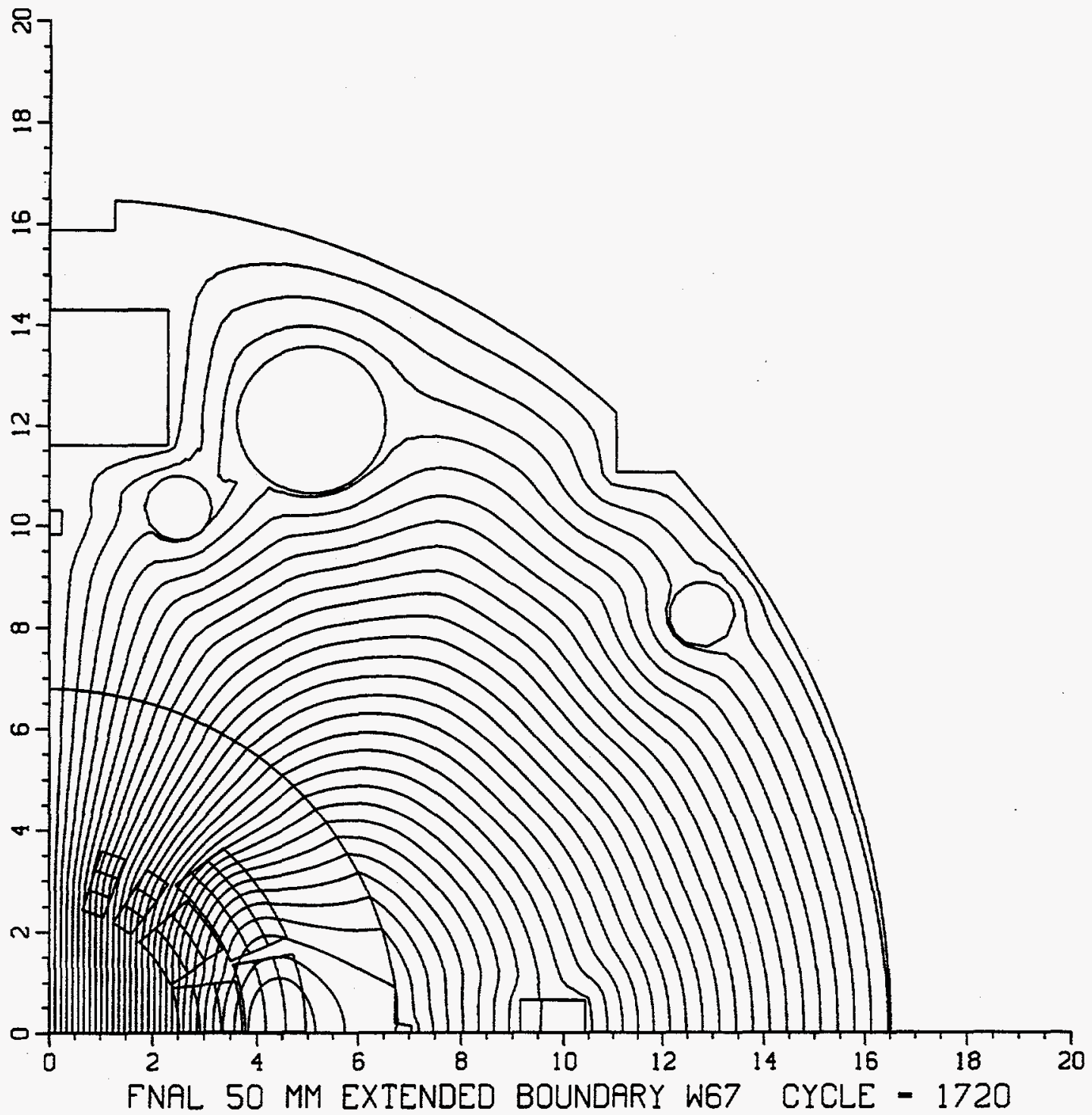

Figure 6.2.3: POISSON model and field lines at 6500 ampere for SSC $50 \mathrm{~mm}$ Dipole with vertically split iron laminations. This magnetic design was used in the prototype magnets built at FNAL. 
the effects of the cryostat wall which modifies the current dependence of the harmonics at high current. POISSON uses a generalized finite difference method whereas MDP uses an integral method. Despite the fact that these two programs use two different methods for solving the problem, it is encouraging to see that both predict a small saturation shift. Similar calculations have been made by Kahn [64] with the computer code PE2D which uses the finite element method and good agreement has been found with the above calculations.

Table 6.2.3: Transfer function and $b_{2}$ variation as function of current. In all cases $b_{2}$ is corrected to start from zero at $3.0 \mathrm{kA}$. FNAL yoke calculations were done only with the code POISSON.

\begin{tabular}{|c|c|c|c|c|c|c|}
\hline I & \multicolumn{3}{|c|}{ T.F. (T/kA) } & \multicolumn{3}{c|}{$b_{2} \times 10^{-4}$} \\
\hline kA & FNAI & \multicolumn{2}{|c|}{ BNL yoke } & FNAL & \multicolumn{2}{c|}{ BNL yoke } \\
& yoke & POISSON & MDP & yoke & POISSON & MDP \\
\hline 3.0 & 1.0450 & 1.0447 & 1.0430 & 0.00 & 0.00 & 0.00 \\
\hline 4.0 & 1.0445 & 1.0441 & 1.0413 & -0.02 & 0.08 & 0.05 \\
\hline 5.0 & 1.0398 & 1.0397 & 1.0364 & -0.04 & 0.22 & 0.16 \\
\hline 5.5 & 1.0339 & 1.0340 & 1.0311 & 0.19 & 0.26 & 0.21 \\
\hline 6.0 & 1.0257 & 1.0262 & 1.0236 & 0.36 & 0.14 & 0.17 \\
\hline 6.25 & 1.0209 & 1.0219 & 1.0194 & 0.38 & 0.07 & 0.11 \\
\hline 6.5 & 1.0159 & 1.0173 & 1.0148 & 0.35 & -0.03 & 0.03 \\
\hline 7.0 & 1.0053 & 1.0073 & 1.0051 & 0.17 & -0.33 & -0.19 \\
\hline 7.6 & 0.9926 & 0.9955 & 0.9935 & -0.15 & -0.77 & -0.60 \\
\hline 8.0 & 0.9845 & 0.9877 & 0.9861 & -0.38 & -1.06 & -0.85 \\
\hline 8.6 & 0.9732 & 0.9766 & 0.9758 & -0.70 & -1.43 & -1.20 \\
\hline
\end{tabular}

The maximum change in the $b_{2}$ and $b_{4}$ harmonics and the drop in transfer function, $\delta(T F)$, at 6.6 tesla (as compared to its value at low field) due to iron saturation as computed by these codes are listed in Table 6.2.4. All higher harmonics remain practically unchanged. In the case of the FNAL yoke, the computations have been done only with the code POISSON. 
Table 6.2.4: Drop in transfer function at 6.6 tesla and the maximum change in $b_{2}$ and $b_{4}$; higher harmonics remain practically unchanged.

\begin{tabular}{|c|c|c|c|}
\hline Harmonic & $\begin{array}{c}\text { POISSON } \\
\text { FNAL yoke }\end{array}$ & $\begin{array}{c}\text { POISSON } \\
\text { BNL yoke }\end{array}$ & $\begin{array}{c}\text { MDP } \\
\text { BNL yoke }\end{array}$ \\
\hline$\delta(T F)$, at $6.6 \mathrm{~T}$ & $2.84 \%$ & $2.62 \%$ & $2.70 \%$ \\
\hline$\delta\left(b_{2}\right)_{\max }, 10^{-4}$ & 0.36 & 0.28 & 0.22 \\
\hline$\delta\left(b_{4}\right)_{\max }, 10^{-4}$ & 0.02 & -0.03 & -0.02 \\
\hline
\end{tabular}

In Table 6.2.5 the results of POISSON calculations are presented for various values of current in the BNL design. In Fig. 6.2.4, the variation in field harmonics as a function of central field is plotted.

The coldmass (see Fig. 6.2.2) is placed in the cryostat. To provide the maximum space for the support posts which minimizes the heat leak, the cold mass is placed above the center of the cryostat, which breaks the top-bottom symmetry. At high field, when the field lines can not be contained in the iron yoke, the cryostat provides an extra return path for flux. A top-bottom asymmetry in the magnet structure is then seen in the magnetic field. The most prominent harmonic to reflect this asymmetry is the skew quadrupole $\left(a_{1}\right)$ term. The presence of the skew quadrupole harmonic at high field and methods to minimize it have been discussed in a previous chapter. 
Table 6.2.5: Results of POISSON computations for the SSC 50 $\mathrm{mm}$ dipole with the horizontally split yoke design built at BNL.

\begin{tabular}{|c|c|c|c|c|c|c|c|c|}
\hline $\begin{array}{c}\mathbf{I} \\
\mathbf{k A}\end{array}$ & $\begin{array}{c}B_{0} \\
\text { tesla }\end{array}$ & $\begin{array}{c}\text { T.F. } \\
\text { T/kA }\end{array}$ & $\begin{array}{c}b_{2} \\
10^{-4}\end{array}$ & $\begin{array}{c}b_{4} \\
10^{-4}\end{array}$ & $\begin{array}{c}b_{6} \\
10^{-4}\end{array}$ & $\begin{array}{c}b_{8} \\
10^{-4}\end{array}$ & $\begin{array}{c}b_{10} \\
10^{-4}\end{array}$ & $\begin{array}{c}b_{12} \\
10^{-4}\end{array}$ \\
\hline$\infty \mu$ & $\infty \mu$ & 1.04493 & 0.020 & -0.046 & 0.000 & 0.047 & 0.015 & -0.001 \\
\hline 3.000 & 3.1341 & 1.04471 & 0.031 & -0.046 & 0.001 & 0.047 & 0.015 & -0.001 \\
\hline 4.000 & 4.1762 & 1.04406 & 0.111 & -0.050 & 0.001 & 0.047 & 0.015 & -0.001 \\
\hline 4.500 & 4.6921 & 1.04268 & 0.140 & -0.055 & 0.001 & 0.047 & 0.015 & -0.001 \\
\hline 4.750 & 4.9464 & 1.04135 & 0.182 & -0.060 & 0.001 & 0.047 & 0.015 & -0.001 \\
\hline 5.000 & 5.1985 & 1.03969 & 0.255 & -0.063 & 0.001 & 0.047 & 0.015 & -0.001 \\
\hline 5.250 & 5.4454 & 1.03721 & 0.299 & $\mid-0.066$ & 0.001 & 0.047 & 0.015 & -0.001 \\
\hline 5.500 & 5.6871 & 1.03402 & 0.291 & -0.069 & 0.001 & 0.048 & 0.015 & -0.001 \\
\hline 5.750 & 5.9240 & 1.03027 & 0.235 & $\mid-0.071$ & 0.001 & 0.048 & 0.015 & -0.001 \\
\hline 6.000 & 6.1573 & 1.02621 & 0.172 & -0.073 & 0.000 & 0.048 & 0.015 & -0.001 \\
\hline 6.250 & 6.3868 & 1.02189 & 0.100 & -0.073 & 0.000 & 0.048 & 0.015 & -0.001 \\
\hline 6.500 & 6.6121 & 1.01725 & -0.003 & -0.072 & 0.000 & 0.048 & 0.015 & -0.001 \\
\hline 7.000 & 7.0513 & 1.00733 & -0.300 & -0.072 & 0.000 & 0.049 & 0.015 & -0.001 \\
\hline 7.600 & 7.5654 & 0.99545 & -0.738 & -0.070 & 0.000 & 0.049 & 0.015 & -0.001 \\
\hline 8.000 & 7.9014 & 0.98767 & -1.032 & $|-0.068|$ & 0.000 & 0.050 & 0.015 & -0.001 \\
\hline 8.600 & 8.3984 & 0.97656 & -1.403 & -0.064 & 0.000 & 0.050 & 0.015 & -0.001 \\
\hline
\end{tabular}


Current dependence in BNL Built SSC $50 \mathrm{~mm}$ Prototype Dipole

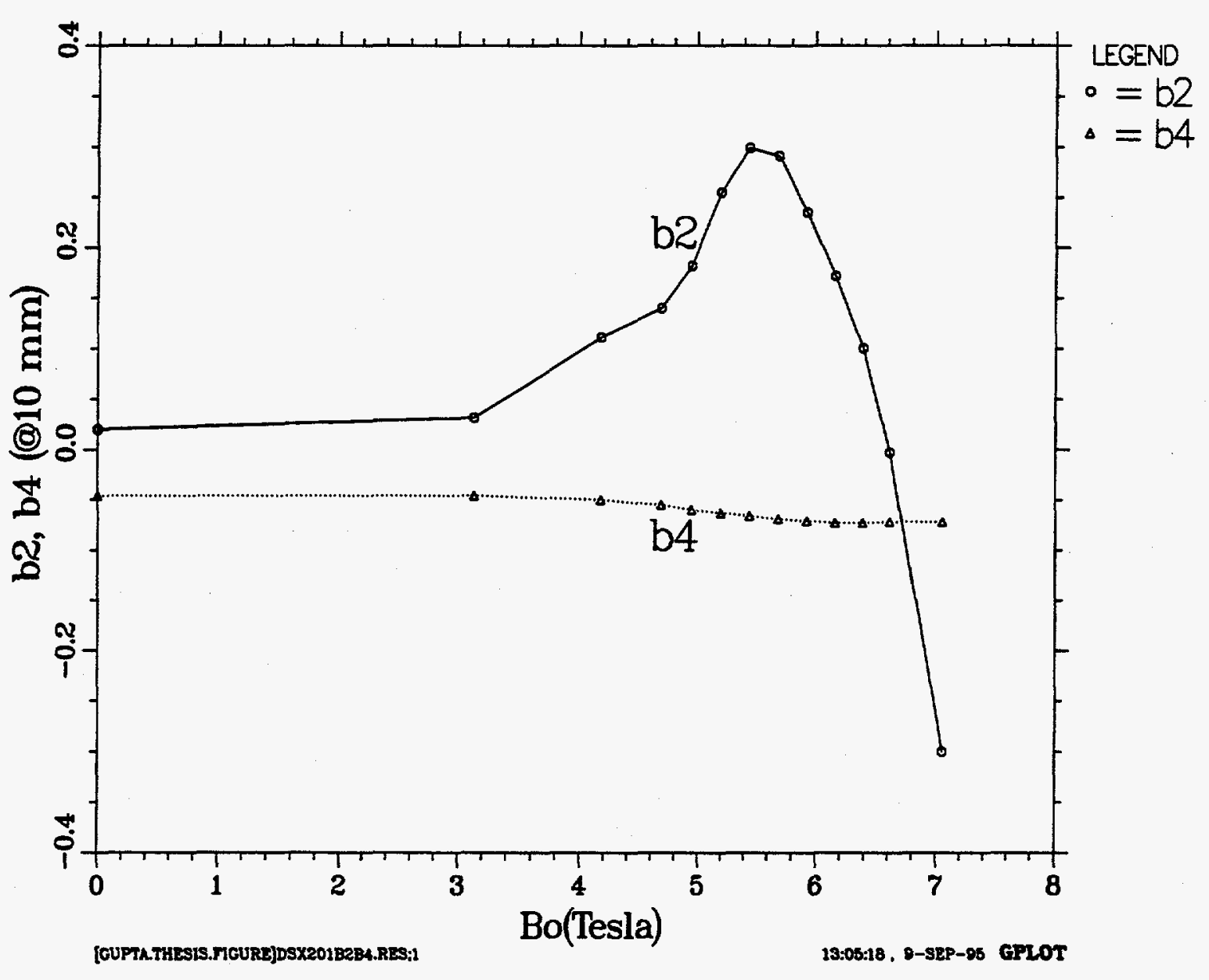

Figure 6.2.4: Variation in Field Harmonics as a function of Current in the SSC $50 \mathrm{~mm}$ BNL built prototype dipole magnet as computed by POISSON. 


\subsubsection{Expected Quench Performance}

The central field at which a given cable looses its superconducting properties $\left(B_{S S}\right.$, with "ss" standing for Short Sample) depends on the current in the cable which is a function of the maximum magnetic field at the conductor (the peak field) and the bath temperature. The superconducting cables for the inner and outer layers are optimized to provide a critical current $(I c)$ at a specified temperature and magnetic field. In a two layer coil design the magnetic design is optimized such that the computed short sample currents in the inner and outer layers are nearly the same. The peak field $\left(B_{p k}\right)$ in the inner and outer layers of the SSC $50 \mathrm{~mm}$ dipole are listed in Table 6.2.6 for two values of central field $\left(B_{o}\right)$. The ratio of $B_{p k}$ to $B_{o}$, the Enhancement Factor, is given in the next column. In each layer, the peak field is found on the upper side of the top-most pole turn. The location of the peak field is listed in the next column. It is expressed as \% of the cable width, measured from the upper-inner corner. The peak field calculations are done using the code MDP. MDP is based on the integral method and therefore is expected to give a more accurate field at the surface of the conductor as compared to codes based on the finite element method which require meshing the conductor.

Table 6.2.6: Peak fields in the SSC $50 \mathrm{~mm}$ dipole as computed using code MDP.

\begin{tabular}{|c|c|c|c|c|c|c|c|}
\hline $\mathrm{I}$ & $B_{0}$ & \multicolumn{3}{|c|}{ Inner } & \multicolumn{3}{c|}{ Outer } \\
\hline $\mathrm{kA}$ & tesla & $B_{p k}, \mathrm{~T}$ & $\frac{B_{p k}}{B_{o}}$ & Location & $B_{p k}, \mathrm{~T}$ & $\frac{B_{p k}}{B_{0}}$ & Location \\
\hline 6.85 & 6.9058 & 7.2374 & 1.048 & $5 \%$ & 6.0016 & 0.869 & $11 \%$ \\
\hline 7.20 & 7.2100 & 7.5595 & 1.048 & $5 \%$ & 6.2660 & 0.869 & $11 \%$ \\
\hline
\end{tabular}

The calculations assume that the superconductor in the wire will have a critical current density $J_{c}(5 T, 4.2 \mathrm{~K})$ of $2750 \mathrm{~A} / \mathrm{mm}^{2}$. The quality of the superconductor is degraded when the wires are made in to a cable and put in the magnet. The calculations presented in Table 6.2.7 have been done assuming $5 \%$ degradation $\left(J_{c}=2612.5\right)$ and $4.35 \mathrm{~K}$ bath temperature.

In Table 6.2.7, the field margin $\left(B_{\text {margin }}\right)$ and the temperature margin $\left(T_{\text {margin }}\right)$ are listed. The temperature margin is defined as the maximum possible computed rise in the operating temperature (over the design value of normal operation, which is $4.35 \mathrm{~K}$ ) before 
Table 6.2.7: Expected quench performance of the SSC $50 \mathrm{~mm}$ dipole with $5 \%$ cable degradation $\left(J_{c}=2612.5 \mathrm{~A} / \mathrm{mm}^{2}\right)$ and at $4.35 \mathrm{~K}$ temperature. $S_{\text {quench }}$ is the computed current density in the copper at quench and $S_{6.7 T}$ at the design field of 6.7 Tesla.

\begin{tabular}{|c|c|c|c|c|c|c|c|}
\hline Layer & Cu/Sc & $B_{\star}$ & $I_{c}$ & $B_{\text {margin }}$ & $T_{\text {margin }}$ & $S_{\text {quench }}$ & $S_{6.7 T}$ \\
$\downarrow$ & Ratio & tesla & $A$ & \%over 6.7T & kelvin & $A / \mathrm{cm}^{2}$ & $A / \mathrm{cm}^{2}$ \\
\hline Inner & 1.7 & 7.149 & 7126 & 6.7 & 0.519 & 736 & 681 \\
& 1.5 & 7.273 & 7273 & 8.6 & 0.625 & 788 & 715 \\
& 1.3 & 7.399 & 7411 & 10.4 & 0.730 & 853 & 759 \\
\hline Outer & 2.0 & 7.268 & 7267 & 8.7 & 0.580 & 919 & 834 \\
& 1.8 & 7.445 & 7470 & 11.1 & 0.709 & 980 & 865 \\
\hline
\end{tabular}

the magnet will quench at the design central field $\left(B_{\text {design }}=6.7\right.$ tesla). The field margin is defined as follows

$$
B_{\text {margin }}(\%)=\frac{B_{a s}-B_{\text {design }}}{B_{\text {design }}} \times 100
$$

The calculations are done for copper to superconductor ratios, CSR or $\mathrm{Cu} / \mathrm{Sc}$, of 2.0 and 1.8 in the outer layer and 1.7, 1.5 and 1.3 in the inner layer. The computed central field $\left(B_{s s}\right)$ at the magnet quench point is listed together with the current in the cable at that point $\left(I_{c}\right)$ and the current density $\left(S_{\text {quench }}\right)$ in the copper available to carry that current after quench. A lower current density in the copper is expected to give better stability. The current density in the copper at 6.7 tesla $\left(S_{6.7 T}\right)$ is also listed. For stability purposes, $S_{6.7 T}$ may be a more appropriate parameter to consider than $S_{q u e n c h}$.

The design values selected were a copper to superconductor ratio of 1.8 in the outer layer and of 1.5 in the inner layer. The quench field $B_{s s}$ of 7.273 tesla in the inner layer gives a field margin of $8.6 \%$ over the design operating field $B_{\text {, }}$ of 6.7 tesla. The quench field of 7.445 tesla in the outer layer gives a field margin of $11.1 \%$. 


\subsubsection{Effect of Manufacturing Errors on the Allowed Harmonics}

For various reasons, the actual value of a parameter used in designing the coil may turn out to be somewhat different than desired. In particular, deviations in the locations of various turns in the coil are very important. This causes changes in the transfer function and the field harmonics. In this section the effect of these errors in various cases are estimated using a procedure developed by P.A. Thompson [130]. The basic four fold symmetry in the dipole coil geometry is retained in this analysis. Though this is not a realistic assumption, it is useful in estimating the size of some errors. In Table 6.2.8 these effects are listed for a nominal $0.05 \mathrm{~mm}$ variation in the given parameter.

First, the change in harmonics due to a change of $+0.05 \mathrm{~mm}$ in the radius of every turn in each current block, one block at a time, is given. The counting of the blocks in the table starts at the inner layer and at the midplane of each layer. Next the effect of changing the wedge size by $+0.05 \mathrm{~mm}$ is estimated. Pole angle is held constant in this calculation by reducing the conductor thickness by an appropriate amount. The counting scheme for the wedges is the same as it is for the current blocks. It is possible that during the molding, the thickness of the cable is not reduced uniformly within a layer. To estimate this effect, a linear increase in the cable thickness is assumed going from the midplane towards the pole, followed by a linear decrease, such that the middle turn is displaced azimuthally by 0.05 $\mathrm{mm}$. The pole angle does not change during this perturbation. This effect is given for the inner and outer layers in the next two rows of the table. The effect of increasing the pole angle by $0.05 \mathrm{~mm}$ in the inner and in the outer layer is shown in the last two rows. In each group the Root Mean Square (RMS) change of these variations is also given. 
Table 6.2.8: The effect of a $0.05 \mathrm{~mm}$ increase in the given parameter on the transfer function and the field harmonics.

\begin{tabular}{|c|c|c|c|c|}
\hline $\begin{array}{l}\text { Parameter } \\
\text { changed }\end{array}$ & $\begin{array}{c}\mathrm{TF} \\
\mathrm{T} / \mathrm{kA}\end{array}$ & $\begin{array}{c}b_{2} \\
10^{-4}\end{array}$ & $\begin{array}{c}b_{4} \\
10^{-4}\end{array}$ & $\begin{array}{c}b_{6} \\
10^{-4}\end{array}$ \\
\hline Radius of Block No. 1 & 0.31 & -0.25 & -0.10 & -0.01 \\
\hline Radius of Block No. 2 & -0.32 & 0.31 & 0.12 & 0.01 \\
\hline Radius of Block No. 3 & -0.12 & 0.36 & -0.02 & -0.01 \\
\hline Radius of Block No. 4 & -0.20 & 0.33 & -0.08 & 0.01 \\
\hline Radius of Block No. 5 & -0.11 & -0.04 & -0.01 & 0.00 \\
\hline Radius of Block No. 6 & -0.78 & 0.22 & 0.03 & 0.00 \\
\hline RMS Blocks & 0.38 & 0.27 & 0.07 & 0.01 \\
\hline Thickness of Wedge No. 1 & -1.56 & -0.48 & 0.02 & 0.01 \\
\hline Thickness of Wedge No. 2 & 0.83 & 0.59 & 0.05 & -0.01 \\
\hline Thickness of Wedge No. 3 & 2.32 & 0.71 & -0.04 & 0.00 \\
\hline Thickness of Wedge No. 4 & -0.57 & -0.11 & 0.00 & 0.00 \\
\hline RMS Wedges & 1.48 & 0.52 & 0.03 & 0.01 \\
\hline Cable thickness inner & 2.63 & 1.08 & 0.05 & -0.01 \\
\hline Cable thickness outer & 1.99 & 0.48 & 0.02 & 0.00 \\
\hline RMS Cable thickness & 2.33 & 0.83 & 0.04 & 0.01 \\
\hline Pole angle inner & -4.01 & -0.45 & 0.06 & -0.01 \\
\hline Pole angle outer & -2.26 & -0.42 & 0.00 & 0.00 \\
\hline RMS Pole angles & 3.25 & 0.43 & 0.04 & 0.01 \\
\hline
\end{tabular}




\subsubsection{Stored Energy and Inductance Calculations}

Stored energy calculations are done with the computer code POISSON [135]. POISSON uses the following formula to compute the stored energy per unit length $\left(E_{l}\right)$ over the cross section area :

$$
E_{l}=\frac{1}{2} \int_{a} J A_{z} d a,
$$

where $A_{z}$ is the vector potential and $J$ is the current density in the mesh triangle having an area da. The integration needs to be performed only over the regions containing current. At low fields when the field $B$ is proportional to $I$ (i.e. when yoke saturation is not significant), the stored energy is expected to be proportional to $B^{2}$ or $I^{2}$.

The stored energy and the inductance are related through the following formula :

$$
\text { Stored Energy }=\frac{1}{2} \text { Inductance } \times(\text { Current })^{2} \text {. }
$$

The inductance decreases at high field as the iron yoke saturates.

The results of POISSON computations for the SSC $50 \mathrm{~mm}$ aperture dipole are given at $6.5 \mathrm{kA}$ in Table 6.2.9 for the stored energy per unit length and the inductance per unit length. The total stored energy and the inductance for a $15 \mathrm{~m}$ long dipole are also given.

Table 6.2.9: Stored Energy and Inductance at $6.5 \mathrm{kA}$ as computed with the code POISSON for the SSC $50 \mathrm{~mm}$ aperture dipole.

\begin{tabular}{|l|l|}
\hline Stored Energy per unit length, $\mathrm{kJ} / \mathrm{m}$ & 105.0 \\
\hline Stored Energy for $15 \mathrm{~m}$ long Dipole, $\mathrm{kJ}$ & 1575.6 \\
\hline Inductance per unit length, $\mathrm{mH} / \mathrm{m}$ & 4.972 \\
\hline Inductance for $15 \mathrm{~m}$ long Dipole, $\mathrm{mH}$ & 74.585 \\
\hline
\end{tabular}




\subsubsection{Lorentz Force Calculations}

The value of Lorentz force per unit of axial length on each turn is obtained from the components of the magnetic field $\left(B_{x}, B_{y}\right)$. These components are calculated using program MDP. Since $B_{x}$ and $B_{y}$ are not uniform in a turn, an average value of these components is obtained from a grid of $10 \times 2$ across the width and thickness of the cable.

The variation in the magnitude of the radial and azimuthal components of the Lorentz forces, namely $F_{\tau}$ and $F_{a}$ (also referred to as $F_{\theta}$ ), with turn number is shown in Fig. 6.2.5. The turn numbers are counted from the midplane. The Lorentz force acts on the coil such that the azimuthal component compresses the coil on the midplane and the radial component expands it outward. Though the radial Lorentz force on the turns in the outer layer is very small, the force on the turns in the inner layer must be transmitted through the outer layer to the structure of the magnet. 


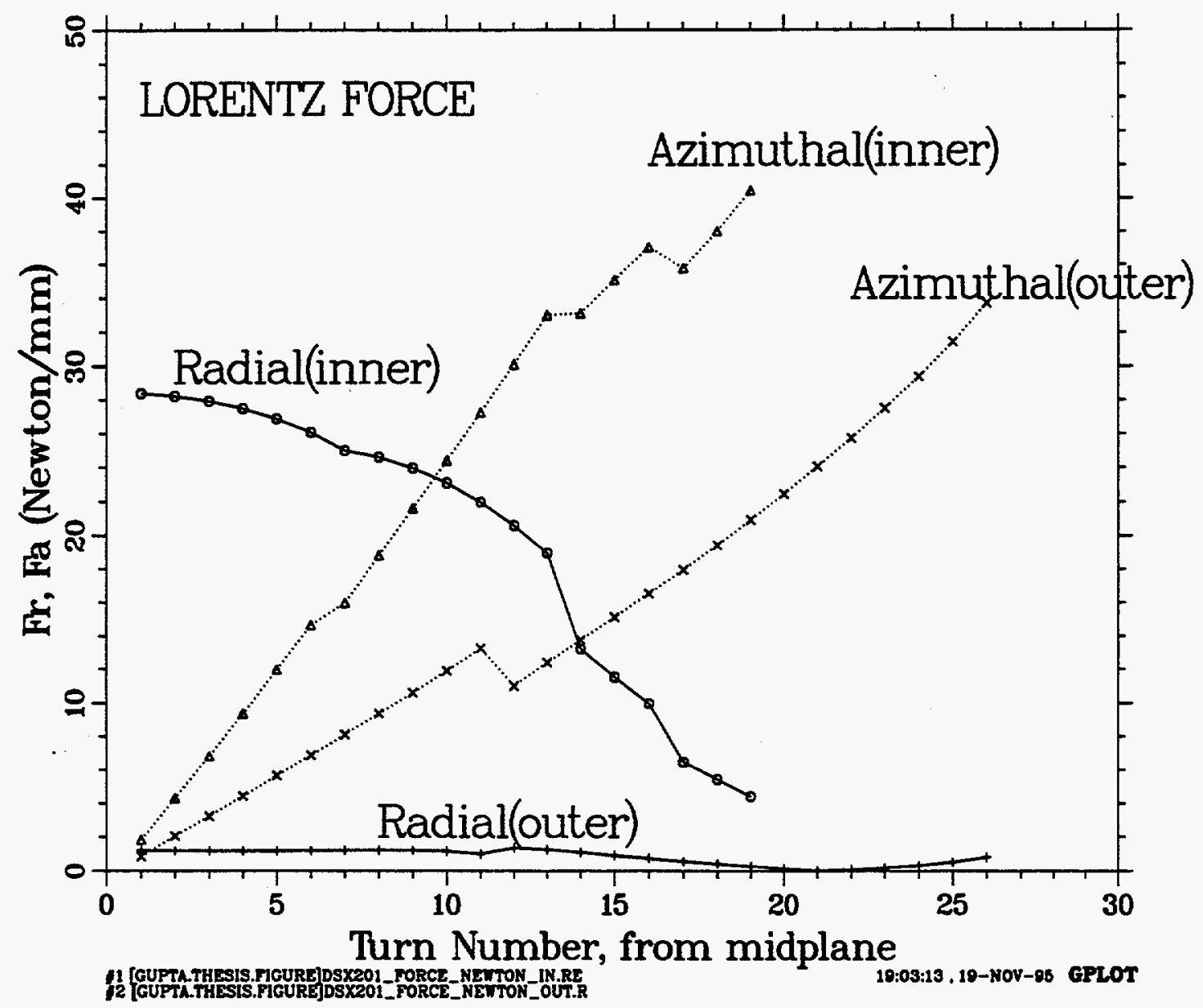

Figure 6.2.5: The magnitude of the components of the Lorentz force on the individual turns in a SSC $50 \mathrm{~mm}$ prototype magnet. The radial component of the force $\left(F_{r}\right)$ pushes the coil outward and the azimuthal component $\left(F_{a}\right)$ compresses the coil towards the midplane (horizontal plane). There are 19 turns in the inner layer and 26 turns in the outer layer of each quadrant. 


\subsubsection{Summary of the Design}

A summary of the coil and iron cross-sections are given respectively in Table 6.2.10 and Table 6.2.11. The coil has two layers and the number of turns is the number of turns in the upper or lower half of a layer. The field margin in this cross section is limited by the inner layer. If the cable used in the inner layer had a copper to superconductor ratio of 1.3 , the margin would be $10.4 \%$ (see Table 6.2.7).

Table 6.2.10: Summary of SSC $50 \mathrm{~mm}$ dipole coil cross section.

\begin{tabular}{|c|c|c|}
\hline Layer $\rightarrow$ & Inner & Outer \\
\hline No. of Turns ....... & 19 & 26 \\
Strand Diameter, mm & 0.808 & 0.648 \\
Strands per turn ..... & 30 & 36 \\
Coil i.d., mm ....... & 49.56 & 74.91 \\
Coil o.d., mm ....... & 75.36 & 99.42 \\
$B_{\text {peak }} / B_{\text {o }} \ldots \ldots \ldots \ldots$. & 1.048 & 0.869 \\
Cu/SC ........... & 1.5 & 1.8 \\
Margin over 6.7 T ... & $8.6 \%$ & $11.1 \%$ \\
\hline
\end{tabular}

Table 6.2.11: Summary of SSC $50 \mathrm{~mm}$ dipole iron cross section. $\delta(T F)$ is the change in transfer function, $\delta b_{2}$ in $b_{2}$ and and $\delta b_{4}$ in $b_{4}$ due to saturation.

\begin{tabular}{|c|l|}
\hline $\begin{array}{l}\text { Inner Diameter, mm } \\
\text { Outer Diameter, mm }\end{array}$ & $\begin{array}{l}135.6 \\
330.2\end{array}$ \\
\hline$\delta(T F)$, at $6.7 \mathrm{~T} \ldots \ldots$ & $2.6 \%$ \\
$\delta b_{2}$, prime unit ...... & 0.3 \\
$\delta b_{4}$, prime unit ...... & 0.03 \\
\hline
\end{tabular}




\subsection{RHIC $130 \mathrm{~mm}$ Aperture Interaction Region Quadrupole Cross-section}

A total of seventy two, $130 \mathrm{~mm}$ aperture quadrupoles are required in six interaction regions of RHIC. Each interaction region includes 4 sets of quadrupole triplets (see Fig. 5.2.11 which shows one of two beam lines at an intersection). The Q1, Q2 and Q3 quadrupoles in each triplet have the same cross section, and magnetic lengths, respectively, of $1.44 \mathrm{~m}, 3.4$ $\mathrm{m}$ and $2.1 \mathrm{~m}$. The major parameters of these quadrupoles are shown in Table 6.3.1.

The particle beam is focussed to a small size at the crossing point in the two low-beta $\left(\beta^{*}=1\right)$ interaction regions to obtain a high luminosity. An unavoidable consequence of this squeeze is the increase in the beam size in these quadrupoles. Moreover, the rapid variation in beam size within the focusing triplet limits the effectiveness of the local and lumped global corrector system [171]. Therefore, good field quality in these quadrupoles is crucial to the high luminosity operation of RHIC. Since the maximum luminosity is desired at the top energy, the field errors are designed to be minimized at the maximum operating gradient.

\subsubsection{Basic Construction}

The cross section of the cold mass is shown in Fig. 6.3.1. Following the design philosophy used in most other RHIC magnets, the yoke is used here as a collar to compress the coils. The yoke aperture consists of concentric circular arcs with two radii instead of the usual circular aperture. As explained in chapter 2 , this reduces the $b_{5}$ saturation by over an order of magnitude. The space between the coil and the yoke is filled with a thin, glass-filled phenolic spacer. The spacer has two radii for the outer surface which closely follows the geometry of the yoke inner surface. However, at eight places a gap is left between the yoke and spacer where partially magnetic tuning shims are placed to correct the measured field harmonics after the coils are assembled in the yoke. The tuning shims are discussed in more detail in a previous chapter. A $6.35 \mathrm{~mm}$ thick stainless steel shell enclosing the yoke is welded together after the collaring keys are inserted in the compressed yoke. The quadrupoles of the inner and outer rings share a common cryostat. The minimum center-to-center distance between adjacent Q1 quadrupoles is $424 \mathrm{~mm}$. 


\section{RHIC INSERTION QUADRUPOLE}

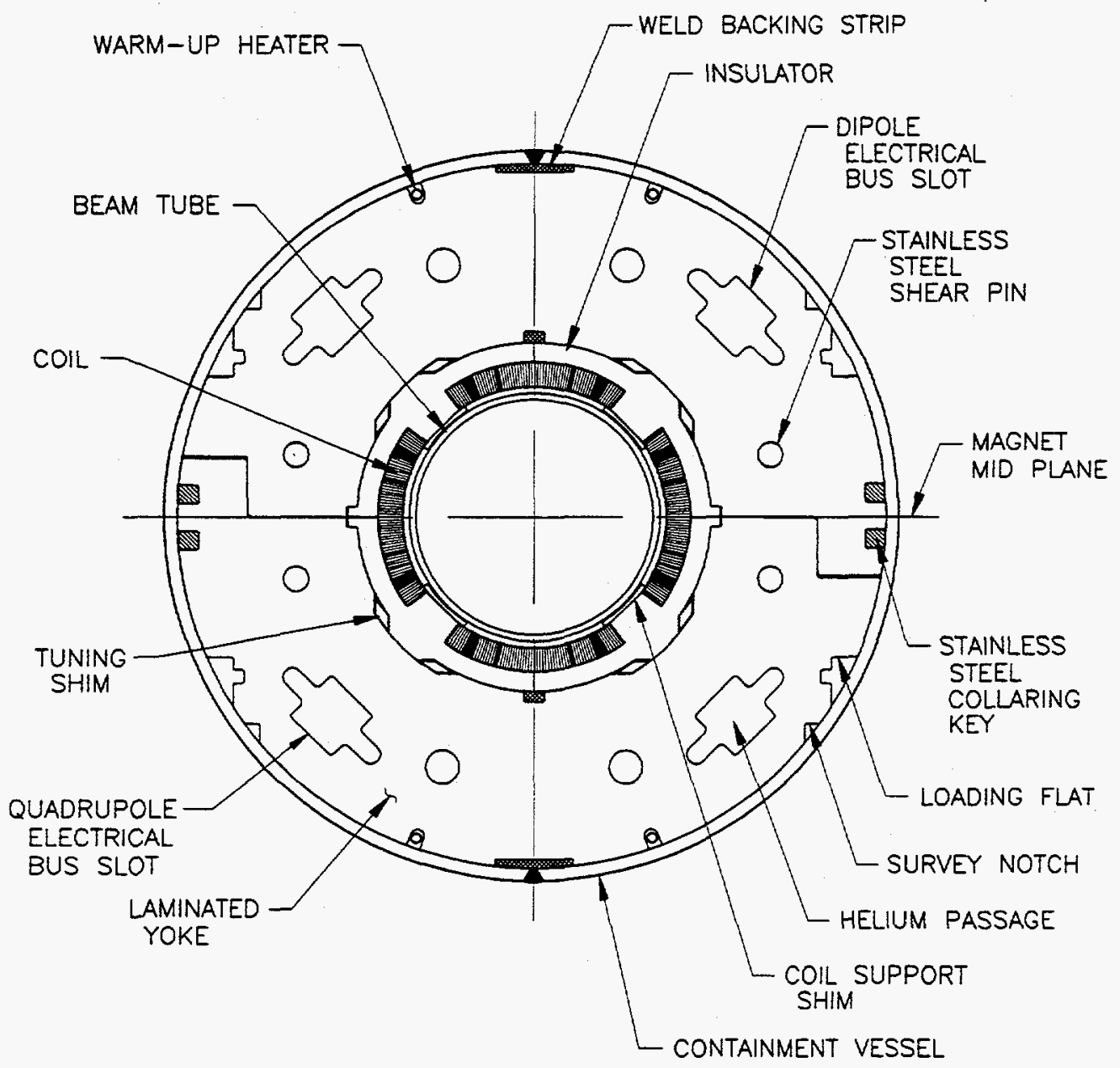

$5 k / s 00488 \mathrm{ml}$ $8 / 29 / 93$

Figure 6.3.1: Cross section of the $130 \mathrm{~mm}$ aperture quadrupoles for a RHIC interaction region. 
Table 6.3.1: The basic design parameters of the $130 \mathrm{~mm}$ aperture quadrupoles for the RHIC interaction regions.

\begin{tabular}{|c|c|}
\hline Parameter & Value \\
\hline Superconducting wire diameter .... & $0.65 \mathrm{~mm}$ \\
\hline Number of wires per cable ........ & 36 \\
\hline Copper to superconductor ratio .... & 1.8 \\
\hline Cable mid-thickness $\ldots \ldots \ldots \ldots \ldots$ & $1.16 \mathrm{~mm}$ \\
\hline Cable width $\ldots \ldots \ldots \ldots \ldots \ldots \ldots$ & $11.7 \mathrm{~mm}$ \\
\hline Cable insulation $\ldots \ldots \ldots \ldots \ldots$ & Kapton CI \\
\hline Critical current at $5.6 \mathrm{~T}, 4.2 \mathrm{~K} \ldots \ldots$ & $\geq 10100 \mathrm{~A}$ \\
\hline Number of turns per pole ......... & 27 \\
\hline Coil inner radius $\ldots \ldots \ldots \ldots \ldots \ldots$ & $65 \mathrm{~mm}$ \\
\hline Coil outer radius $\ldots \ldots \ldots \ldots \ldots \ldots$ & $77 \mathrm{~mm}$ \\
\hline Yoke lamination thickness...... & $6.35 \mathrm{~mm}$ \\
\hline Yoke inner radius at midplane ..... & $87 \mathrm{~mm}$ \\
\hline Yoke inner radius at pole ......... & $92 \mathrm{~mm}$ \\
\hline Yoke outer radius $. . . \ldots \ldots \ldots \ldots . .$. & $175.26 \mathrm{~mm}$ \\
\hline Magnetic length, Q1, Q2, Q3 ..... & $1.44,3.4,2.1 \mathrm{~m}$ \\
\hline Stored energy in Q2 magnet ...... & $165 \mathrm{~kJ}$ \\
\hline Minimum beam spacing in triplet .. & $424 \mathrm{~mm}$ \\
\hline Maximum design current $\ldots .$. & $5.05 \mathrm{kA}$ \\
\hline Maximum design gradient $\ldots \ldots \ldots$ & $48.1 \mathrm{~T} / \mathrm{m}$ \\
\hline Operating temperature $\ldots \ldots \ldots \ldots$ & $4.6 \mathrm{~K}$ \\
\hline Computed quench current at $4.6 \mathrm{~K}$ & $8.26 \mathrm{kA}$ \\
\hline Computed quench gradient at $4.6 \mathrm{~K}$ & $75.3 \mathrm{~T} / \mathrm{m}$ \\
\hline Gradient margin $\ldots \ldots \ldots \ldots \ldots \ldots$ & $57 \%$ \\
\hline
\end{tabular}


Table 6.3.2: The basic coil design parameters of the $130 \mathrm{~mm}$ aperture quadrupoles for the RHIC intersection regions. The harmonics $b_{n}$ for the final cross section are given at $40 \mathrm{~mm}$ reference radius. "Circular iron aperture" rows show the expected harmonics for a circular iron aperture with a yoke inner radius of $87 \mathrm{~mm}$. "Actual iron aperture" rows show the expected harmonics for the two radii $(87 \mathrm{~mm}$ and $92 \mathrm{~mm})$ design. The table also includes the harmonics due to nominal tuning shims and the effect of the asymmetric deformation in the yoke aperture during collaring.

\begin{tabular}{|ll|}
\hline \multicolumn{1}{|c|}{ Parameter } & \multicolumn{1}{c|}{ Value } \\
\hline Number of layer(s) $\ldots \ldots \ldots \ldots \ldots \ldots$. & 1 \\
Number of wedges $\ldots \ldots \ldots \ldots \ldots \ldots \ldots$ & 2 \\
Turn configuration (from midplane) . & $13,8,6$ \\
Wedge 1, Min./Max. thickness, $\mathrm{mm}$. & $1.143 / 1.143$ \\
Wedge 2, Min./Max. thickness, mm . & $4.227 / 6.35$ \\
Coil-to-midplane gap $\left(0^{\circ}, 180^{\circ}\right), \mathrm{mm}$. & $0.356 \mathrm{~mm}$ \\
Coil-to-midplane gap $\left(90^{\circ}, 270^{\circ}\right), \mathrm{mm}$ & $0.279 \mathrm{~mm}$ \\
Coil inner radius $\ldots \ldots \ldots \ldots \ldots \ldots \ldots$ & $65 \mathrm{~mm}$ \\
Coil outer radius $\ldots \ldots \ldots \ldots \ldots \ldots \ldots$ & $77 \mathrm{~mm}$ \\
\hline$b_{3}$ for circular iron aperture $\ldots \ldots \ldots \ldots$ & 3.0 \\
$b_{3}$ for actual iron aperture $\ldots \ldots \ldots \ldots$ & 0.0 \\
$b_{5}$ for circular iron aperture $\ldots \ldots \ldots \ldots$ & -29.6 \\
$b_{5}$ for actual iron aperture $\ldots \ldots \ldots \ldots$. & -1.2 \\
$b_{7}$ for circular iron aperture $\ldots \ldots \ldots \ldots$ & -0.22 \\
$b_{7}$ for actual iron aperture $\ldots \ldots \ldots \ldots$ & -0.1 \\
$b_{9}$ for circular iron aperture $\ldots \ldots \ldots \ldots$ & 1.1 \\
$b_{9}$ for actual iron aperture $\ldots \ldots \ldots \ldots$ & 0.0 \\
\hline
\end{tabular}

\subsubsection{Coil Cross Section}

One octant of the coil cross section is shown in Fig. 6.3.2. The coil has a total of 27 cables per octant in three blocks. The coil is designed using a superconducting cable which is used in the outer layer of the SSC $50 \mathrm{~mm}$ dipole magnet (see Table 6.2.1). This cable is 
$\sim 20 \%$ wider than the one which is used in the RHIC arc dipole and quadrupole magnets. The additional superconductor in this wider cable gave a sufficient margin over the original design gradient of $59 \mathrm{~T} / \mathrm{m}$ in a single layer coil. As shown, in Table 6.3.1, the design gradient in the present RHIC lattice has been reduced to $48.1 \mathrm{~T} / \mathrm{m}$. The specifications for the critical current $I_{c}$ at $4.2 \mathrm{~K}$ and at $5.6 \mathrm{~T}$ in wire and in cable are respectively $286 \mathrm{~A}$ and $10100 \mathrm{~A}$. The cable is almost fully keystoned for the $130 \mathrm{~mm}$ coil aperture.

The coil is optimized using the computer program PAR2DOPT [130]. This is an analytic program which computes and optimizes field harmonics for a coil in a circular iron aperture. As mentioned earlier, the iron aperture is not circular in this design. This means that a coil optimized for zero harmonics in a circular aperture would have non-zero harmonics here. Therefore, the coil is designed with non-zero harmonics in a circular aperture to cancel the harmonics generated by this non-circular iron aperture. These required non-zero values (also referred to as offsets) are computed using the code POISSON.

The optimized coil cross section is shown in Fig. 6.3.2. The coil has 27 turns in the three blocks (two wedges) of each octant with the distribution of the number of turns in the three blocks being 13,8 and 6 . The two wedges are mechanically symmetric. In addition, the smaller wedge (the one which is closer to the midplane) is rectangular. The various mechanical and magnetic parameters of this cross section are shown in Table 6.3.2. The presence of $b_{3}$ and $b_{7}$, which are normally non-allowed harmonics in quadrupoles, is discussed later. In addition to low harmonic content and a lower value of maximum field on the conductor, this particular cross section was chosen for its good flexibility (tunability) in accommodating changes in field harmonics and mechanical parameters such as the thickness of the cable.

The good tunability of coil cross section and the one rectangular wedge turned out to be very useful in obtaining a good field quality in pre-production model magnets when a decision to change the cable insulation was made. The new Kapton-CI insulation increased the thickness of the insulated cable by $0.023 \mathrm{~mm}$ compared to the original design. This would have resulted in an $0.635 \mathrm{~mm}$ increase in coil size since there are 27 turns in the cross section. This is about an order of magnitude more than the tolerance required to maintain the desired pre-compression on the coils. However, since the shape of one of the wedges was rectangular, it could be easily rolled to a $0.635 \mathrm{~mm}$ smaller size. This reduction in wedge size compensated for the thicker insulation, and the original coil size and coil pole angle in the magnet could be maintained. This avoided an adjustment in the number of 


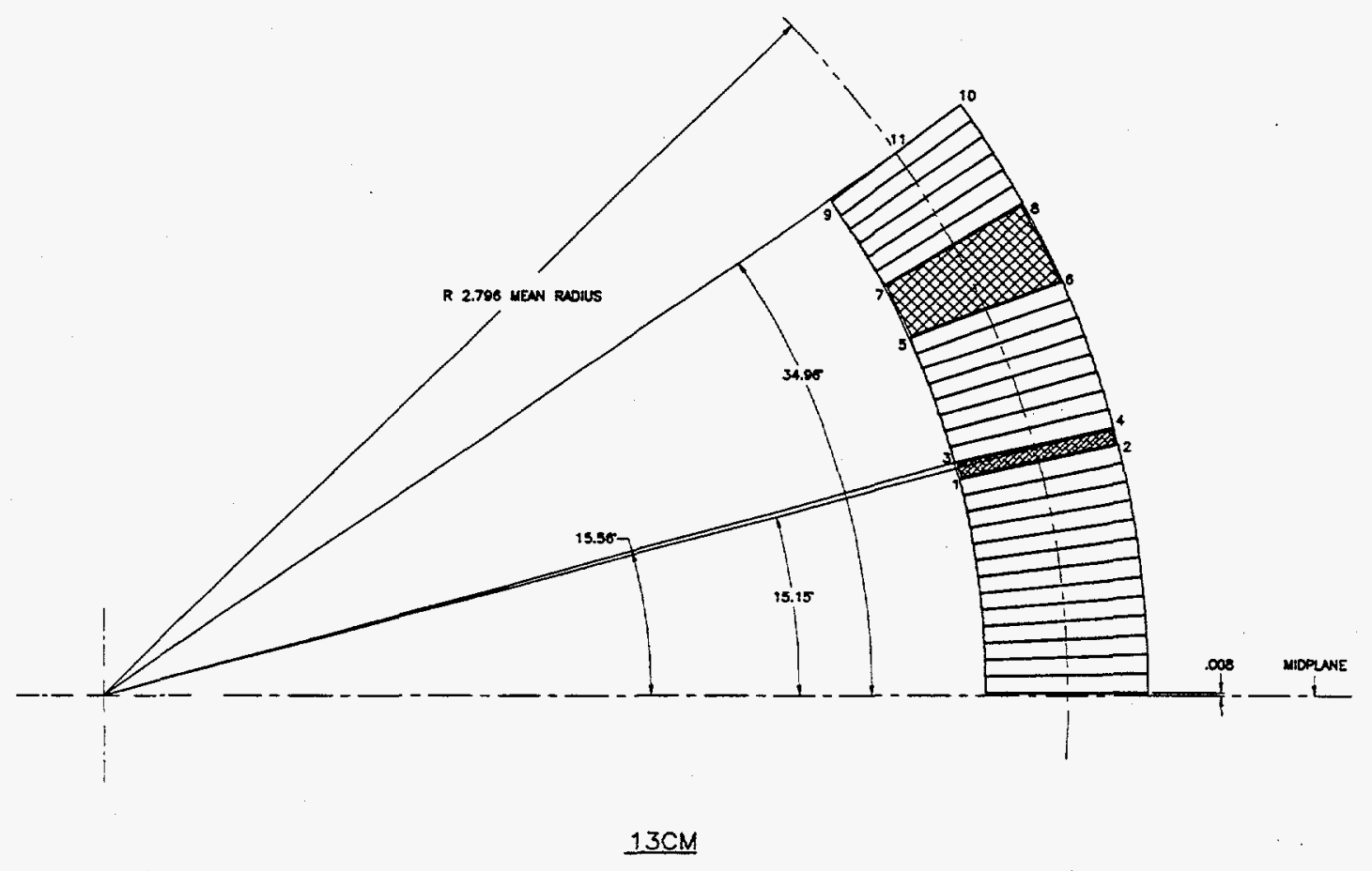

Figure 6.3.2: Optimized coil cross section for the RHIC $130 \mathrm{~mm}$ aperture insertion quadrupole. 
parts in the body and the end section of the magnet. In this selected cross section, the above change did not produce a significant change in $b_{5}$, the first allowed harmonic with quadrupole symmetry. This straightforward accommodation of a large mechanical change without a corresponding deterioration in the field quality was possible only because the cross section was optimised and chosen to permit such tunability. The value of the next allowed harmonic $b_{9}$, created by the large change, is about 1 unit. This was removed in the next iteration.

In an attempt to maximize the use of existing tooling, these quadrupoles are collared like dipoles. However, during the collaring an elliptical deformation is created in the iron and also in the coil since the yoke inner surface defines the coil outer surface. The turns on the horizontal axis are displaced inward and an overall ovality is created as the vertical axis tends to elongate. This deformation breaks an ideal quadrupole symmetry and creates the non-allowed harmonics $b_{3}, b_{7}$, etc. A large value ( $\sim 6$ unit) of $b_{3}$ harmonic was observed in the pre-production quadrupoles due to this yoke deformation (asymmetry). A method was developed to remove the harmonics created by this asymmetry. A deliberate difference between the horizontal and vertical midplane gap (see Table 6.3.2) in the coil cross section was introduced. This difference in midplane gap breaks the horizontal/vertical symmetry in the coil cross section and generates the harmonics $b_{3}, b_{7}$, etc. The amount of this second asymmetry (coil asymmetry) can be adjusted to properly compensate for the first asymmetry (yoke asymmetry) created in the collaring press. The calculations and measurements showed that if the half-gap on the horizontal midplane is made about $0.1 \mathrm{~mm}$ larger than the half-gap on the vertical midplane, then the $b_{3}$ due to yoke asymmetry can be compensated. This was incorporated in eight quadrupoles. However, the asymmetry in coil assembly also produces a small (-0.35 unit) but significant $b_{7}$. Therefore, in later magnets, only part of the correction is applied using this method. The rest is obtained using magnetic tuning shims. The sign of $b_{3}$ and $b_{7}$ in the two methods is opposite. Therefore, in principle when an asymmetric midplane shim is used in conjunction with the magnetic tuning shims, both $b_{3}$ and $b_{7}$ could be made zero. However, that would take away a significant part of the tuning shim capacity which could be used to compensate other harmonics. In the final design the target $b_{3}$ is zero and $b_{7}$ is about -0.1 unit. 


\subsubsection{Yoke Cross Section}

In cosine $\theta$ magnets, the iron aperture is circular. However, in order to reduce the $b_{5}$ due to saturation the conventional circular shape has been modified in this design. The yoke aperture is defined by arcs of two concentric circles having radii of $87 \mathrm{~mm}$ at the midplane and $92 \mathrm{~mm}$ at the pole. This method has been very effective in reducing the $b_{5}$ due to saturation from 15 to 1 unit. The basic principle behind it is that it forces an early saturation at the yoke midplane (unless the yoke outer diameter is too large) and delays/reduces the saturation at the pole. Saturation control holes which were found very effective in dipoles are not so effective in the quadrupoles. The nominal transition from 87 to $92 \mathrm{~mm}$ occurs at about 30 degrees in the first octant (the angle in the other octants is defined by quadrupole symmetry). At $\theta=30$ degree (and similarly in other octants) there is a straight face perpendicular to the midplane, extending from the smaller yoke radius of 87 $\mathrm{mm}$ to the larger iron radius of $92 \mathrm{~mm}$. The position angle and the difference between these two radii are used as parameters in the optimization process to reduce the $b_{5}$ saturation. The size of the locating notch, shown in Fig. 6.3.1, at the midplane of the yoke aperture is $5 \mathrm{~mm}$ deep and $10 \mathrm{~mm}$ wide. Four slots - two for helium bypass and two for bus work - are located symmetrically at $45^{\circ}, 135^{\circ}, 225^{\circ}$ and $315^{\circ}$ as shown in the figure. The size, shape and location of these slots are determined by the magnetic, mechanical and cooling requirements. Other structures in the yoke are also shown in the figure. Though several of these structures break a strict 8-fold quadrupole symmetry, their location is such that their influence on the iron saturation is minimal. These structures include the holes in the yoke for the stainless steel (non-magnetic) pins and and cutouts for the stainless steel keys. The results of computer calculations show that these holes and cutouts in the yoke generate symmetry breaking harmonics of less than 0.1 unit in the design range of operation. The presence of another quadrupole in the other ring may influence the field at the center of the magnet at high currents. This would be a maximum when the separation between the two magnets is a minimum. Moreover, it is maximum when both of these magnets run at the same high gradient. Computer calculations show that these cross talk induced harmonics are less than 0.1 unit.

The calculations have been performed using the computer codes POISSON, PE2D and MDP. Good agreement has been found between the results obtained by these three codes; this is important because the yoke saturation has been significantly altered in this design by the 2-radius aperture. A computer model of one quadrant of the magnet for the code 


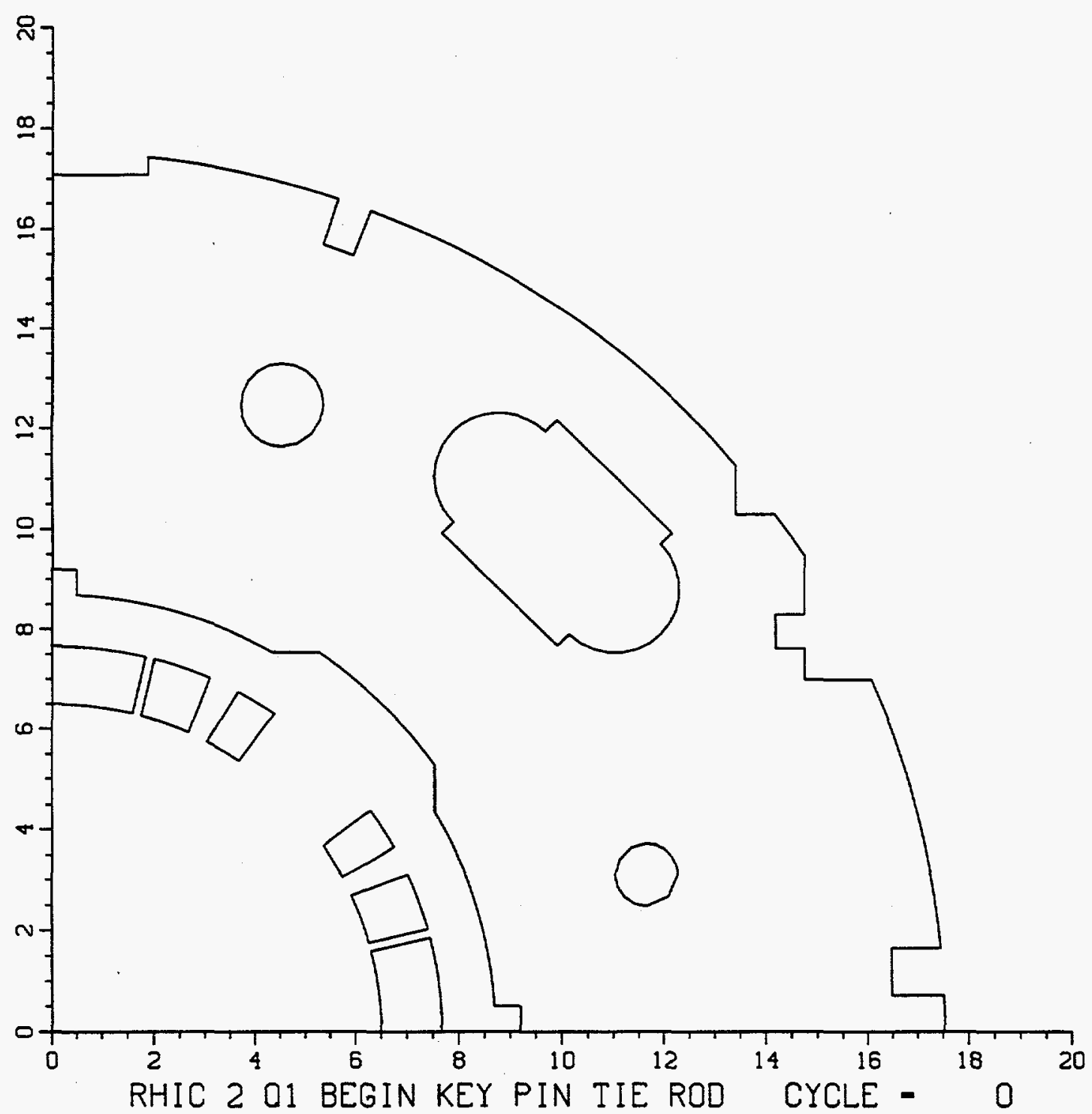

Figure 6.3.3: POISSON model for $1 / 4$ of the cold mass cross section of the RHIC $130 \mathrm{~mm}$ aperture insertion quadrupole. The rest of the cold mass is described by the symmetry. A perfect quadrupole has an eight fold symmetry; this is partly broken here by the yoke split on the horizontal midplane. 
Table 6.3.3: Harmonics in the straight section of the RHIC $130 \mathrm{~mm}$ aperture insertion quadrupoles as computed for a POISSON model of a quadrant of the magnet. The harmonics are given at $40 \mathrm{~mm}$ radius. $\infty \mu$ represents the calculations when the permeability in the iron is assumed to be $\infty$. Grad denotes the field gradient. A dependence of the harmonics $b_{3}$ and $b_{7}$ on the current reflects the absence of an ideal 8-fold quadrupole symmetry.

\begin{tabular}{|c|c|c|c|c|c|c|c|c|}
\hline $\mathrm{I}$ & $\begin{array}{c}\text { Grad } \\
\mathrm{kA}\end{array}$ & $\begin{array}{c}\text { T.F. } \\
\mathrm{T} / \mathrm{m}\end{array}$ & $\begin{array}{c}b_{3} \\
\mathrm{~T} / \mathrm{m} / \mathrm{kA}\end{array}$ & $\begin{array}{c}b_{5} \\
0^{-4}\end{array}$ & $\begin{array}{c}b_{7} \\
10^{-4}\end{array}$ & $\begin{array}{c}b_{9} \\
10^{-4}\end{array}$ & $\begin{array}{c}b_{11} \\
10^{-4}\end{array}$ & $\begin{array}{c}b_{13} \\
10^{-4}\end{array}$ \\
\hline$\infty \mu$ & $\infty \mu$ & 9.574 & 0.00 & -1.20 & 0.00 & -0.10 & 0.00 & 0.0 \\
\hline 1.0 & 9.5730 & 9.573 & 0.00 & -1.22 & 0.00 & -0.10 & 0.00 & 0.00 \\
\hline 3.0 & 28.7165 & 9.5722 & 0.00 & -1.23 & 0.00 & -0.10 & 0.00 & 0.00 \\
\hline 4.0 & 38.2583 & 9.5646 & 0.03 & -1.16 & -0.01 & -0.05 & -0.01 & 0.00 \\
\hline 5.0 & 47.6639 & 9.5328 & 0.10 & -1.11 & 0.00 & 0.13 & -0.01 & -0.01 \\
\hline 5.5 & 52.2567 & 9.5012 & 0.15 & -1.28 & -0.01 & 0.26 & 0.00 & -0.01 \\
\hline 6.0 & 56.7096 & 9.4516 & 0.10 & -1.36 & -0.02 & 0.39 & 0.00 & -0.01 \\
\hline 6.5 & 60.9980 & 9.3843 & 0.08 & -1.32 & -0.03 & 0.52 & 0.00 & -0.01 \\
\hline 7.0 & 65.1189 & 9.3027 & 0.04 & -1.58 & -0.04 & 0.63 & -0.01 & -0.01 \\
\hline 7.5 & 69.0485 & 9.2065 & 0.02 & -2.40 & -0.05 & 0.72 & -0.01 & 0.00 \\
\hline 8.0 & 72.8282 & 9.1035 & -0.11 & -3.70 & -0.07 & 0.78 & -0.01 & 0.00 \\
\hline 8.5 & 76.5207 & 9.0024 & -0.40 & -5.21 & -0.09 & 0.83 & -0.01 & 0.01 \\
\hline 9.0 & 80.1618 & 8.9069 & -0.65 & -6.76 & -0.10 & 0.86 & -0.01 & 0.01 \\
\hline 9.5 & 83.7541 & 8.8162 & -0.76 & -8.30 & -0.11 & 0.88 & -0.01 & 0.02 \\
\hline
\end{tabular}


POISSON is shown in Fig. 6.3.3. The results of calculations showing the current dependence of the harmonics allowed by the actual geometry are given in Table 6.3.3. The harmonics $b_{3}, b_{7}$ and $b_{11}$ are not allowed in a strictly 8-fold quadrupole symmetry. The dependence of them on current refiects the absence of the ideal 8-fold symmetry. The yoke is designed such that the magnitude of these harmonics is small even at high currents. The computed dependence of the harmonics $b_{3}, b_{5}$ and $b_{9}$ on current is plotted in Fig. 6.3.4.

The calculations described above are for one magnet by itself, i.e. no other magnet is present in the vicinity. However, in the layout of the RHIC lattice, there is always another $130 \mathrm{~mm}$ aperture quadrupole located nearby. The minimum separation between the outer yoke diameter of the two is $\sim 73.5 \mathrm{~mm}$. The thickness of the iron yoke in these quadrupoles, though sufficient to contain the field lines at the design current, is not sufficient to contain them at the quench current of $8.26 \mathrm{kA}$. In this situation, the non-allowed harmonics show a dependence on current at high field. This is commonly referred to as cross talk and it implies that the field of one magnet is influencing the field in the aperture of the other magnet.

In RHIC, the ratio of rigidities of the two counter rotating beams varies in the range of $1: 1$ to $1: 0.4$. That means that the excitation of two side-by-side quadrupoles can, in general, be different. The direction of the field in the two is the same. In this case, the amount of cross talk between the two quadrupoles is a maximum when both are excited at the same high current (1:1 case). Therefore, in the calculations presented here for $4: 0$ non-allowed field harmonics, only the 1:1 case is considered. The cross talk is a maxin. at the Q1 location where the two quadrupoles are at their minimum separation, wits a center-to-center distance being $424 \mathrm{~mm}$. Moreover, since the two quadrupoles at the Q1 location are not parallel to each other, the separation (and hence the amount of cross talk) between them is different at the two ends of these magnets. The non-allowed harmonics (in particular $b_{o}$, which is affected the most) are a maximum when the separation between the two quadrupoles is a minimum. The results of calculations for this case using the code POISSON are given in Table 6.3.4. The maximum operating current in the present design is about $5.05 \mathrm{kAmp}$. The amount of cross talk is negligible in the design range of operation and is very small up to the expected quench current $(8.26 \mathrm{kA})$. The field lines at quench in one of two side-by-side quadrupoles are shown in Fig. 6.3.5 (the other quadrupole, not shown, is on the left side of the one shown). 


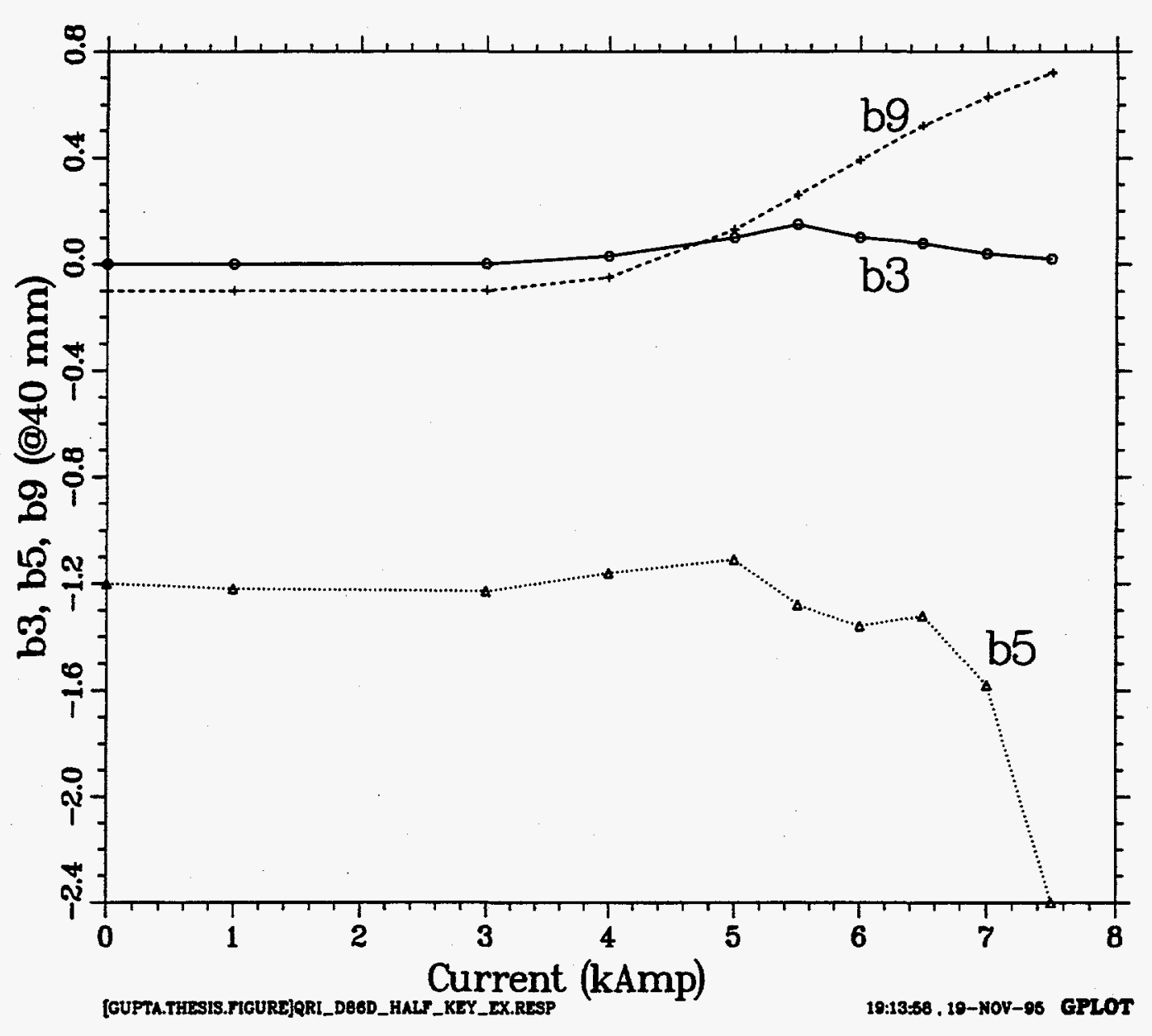

Figure 6.3.4: Variation in the $b_{3}, b_{5}$ and $b_{9}$ harmonics as a function of current in $130 \mathrm{~mm}$ aperture RHIC insertion quadrupole as computed with the code POISSON. The computer model is shown in Fig. 6.3.3. 


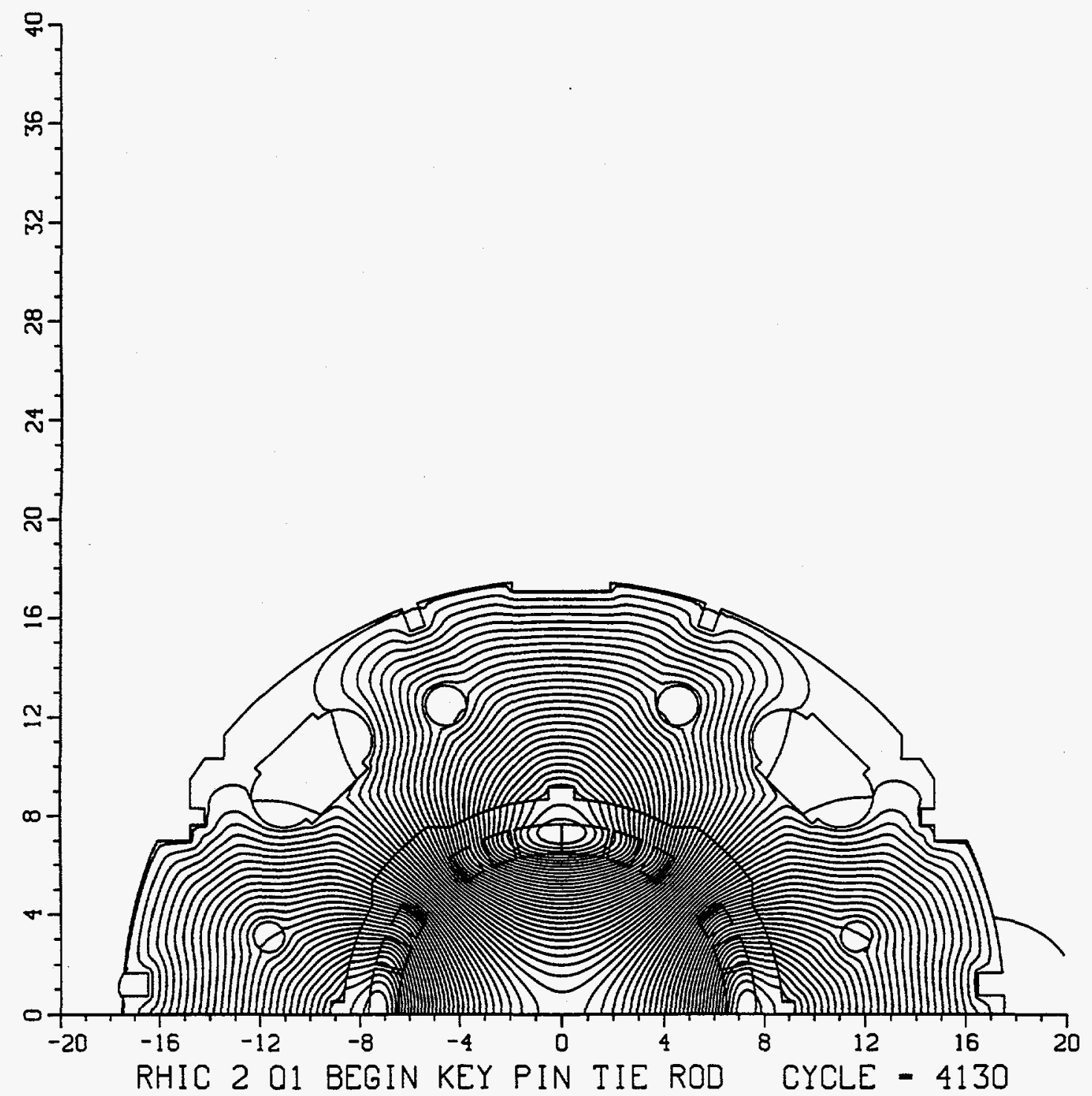

Figure 6.3.5: The field lines at quench in one of two side-by-side quadrupoles. The other quadrupole, not shown, is on the left side of the one shown. The minimum center to center distance between the $t$ wo quadrupoles is $42.4 \mathrm{~cm}$. 
Table 6.3.4: Harmonics in the straight section of the RHIC $130 \mathrm{~mm}$ aperture quadrupoles at the Q1 location where the separation between the two side-by-side quadrupoles is a minimum $(424 \mathrm{~mm})$. The harmonics are given at $40 \mathrm{~mm}$ radius. $\infty \mu$ represents the calculations when the permeability in the iron is assumed to be $\infty$. Grad denotes the field gradient. These computations are made using the code POISSON.

\begin{tabular}{|c|c|c|c|c|c|c|c|c|c|c|c|}
\hline I & Grad & T.F. & $b_{0}$ & $b_{2}$ & $b_{3}$ & $b_{4}$ & $b_{5}$ & $b_{6}$ & $b_{7}$ & $b_{8}$ & $b_{9}$ \\
kAmp & T/M & T/M/kA & $10^{-4}$ & $10^{-4}$ & $10^{-4}$ & $10^{-4}$ & $10^{-4}$ & $10^{-4}$ & $10^{-4}$ & $10^{-4}$ & $10^{-4}$ \\
\hline$\infty \mu$ & $\infty \mu$ & 9.5742 & 0.00 & 0.00 & 0.00 & 0.00 & -1.20 & 0.00 & -0.10 & 0.00 & 0.00 \\
\hline 1.0 & 9.5735 & 9.5735 & -0.03 & 0.01 & 0.01 & 0.01 & -1.22 & 0.01 & -0.10 & 0.00 & 0.00 \\
\hline 3.0 & 28.7171 & 9.5724 & -0.03 & 0.02 & 0.00 & 0.01 & -1.23 & 0.01 & -0.10 & 0.00 & 0.00 \\
\hline 4.0 & 38.2591 & 9.5648 & 0.14 & 0.02 & 0.07 & -0.02 & -1.16 & 0.01 & -0.11 & 0.01 & 0.05 \\
\hline 5.0 & 47.6671 & 9.5334 & 0.14 & 0.01 & 0.05 & -0.02 & -1.09 & 0.01 & -0.11 & 0.01 & 0.24 \\
\hline 5.5 & 52.2588 & 9.5016 & 0.14 & 0.07 & 0.05 & -0.02 & -1.25 & 0.01 & -0.12 & 0.00 & 0.37 \\
\hline 6.0 & 56.7113 & 9.4519 & 0.25 & 0.08 & 0.03 & -0.01 & -1.33 & 0.01 & -0.13 & 0.01 & 0.50 \\
\hline 6.5 & 61.0000 & 9.3846 & 0.13 & 0.03 & 0.01 & 0.01 & -1.31 & 0.01 & -0.14 & 0.01 & 0.63 \\
\hline 7.0 & 65.1185 & 9.3026 & 0.42 & 0.05 & -0.02 & 0.02 & -1.60 & 0.01 & -0.15 & 0.01 & 0.74 \\
\hline 7.5 & 69.0458 & 9.2061 & 0.82 & 0.09 & -0.01 & 0.04 & -2.44 & 0.01 & -0.15 & 0.01 & 0.83 \\
\hline 8.0 & 72.8246 & 9.1031 & 1.61 & 0.19 & -0.16 & 0.07 & -3.74 & 0.02 & -0.17 & 0.01 & 0.89 \\
\hline 8.5 & 76.5132 & 9.0016 & 2.78 & 0.32 & -0.47 & 0.12 & -5.27 & 0.03 & -0.19 & 0.01 & 0.93 \\
\hline 9.0 & 80.1467 & 8.9052 & 4.06 & 0.46 & -0.73 & 0.17 & -6.84 & 0.03 & -0.21 & 0.01 & 0.97 \\
\hline 9.5 & 83.7381 & 8.8145 & 5.53 & 0.59 & -0.87 & 0.22 & -8.40 & 0.04 & -0.21 & 0.01 & 0.990 \\
\hline
\end{tabular}




\subsubsection{Expected Quench Performance}

To compute the gradient at quench, the maximum field on the conductor (the peak field) is first calculated. The location of the peak field is on the pole-most surface of the pole turn. It is $16 \%$ radially outward from the coil inner radius. The value of this field together with the gradient at $7000 \mathrm{~A}$ and $8000 \mathrm{~A}$ is given in Table 6.3.5.

Table 6.3.5: Peak field on the cable in the $130 \mathrm{~mm}$ aperture quadrupole. The peak field in the coil is on the pole turn.

\begin{tabular}{|c|c|c|}
\hline Current & Gradient & Field \\
\hline $7000 \mathrm{~A}$ & $65.07 \mathrm{~T} / \mathrm{m}$ & $4.991 \mathrm{~T}$ \\
\hline $8000 \mathrm{~A}$ & $73.16 \mathrm{~T} / \mathrm{m}$ & $5.626 \mathrm{~T}$ \\
\hline
\end{tabular}

The specification for the critical current in the wire is $\geq 286$ amperes at $4.2 \mathrm{~K}$ temperature and 5 Tesla field. In this 36 strand cable, after a $2 \%$ degradation, the minimum critical current in the cable is specified to be 10,100 amperes. This gives a computed quench gradient of $75.3 \mathrm{~T} / \mathrm{m}$ at $4.6 \mathrm{~K}$, which is $57 \%$ over the design value of $48.1 \mathrm{~T} / \mathrm{m}$. The computed quench current is 8260 amperes which is $\sim 64 \%$ above the design value of 5050 amperes. The current density in the copper at quench is $1133 \mathrm{~A} / \mathrm{mm}^{2}$ and at design field is about 670 $\mathrm{A} / \mathrm{mm}^{2}$. The critical current in the cable actually used in manufacturing the magnets is about 12,000 ampere, which is much better than the minimum specified value. This would give a quench gradient of $80.1 \mathrm{~T} / \mathrm{m}$ and quench current of 8860 ampere. 


\subsection{Conclusions on the Optimized Cross Section Designs}

It is shown in this chapter that the methods described in the previous four chapters can be implemented to design and construct high field quality magnets. These methods have been used in the magnetic design of the cross section for the prototype of the SSC $50 \mathrm{~mm}$ aperture collider dipole magnets built at BNL and Fermilab. These methods are also used in the design of a number of magnets for RHIC but only the design of the $130 \mathrm{~mm}$ aperture quadrupole magnets has been included here to describe the procedure in fair detail.

A good design would require that besides being optimized for field quality, the cross section is also optimized to give a high quench field, to incorporate the required cooling and mechanical considerations and to simplify the manufacturing to make it as error free as possible. For example, the copper wedges are made completely symmetric in the inner layer of the SSC $50 \mathrm{~mm}$ aperture design (and in both layers in the Fermilab design) and the two nearly equal size wedges are made exactly equal. In the RHIC $130 \mathrm{~mm}$ aperture quadrupole design, not only are the wedges symmetric but the smaller of the two wedges is rectangular. None of these mechanical manufacturing considerations resulted in a degradation in field quality; it merely required optimizing the cross section design with certain guidelines.

In the SSC $50 \mathrm{~mm}$ dipole magnet design, the iron saturation is controlled by choosing the size, location and material of the yoke-yoke alignment key at the midplane for the BNLbuilt, horizontally split yoke design. In the Fermilab-built, vertically split design a cutout is introduced at the midplane to obtain similar performance. The iron saturation in the RHIC insertion quadrupoles is controlled by the 2-radius method.

The RHIC $130 \mathrm{~mm}$ aperture quadrupoles are collared with a 2-fold symmetry. The collaring process creates an elliptical-type deformation which creates a large $b_{3}$ harmonic. This can be removed by making the coil midplane gap larger at the horizontal plane than at the vertical plane. This, however, creates $a b_{7}$ harmonic. It is shown that a proper combination of an asymmetric midplane gap and an asymmetric tuning shim (which also creates a $b_{7}$ but with opposite sign) makes both $b_{3}$ and $b_{7}$ small. 


\section{Chapter 7. \\ CONCLUSIONS AND SUGGESTIONS FOR FUTURE WORK}

The field quality in superconducting magnets has been improved to a level that it does not appear to be a limiting factor on the performance of RHIC. The many methods developed, improved and adopted during the course of this work have contributed significantly to that performance. One can not only design and construct magnets with better field quality than in one made before but can also improve on that quality after construction. The relative field error $\left(\frac{\Delta B}{B}\right)$ can now be made as low as a few parts in $10^{-5}$ at $2 / 3$ of the coil radius. This is about an order of magnitude better than what is generally expected for superconducting magnets. This extra high field quality is crucial to the luminosity performance of RHIC.

The research work described here covers a number of areas which all must be addressed to build the production magnets with a high field quality. The work has been limited to the magnetic design of the cross section which in most cases essentially determines the field quality performance of the whole magnet since these magnets are generally long. Though the conclusions to be presented in this chapter have been discussed at the end of each chapter, a summary of them might be useful to present a complete picture. The lessons learned from these experiences may be useful in the design of new magnets. The possibilities of future improvements will also be presented.

In the first chapter, a review of the field is presented. Apart from giving a brief description of the RHIC project a summary of various aspects of a superconducting magnet is presented. The section on magnetic field analysis in accelerator magnets is of particular relevance to the present work. In this section various analytic expressions are given. Most of these expressions have been derived by other authors but more are derived here to give a better foundation to the research and development work to follow in the next five chapters. These expressions are useful to provide a general guidance which is needed to develop a systematic, efficient and logical approach to develop and adopt methods of improving the field quality in superconducting magnets.

In chapter 2 , techniques to improve the computational and analysis procedure are presented. It is useful to evaluate mechanical deformations on the coil and yoke cross section under large compressive forces. This has impacts on both magnetic and mechanical design 
and analysis. A computer aided cross section measurement and analysis method has been developed. The method creates a digital image of the cross section using inexpensive commercial software and hardware. This image can be compared with the original design or expected mechanical deformations. The image can be transferred to the AutoCAD software which facilitates a variety of analyses. However, this method was not used during the R\&D phase of the RHIC magnet program because at that time the resolution of the image in the supported software was at the border line of the requirement and alternate methods were already in use. However, the development in computer technology in the past several years makes this method quite attractive now. The major advantage of this method is the availability of a digitized image on the computer which can be used for a variety of interactive analyses.

The POISSON group codes have been used throughout this research work to perform most of the field calculations. These codes have been extensively improved to enhance their capability to make the complicated and reliable computer models required in such applications. The use of these improved techniques allows efficient use of a limited number of mesh points to define the problem. However, there is still a lot of scope for improving both the pre- and post- processors of the POISSON group codes.

Chapter 3 deals with the saturation induced harmonics due to the non-linear properties of iron. It has been demonstrated here that the saturation induced harmonics can be practically eliminated by improving the yoke design. In RHIC-type magnet designs, where the iron contribution enhances the field by $50 \%$ over the field produced by superconducting coils, the saturation induced harmonics would be expected to be large. However, the use of holes, cutouts and the choice of material for keys and pins (magnetic low carbon steel or nonmagnetic stainless steel) have resulted in reducing the current dependence in the sextupole and decapole harmonics by about an order of magnitude. In a new magnet design one would incorporate such features from the beginning. The improvements in the computer codes and modelling and good agreement with the reliable measurements provides the confidence that one can indeed design a yoke in which the saturation induced harmonics are small.

In SSC dipole magnets the saturation in the sextupole harmonic was reduced by compensating pole saturation with forced midplane saturation. This was done by either using stainless steel keys (BNL design) or a cutout at the midplane (Fermilab design). In some SSC magnets, an appreciable amount of $b_{2}$ was generated by coil deformation from Lorentz forces. This should not cause a deterioration in field quality in the magnets since one can 
minimize the overall change in harmonics from both sources (iron saturation and coil deformation due to Lorentz forces) as both are high field effects. In the BNL-built SSC 50 mm prototype magnets the change in sextupole harmonic was nearly zero $(\sim 0.1$ unit at 10 $\mathrm{mm}$ ) in the entire range of operation.

The two-radius method for controlling iron saturation at the yoke inner surface has been used in the design of the $130 \mathrm{~mm}$ RHIC insertion quadrupole. This has resulted in reducing $b_{5}$ by an order of magnitude as compared to the one-radius method.

The efficiency of a particular saturation control method depends on the details of a particular design. Though not used explicitly in the development of the various yoke designs, towards the end of this research work it has been found that a useful barometer to evaluate the quality of a particular yoke design is to examine the variation in the function $\left(\frac{\mu-1}{\mu+1}\right)$ with azimuthal angle at the yoke inner surface. In a good design the variation in this function will remain small over the entire operating range. However, in most cases it may be sufficient to examine this function at a high field only.

An asymmetric placement of the dipole coldmass in the cryostat may result in introducing a significant skew quadrupole harmonic at high fields. This can be compensated by deliberately making the lower yoke half heavier than the upper. In RHIC dipoles this technique has resulted in reducing the change in quadrupole harmonic by about a factor of 2 at the design field.

Chapter 4 deals with the methods used and developed to improve the geometric harmonics (the harmonics which are related to magnet geometry and not to yoke or superconductor effects). The coil cross sections for various $R H I C$ magnets have been designed in such a way that they allow for a small adjustment in field harmonics even during the production phase with no change in the copper wedges. In the case of the $80 \mathrm{~mm}$ aperture RHIC arc dipole magnets, it provided the iteration which was necessary to remove the measured harmonics due to the change in tooling between the BNL and the industry-built magnets. With an adjustment in the coil pole shim and the midplane gap one can control two harmonics. This adjustment may be needed either to correct for a drift in the manufacturing process or to iterate the initial coil cross section. An adjustment in the midplane gap is more effective in controlling the decapole harmonic. Control of the geometric decapole with this method coupled with a reduction in the saturation-induced decapole harmonic has resulted in reducing this harmonic to a level that use of the decapole correctors installed in RHIC may not be required. 
In order to iterate the coil cross section reliably, mechanical changes should be minimized between iterations. This disciplined approach has resulted in obtaining the desired changes in field harmonics in RHIC magnets when a coil cross section iteration is made.

The RHIC quadrupoles are collared like dipoles for cost and simplicity reasons. This has been generally avoided in the past because it breaks the pure quadrupole symmetry and generates a large octupole harmonic. An asymmetric coil midplane gap between the horizontal and vertical planes has been used in the RHIC quadrupoles to remove practically all octupole harmonic. This demonstrates that one can have the simplicity of the dipoletype, 2-fold collaring method while maintaining a good field quality in quadrupoles.

In chapter 5, methods to compensate for construction errors are presented. The field quality performance in well designed magnets (after using some of the techniques described above) will now depend on the actual engineering tolerances. These are generally at the level of $50 \mu \mathrm{m}$. These usual mechanical errors produce relative field errors $\left(\frac{\Delta B}{B}\right)$ which are a few parts in $10^{-4}$ at $2 / 3$ of the coil radius. A tuning shim method has been developed for the RHIC $130 \mathrm{~mm}$ aperture quadrupoles which is expected to reduce the relative field errors to a few parts in $10^{-5}$. The eight tuning shims compensate for eight measured field harmonics by adjusting the amount of iron in them. This tuning shim compensation is applied in the body of the magnet. Good agreement has been found between calculations and measurements in the magnets with tuning shims made so far. A second method is to make a lumped correction at the ends by adjusting the amount of iron laminations in the magnet ends.

Similar error compensation schemes may be considered for a large scale magnet production program. This would reduce manufacturing tolerances while assuring good field quality. A plan would have to be developed so that it could be efficiently adopted in an industrial magnet production environment.

The techniques and the methods described above have been implemented in the design and construction of RHIC and SSC magnets. The use of these methods is described in detail in chapter 6 where the magnetic design of the following two selected cases is presented : (a) the SSC $50 \mathrm{~mm}$ aperture main collider dipole magnet prototype and (b) the $130 \mathrm{~mm}$ aperture RHIC insertion quadrupole magnets. These magnets are designed to have good field quality and a high quench field. Moreover, the design incorporates various features which are expected to make the relevant part of the manufacturing as simple and as error free as possible. 
The RHIC $130 \mathrm{~mm}$ aperture quadrupole coil cross section has good flexibility in the design. This has allowed a number of iterations to be carried out without changing wedges. This is an efficient way of trying out coil cross section iterations which saves both on the cost and time. Moreover, since the change in the geometry of the coil cross section is either none or small, the magnet ends may not change. In the case when the required iteration can be accomplished by changing only the midplane gaps and the coil pole shims, previously built coils can be used.

Most of the methods described here have been successfully applied to the magnetic design of various RHIC and SSC magnets. The general techniques, improvements and design philosophy presented can however be used in any magnet program. 


\section{References}

1. D. Abeshouse, H. Hahn, Perturbation Method for Cosine theta Magnet with Nonlinear Iron Shields, The Fourth International Conference on Magnet Technology, Brookhaven National Laboratory, NY, USA, MT-4, pp. 594-599 (1972).

2. J. Allinger, et al., High Field Superconducting Magnets for Accelerators and Particle Beams, 1974 Applied Superconductivity Conference; IEEE Transaction on Magnetics, Vol. MAG-11, No. 2, pp.467-470 (1974).

3. J. Allinger, et al., ISABELLE Ring Magnets, 1976 Applied Superconductivity Conference; IEEE Transaction on Magnetics, Volume MAG-13, No. 1, pp. 275-278 (1976).

4. M. Anerella, et al., Construction and Test Results from $15 \mathrm{~m}$-Long, $50 \mathrm{~mm}$ Aperture SSC Dipole Models, Supercollider 4, Proceedings of the Fourth International Industrial Symposium on the Super Collider, March 4-6 1992, New Orleans, USA, pp. 535-549 (1992).

5. M. Anerella, et al., Improved Cable Insulation for Superconducting Magnets, Proceedings of the 1993 Particle Accelerator Conference, Washington, D.C., pp. 2790-2792 (1993).

6. M. Anerella, et al., Industrial Production of RHIC Magnets, Proceedings of the Fourteenth International Conference on Magnet Technology (MT-14), Tampere, Finland, June 11-16 (1995).

7. J. Swanson, ANSYS Finite Element Code Revision 5.0, Swanson, Analysis System, Inc. (1992).

8. AUTOCAD is a commercial software package available from Autodesk, Inc.; AUTOCAD is a registered Trademark of Autodesk, Inc. (1992).

9. D. Beavis, et al., Letter of intent for a forward angle and midrapidity hadron spectrometer at RHIC, RHIC-LOI-8, Brookhaven National Laboratory (1992).

10. D. Bein and J. Zbasnik, Utilization of Gamma Ray Inspection System for Tomographic Imaging and Dimensional Analysis of Complete Model Magnet Cold Masses and Collared Coil Sections, Supercollider 4, Proceedings of the Fourth International Industrial Symposium on the Super Collider, New Orleans, USA, pp. 225-233 (1992).

11. G.F. Bertsch, Searching for the Quark-Gluon Plasma, Science, Vol. 265, pp. 480-481 (1994). 
12. R.A. Beth, Complex representation and computation of two-dimensional magnetic fields, Journal of Applied Physics, Vol. 37, No. 7, p. 2568 (1966).

13. R.A. Beth, An Integral Formula for Two-Dimensional Fields, Journal of Applied Physics, Vol. 38, No. 12, pp. 4689-4692 (1967).

14. R.A. Beth, Elliptical and Circular Current Sheets to Produce a Prescribed Internal Field, 1967 National Accelerator Conference, IEEE Trans. of Nucl. Sci., No. 3, pp. 368-370 (1967).

15. R.A. Beth, Analytic design of superconducting multipolar magnets, Proc. 1968 Summer Study on Superconducting Devices and Accelerators, Brookhaven National Laboratory p. 843 (1968).

16. R.A. Beth, Evaluation of Current-Produced Two-Dimensional Magnetic Fields, Journal of Applied Physics, Vol. 40, No. 12, pp. $4782-4786$ (1969).

17. R.A. Beth, Currents and Coil Forces as Contour Integrals in Two-Dimensional Magnetic Field, Journal of Applied Physics, Vol 40, No. 6, pp. 2445-2449, (1969).

18. -R.A. Beth, Induced EMFS in Two-Dimensional Fields, The Fourth International Conference on Magnet Technology, MT-4, Brookhaven National Laboratory, NY, USA (1972).

19. K.J. Binns and P.J. Lawrenson, Analysis and Computation of Electric and Magnetic Field Problems, Published by Pergamon Press, Second edition (1973).

20. E.J. Bleser, et al., Superconducting Magnets for the CBA Project, Nuclear Instruments and Methods in Physics Research A235, pp. 435-463 (1985).

21. J.P. Blewett, Iron Shielding for Air Core Magnets, Proc. 1968 Summer Study on Superconducting Devices and Accelerators, Brookhaven National Laboratory, p. 1042 (1968).

22. H. Brechna, Superconducting Magnet System, Technische Physik in Einzeldarstellungen Band 18, Springer-Verlag, Berlin (1973).

23. H. Brechna, et al., Workshop on Magnets: Superconducting Magnets for High Energy Accelerators, The Seventh International Conference on Magnet Technology, MT-7, Karlsruhe Nuclear Research Center, Germany, pp. 2355-2365 (1981).

24. G. Brianti, General Aspects and Present Limits in the Accelerator Technology, The Ninth International Conference on Magnet Technology, MT-9, Zurich, Switzerland, pp. 53-61 (1985). 
25. K.L. Brown and R.V. Servranckx, First- and Second-Order Charged Particle Optics, AIP Conference Proceedings No. 127, pp. 62-138 (1983).

26. CAD OVERLAY GS is a commercial software package available from Image System Technology; CAD Overlay GS is a registered Trademark of Image System Technology (1992).

27. S. Caspi, et al., Incorporation of Boundary Condition into the POISSON Program, The Ninth International Conference on Magnet Technology, MT-9, Zurich, Switzerland, pp. 1560-1566 (1985).

28. S. Caspi, et al., The effects of filament magnetization in superconducting magnets as computed by Poisson, 1986 Applied Superconductivity Conference, Baltimore, Maryland, USA, IEEE Transactions on Magnetics, March 1987, Volume MAG-23, No. 2 , pp. 510-513 (1986).

29. R.V. Churchill, Complex Variables and Applications, Published by McGraw-Hill Book Company, Inc (1950).

30. J.H. Coupland, Dipole, quadrupole and higher order fields from simple coils, Nuclear Instruments and Methods, 78, pp. 181-184 (1970).

31. J. Coupland, et al., Very High Field Synchrotron Magnets with Iron Yokes, Nuclear Instruments and Methods 106, p. 595 (1973).

32. E.D. Courant and H.S. Snyder, Theory of the Alternating Gradient Synchrotron, Annals of Physics, Vol 3, pp 1-48 (1958).

33. P. Dahl, et al., Superconducting Magnet Models for ISABELLE, 1973 Particle Accelerator Conference, San Francisco, USA, pp. 688-692 (1973).

34. P. Dahl, et al., Performance of three $4.5 \mathrm{~m}$ Dipoles for SSC Reference Design D, The Ninth International Conference on Magnet Technology, MT-9, Zurich, Switzerland, pp. 80-83 (1985).

35. P. Dahl, et al., Construction of Cold Mass Assembly for Full-Length Dipoles for the SSC Accelerator, 1986 Applied Superconductivity Conference, Baltimore, Maryland, USA, IEEE Transactions on Magnetics, Volume MAG-23, No. 2, pp. 1215-1218 (1987).

36. P. Dahl, et al., Performance of Initial Full-length RHIC Dipoles, The Tenth International Conference on Magnet Technology, MT-10, Boston, MA, USA, pp. 723-725 (1987).

37. P. Dahl, et al., Test Results from 1.8-m SSC Model Dipoles, The Tenth International Conference on Magnet Technology, MT-10, Boston, MA, USA, pp. 816-819 (1987). 
38. P. Dahl, Superconducting Magnet System, 1989 US Particle Accelerator School, AIP Conference Proceedings 184, pp. 1327-1376 (1989).

39. G. Danby, Ultraprecise Magnet Design and Shimming, 1987 Particle Accelerator Conference, Washington DC, pp. 1517-1519 (1987).

40. C. Daum, Three-dimensional computation of magnetic fields and Lorentz forces of an $L H C$ dipole magnet, NIKHEF-H/89-12, LHC Note No. 94 (1989).

41. D. Dell'Orco, S. Caspi, et al., A $50 \mathrm{~mm}$ Bore Superconducting Dipole with a Unique Iron Yoke Structure, Published in the proceedings of the 1992 Applied Superconductivity Conference, Chicago (1992).

42. H. Desportes, Superconducting Magnets for Accelerators, Beam Lines, and Detectors, The Seventh International Conference on Magnet Technology, MT-7, Karlsruhe Nuclear Research Center, Germany, pp. 1560-1566 (1981).

43. A. Devred, Quench Origins, The Physics of Particle Accelerators, AIP Conference Proceedings 249, pp. $1262-1308$ (1990).

44. A. Devred, et al., About the Mechanics of SSC Dipole Magnet Prototypes, The Physics of Particle Accelerators, AIP Conference Proceedings 249, pp. 1309-1374 (1990).

45. A. Devred, et al., Status of 4-cm Aperture, 17-m-long SSC Dipole Magnet R\&D Dipole Magnet Program at BNL Part I: Magnet Assembly, Supercollider 3, Proceedings of the Third International Industrial Symposium on the Super Collider, Atlanta, USA, pp. 549-573 (1991).

46. A. Devred, et al., Review of SSC Dipole Magnet Mechanics and Quench Performance, Supercollider 4, Proceedings of the Fourth International Industrial Symposium on the Super Collider, New Orleans, USA, pp. 113-136 (1992).

47. G.E. Fischer, Iron Dominated Magnets, 1985 SLAC Summer School, AIP Conference Proceedings 153, pp. 1120-1227 (1985).

48. H.E. Fisk, et al., The Ironless Cos theta Magnet Option for the SSC, 1985 Particle Accelerator Conference in Vancouver, Canada, pp. 3456-3461 (1985).

49. G. Ganetis, et al., Field Measuring Probe for SSC Magnets, 1987 Particle Accelerator Conference, Washington DC, pp. 1393-1395 (1987).

50. M. Green, Control of the Fields due to Superconductor Magnetization in the SSC Magnets, IEEE Transactions on Magnetics, Vol. MAG-23, No. 2, pp. 506-509 (1987). 
51. A. Greene, et al., The Magnet System of the Relativistic Heavy Ion Collider (RHIC), Fourteenth International Conference on Magnet Technology (MT-14), Tampere, Finland, June 11-16 (1995).

52. E. Gregory, Conventional Wire and Cable Technology, The Physics of Particle Accelerators, AIP Conference Proceedings 249, pp. 1198-1229 (1990).

53. R.C. Gupta, J.I.M. Botman, M.K. Craddock, A High Transition Energy Lattice for a TRIUMF KAON Factory Synchrotron, Western Region Nuclear Physics Conference, Lake Louise, Canada (1984).

54. R.C. Gupta, J.I.M. Botman, M.K. Craddock, High Transition Energy Magnet Lattices, Proceedings of the 1985 Particle Accelerator Conference, Vancouver, Canada; IEEE Transaction on Nuclear Science, Volume NS-32, Oct 1985, pp. 2308-2310 (1985).

55. R.C. Gupta, G.H. Morgan, A Design for a High Field Combined Function Superferric Magnet, Proceedings of the 1985 Particle Accelerator Conference, Vancouver, Canada; IEEE Transaction on Nuclear Science, Volume NS-32, Oct 1985, pp. 3687-88 (1985).

56. R.C. Gupta, Modifications in the AUTOMESH and other POISSON Group Codes, Proceedings of the Workshop in Electromagnetic Field Computations, Schnectady, NY, pp. H38-H42 (1986).

57. R.C. Gupta, Improvements in the Mesh Generator of the POISSON Group Codes, Proceedings of the 1987 Particle Accelerator Conference, Washington DC, pp. 1449-1451 (1987).

58. R.C. Gupta, G.H. Morgan, P.A. Thompson, A Single Layer Coil Superconducting Magnet for SSC, Proceedings of the 1987 Particle Accelerator Conference, Washington DC, pp. 1413-1415 (1987).

59. R.C. Gupta, G.H. Morgan, P.J. Wanderer, A Comparison of Calculations and Measurements of the Iron Saturation Characteristic of the SSC Design D Dipole Magnet, Proceedings of the 1987 Particle Accelerator Conference, Washington DC, pp. 14051407 (1987).

60. R.C. Gupta, S.Y. Lee, Y.Y. Lee, X.F. Zhao, Beam Transfer Lines for the AGS Booster, Proceedings of the 1987 Particle Accelerator Conference, Washington DC, pp. 11931195 (1987). 
61. R.C. Gupta, G.H. Morgan, Collarless, Close-in, Shaped Iron Aperture Designs for the SSC Dipole, Proceedings of the 1989 Particle Accelerator Conference, Chicago, pp. 500-502 (1989).

62. R.C. Gupta, S.A. Kahn and G.H. Morgan, Coil and Iron Design for SSC $50 \mathrm{~mm}$ Magnet, Proceedings of the 1990 American Society of Mechanical Engineers (ASME) Winter Annual Meeting in Dallas (1990).

63. R.C. Gupta, POISSON-BNL, Computer Codes for Particle Accelerator Design and Analysis : A Compendium, Los Alamos Accelerator Code Group, Second Addition, LA-UR-90-1766 (1990).

64. R.C. Gupta, S.A. Kahn and G.H. Morgan, A Comparison of Calculations and Measurements of the Field Harmonics as a Function of Current in the SSC Dipole Magnets, Proceedings of the 1991 IEEE Particle Accelerator Conference, San Francisco, pp. 42-44 (1991).

65. R.C. Gupta, et al., RHIC Insertion Magnets, Proceedings of the 1991 IEEE Particle Accelerator Conference, San Francisco, pp. 2239-2241 (1991).

66. R.C. Gupta, S.A. Kahn and G.H. Morgan, SSC $50 \mathrm{~mm}$ Dipole Cross section, Proceedings of the $3^{\text {rd }}$ International Industrial Symposium on Super Collider (IISSC), Atlanta, pp. 587-600 (1991).

67. R.C. Gupta, Correcting Field Harmonics after Design in Superconducting Magnets, Proceedings of the $4^{\text {th }}$ International Industrial Symposium on Super Collider (IISSC), New Orleans, pp. 773-780 (1992).

68. R. Gupta, Iron Shims to Correct the Measured Harmonics in $130 \mathrm{~mm}$ Aperture RHIC Insertion Quadrupoles, Magnet Division Internal Note 480-16 (RHIC-MD-185), Dec. 10, 1992, Unpublished (1992).

69. R. Gupta, et al., Large Aperture Quadrupoles for RHIC Interaction Regions, Proceedings of the 1993 Particle Accelerator Conference, Washington, D.C., pp. 2745-2747 (1993).

70. R.C. Gupta, A.K. Jain, Variation in $a_{1}$ saturation in SSC Collider Dipoles, Proceedings of the 1993 Particle Accelerator Conference, Washington, D.C., pp. 2778-2780 (1993).

71. R. Gupta, et al., Field Quality Improvements in Superconducting Magnets for RHIC, Proceedings of the 1994 European Particle Accelerator Conference, London, UK, pp. 2928-2930 (1994). 
72. R. Gupta, et al., Field Quality Control Through the Production Phase of RHIC Arc Dipoles, Proceedings of the 1995 International Particle Accelerator Conference, Dallas, Texas (1995).

73. R. Gupta, et al., Tuning Shims for High Field Quality in Superconducting Magnets, Proceedings of the Fourteenth International Conference on Magnet Technology (MT14), Tampere, Finland, June 11-16 (1995).

74. R. Gupta, Estimating and Adjusting Field Quality in Superconducting Accelerator Magnets, Proceedings of the LHC Collective Effects Workshop, Montreux, 1995; Submitted to the Particle Accelerators (1995).

75. R. Gupta, Field Quality in the Superconducting Magnets for Large Particle Accelerators, Proceedings of the 1996 European Particle Accelerator Conference at Sitges, Spain (1996).

76. H. Gurol, General Dynamics, Private communication, (1993).

77. H. Hahn, et al., Upgrade Coil Configuration for Isabelle Magnets, The Seventh International Conference on Magnet Technology, MT-7, Karlsruhe Nuclear Research Center, Germany, pp. 1575-1578 (1981).

78. H. Hahn, ISABELLE - A Progress Report, IEEE Transaction on Magnetics, January 1981, Vol MAG-17, No. 1, 1981 Applied Superconductivity Conference, pp. 702-708 (1981).

79. K. Halbach, A Program for Inversion of System Analysis and its Application to the Design of Magnets, Proceedings of the second conference on Magnet Technology, Oxford, England (1967).

80. K. Halbach, Application of Conformal Mapping to Evaluation and Design of Magnets Containing Iron with Nonlinear $B(H)$ Characteristics, Nuclear Instruments and Methods 64, pp. 278-284 (1968).

81. K. Halbach, Fields and first order perturbation effects in two-dimensional conductor dominated magnets, Nuclear Instruments and Methods, 78, p. 185 (1970).

82. K. Halbach, Speciality Magnets, 1985 SLAC Summer School, AIP Conference Proceedings 153 , pp. 1277-1295 (1985).

83. R. Hannaford, et al., Resolution to difficulties experienced in SSC cable Fabrication during the initial scale-up period, 1990 Applied Superconductivity Conference, Snowmass, 
Co, USA; IEEE Transaction on Magnetics, March 1991, Vol 27, No. 2, pp. 2024-2026 (1990).

84. M.A. Harrison, The RHIC Project, Proceedings of the 1994 European Particle Accelerator Conference, London, UK, pp. 156-160 (1994).

85. M.A. Harrison, RHIC Status and Plan, Proceedings of the 1995 International Particle Accelerator Conference, Dallas, Texas (1995).

86. W.V. Hassenzahl, R.B. Meuser, C. Taylor, Technology of Superconducting Accelerator Dipoles, 1982 Summer School on High Energy Particle Accelerators, AIP Conference Proceedings 105, pp. 732-800 (1982).

87. J. Herrera, et al., Random Errors in the Magnetic Field Coefficients of Superconducting Magnets, pp. 3689-3691, 1985 Particle Accelerator Conference in Vancotiver, Canada, May 13-16, 1985.

88. J. Herrera, et al., Measurement of the Magnetic Field Coefficients of Particle Accelerator Magnets, 1989 Particle Accelerator Conference in Chicago, USA, pp. 1774-1776 (1989).

89. H. Hillmann, Large Scale Fabrication of Superconductors, The Seventh International Conference on Magnet Technology, MT-7, Karlsruhe Nuclear Research Center, Germany, pp. 1614-1621 (1991).

90. R.F. Holsinger, original version of the User's Guide to POISSON Group Codes. This and a new version of the User's Guide and Reference Manual for POISSON Group Codes can be obtained from the Los Alamos Accelerator Code Group, Los Alamos National Laboratory Publication No. LA-UR-87-126, or latest version (1967).

91. "Paintbrush" is a commercial software package available from the Hewlett Packard Corporation; "HP Paintbrush" is a registered Trademark of Hewlett Packard Corporation. Scan Gallery version 5 has been used here. (1992).

92. "SCANJET Plus" is a package of commercial hardware and software which is available from the Hewlett Packard Corporation; "HP ScanJet Plus" is a Registered Trademark of Hewlett Packard Corp (1992).

93. B.B. Gamble, et al., Prospects for HTS Applications, Fourteenth International Conference on Magnet Technology (MT-14), Tampere, Finland, June 11-16 (1995).

94. F.R. Huson et al., Superferric magnet option for the SSC, 1985 Particle Accelerator Conference in Vancouver, Canada, pp. 3462-3465 (1985). 
95. J.D. Jackson, Classical Electrodynamics, Second Edition, Published by John Wiley \& Sons (1975).

96. J.D. Jackson, editor, Conceptual Design of the Superconducting Super Collider, SSCSR-1020 (1986).

97. A. Jain, R. Gupta, et al., Skew Quadrapole in RHIC Dipole Magnets at High Fields, Fourteenth International Conference on Magnet Technology (MT-14), Tampere, Finland, June 11-16 (1995).

98. S. Kahn, et al., Field Quality Aspects of CBA Superconducting Magnets, 1983 Particle Accelerator Conference, Santa Fe, USA, pp. 3469-3471 (1983).

99. S. Kahn, R. Gupta, G. Morgan, P. Thompson, Comparison of Magnetic Field Calculations to Measurements on a CBA 2-in-1 Magnet System, Proceedings of the 1985 Particle Accelerator Conference, Vancouver; IEEE Transaction on Nuclear Science, Volume NS-32 (1985).

100. S.A. Kahn and G.H. Morgan, Magnetic Properties of Iron Yoke Laminations for SSC Dipole Magnets, 1991 IEEE Particle Accelerator Conference, San Francisco, pp. 21702172 (1991).

101. S. Kahn, Private communication (1992).

102. S.A. Kahn, R.C. Gupta, A.K. Jain, G.H. Morgan, P.A. Thompson, Calculations of Magnetic Field for the End Design of the RHIC Arc Dipole, Proceedings of the 1993 Particle Accelerator Conference, Washington, D.C., pp. 2754-2756 (1993).

103. J.F. Kallsen, et al., SSC Type N6Ti Superconductor Research Program at Teledyne SC, 1990 Applied Superconductivity Conference, September 24-28, 1990, Snowmass, Co, USA; IEEE Transaction on Magnetics, March 1991, Vol 27, No. 2, pp. 1799-1802 (1990).

104. "Kapton" is a registered trademark of E.I. Dupont de Nemours \& Co.

105. T.K. Khoe, R.J. Lari, FORGY, A Companion Computer Program of TRIM to Calculate Forces and Energy in Electromagnets, The Fourth International Conference on Magnet Technology, MT-4, Brookhaven National Laboratory, NY, USA, pp. 585-593 (1972).

106. H. Kirk, et al., Magnetic Field Properties of the Isabelle Project Superconducting Dipole Magnets, 1981 Particle Accelerator Conference, Washington, D.C., pp. 3237-3239 (1981). 
107. H.G. Kirk, et al., End Fields of CBA Superconducting Magnets, 1983 Particle Accelerator Conference, Santa Fe, USA, pp. 3375-3377 (1983).

108. Koska W., et al., Test of Fermilab Built $\$ 0 \mathrm{~mm}$ Aperture Full Length SSC Dipole Magnets, The Twelfth International Conference on Magnet Technology, MT-12, Leningrad, USSR, pp. 303-306 (1991).

109. J. Kuzminski, et al., Test Results of BNL built 40-mm aperture, 17-m-long SSC Collider Dipole Magnets, The Twelfth International Conference on Magnet Technology, MT-12, Leningrad, USSR, pp. 311-314 (1991).

110. D.C. Larbalestier, Recent Advances in Practical Superconductors, The Ninth International Conference on Magnet Technology, MT-9, Zurich, Switzerland, pp. 453-458 (1985).

111. Y.Y. Lee, et al., The AGS Booster Lattice, 1987 Particle Accelerator Conference, Washington DC (1987).

112. Ludlam T.W. and Stevens A.J., A Brief Description of The Relativistic Heavy Ion Collider Facility, Informal Report BNL-49177 (1993).

113. R. Meinke, Superconducting Magnet System for HERA, 1990 Applied Superconductivity Conference, September 24-28, 1990, Snowmass, Co, USA; IEEE Transaction on Magnetics, March 1991, Vol 27, No. 2, pp. 1728-1734 (1990).

114. R. Meinke, P. Schmuser and Y. Zhao, Methods of Harmonics Measurements in the Superconducting HERA Magnets and Analysis of Systematic Errors, DESY HERA 9113 (1991).

115. F.E. Mills and G. Morgan, A Flux Theorem for the Design of Magnet Coil Ends, Particle Accelerators, Vol 5, pp. 227-235 (1973).

116. G.H. Morgan, Two-dimensional, uniform current density, air-core coil configurations for the production of specified magnetic fields, IEEE Trans. Nucl. Sci., NS-16, No. 3, Part 1, p. 843 (1969).

117. G.H. Morgan, Stationary Coil for Measuring the Harmonics in Pulsed Transport Magnets, The Fourth International Conference on Magnet Technology, MT-4, Brookhaven National Laboratory, NY, USA, pp. 787-790 (1972).

118. G. Morgan, Use of an Elliptical Aperture to Control Saturation in Closely-Coupled, Cold Iron Superconducting Dipole Magnets, 1985 Particle Accelerator Conference in 
Vancouver, Canada, IEEE Trans. on Nuclear Science, Vol. NS-32, No. 5, pp. 36953697 (1985).

119. G.H. Morgan, Shaping of Magnetic Fields in Beam Transport Magnets, The Physics of Particle Accelerators, AIP Conference Proceedings 249, pp. 1242-1261 (1990).

120. G.H. Morgan, et al., Construction and results of the $50 \mathrm{~mm}$ short RED dipole magnets, Proceedings of the 1991 IEEE Particle Accelerator Conference, San Francisco (1991).

121. G.H. Morgan, A Computer Program for the 2-D Magnetostatic Problem Based on Integral Equations for the Field of the Conductors and Boundary Elements, The Twelfth International Conference on Magnet Technology, MT-12, Leningrad, USSR, pp. 912-915 (1991).

122. S. Mulhall Private communication, (1993).

123. J.F. Muratore, et al., Construction and Test Results from $1.8 \mathrm{~m}$-Long, $50 \mathrm{~mm}$ Aperture SSC Dipole Models, Supercollider 4, Proceedings of the Fourth International Industrial Symposium on the Super Collider, March 4-6 1992, New Orleans, USA, pp. 559-573 (1992).

124. M.J. Newman, C.W. Trowbridge, L.R. Turner, GFUN: An Interactive Program as an AID to Magnet Design, The Fourth International Conference on Magnet Technology, MT-4, Brookhaven National Laboratory, NY, USA, pp. 617-626 (1972).

125. The RHIC Project and its scope have been endorsed by the DOE/NSF Nuclear Science Advisory Committee (NSAC) in its Dec., 1989 Long Range Plan for Nuclear Science (1989).

126. D. Orrell, Private Communication (1991).

127. R.B. Palmer, et al., Status Report on Isabelle Magnets, 1982 Applied Superconductivity Conference, Knoxville, TN, USA; IEEE Transaction on Magnetics, May 1983, Vol MAG19, No. 3, pp. 189-194 (1992).

128. R. Palmer and A.V. Tollestrup, Superconducting Magnet Technology for Accelerators, Ann. Rev. Nucl. Part. Sci. 34, pp. 247-284 (1984).

129. W.K.H. Panofsky and M. Phillips, Classical Electricity and Magnetism, Second Edition, Published by Addison-Wesley (1962).

130. R. Fernow, G. Morgan, R. Palmer, P. Thompson, PAR2DOPT is a coil design program used at the Brookhaven National Laboratory for superconducting magnets; P. Thompson is responsible for its current form, unpublished (1995). 
131. G. Parzen G. and K. Jellett, Coil Configurations in Superconducting Dipoles and Quadrupoles, The Fourth International Conference on Magnet Technology, MT-4, Brookhaven National Laboratory, NY, USA, pp. 642-649 (1972).

132. J. Peoples, Status of the SSC superconducting magnet program, IEEE Transaction on Magnetics, March 1989, Vol 25, No. 2, 1988 Applied Superconductivity Conference, pp. $1444-1450$ (1988).

133. E.G. Pewitt, editor, Requirements and Specifications for $50 \mathrm{~mm}$ Collider Dipole Magnet, Fermilab Publication (1990).

134. PHENIX, PHENIX Conceptual Design Report, 29 January 1993. Contact PHENIX Collaboration Office at BNL or Research Library at BNL (1993).

135. Los Alamos Accelerator Code Group, User's Guide and Reference Manual for POISSON Group Codes, the standard version of POISSON Group Codes along with these manuals can be obtained from the Los Alamos Accelerator Code Group, Los Alamos National Laboratory Publication No. LA-UR-87-126, or latest version (1987).

136. A number of Quark Matter Conferences have been held which are relevant to physics at RHIC. See for example, The Proceedings of the Ninth International Conference on UltraRelativistic Nucleus-Nucleus Collisions (Quark Matter, '91), T. Awes et al., editors, Published by North Holland (1992).

137. I.I. Rabi, A method of producing uniform magnetic fields, Review of Scientific Instruments, 5, p. 78 (1934).

138. P. Radusewicz, et al., Results from a Partial Lifetime Test of a 40-mm aperture, 17m-Long SSC Model Dipole, Supercollider 4, Proceedings of the Fourth International Industrial Symposium on the Super Collider, March 4-6 1992, New Orleans, USA, pp. 551-558 (1992).

139. P. Reardon, Cold Iron Cos theta Magnet Option for the SSC, 1985 Particle Accelerator Conference in Vancouver, Canada, pp. 3466-3470 (1985).

140. Conceptual Design of the Relativistic Heavy Ion Collider RHIC, Brookhaven National Laboratory Report No. BNL 52195 (1989).

141. S. Russenschuck, et al., Tracing Back Magnetic Field Imperfection in LHC Magnets by Means of the Inverse Problem Approach, Proceedings of the Thirteenth Magnet Technology Conference (MT-13), Victoria, Canada (1993). 
142. W.B. Sampson, New Superconducting Material for Magnet Applications, The Fourth International Conference on Magnet Technology, MT-4, Brookhaven National Laboratory, NY, USA, pp. 487-492 (1972).

143. J.R. Sanford and D.M. Matthews, editors, Site-Specific Conceptual Design of the Superconducting Super Collider, SSCL-SR-1056 (1990).

144. P. Schmuser, Superconducting Magnets for Particle Accelerators, The Physics of Particle Accelerators, AIP Conference Proceedings 249, pp. 1099-1158 (1990).

145. E.V. Shuryak, Quantum Chromodynamics and The Theory of Superdense Matter, Physics Reports, Vol. 61, p. 71 (1980).

146. The STAR collaboration, Conceptual Design Report for the Solenoidal Tracker at RHIC, PUB-5347, LBL, June 15 (1992).

147. J. Starit, et al., Full Length Prototype SSC Dipole Test Results, 1986 Applied Superconductivity Conference, Baltimore, Maryland, USA, IEEE Transactions on Magnetics, March 1987, Volume MAG-23, No. 2, pp. 1208-1214 (1986).

148. J. Strait, et al., Test of Prototype SSC Magnets, 1987 Particle Accelerator Conference in Washington, D.C., pp. 1537-1539 (1987).

149. J. Strait, et al., Tests of Prototype SSC Magnets, The Tenth International Conference on Magnet Technology, MT-10, Boston, MA, USA, pp. 730-733 (1987).

150. A.S. Stratton, Electromagnetic Theory, Published by McGraw-Hill Book Company, Inc (1941).

151. B.P. Strauss, et al., Results of Magnet Prototype Evaluation for the Fermilab Energy Doubler Project, IEEE Transaction on Magnetics, 1974 Applied Superconductivity Conference, pp. 451-454, Volume MAG-11, No. 2 (1974).

152. M.J. Tannenbaum, et al., Magnetic Properties of the Iron Laminations for CBA Magnets, 1983 Particle Accelerator Conference, Santa Fe, USA, pp. 3472-3474 (1983).

153. T. Ludlam and A. Schwarzchild, Task Force for Relativistic Heavy Ion Physics, Nucl. Phys., Vol. A418, 657c (1984).

154. D.B. Thomas, M.N. Wilson, Filamentary Superconductor for Pulsed Application, The Fourth International Conference on Magnet Technology, MT-4, Brookhaven National Laboratory, NY, USA, pp. 493-497 (1972). 
155. R. Thomas, Performance of field measuring probes for SSC magnets, Proc. $5^{\text {th }}$ International Industrial Symposium on the Super Collider, San Francisco, California, USA, May 6-8, 1993, in Supercollider 5, pp. 715-718 (1994).

156. P. Thompson, et al., Superconducting Magnet System for RHIC, 1985 Particle Accelerator Conference in Vancouver, Canada, pp. 3698-3670 (1985).

157. P.A. Thompson, R.C. Gupta, et al.; Iron Saturation Control in RHIC Dipole Magnets, Proceedings of the 1991 IEEE Particle Accelerator Conference, San Francisco (1991).

158. P.A. Thompson, R.C. Gupta, et al., Revised Cross section for RHIC Dipole Magnets: Proceedings of the 1991 IEEE Particle Accelerator Conference, San Francisco (1991).

159. P. Thompson, Private communication, (1992).

160. P. Thompson, et al., B Series RHIC Arc Quadrupoles, Proceedings of the 1993 Particle Accelerator Conference, Washington, D.C., pp. 2766-2768 (1993).

161. A.V. Tollestrup, Superconducting Magnets, 1981 Summer School on High Energy Particle Accelerators, AIP Conference Proceedings 87, pp. 699-804 (1981).

162. C.W. Trowbridge, Progress in Magnet Design by Computer, The Fourth Internationa: Conference on Magnet Technology, MT-4, Brookhaven National Laboratory, NY, USA, pp. 555-565 (1972).

163. C.W. Trowbridge, Status of Electromagnetic Field Computation, The Ninth International Conference on Magnet Technology, MT-9, Zurich, Switzerland, pp. 707-713 (1985).

164. P. Wanderer, et al., Study of Factors which Affect Training in Isabelle R\&D Magnets, 1981 Particle Accelerator Conference, Washington, D.C., pp. 3208-3210 (1981).

165. P. Wanderer, Performance of SSC RED Dipoles, The Physics of Particle Accelerators, AIP Conference Proceedings 249, pp. 1374-1388 (1990).

166. P. Wanderer, et al., Experiments with all-Kapton insulation and axial prestress in 1.8 m-long SSC RED magnets, 1991 IEEE Particle Accelerator Conference, San Francisco, pp. 2164-2166 (1991).

167. P. Wanderer, et al., Results of Magnetic Measurements of $40 \mathrm{~mm}$ Aperture 17-m Long SSC Model Collider Dipole Magnets, The Twelfth International Conference on Magnet Technology, MT-12, Leningrad, USSR, pp. 307-310 (1991).

168. P. Wanderer, et al., Effect of Prestress on Performance of A $1.8 \mathrm{~m}$ SSC RED Dipole, Supercollider 3, Proceedings of the Third International Industrial Symposium on the Super Collider, Atlanta, USA, pp. 325-333 (1991). 
169. P. Wanderer, et al., A Summary of SSC Dipole Magnet Field Quality Measurements, Supercollider 4, Proceedings of the Fourth International Industrial Symposium on the Super Collider, New Orleans, USA, pp. 137-149 (1992).

170. P. Wanderer, et al., Construction and Testing of Arc Dipoles and Quadrupoles for the Relativistic Heavy Ion Collider (RHIC) at BNL, Proceedings of the 1995 International Particle Accelerator Conference, Dallas, Texas (1995).

171. J. Wei, R. Gupta, S. Peggs, Magnetic Correction for RHIC Triplets, Proceedings of the 1993 Particle Accelerator Conference, Washington, D.C., pp. 258-260 (1993).

172. J. Wei, R. Gupta, et al., Field Quality Evaluation of the Superconducting Magnets of the Relativistic Heavy Ion Collider, Proceedings of the 1995 International Particle Accelerator Conference, Dallas, Texas (1995).

173. F. Wilczek, $10^{12}$ Degree in the Shade, The Sciences, Jan./Feb. 1994 pp. 22-30 (1994).

174. E. Willen, et al., Magnetic Properties of Isabelle Superconducting Quadrupoles, Proceedings of the 1981 Particle Accelerator Conference, Washington, D.C., pp. 3300-3302 (1981).

175. E. Willen, P. Dahl, J. Herrera, Superconducting Magnets, 1985 SLAC Summer School, AIP Conference Proceedings 153, pp. 1228-1276 (1985).

176. M.N. Wilson, Rutherford Lab. Report RHEL/M/A26.

177. M.N. Wilson, Superconducting Magnets, Oxford Science Publication, Clarendon Press, Oxford (1983).

178. A.M. Winslow, Numerical Solution of Quasilinear POISSON Equation in a Nonuniform Triangular Mesh, Journal of Comp. Physics, 2, pp. 149-172 (1967).

179. S. Wolf, Superconducting Accelerator Magnet Design, The Physics of Particle Accelerators, AIP Conference Proceedings 249, pp. 1159-1197 (1990).

180. S. Wolf, Review of Accelerator Magnet Design in the World, The Physics of Particle Accelerators, AIP Conference Proceedings 249, pp. 1389-1401 (1990). 


\section{List of Figures}

Figure 1.2.1. Phase diagram of nuclear matter and nuclear collisions. . . . . . 3

Figure 1.4.1. Drawing of the cross section of the RHIC arc dipole in

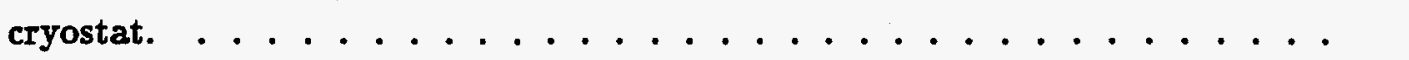

Figure 1.4.2. Critical current density as a function of applied field in superconducting wire. .................. 11

Figure 1.5.1. Computation of the field produced by a line current. . . . . . . . 33

Figure 1.5.2. Computation of the field produced by a current sheet. . . . . . . . 49

Figure 1.5.3. Computation of the field produced by a uniform current in two intersecting circles. . . . . . . . . . . . . . 58

Figure 1.5.4. Field from Beth's current sheet. . . . . . . . . . . . . . . . 64

Figure 2.2.1. Raster image of the cross section of a coil obtained after cutting an SSC R\&D dipole. ................. 73

Figure 2.2.2. Raster image of a RHIC prototype dipole yoke lamination. ...................... 76

Figure 2.3.1. AUTOMESH input No. 1 for the improved POISSON. . . . . . . 81

Figure 2.3.2. POISSON model generated by method No. 1. . . . . . . . . . . 82

Figure 2.3.3. AUTOMESH input No. 2 for the improved POISSON. . . . . . . . 85

Figure 2.3.4. POISSON model generated by method No. 2. . . . . . . . . . . 86

Figure 2.3.5. The improved mesh generated by method No. 2. . . . . . . . . 87

Figure 2.3.6. AUTOMESH input No. 3 for the improved POISSON. . . . . . . . 89

Figure 2.3.7. Improved POISSON model generated by method No. $3 . \quad$. . . . . 90

Figure 3.1.1. The B-H table used in POISSON calculations. . . . . . . . . . . 94

Figure 3.1.2. Field lines at $1000 \mathrm{~A}$ in the RHIC arc dipole magnet. . . . . . . 100

Figure 3.1.3. Field lines at $7000 \mathrm{~A}$ in the RHC arc dipole magnet. . . . . . . 101

Figure 3.2.1. POISSON model of the RHIC arc dipole magnet. . . . . . . . . 103

Figure 3.2.2. POISSON model of the SSC collider dipole magnet. . . . . . . . . 104 
Figure 3.2.3. The computed transfer function as a function of yoke inner radius in the $\mathrm{RHIC}$ arc dipole magnet. . . . . . . . . . . . 106

Figure 3.2.4. Computed saturation induced allowed harmonics as a

function of the yoke inner radius in the RHIC arc dipole magnet. . . . . . . 107

Figure 3.2.5. Computed transfer function as a function of the yoke inner radius in the SSC dipole magnet. . . . . . . . . . . . . . 108

Figure 3.2.6. Computed saturation induced allowed harmonics as a function of the yoke inner radius in the SSC dipole magnet. . . . . . . . 109

Figure 3.2.7. The computed change in transfer function and $b_{2}$ as a function of the yoke outer radius in the SSC dipole magnet. . . . . . . . . 112

Figure 3.2.8. Fringe field on the horizontal axis of the SSC dipole. . . . . . . 114

Figure 3.2.9. Fringe field on the vertical axis of the SSC collider dipole. ........................ 115

Figure 3.2.10. The saturation induced harmonics as a function of bypass hole angular location in the RHIC arc dipole. . . . . . . . . . . . 117

Figure 3.2.11. POISSON model of the RHIC insertion dipole DO. . . . . . . . 119

Figure 3.2.12. The saturation induced $b_{2}$ harmonic as a function of the bypass hole location in the RHIC DO dipole. . . . . . . . . . . . 120

Figure 3.2.13. The saturation induced $b_{4}$ and $b_{6}$ harmonics as a function of the bypass hole location in the RHIC DO dipole. . . . . . . . . . 121

Figure 3.2.14. Reduction in saturation induced harmonics by saturation suppressor holes in the RHIC arc dipole. . . . . . . . . . . . . . . 124

Figure 3.2.15. Variation in saturation induced harmonics as a function of the azimuthal location of the saturation suppressor hole. . . . . . . . . 126

Figure 3.2.16. Variation in saturation induced harmonics as a function of the radial location of the saturation suppressor hole. . . . . . . . 127

Figure 3.2.17. Variation in saturation induced harmonics as a function of the size of the saturation suppressor hole. . . . . . . . . . . 128 
Figure 3.2.18. Influence of the yoke alignment key material on the saturation induced harmonics in the SSC dipoles.

Figure 3.2.19. Influence of the yoke collaring key material on the saturation induced harmonics in the RHIC arc dipoles.

Figure 3.2.20. The saturation induced harmonics with midplane tooth and notch in the RHIC arc dipoles.

Figure 3.2.21. A schematic diagram of cutout and bump at the yoke inner radius.

Figure 3.2.22. Low field transfer function as a function of the angular positions of cutout and bump at the yoke inner radius.

Figure 3.2.23. Low field and saturation induced $b_{2}$ as a function of the angular positions of cutout and bump at the yoke inner radius.

Figure 3.2.24. Low field and saturation induced $b_{4}$ as a function of the angular positions of cutout and bump at the yoke inner radius.

Figure 3.2.25. Low field and saturation induced $b_{6}$ as a function of the angular positions of cutout and bump at the yoke inner radius.

Figure 3.2.26. POISSON model of the proposed elliptical aperture SSC dipole.

Figure 3.2.27. The saturation induced $b_{2}$ and $b_{4}$ in the proposed elliptical aperture SSC dipole.

Figure 3.2.28. The saturation induced harmonic as a function of the angular position where the yoke inner radius changes.

Figure 3.2.29. POISSON model of an octant of the RHIC insertion quadrupole.

Figure 3.2.30. Transfer function as a function of current in the one and two-radius aperture cases.

Figure 3.2.31. $\quad b_{5}$ as a function of current in the one and two-radius aperture cases.

Figure 3.2.32. $\quad b_{9}$ as a function of current in the one and two-radius aperture cases. 
Figure 3.3.1. Drawing of the coldmass of the RHIC arc dipole magnet. . . . . . 160

Figure 3.3.2. The measured $b_{2}$ harmonic as a function of current in various designs of $\mathrm{RHIC}$ arc dipole magnets. . . . . . . . . . . . 162

Figure 3.3.3. The measured $b_{4}$ harmonic as a function of current in various designs of $\mathrm{RHIC}$ arc dipole magnets. . . . . . . . . . . . 163

Figure 3.3.4. The measured $b_{6}$ harmonic as a function of current in various designs of $\mathrm{RHIC}$ arc dipole magnets. . . . . . . . . . . . . . 164

Figure 3.4.1. Drawings of the coldmass of the RHIC insertion dipole Do.

Figure 3.4.2. $b_{1}$ as a function of field when the current is the same in two side-by-side $D O$ magnets. . . . . . . . . . . . . . .

Figure 3.4.3. $\quad b_{1}$ as a function of field when the current is in the ratio of $2.5: 1$ in two side-by-side $D O$ magnets. . . . . . . . . . . . . . 173

Figure 3.4.4. Field lines in the $40 \mathrm{~mm}$ aperture, SSC, 2-in-1 magnet. . . . . . 175

Figure 3.4.5. Current dependence in $a_{1}$ in several SSC dipole magnets. . . . . 177

Figure 3.4.6. Field lines in the SSC $50 \mathrm{~mm}$ dipole magnet with asymmetric placement of coldmass in cryostat. . . . . . . . . . . . . 178

Figure 3.5.1. The axial variation in $a_{1}$ saturation in several SSC magnets.

Figure 3.5.2. Correlation between the axial variation of $a_{1}$ saturation and the top-bottom yoke-weight asymmetry in SSC dipole DCA213. . . . . . . . . . . . . . . 184

Figure 3.5.3. Correlation between geometric $a_{1}$ and saturation $a_{1}$. . . . . . 187

Figure 3.6.1. Drawing of the RHIC arc dipole coldmass asymmetrically placed inside the cryostat. . . . . . . . . . . . . . . . . 191

Figure 3.6.2. The measured current dependence of $a_{1}$ in two RHIC arc dipoles. .......................... 192

Figure 3.6.3. The correlation between local yoke weight asymmetry and local $a_{1}$ saturation in RHIC dipoles. . . . . . . . . . . . . . . 194 
Figure 3.6.4. The calculated $a_{1}$ saturation for various yoke weight asymmetries in RHIC arc dipoles.

Figure 3.6.5. Yoke weight asymmetry in various RHIC arc dipole magnet

Figure 3.6.6. The correlation in integral yoke weight asymmetry and integral $a_{1}$ saturation in two types of RHIC arc dipole designs.

Figure 3.7.1. Average values of allowed harmonics as a function of current in the production series of RHIC arc dipole magnets.

Figure 3.7.2. Magnetization inside the iron before and after yoke optimization as per PE2D model of the RHIC arc dipole magnets.

Figure 3.7.3. $\left(\frac{\mu-1}{\mu+1}\right)$ inside the iron before and after yoke optimization as per PE2D model of the RHIC arc dipole magnets. . . . . . . . . . .

Figure 4.1.1. Drawing of the coil cross section of the RHIC $100 \mathrm{~mm}$ aperture D0 Dipole.

Figure 5.2.1. Drawing of the RHIC insertion quadrupole with the locations of eight tuning shims indicated. . . . . . . . . . . . . 225

Figure 5.2.2. Detailed drawing of a tuning shim. . . . . . . . . . . . 226

Figure 5.2.3. Maximum change in the $\left(a_{2}\right.$ and $\left.b_{2}\right),\left(a_{3}\right.$ and $\left.b_{3}\right)$, and $\left(a_{4}\right.$ and $b_{4}$ ) harmonics from a given thickness of eight tuning shims.

Figure 5.2.4. Maximum change in the $a_{5}$ and $b_{5}$ harmonics from a given thickness of eight tuning shims.

Figure 5.2.5. Maximum change in the $a_{9}$ and $b_{9}$ harmonics from a given thickness of eight tuning shims.

Figure 5.2.6. Current dependence in the skew harmonics generated by the tuning shims. . . . . . . . . . . . . . . . . . 246

Figure 5.2.7. Current dependence in the normal harmonics generated by the tuning shims. . . . . . . . . . . . . . . 247

Figure 5.2.8. Comparison between calculations and measurements for the $a_{2}, b_{2}, a_{3}, b_{3}, a_{4}, b_{4}$ harmonics generated by the tuning shims. 
Figure 5.2.9. Comparison between calculations and measurements for the $a_{6}, b_{6}, a_{7}, b_{7}, a_{8}, b_{8}$ harmonics generated by the tuning shims.

Figure 5.2.10. Comparison between calculations and measurements for the transfer function and the $a_{5}, b_{5}, a_{9}, b_{9}$ harmonics generated by the tuning shims. .................... 254

Figure 5.2.11. Beam size in the insertion region for $\beta^{*}=1 . \ldots \ldots$

Figure 5.3.1. Conceptual diagram for correcting $a_{1}$ and transfer function errors in dipole ends. . . . . . . . . . . . . . . 259

Figure 6.2.1. Drawing of the collared coil cross section of the SSC dipole. ...................... 268

Figure 6.2.2. Drawing of the coldmass of the SSC dipole. . . . . . . . . . 272

Figure 6.2.3. Field lines in the SSC $50 \mathrm{~mm}$ dipole at $6500 \mathrm{~A}$. . . . . . . . . 273

Figure 6.2.4. Current dependence in the allowed harmonics of the SSC

dipole. ..................... 277

Figure 6.2.5. Lorentz forces in the SSC dipole. . . . . . . . . . . . . . . . 284

Figure 6.3.1. Drawing of the RHIC insertion quadrupole magnet cross section. ..................... 287

Figure 6.3.2. Drawing of the coil cross section for the RHIC insertion quadrupole. ..................... 291

Figure 6.3.3. POISSON model of a quadrant of the $130 \mathrm{~mm}$ aperture RHIC insertion quadrupole. . . . . . . . . . . . . . . 294

Figure 6.3.4. Computed harmonics in the $130 \mathrm{~mm}$ aperture RHIC insertion quadrupole. . . . . . . . . . . . . . . . 297

Figure 6.3.5. Field lines in the RHIC insertion quadrupole. . . . . . . . . . . 298 


\section{List of Tables}

Table 1.3.1. Major parameters of the RHIC machine. . . . . . . . . . . 6

Table 1.4.1. Specifications of the superconducting cable for the RHC arc dipole and quadrupole magnets. . . . . . . . . . . . . . . 12

Table 1.4.2. Measured normal harmonics in the RHIC arc dipole mag-

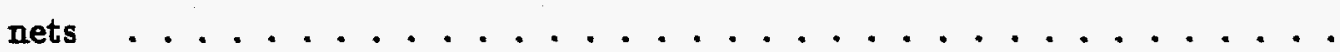

Table 1.4.3. Measured skew harmonics in the RHIC arc dipole mag-

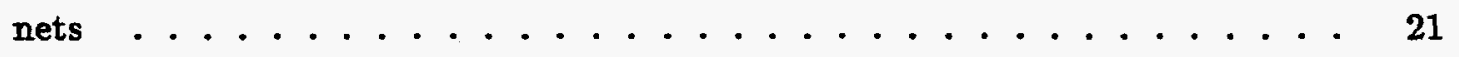

Table 3.1.1. The B-H table used in the POISSON calculations. . . . . . . . . 95

Table 3.2.1. Computed transfer function as a function of the yoke inner radius in the $\mathrm{RHIC}$ arc dipole magnet. . . . . . . . . . . . . 108

Table 3.2.2. Computed saturation induced allowed harmonics as a function of the yoke inner radius in the RHIC arc dipole magnet. . . . . . . 108

Table 3.2.3. The computed change in transfer function, $b_{2}$ and $b_{4}$ as a function of the yoke outer radius in the SSC dipole magnet. . . . . . . 111

Table 3.2.4. Computed fringe field at $6500 \mathrm{~A}$ in the SSC dipole magnet.

Table 3.2.5. The saturation induced harmonics as a function of the bypass hole angular location in the RHIC arc dipole. . . . . . . . . . . 118

Table 3.2.6. The saturation induced harmonics as a function of the bypass hole location in the RHIC DO dipole. . . . . . . . . . . . 122

Table 3.2.7. The saturation induced harmonics as a function of various parameters of the saturation suppressor hole. . . . . . . . . . 125

Table 3.2.8. The saturation induced harmonics in the SSC dipoles when the yoke alignment keys are made of stainless steel. . . . . . . . . 131

Table 3.2.9. The saturation induced harmonics in the RHIC arc dipoles when the yoke alignment keys are made of iron.

Table 3.2.10. The saturation induced harmonics in the RHIC arc dipoles when the yoke collaring keys are made of stainless steel. 
Table 3.2.11. The saturation induced harmonics in the RHIC arc dipoles when the yoke collaring keys are made of iron. . . . . . . . . . 133

Table 3.2.12. The saturation induced harmonics with a midplane notch in the $\mathrm{RHIC}$ arc dipoles. . . . . . . . . . . . . . . . . 138

Table 3.2.13. The saturation induced harmonics with a midplane tooth in the $\mathrm{RHIC}$ arc dipoles. . . . . . . . . . . . . . . . 138

Table 3.2.14. The saturation induced harmonics in the proposed elliptical aperture SSC dipole. . . . . . . . . . . . . . . . . . 148

Table 3.2.15. The saturation induced harmonic as a function of angular position at which the yoke inner radius changes. . . . . . . . . 150

Table 3.2.16. Current dependence in harmonics with a yoke inner radius of $87 \mathrm{~mm} . \ldots \ldots . . \ldots \ldots 7$

Table 3.2.17. Current dependence in harmonics with a yoke inner radius of $92 \mathrm{~mm} . . \ldots \ldots 157$

Table 3.2.18. Current dependence in harmonics with a yoke inner surface defined by two radii. . . . . . . . . . . . . . 158

Table 3.3.1. Measured and computed $b_{2}$ and $b_{4}$ as a function of current in various designs of $\mathrm{RHIC}$ arc dipoles. . . . . . . . . . . . . 166

Table 3.5.1. The $a_{1}$ harmonic in several SSC dipoles. . . . . . . . . . . . . 186

Table 4.3.1. Harmonics generated by a $25 \mu m$ change in wedge thickness in the SSC dipole. . . . . . . . . . . . . . . . . 211

Table 4.3.2. Harmonics generated by a $25 \mu \mathrm{m}$ change in wedge thickness in the RHIC arc dipole. . . . . . . . . . . . . . . . . . . 211

Table 4.4.1. Computed and measured harmonics due to a change in coil midplane gap in the RHIC arc dipole. . . . . . . . . . . . . . . 213

Table 4.5.1. Computed and measured harmonics caused by an asymmetric change in coil midplane gap in a $\mathrm{RHIC}$ insertion quadrupole.

Table 4.6.1. A summary of iterations in RHIC insertion quadrupoles without a change in any wedge. 
Table 5.2.1. Harmonics from $1.52 \mathrm{~mm}$ thick iron tuning shims. . . . . . . . . . 234

Table 5.2.2. Harmonics from $3.05 \mathrm{~mm}$ thick iron tuning shims. . . . . . . . . 235

Table 5.2.3. Harmonics from $4.57 \mathrm{~mm}$ thick iron tuning shims. . . . . . . . . 236

Table 5.2.4. Harmonics from $6.10 \mathrm{~mm}$ thick iron tuning shims. . . . . . . . . 237

Table 5.2.5. Maximum change in harmonics from a given thickness of

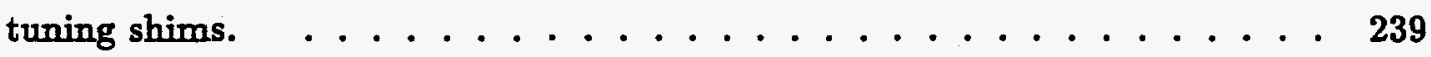

Table 5.2.6. Current dependence in skew harmonics generated by the tuning shims. ................... 244

Table 5.2.7. Current dependence in normal harmonics generated by the tuning shims. . . . . . . . . . . . . . . . 245

Table 5.2.8. Maximum low and high field harmonic correction from the tuning shims. .................... 248

Table 6.2.1. Cable used in SSC $50 \mathrm{~mm}$ dipoles. . . . . . . . . . . . . . 267

Table 6.2.2. Low field harmonics in the SSC dipoles. . . . . . . . . . . . 270

Table 6.2.3. Computed current dependence in transfer function and $b_{2}$ in the SSC dipoles. . . . . . . . . . . . . . . . 274

Table 6.2.4. Maximum change in harmonics due to yoke saturation in the SSC dipoles. . . . . . . . . . . . . . . . . 275

Table 6.2.5. POISSON calculations of the BNL built SSC dipole. . . . . . . 276

Table 6.2.6. Peak field in the coil of the SSC dipole. . . . . . . . . . . 278

Table 6.2.7. Expected quench performance of the SSC dipole. . . . . . . . 279

Table 6.2.8. Allowed harmonics due to $0.05 \mathrm{~mm}$ errors in the SSC dipole. ........................ 281

Table 6.2.9. Stored Energy and Inductance in the SSC dipole. . . . . . . . . 282

Table 6.2.10. Summary of the SSC dipole coil cross section. . . . . . . . . . . 285

Table 6.2.11. Summary of changes in the SSC dipole due to saturation. . . . . . . . . . . . . . . . . . . 285 
Table 6.3.1. Summary of the RHIC insertion quadrupole design parameters.

Table 6.3.2. Coil design for the RHC insertion quadrupoles. . . . . . . . . . 289

Table 6.3.3. Computed harmonics in the RHIC insertion quadrupole. . . . . . . 295

Table 6.3.4. Computed harmonics with two RHIC insertion Q1 quadrupoles at minimum separation. . . . . . . . . . . . . 299

Table 6.3.5. Peak field in the RHIC insertion quadrupole. . . . . . . . . . . 300 